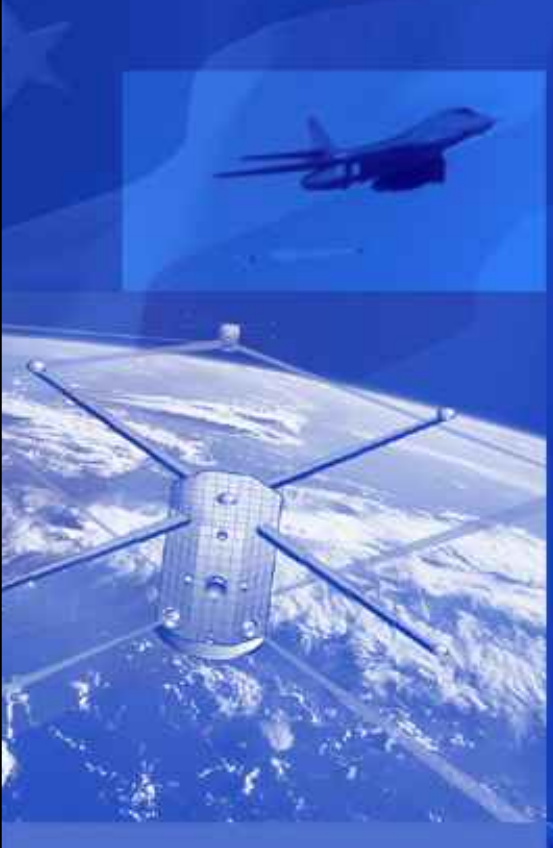

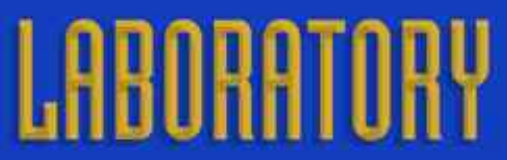

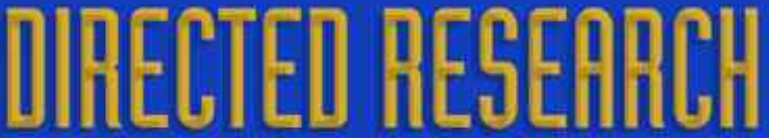

\section{AND DEVELLOPMENT}

Science and Technology Mission Technologies

Grand Challenges
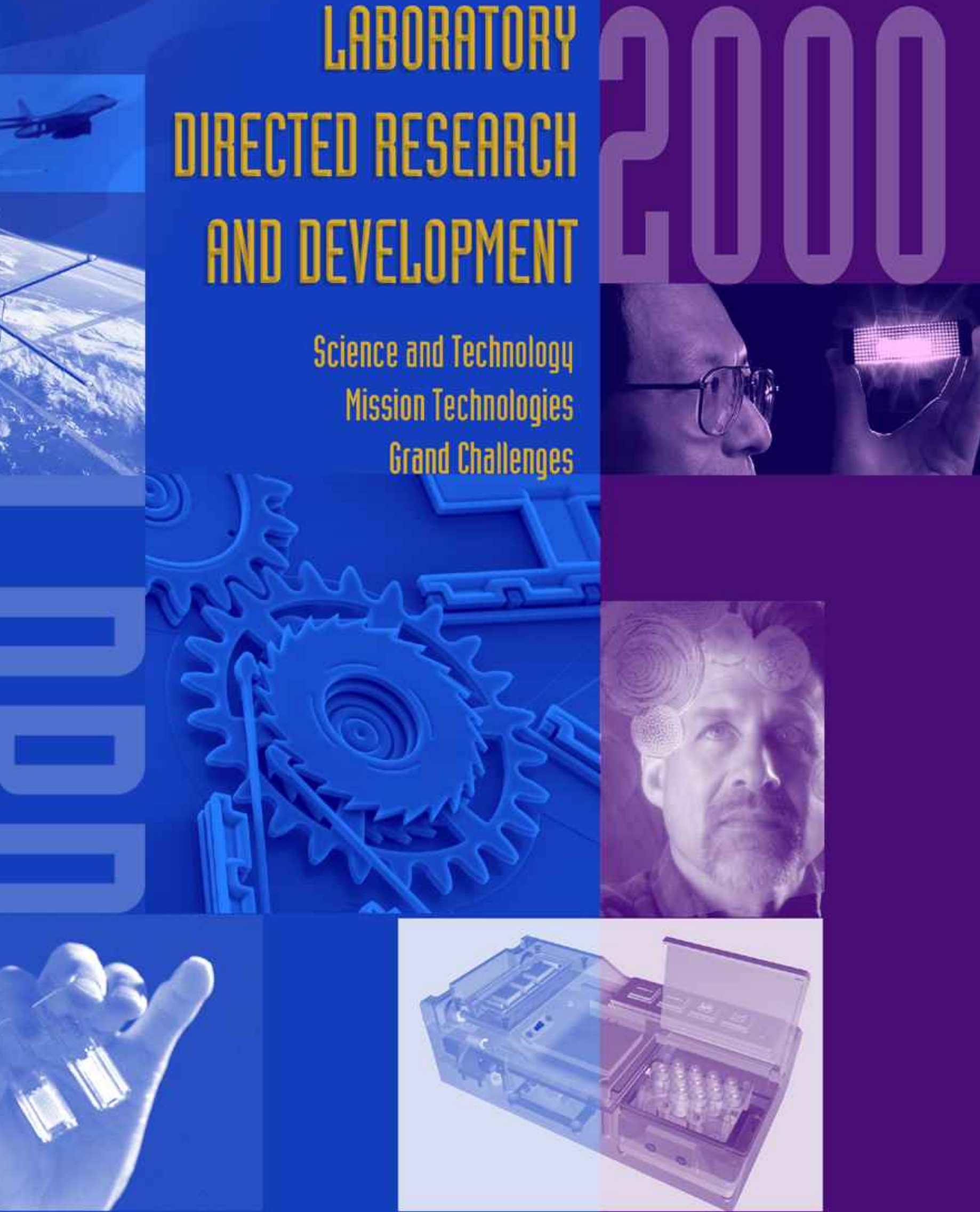

Sandia

National

Laboratories 


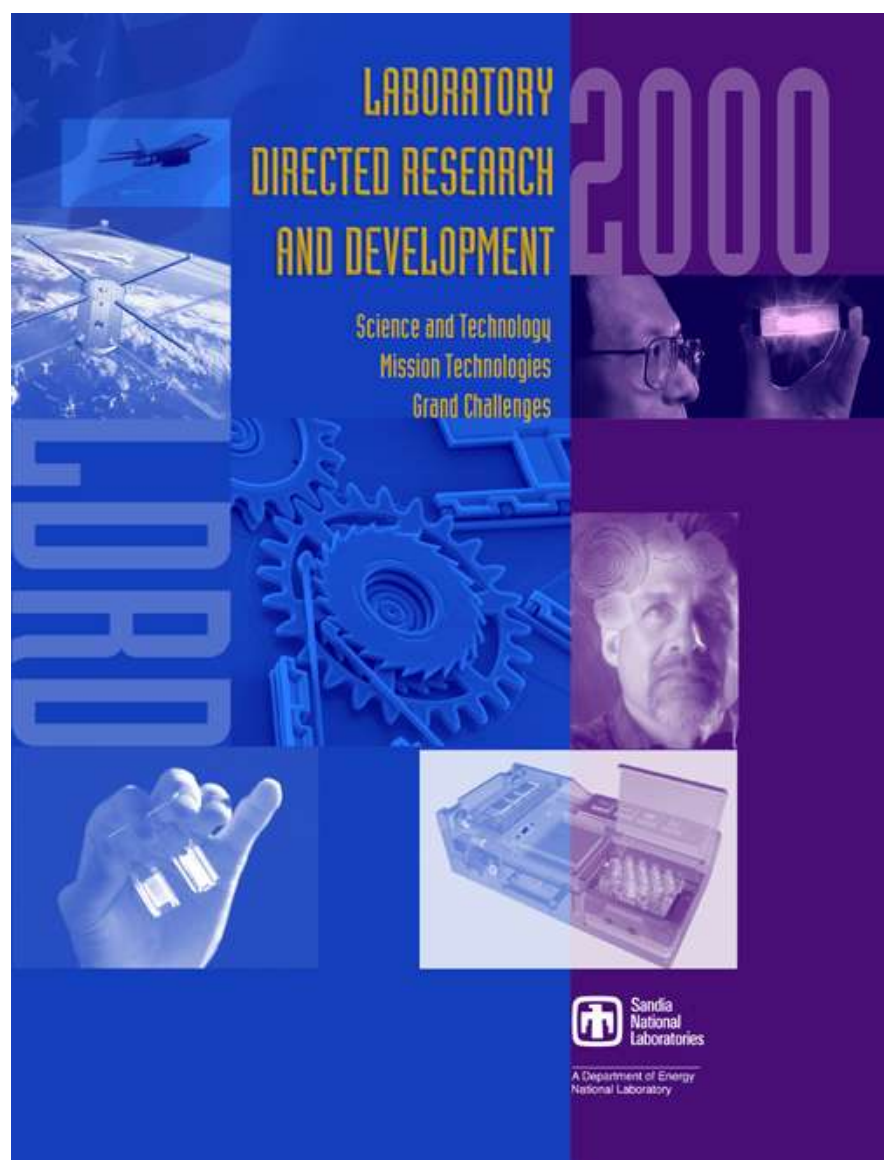

Cover (Above)

The LDRD Program supports the DOE's and the Laboratories' missions through Sandia's four primary strategic objectives: nuclear weapons, nonproliferation and materials control, energy and critical infrastructure, and emerging national security threats. To meet these objectives, LDRD promotes creative and innovative research and development that Labs Director Ambassador C.

Paul Robinson describes as "... the lifeblood of the Laboratories." Over $90 \%$ of all Sandia National Laboratories' LDRD projects provide both direct and indirect benefit to the national security and weapons missions of the Laboratories and the DOE.

The Science and Technology (S\&T) Research Foundations category prioritizes investigations to ensure overall relevance to the Labs' nuclear weapons mission while building upon anticipated future directions of the Laboratories. LDRD is a key component of the weapons research effort that enables Sandia's continuing ability to meet its commitments in surety, microsystems, manufacturing, radiation physics, and neutron generators research and development.

In addition, LDRD's research investigations also promote innovation aligned with the nonweapons missions of the Laboratories. These Roadmap Technologies research category investments derive their scientific and technology basis from the S\&T competencies, are strategically important for future Sandia national security missions needs, and represent a spectrum of research leading to development and application.

\section{Abstract}

This report summarizes progress from the Laboratory Directed Research and Development (LDRD) program during fiscal year 2000. In addition to a programmatic and financial overview, the report includes progress reports from 244 individual R\&D projects in 13 categories.

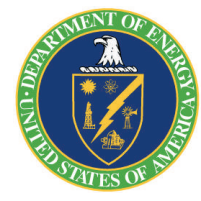

This work was supported by the United States Department of Energy under Contract DE-AC04-94-AL85000.

Sandia is a multiprogram laboratory operated by Sandia Corporation, a Lockheed Martin Company, for the United States Department of Energy.

SAND 2001-1126

March, 2001

LDRD Annual Report Staff:
Chuck Meyers Cynthia Harvey Donna Chavez Bryon Cloer

Carol Whiddon Joy Bemesderfer Donna Drayer Douglas Prout 


\section{Table of Contents}

12 “...exceptional service in the national interest."

13 Laboratory Directed Research and Development (LDRD) Program Overview

\section{Materials Science \& Technology}

32 Functional Materials for Microsystems: Smart Self-Assembled Photochromic Films

34 Innovative Experimental and Computational Diagnostics for Monitoring Corrosion in Weapons Environments

37 Self-Healing Molecular Assemblies for Control of Friction and Adhesion in MEMS

39 Linking Atomistic Computations with PhaseField Modeling

41 A Combinatorial Microlab Investigation of Critical Copper-Corrosion Mechanisms

43 Self-Assembled Templates for Fabricating Novel Nanoarrays and Controlling Materials Growth

45 Wetting and Spreading Dynamics of Solder and Braze Alloys

48 Improved Materials-Aging Diagnostics and Mechanisms Through 2-D Hyperspectral Imaging Methods and Algorithms

52 Microscale Shock-Wave Physics Using Photonic Driver Techniques

$54 \quad$ Reactivity of Metal-Oxide Surfaces

57 Exploiting LENS ${ }^{\text {TM }}$ Technology Through Novel Materials

60 Molecular Characterization of EnergeticMaterial Initiation
63 Nonvolatile Protonic Memory

66 Next-Generation Output-Based Process Control: An Integration of Modeling, Sensors, and Intelligent Data Analysis

68 Functional Materials for Electrochemomechanical Actuation of Microvalves and Micropumps

70 Making the Connection Between Microstructure and Mechanics

72 Switchable Hydrophobic-Hydrophilic Surfaces

$74 \quad$ Nanostructured Materials Integrated in Microfabricated Optical Devices

76 All-Ceramic Thin-Film Battery

\section{Computer Science}

79 Heterogeneous Simulation

81 Volumetric Video Motion Sensing for Unobtrusive Human-Computer Interactions

83 Hybrid Sparse-Dense Incomplete Factorization Preconditioners

85 Advanced Large-Eddy Simulation Algorithms for Coupled-Flow Physics and Complex Geometry

87 Molecular Simulation of Reacting Systems

89 Massively Parallel Global Climate Model for Paleoclimate Applications

91 From Atom-Picoseconds to Centimeter-Years in Simulation and Experiment

93 Parallel Combinatorial Optimization for Scheduling Problems 


\section{Table of Contents}

96 Multilevel Techniques for Unstructured Grid Problems on Massively Parallel Computers

99 Visual Explanation and Insight

102 Emergent Behavior of Large Swarms of Intelligent Agents

104 Algorithmic Advances in Computational Structural Biology

106 Predicting Function of Biological Macromolecules

108 Large-Scale Nonlinear Optimization Arising from PDE Models

110 Parallel Methods for Coupling Circuit- and Device-Scale Simulations

112 A Java-Based Tool for Multifidelity Modeling of Coupled Systems

114 Gamma-Ray Bursts and the Particle Mass Scale

\section{Electronics \& Photonics}

117 Monolithic Integration of VCSELs and Detectors for Microsystems

119 Post-Processed Integrated Microsystems

122 Development of Radiation-Hard Sensors for Space-Based Visible and Infrared Sensing Applications

124 Silicon Three-Dimensional Photonic Crystal and Its Applications

126 Monolithic Micromachined Variable Tuners for Rapid Prototyping and Optimization of Microwave Circuits
129 Quantum Tunneling Transistors for Practical Applications

131 Development of Magnetically Excited Flexural Plate-Wave Devices for Implementation as Physical, Chemical, and Acoustic Sensors, and as Integrated Micropumps for Sensored Systems

134 Novel Acoustically Driven Microoptoelectronic Devices

136 Photonics Integration Devices and Technologies

139 Stress-Free Amorphous Diamond for HighSensitivity Microsensors with Integrated Microstructures

$142 \mathrm{AlGaN}$ Materials Engineering for Integrated Multifunction Systems

144 Semiconductor Current Filament Lasers

147 SOI-Based High-Aspect-Ratio Si Bulk Micromachining for MEMS Applications

150 Defining the Frontiers of Vertical, ExternalCavity, Surface-Emitting Lasers

153 Photoresist Technology for $70 \mathrm{~nm}$ Lithography with $157 \mathrm{~nm}$ Radiation

155 Growth and Characterization of Quantum Dots and Quantum Dot Devices

$158 \mathrm{THz}$ Technologies for Ultrahigh-Data-Rate Communications

160 New Architectures for Micro-Total-Analytical Systems

162 Heterogeneous Integration of Optoelectronic Arrays and Microelectronics 


\section{Table of Contents}

164 Biosensors Based on the Electrical Impedance of Tethered Lipid Bilayers on Planar Electrodes

166 Miniature Sensors for BW Agents Using FattyAcid Profiles

168 Enhanced-Sensitivity Acoustic-Wave Biosensor Arrays

171 High-Al-Content AlGaInN Devices for NextGeneration Electronic and Optoelectronic Applications

173 Microfabrication of Electromagnetic Devices

175 GaAs MOEMS Technology

178 Integrated Microsensors for Autonomous Microrobots

180 Silicon-Integrated Planar Microbatteries

182 High-Efficiency Optical MEMS by the Integration of Photonic Lattices with Surface MEMS

\section{Engineering Modeling \& Simulation}

185 Development of In Situ Diagnostics for Simultaneous Measurement of Transient Gas Species and Soot in Large Fires

188 Structural Simulations Using Multiresolution Material Models

190 Mechanisms of Adiabatic Shear Failure

192 Evolvable Hardware

194 Crack Nucleation and Growth: Combining Validated Atomistic and Continuum Modeling
198 Innovative Measurement Diagnostics for Fluid/ Solid and Fluid/Fluid Interactions in Rotating Flowfields

201 High-Resolution Modeling of Multiscale Transient Phenomena in Turbulent Boundary Layers

203 Dispersive Measurements of Velocity in Heterogeneous Materials

206 A Physically Based Computational Method for Predicting Generalized Fracture

209 Micromechanical Failure Analyses for FiniteElement Polymer Modeling

\section{Advanced Manufacturing}

212 Microdiagnostic MEMS Lab-on-a-Chip

214 Real-Time Error Correction Using Electromagnetic-Bearing Spindles

216 Thin-Film Deposition Processes Incorporating In Situ Monitoring Capabilities

218 Solid-State Neutron Generator for Use in Nuclear Weapons

220 Scripting for Video Inspection

223 Advanced Machining Processes for Microfabrication

225 Advanced Production Planning Models

228 Volumetric Displacement Control (VDC) of Manufacturing Tools

230 Process-Based Quality Tools to Verify Cleaning and Surface Preparation 


\section{Table of Contents}

232 Fabrication of Three-Dimensional

Microstructures Using Soft Lithography

234 Automatic Design of Practical Fixtures

236 IMEMS Packaging and Interconnection

Technology

238 Microreplication: Precision Metal Parts from Electroformed Master Molds

240 Assembly of LIGA Using Electric Fields

241 Computer Numerically Controlled

Micromachines

244 Electromicrofluidic Packaging

\section{Information Systems \& Technology}

247 Weighted-Nearest-Neighbor (WNN) Decision Making for Data Mining

249 Varying QoS for Fixed and Mobile Networks

251 A Real-Time Decision-Support Framework to Guide Facility Response to Abnormal Events

253 Physical Model-Based Fusion of Sensor Array Data

255 Large-Scale Distributed Information System Modeling and Simulation

258 High-Surety SCADA for the Critical Energy Infrastructures

260 Agent-Based Mediation and Cooperative Information Systems

262 Dynamically Self-Configurable Network Protocol
264 Optical Backplane/Interconnect for Super-HighSpeed Communication

266 PUSH Technology Demonstration

268 Controlling Information: Its Flow, Fusion, and Coordination

271 Low-Power, Reduced-Computation, Public-Key Protocols

273 Ten-to-One-Hundred-Gigabit/Second NetworkEnabling R\&D

276 Intrusion Detection for Asynchronous Transfer Mode (ATM) Networks

278 Conversational Policies for Multiagent Systems

280 Fuzzy Data Mining

\section{Directed Energy}

283 Surface Decontamination of Bacterial Protein Toxins by RF Power

284 Intense White-Light Pulse Propagation in Air Using Self-Guided Optical Filamentation: Applications to Remote Sensing and Countermeasures

286 HPM Vulnerability Assessment and Tests

288 Proton Beam Directed-Energy Weapon

290 Intense Directed-Energy Theory Initiative

\section{Sensing \& Intelligent Controls}

293 Miniature Bioaerosol Concentrator 


\section{Table of Contents}

295 Recognizing Partially Obscured Targets by Combining Multiple Data Sources Using Evidential Reasoning

298 Computational Engineering of Sensor Materials and Integration with a Novel Biological Weapon Detection System

300 Biological Weapon Detector Using Bioaffinity Array Impedance Analysis with Chemical Amplification Through Redox Recycling

302 ATR / Exploitation Utilizing UltrahighResolution, Complex SAR Imaging

304 Thin-Skin Deployable Mirrors for Remote Sensing Systems

307 Dispersible Granular Sensor (Smart Sand) for Landmine Detection Based on TNT Immunoassay

309 Characterization of Underground Facilities in an Urban Environment

311 Dexterous Robotic Manipulation of Hazardous Materials in Unstructured Environments

313 Autonomous Dynamic Soaring Platform for Distributed Mobile Sensor Arrays

315 Miniature UV Fluorescence-Based Biological Agent Sensor

317 Enabling Human Skills with Cooperative Automation

319 Cloud to CAD

321 Sparse Geophysical Networks for Monitoring Deep Targets

323 High-Speed 2-D Hadamard Transform Spectral Imager for IR Applications
325 Micro-High-G Acceleration Recorder

327 Dynamic Range Imaging for Terrain Mapping, Position Determination, and Obstacle Avoidance in Autonomous Navigation

329 Induced Molecular Markers for Pathogen Detection: Microincubators for Rapid Toxin Expression

331 Distributed Reconfigurable Homogeneous Microrobotic Systems

334 Robust Planning for Autonomous Navigation of Mobile Robots in Unstructured, Dynamic Environments

\section{Energy \& Power Systems}

337 Adaptive 3-D Sensing

339 Designed Ionophores for Liquid-Membrane Separation and Extraction of Metal Ions

343 An Electromagnetic Imaging System for Environmental Site Reconnaissance

345 Advanced Geosphere Transport Simulation

348 A Novel Microcombustor for Sensor and Thermal-Energy Management Applications in Microsystems

350 A Microgas Chromatograph (mGC)-Based Controller for Energy-Intensive Processes

352 A MEMS Microelectric Generator

354 Nanostructured Silicon Surfaces for CostEffective Photovoltaic Efficiency Improvements

357 Control Strategies for Homogeneous ChargeCompression Ignition (HCCI) Engines 


\section{Table of Contents}

359 Silicon/Carbon Nanocomposites for

Rechargeable Battery Applications

\section{Surety Science}

362 Hybrid Processing of Measurable and

Subjective Information in Surety Analysis

364 Approximate Public-Key Authentication with Information Hiding

366 New Network Analysis Approaches to Evaluate Infrastructure Risk and Reliability

369 Improved Tools for Identifying and Quantifying Potentially Dangerous Human Actions

371 Advanced Signal Processing for Thermal Flaw Detection

374 Production Surety and Disruption Vulnerability Analysis

376 An Optically Triggered Semiconductor Switch for Firing Systems

378 Source Code Assurance Tool

380 Physical Models for Predicting the Effect of Atmospheric Corrosion on Microelectronic Reliability

383 Security of Bulk Power Systems

385 A Massively Parallel Microsimulation Model of Infrastructure Interdependency

388 Network Intrusion Detection Using Adaptive Critic Neural Networks

390 Key Management Techniques for Authenticating Highly Secure SCADA Systems in the Electric Power Industry
392 Self-Assembled Tamper-Detection Seals

395 Virtual Reliability Realization System

397 Ultrahigh-Resolution Radiography for Detailed Inspection of Weapon Components and Systems

\section{Grand Challenges}

401 Engineering Complex Distributed Systems

403 Information Collection

404 Open Access (Nanosat)

409 Autonomous MicroChem Laboratory $\left(\mu \mathrm{ChemLab}{ }^{\mathrm{TM}}\right)$

412 Molecular Integrated Microsystems (MIMS)

\section{Corporate Objectives}

416 Chemiresistors Based on Metal-Loaded

Polymers for Solvent Spill Detection

418 Advance Neutron-Tube Design and Producibility

420 Surface Hardening by Nanoparticle Precipitation and Atomic Clustering in $\mathrm{Ni}(\mathrm{Al}, \mathrm{O})$

422 Dynamical Properties of Polymers: Computational Modeling

423 Broadening Mechanism in 2-D Excitonic and Electron Gases

425 Direct Fabrication of Multifunctional Nanocomposites Via Supramolecular SelfAssembly 


\section{Table of Contents}

428 Information Extraction from Hyperspectral Images Obtained from Satellites

430 Advanced Radiation Sources: Rayleigh-Taylor Mitigation Via Perturbation Reduction

433 Capillary Elastohydrodynamics in Manufacturing Processes

436 Real-Time Design of Improved PowderPressing Dies Using Finite-Element Method Modeling

439 Calculation and Interpretation of the Energies that Underlie Transition-Metal Surface Structure

441 Interfacial Reactions in Ceramic Systems

443 Poco Switch Tubes

444 Ultraminiaturization of RF Circuitry

446 A Stronglink Prime Mover Based on an Electrokinetic Pump

448 Laser-Assisted Microgas Metal Arc Welding

450 Surface-Micromachined Mechanical Timer

453 Pseudostationary Separation Materials for Highly Parallel Microseparations

455 Novel Coatings for Microelectromechanical (MEM) Devices

458 Investigation of the Feasibility of RTL Concept for Fusion Energy

460 Rapid Discovery of Materials for Energy Systems

462 In Situ Formation of Reactive Barriers for Radionuclide, Heavy Metal, and Organic Stabilization
464 MPP Direct Numerical Simulation of Diesel Autoignition

466 A Functional Parallel Discrete-Event Simulation of a Snoopy Coprocessor Software Monitor

468 New Complexing Agent for Co(II) Analysis

470 Infrared Polarization for Target Identification

472 Characterization of Polymer-Metal Interface Properties for LIGA-Based Microsystem Technologies

474 Fault-Tolerant Computing Services for Heterogeneous Clusters

476 DNA Microarray Technology

479 Shock Response of Diamond Crystals

480 The Use of Active Fiber Composites for the Health Monitoring of Wind Turbine Blades

482 Information-Sharing Security Module

484 Adaptive Sensor Optimization and Cognitive Image Processing Using Autonomous Optical Neuroprocessors

486 Integration of Sandia Microsensors to the Internet for Logistics Applications

488 Automatic Target Recognition in Three Dimensions

490 Modeling the Jini Infrastructure Protocol

492 Intelligent Targeting for Remote Weapons

494 Microfuel Reformer: Hydrogen for Microfuel Cells

497 Advanced Techniques for Real-Time Visualization of Data-Intensive Missions 


\section{Table of Contents}

499 Early Diagnosis of Human Exposure to Biological Agents

502 Multisensor Fusion for Characterizing Underground Facilities (UGFs)

504 Developing Land Surface-Temperature Maps from Multispectral Thermal Imager (MTI) Data

506 Enterprise Modeling of Medical Surveillance Network

508 The Thermal History of Charred Materials by Raman Spectroscopy

510 Optical Interconnections to Focal Plane Arrays

512 Change Detection in Flash Memory

514 Study of Effectiveness of Spectro-Polarization Parameters in Military Target Detection

516 Damaged Chip

519 Engineered Window Glass for Architectural Surety Applications

521 Layered Pyrotechnic Smoke Materials for Barriers

523 Miniaturized Flying Penetrator

525 Preconcentration of Volatile Chemical Agents and Explosives

527 Accelerated Beta Decay for the Reduction of Legacy Wastes

529 Kinetic-Energy Lightning Generator

531 Comprehensive Representation of Human Decision Process: Sensor Shooter Problem

533 Spectroscopic Detection of Pathogens
534 Technical Solutions to National Security Issues Based on Limited Natural Resources

535 Sustainable Bioelectronic Microsystems: Life on a Chip

537 Near-Real-Time Surveillance Against Bioterror Attack

539 Investigation of Modeling Approaches for Nuclear Futures

541 Understanding and Managing Threats to the Social Fabric of the United States

543 Physical and Functional Modeling of a Bioelectronic Microsystem

544 Analytic Models for Cooperative Utilization of Critical Resources

546 Cryopreservation of Biological Tissue

548 Investigation of Nanoscience Technologies

549 Magnetic Polysilicon MEMS Devices

551 Autonomous Microexplosive Subsurface Tracing System

553 New Metal-Organic Chemical Vapor Deposition Reactor Designs and Optimization

555 Composite-Wire Plasma Formation and Evolution

557 Scattering from Nanostructured Materials

559 The Liquid-Crystal Physics of EvaporationInduced Self-Assembly

560 Electrochemical Interrogation of Cellular Membrane Transport Processes

562 A Micromethanol Steam Reformer for a Hydrogen-Based Microfuel Cell 


\section{Table of Contents}

564 Spatial Anomalies in Magnetically Insulated Vacuum Structures

Physical Phenomena Underpinning Simulation

567 Radiation-Induced Prompt Photocurrents in Microelectronics: Physics

569 Physical Basis for Interfacial TractionSeparation Models

571 Diagnostics for Joining Solidification/ Microstructural Simulations

574 Effects of Microstructural Variables on the Shock-Wave Response of PZT 95/5

577 Mechanisms of Dislocation-Grain-Boundary Interaction

579 Energetic-Material Burn and Detonation at the Mesoscale

581 Small-Scale Multiaxial Deformation Experiments on Solder for High-Fidelity Model Development

583 Appendix A Project Number/Title Index

593 Appendix B Awards/Recognition List

595 Appendix C Project PerformanceMeasures

609 Appendix D DOE Critical Technologies

623 Appendix E Major National Programs

637 Appendix F Dual-Benefit Areas and Single-Area Categories 
“...exceptional service in the national interest."

Science and technology are at the heart of United States industrial competitiveness, national security, energy resources, environmental quality, and leadership in fundamental and applied science.

At Sandia, the Laboratory Directed Research and Development Program provides the knowledge that drives our future. We initiate research and development that spawn the knowledge that revolutionized technology. Areas of emphasis center on our core technical competencies and the major strategic thrusts of Sandia's Institutional Plan. Leading-edge experiments that validate our work are constructed and operated on schedule, within budget, and in a safe and environmentally responsible manner.

Our work continues to produce many scientific and technological breakthroughs that lead to new technologies, markets, and businesses for the nation.

To all those who have contributed so generously of their time and talent, thanks and congratulations for a job well done. 


\section{Laboratory Directed Research and Development (LDRD) Program Overview}

\section{B. K. Cloer}

“Sandia's nuclear weapons mission requires extraordinarily strong science and technology... research and development has strengthened Sandia's ability to fulfill its mission for the DOE."

Ambassador C. Paul Robinson President, Sandia National Laboratories Sandia National Laboratories LDRD FY 2001

Program Review, September 2000

"The LDRD Program is designed and managed to assure that the right science and the right technology are available to provide the differentiating strengths for meeting the Labs' mission needs."

Al D. Romig

Vice President for the Science and Technology Stragetic Management Unit (SMU), Sandia National Laboratories Sandia National Laboratories LDRD FY 2001

Program Review, September 2000

\section{Program Strategy}

Sandia National Laboratories is a national resource that provides world-class science, technology, and engineering. The Laboratories' capabilities must remain on the cutting edge since the safety, security, and reliability of the U.S. nuclear weapons stockpile depends directly on them.

Sandia's LDRD Program supports the DOE's missions through Sandia's four primary strategic objectives: nuclear weapons, nonproliferation and materials control, energy and critical infrastructure, and emerging national security threats. To meet these objectives, LDRD promotes creative and innovative research and development that Labs' Director Ambassador C. Paul Robinson says “... has significantly strengthened Sandia's ability to fulfill its mission for the DOE."

\section{FY 2000 Funding levels (\$M)}

\section{$\$ 27.3$}

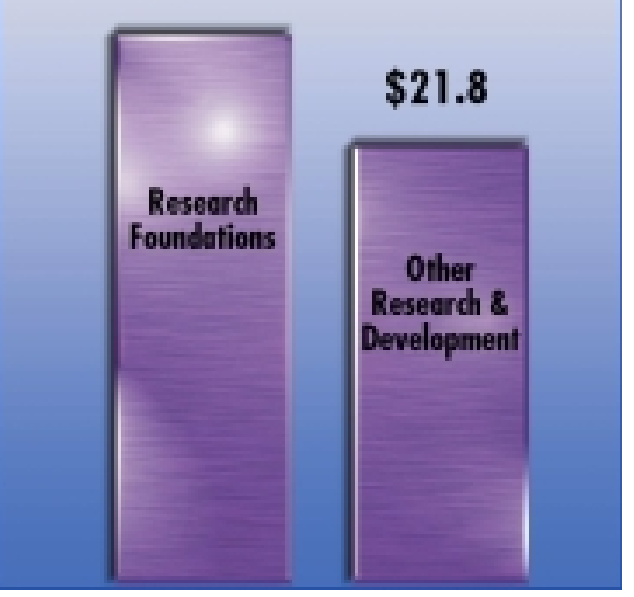

FY 2000 Selected Statistics

Full-time Stoff 239

Smallest Project (SK) 3

Average Project (\$K) 199

Averoge Project (FTE) 0.97

Lorgest Project [\$M] 2.47

Ist Year Projects

2nd Year Projects

3rd Yeor Projects

4th Year Projects

\section{FY 2000 \\ Performance}

Refereed Publicotions

Other Communications

Potent Disclosures

Potent Applications

Potents 
Sandia's LDRD Program provides the flexibility to invest in long-term, high-risk research activities that attract the best research talent from across the Laboratories. LDRD research provides an opportunity for this talent to explore innovative scientific and technological opportunities that hold high potential for payoff in future applications. As a result, LDRD advances strengthen Sandia's science and technology base and provide considerable support to DOE and Laboratories' mission needs.

Authorized by federal law and implemented by DOE under DOE Order 413.2, LDRD is Sandia's sole source of discretionary research funds. In FY 2000, congressional appropriations capped the LDRD Program at four percent of operating budget and eliminated the assessment on environmental management funds. This FY 2000 LDRD Program reduction resulted in cancellation of some research projects and the scaling back of others, thus reducing the breadth and depth of fundamental and exploratory science that supports our Labs' missions. In addition, the FY 2000 LDRD Program reduction had a large negative impact on the morale of the LDRD participants. Stable research funding at a sufficient level is essential to support and strengthen Sandia's mission responsibilities.

\section{Program Performance}

The FY 2000 LDRD Program funded 245 projects (selected from 1116 possibilities, including ideas and continuations) with a budget of $\$ 50.98$ million. The program was divided into five major investment categories (including subdivisions):

- Research Foundations (Computer Sciences, Electronics and Photonics, Engineering Sciences Modeling and Simulation, Materials Science and Technology)

- Roadmap Technologies (Advanced Manufacturing,

Directed Energy, Energy and Power Systems, Information Systems and Technology, Physical Phenomena Underpinning Simulation, Sensing and Intelligent Controls, Surety Sciences)

- Corporate Objectives (Nuclear Weapons, Nonproliferation and Materials Control, Energy and Critical Infrastructure, Emerging Threats, Science and Technology, Partnerships)

- Grand Challenges

- Development Reserve
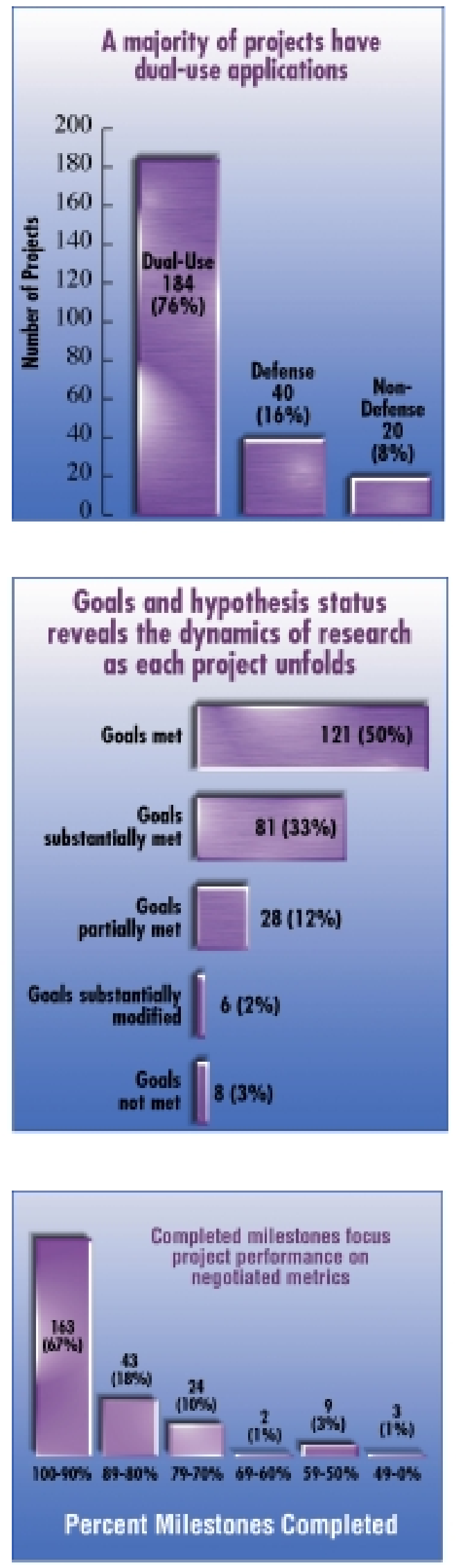
A strong LDRD Program provides critical support to the Laboratories' missions by advancing the technologies essential to the Labs' future. In FY 2000, atomic energy defense activities were supported by 79 percent of LDRD projects, although weapons-related activities provided only 49.4 percent of FY 2000 LDRD funding. More than 95 percent of LDRD projects provided benefits in basic and applied research to national security needs.

Sandia's LDRD Program utilizes several types of measures to identify research performance, including:

- quality of science, technology, and engineering

- relevance to national needs and agency missions

- quality of research management.

Quality of science is indicated by expert advisory committees, peer reviews, sustained progress, and recognition by the scientific community. Relevance is indicated by sustained advancement of fundamental science and advances in support of missions. The quality of LDRD research management is indicated by well-developed research processes; established project goals and milestones; and effective decision making in managing and redirecting projects.

Sandia LDRD received significant scientific recognition from industry, government, and professional societies in FY 1999 (the latest year for complete results). Two of the three R\&D 100 awards received by the Laboratories in 1999 were supported by LDRD projects, and since 1993, 21 of the Labs' 34 awards have been supported by LDRD. Citation analysis provides a measure for the impact of research advancement. In 1999, Sandia LDRD journal articles across all fields represented $15 \%$ of all Sandia articles and outranked overall Sandia articles in terms of relative impact: 1.66 to 1.27 (as measured by the ISI's [Institute for Scientific Information] Science Citation Impact database).

Each foundational LDRD investment area maintains external review boards to provide feedback on research quality. As an example, LDRD Materials research results were externally peer-reviewed by the Materials Science and Technology (S\&T) Council Review Board on January 25-26, 2000. Excerpts from the Board's report include:

- “...scientifically tailored materials and processes ... yield significant results that are world renowned, and add to Sandia's world stature...."
Sandia's LDRD Program utilizes several types of measures to identify research performance...

...since 1993, 21 of the Labs' 34 [R\&D 100] awards have been supported by LDRD.

“...scientifically tailored materials and processes ... yield significant results that are world renowned, and add to Sandia's world stature...." 
- "The LDRD seed projects appear to be thoughtful, highly promising investments-congratulations."

External reviews also provide important perspective and recommendations on the relevance of LDRD research. As an example of relevance, the Materials S\&T Council Review Board highlighted the support of LDRD research to the Labs' missions:

- “...The Board congratulates Sandia for the exemplary high technical quality of research undertaken to resolve fundamental scientific questions and technical challenges to enable applications to Sandia mission areas...."

- “...linkages are technically valid and mission relevant...."

- Dr. Christine S. Sloane, Board Chair, concluded, "...LDRD seeds critical future capabilities...."

The Sandia LDRD Program has developed a process that encourages Labwide participation (i.e., involves executive leadership, program directors, and research staff throughout the Labs) to produce new knowledge with long-term benefits. Because research outcomes are frequently unpredictable, targeted levels of research performance and milestones cannot be $100 \%$ specified beforehand. However, research goals and milestones are established for each project to inspire extraordinary levels of innovation from the research teams and staff. As such, each project team is encouraged to create a deliberate mismatch between the group's research aspirations and its present knowledge and resources.

The Sandia LDRD Program has been tracking the number of completed milestones and the number of goals met since the early 1990s. Project milestones are determined for every $\$ 300 \mathrm{~K}$ of funding and identify specific accomplishments in the progression of research. Goals refer to certain capabilities that are desired within the overall framework of a project's research activities. In scientific inquiry, meeting all of the intended goals indicates that the research challenges have been set too low. From FY 1995 through FY 1999, LDRD projects maintained a risk balance that resulted in a 70 percent average completion rate for milestones and a 52 percent average rate for goals met. In addition, the percentage of research goals met has declined over the past four years. This provides a positive indication of the high level of "stretch" anticipated by LDRD participants in their research activities.

“...LDRD seeds critical future capabilities...."

The Sandia LDRD Program has developed a process that encourages Labwide participation to produce new knowledge with long-term benefits. 


$\begin{array}{ccc}\text { FY } & \text { Completed Milestones } & \text { Goals Met } \\ \text { FY95 } & 70 \% & 55 \% \\ \text { FY96 } & 72 \% & 69 \% \\ \text { FY97 } & 72 \% & 50 \% \\ \text { FY98 } & 69 \% & 45 \% \\ \text { FY99 } & 66 \% & 40 \%\end{array}$

During FY 2000, the General Accounting Office (GAO) conducted a review of the DOE LDRD Program. In the exit briefing to Sandia National Laboratories on June 20, 2000, the GAO provided very positive commentary on the Labs' LDRD process. The GAO investigative team concluded that Sandia's LDRD Program employed a "...very robust process geared to ensure that projects meet the LDRD standard..." They stated further that "...we understand that the approach of Sandia's President to the management of LDRD is based on principles of trust and encouragement with delegation of authority."

\section{Additional Reading}

A comprehensive discussion of DOE and Sandia's LDRD Program management and funding sources may be found in the "Sandia National Laboratories FY 2000-2005 Institutional Plan," dated December 1999.
The GAO investigative team concluded that Sandia's LDRD Program employed a "...very robust process geared to ensure that projects meet the LDRD standard...." 
The Sandia LDRD Program's Impact on Science and Technology

\author{
Al D. Romig \\ Vice President for the Science and Technology Strategic \\ Management Unit
}

Sandia National Laboratories is a national security laboratory that utilizes excellent science and technology (S\&T) to achieve its missions. Sandia has a primary mission of ensuring the safety, security, and reliability of the nation's nuclear weapons stockpile in the indefinite absence of underground testing. Sandia's S\&T competencies are also leveraged to support several missions that are synergistic to its primary mission. These synergistic missions support and enhance the S\&T capabilities of Sandia.

Sandia invests over $\$ 100$ million in annual R\&D funds derived primarily from three revenue sources: LDRD research foundation funds, DOE Defense Programs science foundation funds, and funding from the DOE Office of Science. The LDRD Program encourages innovative, long-term, high-risk research that provides vital advances to support the weapons and national security programs of the Labs. This is consistent with Sandia's S\&T strategic objective for pursuing "science with the mission in mind," thereby ensuring that the right science and technology are available and provide differentiating strengths to Sandia's Strategic Business Units (SBUs) and Strategic Management Unit (SMU).

Sandia tracks its LDRD research by determining the scope of research activities for project portfolio-including incremental, radical, or fundamental investigations. In FY 2000, the R\&D type profile for Sandia's LDRD research was distributed as follows: 6 percent incremental, 21 percent incremental-radical, 22 percent radical-incremental, 26 percent radical, 12 percent radical-fundamental, 9 percent fundamental-radical, and 4 percent fundamental.

Incremental research exploits existing scientific and engineering knowledge in new ways to produce results that may be usefully adopted in the next two years.

Radical research focuses on the creation of new knowledge - new either to Sandia or to the world - that is then combined with existing knowledge to address a specific goal(s). "Success" rates for radical research are typically up to 40 percent, and results may be usefully adopted in two to seven years.

\section{Sandia...utilizes excellent \\ science and technology to achieve its missions.}

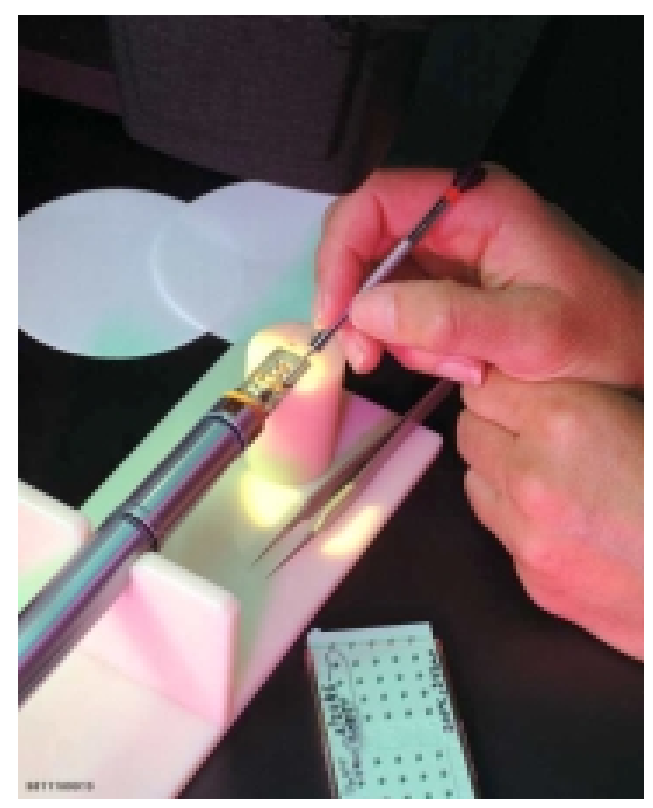

Examining materials with a transmission electron microscope. 
Fundamental research intends to create absolutely new knowledge that extends Sandia's understanding in a scientific or engineering arena. The time to useful adoption of fundamental research can stretch up to ten or more years. Fundamental research is discovery-driven science and, by definition, there are no failures-scientists may not get the results they expect, but learning is greatly increased.

\section{Science and Technology Investment Categories}

For FY 2001, the LDRD Program will be redesigned to align with the "mission challenges" defined by the Labs' business areas and by key science and technologies that affect the Labs' future. The foundations for accomplishing Sandia's missions are defined by the Labs' S\&T foundational competencies. For FY 2001, these competencies will include the traditional LDRD research foundations:

- Computational and Information Sciences

- Electronics and Photonics

- Engineering Sciences

- Materials Science and Technology

The Computational and Information Sciences investment area develops next-generation computing and information capabilities that benefit each of Sandia's business areas. Many computational science advances that were begun in the 1980s (e.g., Teraflops and CPlant) have enabled current capabilities utilized in weapons work. In addition, advances in massively parallel (MP) computing research have greatly expanded the usefulness of high-definition models, the manipulation of very large datasets, and the creation of technologies that make highperformance computers easier to use.

The Electronics and Photonics investment area conducts innovative research that builds on the Labs' differentiating strengths in microelectronics, photonics, MEMS

(microelectromechanical systems), and sensors. In particular, this investment area explores new silicon and compound semiconductor concepts that enable the realization of small, low-power, highly integrated electronics packages called integrated microsystems - these serve as the building blocks required in many of the Labs' mission-related systems. LDRD research has also supported Sandia's world leadership in vertical-cavity surface-emitting lasers (VCSELs) and has promoted next-generation research for optoelectronic applications.
Fundamental research intends to create absolutely new knowledge that extends Sandia's understanding in a scientific or engineering arena.

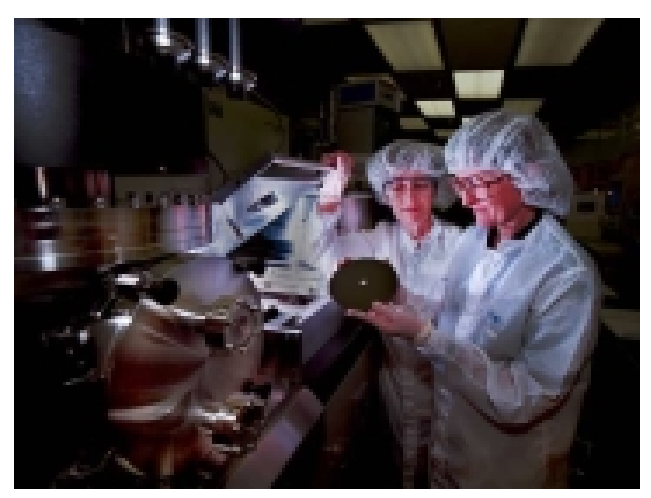

Building versatile microfluidic devices.

LDRD research has supported Sandia's world leadership in vertical-cavity surface-emitting lasers (VCSELs) and has promoted next-generation research for optoelectronic applications. 
The goal of the Engineering Sciences Modeling and Simulation investment area is to dramatically change the process for life-cycle engineering of Sandia products. Improvement is sought by significantly increasing the role of validated engineering modeling and simulation in the full life cycle of systems while achieving mission goals with reduced resources, reduced costs, and increased system and component reliability. This investment area conducts research in fluid and thermal sciences, solid and material mechanics, structural dynamics, reactive processes, and aerosciences. Translation of phenomenology to validated simulation capabilities is the key to advance our understanding in the engineering disciplines critical to Sandia's missions.

The Materials Science and Technology investment area conducts materials and processing research that supports Sandia's strategic S\&T focus areas. Materials research themes include materials-degradation diagnostics and aging mechanisms (S\&T goals); microstructural control and advanced concepts for model-based process control (Revolution in Engineering and Manufacturing goals); and functional materials interfaces, packaging, and power systems (Integrated Microsystems goals). In addition, corrosion research addresses long-term needs of the weapons program, but also has application to basic energy sciences research.

In FY 2001, the S\&T investment category will also include additional investment areas relevant to national security missions' needs, including:

- Advanced Manufacturing

- Biotechnology

- MESA (Microsystems and Engineering Sciences Applications) Technologies

- Pulsed-Power Sciences

The Advanced Manufacturing investment area funds research that has the potential to dramatically reduce the volume of weapon components and/or to reduce production defects. This research supports the process-based quality objective for manufacturing weapons-related products with little or no product inspection. A number of LDRD innovations have demonstrated sufficient efficacy to receive funding from the nuclear weapons program.

The Biotechnology investment area is a new Sandia LDRD research area that will invest in unique crossdisciplinary physical science, information science, and biotechnical investigations to create solutions supportive of future mission needs. In FY 2001, investigations will address
Translation of phenomenology to validated simulation capabilities is the key to advance our understanding in the engineering disciplines critical to Sandia's missions.

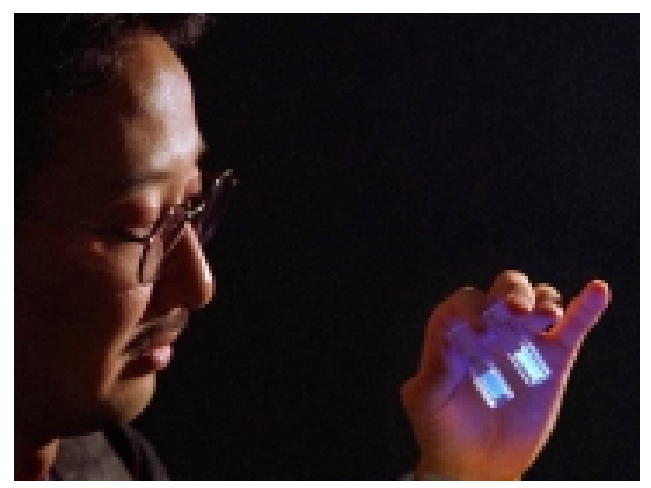

Developing biochemical techniques to detect contaminants. 
computational biochemistry, protein folding, nanotechnology, biosensors, and biochemical protection issues. Biotechnology advances have the potential to lead to new materials and new approaches for future stockpile upgrades.

Nanotechnology is an enabling research area that promises significant benefits to many of the Labs' mission areas and, in particular, the Labs' science-based stockpile stewardship programs. Nanotechnology is a critical component of Sandia's long-term vision for MESA (Microsystems and Engineering Sciences Applications) as we progress to increasing miniaturization of components and the use of tailored functional materials for increased performance, safety, and reliability. In addition, Sandia will research the coupling of nanotechnology and biotechnology through applications of microsystems, scanning probes, materials nanofabrication, and materials integration into microsystems. With the current advances in nanotechnology and biotechnology, there will clearly be additional opportunities benefiting the DOE mission areas of national security, energy, and environment.

The Pulsed-Power Sciences investment area conducts research that has the potential to enhance national security by advancing nuclear and conventional deterrents as well as expanding energy options. These investigations evaluate highrisk, innovative approaches in theoretical and experimental electromagnetics; innovations in z-pinches, radiography, and particle beams; high magnetic fields; and diagnostics. In addition, researchers are investigating impulse response models for hostile environments to improve our understanding of next-generation drivers for weapons effects applications.

\section{Mission Technology Investment Category}

For FY 2001, a new investment category, Mission Technologies (MT), has been created to support the science and technology needs of Sandia's Energy and Critical Infrastructures (E\&CI), Emerging Threats (ET), and Nonproliferation and Materials Control (NP\&MC) business areas.

The Energy and Critical Infrastructures investment area develops enhancements to the surety (i.e., safety, security, and reliability) of the nation's energy and other critical infrastructures. An important focus within this area is research that seeks to improve our understanding of the interdependencies between and among critical infrastructures. This area also invests in explorations of chemical and physical processes to promote
Nanotechnology is an enabling research area that promises significant benefits to many of the Labs' mission areas and, in particular, the Labs' sciencebased stockpile stewardship programs.

With the current advances in nanotechnology and biotechnology, there will clearly be additional opportunities benefiting the DOE mission areas of national security, energy, and environment. 
energy efficiency, fuel-use optimization, and environmental protection.

The Emerging Threats investment area investigates highimpact responses to emerging national security threats such as difficult targets, homeland defense, minimally manned warfare, and focused logistics. This area researches new technologies and novel integrations of diverse technologies that have the potential to mitigate threats to the nation's security.

The Nonproliferation \& Materials Control investment area invests in research that enables Sandia to address new national security challenges. LDRD projects include innovative approaches to remote sensing and verification, physical security, chemical-biological nonproliferation, and materials management and international cooperation.

\section{Grand Challenges}

LDRD Grand Challenges are imaginative, integrated investigations that apply many of Sandia's competencies in the search for solutions to the nation's current and future security concerns. The combination of individual LDRD research projects and a few selected Grand Challenges support a wellrounded LDRD portfolio. Grand Challenges are linked to the future strategy of the Labs by requiring at least one technical vice president's endorsement as being a key to achieving the future goals of their business area. Over the years, LDRD research projects have generated many innovative ideas and advances.

Two examples of Grand Challenge research are the current Molecular Integrated Microsystems (MIMS) project and the recently completed $\mu$ ChemLab ${ }^{\mathrm{TM}}$ project. The MIMS project is exploring novel nanomaterials for next-generation microsystems. These nanomaterials can be dynamically programmed to provide new architectures for biochemical analysis and weapons safety. In FY 2001, research efforts will concentrate on materials optimization and device design. It is anticipated that by FY 2002, Sandia will be fabricating and testing proof-of-principle devices based on these new materials. The first licensing agreement for MIMS technology is under consideration.

The $\mu$ ChemLab ${ }^{\mathrm{TM}}$ Grand Challenge project has produced technologies with the potential to impact Sandia's weapons program in such areas as MEMS, optoelectronics, electrokinetic pumping (EKP), and stockpile monitoring.

\author{
Grand Challenges are linked to \\ the future strategy of the \\ Labs....
}


Recent applications of $\mu$ ChemLab ${ }^{\mathrm{TM}}$ technology have demonstrated outstanding results with detection tests involving agents amidst a variety of interferants. The $\mu$ ChemLab ${ }^{\mathrm{TM}}$ Grand Challenge (and related efforts beginning in the early 1990s) has produced nearly 70 patentable technical advances, and negotiations to license selected technologies are in progress.

\section{Conclusion}

Science and technology are at the heart of the nation's security and international leadership.

Science and technology are at the heart of the nation's security and international leadership. The LDRD Program initiates critical research and development that produce the knowledge for revolutionizing our science and technology capabilities. This, in turn, enhances the continued growth, prosperity, and security of the nation. 
The Impact of LDRD on Sandia's Nuclear Weapons Program

Cliff Renschler

Stockpile Stewardship Office Manager

(On behalf of Tom Hunter, Senior Vice President, Nuclear

Weapons Program)

The Nuclear Weapons (NW) program at Sandia National Laboratories derives significant benefit from Sandia's LDRD Program. LDRD is a key driver for the fundamental and applied research that allows Sandia to meet its weapons and national security mission commitments. In addition, the LDRD Program supports three essential needs of the national security mission: (1) recruiting and retention, (2) troubleshooting as a hedge against surprise, and (3) executing the Labs' weapons mission assignments.

LDRD is managed to ensure a high degree of relevancy between the Labs' NW Strategic Business Unit (SBU) and the Science and Technology (S\&T) Strategic Management Unit (SMU) efforts. The S\&T/NW LDRD investment area selects research projects that are focused on weapons-centric technologies and that enhance prospects for the successful transfer of components and processes to the weapons Advanced \& Exploratory (A\&E) development program. An NW advocate serves on every LDRD Investment Area Team (IAT) and assesses proposals for weapons relevancy.

LDRD projects source the science and technology supply chain that feeds the development and prove-out stages for the Labs' NW A\&E program. From there, the innovations proceed to full-scale engineering design and development and the first production units. In the final stage, weapons components are produced are at Kansas City or Sandia. Examples of past LDRD advances that have impacted Sandia's weapons work

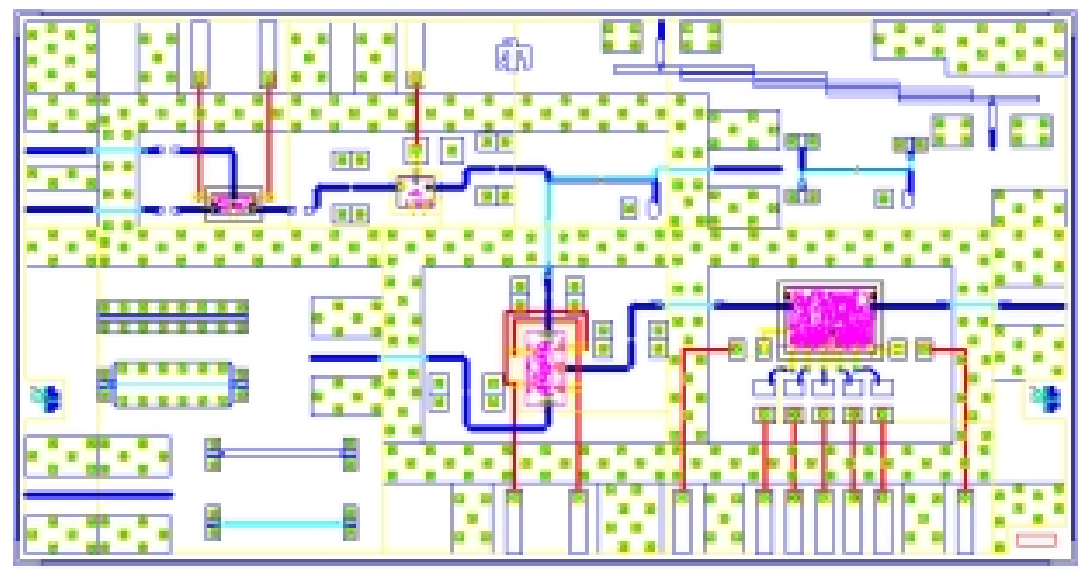

LDRD is a key driver for the fundamental and applied research that allows Sandia to meet its weapons and national security mission commitments.
Innovating high-density, high-speed interconnection systems. 
are found in the development of a dozen or more components currently in the stockpile, including radiation-hardened microelectronics for War Reserve applications and ceramics for neutron generators.

In addition, examples of current LDRD research with the potential to impact future weapons work include the ultraminiaturized radar project and the accurate timing project (an exploration to provide accurate timing in high-radiation environments without external power). Both projects utilize Sandia's MEMS and substrate technologies expertise to produce innovative solutions. The ultraminiaturized radar research is enabling extremely high-density interconnects and ultrahigh-speed switching to produce smaller system volumes and cost savings. The accurate timing research is enabling greater timing accuracy in a device that is hardened against exoatmospheric radiation and nearby nuclear detonations.

In FY 2002 and beyond, LDRD research investigations will be guided by "The Fearless Forecast," a set of long-term weapons needs defined by Sandia weapons engineers. This document will provide principal investigators throughout the Labs with a list of potential fundamental, radical, and incremental research issues to encourage innovation in the needs areas critical to Sandia's weapons program.

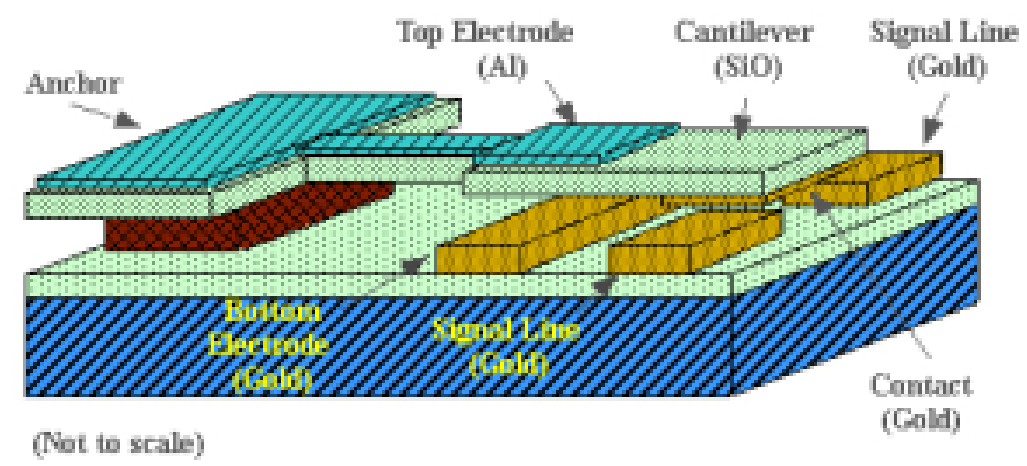

...examples of current LDRD research with the potential to impact future weapons work include the ultraminiaturized radar project and the accurate timing project....

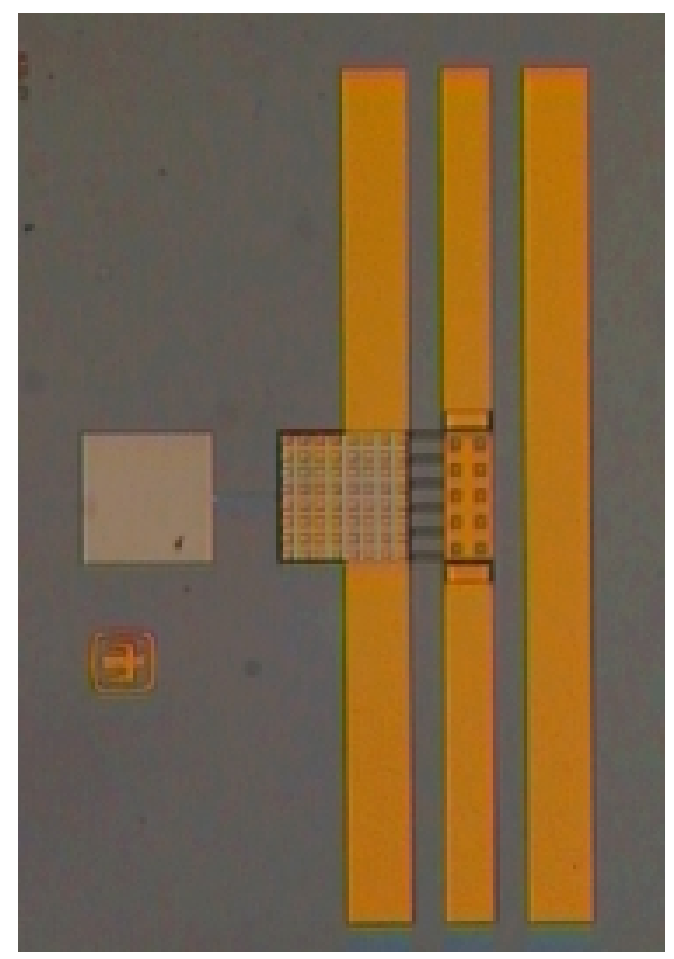

Developing ultraminiature RF circuitry for future missions. 
The LDRD Program Supports Responses to Emerging Threats

\section{Dan M. Rondeau}

Deputy to the Vice President for the Emerging Threats Strategic Business Unit

The Emerging Threats Strategic Business Unit (SBU) develops systems solutions to respond to a wide range of threats against the nation's security. The changing threat environment includes weapons of mass destruction, major theater warfare, weapons proliferation, terrorism, information security, and military operations other than war. Many of the response solutions are derived from capabilities developed in weapons life-cycle applications. For example, advanced computational modeling and simulation, advanced microelectronics, and microelectromechanical systems (MEMS) support advanced capabilities for attacking difficult targets, conducting minimally manned warfare, ensuring homeland defense, and developing focused logistics.

Attack of difficult targets researches numerous technologies that can "see, decide, and act" to locate, characterize, and destroy threats. Minimally manned warfare investigates technologies that improve warfighter effectiveness while reducing personal risk. Numerous LDRD advances have supported this thrust, including robotic sensing platforms, collective swarms, and direct-fire robot systems, among others. In addition, focused logistics explores technologies and processes to reduce operation and maintenance costs, thereby freeing up money for force modernization.

One of Sandia's differentiating strengths has been its systems integration capability for the development of products and processes with high technical content. The LDRD Program is a key science and technology content provider enabling that strength.

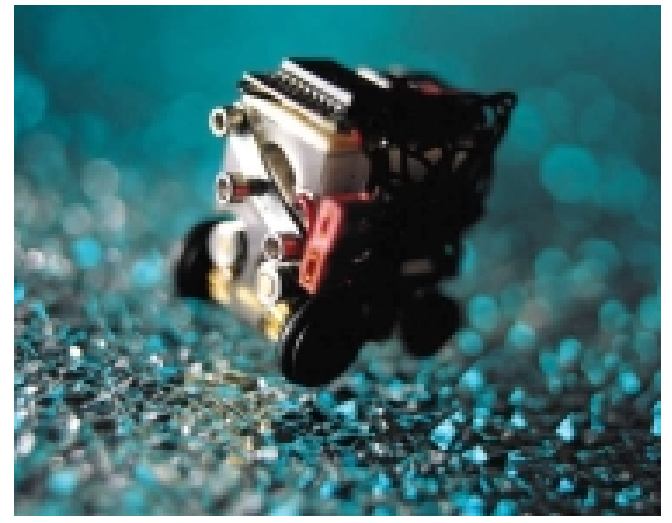

Miniature robots incorporate "see, decide, act" capabilities.

One of Sandia's differentiating strengths has been its systems integration capability for the development of products and processes with high technical content. 
The Sandia LDRD Program's Impact on Energy and

\section{Critical Infrastructures}

Robert J. Eagan

Vice President for the Energy and Critical Infrastructures

Strategic Business Unit

The chief goal of the Energy and Critical Infrastructures $(\mathrm{E} \& \mathrm{CI})$ investment area is to ensure national security through research that supports affordable, reliable, and clean energy. The portfolio of power sources that E\&CI researches includes nuclear energy, renewable energy, fossil fuels, and pulsed power. In natural extensions to the $\mathrm{E} \& \mathrm{CI}$ goal, research teams undertake the crucial national issues of energy efficiency, environmental stewardship, and infrastructure surety. A new research focus for this area is the examination of international water resources as an important national security issue.

LDRD research supports innovative solutions for some of the greatest challenges facing the nation's critical infrastructures. A single component of the nation's infrastructure, for example the electric power grid, is complex in its own right. But when linked together with other infrastructures, complexity grows by levels of magnitude. Maintaining the security of these linkages provides an excellent opportunity to apply Sandia's extensive experience in systems engineering, experience gained from-and fed back to-nuclear weapons systems.

Using Sandia's weapons security skills and experience with sensors, microelectronics, and allied applied technologies, research is also focusing on reducing

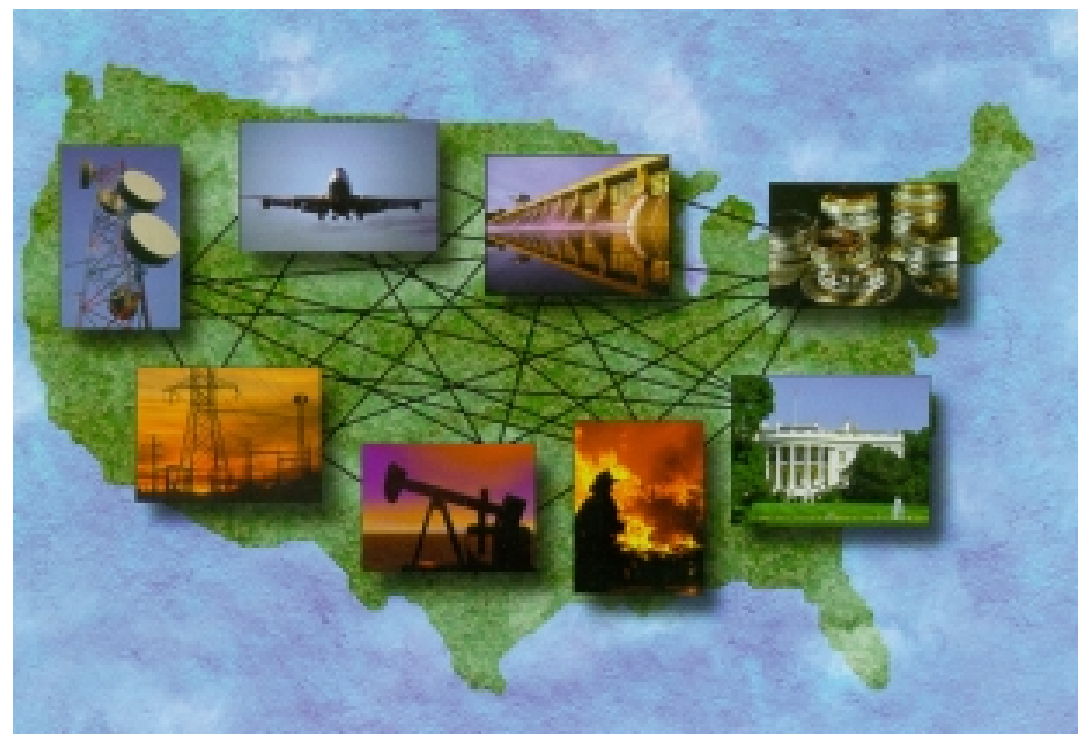

LDRD research supports innovative solutions for some of the greatest challenges facing the nation's critical infrastructures.

Developing technologies to protect the nation's infrastructure. 
international conflicts over water supplies. Protection of water sources and supply can include protection against unauthorized access and the detection of chemical pollutants or biological agents. LDRD research supports the verification of water allocation agreements by developing automated monitoring technologies to support treaty compliance issuesthus minimizing international conflicts over water resources.
LDRD research supports the verification of water allocation agreements by developing automated monitoring technologies to support treaty compliance issues-thus minimizing international conflicts over water resources. 
The LDRD Program Strengthens the Foundation for Sandia's Nonproliferation and Materials Control Mission

Ron W. Moya

Operations Manager for the Nonproliferation and Materials Control Strategic Business Unit

Currently, Sandia's total nonproliferation program is the largest undertaken by the U.S. government. In fact, Sandia's arms control, monitoring, and nonproliferation technical work forms the largest single program undertaken by any technical institution in the world. The LDRD Program plays a vital role in ensuring that Sandia can meet its nonproliferation and materials control responsibilities regardless of changing regional tensions and conflicts, evolving terrorist threats, increasing or decreasing interest in nonproliferation, and the nation's ever-changing relationships with other world powers.

The Nonproliferation and Materials Control (NP\&MC) investment area investigates science and technology to reduce the nation's vulnerability to weapons of mass destruction and internal threats. NP\&MC research addresses five thrusts, including materials management, remote sensing and verification, proliferation assessment, physical surety, and chemical-biological nonproliferation. Forty percent of the research projects in this area address needs with applications to multiple Sandia mission areas, including an emphasis on robust nuclear weapons systems.

The Materials Management and International Cooperation thrust encourages innovation in the safety, security, and transparency of nuclear materials worldwide. Scientific and technological advances in preventing the spread of weapons of mass destruction are emphasized. Current research challenges include developing proliferation assessment models and establishing information barriers for arms control applications.

The Chemical-Biological Nonproliferation thrust explores advanced technologies for the detection of and defense against chemical and biological threats. Research in this area concentrates on biological agent detection and identification, disease surveillance, and the protection of critical facilities against chemical-biological attacks.

The Physical Surety thrust promotes innovative, costeffective system solutions to protect vital assets while mitigating injury and loss of life. Research activities focus on developing advanced sensor systems, performing advanced modeling of security systems, and studying architectural

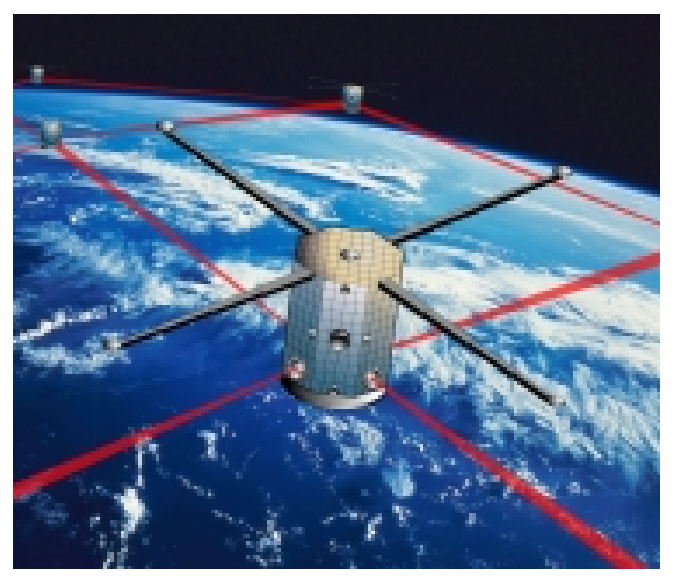

A cluster of nanosatellites creates a sparse aperture in space.

...Sandia's arms control, monitoring, and nonproliferation technical work forms the largest single program undertaken by any technical institution in the world.

The LDRD Program plays a vital role in ensuring that Sandia can meet its nonproliferation and materials control responsibilities.... 
surety technologies.

The Remote Sensing and Verification thrust researches space- and ground-based capabilities that support advanced treaty monitoring and detection of weapons of mass destruction proliferation capabilities. The research challenges for this team include enhancing the knowledge of signal collection, conversion, processing, and exploitation technologies, and developing astrodynamics and Attitude Determination and Control (ADC) technologies.

The Proliferation Assessment thrust investigates innovative solutions that advance intelligence capabilities. Exploration activities include data exploitation, predictive models, sensors, and methods for collecting information from denied areas.

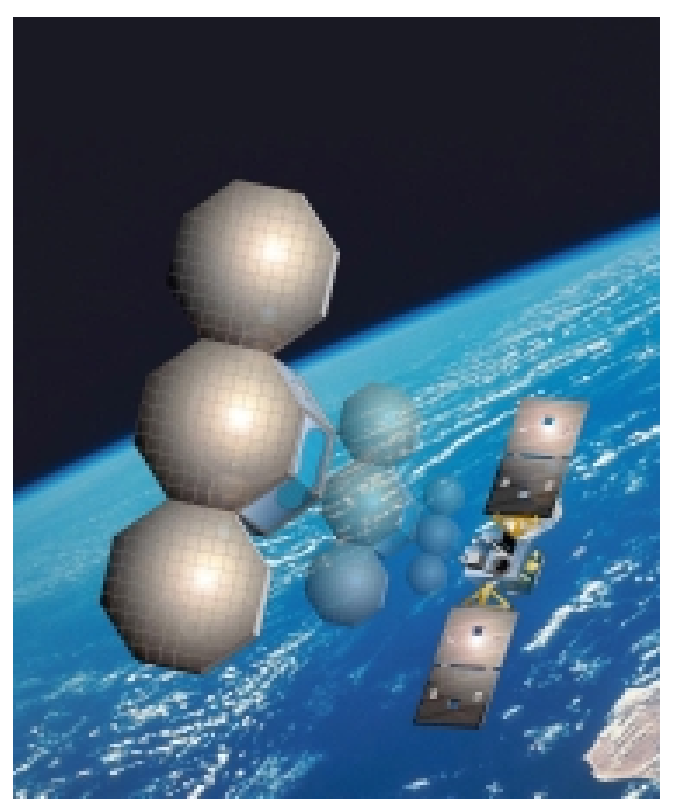

Future nanosats may perform very complex functions. 


\section{Materials Science and Technology}

The Materials Science and Technology (MS\&T) investment area researches materials that behave in a predictable manner during all phases of their life cycle. Research investigations focus on innovations in surety science and technology to improve our understanding of changes in critical materials properties.

Research efforts also support the "Revolution in Engineering and Manufacturing" initiative to develop highly sophisticated materials processes for manufacturing affordable, high-performance hardware in low volume with no defects. In addition, research efforts focus on scientifically tailored materials that utilize optical, electromechanical, magnetic, and numerous chemical functions to sense, think, act, and communicate.

The "Reactivity of Metal-Oxide Surfaces" project uses a combination of experimental and theoretical techniques to explore atomic-scale defect chemistry on technologically important surfaces. The formation,

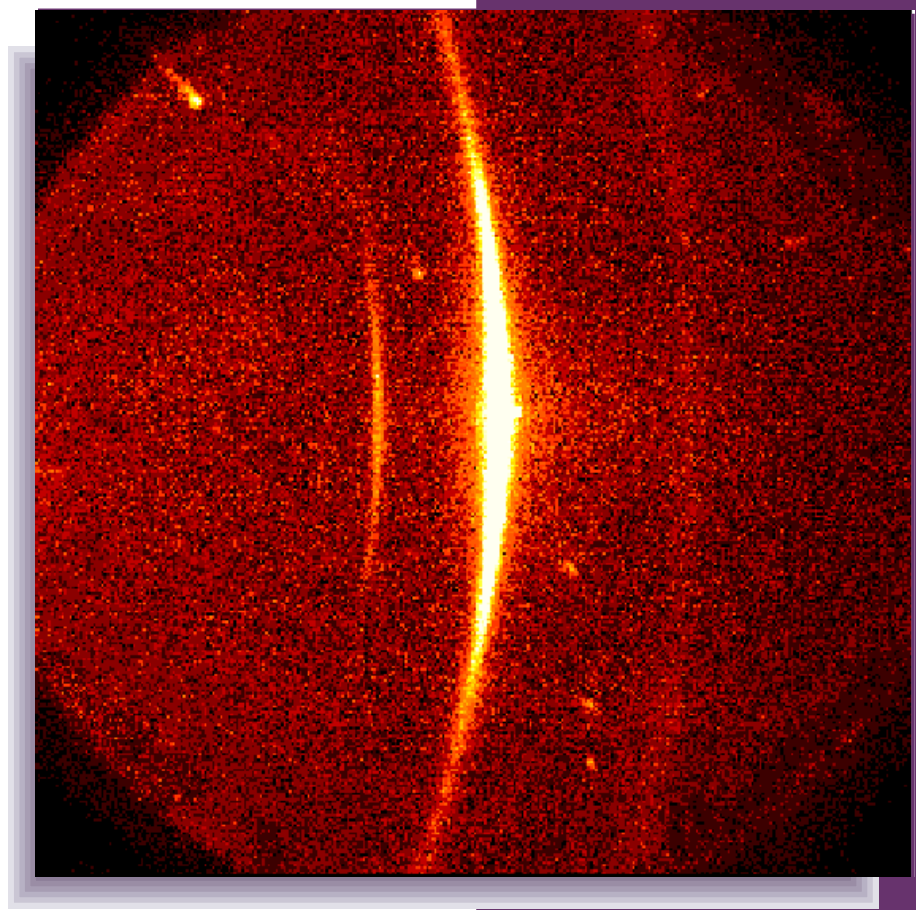

mobility, reactivity, and dissolution of atomic-scale surface defects are important drivers in materials issues including catalysis, corrosion, thinfilm growth, and semiconductor processing. This research is investigating the role of particularly reactive localized surface defects as a driver of the surface chemistry of many materials. Experimental results are being examined against theoretical studies to improve the development of surface-defect reactivity models. A patent disclosure has been filed based on research results from this project. 
10300

Functional Materials for Microsystems: Smart Self-Assembled Photochromic Films

A. R. Burns, R. W. Carpick, M. H. Crawford, D. Y. Sasaki, C. J. Brinker, F. B. Van Swol

Sandia will scientifically tailor "smart" interfacial films and 3-D composite nanostructures to exhibit photochromic responses to specific, highly localized chemical and/or mechanical stimuli and to integrate them into optical microsystems. The project will involve the design of functionalized chromophoric self-assembled materials (SAMs) that possess intense and environmentally sensitive optical properties (absorbence, fluorescence), thus enabling their use as detectors of specific stimuli and transducers when interfaced with optical probes. Particularly strong candidates for initial studies are the conjugated polydiacetylenes (PDAs). We will immobilize the organic functional material in an ordered, mesostructured inorganic host matrix that will serve as a perm-selective barrier to chemical and biological agents and provide structural support for improved material durability. Construction of these hybrid organic/inorganic layers will be facilitated by advanced self-assembly techniques. Multitask scanning probes (atomic force microscope [AFM], near-field scanning optical microscopy [NSOM]) offering simultaneous optical and interfacial force capabilities will drive the chromophoric materials with localized and specific interactions for detailed characterization of physical mechanisms and parameters. We will directly interface the composite films with microscale devices as optical elements (e.g., intracavity mirrors, diffraction gratings), taking advantage of the very high sensitivity of device performance to real-time dielectric changes in the films.

We used AFM to simultaneously actuate and characterize the nanometer-level structural changes associated with the blue-to-red-phase transformation. The rate of transformation directly correlated with the shear forces exerted by the AFM probe perpendicular to the backbone, knocking the side-chains toward the substrate. To understand how side-chain rotations induce the transformation, we initiated molecular mechanics and electronic structure calculations. So far, we have found that $>40$-degree dihedral angle rotations within the conjugated backbone are required to induce a color change. Cubic, hexagonal, and lamellar PDA/silica nanocomposites

\section{The project will involve the design of functionalized} chromophoric self-assembled materials (SAMs) that possess intense and environmentally sensitive optical properties (absorbence, fluorescence), thus enabling their use as detectors of specific stimuli and transducers when interfaced with optical probes. 
were created by evaporation-driven self-assembly of silicates that we organized by diacetylene amphiphiles with ethyleneoxide head groups. We found that the size of the head groups directly influenced the curvature of the mesophase: Structures with small head groups readily polymerized, whereas structures with larger ones did not. We demonstrated that polymerized diacetylene not only exhibited full blue-to-red chromism for all the structure morphologies, but was also reversible. Work in optical systems revealed a disappointing blue-red fluorescence dynamic range, indicating that further film development is required.

\section{Refereed}

Burns, A. R., R. W. Carpick, D. Y. Sasaki, J. A. Shelnutt, and R. Haddad. 2000. "Shear-Induced Mechanochromism in Polydiacetylene Monolayers." Tribology Lett., accepted.

Carpick, R. W., D. Y. Sasaki, and A. R. Burns. 2000. "First Observation of Mechanochromism at the Nanometer Scale." Langmuir 16 (2000): 1270.

Carpick, R. W., D. Y. Sasaki, and A. R. Burns. 1999. "Large Friction Anisotropy of a Polydiacetylene Monolayer.” Tribology Lett. 7 (1999): 79.

Carpick, R. W., T. M. Mayer, D. Y. Sasaki, and A. R. Burns. 2000. "Spectroscopic Ellipsometry and Fluorescence Study of Thermochromism in an Ultrathin Polydiacetylene Film: Reversibility and Transition Kinetics.” Langmuir 16 (2000): 4639.

Sasaki, D. Y., R. W. Carpick, and A. R. Burns. 2000. "High Molecular Orientation in Mono- and Tri-layer Polydiacetylene Films Imaged by Atomic Force Microscopy." J. Colloid and Interface Sci. 229 (2000): 490.

\section{Other Communications}

Burns, A. R., R. W. Carpick, and D. Y. Sasaki. 2000.

"Mechanochromism and Friction Anisotropy in Functional Organic Films." Paper presented to the American Physical Society Meeting, Minneapolis, MN, March.

Burns, A. R., R. W. Carpick, and D. Y. Sasaki. 1999. "Nanometer-Scale Science of Conjugated Polymer Interfaces." Paper presented to the American Vacuum Society International Symposium, Seattle, WA, October.

Burns, A. R., Y. Lu, R. W. Carpick, D. Y. Sasaki, and C. J. Brinker. 1999. "Nanoscale Organization of Functional Materials Via Biomimetic Composite Assemblies." Paper presented to the Materials Research Society Fall Meeting, Boston, MA, November. 
10301

\section{Innovative Experimental and Computational Diagnostics for Monitoring Corrosion in Weapons Environments}

\author{
F. D. Wall, N. A. Missert, C. C. Battaile, J. C. Barbour
}

We are advancing Sandia's capabilities to detect and quantify aluminum (Al) alloy degradation in atmospheric environments by leveraging our expertise in nanofabrication technology to produce electrode microstructures and microelectrochemical test platforms amenable to testing in thin adsorbed water layers. Planar arrays of individually addressable microelectrodes are used to perform both passive and perturbing electrochemical testing under conditions in which conventional electrochemical techniques are not applicable. Precisely engineered sample microstructures make it possible to systematically vary material characteristics and derive causal relationships with atmospheric degradation. Secondary phases in Al alloys can polarize surrounding material due to galvanic interactions, and many proposed degradation mechanisms include galvanic driving force as a critical component. To understand how secondary phases affect corrosion, it is necessary to describe the potential and current distributions on a sample surface. We are developing a mesoscale electrical model to incorporate arbitrary secondphase distributions and variable adsorbed electrolyte morphology to predict combinations of environment and microstructure that enhance the propensity for galvanic degradation. We are taking into account particle-particle interactions as well as particle-matrix interactions. To predict material performance in atmosphere, it is also necessary to describe the adsorbed environment. Surface acoustic-wave (SAW) technology provides a means of detecting a fraction of a monolayer change in adsorbed species. By nanofabricating electrodes on a SAW platform, we can determine how microstructure and geometry impact water adsorption. SAW devices can also be used to sense changes in the acoustic properties of material oxides due to damage accumulation and may operate as early warning sensors for atmospheric degradation. We will also make efforts to spatially resolve SAW signals to determine where, as well as when, damage initiates. The focusing of specimen design, electrochemical measurements, modeling, and environmental characterization to the mesoscale level is critical to lifetime predictions in stockpile environments.
The focusing of specimen design, electrochemical measurements, modeling, and environmental characterization to the mesoscale level is critical to lifetime predictions in stockpile environments. 
- Nanoengineered thin-film electrodes. We constructed a 19-electrode microelectrochemical cell consisting of $12 \mathrm{Al}$ electrodes arranged as a square with 3 electrodes per edge, a central copper $(\mathrm{Cu})$ electrode, and 6 spatially distributed palladium (Pd) electrodes. For initial experimentation, the $\mathrm{Al}$ serves as the anode, the $\mathrm{Cu}$ as the cathode (simulating a noble phase), and the Pd as sense electrodes to monitor the potential field. We obtained a multimicroelectrode analyzer (MMA) to allow simultaneous measurements of multiple current and potential signals. The test environments used to date are deionized water and dilute aqueous sodium chloride ( $\mathrm{NaClaq}$ ). We apply the solution as a small droplet and continue measurements until complete dryout of the solution occurs. Metastable pitting on one of the Al electrodes causes a decrease in the current available for the other electrodes and results in the $\mathrm{Cu}$ generating a symmetric cathodic current. The six reference electrodes all measure the same potential fluctuations but are offset from one another due to the resistivity of the solution between electrode locations. When the solution is allowed to dry out, the ionic strength of the solution increases and can result in exacerbated corrosive attack. To date, we have not quantified the sensitivity of this electrode-to-electrochemical activity as a function of relative humidity (RH). We will use a newly completed atmospheric test facility to determine the RH levels at which this electrode is functional. Based on those results, we may construct a similar electrode with much smaller inter-electrode spacing to accommodate testing in low-humidity environments.

- Multilayer thin-foil electrodes. We constructed reusable atmospheric sensors from thin-metal foils (25 microns) separated by a thin (10 microns) insulating coating. The foils are stacked and encased in an epoxy resin for mechanical rigidity. After a corrosion experiment is complete, the corrosion layer can be removed by mechanical polishing, leaving a fresh surface for subsequent testing. The foil-based electrodes have proven invaluable in advancing our microelectrochemical testing effort. To date, we have constructed several electrodes that either test interactions between different materials (i.e., galvanic couple between $\mathrm{Al}$ and $\mathrm{Cu}$ ) or test the electrochemical behavior of a single material as a function of imposed electrochemical stresses. Both electrode designs have proven capable of measuring electrochemical signals in adsorbed electrolytes. The galvanic couple between $\mathrm{Al}$ and $\mathrm{Cu}$ became electrochemically active at an $\mathrm{RH}$ between $50 \%$ and $65 \%$, which is in agreement with ...we have constructed several electrodes that either test interactions between different materials (i.e., galvanic couple between $\mathrm{Al}$ and $\mathrm{Cu}$ ) or test the electrochemical behavior of a single material as a function of imposed electrochemical stresses. 
adsorption theory. We performed standard electrochemical measurements (open circuit, potential scan, and impedance spectroscopy) using an $\mathrm{Al}$ and platinum $(\mathrm{Pt})$ sample exposed to a $90 \%$ RH environment with no contaminants. These atmospheric electrochemical tests will enable quantification of material degradation as a function of adsorbed moisture and contaminant levels. This information will be fed directly into the 3-D electrical model to predict the galvanic corrosion behavior of more complex systems.

- 3-D mesoscale electrical model. Previously, we used a mesoscale electrical model to predict the propensity for the initiation of localized corrosion in a metal system given the geometric arrangement of noble particles, the solution conductivity, and the polarization behavior of the relevant phases. The model is updated and now has the following additional capabilities: (1) prediction of potential and current fields for a system undergoing active pitting, (2) calculation of potential field data given the corrosion current values as a function of position at the metal/electrolyte interface, and (3) prediction of the resistivity of a mixed-phase system given conductivity and geometric data. We are working to predict maximum pit size (depth, radius) that can be supported for a given electrode geometry and corrosive environment. We made predictions for pit behavior as a function of solution layer thickness; as the thickness decreases, pits become ohmically limited and switch off. We will refine future predictions by inputting boundary conditions from the three-electrode measurements made under atmospheric conditions. 
10302

Self-Healing Molecular Assemblies for Control of Friction and Adhesion in MEMS

M. T. Dugger, D. Y. Sasaki, M. J. Stevens

The major barrier to implementing microelectro-

mechanical systems (MEMS) technologies is the inability to lubricate the surface of micromechanical structures to prevent adhesion and to reduce friction and wear. Sandia seeks a new class of molecular coatings that offer self-healing lubrication properties and a built-in mechanism to release shear energy through reversible chemical cleavage. We developed molecular assemblies that form liquid crystalline lamellar structures, and hyperbranched polymer films that form a densely crosslinked surface over a more compliant molecular base. We are focusing on the two most promising candidate surface treatments investigated during the past two years. We are investigating damage generation and kinetics of healing for these systems with scanning probe microscope (SPM) techniques. We are testing the ability to coat complex shapes and hidden interfaces by forming these coatings on actual MEMS devices and will obtain quantitative friction and durability data. We will use molecular dynamics (MD) simulation techniques to investigate the lubricants' response to shear and compressive forces and to determine the molecular mechanisms involved in ordering, disruption of that order, and re-ordering.

We prepared several new double-tailed fluorocarbon amphiphiles to enhance self-assembling properties.

Amphiphiles prepared with glycerol and glutamate backbones yielded dramatically improved self-assembling properties compared to their single-chain counterparts. Transmission electron microscopy (TEM) of structures formed from bis(F8)glu-ole in decalin exhibit the presence of cylindrical micellar structures, confirmed by nuclear magnetic resonance (NMR). Contact angle measurements of the double-tailed amphiphiles on octadecylsilane-coated silicon ( $\mathrm{Si}$ ) indicate good wettability, suggesting the ability to produce conformal coatings. We observed thermotropic liquid crystalline behavior in three of the materials investigated.

Friction tests of fluorinated hyperbranched polyacrylic acid (PAA) films on gold exhibited higher friction but better durability than silane coatings. We developed a linker layer so that PAA films could be grown on silica, and we accomplished
Sandia seeks a new class of molecular coatings that offer self-healing lubrication properties and a built-in mechanism to release shear energy through reversible chemical cleavage. 
fluorination with either a fluorinated octylamine or a perfluorinated polyether. We observed no discernible thermal degradation in octylamine films, and only slight degradation in the polyether modified films.

We built an SPM with detection geometry optimized for lateral force measurement, which permits lubricant films to be rubbed with an atomic force microscope (AFM) tip and then imaged with a different set of conditions. This will improve resolution of differences in friction response after mechanical damage. We set up MD code to treat bilayer lubricant systems between two walls. Initial simulations of dense, well-packed linear amphiphiles exhibit behavior similar to atomically flat solid surfaces sliding over each other; on a plot of viscosity versus shear rate, they exhibit a shear rate exponent of - 0.94 . For the dense system investigated, we expected an exponent of near negative one $(-1)$.

\section{Refereed}

Dugger, M. T. 1999. "Micromachine Tribology: Surface Modification and Metrology Challenges." Paper presented to a special seminar at Argonne National Laboratory, Argonne, IL, 30 September.

Dugger, M. T. 2000. "Quantification of Friction in Microsystem Contacts: A Step Toward Predictive Capability." Nanotribology: Critical Assessment and Res. Needs, Proc. NIST Workshop (Gaithersburg, MD, 13-15 March).

Dugger, M. T., G. A. Poulter, and J. A. Ohlhausen. 2000. "Development of a Surface-Micromachined Device to Quantify Friction on Polysilicon Sidewall Surfaces." Paper presented to the STLE Annual Meeting, Nashville, TN, 8 May.
Dugger, M. T., G. A. Poulter, and J. A. Ohlhausen. 1999. "Surface Passivation for Reduced Friction and Wear in Surface-Micromachined Devices." Paper presented to the Materials Research Society Fall Meeting, Boston, MA, 29 November.

Dugger, M. T., J. A. Ohlhausen, and G. A. Poulter. 1999. "Environmental Effects on the Tribological Behavior of Silane-Treated Micromachines." Paper presented to the American Vacuum Society International Symposium, Seattle, WA, 25 October. 
10303

\section{Linking Atomistic Computations with Phase- Field Modeling}

J. J. Hoyt, V. Tikare, F. M. Hosking

The phase-field model (PFM) has proven to be a very powerful tool for modeling microstructural evolution during complex materials processing techniques. Although the method can be used to study grain growth and particle coarsening, the PFM is particularly effective in predicting dendrite formation and solute redistribution during solidification and is therefore very useful in the numerical modeling of joining operationswelding, soldering, and brazing. To date, most of the work utilizing the phase-field concept has focused on the development of the numerical technique and the study of generic microstructures; microstructure modeling applied to specific alloy systems has been rare. The lack of direct comparison between model predictions and actual microstructures in real alloys stems from the fact that many materials parameters-mobilities, free energies, surface energies, gradient energy coefficients, etc.-are required as input in the PFM, and often these parameters are difficult to obtain experimentally. Furthermore, current theories of dendrite solidification predict that the velocity of growth is a very strong function of the anisotropy in the solid-liquid interfacial free energy or the kinetic coefficient. These anisotropy factors are typically quite small and have yet to be measured in any metal system. The purpose of this project is to employ atomistic calculations to obtain numerical values of all parameters necessary to model microstructural evolution.

Our work resulted in the first atomistic simulation method that is capable of determining the anisotropy of the solid-liquid interfacial free energy in metals and alloys. It is well known from solidification theory that the growth rate of an isolated dendrite is extremely sensitive to the very small (on the order of 1\%) anisotropy in the interfacial energy. Thus, to model the complex morphologies that form during solidification of alloys, one is faced with the daunting task of measuring or computing anisotropies to a very high accuracy. Prior to our work, only two experiments and no computation schemes had been successful in extracting the anisotropy.

Our method, developed in collaboration with researchers from Northeastern and Northwestern universities, involves monitoring the fluctuations in the position of the
The purpose of this project is to employ atomistic calculations to obtain numerical values of all parameters necessary to model microstructural evolution....Our work resulted in the first atomistic simulation method that is capable of determining the anisotropy of the solid-liquid interfacial free energy in metals and alloys. 
solid-liquid interface for a system in equilibrium at its melting temperature. We implemented an algorithm that can accurately track the interface position during molecular dynamics (MD) simulation. If $\mathrm{A}(\mathrm{k})$ represents the Fourier transform of the position of the solid-liquid interface, then it can be shown that $\left.=\mathrm{k}_{\mathrm{B}} \mathrm{T} /(\mathrm{g}+\mathrm{gamma})^{\prime \prime}\right)\left(1 / \mathrm{k}^{2}\right)$, where the angular brackets denote an average over many configurations, $\mathrm{k}_{\mathrm{B}} \mathrm{T}$ is Boltzmann's constant times temperature, $g$ is the surface-free energy, and $g^{\prime \prime}$ is the second derivative of the interfacial energy with respect to the angle between the interface normal and the 100 direction of the crystal. The above expression is valid for a one-dimensional interface; therefore, in the simulations we maintain a dimension parallel to the solid-liquid boundary as thin as possible; $t$ represents this small thickness. The combination $g+g^{\prime \prime}$ is commonly referred to as the interface stiffness.

The key to our computational scheme is the following. It is the stiffness, not $g$ alone, that controls the amplitude of fluctuations, and even though the anisotropy in $g$ is very small, the anisotropy in stiffness is very appreciable (on the order of $30 \%$ between 100 and 110). Therefore, by comparing the fluctuation spectrum about the 100 and 110 crystal orientation, we can extract the orientation dependence of the solid-liquid interfacial energy. We applied the method to simulations of pure nickel $(\mathrm{Ni})$ and copper $(\mathrm{Cu})$ using interatomic potentials based on the embedded atom method (EAM) and were able to compute, for the first time, an anisotropy of just $3.8 \%$ for $\mathrm{Ni}$ and $2.7 \%$ for $\mathrm{Cu}$. Future simulations will compute the anisotropy in other elements important in brazing, namely gold $\mathrm{Au}$ ) and silver $(\mathrm{Ag})$, and we shall extend the approach to the case of binary alloys starting with $\mathrm{Cu}-\mathrm{Ni}$.
We implemented an algorithm that can accurately track the interface position during molecular dynamics (MD) simulation. 
10304

\section{A Combinatorial Microlab Investigation of Critical Copper-Corrosion Mechanisms}

J. C. Barbour, J. W. Braithwaite, W. G. Breiland, N. A. Missert, J.

P. Sullivan, K. R. Zavadil, A. F. Wright

An important aspect in developing the capability to predict stockpile reliability is a physics-based understanding of atmospheric corrosion. Corrosion modeling is hindered by limited knowledge of primary mechanisms and large numbers of coupled chemical reactions, which depend on complex interactions of materials with environment and functionality. This multidimensional problem requires fundamentally new experimental approaches that can provide timely quantitative information on critical phenomena occurring in corrosionphase space. Sandia is combining parallel miniature experimentation with ultrasensitive microanalytical techniques to efficiently explore this phase space and to identify mechanisms and kinetics for copper $(\mathrm{Cu})$ sulfidation in the "Microdomain Laboratory." This approach differs from convention by focusing on microscopic length scales, the relevant scale for corrosion. Combinatorial experiments (arrays of microlabs) will quantify the direct and synergistic effects of morphological and metallurgical variables (alloying, defect density in the Cu oxide and bulk, diffusivities, porosity), environmental variables (sulfur content in air, light exposure, water-droplet size, and distribution versus humidity), and functionality (e.g., electric-current conduction). Novel diagnostics include conductivity microsensors to locally quantify $\mathrm{pH}, \mathrm{pO}_{2}$, and $\mathrm{pH}_{2} \mathrm{~S}$, in situ electrical conductivity, and light scattering to monitor real-time evolution of corrosion reactions, local ion-selective potentiometric monitoring of reaction products, and ultimately microcalorimetry sensors. This project enables a new and rapid approach to determine physical models for complex chemical processes of key importance to DOE technology needs.

The results highlighted this year are (1) the development of a stagnation cell reactor for more controlled sulfidation and better-understood gas-flow geometry, (2) the measurement of $\mathrm{Cu}$ diffusivity in $\mathrm{Cu}_{2} \mathrm{~S}$ (copper sulfide) via markers that showed that $\mathrm{Cu}$ was the fastest-diffusing species over a wide range of relative humidity $(\mathrm{RH})$, and (3) the development of potentiometric microsensors. To characterize the potential effect of gas-phase mass transport, we measured
This project enables a new and rapid approach to determine physical models for complex chemical processes of key importance to DOE technology needs. 
and mathematically modeled sulfidation kinetics using two reactor configurations with well-defined flow characteristics: a laminar-flow tubular reactor and a direct-impingement "stagnation-flow" reactor whose design purpose was to minimize gas-phase mass transport control. We drew several conclusions from this work: (1) first-order kinetics were indicated from monitoring $\mathrm{H}_{2} \mathrm{~S}$ (hydrogen sulfide) outlet concentration, and (2) the sulfidation reaction appeared to be nearly, but not quite, limited by gas-phase diffusion. We then performed marker experiments and showed that $\mathrm{Cu}$ was the dominant diffusing species through $\mathrm{Cu}_{2} \mathrm{~S}$ in $0.5 \% \mathrm{RH}$ and $80 \%$ $\mathrm{RH}$ atmospheres containing 150 parts per billion (ppb) $\mathrm{H}_{2} \mathrm{~S}$. More important, we found that thicker layers of $\mathrm{Cu}_{2} \mathrm{~S}$ were formed at $0.5 \% \mathrm{RH}$ than at $80 \% \mathrm{RH}$ because the growth kinetics never became solid-state diffusion-limited for the low ...we found that thicker layers of $\mathrm{Cu}_{2} \mathrm{~S}$ were formed at $0.5 \% \mathrm{RH}$ than at $80 \%$ RH because the growth kinetics never became solid-state diffusion-limited for the low RHs (up to 40 hours of exposure). RHs (up to 40 hours of exposure). For high RH, we determined the $\mathrm{Cu}$ diffusivity to be $10^{-16} \mathrm{~cm}^{2} /$ second when the growth of the $\mathrm{Cu}_{2} \mathrm{~S}$ layer became solid-state diffusion-limited. In addition, we performed water-layer distribution experiments and determined that techniques with resolution better than 1 $\mathrm{mm}$ will be required to map the water distribution as a function of $\mathrm{RH}$. We developed microelectrode arrays and made $\mathrm{pH}$ measurements on thin, continuous water layers on a $\mathrm{Cu}_{2} \mathrm{O} / \mathrm{Cu}$ surface. We then demonstrated electrodeposition of ionselective media on the microelectrode array surfaces. Further, we made real-time $\mathrm{pH}$ changes using a pulsed dissolved $\mathrm{O}_{2}$ (dioxygen) scheme.

\section{Refereed}

Barbour, J. C., J. P. Sullivan, J. W. Braithwaite, N. Missert, J. S. Nelson, R. G. Dunn, K. G. Minor, G. Copeland, and S. Lucero. 1999. "A Combinatorial Approach to Determine Mechanisms of Atmospheric Copper Sulfidation." Corrosion and Reliability of Electronic Mater. and Devices, The Electrochem. Soc. Proc. Series, PV 99-29, edited by R. B. Comizzoli, R. P. Frankenthal, and J. D. Sinclair, Pennington, NJ. SAND99-2620C.

Barbour, J. C., J. W. Braithwaite, and A. F. Wright. 2000. "Determination of Solid-State Sulfidation Mechanisms in Ion-Implanted Copper." Nucl. Instru. and Meth. in Phys. Res. B, Proc. Ion Beam Modification of Mater. Conf. 2000.
Barbour, J. C., J. W. Braithwaite, and N. R. Sorensen. 2000. "A Marker Experiment to Identify the Dominant Moving Species in Atmospheric Copper Sulfidation." Appl. Phys. Lett. (October).

\section{Other Communications}

Barbour, J. C., J. W. Braithwaite, J. P. Sullivan, N. Missert, W. G. Breiland, J. S. Nelson, and K. R. Zavadil. 2000. "A Combinatorial Microlab Investigation of Atmospheric Copper Sulfidation." (Invited) Paper presented to the Spring 2000 Materials Research Society Meeting, San Francisco, CA, 24-28 April.

Campin, M. A., J. H. Zhu, and J. C. Barbour. 1999. "Transmission Electron Microscopy Investigation of Copper and Copper Oxides." Paper presented to the 11th Annual Rio Grande Regional Symposium on Advanced Materials, Albuquerque, NM, 11 October. 
10305

Self-Assembled Templates for Fabricating Novel Nanoarrays and Controlling Materials Growth

A. K. Schmid, M. E. Bartram, P. J. Feibelman, N. C. Bartelt, K. R. Zavadil, C. J. Brinker, N. A. Missert

Novel and exciting phenomena that have the potential to revolutionize science, materials, and next-generation production methods are manifested as structural dimensions approach the nanometer level. However, advances in lithography are insufficient to achieve this feature scale. As a result, molecular self-assembly has attracted a great deal of interest since this provides a possible spontaneous mechanism by which nanometer-sized arrays can be formed without mechanical intervention. Although these "low-tech" processes are highly dependent on system/material specifics, Sandia believes that new techniques and the science that underlies them can be developed in a manner that allows us to extend the natural order of self-assembling systems. We based our approach on previous work in which we fabricated templates of unprecedented size and regularity. We will then apply these templates to form nanoarrays based on a wide range of materials with tunable properties and nanoscale selective area overgrowth patterns for improving the materials quality of thin films.

We achieved the ability to tailor the nanostructure array periodicity and symmetry in the $\mathrm{Ag} / \mathrm{Ru}(0001)$ (silver/ ruthenium) system by supersaturation of the Ag film. This produces different and controllable levels of strain that lead to the arrays by exposure to sulfur $(\mathrm{S}) . \mathrm{SiO}_{2}$ (silicon dioxide) films deposited via sol-gel processes on sapphire and $\mathrm{Si}(100)$ substrates were characterized with $\mathrm{x}$-ray photoelectron spectroscopy (XPS) as a function of both azimuthal detection angle and temperature and with scanning electron microscopy (SEM). The results suggest strongly that these films are not well suited for selective area growth (SAG) templating as we originally envisioned. Although contrary to previous SEM measurements in which the film surfaces were thought to be featureless, our XPS and SEM measurements are consistent with the presence of large openings in the thin films (nonuniform wetting) having dimensions on the order of microns over one-fourth to one-half of the surface. We synthesized highly ordered mesoporous silica films with
We achieved the ability to tailor the nanostructure array periodicity and symmetry in the Ag/Ru (0001) (silver/ruthenium) system by supersaturation of the Ag film. This produces different and controllable levels of strain that lead to the arrays by exposure to sulfur $(S)$. 
variable pore size without organic swelling agents. The structure-directing agents were amphiphilic polystyrene-blockpoly(ethylene oxide) (PS-b-PEO) diblock copolymers.

\section{Refereed}

Fan, H. Y., Y. F. Lu, A. Stump, S. T. Reed, T. Baer, R. Schunk, V. Perez-Luna, G. P. Lopez, and C. J.

Brinker. 2000. "Rapid Prototyping of Patterned

Functional Nanostructures.” Nature 405 (4 May): 56-

60.

Lu, Y. F., H. Y. Fan, N. Doke, D. A. Loy, R. A. Assink, D. A. La Van, and C. J. Brinker. 2000.

"Evaporation-Induced Self-Assembly of Hybrid Bridged Silsesquioxane Film and Particulate Mesophases with Integral Organic Functionality." $J$. Amer. Chem. Soc. 122 (7 June): 5258-5261. 
10306

Wetting and Spreading Dynamics of Solder and Braze Alloys

G. L. Kellogg, R. Q. Hwang, N. D. Shinn, G. S. Grest, E. B. Webb, III, D. R. Jennison, J. J. Stephens, Jr., F. M. Hosking

The purpose of this project is twofold: (1) to develop a scientific understanding of the microscopic processes that control wetting and spreading dynamics in materials systems related to soldering and brazing, and (2) to design improved active braze alloys by investigating the thermodynamic aspects of reactive wetting of eutectic silver copper ( $\mathrm{AgCu}$ ) alloys on ceramic substrates. The first task exploits new advances in experimental surface techniques and atomistic modeling methods to study wetting and spreading phenomena on length scales that range from angstroms to microns. Sandia is using the unique imaging capabilities of the low-energy electron microscope (LEEM) and scanning-tunneling microscope (STM) to characterize the interfacial region when materials such as lead $(\mathrm{Pb})$ and tin ( $\mathrm{Sn})$ are deposited on Cu surfaces. We also use LEEM to observe the spreading behavior of small molten droplets on fully characterized metal surfaces. We use results from these studies to test the validity of atomic potentials being developed to simulate the dynamics of wetting and flow. We currently have accurate, empirical potentials that can be applied in large-scale simulations involving $\mathrm{Pb}, \mathrm{Cu}$, and $\mathrm{Ag}$. In the second task we will develop new active-metal-braze alloy compositions based on thermodynamic considerations and test the proposed materials for improved performance. The goal is to achieve strong, hermetic seals in the brazing of kovar to alumina. The two tasks are merging with our recent successful LEEM studies of model alumina oxides grown on NiAl (nickel aluminum) substrates and investigations of empirical potentials for Sn, vanadium (V), and titanium (Ti).

Fundamental investigations of active-braze materials on model alumina surfaces under well-controlled conditions and realistic simulations will provide a science-based approach for the design of improved, active-braze materials.

We extended our LEEM investigations of $\mathrm{Pb}$ interactions with $\mathrm{Cu}$ surfaces to the (111) crystal plane and discovered remarkable behavior during submonolayer film growth. Two distinct phases (a surface alloy and a $\mathrm{Pb}$ overlayer phase) are present in the submonolayer coverage regime. With increasing $\mathrm{Pb}$ coverage and at constant temperature, we

\author{
Fundamental investigations of \\ active-braze materials on model \\ alumina surfaces under well- \\ controlled conditions and \\ realistic simulations will provide \\ a science-based approach for \\ the design of improved, active- \\ braze materials.
}


observed a progression through a series of domain structures (droplets to stripes to inverted droplets), verifying an earlier theory of domain evolution in two-phase systems with dipolar interactions. We grew 3-D Pb islands on top of the overlayer and compared their melting behavior to $\mathrm{Pb}$ on $\mathrm{Cu}(100)$. We continued our LEEM studies of alloying dynamics for the system of $\mathrm{Sn}$ on $\mathrm{Cu}(111)$ and discovered a novel process in which alloying proceeds by the migration of large $(100,000$ atom) clusters of $\mathrm{Sn}$ on the surface. We developed accurate, empirical potentials that can be applied in large-scale simulations for $\mathrm{Pb}, \mathrm{Cu}$, and $\mathrm{Ag}$, and are simulating the wetting of $\mathrm{Pb}$ on $\mathrm{Cu}$ and $\mathrm{Ag}$ on $\mathrm{Cu}$. We are also investigating potentials for $\mathrm{Sn}, \mathrm{V}$, and $\mathrm{Ti}$, which will be used to more directly address active solder and braze systems. We acquired, through collaboration with Louisiana State University, a massively parallel (MP) code for performing wetting simulations of metals on oxide surfaces. For experimental studies of metal wetting and flow on oxide surfaces, we produced uniform alumina films of self-limiting thickness by oxidizing $\mathrm{NiAl}(110)$ surfaces. We characterized the domain structure of the model oxides with the LEEM. We designed and tested new active $\mathrm{Cu}$ / $\mathrm{Ag} / \mathrm{Zr}$ (zirconium) active-braze alloys that demonstrated improved performance in the joining of kovar to alumina. Currently, we are preparing a technical advance for a family of braze alloys containing $\mathrm{Cu}_{10} \mathrm{Zr}_{7}$ and associated compounds and a $\mathrm{Cu}-\mathrm{Ag}$ alloy.

\section{Refereed}

Kellogg, G. L., and R. A. Plass. 2000. "Low-Energy Electron Microscope Investigations of $\mathrm{Pb}$ Film Growth on Cu Surfaces." (Invited) Paper presented to the 47th International Symposium of the American Vacuum Society, Boston, MA, 2-6 October.

Kellogg, G. L., and R. A. Plass. 2000. "Low-Energy Electron Microscope Investigations of Surface Alloying and De-Alloying: Pb on $\mathrm{Cu}(100)$." Paper presented to the 60th Annual Physical Electronics Conference, Baton Rouge, LA, 21-23 June.
We continued our LEEM studies of alloying dynamics for the system of $\mathrm{Sn}$ on $\mathrm{Cu}(111)$ and discovered a novel process in which alloying proceeds by the migration of large (100,000 atom) clusters of Sn on the surface. We developed accurate, empirical potentials that can be applied in large-scale simulations for $\mathrm{Pb}, \mathrm{Cu}$, and $\mathrm{Ag}$, and are simulating the wetting of $\mathrm{Pb}$ on $\mathrm{Cu}$ and $\mathrm{Ag}$ on $\mathrm{Cu}$.
Kellogg, G. L., and R. A. Plass. 2000. "MesoscopicScale Observations of Surface Alloying, Surface Phase Transitions, Domain Coarsening, and 3-D Island Growth: $\mathrm{Pb}$ on $\mathrm{Cu}(100)$." (Invited) Surf. Rev. and Lett., Proc. U.S.-Japan Seminar on Mesoscopic Scale Phenomena on Surfaces 7 (Park City, UT, 2-8 April).

Kellogg, G. L., and R. A. Plass. 2000. "The Relationship Between the Growth Shape of ThreeDimensional $\mathrm{Pb}$ Islands on $\mathrm{Cu}(100)$ and the Domain Orientation of the Underlying c $(5 * 2 \mathrm{x} * 2) \mathrm{R} 45$ Structure." Surface Sci. Lett., accepted. 
Plass, R. A., and G. L. Kellogg. 2000. "Low-Energy Electron Microscope Investigations of Submonolayer $\mathrm{Pb}$ Film Growth on $\mathrm{Cu}(100)$ and $\mathrm{Cu}(111)$." Paper presented to the 2nd LEEM/PEEM Workshop, Paris, France, 26-28 September.

Plass, R. A., and G. L. Kellogg. 2000. "Surface Morphology Changes During Pb Deposition on $\mathrm{Cu}(100)$ : Evidence for Surface Alloyed $\mathrm{Cu}(100)$ c(2x2) Pb." Surface Sci., accepted.

Plass, R. A., J. Last, N. C. Bartelt, and G. L. Kellogg. 2000. "The Evolution of Self-Organized Domain Structures: $\mathrm{Pb}$ on $\mathrm{Cu}(111)$." Science, submitted.

Plass, R. A., N. C. Bartelt, and G. L. Kellogg. 2000. "Direct Observations of Ordered Domain Structures and their Dynamics: $\mathrm{Pb}$ on $\mathrm{Cu}(111)$." Paper presented to the 47th International Symposium of the American Vacuum Society, Boston, MA, 2-6 October.

Plass, R. A., N. C. Bartelt, and G. L. Kellogg. 2000. "Direct Observations of Self-Assembled Domain Structures and Their Dynamics: $\mathrm{Pb}$ on $\mathrm{Cu}(111)$." (Invited) Paper presented to the 2000 Fall Meeting of the Materials Research Society, Boston, MA, 27-30 November.

Schmid, A. K., N. C. Bartelt, and R. Q. Hwang. 2000. "Alloying at Surfaces by the Migration of Reactive Two-Dimensional Islands." Science, accepted.

Schmid, A. K., N. C. Bartelt, and R. Q. Hwang. 2000. "Bronze Formation and the Motion of Mesoscopic Tin Clusters on a Copper Surface." (Invited) Surf. Rev. and Lett., Proc. U. S.-Japan Seminar on Mesoscopic Scale Processes on Surfaces 7 (Park City, UT, 2-8 April).
Schmid, A. K., N. C. Bartelt, and R. Q. Hwang. 2000. "Bronze Formation as an Engine for the Motion of 100,000 Atom Tin Clusters on Copper Surfaces." (Invited) Paper presented to the Materials Research Society Spring Meeting, San Francisco, CA, April.

Schmid, A. K., N. C. Bartelt, and R. Q. Hwang. 2000. "Bronze Formation Through the Motion of Tin Islands on $\mathrm{Cu}(111)$." Paper presented to the 47th International Symposium of the American Vacuum Society, Boston, MA, 2-6 October.

Schmid, A. K., N. C. Bartelt, and R. Q. Hwang. 2000. "Kinetics of Alloying at Surfaces: The Mechanism of Bronze Formation by Sn Deposition onto $\mathrm{Cu}(111) . "$ Paper presented to the Fall Meeting of the Materials Research Society, Boston, MA, 27-30 November.

Webb, III, E. B., and G. S. Grest. 2000. "Large-Scale Molecular Dynamics Simulations of Liquid Metal Wetting and Spreading." Paper presented to the Materials Research Society Annual Spring Meeting, San Francisco, CA, April.

Webb, III, E. B., and G. S. Grest. 2000. "Liquid/ Vapor Surface Tension of Metals: Embedded Atom Method with Charge Gradient Corrections." Phys. Rev. Lett., submitted.

Webb, III, E. B., and G. S. Grest. 2000. "Surface Tension of Liquid Metals: Predictions of Embedded Atom Method with Charge Gradient Corrections." Paper presented to the American Physical Society Annual March Meeting, Minneapolis, MN, March. 
10307

\section{Improved Materials-Aging Diagnostics and Mechanisms Through 2-D Hyperspectral Imaging Methods and Algorithms}

D. M. Haaland, D. K. Melgaard, C. A. Drewien, M. C. Celina, E. V. Thomas, C. M. Wehlburg, F. W. Koehler, C. M. Boney, J. C. Wehlburg

Twenty years ago, Sandia developed new quantitative multivariate spectral analysis methods that caused a quantum leap in the capabilities of quantitative spectroscopy over previous univariate methods. We have now achieved a more significant revolution in the capabilities of quantitative spectral analyses. By combining the massive amounts of data generated using new commercial two-dimensional (2-D) infrared (IR) imaging spectrometers with the Sandia invention of powerful new hyperspectral information extraction algorithms, we developed the ability to perform quantitative spectroscopy without the need for calibration standards. Thus, we can now rapidly obtain 2-D spectral images of aging polymeric and energetic materials and directly extract purecomponent spectra of all spectrally active chemical species in samples, which include the spectra of degradation products. This information, combined with our new hyperspectral analysis algorithms, allows us to obtain accurate compositional maps of materials with 10-micrometer spatial resolution. This new work has directly impacted materialsdegradation diagnostics by distinguishing multiple aging mechanisms and by greatly improving IR sensitivity and chemical selectivity. The chemical specificity and spatial mapping of 2-D IR spectroscopy will allow quantification of degradation products to help us understand degradation mechanisms, kinetics, and diffusion.

The methods developed during this project led to the concept of using similar techniques for designing and constructing a new multifluorophore microarray scanner for improved functional genomics studies. Microarray technology can be used to develop new biosensors for biowarfare agent detection and for understanding the genetic basis of cancer. We also initiated the development of the new microarray scanner using Sandia's existing information-efficient spectral imaging system (ISIS) that was originally used for satellite hyperspectral imaging.
The methods developed during this project led to the concept of using similar techniques for designing and constructing a new multifluorophore microarray scanner for improved functional genomics studies. Microarray technology can be used to develop new biosensors for biowarfare agent detection and for understanding the genetic basis of cancer. 
We submitted two patent applications on the new algorithms. We identified the source of the problem with the prediction phase of our new proprietary hybrid algorithm. With this knowledge, we generated three new algorithms to correct the problem so that the true prediction performance of the algorithm is vastly improved without performing timeconsuming recalibration. We submitted a technical advance detailing these three new improved algorithms. We also tested the proposed methods for extrapolating hyperspectral image spectral data to pure-component spectra using two different types of samples, one of which involves aged neoprene samples with weapons applications. The commercially available pixel-purity-index method is very slow to compute and does not provide satisfactory results for the more difficult aged-polymer sample. The Simplisma and alternating constrained least-squares multivariate-curve-resolution (MCR) methods yield similar pure-component spectral estimates, which have yielded new insights into the oxidative degradation of neoprene polymer samples. The Simplisma algorithm is the faster of the two methods, but the MCR method is expected to be more general. We can combine the Simplisma and MCR techniques by making the Simplisma results the initial estimates for the iterative MCR routine. This procedure improves the convergence performance of MCR and decreases computation times. We programmed the Simplisma and MCR methods in MATLAB ahead of schedule and made progress in combining spatial and spectral information in the images. We also developed new methods for reducing the detrimental effects of both pathlength variations in the samples and correlated spectral errors in the hyperspectral image data.

In the new augmented program to develop a new multifluorophore hyperspectral microarray scanner, we made significant progress, particularly in several areas, to reconfigure the ISIS spectrometer to facilitate imaging of deoxyribonucleic acid (DNA) microarrays. We converted ISIS to a small-sample imager, obtained a scanning stage, and began to reconfigure the ISIS handshake with the stage. We calibrated the spectrometer for wavelength and intensity and collected data to characterize the spectrometer stability (wavelength, temperature, intensity). Finally, we collected initial data on a test sample of Rhodamine 590 dye and bar target to begin to characterize the spectrometer's imaging capabilities and limitations.
In the new augmented program to develop a new multifluorophore hyperspectral microarray scanner, we made significant progress, particularly in several areas, to reconfigure the ISIS spectrometer to facilitate imaging of deoxyribonucleic acid (DNA) microarrays. 


\section{Refereed}

Haaland, D. M., and D. K. Melgaard. 2000. "A New Classical Least-Squares/Partial Least-Squares Hybrid Algorithm for Spectral Analyses." Appl. Spectroscopy, accepted.

Haaland, D. M., and D. K. Melgaard. 2000. "New Prediction-Augmented Classical Least-Squares (PACLS) Methods: Application to Unmodeled Interferants." Appl. Spectroscopy 54 (September): 1303-1312.

\section{Other Communications}

Haaland, D. M., and D. K. Melgaard. 1999.

"Accounting for Unmodeled Spectral Interferences in the Quantitative Prediction of Unknown Samples Using a New CLS/PLS Hybrid Multivariate Calibration Method." Paper presented to the Federation of Analytical Chemistry and Spectroscopy Societies, Vancouver, BC, Canada, 24-29 October.

Haaland, D. M., and D. K. Melgaard. 1999. "A New CLS/PLS Hybrid Algorithm for Improved Multivariate Calibration Capabilities." Paper presented to the Federation of Analytical Chemistry and Spectroscopy Societies, Vancouver, BC, Canada, 24-29 October.

Haaland, D. M., and D. K. Melgaard. 1999. "Prediction-Augmented Classical Least-Squares Methods for Maintaining Calibrations on a Rapidly Drifting Spectrometer." Paper presented to the Federation of Analytical Chemistry and Spectroscopy Societies, Vancouver, BC, Canada, 24-29 October.

Haaland, D. M., and D. K. Melgaard. 2000. "Use of Random Numbers to Improve Classical Least-Squares Predictions: Violating the Garbage In-Garbage Out Rule." Paper presented to the Federation of Analytical Chemistry and Spectroscopy Societies, Nashville, TN, 25-30 September.

Haaland, D. M., C. M. Wehlburg, and D. K. Melgaard. 1999. "Addition of Known Spectral Shapes to a New CLS/PLS Hybrid Algorithm to Improve the Capabilities of Multivariate Spectral Calibrations." Paper presented to the Eastern Analytical Symposium and Exposition, Somerset, NJ, 14-19 November.
Haaland, D. M., C. M. Wehlburg, and D. K. Melgaard. 2000. "New Multivariate Calibration Algorithms for Improved Quantitative Spectral Analyses." Paper presented to the Nicolet Instrument Corporation, Madison, WI, 15-16 May.

Haaland, D. M., F. W. Koehler, and M. C. Celina. 2000. "Multivariate Methods Applied to Hyperspectral FT-IR Imaging for Quantitative Analysis." Paper presented to the SPIE Bios 2001 Spectral Imaging Conference, San Jose, CA, January.

Haaland, D. M., F. W. Koehler, E. V. Thomas, and M. C. Celina. 2000. "Multivariate Calibration Applied to Hyperspectral FT-IR Imaging: Integrating Spectral and Spatial Information." Paper presented to the Federation of Analytical Chemistry and Spectroscopy Societies, Nashville, TN, 25-30 September.

Haaland, D. M., F. W. Koehler, E. V. Thomas, M. C. Celina, and M. R. Keenan. 2000. "Multivariate Calibration for Improved Analysis of Hyperspectral Images." Paper presented to the Pittsburgh Conference, New Orleans, LA, 12-17 March.

Koehler, F. W., and D. M. Haaland. 1999.

"Quantitative Determination of 2-D Hyperspectral Image Data." Paper presented to the Federation of Analytical Chemistry and Spectroscopy Societies, Vancouver, BC, Canada, 24-29 October.

Koehler, F. W., D. M. Haaland, and M. Celina. 2000. "Quantitative Determination of 2-D FT-IR Imaging Polymer Data." Paper presented to the Pittsburgh Conference, New Orleans, LA, 12-17 March.

Koehler, IV, F. W., M. C. Celina, and D. M. Haaland. 2000. "Quantitative Analysis Without Standards of EPDM Polymer Degradation Products by FT-IR Spectroscopy." Paper presented to the Eastern Analytical Symposium and Exposition, Atlantic City, NJ, 29 October-3 November. 
Melgaard, D. K., and D. M. Haaland. 2000.

"Concentration Residual Augmented Classical Least-

Squares: Part I. Algorithm Description and

Demonstration Using Simulated Data." Paper

presented to the Federation of Analytical Chemistry

and Spectroscopy Societies, Nashville, TN, 25-30

September.

Melgaard, D. K., D. M. Haaland, and C. M. Wehlburg. 2000. "Concentration Residual Augmented Classical Least-Squares: Part II. A Classical Least-SquaresType Method with Prediction Ability Comparable to PLS." Paper presented to the Federation of Analytical Chemistry and Spectroscopy Societies, Nashville, TN, 25-30 September. 
10308

\section{Microscale Shock-Wave Physics Using Photonic Driver Techniques}

R. E. Setchell, A. V. Farnsworth, Jr., W. M. Trott

The goal of this project is to establish a new capability for conducting shock-wave physics experiments at low perexperiment costs through a reduction in experimental length and time scales. Based on the earlier development of laserdriven optical detonators at Sandia, this new capability utilizes the rapid absorption of optical energy from a large, $Q$-switched, solid-state laser to accelerate a small, planar disk of a material of interest (a launch process known as photonic driving). The disk (or "flyer") subsequently impacts another material, generating shock waves in both materials having amplitudes and durations that depend on the two materials, the impact velocity, and the disk thickness. Subnanosecond interferometric diagnostics were developed previously to examine the motion and impact of laser-driven flyers. Conducting "microscale" shock physics experiments poses significant challenges in terms of flyer and target fabrication and the means to ensure controlled, well-defined shock-loading conditions. To address a broad range of materials and stress states, photonic driving levels must be scaled up considerably from optical detonator conditions. However, progressively increasing challenges in terms of laser-induced damage mechanisms in optics, thermally driven phase transitions and instabilities in laser-driven materials, and beam-shaping requirements accompanies the desire to scale up driving levels.

We addressed both practical applications of the capability established previously and the extension of photonic driving levels to two orders of magnitude above optical detonator conditions. We examined design options for flyer assemblies that we simulated using a CTH-based hydrocode. The first practical application of interest is to examine the shock response of polycrystalline silicon ( $\mathrm{Si}$ ). Working with individuals in Sandia's thin-film deposition group, we examined various ways to fabricate appropriate flyer assemblies for these studies. Material compatibility and process temperature issues led us to an approach that involves the hightemperature deposition of a 4-micron layer of Si on a 30micron-thick layer of molybdenum (Mo) previously deposited on an optical substrate. We utilized masking and selective etching to produce separate islands of the polysilicon/Mo
The goal of this project is to establish a new capability for conducting shock-wave physics experiments at low perexperiment costs through a reduction in experimental length and time scales. 
structures, each representing a "flyer" for a shock experiment. Residual stress effects are still an issue in these structures, but we have launched prototypes and believe that we are close to achieving the first shock Hugoniot measurements on polysilicon. A second application is to conduct validation experiments using flyers made from materials having wellestablished shock properties. We pursued methods for fabricating small flyers made from 6061-T6 aluminum (Al), a material that has been extensively studied under shock loading. We emphasized the fabrication of $0.8 \mathrm{~mm}$-diameter, $0.1 \mathrm{~mm}$ thick flyers suitable for "first-year" driving levels, as these are more challenging and require better process controls than the larger flyers to be used at higher levels. We progressed from making a few disks at a time with poor thickness control to making up to twenty disks at a time, diffusion-bonded onto an optical substrate, with very good thickness control.

To achieve our goal for photonic driving levels, we designed a new diffractive beam-shaping optic for open-air delivery specifically for the lasers used in this project. Commercial fabrication of this device is still in progress. However, we used a similar diffractive element designed for a different application for tests at the higher driving levels. Even though this device was not optimized for our lasers, we achieved successful launching of $2.5 \mathrm{~mm}$-diameter, $0.1 \mathrm{~mm}$ thick $\mathrm{Al}$ flyers at conditions very close to our goal.

Finally, we used the CTH-based hydrocode originally developed for optical detonators to examine the effects of varying the composition and thickness of multicomponent launch layers. We first performed one-dimensional (1-D) calculations to quickly assess the effects of these parameters on the strength and persistence of waves reverberating within the flyer and on the temperature profiles within the flyer at times near the end of the acceleration process. These were followed by more detailed 2-D calculations that added the important effects of material yielding and plasma escape at the flyer boundaries. We will next perform comparative experiments utilizing spatially resolved diagnostics ("line VISAR" [velocity interferometry system for any reflector]).

\section{Other Communications}

Trott, W. M., R. E. Setchell, and A. V. Farnsworth, Jr. 2000. "Investigation of the Effects of Target Material Strength on the Efficiency of Acceleration of Thick
We utilized masking and selective etching to produce separate islands of the polysilicon/Mo structures, each representing a "flyer" for a shock experiment. Residual stress effects are still an issue in these structures, but we have launched prototypes and believe that we are close to achieving the first shock Hugoniot measurements on polysilicon.
Laser-Driven Flyers." Proc. APS Shock Compression of Condensed Matter-1999, Part 2 (Snowbird, UT, 27 June-2 July 1999): 1203-1206. 
10309

\section{Reactivity of Metal-Oxide Surfaces}

A. G. Sault, B. S. Swartzentruber, D. R. Jennison, J. A. Ruffner, R.

Q. Hwang

Sandia is using a combination of experimental and theoretical techniques to explore atomic-scale defect chemistry on technologically important surfaces, with the goal of determining the salient factors that control defect chemistry. The formation, mobility, reactivity, and dissolution of atomicscale surface defects play important roles in materials areas such as catalysis, corrosion, thin-film growth, semiconductor processing, sensors, and magnetic devices. Important defects include single adatoms, surface steps and kinks, dislocations, and vacancies. It is generally believed that a small number of localized surface defects may be particularly reactive and thereby dominate the surface chemistry of many materials. While past studies of this important problem have been limited by an absence of appropriate experimental probes for imaging atomic-scale defects, the recent advent of specialized scanning probe microscopies (SPM) largely overcomes this problem and now allows more detailed studies of defect chemistry than previously possible. The unique atom-tracking scanningtunneling microscope (AT-STM) recently developed at Sandia is a particularly powerful new technique that allows measurement of atomic-scale kinetic processes on a time scale that is more than three orders of magnitude faster than previous techniques. Similarly, a lack of sufficient computing power has severely limited previous theoretical studies of defect properties. The recent development of massively parallel (MP) computational techniques at Sandia, which now allow calculations on systems containing hundreds of atoms per unit cell, will enable studies of surface defects with a level of detail and sophistication far greater than previously possible. By studying well-defined, well-ordered surfaces, we can greatly simplify the study of defect chemistry and obtain detailed fundamental information on defect formation and reactivity. Coupling experimental results with theoretical studies will allow the development of models that explain, predict, and ultimately help to control surface-defect reactivity.

We studied the effect of oxygen $(\mathrm{O})$ flow during deposition on the reactivity of $2000 \AA$-oriented $\mathrm{Mg}_{3}\left(\mathrm{VO}_{4}\right)_{2}$ films. Infrared (IR) spectra show a progressive transition from the fully oxidized state to a reduced $\mathrm{Mg}_{3} \mathrm{~V}_{2} \mathrm{O}_{6}$ phase as $\mathrm{O}$ flow
The unique atom-tracking scanning-tunneling microscope (AT-STM) recently developed at Sandia is a particularly powerful new technique that allows measurement of atomicscale kinetic processes on a time

scale that is more than three orders of magnitude faster than previous techniques....By studying well-defined, wellordered surfaces, we can greatly simplify the study of defect chemistry and obtain detailed fundamental information on defect formation and reactivity. 
decreases. X-ray photoelectron spectroscopy studies show increasing reactivity toward reduction by propane as the films become more $\mathrm{O}$ deficient. This reactivity can be mitigated by oxidation treatments prior to reduction. Thus, by combining control of $\mathrm{O}$ levels during deposition with appropriate pretreatment, we can tailor the reactivity of these films toward molecules of interest for oxidative dehydrogenation (ODH). Comparison of the behavior of oriented films with amorphous films reveals higher reactivity for the oriented films, providing the first evidence ever reported for structure sensitivity for mixed metal-oxide catalysts. We devoted substantial effort to attempts to measure catalytic activity for ODH on these films. Due to extremely low surface areas and low specific activity, these efforts have been unsuccessful to date.

When palladium $(\mathrm{Pd})$ atoms are deposited onto a $\mathrm{Cu}(001)$ (copper) surface, they readily place exchange with the surface $\mathrm{Cu}$ atoms, forming a 2-D surface alloy phase. At low coverage, $\mathrm{Pd}$ atoms are distributed randomly in the surface layer. At coverages approaching 0.5 ML (monolayer), an ordered (2x2) alloy phase forms. Using AT-STM, we directly measured the diffusion of $\mathrm{Pd}$ atoms as a function of temperature. The position of individual $\mathrm{Pd}$ atoms is recorded as a function of time with millisecond resolution, thus recording the diffusion path of the incorporated atom. From these data, we determine the relative binding energies of the sites visited by the atom, as well as the local diffusion barriers. We find that a large percentage $(\sim 25 \%)$ of the diffusion events involve displacements greater than a single lattice spacing-so-called "long jumps." The distribution of jump lengths is well described by a process in which the Pd atoms diffuse via exchange with the equilibrium population of surface vacancies. Strained thin films often contain ordered networks of misfit dislocations that determine their chemical and mechanical properties. We consider reaction of sulfur $(\mathrm{S})$ with two-monolayer films of $\mathrm{Cu}$ on $\mathrm{Ru}(0001)$ (ruthenium). These films contain a network of parallel partial dislocations, with threading edge dislocations where partial dislocations meet. S reacts with the threading dislocations and dissociates them. The increase in threading dislocation density is accommodated by modifying the dislocation network. The induction period of the reaction between $\mathrm{Cu}$ and $\mathrm{S}$ was visualized with STM. Copper sulfide formation started only after adsorption of $0.2 \mathrm{ML}$ of $\mathrm{S}$ on a two-atom-thick $\mathrm{Cu}$ layer. $\mathrm{S}$ decorated the $\mathrm{Cu}$ surface by adsorbing at pseudo fourfold hollow sites of the $\mathrm{Cu}$ phase and restructured the $\mathrm{Cu}$ layer to create additional adsorption sites.
Comparison of the behavior of oriented films with amorphous films reveals higher reactivity for the oriented films, providing the first evidence ever reported for structure sensitivity for mixed metal-oxide catalysts. 
We observed several self-organized structures of adsorbed S on the strained $\mathrm{Cu}$ layer. Significant structural changes of both adsorbate and adsorbant occurred within the induction period of this surface chemical reaction.

\section{Refereed}

de la Figuera, J., K. Pohl, A. K. Schmid, N. C. Bartelt, J. Hrbek, and R. Q. Hwang. 1999. "Multiplication of Threading Dislocations in Strained Metal Films Under Sulfur Exposure.” Surface Sci. 435 (2 August): 93-98.

Hrbek, J., J. de la Figuera, K. Pohl, T. Jirsak, J. A. Rodriguez, A. K. Schmid, N. C. Bartelt, and R. Q. Hwang. 1999. "A Prelude to Surface Chemical Reaction: Imaging the Induction Period of Sulfur Interaction with a Strained Cu Layer." J. Phys. Chem. $B 103$ (48) (2 December): 10557-10561. 
10310

\section{Exploiting LENS ${ }^{\mathrm{TM}}$ Technology Through Novel Materials}

\section{J. A. Brooks}

Laser-Engineered Net Shaping (LENS $\left.{ }^{T M}\right)$ is a directfabrication process in which metal powders are deposited into a laser-melted pool, with succeeding layers being deposited to build up complex engineering shapes. This process is a rapid, low-cost, low-footprint direct-fabrication technique that lends itself to the concept of advanced manufacturing. However, previous work developed LENS ${ }^{T M}$ as an advanced manufacturing tool rather than exploiting its potentially unique attributes. These attributes include real-time control of microstructure, tailored material properties at different locations in the same part, the production of graded thermal expansion parts, etc. In this project Sandia characterized the important metallurgical parameters, solidification velocity, and cooling, and developed process models that are being used as input to microstructural models. We used tool steels and stainless steels as model systems to demonstrate the concept and feasibility of utilizing the high solidification velocities and cooling rates to design microstructures and properties to meet functionality. We developed process maps for controlling residual stresses and melt-pool size that are critical for controlling part dimensions and quality. We also showed that starting powder characteristics can greatly affect build quality. Limited work on ceramic materials indicates that novel material can be fabricated using this technology. This work has added a new dimension in designing and fabricating engineering components with novel microstructures and properties using this rapid prototyping technology.

We designed and fabricated austenitic stainless steels to determine if single-phase microstructures, rather than the twophase structures reported in the literature, could be achieved. We used solidification and microstructural models, along with thermodynamic base programs, to design alloys that produced a single-phase microstructure using two different solidification and transformation sequences. We achieved single-phase microstructures directly as a product of solidification. We also achieved nearly ferrite-free microstructures as a product of a solid-state transformation. This microstructure was more sensitive to cooling rates, controlled largely by part configuration and small variations in alloy composition.
This work has added a new dimension in designing and fabricating engineering components with novel microstructures and properties using this rapid prototyping technology. 
However, the fine-grain microstructure was very homogeneous with no measurable microsegregation. Extensive electron microscopy studies indicated that the solid-state transformation from ferrite to austenite was likely diffusive rather than massive in nature. This work, along with studies on tool steels in which we used carbide coarsening and process models to predict microstructure and hardness, has clearly demonstrated the power of using process and microstructural models to design novel materials to meet engineering requirements.

We also developed process maps using thermomechanical models for the fabrication of thin-walled structures of stainless steel. We used temperature predictions obtained from a thermal model as inputs to a mechanical model to predict residual stress. These models can be used to determine optimal process variables for obtaining consistent melt-pool lengths while limiting residual stresses. We also validated by using 304 stainless steel that substrate preheating, as predicted by the model, significantly reduces residual stresses. The lower residual stresses are due primarily to the reduction in yield strength at the preheat temperature, although the reduction in thermal gradients with preheating provides some additional contribution.

Work on ceramic materials showed that the materials could be melted and deposited using the laser process. However, we observed cracking when we attempted multiple passes of material. We could deposit single lines of titanium (Ti) on aluminum oxide (AlO) substrates with success, but again it was not possible to build full three-dimensional (3-D) structures. Adding ceramic powders to Ti also yielded success in making a metal matrix composite. We successfully added three different materials $\left(\mathrm{Al}_{2} \mathrm{O}_{3}, \mathrm{BN}, \mathrm{SiC}\right)$ to $\mathrm{Ti}$ in amounts up to $20 \%$ by volume. We envision that the development of this technology would allow for an engineer to design regions of a component to be stiffer by the addition of these ceramic powders. Additional work would be required to determine if higher-volume fractions of ceramics could be deposited with a metal matrix.

A very important finding is that porosity within the initial starting feed powder is a primary source of porosity in the final build. This results even though the particles are completely melted during the LENS ${ }^{\mathrm{TM}}$ process. A statistical study in which we characterized different starting powders using imaging analysis techniques showed that a large range in porosity can exist with different powder lots. In four different lots of 304L stainless-steel powder, the measurable porosity
This work, along with studies on tool steels in which we used carbide coarsening and process models to predict microstructure and hardness, has clearly demonstrated the power of using process and microstructural models to design novel materials to meet engineering requirements. 
ranged from over $5 \%$ to only $\sim 0.1 \%$. Direct correlations were obtained between the porosity content of the starting powder and the porosity content of the final LENS ${ }^{\mathrm{TM}}$-fabricated structures. However, we established no measurable correlation between build porosity and LENS ${ }^{\mathrm{TM}}$ processing conditions. In one high-strength steel the starting powder contained 5\% porosity, and that of the build was $\sim 3.7 \%$. In this case, porosity content severely reduced tensile ductility. These results show that powder specifications for LENS ${ }^{\mathrm{TM}}$ processing must include not only particle size and morphology, but also internal porosity.

\section{Refereed}

Brooks, J. A., T. J. Headley, and C. V. Robin. 2000.

"Microstructures of Laser-Deposited 304L Austenitic Stainless Steel." Symp. Y, Net Shape Processing by Additive Processes, Proc. Mater. Res. Soc. 1 (San Francisco, CA, April): 1.

Griffith, M. L. 2000. "Laser-Engineered Net Shaping." Chapter 9, Handbook of Rapid Prototyping and Layered Manufacturing: Technologies, Fundamentals, and Applications, edited by Ming Leu, San Diego, CA.

Griffith, M. L., M. T. Ensz, J. D. Puskar, C. V. Robino, L. A. Brooks, J. A. Philliber, J. E. Smugeresky, and W. H. Hofmeister. 2000. "Understanding the Microstructure and Properties of Components Fabricated by Laser-Engineered Net Shaping (LENS ${ }^{\mathrm{TM}}$ )." Symp. Y, Net Shape Processing by Additive Processes, Proc. Mater. Res. Soc. Mtg. 1 (San Francisco, CA, April): 1.

Susana, D. F., J. D. Puskara, J. A. Brooks, and C. V. Robino. 2000. "Porosity in Stainless-Steel LENS" Powders and Deposits." Proc. Solid Freeform Fabrication Symp. 20001 (Austin, TX, August): 1.
Vasinonta, A., J. L. Beuth, and M. L. Griffith. 2000. "A Process Map for Consistent Build Conditions in the Solid Freeform Fabrication of Thin-Walled Structures." J. Manuf. Sci. and Eng., accepted.

Vasinonta, A., J. L. Beuth, and M. L. Griffith. 2000. "Process Maps for Controlling Residual Stress and Melt Pool Size in Laser-Based SFF Processes." Proc. Solid Freeform Fabrication Symp. 20001 (Austin, TX, August): 1.

\section{Other Communications}

Griffith, M. L. 2000. "The Development of Residual Stress in LENS ${ }^{\text {TM }}$ Fabrication." Paper presented to the Mineral, Metals, and Materials Society Conference, Nashville, TN, March.

Robino, C. V., J. A. Brooks, T. J. Headley, R. Dykhuizen, and M. L. Griffith. 2000. "Microstructural Modeling of H-13 LENS ${ }^{\text {TM }}$ Deposits." Paper presented to the Mineral, Metals, and Materials Society Conference, Nashville, TN, March. 


\section{1}

\section{Molecular Characterization of Energetic- Material Initiation}

A. M. Renlund, A. S. Tappan, J. C. Miller

Sandia has responsibility for a diverse mix of energetic materials (EMs) and components. Determining microscale chemical and physical responses of EMs to abnormal environments is fundamental to understanding the safety of these EMs. The explosive HMX (1,3,5,7-tetranitro-1,3,5,7tetraazacyclooctane) is an EM used in many applications and components for which Sandia is responsible. The hightemperature polymorph (designated $\delta$-HMX) is of special concern due to our recent discovery that it may control reaction progress for thermal initiation. The solid-solid-phase transition of HMX is dependent on pressure and temperature, with high pressures favoring the less-sensitive $\beta$-HMX and high temperatures favoring the more-sensitive $\delta$-HMX. We believe that we have established a clear link between the HMX $\beta$ - $\delta$ phase transition and increased reactivity, sensitivity to initiation, and subsequent burning. However, sampling techniques used in our preliminary tests are not amenable to most component configurations. We are developing an in situ probe to monitor the phase transition using Raman spectroscopy to distinguish the phases. Probe development and incorporation of the probe into our experimental apparatus are the main focus of this effort. Following development of the experimental probe, we will study the phase behavior of HMX at elevated temperatures and pressures relevant to cookoff environments. Understanding the rate and extent of the solidsolid-phase transition as functions of confinement, pressure, temperature, particle size, and density at time of cookoff is vital to improving our safety analyses of the material. In addition to the forward-phase transition, which occurs as HMX is heated, the reverse-phase transition, which occurs as HMX is cooled, is of great interest. A third phase, designated $\alpha$ $H M X$, also more sensitive than b-HMX, can form upon cooling of $\delta$-HMX. We observed $\beta$-, $\delta$-, and $\alpha-H M X$ in post-mortem analysis of samples, frequently observing two phases in the same sample. We will apply this experimental development to monitor the kinetics of the phase transition and how the phase transition affects subsequent decomposition and cookoff.

We conducted initial studies on HMX in what is called the "displacement-controlled" variation of our experiment, in
We believe that we have established a clear link between the $H M X \beta-\delta$ phase transition and increased reactivity, sensitivity to initiation, and subsequent burning. 
which we confined the HMX sample axially at relatively constant displacement. Because the expansion that accompanies and allows the phase transition was heavily inhibited in this configuration and did not occur in much of the sample, the phase transition was undetectable by Raman spectroscopy. For this reason, we decided to modify the experiment to a "load-controlled" configuration, in which the pressure experienced by the HMX sample was held at a relatively constant value for the duration of the experiment. This arrangement allowed the pellet to expand, and therefore a greater proportion of the sample could undergo the phase transition. In this new configuration, we conducted a series of very successful experiments in which we heated HMX samples to a temperature above that which induces the phase transition, held it for a time, and then cooled it at a controlled rate. The controlled variable in these experiments was the value of the constant pressure at which the sample was confined during a single experiment. These experiments provided critical data on both the $\delta$ - to $\beta$-HMX "forward"-phase transition and the $\delta$ - to $\beta$-HMX and $\delta$ - to $\alpha$-HMX "reverse"-phase transitions. We constructed pressure-temperature diagrams for both the forward- and reverse-phase transitions, which led to several conclusions. First, because Raman spectroscopy samples only a small portion of the pellet, there was considerable scatter in the data. A comparison of expansion data, a rough approximation of phase transition, and Raman data revealed that trends obvious in the expansion data were not as apparent in the Raman data. Second, the reverse-phase transition data showed a greater correlation coefficient than the data for the forwardphase transition, indicating that this anomalous behavior may have been caused by greater inconsistencies experienced in the heating cycle than those experienced in the cooling cycle. We determined conclusively that, even in a very small HMX sample, we saw very different phase-transition characteristics throughout the sample. Post-mortem analysis revealed that within the same sample, multiple phases of HMX were present. We assumed this to be due to the thermal and pressure gradients experienced by the sample during an experiment and to have important implications in real-world cookoff scenarios. Because of efforts necessary to modify the experimental apparatus to accommodate load-controlled experiments, we deemed the incorporation of second harmonic generation into the experiment to be less important and not completed. In addition, the conflicting results in HMX studies precluded expanding the experiments to include HMX-containing plastic- ...we conducted a series of very successful experiments in which we heated HMX samples to a temperature above that which induces the phase transition, held it for a time, and then cooled it at a controlled rate....These experiments provided critical data on both the $\delta$ - to $\beta$-HMX "forward"phase transition and the $\delta$ - to $\beta$ $H M X$ and $\delta$ - to $\alpha-H M X$ "reverse"-phase transitions. 
bonded explosives. The successful development of a Raman spectroscopy probe and incorporation of it into our experimental apparatus have provided us with a valuable tool to improve our safety analyses of this material.

\section{Other Communications}

Tappan, A. S., A. M. Renlund, and J. C. Miller. 2000.

"LDRD Final Report: Raman Spectroscopic Measurements to Monitor the HMX $\beta-\delta$ Phase Transition." Sandia Technical Report SAND20002927, Sandia National Laboratories, Albuquerque, NM (October).

Tappan, A. S., A. M. Renlund, J. H. Gieske, and J. C. Miller. 2000. "Real-Time Raman Spectroscopic and Ultrasonic Measurements to Monitor the HMX $\beta-\delta$ Phase Transition and Physical Changes in Thermally Damaged Energetic Materials." Proc. 2000 JANNAF Propulsion Sys. Hazards Subcommittee Mtg.

(Monterey, CA, November).
The successful development of a Raman spectroscopy probe and incorporation of it into our experimental apparatus have provided us with a valuable tool to improve our safety analyses of this material. 
10312

\section{Nonvolatile Protonic Memory}

R. A. Anderson, C. H. Seager, A. G. Sault, B. L. Draper, G. V. Herrera, T. D. Dunbar, H. D. Stewart

Sandia discovered a new principle for memory devices in which the polarization state in the oxide layer of silicon/ silicon dioxide/silicon ( $\mathrm{Si} / \mathrm{SiO} / \mathrm{Si}$ ) structures can be changed by an applied electric field. This sandwich is readily fabricated by conventional processing into field-effect transistor (FET) devices that respond to the oxide polarization. Because of their simplicity, process compatibility, and apparent radiation hardness, such devices may be suited to low-power, pseudononvolatile memory applications. We were tasked with understanding the memory phenomenon to facilitate its optimization and to resolve fabrication issues. A crucial step is a $600^{\circ} \mathrm{C}$ hydrogen $(H)$ anneal, during which $H$ enters the oxide around the periphery of the polysilicon cap. We attribute memory to the field-induced drift of mobile protons that are presumed to be created during this step. Device-to-device variability remains problematic, as does the length of time required to write a memory state, currently several seconds with roughly $50 \mathrm{~nm}$-thick oxide layers. Neither the H-anneal processing step nor the physics underlying memory activity are well understood, and the central assumption of mobile protons remains controversial. We studied capacitor structures that we processed similarly to FET devices used in a complementary device-oriented effort at the University of New Mexico (UNM). Our specimens permitted direct measurements of the interfacial electric field that controls FET memory devices, and we developed a variety of automated voltage-dependent capacitance measurements that probed the oxide polarization in response to applied electric fields. We characterized memory-retention stability and oxide thickness effects in memory-writing rates by using these methods, and we continued to seek evidence for isotopic-mass shifts in the polarization rates of specimens annealed in deuterium (D) or $H$. We also investigated a new experimental technique in which we exposed specimens to electron and ion irradiation while holding them at fixed bias voltage. These experiments revealed charge-trapping behavior that affected the memory-writing process, and the results may help to resolve the physics of the memory effect.
Sandia discovered a new principle for memory devices in which the polarization state in the oxide layer of silicon/silicon dioxide/silicon ( $\left.\mathrm{Si} / \mathrm{SiO}_{2} / \mathrm{Si}\right)$ structures can be changed by an applied electric field. 
Our investigations concentrated on characterizing and understanding the memory effect in protonated buried-oxide devices, and we made good progress, although the underlying physics remains elusive. We again compared polarization rates in D- and H-treated capacitors and again found no indication of true mass-dependent carrier motion, although isotopic effects may influence the $\mathrm{H}$-anneal processing step. We investigated memory-retention stability and found that the polarization state written by applying negative gate bias has long-term stability under powered-down (zero gate bias) conditions. However, the contrasting state written with positive gate bias slowly relaxes after the bias is dropped to zero. We found similar behavior in FET structures fabricated in the device effort at UNM. We estimate that a practical memory-retention period would be tens of hours if the memory circuitry were operated at room temperature, and less at higher operating temperatures. The major accomplishments came from tests to implant protons directly into the oxide of our capacitor structures. These experiments, combined with electron-beam irradiation, provided new methods of studying charge transport in the oxide layer of our capacitors. Electrons that enter the oxide layer with a few hundred $\mathrm{eV}$ of remnant energy create a cloud of electrons and holes that can selectively be driven deeper with applied bias. We discovered that applying a negative gate voltage during electron irradiation caused the post-treatment memory-writing behavior to be shifted by an amount that depended on the applied voltage and found the shifts to be reversible with further electron irradiation at other voltages. The curves appear to be rigidly shifted, as might be expected from the superposition of a fixed internal field arising from trapped space charge. Details in these results appeared to contradict some simple ideas of proton motion. In the protonimplantation tests, we used an ion energy that was just able to penetrate the capping polysilicon layer, which presumably reduced the remnant energies in the oxide below the lattice damage threshold. Our intent was to create memory activity in nonprotonated capacitor structures, but we found no combination of conditions that resulted in memory activity. By contrast, implanting protons into a memory-active capacitor permanently reduced the activity. This latter method, when used on specimens that have been prepared in different polarization states, may constitute a sensitive probe of proton motion that could test the mobile proton hypothesis. We acquired a small amount of such data. Our continued efforts in numerical transport modeling emphasized a need for true three-
The major accomplishments came from tests to implant protons directly into the oxide of our capacitor structures. These experiments, combined with electron-beam irradiation, provided new methods of studying charge transport in the oxide layer of our capacitors. 
dimensional codes, due to the coarse spacing of individual charge carriers, to achieve realism. We addressed effects of process variations by testing capacitor structures on a new wafer from the FET device effort at UNM. We found the rates of polarization change in these new specimens with $40 \%$ thinner buried-oxide layers to be closely similar, at comparable oxide fields, to our previous results from specimens having approximately $70 \mathrm{~nm}$-thick oxides. Oxide layers that are substantially thinner than these, however, would have been needed to rule out beneficial oxide-thickness effects. We also found that applying fields that approached the oxide breakdown strength did not greatly accelerate the writing process. On the device side, we discovered that appropriately designed FET gates can switch current well beyond logic levels, which could enable low-power solid-state switches with built-in memory.

\section{Refereed}

Devine, R. A. B., and G. V. Herrera. 2000. "Electric

Field-Induced Transport of Protons in Amorphous

$\mathrm{SiO}_{2}$ Films." Phys. Rev. B, submitted.

Devine, R. A. B., and G. V. Herrera. 2000. "Retention and Switching Kinetics of Protonated Gate FieldEffect Transistors." J. Appl. Phys. 88 (1 September): 2862-2867.

Devine, R. A. B., K. Vanheusden, and G. V. Herrera. 2000. "Correlation of Mobile and Fixed-Charge Creation in Protonated Field-Effect Transistors." Appl. Phys. Lett. 77 (24 July): 579-581. ...we discovered that appropriately designed FET gates can switch current well beyond logic levels, which could enable low-power solid-state switches with built-in memory. 
10734

\section{Next-Generation Output-Based Process} Control: An Integration of Modeling, Sensors, and Intelligent Data Analysis

R. A. Neiser, Jr., D. M. Haaland, M. F. Smith, F. W. Spencer, E. V. Thomas, R. C. Dykhuizen

The goal of process-based quality is to accept War Reserve (WR) product without inspection. For simple processes, traditional feedback control of process input variables might achieve this goal. However, input-based (upstream) control systems are a major source of Defense Program (DP) production problems for complex processes, because it is impractical, if not impossible, to control all of the factors that affect process output. With recent advances in sensor technology, process modeling, intelligent data analysis, and computing power, a broadly applicable next-generation control technology that monitors and controls process output rather than input may now be possible.

Substantial technical challenges must be overcome to realize this goal. For real-time, output-based (downstream) process control, it is necessary to rapidly interpret and correctly respond to large amounts of temporally and spatially varying data, which requires an innovative new approach to data analysis. Highly complex relationships between process inputs and outputs must also be correctly modeled, and costeffective robust sensors must be developed. In addition, relationships between sensor observations and product microstructure, properties, and performance must be understood and integrated into the control system. Sandia developed an algorithm that could substantially simplify the amount of data required to effect real-time process control. We will test this new algorithm. If successful, we anticipate that the algorithm will be implemented in a WR production system for flame-spraying neutron generators (NGs).

For some complex processes, e.g., thermal spray, a first-generation downstream process controller based on inflight monitoring and control of the spray plume may be achievable. For example, we already have considerable expertise in state-of-the-art diagnostic sensors and process modeling. To extract needed information from large, rapidly varying, complex data in real time, we will build upon concepts developed for Chem-Lab-on-a-Chip to create a new statistical methodology that combines response surface modeling with multivariate statistical process control (SPC).
With recent advances in sensor technology, process modeling, intelligent data analysis, and computing power, a broadly applicable next-generation control technology that monitors and controls process output rather than input may now be possible. 
A significant decision was to choose to develop an output-based feedback-control algorithm for the wire-fed flame-spray process. A key reason is that the DP impact of a successful control scheme will be high. Flame spray is used to coat NGs at Sandia, and recent production problems demonstrate that feedback control of particle temperature and velocity will greatly increase the robustness of the process.

A major accomplishment is the development of a novel, simple-to-use control algorithm that we believe can successfully provide output-based feedback control in many manufacturing processes, not just in the field of thermal spray. The approach is titled "A Simplex Method Adapted for Systems with Noisy Function Evaluations." This type of control methodology is new, and we are submitting a technical advance.

Using a new sensor that is inexpensive enough for use in many production systems, we constructed and analyzed process maps for two different flame-sprayed wires. These data indicate that we can use variations in gas flows to compensate for drifts in particle temperature and velocity that occur during the process. Adjusting gas flows to correct for drifts is desirable because they are easy to adjust on a real-time basis.

Additional work to identify the sources of process drift is under way. Output-control algorithms can compensate for drifts only up to a certain point. Beyond this point, the source of the drift has to be identified and corrected. We identified two significant sources of process drift. The first is associated with nozzle hardware and orientation within the flame-spray device. Swapping out hardware can lead to significant changes in particle temperatures and velocities. A second source is wire feedstock variation. We found that nominally identical wires from the same vendor as well as vendor-to-vendor variations can also cause substantial variations in particle characteristics. A primary goal is to test whether the Simplex Method can use gas-flow adjustments to dynamically compensate for these two sources of process instability.

\section{A major accomplishment} is the development of a novel, simple-to-use control algorithm that we believe can successfully provide output-based feedback control in many manufacturing processes. ... 
10754

\section{Functional Materials for}

\section{Electrochemomechanical Actuation of}

\section{Microvalves and Micropumps}

\section{L. Whinnery, Jr., S. H. Goods, D. A. Chinn, M. J. Stevens, J. S.} Korellis

It is generally recognized that integrated microsystems for Defense Program (DP) applications, chemical sensing, and medical devices will not be possible without development of efficient microactuators that can be used as valves and pumps. Conventionally actuated devices affect the operating characteristics of microsystems and cannot be miniaturized without significant loss in performance. Sandia's approach is to take advantage of electrochemomechanical (ECM) functions of conductive polymers and use them to produce work in microactuators. We are tailoring molecular structure and composition of conductive polymer gels to maximize physical/ mechanical response to electrochemical stimulation. Much of our initial work focused on the synthesis and mechanical characterization of polymer gels. ECM response (extension, force generation, response rate) of conductive polymer gels will continue to be systematically characterized with respect to applied potential, temperature, and gel-electrode geometry. The computational aspects of this project continue; specifically, calculation of the dependence of osmotic pressure on the charging of conducting polymers is of continuing interest. Finally, we will design and test polymer actuator geometry and microelectrodes to optimize microscale performance, including response time, displacement, and force. Our principal goals are (1) to obtain fundamental understanding of structure/property/response relationships of conductive polymers with respect to ECM phenomena, and (2) to build a prototypical ECM-based valve that is inexpensive, easily manufactured, and adaptable to integrated microsystems.

We synthesized and conducted preliminary testing on thiophene-based polymer gels by a variety of methods to control the sample size and shape as well as polymer morphology. We determined that there is a fourfold decrease in gel particle size by changing polymerization solvents from chloroform to acetonitrile. We developed an electrochemical cell that consists of a hollow polypropylene porous frit that contains the conductive polymer gel and allows for the free
We are tailoring molecular structure and composition of conductive polymer gels to maximize physical/mechanical response to electrochemical stimulation. 
flow of ions and solvent while permitting expansion of the gel in the vertical direction. We further developed a test apparatus that allows for the systematic characterization of force, displacement, and response rate of gel samples. This apparatus has sufficient precision to measure $<0.1$ micron of displacement and $<0.1$ gram of mechanical load while the gel specimen is immersed in the electrochemical solution. We can simultaneously record potential (V), displacement/force, temperature, and current, and can thus compute the power required to actuate the gel to full expansion. We successfully demonstrated the ECM function of a gel system when it is subjected to a $+0.8 /-0.5 \mathrm{~V}$ square waveform having a 2-, 10-, or 20-minute period. With a 20-minute period under these conditions, the gel exhibits a maximum swell of $2 \%$ per 10 minute expansion interval $(+0.8 \mathrm{~V})$ and a stable response over 20 hours. Under fixed displacement conditions, samples generate upwards of $3 \mathrm{kPa}$ of pressure when at full expansion. We developed and built new electrodes with increased surface area and increased contact area with the gel specimens. These new electrodes dramatically decrease damage to the gel and reduce internal gel stresses generated during cell assembly.
We further developed a test apparatus that allows for the systematic characterization of force, displacement, and response rate of gel samples. This apparatus has sufficient precision to measure $<0.1$ micron of displacement and $<0.1$ gram of mechanical load while the gel specimen is immersed in the electrochemical solution.

\section{Refereed}

Irvin, D. J., S. H. Goods, and L. L. Whinnery. 2000.

"Direct Measurement of Extension and Force in Conductive Polymer Gel Actuators." Chemistry of Mater., submitted. 
10761

\section{Making the Connection Between Microstructure and Mechanics}

E. A. Holm, C. C. Battaile, T. E. Buchheit, G. W. Wellman

The purpose of microstructural control is to optimize materials properties. To that end, Sandia has developed sophisticated and successful computational models of both microstructural evolution and mechanical response. However, there is currently no way to couple these models to quantitatively predict the properties of a given microstructure.

This problem arises because continuum response models, such as finite-element (FE), finite-volume, or material point methods, do not incorporate a real length scale. Thus, two self-similar polycrystals have identical mechanical properties regardless of grain size, in conflict with theory and observations.

In this project, we take a tiered-risk approach to incorporate microstructure and its resultant length scales in mechanical response simulations. Techniques considered will include low-risk methods that are guaranteed to work (albeit with shortcomings) as well as higher-payoff, higher-risk methods. The initial set of methods will include incorporating a local length-scale parameter in the constitutive response model, direct simulation of a grain-boundary phase, straingradient polycrystal plasticity, and a subgrain breakup model. We will validate these techniques on a variety of systems for which theoretical analyses and/or experimental data exist, including tension and bending experiments on bicrystals and polycrystals. The results can be used to generate improved constitutive models that explicitly depend on microstructure and to provide insight into microstructural deformation and failure processes.

Because mechanical state drives microstructural evolution, a strain-enhanced grain growth model will be added to the mechanical response simulation. The successful coupled model will predict both properties as a function of microstructure, and microstructural development as a function of processing conditions.

Incorporating microstructure into mechanical response simulations will provide a new tool for scientific insight and model development in materials science, engineering science, and process design and control.

\author{
Incorporating microstructure \\ into mechanical response \\ simulations will provide a new \\ tool for scientific insight and \\ model development in materials \\ science, engineering science, \\ and process design and control.
}


We compiled and prioritized a list of candidate methods

for including a physical length scale in polycrystal plasticity

finite-element (PPFEM) calculations. The initial method chosen was a hardness gradient method, with a single, adjustable-length-scale parameter related to a boundary influence width. We developed and implemented a constitutive model incorporating a hardness gradient near grain boundaries in Sandia's JAS3D PPFEM code. Parametric tests indicate that this model qualitatively reproduces grain-size effects in polycrystalline materials. We are quantitatively testing this model in tension and bending configurations.

Concurrent with plasticity modeling, we developed tools for simulating strain-enhanced microstructural evolution. We generated a library of representative three-dimensional grain structures. We coupled a grain growth model that performs front-tracking motion on boundary nodes with PPFEM codes. We also modeled the effects of elastic and plastic strain on boundary motion and incorporated them into the front-tracking code. We observed a broad range of responses, from texture development to recrystallization, in various strain regimes.

We shifted project focus to include a greater emphasis on physics-based models for plasticity in polycrystalline materials.

\section{Refereed}

Battaile, C. C., E. A. Holm, T. E. Buchheit, G. W. Wellman, and M. K. Neilsen. 1999. "Coupled Simulations of Mechanical Deformation and Microstructural Evolution Using Polycrystal Plasticity and Monte Carlo Potts Models." Proc. Mater. Res. Soc. Symp. 538 (Boston, MA, December): 269-273.

Battaile, C. C., T. E. Buchheit, E. A. Holm, G. W. Wellman, and M. K. Neilsen. 1999. "Simulating Microstructural Evolution in Deformed Polycrystals." (Invited) Paper presented to the TMS Fall Meeting, Cincinnati, $\mathrm{OH}$, November.
We developed and implemented a constitutive model incorporating a hardness gradient near grain boundaries in Sandia's JAS3D PPFEM code. Parametric tests indicate that this model qualitatively reproduces grain-size effects in polycrystalline materials.
Buchheit, T. E., and G. W. Wellman. 1999. "Predicted Evolution of a Flow Surface by a Polycrystal Deformation Model." Paper presented to the Materials Research Society Fall Meeting, Boston, MA, December.

Buchheit, T. E., D. A. La Van, G. W. Wellman, and M. K. Neilsen. 1999. "Comparison Between Simulation and Experiment of a Polycrystal Plasticity Deformation Model." Paper presented to the TMS Fall Meeting, Cincinnati, OH, November. 
10779

\section{Switchable Hydrophobic-Hydrophilic}

\section{Surfaces}

B. C. Bunker, M. S. Kent, J. G. Curro, R. P. Manginell, S. H.

Kravitz, D. L. Huber, J. G. Kushmerick

The ability to perform chemical and biological analyses

on a microchip requires understanding how to transport, separate, and detect species in nanoliter quantities of liquid confined in channels having dimensions of microns. At such small length scales, surface effects play a dominant role. If smart coatings could be developed whose surface properties and chemical interactions could be switched in a controlled fashion, it might be possible to create complex analytical surfaces within a simple architecture of interconnected channels. We showed that fluids can be pumped and mixed in microchannels by manipulating whether channel surfaces attract or repel water. Switching of interfacial energies could also be used to control selective adsorption for advanced separations and sensor devices.

The objective of this project is to investigate mechanisms for switching the interfacial energies of organic coatings using phase transitions inducted via on-chip stimuli. For example, the all-trans-to-helical transition in polyethylene oxide (PEO) transforms a hydrophobic, protein-adsorbing surface into a hydrophilic surface that repels proteins. Similarly, changes in matter contact angle sufficient to pump liquids in microchannels are known to accompany the reversible collapse transition in poly(n-isopropylacrylamide) (PNIPAM).

The work involves (1) attaching polymers known to undergo a hydrophobic-hydrophilic transition to substrate surfaces, (2) examining various mechanisms for stimulating the desired phase transition (via temperature, applied voltages, or light), (3) characterizing conformation changes in polymers and the impact of transitions on surface energies, (4) modeling chain conformations, the kinetics of phase transformations, and surface energies using Monte Carlo and molecular dynamics (MD) simulations, and (5) fabricating and using microchannels for direct measurement of film switching and droplet motion. The impact of the project will be to establish protocols for the development of switchable organic coatings for pumps, valves, adsorbers, and sensors in microanalytical systems.
The objective of this project is to investigate mechanisms for switching the interfacial energies of organic coatings using phase transitions inducted via on-chip stimuli....The impact of the project will be to establish protocols for the development of switchable organic coatings for pumps, valves, adsorbers, and sensors in microanalytical systems. 
We attached PEO to gold ( $\mathrm{Au}$ ) substrates via an alkane thiol terminated with three ethylene glycol units (called EG3). PEO was attached to silicon ( $\mathrm{Si}$ ) substrates via thermal condensation of a multi-armed "star" PEO and by reacting single PEO chains with chlorinated Si surfaces.

We prepared PNIPAM films on Si via grafting existing polymers to surfaces functionalized with self-assembled monolayers (SAMs) and via surface-initiated free-radical polymerization in which the initiator was attached to a SAM.

All switching of polymer film conformations to date has involved thermal stimulation. We heated several modifications of PEO films above the helical-amorphous phase transition at $60^{\circ} \mathrm{C}$. All PEO films examined so far exhibit irreversible switching due to decomposition (EG3) or changes in chain entanglement and surface morphology ("star" PEO). PNIPAM films exhibit reversible switching at $35^{\circ} \mathrm{C}$, with a water contact angle change of as much as $25^{\circ}$. We used neutron-reflectivity experiments to monitor swelling and collapse of surface films for temperatures on either side of the We performed MD simulations to help calculate solvation potentials to be used in selfconsistent polymer reference interaction site models (PRISM). We then used PRISM to model the phase transition in PNIPAM transition temperature.

We performed MD simulations to help calculate solvation potentials to be used in self-consistent polymer reference interaction site models (PRISM). We then used PRISM to model the phase transition in PNIPAM for chains containing up to 80 monomers.

We developed a flexible modular design for driving liquids with thermally switched films in which Au wires are deposited on thin silicon nitride $(\mathrm{SiN})$ membranes to minimize undesired thermal gradients. 
10780

\section{Nanostructured Materials Integrated in Microfabricated Optical Devices}

D. Y. Sasaki, M. E. Warren, S. A. Kemme, A. R. Burns, S. M. Brozik, M. B. Sinclair, C. J. Brinker

This project will combine the emerging strength of nanocomposite materials at Sandia with the recognized expertise and processing capabilities involving microfabricated optical-device structures for the development of microsensor arrays and unique tunable microoptical devices. The project will involve the design, development, and characterization of molecular-(nanoscale) to micron-scale structures to achieve desired material and device function. At the core of the material is a liquid crystalline $(L C)$ lipid bilayer that mimics the properties of a biological cell membrane. The bilayer is functionalized with molecular receptors, for specific recognition of chemical and biological species, and with optical reporters that signal the occurrence of the recognition event. These molecular and supramolecular structures will be supported on a structurally oriented, mesoporous, inorganic matrix. The matrix offers structural stability and physical protection of the LC materials and provides a platform for forming dense LC structures for greater signal output. Together the materials form a unique nanocomposite with molecularly dynamic organic components for rapid optical response to specific analytes coupled to a robust inorganic scaffold with highly defined and tunable surface area and pore volume. Integration of the nanocomposites to microfabricated optical devices will occur in two stages. In the first stage, we will couple the materials onto substrate-mode platforms for combinatoric sensing arrays. The second stage will then further the materials integration by forming the material into an optical structure yielding devices that can tune their properties through chemical signaling. Multiple materials and optics issues must be addressed to successfully merge these disparate research areas. Results from this work alone will make great strides in creating new micron-scale sensing arrays for biopathogens and combinatorial approaches to materials and drug discovery, and unique microfabricated optical structures with rapid and tunable switching properties.

We focused our efforts on developing methods to form and characterize bilayer films on surfaces, lipid bilayer opticalsensor materials, and photodefinable nanocomposite materials.
This project will combine the emerging strength of nanocomposite materials at Sandia with the recognized expertise and processing capabilities involving microfabricated optical-device structures for the development of microsensor arrays and unique tunable microoptical devices....Results from this work alone will make great strides in creating new micron-scale sensing arrays for biopathogens and combinatorial approaches to materials and drug discovery, and unique microfabricated optical structures with rapid and tunable switching properties. 
We prepared lipid bilayers on mica surfaces using a vesicle fusion technique and monitored the bilayer formation by atomic force microscopy (AFM) in a solution cell. To improve bilayer stability on solid surfaces, we prepared tethering molecules by multistep synthetic procedures to provide (1) a lipid-mimetic structure for facile membrane incorporation, (2) a long hydrophilic spacer to allow for a hydration layer between the membrane and the substrate, and (3) activated headgroup for covalent coupling to the substrate. We prepared a new optical-sensor film functionalized with 18-crown-6 receptors and evaluated it with a range of cations. The material exhibited a selective response for $\mathrm{Pb}$ (II) (lead) with detection limits at sub-ppm (parts per million) level. AFM imaging of this lipid bilayer could readily distinguish the aggregated receptors in the phosphatidylcholine membrane. Photodefinable nanocomposite materials were also under development with polydiacetylene and photoacid-catalyzed mesostructured sol-gel materials. With the polydiacetylene films, we were able to induce colored transitions through photo-illumination and chemical and physical stress. We generated refined optical structures using a combination of photolithography with thermal treatment. We also achieved gray-scale patterning with photoacid generators in silica sol-gels. Ultraviolet (UV) illumination of the sol catalyzed the condensation of the exposed regions of the film. The crosslink density increased with increasing exposure time, causing syneresis in those areas, film shrinkage, and a concomitant increase in index of refraction. Furthermore, the index of refraction could be tuned by varying the UV exposure times.

\section{Refereed}

Sasaki, D. Y., and T. A. Waggoner. 2000. "SurfaceCompatible Sol-Gel Materials for Immobilized Lipid Bilayers." Paper presented to the American Chemical Society Spring 2000 National Meeting, Washington, DC, March. SAND99-2933A.

Sasaki, D. Y., J. A. Last, and T. A. Waggoner. 2000. "AFM Imaging of Molecular Reorganization in Lipid Bilayers Induced Through Ion Recognition.” Paper presented to the American Chemical Society Spring 2000 National Meeting, Washington, DC, March. SAND99-2936A.
We generated refined optical structures using a combination of photolithography with thermal treatment. We also achieved gray-scale patterning with photoacid generators in silica sol-gels. 
10789

\section{All-Ceramic Thin-Film Battery}

T. J. Boyle, D. Ingersoll, R. T. Cygan, J. A. Voigt, M. A. Rodriguez, J. A. Ruffner, K. Rahimian

The production of a thin-film all-ceramic battery (ACB) that has power sources that generate the necessary power and energy to operate microsystems without substantially increasing the size of the device is required to achieve autonomy. Thin-film batteries are an attractive power source for these applications; however, currently they are inadequate to operate the microsystems effectively. Sandia intends to develop a rechargeable thin-film lithium (Li)-ion ACB using solution route deposition methods. The ACB will have higher power output, longer run times, and a greatly simplified production scheme in comparison to existing technology. Early goals are to produce a working cathode of oriented $\mathrm{LiCoO}_{2}$, an anode of $\mathrm{Sn}^{\circ} / \mathrm{SnO}_{2}$ (tin/tin-oxide) and an electrolyte consisting of either a silicon ( $\mathrm{Si}$ ) polymer or Li-Ln ceramic oxide with low resistivities. Once sufficiently developed, these ACBs will have the versatility to power any number of small systems, such as microsystems and miniature autonomous robotics.

We generated the individual components, which include the cathode $\left(\mathrm{LiCoO}_{2}\right)$, the anode $\left(\mathrm{SnO} / \mathrm{Sn}^{\circ}\right)$, and the electrolyte $\left(\mathrm{LiLn}\left(\mathrm{PO}_{3}\right)_{4}\right)$. We developed novel or commercially available precursors and used them to generate the precursor solutions for production of the individual components of the ACB. We prepared individual precursor solutions under controlled atmospheres. We synthesized the individual layers and characterized them prior to combining them to ensure that the optimized material will be used. We made all the films by spin-coating or drop-coating methods under ambient atmospheric conditions.

We generated films of the $\mathrm{SnO}_{2}$ anode using $\mathrm{Sn}(\mathrm{OAr})_{2}$ compounds that we synthesized in our laboratory. The properties of the anode are currently under investigation but should prove to be acceptable. Processing produces only the oxide, whereas some $\mathrm{Sn}^{\circ}$ is desirable; however, this is not critical since $\mathrm{Sn}^{\circ}$ will form after the first cycle. We are investigating alternative processing gases or incorporation of $\mathrm{Sn}^{\circ}$ to optimize the final material.

The electrolyte has not been synthesized cleanly as a powder but appears to be phase pure as a film. We will repeat
Sandia intends to develop a rechargeable thin-film lithium (Li)-ion ACB using solution route deposition methods. The $A C B$ will have higher power output, longer run times, and a greatly simplified production scheme in comparison to existing technology.... Once sufficiently developed, these $A C B$ s will have the versatility to power any number of small systems, such as microsystems and miniature autonomous robotics. 
testing of the film with protected platinum $(\mathrm{Pt})$ to determine the properties.

We studied the powders-glasses versus crystals-and proved that they have high resistivities. Tests on the films are under way. Alternative materials are continually being investigated in an effort to generate an all-ceramic material.

We successfully produced the cathode on films of Pt, gold $(\mathrm{Au})$, ruthenium $(\mathrm{Ru})$, and rhenium $(\mathrm{Re})$. Currently, $\mathrm{Au}$ substrates are under investigation due to the improved orientation matches between the substrate and layered oxide material. We circumvented the orientation of the $\mathrm{LiCoO}_{2}$ by producing thicker films in other research efforts and will use them here. We are investigating sputtering-generated $\mathrm{LiCoO}_{2}$ materials coupled with sol-gel routes. We tested the sol-gel material electrically by cyclic voltammetry and found it to be reversibly cyclable. We observed several oxidation waves that did not have corresponding reduction waves in the first cycle. These waves were not present during the second cycle.

Investigations to understand this phenomenon are under way. We will investigate alternative substrates and processing in case the orientation becomes a necessity.

We successfully implemented computer modeling to assist in directing the appropriate substrates. We are going to explore further studies to understand the different interfaces.
We successfully produced the cathode on films of Pt, gold $(A u)$, ruthenium $(R u)$, and rhenium (Re). Currently, Au substrates are under investigation due to the improved orientation matches between the substrate and layered oxide material. 


\section{Computer Sciences}

The goal of the Computer

Sciences investment area is to develop next-generation computing capabilities that will provide a strategic benefit to Sandia's business areas. Recent advances in massively parallel computing technology are greatly expanding the usefulness of computer simulations. Research focus areas have included largescale simulations technologies to enable system-scale modeling, scalable algorithms and methods for deciphering problems with large numbers of unknowns, and biotechnology investigations that are synergistic with the Labs' national security mission.

The current trend of information collection and storage has led to a quantity of information that far exceeds the analyst's ability to comprehend it. Conventional tools exist for exploring small amounts of data, but these approaches break down when trying to decipher emerging trends and relationships within large datasets. Sandia's knowledgemining and -management tool, VxInsight $^{\mathrm{TM}}$, explores new methods for visually presenting the structural context for large sets of related data. Through visual exploration, users can detect emerging trends over time, observe interactions of scientific fields, and grasp the overall structure of large datasets. Collaborative work at Sandia has led to the

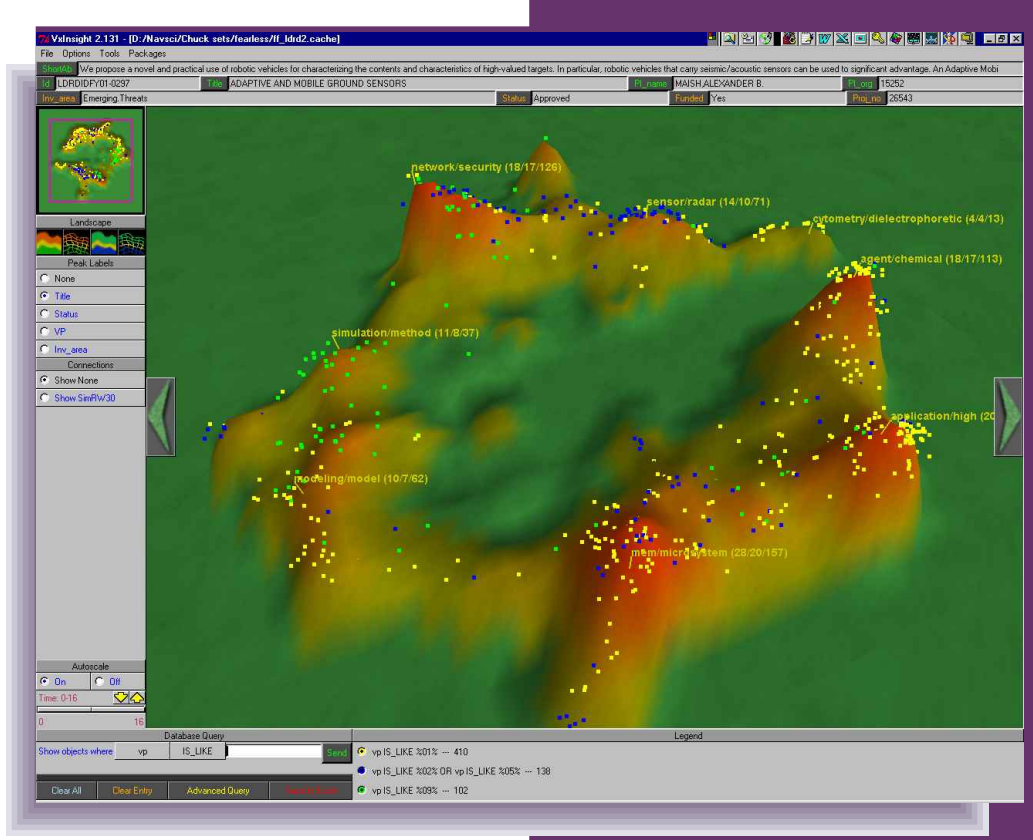

use of VxInsight ${ }^{\mathrm{TM}}$ for exploring transaction data for intelligence agencies, tracking competitive research using patent data, and following research trends in published articles. In addition, VxInsight ${ }^{\mathrm{TM}}$ is being used to determine causal factors for diseases affecting both local and national populations. Further research is needed to begin to understand how this and other tools will become the basis for a $21^{\text {st }}$-century knowledgebased society. This tool has already received two copyrights and a patent. 
10313

\section{Heterogeneous Simulation}

F. J. Oppel, III, E. Gottlieb, S. J. Plimpton, P. G. Xavier

No generalized simulation framework exists to enable modeling behaviors of intelligent machines that depend on physical environments. This requires a heterogeneous simulation environment to model these intelligent machines operating in a real world containing different environments.

Sandia will develop a framework for building and testing heterogeneous simulations. This research enables the integration of several models residing on different platforms that often contain varying model fidelity. It will interface with existing simulation packages through either on-line access or off-line data access. The framework will incorporate diverse simulation environments ranging from continuous simulations (modeling physics such as dynamics, radiation, plumes, etc.) to event-based simulations (modeling agent behaviors, state machines, etc.).

Sandia's design for the "on-line" connector module enables connecting modules for data flow using messagepassing interface (MPI) on multiple central processing units (CPUs) to increase system performance. Our effort to implement and test the "on-line" connector module demonstrated using world modules of rigid-body dynamics to determine the uncoupled dynamic motion of numerous bodies as a test case. These instances of rigid-body dynamics modules were explicitly located on several CPUs through a Tool Command Language (TCL) script that resulted in a performance increase. Finally, we demonstrated incorporating the "on-line" connector module with the multimodel scheme to simulate intelligent agent/machine behaviors in a heterogeneous environment.

We are developing a simulation framework (Umbra) to enable building simulation environments to examine the behaviors of intelligent agents/machines in complex heterogeneous environments. We extended previous achievements (basic Umbra framework design and multimodel methodology) to include the on-line multiprocessing method.

The design of an "on-line" connector enables data to flow between Umbra modules executing on different CPUs. The "on-line" connector acts as an MPI port between Umbra modules to enable distributing Umbra modules that require higher CPU activity (e.g., world modules) onto other CPUs to
Sandia will develop a framework for building and testing heterogeneous simulations. This research enables the integration of several models residing on different platforms that often contain varying model fidelity. It will interface with existing simulation packages through either on-line access or off-line data access. 
improve the overall simulation performance. The MPI protocol, along with a Linux version of Umbra, enables us to meet our Computational Plant (CPlant) target requirement. To avoid communication deadlock between CPUs, we enforced a global scheduling scheme. We accomplished this by building dummy/ ghost modules on each process that stand in for the modules running in other processes. The normal Umbra scheduling scheme (based on feed-forward data flow) then creates a consistent schedule for each process. We developed a simple MPI extension to TCL to explicitly define the module CPU targets through a high-level script.

We implemented this multiprocessor methodology and verified it on an eight-CPU SGI Onyx containing MPI. Our test problem consisted of calculating the dynamic motion of numerous rigid bodies that are uncoupled, which illustrates how rigid-body motion could be used to determine vehicle motion over terrain. As the number of bodies increased, a higher CPU activity was required. Spreading the modules across more CPUs using MPI connectors dramatically improved the system simulation performance.

The last achievement demonstrated using the multimodel and on-line approach together. On a single-CPU system, a heterogeneous simulation utilizing the multimodel approach can contain many different physical models requiring more CPU performance. The on-line approach allows modules to execute on different CPUs, which increases the CPU performance. This demonstration simulated several intelligent machine behaviors executing in a multiple physics world. Executing this simulation on a single CPU was very inefficient with respect to user interaction during run time. It essentially was a batch-type execution. Adding the on-line approach enabled placing many modules over multiple CPUs, which increased the simulation speed.
Spreading the modules across more CPUs using MPI connectors dramatically improved the system simulation performance. 
10314

\section{Volumetric Video Motion Sensing for} Unobtrusive Human-Computer Interactions

\section{E. Small, C. F. Diegert, J. J. Carlson, J. P. Luck}

This project will develop sensing, parallel processing, and geometric reasoning algorithms to enable unobtrusive human-computer interactions. Our approach is to leverage two unique Sandia capabilities: volumetric video motion detection (VVMD) and large-scale computing. We will demonstrate unprecedented human-machine interactions through dramatic advances to the VVMD. By developing new parallel processing algorithms and a new PC cluster deployment architecture, we will extend the resolution of the current VVMD platform to its attainable limits. We will move from platforms that make video-rate constructions of several hundred volume elements (voxels) to platforms that update millions of voxels in real time. We will also develop robust geometric reasoning algorithms to interpret the constructions.

We will demonstrate two scales of human-computer interaction. On a small scale, the VVMD will monitor a small volume (several cubic feet) directly in front of a computer monitor. A person will interact with the computer through a combination of gestures and voice commands. This system could replace or augment a keyboard and mouse. On a larger scale, the VVMD will monitor the position and orientation of one or more humans in a room-sized volume. Differentiating itself from other position/orientation sensors, the VVMD allows participants to maneuver untethered, thus allowing more natural human-machine interactions. By combining a VVMD at this scale with a state-of-the-art video wall and visualization platform, participants could use simple gestures to browse through high-fidelity simulation results and to collaborate with remote participants. Another application at this scale is a robotic work cell, where an operator can point at an object and gesture to cause the robot to move the object to a new position.

We shifted away from high-resolution visualization of the volumetric data and used the VVMD to track a highdimensional kinematic model of a human hand.

- Interaction with a computer simulation using gesture and voice. We demonstrated how we could use a voicerecognition system to add context to gestural commands. In our robot-interaction scenario, a user tells the system (using his
This project will develop sensing, parallel processing, and geometric reasoning algorithms to enable unobtrusive human-computer interactions. Our approach is to leverage two unique Sandia capabilities: volumetric video motion detection (VVMD) and large-scale computing. 
voice) to put an object "over there," and the VVMD system can interpret where "over there" is. The simulation contained a three-dimensional (3-D) model of the room, a model of the robot (Fanuc Robotics industrial robot), and the volumetric data and gesture data being generated by the sensor. The simulation is updated in real time with the information from the sensor, robot, and cameras. We are continuing to develop simulation interaction techniques and will demonstrate gestural manipulation of a weapons part.

- Real-time dynamic gesture recognition. We developed a back-propagation-neural-network technique to recognize 3-D gestures in real time.

- Fitting a human kinematic model to 3-D volumetric

data. We developed a technique to fit a 14-degree-of-freedom human kinematic model to the volumetric data.

- Deployment of 3-D room. A new facility leveraged an existing distributed parallel cluster with built-in video-framegrabbing capability. This resulted in a budget savings and allowed us to expand the number of cameras we can integrate into a single system. We developed autocalibration techniques to allow us to rapidly add and subtract cameras from the system.

\section{Refereed}

Luck, J. P., and D. E. Small. 2000. "Real-Time

Tracking of Articulated Human Models Using a 3-D Shape-from-Silhouette Method." Robot Vision 2001, accepted.
In our robot-interaction scenario, a user tells the system (using his voice) to put an object "over there," and the VVMD system can interpret where "over there" is. 
10315

\section{Hybrid Sparse-Dense Incomplete}

\section{Factorization Preconditioners}

M. A. Heroux, P. R. Schunk, R. R. Rao, J. N. Shadid

Development of robust scalable linear equation solvers persists as one of the most important and elusive goals in large-scale scientific and engineering computing. With continued progress in parallel applications development and steady advances in availability of parallel computers, the lack of robust scalable solvers increasingly becomes a critical roadblock to progress in the use of numerical simulation for engineering and scientific disciplines. In the past few years, many key Sandia applications have been ported to parallel computers, a large number of them requiring a linear solver. With few exceptions, these codes face the difficult task of finding a robust parallel solver. Since preconditioned iterative solvers are the methods of choice and because basic iterative methods have reached a high level of maturity, the real heart of the challenge is the need for robust parallel preconditioners.

This project proposes to develop a set of preconditioners in the AZTEC framework that directly addresses the problems of robustness and scalability for key applications on current and future parallel computers. This work is unique in that it brings together the strengths of existing sparse solver technology into a single preconditioner framework that can be tuned to match the needs of the most challenging problems of today. By imposing a block partitioning on the linear operator (or a recursive set of such partitionings) that matches the underlying physics of the application, and then developing block preconditioners based on this partitioning, we open up the ability to have a full spectrum of robustness and scalability. In addition, within this framework we can exploit in a very natural way the memory hierarchies of parallel computers like CPlant (Computational Plant Cluster Computer Project) and can simultaneously leverage the past investment in both distributed and sharedmemory parallel programming techniques.

We made significant progress in improving the solution capabilities of GOMA. We are now able to solve many more difficult engineering problems than when we started and have a good understanding of the immediate needs to improve our capabilities even more. Furthermore, we have gained external recognition for our efforts. Colleagues in the computational
Development of robust scalable linear equation solvers persists as one of the most important and elusive goals in large-scale scientific and engineering computing....This project proposes to develop a set of preconditioners in the AZTEC framework that directly addresses the problems of robustness and scalability for key applications on current and future parallel computers. This work is unique in that it brings together the strengths of existing sparse solver technology into a single preconditioner framework that can be tuned to match the needs of the most challenging problems of today. 
fluid dynamics (CFD) field are interested in our progress and in our understanding of the historical background in this area. In addition to the direct improvements in GOMA, we made tremendous progress in providing our algorithmic improvements as general-purpose capabilities via the Trilinos framework. GOMA has been an excellent test-bed and early adopter of Trilinos, and that has made Trilinos a more useful tool to other applications.

Algorithmic development in this project focused on improving the quality of incomplete lower-upper (ILU) factorizations. These ILU factorizations are a necessity for large-scale parallel CFD simulations; at the same time, they are the most problematic phase of the computation since these factorizations often fail, leaving the user with little guidance on how to continue. We developed an ability to estimate the quality of the ILU factorization and to provide that information to the user along with guidance on how to proceed if the factorization is detected to be bad. We developed a parallel implementation of this ILU factorization that can always produce a well-conditioned ILU factor, although we are still not able to solve every problem in GOMA.

We also made significant progress toward understanding the role of shared-memory parallel programming within distributed-memory parallel programming and can clearly state where shared-memory parallelism can and cannot be useful in an application that is already implemented using distributed memory.

Finally, we made progress in understanding the role of parallel data repartitioning in improving the quality of the preconditioner. We plan to incorporate our understanding into the GOMA-Trilinos interface.

\section{Other Communications}

Heroux, M. A. 2000. "Exploiting Shared-Memory Parallelism Within a Distributed-Memory Parallel Application." Paper presented to the SIAM Conference on Computational Science and Engineering, Washington, DC, September.

Heroux, M. A., P. R. Schunk, S. R. Subia, T. A. Baer, R. R. Rao, and P. A. Sackinger. 2000. "Iterative Solvers and Preconditioners for Fully Coupled Finite Element Formulations of Incompressible Fluid
In addition to the direct improvements in GOMA, we made tremendous progress in providing our algorithmic improvements as generalpurpose capabilities via the Trilinos framework. GOMA has been an excellent test-bed and early adopter of Trilinos, and that has made Trilinos a more useful tool to other applications.
Mechanics and Related Transport Problems." Paper presented to the Finite Elements in Fluids Conference, Austin, TX, April.

Heroux, M. A., P. R. Schunk, S. R. Subia, T. A. Baer, R. R. Rao, and P. A. Sackinger. 2000. "Iterative Solvers and Preconditioners for Fully Coupled Finite Element Formulations of Incompressible Fluid Mechanics and Related Transport Problems." Paper presented to the SIAM Conference on Computational Science and Engineering, Washington, DC, September. 
10316

\section{Advanced Large-Eddy Simulation Algorithms for Coupled-Flow Physics and Complex}

\section{Geometry}

R. C. Schmidt, T. E. Voth, A. R. Kerstein, T. M. Smith, P. E.

Desjardin

The objective of this work is to advance the algorithms and methods for large-eddy simulation (LES) for unstructured grids, irregular geometry, and coupled physics. The emphasis for the effort is on developing the tools, the knowledge, and the experience needed to perform accurate and computationally affordable LES calculations of engineering problems with coupled physics in complex geometries. LES is a promising alternative to traditional turbulence models because little empiricism is required for a broad spectrum of flow regimes, and transitional and time-dependent flows can be treated. However, although LES has been demonstrated for simple geometries, the treatment of complex geometries with unstructured grids is just beginning to be explored. The interaction between dispersive and diffusive errors, grid anisotropy, filters and filter scales, and subgrid scale (SGS) models has not been quantified in a rigorous way, resulting in uncertainty for many LES computations. These issues are compounded by Sandia applications that demand unstructured grids and accurate treatment of coupled physical processes. This research seeks to advance LES models and methods, reduce the uncertainty, and improve the reliability of LES by quantifying the effects of filters and filter scales, underresolved flowfields, diffusive and dispersive errors, and stochastic subgrid scale (SGS) models on unstructured grid turbulence simulations. This effort will rely on Sandia's largescale computational capability, advanced numerical analysis techniques, and controlled numerical experiments to develop an LES toolkit with models, algorithms, standard libraries for filters and derived flow statistics, and a suite of validation and verification problems. The LES toolkit will directly impact multiple Sandia applications codes (ALEGRA, CTH, MPSalsa, GILA, and FUEGO) and will enhance Sandia's leadership in large-scale computing and turbulent simulation.

This work was focused within the framework of MPSalsa, an unstructured-grid finite-element (FE) code for simulating reacting flow. We made major revisions and modifications to incorporate a range of LES subgrid models,

\section{The LES toolkit will directly} impact multiple Sandia applications codes (ALEGRA, CTH, MPSalsa, GILA, and FUEGO) and will enhance Sandia's leadership in largescale computing and turbulent simulation.

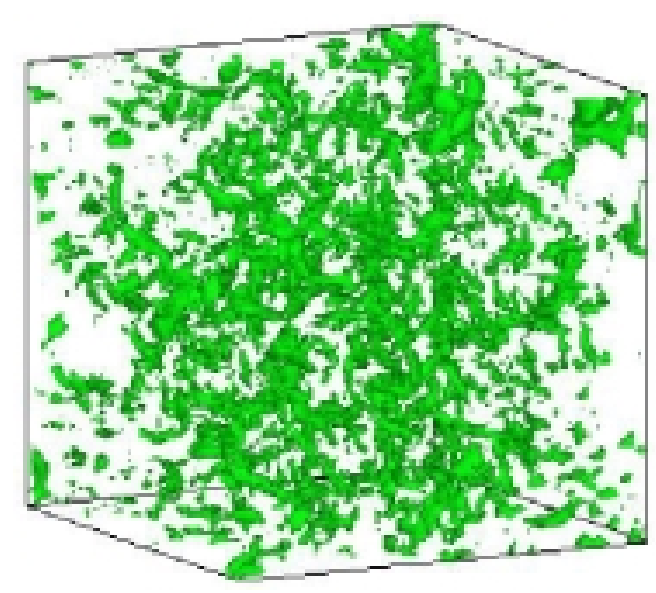

The file illustrates how the decaying isotropic turbulence problem provides a useful testbed for investigating various unstructured grid LES issues. 
unstructured grid filters, statistics-gathering routines, and new boundary conditions (BCs) necessary for the testing and model development in this project. Specific modifications include (1) a second constant coefficient subgrid model (Ksgs), (2) two dynamic LES subgrid models (Smagorinsky, Ksgs), (3) two explicit unstructured grid filters (LMP, RKPM), (4) an additional combustion model (flamelet model with scalesimilarity mixing), (5) routines to compute and store LES statistics for later analysis, (6) routines to compute and store gradient-based auxiliary variables, and (7) code revisions to treat three-dimensional (3-D) periodic BCs (nontrivial for unstructured-grid, parallel code).

In addition to MPSalsa code infrastructure, we rewrote and debugged the following LES post-processing tools (for use with the unstructured-grid EXODUS II database standard): (1) NODAL_STATS, for computing time average moments for stationary data (16 different quantities), (2) ISO_STATS, for computing statistics for spatially homogeneous flows, and (3) EXO_PERIODIC, for adding the redundant node data to special EXODUS files set up for periodic BCs (for proper visualization).

We performed numerical experiments to test and evaluate the performance of different models and filters. To date, we have tested all models and filters by doing simulations of the classic experiment of Comte-Bellot and Corrsin on the decay of isotropic turbulence, with mesh refinement varied from 163 to 1283 . We tested turbulent combustion models on a benchmark reacting plume problem (2-D axisymmetric) and compared them to other Sandia code predictions. Work on the turbulent channel flow, cylinder-in-crossflow, and turbulent non-premixed reacting jet problems is in progress. ...we have tested all models and filters by doing simulations of the classic experiment of ComteBellot and Corrsin on the decay of isotropic turbulence, with mesh refinement varied from 163 to 1283. We tested turbulent combustion models on a benchmark reacting plume problem (2-D axisymmetric) and compared them to other Sandia code predictions. 


\section{7}

\section{Molecular Simulation of Reacting Systems}

A. P. Thompson, F. B. Van Swol

Reacting systems are critical in a wide variety of materials problems central to Sandia's missions, including polymer oxidation, epoxy curing (encapsulation), corrosion, reactive spreading and wetting (solder), and reactive equilibria in hazardous waste. In many cases, significant resources (e.g., Accelerated Strategic Computing Initiative [ASCI] polymers aging project) were focused on molecular simulation of equilibrium and transport properties of materials in which chemical reactions play an important role, but the reaction events have been either completely ignored or greatly simplified. For example, in polymer aging it is likely that on some short length scale the oxygen reaction and diffusion processes are linked. Any truly predictive model of the chemical and physical processes underlying aging must include this reactive/diffusive linkage.

Sandia continued two major efforts to incorporate reaction models into existing molecular simulation codes. For continuous forcefield molecular dynamics (MD), we extended the Monte Carlo reaction capability to include bond-breaking. To do this within the spatial decomposition parallel processing framework of LAMMPS (Large-Scale Atomic/Molecular Massively Parallel Simulator), we implemented a general checkerboarding procedure for avoiding conflicts between reactions on neighboring processors. We used the code to examine a model hydrocarbon system in which the molecular weight can grow by linear growth and decrease by random chain scission. In addition, we continued our study of linear polymer growth, determining that polymer trapping occurs in two dimensions (2-D), but not in 3-D.

For the discontinuous forcefield MD (tethered hardsphere-based polymers), we implemented the polymerization reaction capability, both forming bonds and breaking bonds. Two options exist for the reaction criterion. One is stochastically based while the other is more firmly based on reaction kinetics using the energy and angles of collisions. The latter approach exploits the event-driven algorithm that underlies hard-sphere dynamics. The tethered chains can now account for bond angle potentials though the use of nonadditive diameters. ...we continued our study of linear polymer growth, determining that polymer trapping occurs in 2-D, but not in 3-D. 
The primary focus of this project is to introduce chemical reaction models into existing materials simulation models. We targeted two different classes of model: continuous forcefield MD and hard-sphere MD.

In the case of the continuous forcefield code, we now have implemented both Monte Carlo bond-breaking and bondmaking capabilities. We did this using a general parallel algorithm, which enables us to simulate large systems without any conflicts between reactions on different processors. We used the code to study two different reacting systems:

(1) Linear polymer growth. We observed that trapping of the reactive end inhibits the polymerization rate in $2-\mathrm{D}$, but not in 3-D.

(2) Hydrocarbon cracking/reforming. We found that at low scission/addition ratios, the average molecular weight passes through a maximum before reaching a stable dynamic equilibrium.

In the case of the hard-sphere MD, we introduced bondmaking events. We used this capability to simulate linear polymer growth. Two options exist for the reaction criterion. One is stochastically based while the other is more firmly based on reaction kinetics using the energy and angles of collisions. The latter approach exploits the event-driven algorithm that underlies hard-sphere dynamics. The tethered chains can now account for bond angle potentials through the use of nonadditive diameters. We used the code to study polymer wall interfaces focusing on both melts and solutions. We will use the results to look at reactions at interfaces.

\section{Refereed}

Diggs, A. B., and F. B. Van Swol. 2000. "The Surface Free Energy of a Hard Chain Fluid at a Planar Hard Wall." J. Chem. Phys., submitted.

Hooper, J. B., J. D. McCoy, J. G. Curro, and F. B. Van Swol. 2000. "Density Functional Theory of Simple Polymers in a Slit Pore: 3. Surface Tension." J. Chem. Phys., accepted.

Van Swol, F. B. 2000. "On the Interface Between Hard Chain Fluids and a Hard Planar Wall." Proc. Mater.

Sci., accepted.
The primary focus of this project is to introduce chemical reaction models into existing materials simulation models. We targeted two different classes of model: continuous forcefield MD and hard-sphere MD. 
10318

\section{Massively Parallel Global Climate Model for Paleoclimate Applications}

M. B. E. Boslough, T. R. Guilinger

Global climate has recently become recognized as a national security issue and has been identified as important to decision-making on many issues relating to stewardship of the planet. The potential economic costs associated with climate change are enormous, but so are the costs of policies designed to avoid or minimize climate change. Predictive climate models must be developed, but the time scales of global climate change preclude validation before the damage is done. Thus, there will always be an element of uncertainty as to how good the models are and whether or not they should be trusted. One of the most notable successes in recent years is the ability of climate modelers to accurately hindcast the time-dependence of global temperature changes in the twentieth century. This breakthrough resulted from the addition to the radiative transport model of the opacity of sulfate aerosol pollution and boosted the credibility of climate modeling in general. Another validation strategy is to take advantage of the earth's geologic history to test the predictability of climate models in hindcast mode using paleoclimatic data. Sandia believes that successful paleoclimate models will lead to further gains in climatemodeling credibility. This project will parallelize a widely used paleoclimate code to take advantage of the massively parallel (MP) environments that are becoming available.

We successfully ported the paleoclimate workhorse code GENESIS to the Computational Plant (CPlant) and converted its parallelism from shared memory to distributed memory using message-passing interface (MPI). This required a fundamental change in the parallel decomposition (now over latitude strips only). With $96 \%$ of the code parallelized (in terms of run time of the serial version), the speedup approached double that of the distribution shared-memory parallel version. Our success led to an invitation to Sandia to become the national laboratory partner in the National Science Foundation (NSF) Partnership for Modeling Earth System History (PMESH). We are now collaborating with Penn State University to develop the latest serial version of GENESIS, which allows us to keep our parallel version current with the latest production release. For example, we now have a parallel NetCDF version that will make post-processing much easier.

\section{Global climate has recently}

become recognized as a national security issue and has been identified as important to decision-making on many issues relating to stewardship of the planet....Sandia believes that successful paleoclimate models will lead to further gains in climate-modeling credibility, and this project will parallelize a widely used paleoclimate code to take advantage of the massively parallel (MP) environments that are becoming available. 
We made significant improvements in the speedup of the parallel version, reaching 8.8 times the speed of the serial version and approaching four times the speed of the sharedmemory version. On the Siberia partition of the CPlant, we can now achieve nearly 10 model years per central processing unit (CPU) day at T31 (spectral truncation limit) resolution. Typical paleoclimate model runs of 20-30 years can now be performed in a couple of days. This compares to 3 model years per day with the old version of GENESIS for the PMESH Cray SV1 at Penn State and gives us a version with which we can initiate some production runs. We identified the following ways to further speed up the code:

(1) During the spectral domain portion of the computation, parallelize over spectral coefficient and perform sums as MPI reduction operations. This will reduce the communication required every timestep. We have already made some progress on this parallelism.

(2) Re-dimension all the global arrays so that only memory that is used is actually allocated. This will decrease latency time for communication as well as pipelining during do loops. The latest version of GENESIS has the option of running at T63 and T106 resolutions, for which our distributedmemory parallelism will be even more advantageous.

Typical paleoclimate model runs of 20-30 years can now be performed in a couple of days. This compares to 3 model years per day with the old version of GENESIS for the PMESH Cray SV1 at Penn State and gives us a version with which we can start doing some production runs. 
10319

\section{From Atom-Picoseconds to Centimeter-Years in Simulation and Experiment}

J. C. Hamilton, M. F. Horstemeyer, A. P. Thompson, S. J. Plimpton

Stockpile stewardship is perhaps Sandia's most important mission. Understanding aging phenomena is critical to this mission. Accelerated aging and examinations of stockpile components play an important role but have their limitations. Accelerated tests at elevated temperatures skew the relative probabilities of various reactions. Examination of components provides a limited database and puts us in a reactive rather than proactive position; we may learn about a problem only when it is almost too late to do anything about it. Consequently, it is a vital part of stockpile stewardship to develop simulation techniques that are accurate and valid over much greater time and length scales than those presently available. Most importantly, the simulation techniques should be tested by experiment at various length and time scales so they can be validated and improved.

We have made major progress toward these goals. We modeled surface diffusion and grain-boundary motion important to aging. The grain-boundary motion problem used a unique combination of atomistic modeling to understand fundamental atom motions combined with a lattice model allowing large length scale predictions. We developed threedimensional (3-D) cavity and transition-state analysis and tested it with excellent agreement against molecular dynamics (MD) simulations of methane and xenon (Xe) penetration in zeolite ZK4. We also used the code to find good initial estimates of low-energy transition states for oxygen $(O)$ and nitrogen $(N)$ in flexible polymers, allowing a comparison of energy and entropy selectivity. We developed a discrete-event stochastic dynamics code to simulate coarse-grained diffusion on the cavity network generated by the transition-state theory (TST) code.

- We studied diffusion of iridium (Ir) clusters on Ir using first-principles electronic structure calculations. We used the nudged elastic band method to find activation energies for diffusion events. We identified a new kind of rotational diffusion mechanism.

- We modeled defaceting transitions of grain boundaries in aluminum $(\mathrm{Al})$ using a unique combination of atomistic calculations and a coarse-grained lattice model. We identified
Stockpile stewardship is perhaps Sandia's most important mission. Understanding aging phenomena is critical to this mission.... Consequently, it is a vital part of stockpile stewardship to develop simulation techniques that are accurate and valid over much greater time and length scales than those presently available. Most importantly, the simulation techniques should be tested by experiment at various length and time scales so they can be validated and improved. 
the fundamental atomic shuffle mechanism responsible for the defaceting phase transition. We used this understanding to derive a coarser-scale lattice model that predicted the transition temperature in excellent agreement with experiment. We also predicted the transition temperature analytically using statistical mechanics.

- We developed 3-D cavity and transition-state analysis and tested it with excellent agreement against MD simulations of methane and Xe penetration in zeolite ZK4. The calculation is memory-limited by the number of gridpoints, making it impossible to achieve sufficient resolution for large samples on a workstation. We overcame this problem by implementing a massively parallel (MP) version of the code that runs efficiently on large numbers of processors (ASCI [Accelerated Strategic Computing Initiative] Red, CPlant [Computational Plant]). This enabled us to fully resolve 100,000 atom polymer systems in about one hour on ASCI Red. We used the code to find good initial estimates of low-energy transition states for $\mathrm{O}$ and $\mathrm{N}$ in flexible polymers, allowing a comparison of energy and entropy selectivity.

- We developed a discrete-event stochastic dynamics

We developed a discrete-event stochastic dynamics code to simulate coarse-grained diffusion on the cavity network generated by the TST code. Using a binary tree for randomly selecting hopping events, we achieved accurate diffusion coefficients for large networks in about one hour on a workstation. code to simulate coarse-grained diffusion on the cavity network generated by the TST code. Using a binary tree for randomly selecting hopping events, we achieved accurate diffusion coefficients for large networks in about one hour on a workstation. Initial application of this code to study $\mathrm{O}$ diffusion in polymer melts made use of an isotropic elastic ansatz to account for polymer motion in a time-average sense.

Calculated diffusion coefficients agreed quite well with the exact solution obtained from brute-force MD. The computational effort was several hours for the TST calculation versus at least a week (wall-calendar time) for the MD simulation on ASCI Red.

Refereed

Daruka, I., and J. C. Hamilton. 2000. "GrainBoundary Defaceting: A First-Order Phase Transition by Atomic Shuffle." Phys. Rev. Lett., submitted.

Hamilton, J. C., M. R. Sorensen, and A. F. Voter. 2000. "Compact Surface-Cluster Diffusion by Concerted Rotation and Translation." Phys. Rev. B. 65: R5125.
Rallabandi, P. S., A. P. Thompson, and D. M. Ford. 2000. "A Molecular Modeling Study of Entropic and Energetic Selectivities in Air Separation with Glassy Polymers." Macromolecules 33: 3142.

Tunca, C., and D. M. Ford. 1999. "A Transition-State Theory Approach to Adsorbate Dynamics at Arbitrary Loadings." J. Chem. Phys. 111: 2751. 
10320

\section{Parallel Combinatorial Optimization for} Scheduling Problems

\author{
C. A. Phillips, D. A. Jones, W. E. Hart, E. A. Kjeldgaard, R. D. Carr
}

Sandia has developed a state-of-the-art massively parallel (MP) combinatorial optimization engine. This is the first fully general mixed-integer programming (MIP) branchand-bound code scalable to thousands of processors. The engine can adaptively incorporate application-specific methods (combinatorial approximation algorithms and genetic algorithms) for both lower and upper bounds to limit the search space explored by the MIP engine. We will use this exact method in a statistically rigorous performance analysis of sequential heuristics. The branch-and-bound code is fully implemented and largely tuned. The branch-and-cut and branch-and-price engines are largely designed but not implemented.

We will use the MIP manufacturing at Sandia, and related scheduling problems for the Product Realization Program, Stockpile Life Extension Program, Revolution in Engineering (formerly ADaPT), and the Computational Plant (CPlant). However, MIP is a core optimization technology, and this capability can be applied in other areas consistent with Sandia's mission: nonproliferation, transportation, infrastructure analysis and design, manufacturing, energy, environment, and tools for MP computation such as meshing and scheduling heterogeneous supercomputers.

Management of multiple loosely coupled threads, each distributed and largely asynchronous, required the development of new cooperative proportional-share threadscheduling tools.

We ported PICO (Parallel Integer and Combinatorial Optimization) to CPlant using the SoPlex linear programming solver and determined changes we must make to SoPlex in the future to make it a more reliable solver for the PICO system. SoPlex is currently the only public-domain solver that PICO supports and is therefore important for usability when PICO is released.

The branch-and-bound portion of PICO now runs well on a reasonable set of MIPlib (mixed-integer programming library) test problems, with good parallel speedup until the basic parallelism in these limited test problems is exhausted. We did considerable testing and tuning of the general branch-
Sandia has developed a state-ofthe-art massively parallel (MP) combinatorial optimization engine. This is the first fully general mixed-integer programming (MIP) branchand-bound code scalable to thousands of processors. 
and-bound engine. In particular, we tuned the interaction between controlling "hub" processes and worker processes. We added parallel code for initialization of pseudocosts (gradients). We added code to update subproblem bounds based on information gained when initializing these gradients.

In the Pantex application, we added specialized feasibility-testing routines and output routines. We added linear programming (LP)-based incumbent-finding routines (routines to find approximate feasible schedules; an incumbent is the best feasible solution found so far). To do this, we added mapping routines that allowed our Pantex classes to access variables in their logical three-dimensional (3-D) form (mapping back and forth to PICO's standard 1-D representation). We designed and coded a simple problem generator for synthetic data based on the Pantex datasets.

We investigated scheduling issues for CPlant and heterogeneous and/or commodity-based supercomputers in general and concluded that efficient scheduling and processor allocation in both batch and interactive modes was an area of great need in DOE.

We evaluated branch-and-price search strategies. In particular, we incorporated branch-and-price into our designs for branch-and-cut. Therefore, we expect the addition of branch-and-price to be reasonably straightforward once branchand-cut is functioning well.

We began the design of a scalable branch-and-cut engine, determining that serial implementation is not a valuable stepping stone for the final design. We have almost completed design of a user interface for the branch-and-cut engine. This interface should allow fast and efficient implementation of problem-specific branching strategies. In particular, our design incorporates the best points of the ABACUS (serial) branchand-cut system into a much more efficient system. Our design also better shields the user from the specifics of PICO implementation, allowing the user to work with logical variables most appropriate for his/her application. We intend to interface PICO with a math-programming language such as AMPL or GAMS. This allows the user to express a problem mathematically, which is superior to the time-consuming and error-prone process of specifying constraint matrices. We will also allow matrix-based interfaces for applications where these are available already.

We have begun the software licensing process.
We investigated scheduling issues for CPlant and heterogeneous and/or commodity-based supercomputers in general and concluded that efficient scheduling and processor allocation in both batch and interactive modes was an area of great need in DOE. 


\section{Other Communications}

Eckstein, J. 2000. “The PICO Parallel Branch-and-

Bound System." Paper presented to the 17th

International Symposium on Mathematical

Programming, Atlanta, GA, August.

Eckstein, J., W. E. Hart, and C. A. Phillips. 2000.

"PICO: An Object-Oriented Framework for Parallel

Branch-and-Bound." Sandia Technical Report

SAND2000-3000, Sandia National Laboratories, Albuquerque, NM (August).

Hart, W. E. 2000. "Solving Mixed-Integer Nonlinear Problems with PICO." Paper presented to the 17th International Symposium on Mathematical Programming, Atlanta, GA, August.

Phillips, C. A. 2000. "Computing Approximate Solutions in Resource-Constrained Project Scheduling." Paper presented to the DIMACS Workshop on Computing Approximate Solutions to NP-Hard Problems, New Brunswick, NJ, February.

Phillips, C. A. 2000. "Parallel Branch-and-Bound for Mixed-Integer Programming." Paper presented to the 12th ACM Symposium on Parallel Algorithms and Architectures, Bar Harbor, ME, July.

Phillips, C. A. 2000. "Solving a Large-Scale MIP with PICO: Production Planning for Pantex." Paper presented to the 17th International Symposium on Mathematical Programming, Atlanta, GA, August. 
10321

\section{Multilevel Techniques for Unstructured Grid Problems on Massively Parallel Computers}

R. S. Tuminaro, J. N. Shadid, C. D. Moen, A. C. Robinson, P. R. Schunk, A. B. Williams

This project resulted in the creation of a multilevel $(M L)$ software package that provides three different ways to construct a multigrid iterative solver for a particular application: (1) smoothed aggregation, (2) finite-element (FE) basis function, and (3) user-defined. Sandia incorporated the main method, parallel smoothed aggregation, into MPSalsa, ALEGRA, and into a FUEGO prototype. It is also available through Sierra's finite-element interface (FEI) and is being used by a Calore. Parallelizing the aggregation phase required several algorithmic enhancements to avoid convergence degradation. On most problems, the ML package has significantly improved the overall performance of what was available in the AZTEC package. In some cases, we achieved convergence where divergence had occurred with previous solvers. The ML framework that we developed for this project should enable future ML methods as well as enhancements to the existing methods.

Most of our work involved improving, optimizing, and generalizing the smoothed aggregation multigrid method.

We completed several different parallel aggregation schemes: (1) subdomain-based aggregation, (2) a coupled aggregation scheme that aggregates interprocessor boundaries first, and (3) a maximal independent set (MIS) algorithm applied to the square of the matrix. In addition, we explored several tuning parameters and modifications to enhance performance. Overall, we identified a hybrid scheme that uses the subdomain-based aggregation on finer grids and then switches to the MIS on coarser grids as the most practical and efficient.

We incorporated several different direct solvers into ML for use on the coarsest grid problem: (1) serial SuperLU (LU is a code that solves linear systems of equations by Gaussian elimination), (2) parallel SuperLU, and (3) for symmetric problems, parallel XXT (a code that solves a linear system of equations by a direct method similar to SuperLU, but computes approximations to the matrix inverse). The efficiency of these coarse-grid solves is most important for MPSalsa when using the two-level domain decomposition scheme.
On most problems, the $M L$ package has significantly improved the overall performance of what was available in the AZTEC package. In some cases, we achieved convergence where divergence had occurred with previous solvers. The ML framework that we developed for this project should enable future ML methods as well as enhancements to the existing methods. 
While benchmarking with the most recently added parallel SuperLU has not been completed, we anticipate that this will significantly improve run times within MPSalsa.

We ran several new application problems with ML. A realistic pulsed-power ALEGRA simulation exercised ML. On this problem, ML often finished in about half the time as the best non-ML solvers. Results generated on four million unknown problems using 512 processors demonstrate good, though not entirely perfect, scalability. In addition to ALEGRA results, MPSalsa generated significantly more results, demonstrating very good scalability on both Stokes and NavierStokes problems. We also ran our first smoothed-aggregation multigrid problem within MPSalsa. This required generalization to nonsymmetric operators and the use of AZTEC smoothers. Overall, the smoothed-aggregation results were significantly better than the non-ML methods. In fact, on several problems we achieved convergence in situations where the non-ML methods diverged. The ML package was also exercised by Calore via Sierra's FEI interface. We obtained promising results on some small model problems. Additional work will continue beyond the scope of this project. We also began addressing some model elasticity problems. This required modifications to how rigid-body modes are handled by the smoothed-aggregation method. Additional work will continue beyond the scope of this project to assess the usefulness of ML within these applications.

We made several other improvements within the ML package. Two notable improvements correspond to the matrixmatrix multiplication software and the eigenvalue estimation routines used in choosing damping factors. We rewrote the matrix-matrix multiply routine to make heavy use of hash tables to speed up the run time. The resulting scheme runs almost an order of magnitude faster than our previous version and reduces the time to multiply matrices to a modest (though still not negligible) fraction of the total time. The improved damping-factor choices use a few iterations of a conjugate gradient $(\mathrm{CG}) /$ Lanczos-type iteration to estimate the largest eigenvalue of the matrix, which can be used to pick good damping factors.
We rewrote the matrix-matrix multiply routine to make heavy use of hash tables to speed up the run time. The resulting scheme runs almost an order of magnitude faster than our previous version and reduces the time to multiply matrices to a modest (though still not negligible) fraction of the total time. 


\section{Refereed}

Tuminaro, R. S., and C. H. Tong. 2000. "ML 2.0

User's Guide." Sandia Technical Report, in process.

Tuminaro, R. S., and C. H. Tong. 2000. "Parallel

Smoothed-Aggregation Multigrid: Aggregation

Strategies on Massively Parallel Machines." Proc.

SuperComputing 2000 ( Dallas, TX, November). 
10323

\section{Visual Explanation and Insight}

B. N. Wylie, M. H. Koller, B. A. Hendrickson, K. W. Boyack, D. K. Johnson

The information age allows people to find, extract, and gather information in astronomical proportions. Commercial databases, the World Wide Web, and data warehouses offer the promise of knowledge and insight if one can harness the overwhelming volume of raw information. Currently, with traditional tools, the task of extracting knowledge from large databases is exceedingly difficult. A number of data-mining tools exist to answer fairly specific questions about data collections, but more often than not, an analyst does not even know what questions to ask. Also, without the understanding of the global picture, specific questions and observations cannot be placed within the proper context. Unfortunately, existing tools are not well suited for studying large-scale structure or interacting with data in an exploratory manner.

VxInsight ${ }^{T M}$ is a powerful and flexible tool for exploring data collections. It works by transforming the data into an intuitive visual format that is easy to interpret and allows natural navigation. Millions of years of evolution have equipped humans with extraordinary powers to visually spot trends and patterns, to identify outliers, and to detect relationships. VxInsight ${ }^{\mathrm{TM}}$ exploits this capability by presenting the data as a landscape, a familiar representation that we are adept at interpreting and that allows very large datasets to be represented. The landscape representation conveys significant information about the implicit structure of the data, providing context for the analyst's exploration and queries of the database.

(1) Develop lazy-evaluation techniques for "detail on demand" visualization of large datasets. VxInsight ${ }^{\mathrm{TM}}$ employs a caching scheme for data that need to be ready on demand. The data manager, through the database connection, retrieves any data not present in the cache. These data are then stored in memory and can be later saved to disk. While this architecture satisfies the "detail on demand" criteria, the data manager does not currently employ any algorithms for discarding data (e.g., least recently used [LRU] queues).

(2) Design a data-management architecture that allows integration of disparate information sources into one virtual data source. VxInsight ${ }^{\mathrm{TM}}$ has been used on wildly disparate
The information age allows people to find, extract, and gather information in astronomical proportions. Commercial databases, the World Wide Web, and data warehouses offer the promise of knowledge and insight if one can harness the overwhelming volume of raw information.... VxInsight ${ }^{T M}$ is a powerful and flexible tool for exploring data collections. It works by transforming the data into an intuitive visual format that is easy to interpret and allows natural navigation. 
datasets ranging from genome sequences to patents to people. VxInsight ${ }^{\mathrm{TM}}$ 's data manager is an abstract class that defines an interface to arbitrary data. To date, this interface has allowed VxInsight ${ }^{\mathrm{TM}}$ to talk to databases, socket channels, Web pages, and Web servers. The data manager abstraction provides a general methodology to retrieve data and is easily extendible. While this software approach allows unique data sources to be tapped with a minimal amount of effort, it does require some additional coding.

(3) Create a transfer-port mechanism, which presents a standardized interface for inter-application communications. We investigated several alternatives for inter-application communication. The first one is interaction through database tables, currently being used on the VxInsight ${ }^{\mathrm{TM}}$-Vista project. VxInsight ${ }^{\mathrm{TM}}$ transfers data into the database that are then retrieved and processed by Vista. An alternative communication mechanism relies on some of the operatingsystem facilities that Microsoft provides. Object Linking and Embedded and Direct Data Exchange (OLE/DDE) are provided to applications running on Windows 95/98/NT/2000. We are currently using DDE to pass data from VxInsight ${ }^{\mathrm{TM}}$ to the Netscape Web browser, which then displays graphs and hyperlinks for the data.

(4) Investigate and implement "feature enhancements" to the existing landscape viewing model. We tabled the work in feature enhancements because of concentration on other areas, including linking to Web databases, working with genomic data, and issues relating to use and deployment.

(5) Develop visual interfaces for user control of similarity/ordination computations. To simplify the processing of new data, we provided visual interfaces to the similarity calculations and ordination (mapping) of the data objects. The nature of computing similarities is often specific to the type of data being processed, so we developed an interface for the most general type of similarities. The user can tell VxInsight ${ }^{\mathrm{TM}}$ which tables and fields in the database they wish to generate similarities on, and VxInsight ${ }^{\mathrm{TM}}$ will lead them through the process.

The ordination (mapping) algorithm is now more intuitive to use and is exposed through the VxInsight ${ }^{\mathrm{TM}}$ graphical user interface (GUI). This means that once the similarities are generated, the mapping process can occur with a simple click of a button.
The ordination (mapping) algorithm is now more intuitive to use and is exposed through the VxInsight ${ }^{T M}$ graphical user interface (GUI). This means that once the similarities are generated, the mapping process can occur with a simple click of a button. 


\section{Refereed}

Davidson, G. S. 2000. "VxInsight ${ }^{\mathrm{TM}}$ and Microarray

Analysis." Paper presented to SWARM 2000

(Regional AAAS Meeting), Las Cruces, NM, 10 April.

Werner-Washburne, M., and G. S. Davidson. 2000.

"VxInsight ${ }^{\mathrm{TM}}$ and Microarray Gene Expression Data

Mining." Paper presented to the DOE Genome

Contractor-Grantee Workshop, Santa Fe, NM, 28

February-2 March.

\section{Other Communications}

Boyack, K. W. 2000. "Video of Map of Science Using

VxInsight ${ }^{\mathrm{TM}}$." Presentation to the National Academy of

Science Committee Meeting (video prepared for

presentation by Dr. Richard Shiffrin, Indiana

University), Washington DC, 5 October.

Boyack, K. W. 1999. "Visualization of Large Databases to Impact R\&D Investment Decisions."

Proc. Delphi Group's E-Business Conf., Internat.

Business Process Summit 1 (Reston, VA, 6-8

October).

Boyack, K. W. 1999. "Visualization of Large

Databases Using VxInsight ${ }^{\mathrm{TM}}$." Paper presented to the

New Mexico Drug Utilization Review Board Meeting,

Santa Fe, NM, 11 November.

Boyack, K. W. 2000. "Visualization of Large

Databases Using VxInsight ${ }^{\mathrm{TM}}$ to Aid in Forecasting

Technology Trends." Paper presented to Lockheed

Martin Information Systems, Orlando, FL, 23 August.

Boyack, K. W. 2000. "Visualization of Literature Databases as a Precursor to Technology Forecasting." Paper presented to Oklahoma State University, Stillwater, OK, 21 January.

Boyack, K. W., B. N. Wylie, G. S. Davidson, and D. K. Johnson. 2000. "Analysis of Patent Databases Using VxInsight ${ }^{\mathrm{TM}}$." Extended abstract submitted to the Workshop on New Paradigms in Information Visualization and Manipulation, 14 September. SAND2000-2266A.
Davidson, G. S. 2000. "Data Visualization and Analysis." Paper presented to the Sandia/AFCS Workshop, Albuquerque, NM, 2 August.

Davidson, G. S. 2000. "New Genome Informatics and Visualization Tools." Paper presented during the Keck Grant site visit to UNM, Albuquerque, NM, 18 April.

Davidson, G. S. 2000. "New Genomic Informatics and Visualization Tools." Paper presented to the UNM Press Conference announcing Keck Grant Award, Albuquerque, NM, 18 July.

Davidson, G. S. 2000. "New Mexico as an Emerging BioInformatics Region." Paper presented to the Southwest Genomics and Biotechnology Alliance Sevilleta Workshop, Sevilleta Wildlife Refuge, NM, 18 June.

Davidson, G. S. 2000. "SNL/NCGR Collaborations with VxInsight ${ }^{\mathrm{TM}}$." Paper presented to the National Center for Genomic Resources, Santa Fe, NM, 7 August.

Davidson, G. S. 2000. "The Data Mining Tool VxInsight ${ }^{\mathrm{TM}}$." Paper presented to a Seminar at University of Texas Southwestern Medical School, Dallas, TX, 24 May.

Davidson, G. S. 2000. "VxInsight" for Data Mining and Knowledge Management." (Invited) Paper presented to a Technical Seminar at Celera, Inc., Gaithersburg, MD, 8 June.

Davidson, G. S., and M. Werner-Washburne. 2000. "Analyzing Microarray Data with VxInsight ${ }^{\mathrm{TM}}$." Paper presented to the Southwest Genomics and Biotechnology Alliance Day of Talks (SWGABADOT 1), Albuquerque, NM, 22 March.

Fleck, J. 2000. "DNA: Using the Code, Powerful Tools Help UNM Researchers Try to Make Sense of Book of Life." Article in the Albuquerque, NM, Albuquerque Journal, Sunday edition (6 August). 
10690

\section{Emergent Behavior of Large Swarms of Intelligent Agents}

R. J. Pryor, B. L. Spletzer

In this project, Sandia performed research in many areas of the behavior of large swarms of intelligent agents. We developed some decentralized learning control methods using genetic programming techniques. We also researched the use of genetic algorithms (GAs) in decentralized control. This novel idea extends GAs from an optimization tool to a method for controlling a large swarm of robots. We conducted tradeoff studies on the ideal number of robots and the type of communication mode that should be used. We implemented several of our decentralized control ideas for controlling large swarms of agents on actual robot hardware platforms. We solved optimal control problems using our decentralized control methods. This extension treats a single processor of a parallel collection of processors as an "intelligent agent."

We researched a GA approach to controlling a large collection of robots. GAs operate on the basis of a probabilistically directed search, recombination, and mutation of trial solutions. GAs can be an efficient approach for the exact or approximate location of a global optimum. In a GA, the independent variables that influence an objective function are coded in a binary format. We evaluated the objective function using this coded representation of the variables. To begin, the variables are first set in a random fashion, but as the GA progresses, the binary coded variables mutate and recombine, based on probability measures, to result in a "better" evaluation of the objective function.

We performed research that studied the trade-offs of numbers of robots. We found that for a simple function with no noise, the number of iterations required for all robots to find the minimum seemed independent of the number of robots. For a simple function with noise, the number of iterations required for all or a majority of robots to find the minimum was dependent on the number of robots. This result, however, is partially dependent on the initial positions of the robots. We found that a local communication mode is best for extremely noisy or sparse functions.

We implemented one of our distributed optimization routines on two robotic platforms. One platform is composed of a collection of microrobots that are less than one cubic inch
In this project, Sandia performed research in many areas of the behavior of large swarms of intelligent agents. We developed some decentralized learning control methods using genetic programming techniques. We also researched the use of genetic algorithms (GAs) in decentralized control. This novel idea extends GAs from an optimization tool to a method for controlling a large swarm of robots. 
in volume. The robots executed the distributed optimization routine to find a block of dry ice placed in a room. The other platform is composed of a collection of robotic all-terrain lunar exploration rover (RATLER ${ }^{\mathrm{TM}}$ ) vehicles. The RATLER ${ }^{\mathrm{TM}}$ vehicles executed the distributed optimization routine to an acoustic speaker placed outside. The hardware demonstrations showed that some routines are simple enough to be downloaded and implemented on 8-bit processors with 4 kilobytes of RAM (random access memory).

We used our decentralized control methods to solve optimal control problems. In this extension of our ideas, the "intelligent agents," or "robots," are feasible solutions to the optimal control problem. The feasible solutions share information, and each feasible solution uses this information to generate a better solution to the control problem. This extension treats a single processor of a parallel collection of processors as an "intelligent agent" and demonstrates that the same routines can be used in the "real" world (i.e., real robots) and in the "cyber" world (i.e., processors).

\section{Refereed}

Hurtado, J. E., R. H. Byrne, S. E. Eskridge, and J. J. Harrington. 2000. "Miniature Mobile Robots for Plume Tracking and Source Localization Research." Proc. IEEE Internat. Conf. of Robotics and Automation W2 (San Francisco, CA, 23-28 April): 70-77.
The feasible solutions share information, and each feasible solution uses this information to generate a better solution to the control problem. This extension treats a single processor of a parallel collection of processors as an "intelligent agent" and demonstrates that the same routines can be used in the "real" world (i.e., real robots) and in the "cyber" world (i.e., processors). 
10728

\section{Algorithmic Advances in Computational Structural Biology}

R. D. Carr, D. C. Roe, A. M. Johnston, S. J. Plimpton

The area of protein structure alignment/fold

recognition/fold classification is at the cutting edge of research in structural genomics. As opposed to the state of research in protein sequence alignment, where globally optimal pairwise alignment can be effectively computed using dynamic programming methods, the state of the art in structure alignment is still at empirical and heuristics stages.

The area of protein structure alignment depends on the ability to measure the similarity of protein structures; however, none of the proposed measures of structure similarity in the literature can be effectively computed. One measure of structure alignment is "contact map overlap." Sandia has developed the first polynomial time algorithms for constructing close to globally optimal pairwise structure alignments of proteins. We also developed algorithms for efficiently finding globally ribonucleic acid (RNA) structure alignment.

There are 12,000 structures in the Brookhaven Protein Database (PDB); each structure has several thousand atomic coordinates. One fold is a cluster of crystal structures in the PDB aligned on structure similarity. The key advance proposed in this project is to use Sandia's Computational Plant (CPlant) supercomputer to compute all pairwise PDB alignments and to obtain a new fold classification based on contact maps and the rigorous algorithms to cluster the folds. This will establish the practicality of our algorithms and will provide a new prediction tool. We will perform this work in collaboration with Stanford University and the University of California-San Francisco. The computational challenge is to pairwise align and cluster the entire PDB. Previous work used a set of only 2000 structures due to the limitation of the computational resources available at the time. Our work will generate about 130 million structure alignments. The algorithmic methods will be both the Stanford method and a new Sandia-Berkeley method based on self-avoiding-walks, which for the first time address the excluded volumes. The successful completion of this project will have a long-term impact on Sandia's work in national security, e.g., in molecular recognition to counter biological warfare $(B W)$ and terrorism.
Sandia has developed the first polynomial time algorithms for constructing close to globally optimal pairwise structure alignments of proteins. We also developed algorithms for efficiently finding globally ribonucleic acid (RNA) structure alignment....The successful completion of this project will have a long-term impact on Sandia's work in national security, e.g., in molecular recognition to counter biological warfare $(B W)$ and terrorism. 
Our two main accomplishments were the development of the TORTILLA software package for protein analysis and the development and implementation of new algorithms for comparing folded protein structures.

TORTILLA is structured loosely as a blackboard model. Multiple proteins and data objects can be loaded into the system (written on the blackboard); then any available algorithm can operate on them.

We included a genetic algorithm for computing good (not optimal) contact map alignments in TORTILLA.

We also developed exact algorithms, using a branchand-cut technique, for classifying protein folds based on their contact map overlap. The result of this work thus far is a prototype implementation of our algorithm, solving problems coming from real proteins, of small to moderate size, obtained from the PDB database.

This is the first time that provably optimal solutions have been found for the structure alignment of real proteins. The biggest difficulty has been the scalability of our algorithms. To improve our time performance, we invented a linear programming technique we call "compact optimization." We also found a way to break up these optimization problems into smaller subproblems, also resulting in a time savings.
Our two main accomplishments were the development of the TORTILLA software package for protein analysis and the development and implementation of new algorithms for comparing folded protein structures.... This is the first time that provably optimal solutions have been found for the structure alignment of real proteins.

\section{Refereed}

Carr, R., and G. Lancia. 2000. "Compact vs.

Exponential-Size LP Relaxations.” J. Operations Res. Lett., submitted. SAND2000-2170.

Carr, R., G. Lancia, B. Walenz, and S. Istrail. 2000. "Branch-and-Cut Algorithms for Independent Set Problems: Integrality Gap and an Application to Protein Structure Alignment." RECOMB Computational Biology Conference and Proceedings, submitted. SAND2000-2171. 
10746

\section{Predicting Function of Biological} Macromolecules

L. J. D. Frink, M. J. Stevens, A. G. Salinger, D. C. Roe, M. P. Sears, C. Springer, H. P. Hjalmarson

The elucidation of protein amino acid sequences has been one goal of the human genome project. Consequently, one effort of the computational biology community has been to predict protein structures given those sequences. Protein structures can also be determined from $x$-ray crystallography, and the protein databank contains a library of known structures. It is commonly hypothesized that the structure of a protein is critical for its function. Sandia will develop and apply novel physics-based molecular modeling capabilities to the prediction of the function of biological macromolecules given their structures. For example, we will extend the coarsegrained molecular dynamics (MD) methods that have been applied to polymeric systems to treat the polyelectrolytes (e.g., deoxyribonucleic acid [DNA]) and polyampholytes (e.g., proteins) found in biological systems. In particular, we will study how the structure, shape, and charge distribution on DNA cause it to pack densely. In addition, we will extend and apply a novel three-dimensional (3-D) density functional theory (DFT) code (TRAMONTO) to predict the function of ion channel proteins in regulating ion transport to cells and the function of binding sites on proteins by calculating free energies in molecular recognition events. We will explore the possibility of calculating solvation free energies at the quantum mechanical level with a coupled electronic structurel DFT code.

We modified the dual-control volume grand canonical MD code (LADERA) to add a steady-state flux to the molecules that are inserted into the system. A study of the accuracy of the Amber, Charmm, Compass, Gromos, and OPLS force fields for protein simulation is nearing completion. This also means that we now can simulate systems using these force fields. We upgraded TRAMONTO toward being able to perform a transport/DFT calculation of a real ion channel protein. We completed MD simulations on DNA packing and simulated the fundamentals of DNA packing, or "condensation." We replicated several experimental results by the simulations. Moreover, the simulations reveal the basic
Sandia will develop and apply novel physics-based molecular modeling capabilities to the prediction of the function of biological macromolecules given their structures. 
mechanism for DNA condensation, which resolves a longstanding basic issue of DNA dynamics.

\section{Refereed}

Stevens, M. J. 2000. "Simple Simulations of DNA

Condensation." Biophysical J., accepted. 
10757

\section{Large-Scale Nonlinear Optimization Arising from PDE Models}

W. E. Hart, B. G. Van Bloeman Waanders, M. S. Eldred, P. T.

Boggs, K. R. Long

Sandia initiated the development of new parallel optimization methods for large-scale applications. In particular, we made significant progress toward the implementation of successive quadratic programming (SQP) algorithms for large-scale optimization with partial differential equation (PDE) constraints, which we expect to significantly reduce the computational requirements for these applications. Instead of the conventional optimization process of converging a nonlinear simulation every time a function evaluation is required, the nonlinear simulation is converged only once for the entire optimization process. Consequently, the parallel optimization methods that we are developing will be able to solve high-fidelity, coupled-physics problems that are too expensive to solve with existing optimization capabilities. A particular focus of our current effort involves the characterization of the information from the PDE that is needed by the optimizer, and we began investigating how these optimization methods could interface with the capabilities of the SIERRA, DAKOTA, and IDEA frameworks.

We interfaced a mature SQP code from CarnegieMellon University (CMU) with MPSalsa using a relatively nonintrusive interface. Additionally, we coupled SQP optimizers in DAKOTA with MPSalsa with a decoupled, black-box approach. We evaluated these two interfaces using simple test functions and are evaluating them both on realworld applications. We are developing a parallel SQP code based on a $\mathrm{C}++$ SQP library, which will enable the investigation of more tightly coupled parallel algorithmic approaches. We completed a comprehensive survey of SQP methods that are relevant for parallel optimization and are preparing a white paper that evaluates parallelization issues for weakly coupled SQP approaches. We also developed a symbolic interface to a parallel simulator (Sundance) to help investigate a number of important algorithmic optimization issues in addition to the review of the various levels of intrusiveness. We will use this simulator to test limitedmemory SQP. Finally, we modified the QP solver (O3D) to incorporate a vector/matrix class interface that can easily be 
extended to include a parallel vector/matrix class (PETRA). We developed a collaboration with academia through the Computer Systems Research Institute (CSRI) to research large-scale transient PDE-constrained simulation. We will focus on transient applications while we evaluate steady-state applications in parallel.

\section{Refereed}

Boggs, P. T., and J. W. Tolle. 2000. "Sequential

Quadratic Programming for Large-Scale Nonlinear Optimization." J. Computational and Appl. Math., accepted.

Boggs, P. T., and K. R. Long. 2000. "A Flexible Implementation of an SQP Algorithm 1." Paper presented to the SIAM National Meeting, Rio Grande, Puerto Rico, July.

Boggs, P. T., and K. R. Long. 2000. "Integrating an Object-Oriented SQP Algorithm into ComponentsBased Frameworks." Paper presented to the 17th International Symposium on Mathematical Programming, Atlanta, GA, August.

\section{Other Communications}

Boggs, P. T., and K. R. Long. 2000. "Tightly Coupled SQP Algorithms from Loosely Coupled Components." Paper presented to the 1st SIAM Conference on Computational Science and Engineering, Washington, DC, September.
We developed a collaboration with academia through the Computer Systems Research Institute (CSRI) to research large-scale transient PDEconstrained simulation. We will focus on transient applications while we evaluate steady-state applications in parallel. 
10777

\section{Parallel Methods for Coupling Circuit- and Device-Scale Simulations}

S. A. Hutchinson, E. R. Keiter, R. J. Hoekstra

Circuit simulation tools (SPICE) have become

invaluable in the development and design of electronic circuits.

Similarly, device-scale simulation tools (Da Vinci) are

commonly used in the design of individual semiconductor

components. Often, however, circuit-level models do not afford

the designer the required fidelity. But while single-device, mesh-level simulations provide the higher fidelity, an understanding of how the device behaves as part of a dynamic circuit is crucial, thus creating the need for a coupled modeling approach.

Typically, the coupling of circuit ordinary differential equation $(O D E)$ and device partial differential equation (PDE) methods to create a "mixed-mode" simulation tool has been problematic. This is due to both numerical and physical considerations. In circuits and devices, the important time scales can vary greatly, leading to numerical stiffness. Further, the nonlinear nature of the two models can be very different when dealing with phenomena such as discontinuities and hysteresis. Finally, the nature of the linear system resulting from a device simulation is generally much nicer numerically than that resulting from a circuit. Thus, solution methods that typically work well for the PDE problem do not necessarily work well for the ODE problem and vice versa. When one considers the need to provide an efficient implementation of coupled solutions on parallel computers, the complications associated with this quickly become very challenging.

Nevertheless, Sandia will investigate numerical solutions associated with such a mixed-mode simulation, with an emphasis on matrix-solution and time-integration techniques for heterogeneous parallel computers (Computational Plant [CPlant]). By separating the solution modes, integration techniques for each can be tailored for robustness. Further, efficiency for this heterogeneous problem will be provided by utilizing advanced load-balancing tools (Zoltan). Bringing such state-of-the-art algorithms and parallel computing tools to bear on this problem will yield a valuable tool for Sandia's electrical modeling and simulation community.

\author{
Bringing such state-of-the-art \\ algorithms and parallel \\ computing tools to bear on this \\ problem will yield a valuable \\ tool for Sandia's electrical \\ modeling and simulation \\ community.
}


We completed the design and implemented the standard interface data structures and communication protocols within the Xyce ${ }^{\mathrm{TM}}$ Parallel Electronic Simulator code to accommodate PDE-based models. This design provides an object-oriented (OO) opaque interface between the nonlinear Jacobian loading routines and the circuit-device specific routines that will accept both lumped-parameter models and PDE models for devices.

We also completed the design and initial implementation of the complete numerical-solution phase, including time integration, nonlinear solver, and linear solver, and associated parallel data structures (via an interface with Trilinos/Petra). This solution-phase design interfaces with the Jacobian loading routines described above and allows for the mixed-mode coupling between the lumped-parameter and PDE models.

We completed an initial 1-D drift-diffusion device model for a diode within the Xyce ${ }^{\mathrm{TM}}$ Parallel Electronic Simulator for the purposes of demonstrating the capabilities of We completed an initial 1-D drift-diffusion device model for a diode within the Xyce ${ }^{T M}$ Parallel Electronic Simulator for the purposes of demonstrating the capabilities of the approach. This will allow us to begin to address some of the issues, both numerical and parallel, associated with our mixed-mode approach. the approach. This will allow us to begin to address some of the issues, both numerical and parallel, associated with our mixedmode approach. 
10778

\section{A Java-Based Tool for Multifidelity Modeling of Coupled Systems}

D. R. Gardner, J. P. Castro, G. L. Hennigan, M. F. Young

Significant effort has been invested in developing highfidelity models for parts of nuclear weapons (e.g., the neutron generator [NG]). However, comparatively little effort has been invested in developing computer modeling tools to examine interactions among weapon subsystems. Computer tools for modeling complex subsystem interactions in a nuclear weapon in varied environments and over a wide range of time scales (from nanoseconds to years) at multiple levels of fidelity will enable designers and surety analysts to perform their jobs "faster, better, and cheaper."

The goal of this project is to develop methods to couple vastly different subsystems and physical models. Sandia will encapsulate these methods within a framework accessed by a JAVA-based Web interface. Users will be able to select modules from a library and modify and connect them, thus creating a system for thermal and electrical analysis. We will model a range of thermal phenomena within each module. Users will be able to couple modules with differing spatial dimensionalities.

Technical challenges being addressed in this project include how to couple models of different spatial dimensionalities. We will also develop appropriate lumpedparameter thermal models for modules and couple them to a three-dimensional (3-D) model.

The methods developed will be broadly applicable to systems ranging from nuclear weapons to power plants. In particular, this work will provide an enhanced capability to model systems of nuclear weapons modules in normal and abnormal environments.

We developed a concept for linking a lumped-parameter model to a 3-D finite-element (FE) model for thermal radiation and completed a trial implementation. We are assessing the algorithm by comparing results from a mixed-fidelity system to results from a 3-D FE system. The fictitious test system consists of a case module containing an arming, fuzing, and firing (AF\&F) system module, a package module, and a safety device module. In the mixed-fidelity version, the AF\&F module is modeled by a 3-D FE model with temperatures calculated using the Coyote thermal analysis code, while the
The goal of this project is to develop methods to couple vastly different subsystems and physical models. Sandia will encapsulate these methods within a framework accessed by a JAVA-based Web interface....The methods developed will be broadly applicable to systems ranging from nuclear weapons to power plants. In particular, this work will provide an enhanced capability to model systems of nuclear weapons modules in normal and abnormal environments. 
remaining modules are modeled with 0-D (lumped-parameter) models. In the 3-D version of the test system, all the modules are modeled by 3-D FE modules with temperatures calculated by the Coyote code. We are currently assessing the algorithm.

We coupled the Spice 3f5 electrical circuit simulator to the 3-D FE model of the AF\&F in the Entero framework. Electrical circuits can be embedded in each of the modules (0-D or 3-D), although circuits cannot currently be connected between modules. This enables users to perform some coupled thermal/electrical analyses.

We enhanced the Entero prototype engineering interface to enable a user to specify a 3-D Coyote thermal radiation model for the AF\&F or a $0-\mathrm{D}$ model. We developed a special editor in the JAVA programming language to assist a user in setting up a Coyote calculation and, more importantly, to capture and format the input for later use in communicating with other modules in a simulation. The interface enables a user to select modules from a library, customize them by specifying physical properties (e.g., specific heat) and the physics to be modeled (e.g., thermal radiation), and assemble them graphically into a system. The completed system can be saved and reloaded for later analysis.

\section{Other Communications}

Gardner, D. R. 2000. "A Brief Introduction to the Entero Project." Paper presented to the CoMeT

Summit Meeting, Albuquerque, NM, May.
We developed a special editor in the JAVA programming language to assist a user in setting up a Coyote calculation and, more importantly, to capture and format the input for later use in communicating with other modules in a simulation. The interface enables a user to select modules from a library, customize them by specifying physical properties and the physics to be modeled, and assemble them graphically into a system. The completed system can be saved and reloaded for later analysis. 
11336

Gamma-Ray Bursts and the Particle Mass Scale

P. C. Gray

Project research involves using number theory and, in particular, large integral numbers $\left(\sim 10^{60}\right.$ and greater $)$ formed from the products of prime numbers, to develop a counting procedure that begins with the relativistic energy/momentum relationship $(p c)^{2}+\left(m c^{2}\right)^{2}=E^{2}$. We then use this counting procedure to investigate and propose mechanisms for certain astrophysical phenomena-in particular, the extremely intense, short-pulse (>10 $10^{50}$ ergs, 10-100-second duration) gamma-ray bursts (GRBs) that are at cosmological distances. The counting procedure involves developing a scale-invariant description of space capable of representing highly energetic fermion/boson pairs of supersymmetric particles. Sandia believes that neutron starts with specific mass values can become unstable toward decay into an ensemble of these fermion/boson pairs with an energy release whose upper bound can approach the rest mass energy of the original body. We then use subsequent scattering of these particles to account for the high radiative yield of the original body. We next use subsequent scattering of these particles to account for the high radiative yield of GRBs (as much as 1053 ergs observed January 23, 1999, for GRB990123), the large variability in their observed energy output, and the expected coincident production of ultrahighenergy cosmic rays.

Sandia's results of this project can be applied to several important specific computations that permit the determination of the mass parameter that we identify as the mass of the muon neutrino. We made this determination by using primitive roots and a specific counting system. From symmetry, we predicted the physical mass of the muon neutrino to be $27.68 \mathrm{meV}$, a value that is consistent with both the available data and the expected range of the particle. The analysis demonstrates how the definition of inverse states extends beyond the previously developed four-dimensional (4-D) index space to eight dimensions, thereby enriching its classification capacity. We can make an explicit evaluation of the Higgs masses and determine the critical power indices. We evaluated the mass parameter of the Higgs boson as an integer composed of many factors. Analysis yields a mass of 9.56 times $10 \mathrm{E} 18 \mathrm{GeV}$. The corresponding super-symmetric Higgs fermion is then 
found directly, using the condition for super-symmetry, to have a physical mass of $\sim 2.64 \times 10 \mathrm{E} 18$. The mass parameters are part of the analysis that needs to be done using the numerical counting system.

\section{Refereed}

Yang, D., B. Alexey, B. Kieth, and R. Charles. 2000.

"Computation with Inverse States in a Finite Field.

The Muon Neutrino Mass, the Unified Strong-

Electrioweak Coupling Constant, and the Higgs

Mass." Sandia Technical Report SAND2000-2043,

Sandia National Laboratories, Albuquerque, NM

(August). 


\section{Electronics and Photonics}

The Electronics and Photonics investment area explores innovative solutions that build on the Labs' strengths in microelectronics, photonics, microelectromechanical systems (MEMS), and sensors. Advances in the realization of small, low-power, highly integrated electronics packages (i.e., integrated microsystems) support the mission needs of the Labs.

Research focus for this investment area have included microdevice concepts that enable advances in microelectronics, photonics, MEMS, and sensors, as well as innovative approaches for achieving higher levels of integration and mixed technology integration. Other research focus areas, including biological interfaces, mechanical actuation, photonics concepts, chemical sensing, and optical recognition, are being investigated for producing high-yield, highreliability microsystems.

Advances in the transistor of the future may not rely on decreasing its size but rather on a radical change in operation made possible by a quantum mechanical transistor created at the Labs. The "Quantum Tunneling Transistors for Practical Application" project has developed a mechanical transistor that is the equivalent of turning on a light bulb

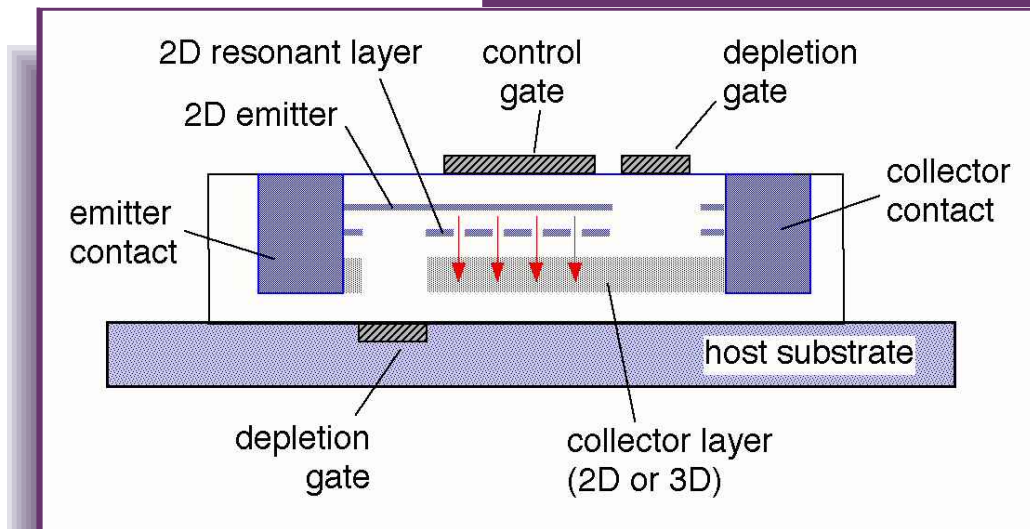

without closing a switch: electrons "tunnel" from path to path through a barrier that, according to classical physics, is impenetrable. "We have demonstrated real circuits that work and are easily fabricated," says Jerry Simmons, leader of the development team. The device, dubbed DELTT (Double Electron Layer Tunneling Transistor), offers promise of significant improvements in the speed of computers and in the accuracy of sensors. According to Paul Berger, chair of a session at the International Electron Devices Meeting last year, "I was impressed by the multitude of possible uses of the DELTT device, as well as by its simplicity of connectivity." Sandia has submitted two patent applications and has received one patent issued for the device. 
10325

Monolithic Integration of VCSELs and Detectors for Microsystems

K. D. Choquette, A. A. Allerman, K. M. Geib, J. A. Nevers, R. D. Briggs, C. T. Sullivan

Vertical-cavity surface-emitting lasers (VCSELs) are high-efficiency light sources appropriate for microsystem applications that will benefit from monolithic integration with compatible photodetectors. Such an integration of functions has applications ranging from near-term use in monitors for surety devices to longer-term enabling of on-chip photonic interconnects and advanced microsystems for sensing, surety, data storage, data routing, communication, and image manipulation, processing, and transmission. Moreover, twodimensional (2-D) arrays are an important extension of VCSEL technology, and intermeshed VCSEL/detector arrays will enable numerous advanced applications. Approaches for VCSEL/photodetector integration include the application of metal-semiconductor-metal (MSM) detectors on the laser substrate and the creation of resonant-cavity photodetectors (RCPDs) using the VCSEL epitaxial layers. This project addresses the basic enabling technologies for these approaches and uses combinations of these approaches to create prototypes that are designed to demonstrate the ability to enable new applications. In addition, this project includes environmental testing (radiation and high temperature) of these devices under conditions appropriate for DOE applications and applications such as optocouplers in spacecraft targeted for outer planetary systems.

This project addresses the basic enabling technologies for these approaches and uses combinations of these approaches to create prototypes that are designed to demonstrate the ability to enable new applications. In addition, this project includes environmental testing (radiation and high temperature) of these devices under conditions appropriate for DOE applications and applications such as optocouplers in spacecraft targeted for outer planetary

systems.

Monolithic integration of VCSEL and photodetector arrays is expected to provide a miniature and potential low-cost interface between digital electronics and optical interconnect and transmission media. We chose both the RCPDs and the MSM back-to-back Schottky photodiode for integration studies based on ease of integrability with selectively oxidized VCSELs, good data communications performance in the $<10$ Gbps data-rate range, and the potential for low-cost packaging with multimode fiber. We designed MSM photodetectors and developed processes with the photodetector on top of the VCSEL to take advantage of the high reflectance from the underlying p-doped distributed Bragg reflector (DBR) of the VCSEL and the relative ease of delineating the narrow electrode fingers of the photodetector. We determined this 
device design to be the best compromise of wide bandwidth, high responsivity, and minimally degraded yield. We made significant progress in integrating VCSELs and MSM detectors. We designed, grew, and tested epitaxial material for the monolithically integrated structure. The epitaxial design incorporated layers for both an optimized VCSEL structure (grown on the substrate) and an optimized MSM structure grown on top of the VCSEL structure, including etch-stop layers, electrical isolation layers, and contact layers. We demonstrated both the operation of the MSM grown on top of the VCSEL (but electrically independent) and operation of the VCSEL after etching away the MSM structure from above it. In both cases, the device operation was comparable to devices fabricated on epitaxial material optimized for each individual device. The present integration limitation is the difficulty of delineating 1-micron features over 12-micron topography for the MSM electrodes after the thermal processes required for VCSEL fabrication, specifically selective oxidation. We are currently investigating the use of refractory Schottky contacts compatible with VCSEL oxidation that would allow the electrodes to be delineated before any etching, resulting in a much higher yield integration of these dissimilar devices.

We successfully fabricated large arrays of integrated VCSELs and RCPDs. The arrays consist of 64 columns with alternating VCSEL and RCPD rows. We included an extra row of RCPDs so that there is a detector on each side of each row of VCSELs, resulting in a 64 x 65 array with a device-to-device pitch of 55 microns. We demonstrated individual VCSELs and RCPDs in the array, demonstrating performance comparable to previous stand-alone devices. We made significant progress on the development of $128 \times 129$ arrays, as well. Implementation of the matrix-addressing scheme proved to be very challenging. The initial array structure design proved to be overly ambitious, requiring significant modifications to both the epitaxial design and the photolithographic mask set. The underlying difficulty is achieving sufficiently low series resistance along the bottom of the VCSELs, such that the column lines that connect the devices all have similar voltage characteristics. To solve the resistivity problem, we pursued a more advanced epitaxial structure incorporating etch-stops and contact layers without sacrificing the optical properties of the DBR mirror. The new epitaxial structure allows us to change the row lines from highly doped semiconductor material to deposited metal traces that we expect to yield significant reductions in series resistance.
We demonstrated both the operation of the MSM grown on top of the VCSEL (but electrically independent) and operation of the VCSEL after etching away the MSM structure from above it. In both cases, the device operation was comparable to devices fabricated on epitaxial material optimized for each individual device. 
10326

\section{Post-Processed Integrated Microsystems}

R. J. Shul, S. H. Kravitz, S. A. Casalnuovo, W. K. Schubert, K. O. Wessendorf, T. R. Christenson

Sandia will develop a low-cost platform for integrated microsystems that is easily configured to meet a wide variety of specific applications-driven needs. Once developed, this platform, which incorporates many of the elements that are common to numerous microsystems, will enable integrated microsystem users access to this technology without paying the high upfront development costs that are now required.

The process starts with the fabrication, or acquisition, of wafers of sparsely placed device or circuit components fabricated in any foundried technology (silicon [Si] complementary metallic oxide semiconductor [CMOS] or bipolar technologies, high-frequency gallium arsenide [GaAs] technologies, microelectromechanical systems [MEMS], etc.). We call these "smart substrates." Using the diverse processing capabilities of Sandia, we then intend to "post-process" highvalue components in open areas on the front of the wafers, or on the back, and to microelectronically integrate the added components with the preplaced circuitry. Examples of postprocessed components include sensors, antennas, surface acoustic-wave (SAW) devices, passive elements, microoptics, and surface-mounted hybrids. The combination of preplaced electronics and post-processed components will enable the development of many new types of integrated microsystems. Targeted applications include integrated sensored systems, tags, and electromechanical systems.

We demonstrated a robust electroplated via technology for through-wafer interconnects for device applications. We tested a series of plated vias electrically and demonstrated electrical continuity and isolation between vias as well as from the Si wafer. We implemented these vias in the fabrication of a four-wafer planar microbattery.

The hybrid SAW chemical microsensor array successfully demonstrated detection of volatile organic compounds (VOCs), semivolatile simulants of chemical warfare $(\mathrm{CW})$ agents, and $\mathrm{CW}$ agents themselves. Device sensitivity, discrimination, and measurement repeatability are sufficiently good to distinguish between interferants and the $\mathrm{CW}$ agents during numerous tests. We successfully tested the
Sandia will develop a low-cost platform for integrated microsystems that is easily configured to meet a wide variety of specific applicationsdriven needs. Once developed, this platform, which incorporates many of the elements that are common to numerous microsystems, will enable integrated microsystem users access to this technology without paying the high upfront development costs that are now required. 
first monolithically fabricated SAW chemical sensor array against VOCs typically used as interferants for CW agents. The device was fully functional with good sensitivity.

We integrated an application-specific integrated circuit (ASIC) with an on-chip chemiresistor-sensor and evaluated it with both fixed-value thermistors (to evaluate the temperaturecompensation circuitry) and with off-chip chemiresistor arrays. Post-processing of the chips to modify the metallization of the array fingers was very successful, as was the printing of the chemiresistors. The ASIC functioned as designed, and we delivered an integrated ASIC and sensor in the form of an integrated robot substrate. We fully evaluated the ASIC for practical layout reasons and small design changes and defined the goals and design changes.

The one-step deep reactive ion etch (DRIE) process used to fabricate mag-FPW (magnetic flexural plate-wave) devices created irregular and irreproducible oval membranes from a rectangular etch window, which led to poor electrical characteristics of the device. We developed a two-step DRIE process to control the foot profile that defines the membrane edge in mag-FPWs. The process includes a two-level mask process that enables the formation of a $50 \mu \mathrm{m}$-wide trench around the perimeter of the membrane. We removed the first mask level and etched the large rectangular FPW well simultaneously with the $50 \mu \mathrm{m}$ trench. The optimized two-step process produces a much more rectangular and reproducible membrane with only very slight curvatures in the edge. The improved two-step DRIE process results in FPWs that are equivalent to the best $\mathrm{KOH}$ (potassium hydroxide)-etched devices, which have perfectly straight membrane edges. The DRIE process is more compatible with post-processing of the FPW on an ASIC substrate. We demonstrated the two-step process with both amorphous diamond (a-D) membranes and silicon nitride ( $\mathrm{SiN})$ membranes.

The lack of an in-house FPW membrane material compatible with post-processing on ASIC substrates has delayed demonstration of post-processed FPWs on ASIC substrates. Low-stress a-D layers are incompatible with the ASIC fabrication process. We identified two promising polymer membrane materials compatible with post-processing, but process development was delayed due to equipment problems.

We finalized FPW ASIC designs but did not obtain ASIC chips from an IC foundry. The FPW ASIC is designed in a discrete bipolar-transistor PC board implementation. The high
We integrated an applicationspecific integrated circuit (ASIC) with an on-chip chemiresistor-sensor and evaluated it with both fixedvalue thermistors (to evaluate the temperature-compensation circuitry) and with off-chip chemiresistor arrays. Postprocessing of the chips to modify the metallization of the array fingers was very successful, as was the printing of the chemiresistors. The ASIC functioned as designed, and we delivered an integrated ASIC and sensor in the form of an integrated robot substrate. 
transconductance per unit bias current and low junction capacitance of the bipolars made this the technology of choice for the first deliverable. To make an IC using a straight CMOS process would make the design nearly impossible to implement. We chose a $\mathrm{Bi}-\mathrm{CMOS}$ process to design this circuit due to the ability to add a custom-designed n-type/p-type/ntype (NPN) donor bipolar to the process. A small amount of design time, layout, and fabrication is still required to complete this deliverable. 
10327

Development of Radiation-Hard Sensors for Space-Based Visible and Infrared Sensing Applications

R. R. Kay, M. K. Hinckley, D. V. Campbell, S. K. Dunlap, J. L. Rienstra

Sandia investigated the design, manufacture, and test of visible image sensors and infrared (IR)-readout integrated circuits (ICs). We fabricated an operable visible imager test device utilizing 40-micron x 40-micron pixels using Sandia's CMOS6RA microelectronics fabrication process. We characterized conversion gain, quantum efficiency, linearity, and noise performance. Although the process is not optimized for photodiode fabrication, we did obtain usable quantum efficiencies for both n-on-p and p-on-n photodiodes. We proved operability of capacitive transimpedance, direct injection (DI), and source follower (SF) unit cell types at ambient and liquidnitrogen temperatures. The higher-than-expected measured noise levels that we observed can be attributed to the integration capacitor reset process.

We designed a test device, referred to as DART (Detector Array Readout Test), to investigate the performance of the building blocks of a visible imager or IR-readout device in Sandia's CMOS6RA microelectronics fabrication process. We designed DART to allow testing of the component blocks individually, as well as to provide an operational device in which all components work together. Thus, DART contains two primary sections: a 128 x 16-pixel focal plane array and a device characterization block. The unit cell topologies chosen for investigation are capacitive transimpedance amplifier (CTIA and CTIAx4), DI, and SF.

We processed the first fabrication lot of DART devices. In several packaged and bonded devices, the photodiode test structures were accessible; in others the transistor test structures were accessible. We packaged and bonded nine devices such that the DART focal plane array was accessible.

We performanced-characterized the nine DART devices for operation of the focal plane array portion of the device. Evaluators provided bias and clock inputs using a Pulse Instruments Model 4700 focal plane tester. We provided optical inputs from a CVI Digikrom monochrometer. The DART devices operated at a frame rate of 500 frames per second. All unit cell types were functional and responsive to
We proved operability of capacitive transimpedance, direct injection (DI), and source follower (SF) unit cell types at ambient and liquid-nitrogen temperatures.

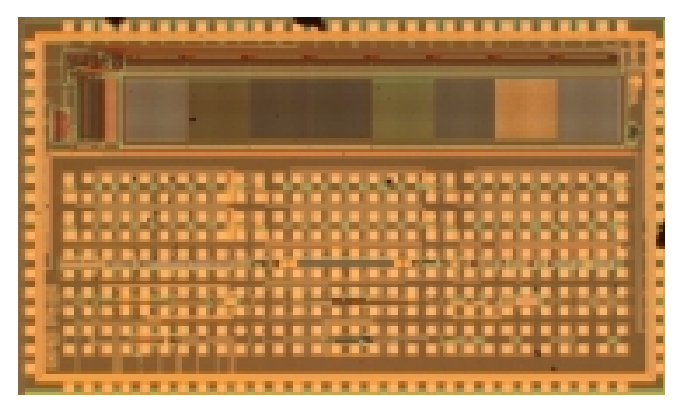

DART die photomicrograph. 
optical input. We found that the responsivity of the DI unit cells was too low to perform quantitative electrooptical measurements; the output of the monochrometer was not of sufficient amplitude to stimulate these unit cells.

We performed screening tests using an oscilloscope to identify device functionality and the number of inoperable pixels within each 16 x 16-pixel subarray. We considered inoperable any pixel that exhibited significantly higher or lower optical response than the average or that displayed markedly noisy behavior. Two devices exhibited only five bad pixels and were selected for further quantitative analysis.

We measured spectral quantum efficiency at ambient and cryogenic temperatures. We measured peak quantum efficiency of $60 \%$ at 550 nanometers wavelength at ambient temperature, with a reduction to $45 \%$ at 85 Kelvin.

We also measured the array output signal at increasing optical irradiance levels. By increasing the slit width of the monochrometer illumination source, the irradiance could be increased in uniform increments to gauge the linearity of pixel output versus input irradiance. The slope of the CTIA and CTIAx 4 responses changes dramatically at about 2800 millivolts. This is because the sample-and-hold switch is unable to discharge the sample-and-hold capacitor when the amplifier output rises above this value. This relatively low voltage limit is due to the body effect increase in threshold voltage. The usable output swing of the CTIA unit cell is about 1.25 volts, and the CTIAx 4 is about 0.7 volt. The SF unit cell has the largest usable output swing of about 1.75 volts.

We collected dark output noise voltage measurements. The noise voltage was referred to input noise using the measured conversion gain. For the CTIA unit cell, we measured an input-referred noise level of 220 electrons. This is a factor of four greater than modeled values. We demonstrated the excess noise to be related to the unit cell reset process. Future designs should incorporate correlated double sampling to reduce this excess noise.
For the CTIA unit cell, we measured an input-referred noise level of 220 electrons. This is a factor of four greater than modeled values. 
10328

\section{Silicon Three-Dimensional Photonic Crystal} and Its Applications

S. Y. Lin, J. G. Fleming, S. K. Lyo

The goals of this project are to advance Sandia's recent technology breakthrough in creating silicon (Si) threedimensional (3-D) photonic crystals and to demonstrate these engineered materials' unique military and commercial applications.

Military applications include infrared (IR) image control (thermal stealth), target identification, and signature recognition. Commercial applications targeted at the wavelength used for optical communications, l micron, will lead to a totally new generation of Si photonic devices that are monolithically integratable with current Si microelectronics, microelectromechanical systems (MEMS), and sensors. This will enable full microsystems on a chip. Examples of discrete Si photonic devices are high-performance low-cost lasers, lightemitting diodes (LEDs), mirrors, waveguides, and optical filters.

Photonic crystals are materials patterned with a periodicity in dielectric constant, which can create a range of forbidden frequencies called a photonic bandgap (PBG). Photons with energies lying in the bandgap can not propagate through the medium. This provides the opportunity to control light for photonic information technology. Photonic crystals can be thought of as optical analog of electronic semiconductors. In this way, waveguides in photonic crystals are the analog of metal lines on integrated circuits (ICs), and physical defects in the photonic crystal correspond to dopants in a semiconductor.

While 3-D photonic structures were proposed over a decade ago, and despite intense efforts, difficulties in fabricating these devices have effectively limited this technology to the microwave regime. Using techniques developed for the fabrication of MEMS, we recently fabricated a 3-D photonic crystal with stop gap centered at 11 microns. At present, we have a unique combination of facilities and expertise to design, fabricate, and test crystals with bandgaps down to between 1 and 2 microns.

Using a new design and a record fabrication of a 10layer photonic crystal structure, we achieved high-Q and, at the same time, high transmission through 3-D Si microcavities.
The goals of this project are to advance Sandia's recent technology breakthrough in creating silicon $(\mathrm{Si})$ threedimensional (3-D) photonic crystals and to demonstrate these engineered materials' unique military and commercial applications.... we have a unique combination of facilities and expertise to design, fabricate, and test crystals with bandgaps down to between 1 and 2 microns. 
We designed, fabricated, and tested two types of 3-D waveguides and waveguide bends. Both types of waveguide have very broad bandwidth, $50 \%$ and $\sim 100 \%$ of the bandgap, and a near-perfect transmission $(\sim 100 \%)$ efficiency.

On the IR imaging side, we fabricated new six- and eight-layer 3-D photonic crystals and took their IR images. This work continues to demonstrate the tremendous potential of 3-D photonic crystals in solar-cell and military image-control applications.

In the area of 3-D optical crystals, we made important progress in controlling light emission from an erbium (Er)doped 3-D photonic crystal. The first challenge that we resolved is the successful integration of Er glass into 3-D photonic crystal. We infiltrated low-temperature glass uniformly into the entire photonic crystal and performed a series of time-resolved photoluminescence experiments. These data, showing modification of spontaneous rate, represent the very first observation of modification of spontaneous emission using a 3-D optical photonic crystal.

\section{Refereed}

Lin, S. Y. 1999. "Waveguide Bends in Three-

Dimensional Layer-by-Layer Photonic Bandgap

Materials." Microwave and Opt. Tech. Lett. 23

(October): 56.

Lin, S. Y., and J. G. Fleming. 1999. "Three-

Dimensional Optical Photonic Crystal.” IEEE J.

Lightwave Technol. 17 (November): 1944.

We infiltrated low-temperature glass uniformly into the entire photonic crystal and performed a series of time-resolved photoluminescence experiments. These data, showing modification of spontaneous rate, represent the very first observation of modification of spontaneous emission using a 3 D optical photonic crystal. 
10329

\section{Monolithic Micromachined Variable Tuners for Rapid Prototyping and Optimization of Microwave Circuits}

\author{
L. R. Sloan, C. P. Tigges, C. T. Sullivan, C. W. Dyck, T. A. Plut
}

Microwave tuners measure and optimize the performance of microwave devices by varying the impedance presented to the device. Once the optimal impedance for a performance parameter (noise, power, efficiency, gain, etc.) is known, a circuit is synthesized to present the proper impedance to the device and translate that impedance to the system environment (usually 50 ohms). Present tuners are greater than 20 cubic inches with coaxial connectors as an interface. Loss incurred in the interface between the tuner and the device diminishes the impedance range of the tuner.

Sandia will develop miniature integratable precisionmachined (MIPM) tuners that can be assembled in a hybrid fashion or monolithically integrated with microwave active devices. This development would minimize interface losses, increase the tuning range, reduce design cycle times and cost, reduce size and weight of microwave circuitry, allow for frequency agility in circuit design, and improve microwave circuit performance.

A key element in the realization of an MIPM tuner is a small, low-loss switch. The switch can be used to connect or disconnect distributed or lumped components along a transmission line to present a large array of impedances. We demonstrated a functional MIPM switch. The switches processed on gallium arsenide (GaAs) are .046" x .046". Loss in the on state is less than $0.3 \mathrm{~dB}$ with a return loss greater than $25 \mathrm{~dB}$ from 0.1-20.1 GHz. Off-state isolation is greater
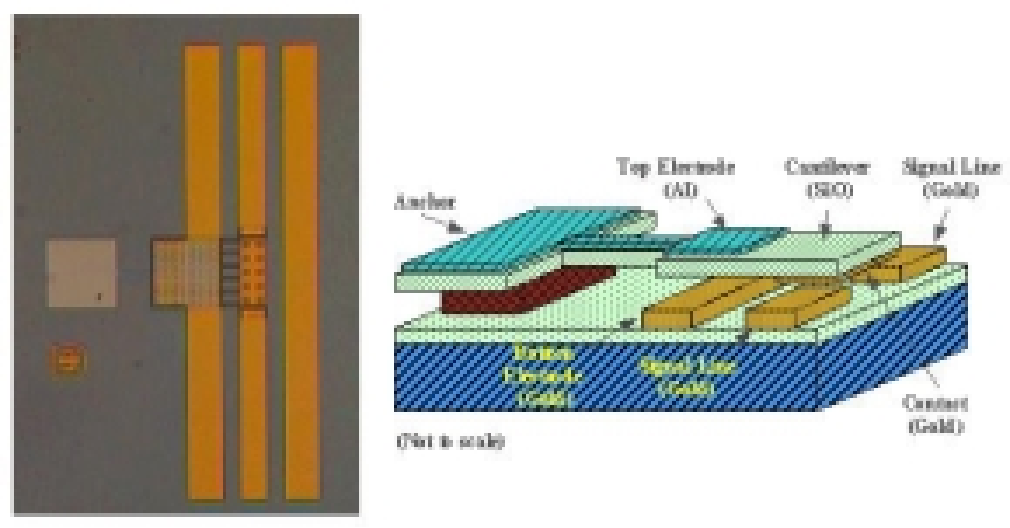

Sandia will develop miniature integratable precision-machined (MIPM) tuners that can be assembled in a hybrid fashion or monolithically integrated with microwave active devices. This development would minimize interface losses, increase the tuning range, reduce design cycle times and cost, reduce size and weight of microwave circuitry, allow for frequency agility in circuit design, and improve microwave circuit performance.

Picture and rendering of MEMS switch. 
than $25 \mathrm{~dB}$. Present efforts focus on improving the manufacturability, yield, packaging, and performance of the MIPM switch. Included in this effort is the collaboration with a commercial microwave foundry (Triquint Semiconductor) to supply commercial microwave active devices that can be used to develop the post-processing techniques of MIPM switches on their die.

The initial focus of this project was the microwave design, parametric modeling, and process development for an MIPM switch. We completed the work and demonstrated a functional switch. The switches had less than $0.3 \mathrm{~dB}$ loss, a return loss greater than $25 \mathrm{~dB}$, and greater than $25 \mathrm{~dB}$ of isolation from $0.1 \mathrm{GHz}$ to $20.1 \mathrm{GHz}$. Actuation voltages were between 23 and 28 volts.

The excellent performance of the switch makes it a viable component for an MIPM tuner, and we are also targeting it for receiver front-end protection circuitry, for switch-based phase shifters for phased-array antennas, and as a control element in radio-frequency (RF) tagging circuits. We completed preliminary designs of these circuits that now require integration of multiple switches with other $\mathrm{RF}$ components. Therefore, we concentrated on improving the manufacturability, performance, and yield of the MIPM switch.

Based on failure analysis, we optimized several processing steps to increase the yield of the MIPM switch. Improvements in the switch fabrication led to meeting militaryspecification wire-bond pull specifications, eliminating an electromechanical fatigue problem in the cantilever, and developing a process to remove the curvature in the switch structure caused by the stresses in the different deposited materials. A new lot incorporating these process changes is near completion and should result in higher yield.

We completed a design and layout of a frequency-agile amplifier using an active device from Triquint Semiconductor and an MIPM switch. Triquint delivered the devices, and we post-processed the MIPM switches.

We designed tunable capacitors and inductors, and process development using deep x-ray lithography is under way.

We wrote a processing simulator to enable viewing arbitrary cross-sections of the switches. Encoded in this software are resist profiles at various temperatures and etch profiles that enable the viewing of the process and examination for any "stringers" that may hinder mechanical movement. 


\section{Other Communications}

Palmer, D. W., L. R. Sloan, T. A. Plut, C. P. Tigges, C. R. Sullivan, and C. E. Sandoval. 2001. "Integration of MEMS and Commercial RFICs." Paper to be presented to the IEEE ECTC Special Wireless Components Session (May).

Sloan, L. R., S. L. Hietala, C. P. Tigges, G. Schuster, and C. E. Sandoval. 2000. "Post-Processed RF Micromechanical Switches on Commercial RFICs." Microwave Theory and Techniques Symp., accepted. 
10330

\section{Quantum Tunneling Transistors for Practical} Applications

J. A. Simmons, J. L. Reno, S. K. Lyo, J. R. Wendt, J. S. Moon, N. E. Harff

Sandia recently demonstrated the Double Electron Layer Tunneling Transistor (DELTT), a three-terminal quantum transistor with excellent electrical isolation between input and output. This is the first three-terminal quantum device that does not depend on lateral size confinement but rather is planar, allowing reliable fabrication. In the past year we demonstrated DELTTs with excellent behavior at $77 \mathrm{~K}$ and verified models predicting the maximum operating temperature. In addition, we also demonstrated circuits (bistable memories and digital logic gates) using the multifunctionality of the DELTT at $77 \mathrm{~K}$.

Because the DELTT is quantum, it should be capable of extremely high speeds. In conjunction with its multifunctionality, this would make it particularly suited for such high-end applications as ultrahigh-speed A-to-D (analogto-digital) converters for use in radar imaging and high-speed transistors for communications. Before the DELTT can fully live up to its exceptional promise, however, a number of engineering obstacles need to be overcome. The most important of these are (1) modification of the growth structure so as to achieve room-temperature operation, and (2) engineering of the device geometry to reduce parasitics and thus to achieve high speed.

Sandia intends to engineer further improvements in the DELTT to achieve room-temperature operation, increase the operating speed, and assess the ultimate speed limitations. We will pursue two different major thrusts. These are (1) the use of different material systems with larger conduction band offsets than AlGaAs/GaAs (aluminum gallium arsenide/gallium arsenide) so as to achieve deeper quantum wells ( $Q W s)$ and larger tunneling barriers for higher temperatures, and (2) the design of DELTTs with very short low-capacitance gates, lowresistance channels, and various other optimized features designed to reduce parasitics and enable high speed.

The most significant advance in this work is the demonstration of an increase by nearly four orders of magnitude in the current density of the three-layer DELTTs, achieved by reducing the thickness of the tunneling barriers.
Sandia recently demonstrated the Double Electron Layer Tunneling Transistor (DELTT), a three-terminal quantum transistor with excellent electrical isolation between input and output. This is the first three-terminal quantum device that does not depend on lateral size confinement but rather is planar, allowing reliable fabrication.

The most significant advance in this work was the demonstration of an increase by nearly four orders of magnitude in the current density of the three-layer DELTTs, achieved by reducing the thickness of the tunneling barriers. 
This is significant, since it implies that these DELTTs will operate at speeds on the order of $100 \mathrm{GHz}$, given that the speed is limited by resistance capacitance (RC) time constants. We also developed a quantitative model for the electrical characteristics of the three-layer DELTT, including the value of the emitter-collector voltage at the resonant current peak, and the voltage gain, as a function of growth structure parameters. We verified this model by electrical and photoluminescence (PL) measurements. We attempted to fabricate three-layer DELTTs with very high gain by placing heavily doped emitters very close to the surface, but were unsuccessful because of an intermittent problem with doping incorporation in Applied Epi, Inc.'s molecular beam epitaxy (MBE) machine. We believe that the doping problem is now fixed and have begun a new cycle of high-gain growth, fabrication, and measurement. Finally, through extensive simulation and design changes, we determined that the incorporation of pseudomorphic InGaAs layers is unlikely to improve the performance of three-layer DELTTs, and that instead it will be necessary to move to the more difficult route of $\mathrm{In} 0.53 \mathrm{Ga} 0.47 \mathrm{As} / \mathrm{In} 0.52 \mathrm{Al} 0.48 \mathrm{As}$ latticematched to indium phosphate (InP) substrates. We are planning calibration runs for these structures at Sandia and have made arrangements with Hughes Research Labs, who grows the world's best material in this system, to collaborate on growing them there as well.

\section{Refereed}

Moon, J. S., J. A. Simmons, J. L. Reno, W. E. Baca, M. A. Blount, V. M. Hietala, and E. D. Jones. 2000.

"Double Barrier Resonant Tunneling Transistor with a Fully Two-Dimensional Emitter." Appl. Phys. Lett., submitted.

Moon, J. S., J. A. Simmons, J. R. Wendt, V. M. Hietala, J. L. Reno, W. E. Baca, and M. A. Blount. 2000. "Dual-Side Wafer Processing and Resonant Tunneling Transistor Applications." Proc. 196th Electrochem. Soc. Mtg. I (Honolulu, HI, 17-22 October).

Reno, J. L., J. A. Simmons, J. S. Moon, M. A. Blount, F. Gelbard, and W. E. Baca. 1999. "Double Electron Layer Tunneling Transistor Modifications for HigherTemperature Operation." Paper presented to the North American Conference on Molecular Beam Epitaxy, Banff, Alberta, Canada, 9-15 October. 
10331

\section{Development of Magnetically Excited Flexural Plate-Wave Devices for Implementation as Physical, Chemical, and Acoustic Sensors, and as Integrated Micropumps for Sensored Systems}

W. K. Schubert, D. R. Adkins, R. J. Shul, C. P. Tigges, J. L. Dohner, M. A. Mitchell

The magnetically excited flexural plate-wave (mag$F P W)$ device has great promise as a versatile sensor platform. FPWs can have better sensitivity at lower operating frequencies than the more common surface acoustic-wave (SAW) device. Lower operating frequency ( < 1 megahertz for the FPW versus several hundred megahertz to a few gigahertz for the SAW device) simplifies the control electronics and makes integration of sensor with electronics easier. Magnetic rather than piezoelectric excitation of the FPW greatly simplifies the device structure and processing by eliminating the need for piezoelectric thin films, also simplifying integration issues. Furthermore, unlike the SAW device, the FPW can operate in both gas- and liquid-phase ambients, greatly increasing the number of potential applications. The versatile mag-FPW resonator structure can be configured to fulfill a number of critical functions in an autonomous sensored system. As a physical sensor, the device can be extremely sensitive to temperature, fluid flow, strain, acceleration, and vibration. By coating the membrane with self-assembled monolayers (SAMs), or polymer films with selective absorption properties (originally developed for SAW sensors), the mass sensitivity of the FPW allows it to be used as biological or chemical sensors. The mag-FPW device can be used as an acoustic transceiver, operating at frequencies from the audio range up to a few hundred kilohert. Yet another critical need in autonomous sensor systems is the ability to pump fluid. Mag-FPW structures can be configured as micropumps, using controlled acoustic streaming to move the fluid. Sandia intends to enhance the acoustic streaming by inducing wave interference through active control of the phase relationship of multiple sources. Successful development of mag-FPW devices as physical, chemical, and acoustic sensors and as micropumps for both liquid-and gas-phase analytes would enable the development of a completely new integratedsensing platform. Full development of this technology in the
Successful development of magFPW devices as physical, chemical, and acoustic sensors and as micropumps for both liquid-and gas-phase analytes would enable the development of a completely new integratedsensing platform.
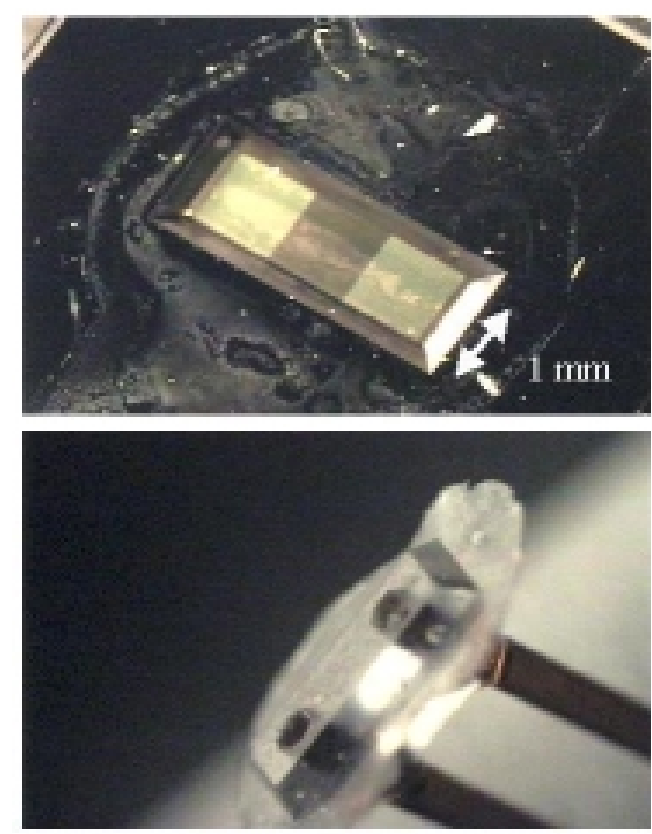

Micrographs showing the bottom view of the membrane and cavity of a micropump structure and the mating lid made from epoxy molded in a micromachined silicon form that exactly matches the pump cavity. The etch depth of the silicon form is slightly less than the full thickness of the wafer so that the lid molded in it will confine the liquid close to the membrane surface. 
areas defined previously would give Sandia strong and unique capabilities in the area of miniature, smart, and sensored systems. This technology is also applicable to miniature chemical-analysis systems.

Development of mag-FPW devices as basic building blocks for integrated physical, chemical, and acoustic sensing platforms requires progress on several interrelated fronts.

We fabricated and tested multiple membrane chemical sensors. We applied polymer films (such as ethyl cellulose) with selective absorption properties to the sensor membrane of these devices. We used the second membrane on the same die as a reference, providing compensation for drift in ambient conditions like temperature, pressure, and humidity.

We also fabricated and tested acoustic streaming test structures utilizing silicon nitride $(\mathrm{SiN})$ membranes. Results indicate that lower-stress membranes (potentially the polymer membranes discussed below) are needed for stronger streaming effects. We produced and used micromachined Si molds to fabricate FPW pump lids from a variety of epoxy materials. We used a release agent to keep the epoxy from adhering to the $\mathrm{Si}$ mold. These lids confine the fluid within a fraction of an acoustic wavelength of the membrane where the acoustic streaming effects are strongest.

We demonstrated FPW operation in liquids (although damping effects were quite high). The observed resonant frequency differences between air and water operation are consistent with theoretical predictions.

We developed a two-step, anisotropic dry Si etch (called the Bosch etch, after the inventor) that is more suitable than wet etching for post-processing FPW devices in reserved areas of wafers already containing the drive electronics. This was a significant accomplishment since lateral dimensional accuracy is more critical for FPW resonators than for any other Bosch-etched device under development. This process produced devices with performance characteristics rivaling those of our best wet-etched devices. We also developed a version of this process that will allow multiple etch depths.

We investigated two primary alternatives to $\mathrm{SiN}$ membrane materials. We produced Bosch-etched FPW devices using a-D (amorphous diamond)-like carbon membranes, which are extremely resistant to the Bosch etch and eliminate the need for an underlying oxide etch stop layer. We also investigated silicon-carbide ( $\mathrm{SiC}$ ) membranes but were not successful in achieving both tensile stress and good Bosch-etch
We developed a two-step, anisotropic dry Si etch (called the Bosch etch, after the inventor) that is more suitable than wet etching for postprocessing FPW devices in reserved areas of wafers already containing the drive electronics.

This was a significant accomplishment since lateral dimensional accuracy is more critical for FPW resonators than for any other Bosch-etched device under development. This process produced devices with performance characteristics rivaling those of our best wetetched devices. We also developed a version of this process that will allow multiple etch depths. 
characteristics. We shifted the alternative membrane effort to an investigation of low-stress polymer membrane materials that have lower processing temperatures $\left(<400^{\circ} \mathrm{C}\right)$ and would facilitate post-processing of FPW devices.

We improved both the resonant mode imaging system and the finite-element modeling (FEM) capabilities and used them in combination to examine several aspects of the FPW resonator operation. Correlation of the FEM predictions with experimental results gives us confidence to test many process requirements and device design modifications theoretically, reducing both development time and expense.

We designed and modified several generations of FPW resonator structures and chemical sensors to accommodate new process protocols and to account for identified process limitations. We also developed vibration sensor designs and procured a mask set. We are preparing to produce the first lot of vibration sensors.

\section{Other Communications}

Adkins, D. R., W. K. Schubert, M. A. Butler, and A. S. Chu. 1999. "Modeling and Imaging Flexural PlateWave Devices." Proc. ASME 1999 Internat. Mech. Eng. Congress and Expo. MEMS-Vol. 1 (Nashville, TN, 14-19 November): 19-23.

Schubert, W. K., D. R. Adkins, M. A. Butler, S. J. Martin, M. A. Mitchell, R. Kottenstette, and K. O. Wessendorf. 1999. "Chemical Sensing with a Magnetically Excited Flexural Plate-Wave Resonator." Chemical Sensors IV, Proc. 1999 Joint Internat. Mtg. of the Electrochem. Soc. 99-23 (Honolulu, HI, 17-22 October): 332-335.
We improved both the resonant mode imaging system and the finite-element modeling (FEM) capabilities and used them in combination to examine several aspects of the FPW resonator operation. Correlation of the FEM predictions with experimental results gives us confidence to test many process requirements and device design modifications theoretically, reducing both development time and expense. 
10332

Novel Acoustically Driven Microoptoelectronic Devices

E. D. Jones, R. L. White, B. L. Wampler, J. L. Reno

This project investigates a novel approach for combining optical and surface acoustic-wave (SAW) techniques. In direct bandgap semiconductors, when a photon is absorbed, an exciton is formed in the focused spot region. If there are no interactions that disturb the exciton, it will emit a photon equal to the semiconductor bandgap energy minus the binding energy of the exciton. However, the presence of a short-pulse-duration SAW can transport the exciton from the original focused spot to another spatial location and then the photon is re-emitted. Because of the ability to control the input and output locations, such a device could strongly impact several Sandia programs, most notably mass sensors and weapon security devices. Because electron-phonon and exciton-phonon interactions are required for carrier movement leading to spatial relocation, there are basic physics issues that need to be understood, specifically, the electron-phonon interaction between the $S A W$, the exciton, and free carriers. Also, knowledge of exciton ionization processes by the SAW is important. Room-temperature operation is also an issue that must ultimately be resolved.

The project relies on excitons that are being transported large distances $(1 \mathrm{~mm})$ and therefore needs stringent sample requirements. Photoluminescence (PL) measurements for the exciton linewidth and lineshape are excellent indicators for sample quality. Initial measurements on molecular beam epitaxy (MBE)-grown GaAs/AlGaAs (gallium arsenide/ aluminum gallium arsenide) structures showed poor PL, and subsequently, we tested a series of structures to identify the source of the problems resulting during growth. We defined and implemented new processing requirements, resulting in a MESA-like design for the SAW and quantum-well (QW) configuration.

We tested and refined the SAW radio-frequency $(\mathrm{RF})$ characteristics by studying the shape and amplitude of the signal from the SAW receiver compared to that launched by the SAW transmitter. We found that with approximately $1 \mathrm{~mm}$ spacing between the SAW transmitter and receiver, the signal decreased by about $40 \mathrm{~dB}$. We surmise that this reduction is
This project investigates a novel approach for combining optical and surface acoustic-wave (SAW) techniques. 
due to the presence of the GaAs QW, optical fiber, associated wiring, mounting, etc.

We studied the exciton PL energy, lineshape, and intensity with and without the SAW operating continuously. Both the exciton PL energy and lineshape were unaffected by the presence of the continuously operated SAW; however, the amplitude decreased, indicating that some excitons were physically moved from the observation point by the SAW. Sample heating by the continuous SAW at high RF amplitudes is a problem. Thus, to confirm the exciton movement hypothesis, we made a series of pulsed SAW measurements with varying pulsewidths and amplitudes. Sample heating effects were greatly reduced by operating with small duty cycles, i.e., the time between each RF pulse (needed to launch the SAW) was very long.

For these pulsed experiments, we used a gated photon counter to measure the strength of the exciton PL signal. Two exciton signals were monitored by the gated photon detector. One gated signal was the exciton luminescence that occurs before (or long after) the pulsed SAW passed through the observation point. The second gated signal was taken at the time the SAW was in the exciton observation area. We determined experimentally the exact timing requirements between the launching of the pulsed SAW and the two gate times for the gated photon detector. After we measured the two exciton PL amplitudes at their respective gate times, we subtracted their amplitudes from each other. In this manner, if the presence of the SAW does not move the excitons from the focal spot area (observation point), the signal amplitude difference would be zero. On the other hand, exciton movement would cause the subtracted exciton signals to increase with increasing SAW amplitude.

We found the difference in amplitude between the two gated exciton signals to linearly increase with increasing amplitude of the pulsed SAW wave. The slope of this relationship represents the coupling efficiency between the RF circuitry and the SAW generator. Measuring this slope represents a new and novel method to measure the overall efficiency of generating a SAW. The fact that the difference amplitude increased with increasing SAW amplitude is proof that exciton movement occurred.
We found the difference in amplitude between the two gated exciton signals to linearly increase with increasing amplitude of the pulsed SAW wave. The slope of this relationship represents the coupling efficiency between the $R F$ circuitry and the SAW generator. Measuring this slope represents a new and novel method to measure the overall efficiency of generating a SAW. The fact that the difference amplitude increased with increasing SAW amplitude is proof that exciton movement occurred. 
10333

\section{Photonics Integration Devices and} Technologies

G. A. Vawter, C. T. Sullivan, O. B. Spahn, A. A. Allerman, J. R.
Wendt, W. W. Chow, S. Y. Lin

We will use Sandia's world-class expertise in selective AlGaAs (aluminum gallium arsenide) oxidation, dry-etching, high-gain semiconductor laser simulation, and recent progress in selective-area regrowth of AlGaAs to create new in-plane lasers with interconnecting passive waveguides for use in highdensity photonic circuits and future integration of photonics with electronics. Selective oxidation and doping of semiconductor heterostructures have made vertical-cavity surface-emitting lasers (VCSELs) into the world's most efficient low-power lasers. We will use technologies developed for VCSELs to improve etched-facet lasers, making them suitable for monolithic integrated microsystems. We will investigate three types of lasers: (1) a ridge laser with resonant coupling to an output waveguide, (2) a selectively oxidized laser with a low active volume and potentially submilli-Amp threshold current, and (3) a one-dimensional (1-D) photonic lattice laser with the potential to exhibit submicro-Amp threshold current.

We will investigate a variety of methods for enhancing laser-reflective feedback and laser-to-waveguide coupling both theoretically and experimentally. In addition to resonant couplers, we will evaluate such novel structures as in-plane refractive lenses formed by selective oxidation, subwavelength diffractive optic lenses, and photonic lattices as laser-towaveguide couplers. Laser-reflective feedback concepts include metal-or dielectric-coated facets, single or multiple airgaps, photonic lattice mirrors, and total-internal-reflectance triangular ring cavities. Finally, we will fabricate integrated passive waveguides by selective-area regrowth, selective oxidation, or high-temperature diffusion of $\mathrm{Al}, \mathrm{Ga}$, and In (indium) within adjoining layers.

This project will result in a high-performance activel passive photonic integrated circuit (PIC) technology enabling solutions appropriate for future monolithic integrated microsystems. The new technology will comprise highly efficient integrated waveguide-coupled lasers and the combined laser optics and time-dependent gain models that will enable future systems to be developed more efficiently.
We will use Sandia's worldclass expertise ...to create new in-plane lasers with interconnecting passive waveguides for use in highdensity photonic circuits and future integration of photonics with electronics.... This project will result in a highperformance activelpassive photonic integrated circuit (PIC) technology enabling solutions appropriate for future monolithic integrated microsystems. 
We demonstrated resonant reflector lasers with lower threshold current than conventional facets. The additional reflection interface of the resonant gap clearly enhances effective reflectivity up to $\mathrm{R}=0.51$, compared to $\mathrm{R}=0.31$ for a cleaved facet, and reduces laser threshold current (lth). We also substantially improved current-voltage characteristics such that reverse-biased lasers have less than $30 \mathrm{pA}$ leakage current and high-breakdown voltage. In preparation for monolithic integration with lasers and waveguides, we tested these devices as waveguide photodiodes with internal responsivity of $0.3-0.6$ $\mathrm{A} / \mathrm{W}$.

We demonstrated a broad, two-dimensional (2-D), photonic-bandgap material, high-reflectance mirror with a very wide bandgap of $20 \%$ of the gap-center frequency. We achieved $99.9 \%$ reflectance with just six periods of photonic crystal. Using an underlayer of aluminum oxide ( $\mathrm{AlO}$ ), this new design reduces out-of-plane scattering losses by nearly an order of magnitude compared to previous AlGaAs underlayers and performs as an efficient waveguide at $1.55 \mu \mathrm{m}$ wavelength. Work on the 1-D laser has progressed with fabrication of a microcavity laser using InGaAsSb (indium gallium arsenide antimony)-active material for $1.25 \mu \mathrm{m}$ wavelength.

We dramatically lowered ohmic contact quality of buried-oxide-waveguide (BOW) lasers through improved doping control of the top contact layer. Improved contacts lowered the threshold voltage by 30 to $40 \%$ and improved yield of lasers with $>40 \%$ wall-plug efficiency. We developed and tested folded-cavity BOW lasers in both triangular and Ushaped configurations.

We developed a very exciting new concept in which etched laser facets can be eliminated in favor of selectively oxidized waveguide gratings. The strong index contrast of the oxidized grating permits strong resonant reflection of $20 \%$ to $80 \%$ using just a few tens of microns of grating length. Diffraction of light output from the laser is also eliminated since the beam never needs to exit the semiconductor material. We completed preliminary design models of an oxidized distributed Bragg reflector (DBR) laser and demonstrated fabrication of a grating.

A final key aspect of photonics integration is the combining of active and passive waveguides within the same chip, or even the same device. This need is underscored by the new DBR laser where the grating section and any external waveguide must not be light-absorbing. One very attractive means to achieve active/passive integration is selective-area
We developed a very exciting new concept in which etched laser facets can be eliminated in favor of selectively oxidized waveguide gratings. 
disordering of quantum wells (QWs). With this technology, we coat areas of a semiconductor wafer with an oxide layer to promote diffusion of $\mathrm{Al}$ at high temperatures. This interdiffusion of $\mathrm{Al}$ in $\mathrm{GaAs} / \mathrm{AlGaAs} \mathrm{QWs}$ raises the QW bandgap, shifting the absorption edge toward the blue, and rendering the material transparent to light emitted by nondisordered QW regions of the same wafer. We demonstrated $60 \mathrm{~nm}$ of absorption-edge blue shift by selective disordering. We demonstrated active/passive waveguide integration by fabricating extended-cavity lasers with both disordered and nondisordered QW waveguides within the Fabry-Perot cavity. We are now at a point in developing compact DBR lasers where we can begin to build gratings onto disordered material and make a true short-cavity DBR suitable for waveguide photonics.
We are now at a point in developing compact DBR lasers where we can begin to build gratings onto disordered material and make a true shortcavity $D B R$ suitable for waveguide photonics. 
10334

\section{Stress-Free Amorphous Diamond for High- Sensitivity Microsensors with Integrated Microstructures}

\author{
J. P. Sullivan, T. A. Friedmann, W. K. Schubert, M. P. De Boer, R. \\ J. Shul, C. I. H. Ashby, D. A. La Van, R. J. Hohlfelder
}

Sandia is creating high-sensitivity microsensors and rugged microelectromechanical systems (MEMS) using its recently discovered stress-free amorphous diamond (a-D). $a-D$ possesses a suite of properties unlike any other microstructural or microsensor material, including the ability to easily tailor film stress from compressive through zero to slightly tensile hardness and stiffness $90 \%$ that of diamond, very high wear resistance, surfaces that are intrinsically hydrophobic, extreme chemical inertness, chemical compatibility with silicon ( $\mathrm{Si}$ ) processing, room-temperature deposition, the ability to control the film conductivity from insulating to conducting, and biocompatibility. We used ultrathin (<0.1 micron) $a-D$ freestanding membranes to fabricate flexural plate-wave (FPW) chemical sensors with sensitivity 50 times greater than existing designs. A unique advantage of $a-D$ is that it is possible to use focused ion beams to locally graphitize the material in order to create embedded conducting lines. The MEMS structures fabricated from $a-D$ are important because they offer one possible solution for addressing stiction in polySi MEMS and can lead to wear-resistant, long-lifetime MEMS. The MEMS structures also permit measurement of the mechanical properties of films of $a-D$-measurements that are not possible using conventional techniques developed for bulk material, as there is no bulk analog of a-D that exists in nature. We also conceived new sensor and MEMS device structures that offer new capabilities for Sandia's programs

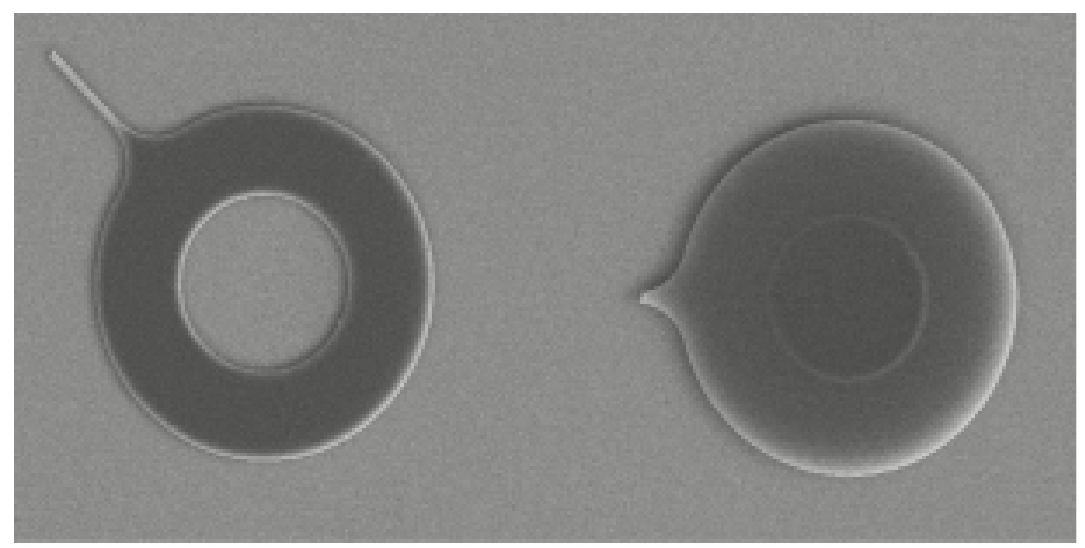

We also conceived new sensor and MEMS device structures that offer new capabilities for Sandia's programs within this project, including acoustic sensors, $a-D$ microrelay structures, hybrid a-D/poly-Si $M E M S$, and bio-MEMS.

This image shows an amorphous diamond uniaxial loading MEMS test sample (a MEMS pull-tab) after mechanical testing. The hole in the left piece is about 50 microns in diameter (about one-half the width of a human hair). The right end of the sample is fixed to the substrate by a si licon dioxide pillar (seen as the dark shadow region below the amorphous diamond). The specimen failed, as expected, in this thin region at a strength of about 9 GPa. These numbers are several times higher than conventional MEMS materials (e.g., polysilicon). The use of microscopic mechanical test specimens is a nice illustration of how MEMS structures can advance the fundamental understanding of materials. 
within this project, including acoustic sensors, $a-D$ microrelay structures, hybrid a-D/poly-Si MEMS, and bio-MEMS.

We demonstrated the chemical-sensing ability of the a-D FPW sensors and the ability to create multilevel a-D MEMS devices. We identified several new applications of a-D in the areas of sensors and MEMS, including a-D acoustic sensors, a-D membrane-based adaptive optic devices, MEMS microswitches, hybrid a-D/poly-Si MEMS structures, and a-D bio-MEMS structures. We developed both internally and externally funded proposals on some of these ideas with good likelihood of leading to new funded programs for Sandia. These developments hinged on improving the deep etch process for fabricating a-D membranes, identifying new materials and processes to enable multilevel MEMS structures, and improving our understanding of structural relaxation in a-D that occurs with annealing. We performed the basic materials research for some of these developments using our fabricated MEMS structures, which is an effective demonstration of the utility of MEMS structures for enabling materials property measurements when no other direct measurement is possible. The MEMS-based measurements reveal that a-D is

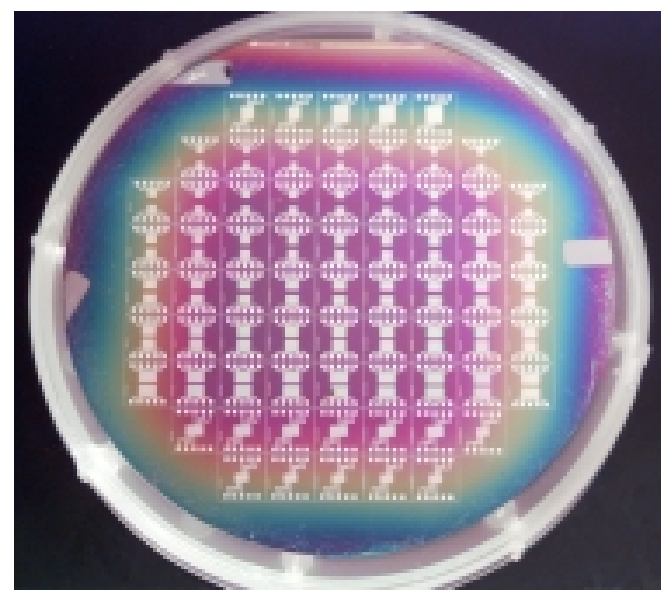

An array of amorphous diamond ( $a-D)$ flexural plate-wave sensor devices is shown on a 4-inch a-D-coated silicon wafer. Each sensor consists of two membranes of $a-D$ formed by deep reactive ion etching through the silicon. Atop these membranes are patterned aluminum lines. These a-D devices have shown the best sensor characteristics compared to other sensor devices, which are based on silicon nitride, that have been processed in the same way. exceptionally stiff (with an elastic modulus exceeding 700 $\mathrm{GPa}$, about 4.5 times that of $\mathrm{Si}$ ) and strong (with a fracture strength of about $8 \mathrm{GPa}$, about two to three times that of $\mathrm{Si}$ ), and there may be anisotropic ordering of the threefold and fourfold carbon components in the film. We also developed designs for advanced MEMS structures, including captured hubs that would permit the creation of rotating gear structures.

\section{Refereed}

Friedmann, T. A., J. P. Sullivan, M. P. de Boer, B. P. Jensen, D. A. La Van, C. I. H. Ashby, J. A. Knapp, D. M. Follstaedt, M. M. Mitchell, and R. G. Dunn. 1999. "Amorphous Diamond MEMS." Paper presented to the Materials Research Society Meeting, Boston, MA, 29 November-3 December.

Friedmann, T. A., J. P. Sullivan, R. J. Hohlfelder, C. I. H. Ashby, M. A. Mitchell, M. M. Bridges, M. P. de Boer, D. A. La Van, and B. P. Jensen. 2000.

"Amorphous Diamond MEMS." Paper presented to the ICMCTF Conference, San Diego, CA, 10-14 April.
La Van, D. A., R. J. Hohlfelder, J. P. Sullivan, T. A. Friedmann, M. A. Mitchell, and C. I. H. Ashby. 2000. "Tensile Properties of Amorphous Diamond Films." Proc. Mater. Res. Soc.: Amorphous and Nanostructured Carbon 593 (Boston, MA, 29 November-3 December): 465-470.

Sullivan, J. P., T. A. Friedmann, W. K. Schubert, D. R. Adkins, C. H. Seager, M. A. Mitchell, R. G. Dunn, and R. J. Shul. 1999. "An Amorphous Diamond-Like Carbon Sensor Device." Paper presented to the Materials Research Society Meeting, Boston, MA, 29 November-3 December. 


\section{Other Communications}

Sullivan, J. P., T. A. Friedmann, M. P. de Boer, D. A. La Van, R. J. Hohlfelder, C. I. H. Ashby, M. A.

Mitchell, and R. G. Dunn. 2000. "Developing a New Material for MEMS: Amorphous Diamond." (Invited)

Paper presented to the Fall 2000 Materials Research

Society Meeting, Boston, MA, 17 November-1

December. 
10688

\section{AIGaN Materials Engineering for Integrated Multifunction Systems}
J. Han, S. R. Lee, J. G. Fleming, S. A. Casalnuovo, D. M. Follstaedt,
C. C. Willan, W. R. Wampler, S. S. I. Tsao, S. S. Mani, M. J. Kelly,
T. R. Guilinger

In response to a worldwide interest in wide-bandgap III-nitride devices and the recent progress in the development of metal-organic chemical vapor deposition (MOCVD) growth of $(\mathrm{Al}, \mathrm{Ga}) \mathrm{N}$ (aluminum gallium nitride) materials at Sandia, we will explore the multifunctional usage of GaN to meet various mission requirements and to position Sandia at the technological forefront of this strategically important material family. Over a wide range of possible applications, we identified the development of compact ultraviolet (UV) optoelectronic devices (emitters and detectors), piezoelectric devices (surface acoustic wave [SAW] sensors), and the integration of GaN with silicon (Si) as the initial thrust areas. All of these areas have thus far remained relatively unexplored and yet are well aligned with Sandia's mission objectives. In addition to maintaining a broad portfolio, this project will explore the full potential of the ceramic-like nature of the IIInitride materials (namely, the physical robustness, chemical inertness, and tolerance to structural defects) to achieve chiplevel integration into microsystems containing various nitride devices and possibly between nitride devices and Si-based electronics.

We focused on the integration of GaN with Si-based microelectronics. Key issues addressed were (1) the growth and defect control of $\mathrm{AlGaN}$ and $\mathrm{GaN}$, and (2) relief of the strain resulting from the heteroepitaxy of $\mathrm{GaN}$ on $\mathrm{Si}$. We investigated stress due to a mismatch of thermal expansion coefficients between $\mathrm{GaN}$ and $\mathrm{Si}$ using a novel in situ stress sensor developed at Sandia. The in situ stress monitor enabled us to quantify the magnitude of thermal mismatch, as opposed to a projection from reported linear thermal expansion coefficients at room temperature. We employed a multipronged approach in overcoming the issue of cracking due to thermal mismatch.

We proved the use of stress engineering to be effective in controlling the growth stress. We showed that, with the employment of stress-management AlGaN interlayers, it is possible to control and allow a buildup of compressive stress during GaN growth. Tensile stress due to a thermal mismatch
In response to a worldwide interest in wide-bandgap IIInitride devices and the recent progress in the development of metal-organic chemical vapor deposition (MOCVD) growth of $(\mathrm{Al}, \mathrm{Ga}) \mathrm{N}$ (aluminum gallium nitride) materials at Sandia, we will explore the multifunctional usage of GaN to meet various mission requirements and to position Sandia at the technological forefront of this strategically important material family. 
can be offset by the "preexisting" compressive stress; we observed that the critical thickness of cracking is subsequently extended. Using this interlayer scheme, we succeeded in preparing thick $\mathrm{AlGaN} / \mathrm{GaN}$ distributed Bragg reflectors (DBRs) and demonstrated optically pumped vertical-cavity lasers in the near-UV range.

We also studied the use of patterned $\mathrm{Si}(111)$ templates, in this case in square posts with trenches of $5 \mathrm{~mm}$ to $80 \mathrm{~mm}$ deep, to study the behavior of the generation and propagation of cracks. It appeared that cracking was generated from incipient structural defects. The linear propagation of cracks can be blocked or isolated by the patterned posts. We observed that cracking can be effectively suppressed for features below $250 \mathrm{~mm}$. We demonstrated a GaN field-effect transistor (FET) on $\mathrm{Si}(111)$ substrates.

Finally, we investigated nanopatterned porous gallium nitride (PGaN) over porous silicon (PS) to produce devicequality AlGa1-xN (to be referred to as $\mathrm{GaN}$ ) material. We grew these $\mathrm{GaN}$ layers onto a composite PS/PGaN. This composite is formed by epitaxially depositing a thin (ca. $0.1 \mu \mathrm{m}) \mathrm{GaN}$ layer onto a fully dense $\mathrm{Si}$ wafer. Because the $\mathrm{GaN}$ is so thin, no cracks form in the GaN on cooldown from deposition temperatures. The GaN and the underlying $\mathrm{Si}$ are made porous in one step by electrochemical anodization in hydrofluoric acid electrolytes. We observed that PS layers served to relieve the thermal mismatched stress, even though careful surface preparation is required to facilitate planar growth of GaN. ...we succeeded in preparing thick AlGaN/GaN distributed Bragg reflectors $(D B R s)$ and demonstrated optically pumped vertical-cavity lasers in the near- $U V$ range.

\section{Refereed}

Han, J., K. E. Waldrip, S. R. Lee, J. J. Figiel, S. J. Hearne, G. A. Petersen, and S. M. Myers. 2000.

"Control and Elimination of Cracking of AlGaN Using LT AlGaN Interlayers." Appl. Phys. Lett. 78 (January 2001): 30 .

Hearne, S. J., J. Han, S. R. Lee, J. A. Floro, D. M. Follstaedt, E. Chason, and S. T. Tsong. 2000. "BrittleDuctile Relaxation Kinetics of Strained AlGaN/GaN Heterostructures." Appl. Phys. Lett. 76 (March): 1534.

Mitchell, C. C., M. E. Coltrin, and J. Han. 2000. "Mass Transport in the Epitaxial Lateral Overgrowth of Gallium Nitride.” J. Crystal Growth, accepted. 
10696

\section{Semiconductor Current Filament Lasers}

F. J. Zutavern, A. G. Baca, W. W. Chow, H. P. Hjalmarson, A. Mar, G. A. Vawter, M. W. O’Malley

Sandia is fabricating current filament semiconductor lasers (CFSL) that have produced 10 times more short-pulse energy than conventional semiconductor lasers (CSLs). This is a completely new type of laser that can potentially produce more than 1,000 times the peak power or short-pulse energy of CSLs. These lasers are created from current filaments in semiinsulating GaAs (gallium arsenide) and are not based on current injection or $p$-n (positive-negative) junctions. Instead, low-field avalanche carrier generation produces a highdensity, charge-neutral plasma channel with the required carrier-density distribution for lasing. Since these current filaments are not limited in volume by a diffusion length (as are junctions), their lasing volume can be much larger than CSLs.

Miniature short-pulse lasers are important to Sandia, the Department of Energy, and others for (1) active optical sensors for weapons systems in limited-visibility environments (optical fuzing, light amplification for detection and ranging [LADAR], range-gated imaging, and secure communications), (2) direct optical ignition of fuels and explosives, (3) micromachining micron-size features over large areas of metals and alloys, (4) ultra-wideband communications, (5) optical recording, and (6) laser surgery. All these applications are limited by the size, cost, and reliability of lasers that produce the desired beam parameters (> $1 \mathrm{~mJ},<1 \mathrm{~ns},<1$ degree). High-intensity subnanosecond pulses discriminate targets from spurious reflections of diffuse obscurants; produce high-resolution images from ballistic photons; pump optical parametric amplifiers for frequency-conversion, gain, and time-gating; and induce plasma breakdown at low energies. Sandia can drive these applications to new horizons when CFSLs become compact, inexpensive, high-energy, highquality, short-pulse lasers.

High-energy, electrically controlled, compact, shortpulse lasers are useful for active optical sensors (LADAR, range imaging, and imaging through clouds, dust, smoke, or turbid water), direct optical ignition of fuels and explosives, optical recording, and micromachining. We developed a new class of semiconductor laser that can potentially produce much more short-pulse energy than (injection-pumped) CSLs
Sandia is fabricating current filament semiconductor lasers (CFSL) that have produced 10 times more short-pulse energy than conventional semiconductor lasers (CSLs). This is a completely new type of laser that can potentially produce more than 1,000 times the peak power or short-pulse energy of CSLs....Sandia can drive these applications to new horizons when CFSLs become compact, inexpensive, highenergy, high-quality, short-pulse lasers. 
because this new laser is not limited in volume or aspect ratio by the depth of a p-n junction. We tested current filament semiconductor lasers (CFSLs) that produced $75 \mathrm{~nJ}$ of $890 \mathrm{~nm}$ radiation in $1.5 \mathrm{~ns}$ ( $50 \mathrm{~W}$ peak), approximately ten times more energy than CSLs. These lasers are created from current filaments in semi-insulating GaAs and, in contrast to CSL, are not based on current injection. Instead, low-field avalanche carrier generation produces a high-density, charge-neutral plasma channel with the required carrier-density distribution for lasing. We observed filaments as long as $3.4 \mathrm{~cm}$ and several hundred microns in diameter in our high-gain GaAs photoconductive switches. Their smallest dimension can be more than 100 times the carrier diffusion length in GaAs. We measured and reported on spectral narrowing, lasing thresholds, beam divergence, temporal narrowing, and energies that imply lasing for several configurations of CFSLs.

Our most stable CFSL is a quasi-vertical device that is $0.5 \mathrm{~mm}$ long. In this "surface-emitting" configuration, we grew metal-coated epitaxial contacts on both surfaces of a semiinsulating GaAs substrate and applied 0.7-2.5 kV. A lowenergy short pulse of light initiates a straight current filament when a CSL is focused to a narrow stripe on the edge of the substrate, across the contacts. Images of the spontaneous radiation from current filaments on $1 \mathrm{~mm}$-long devices show that the filaments are $40 \mathrm{~mm}$ wide by $1 \mathrm{~mm}$ long when conducting $20 \mathrm{~A}$. The active region of the laser is this plasma channel between the contacts. Light exits the cavity through holes in the metallic layers on the surfaces that may be coated with metallic or dielectric reflective layers. The cavity length is limited by the substrate thickness. Other configurations include a lateral edge-emitting device with surface contacts and a lateral edge-emitting device with edge contacts. The highest optical output energy came from an edge-emitting, surfacecontacted device.

We recorded spontaneous and stimulated emission and compared an off-axis spectrum (spontaneous) with an on-axis spectrum (stimulated). The spontaneous emission is $\sim 50 \mathrm{~nm}$ wide. The envelope of stimulated emission is $\sim 5 \mathrm{~nm}$ wide. The width of the peak-stimulated emission at $898 \mathrm{~nm}$ was $0.1 \mathrm{~nm}$. Beam divergence was 3 degrees for a $2.5 \mathrm{~mm}$ filament and 9 degrees for a $0.5 \mathrm{~mm}$-long device (full angle, 1/e diameters). Output energy ranged from 5-30 $\mathrm{nJ}$ in 1-2 ns-wide pulses (2-30 W peak) where the current ranged from 5-95 A in 6 nswide pulses. Temporal narrowing was evident in that optical pulsewidths were shorter and independent of current
We developed a new class of semiconductor laser that can potentially produce much more short-pulse energy than (injection-pumped) CSLs because this new laser is not limited in volume or aspect ratio by the depth of a $p$ - $n$ junction. 
pulsewidths when varied from 6 to $15 \mathrm{~ns}$. We also measured thresholds to lasing, i.e., thresholds in optical output energy versus filament current for devices with no reflective coatings, a low-reflectivity $(\mathrm{R})$ coating on the backside, a high-R coating on the backside, and a low-R coating on the front side. These data represent the highest energies observed at these currents with $0.5 \mathrm{~mm}$-long lasers (40 $\mathrm{nJ}$ ). Instabilities and inhomogeneities in current filament formation produce a range of output energies and a variety of spectra. Methods to improve device operation such as electrical and optical confinement should be pursued.

\section{Other Communications}

Zutavern, F. J., A. G. Baca, W. W. Chow, M. J.

Hafich, H. P. Hjalmarson, G. M. Loubriel, A. Mar, M.

W. O'Malley, and G. A. Vawter. 2000. "Current

Filament Semiconductor Lasers." Paper presented to

the Conference on Lasers and Electro-optics (CLEO

2000), San Francisco, CA, 11 May.

Zutavern, F. J., A. G. Baca, W. W. Chow, M. J.

Hafich, H. P. Hjalmarson, G. M. Loubriel, A. Mar, M. W. O'Malley, and G. A. Vawter. 2000.

"Semiconductor Electron-Hole Plasma Lasers." Paper presented to the International Semiconductor Laser Conference (ISCL), Monterey, CA, 26 September.
We also measured thresholds to lasing, i.e., thresholds in optical output energy versus filament current for devices with no reflective coatings, a lowreflectivity $(R)$ coating on the backside, a high-R coating on the backside, and a low-R coating on the front side. These data represent the highest energies observed at these currents with $0.5 \mathrm{~mm}$-long lasers (40 nJ). 
10733

SOI-Based High-Aspect-Ratio Si Bulk Micromachining for MEMS Applications

R. J. Shul, J. J. Sniegowski, C. P. Tigges, T. R. Christenson, M. A. Polosky, C. T. Sullivan

Nearly all microactuator types rely on elastic suspension for reliable and effective operation. The exceptionally high yield strength and linear mechanical response possessed by single-crystal silicon (Si) renders it an appropriate material for the fabrication of springs, particularly if they may be patterned into arbitrary prismatic geometry. Using a deep reactive ion etch (DRIE) process and silicon-on-insulator (SOI) wafers, Sandia will fabricate released, movable, single-crystal Si structures 10-100 $\mu$ m thick with lateral dimensions $<5 \mu \mathrm{m}$. The integration of these structures with, for example, surface-machined microelectromechanical systems (MEMS) or LIGA (the German term Lithographie, Galvanoformung, und Abformung, for lithography, electroplating, and molding), enables a considerable design leverage in diverse applications such as clasps, sensors, inertial resonators, microengines, and position actuators.

The attraction of high-aspect-ratio Si is that the parts can have relatively high longitudinal (out-of-plane) stiffness and mass. With this technology, we anticipate the fabrication of thick actuators with increased force and comparable throw as compared to surface-micromachined MEMS. Additionally, we will develop a two-mask lithographic process to form thick comb drives and stators (providing high force) with thin springs (low spring constants) on a single device to achieve low-voltage actuation, a very desirable goal for microstronglinks. Also enabled is the fabrication of high-mass structures with micron resolution for accelerometers or gyros in addition to extremely rigid, lightweight, high-strength structural components for systems applications. The development of this technology will also allow fabrication of a simple and robust microstronglink discriminator/optical redirect design. The simplicity is a direct consequence of the use of thick $\mathrm{Si}$, which reduces the number of required levels for the device structure and permits a simpler configuration of the optical redirect elements and interconnect.

We designed a diagnostic-based mask layout to develop both DRIE and wet-release etch processes for SOI structures.
Using a deep reactive ion etch (DRIE) process and silicon-oninsulator (SOI) wafers, Sandia will fabricate released, movable, single-crystal $\mathrm{Si}$ structures 10-100 $\mathrm{mm}$ thick with lateral dimensions $<5 \mu \mathrm{m}$.

The integration of these structures with, for example, surface-machined microelectromechanical systems (MEMS) or LIGA (lithography, electroplating, and molding), enables a considerable design leverage in diverse applications such as clasps, sensors, inertial resonators, microengines, and position actuators. 
The majority of devices were comb-drive structures, which consist of lines and spaces ranging from 1 to $3 \mu \mathrm{m}$ with spring widths of 1,2 , and $4 \mu \mathrm{m}$ and lengths of 25,50 , and 100 $\mu \mathrm{m}$. In addition, we developed and optimized a DRIE process for single-level SOI structures. The DRIE process yields anisotropic profiles with smooth wall and field etch morphologies. Optimized etch time minimizes the effect of aspect-ratio-dependent etching (ARDE) and lateral undercutting of the features. The etch time is defined to remove all of the Si between structures spaced 1 to $5 \mu \mathrm{m}$. Less etch time results in inefficient $\mathrm{Si}$ removal, while increased etch time results in a loss of dimensional control on the narrower features. Under the present etch conditions, dimensional control was lost on the $1 \mu \mathrm{m}$-wide fingers due to inefficient passivation of the Si sidewalls. Another complication in the DRIE process is the formation of wet etch-release holes during the DRIE process. The etch-release holes must be large enough to minimize ARDE and allow complete Si etching to the buried oxide, but also to maintain the structural integrity of the device. We designed a "honeycomb" structure $\sim 3 \mu \mathrm{m}$ across with $\sim 2$ $\mu \mathrm{m}$-thick walls for this purpose.

We developed and applied a wet etch-release process to $3 \mu \mathrm{m}$ and $25 \mu \mathrm{m}$-thick SOI actuators. The selectivity of the wet etch for oxide to $\mathrm{Si}$ was high (> 1000:1). The oxide etch rate was $~ 1000 \AA / \mathrm{min}$ in both the lateral and vertical directions. We laid out the "honeycomb" etch-release structures to reduce the required undercut distance for releasing the comb beam structures but not to release the anchors. The process consisted of a buffered oxide etch (BOE) (1:6, HF:NH4F) at room temperature with no agitation. We also added a wetting agent to the etch chemistry to improve etching in high-density feature areas.

We tested released structures using a computercontrolled probe station. Actuators, $25 \mu \mathrm{m}$ thick, were functional at voltages of $\sim 100 \mathrm{~V}$. Although the yield was relatively low, $<15$ percent, several different structures were functional. Improved handling as well as optimization of DRIE and wet etch-release processes are expected to improve yield significantly. The structures required no additional processing, such as the application of antistiction coatings or sublimation drying, prior to testing. This implies reduced stiction of the devices to the underlying substrate as compared to surfacemicromachine (SMM) structures. This may be attributed to the higher stiffness of the bulk single-crystal structures in the Z-directions as compared to polysilicon structures that are
We developed and applied a wet etch-release process to $3 \mu \mathrm{m}$ and 25 um-thick SOI actuators. 
typically $<10 \mu \mathrm{m}$ thick. In addition to comb-drive structures, we also fabricated cantilevered optical waveguide switching devices using electrostatic actuators formed in SOI materials for high-density optical switch matrices.

We also evaluated a multiwafer

We also evaluated a multiwafer integration process that relies on the DRIE process. We demonstrated vertical integration of four Si wafers using DRIE-etched via holes and integration process that relies on the DRIE process. press-fit precision-alignment pins. Vertical Si wafer integration using Si fusion bonding allows increased complexity and capability of microstructures. We anticipate that mechanical alignment features may be implemented with multilevel etched substrates that can maintain alignment of two etched substrates during fusion bonding sequences. 
10744

\section{Defining the Frontiers of Vertical, External- Cavity, Surface-Emitting Lasers}

W. J. Alford, R. E. Allman, A. A. Allerman, W. W. Chow, M. H. Crawford, T. D. Raymond, C. C. Wong

New sources based on diode laser technology are needed to improve wavelength coverage and beam quality for applications in remote sensing, local sensing, displays, and data storage. Vertical external-cavity surface-emitting lasers (VECSELs) provide significant flexibility in output wavelength and power over vertical-cavity surface-emitting lasers (VCSELs) and yet maintain the good beam quality inherent in VCSELs. The external cavity permits single-transverse-mode operation with large-diameter beams and also provides space for nonlinear optical elements within the laser cavity that can shift the output wavelength of near-infrared (NIR) lasers into the visible, ultraviolet (UV), or mid-IR. Sandia previously established itself as a leader in VECSEL research by demonstrating record IR efficiencies and the first blue-emitting $V E C S E L$. We are advancing the understanding and capabilities of VECSELs in three important areas: (1) optically pumped, wavelength-converted VECSELs, (2) optically pumped, highpower (> $1 \mathrm{~W}$ ) VECSELs, and (3) electrically pumped VECSELs (E-VECSELs).

Optical pumping demonstrated $>1 \mathrm{~W}$ output power and continues to lead the way in our understanding of VECSELs. Wavelength-converted VECSELs shift the output wavelength of NIR lasers into the visible with frequency doubling. We will produce mid-IR wavelengths by inserting an optical parametric oscillator into the VECSEL cavity. Highpower VECSELs require optimized quantum-well $(Q W)$ gain regions and sophisticated thermal management. We are using thermal and active region modeling to guide the VECSEL designs. Direct electrical pumping of VECSELs is a technically formidable problem and certainly presents the largest payoffs if realized. Our approach to electrically pumped devices is to design structures with lateral-current-spreading layers. We demonstrated the first intracavity-doubled E-VECSEL by producing microwatts of $430 \mathrm{~nm}$ light.

Our previous work with optically pumped VECSELs saw $\sim 0.25 \mathrm{~W}$ output with $\sim 40$ percent efficiency when pumping with a pump beam diameter of $\sim 120$ microns. The laser cavity for this work consisted of a curved dielectric mirror
We demonstrated the first intracavity-doubled E-VECSEL by producing microwatts of 430 nm light. 
and a gallium arsenide (GaAs) wafer on which we grew a highreflectivity distributed Bragg reflector (DBR) and an InGaAs (indium gallium arsenide) QW gain region. Since the pump power density is nearly optimized for these results, an obvious path to higher power is to scale the pump beam diameter while keeping the pump power density unchanged. Unfortunately, scaling the pump diameter to larger values results in higher temperatures in the active region, and this leads to reduced efficiency. The previous $40 \%$ efficient work used a quite simple thermal setup. However, when we use this setup with pump beam diameters of $\sim 300-500$ microns, the efficiency is a few percent or less. To improve the heat removal from the active region when pumping with large pump diameters, we used a sapphire window optically contacted to the top surface of the wafer to remove heat out the top as well as through the substrate. The sapphire window improved output efficiency by almost a factor of five and led to $>1 \mathrm{~W}$ output power at $\sim 980$ $\mathrm{nm}$.

We made progress in moving to an $860 \mathrm{~nm}$ wavelength. We demonstrated higher-efficiency $860 \mathrm{~nm}$ operation than has been seen by Ferguson's group in Scotland. We also made progress in the frequency doubling of optically pumped VECSELs. We demonstrated $\sim 6 \mathrm{~mW}$ of violet $(430 \mathrm{~nm})$ light from an intracavity-doubled VECSEL using GaAs QWs for the gain medium. We carried out this work with a krypton ion laser as the pump source, but these devices can be diode-pumped at $670 \mathrm{~nm}$.

In an effort to get better control of the lasing wavelength, we grew wafers with a partially reflective top DBR. This DBR and the high-reflecting DBR serve as an intracavity etalon and reduce the wavelength range over which the laser can operate. A wafer utilizing this design served in a frequency-doubled VECSEL for use in a flow visualization project that required $>5 \mathrm{~mW}$ of $490 \mathrm{~nm}$ with good beam quality and stable amplitude.

Extending the optically pumped VECSEL technology to an electrically injected version (E-VECSEL) could potentially generate a much more compact unit (no pump laser or optics) with higher efficiency and more stable alignment. Our efforts focus on reducing the threshold and improving the efficiency of the E-VECSELs. One critical area of improvement involved the implementation of an aluminumoxide-current blocking layer with a diameter slightly less than
Extending the optically pumped VECSEL technology to an electrically injected version (E-VECSEL) could potentially generate a much more compact unit (no pump laser or optics) with higher efficiency and more stable alignment. Our efforts focus on reducing the threshold and improving the efficiency of the E-VECSELS. 
the metal electrode placed directly above the QW active region. This layer channels injected carriers toward the center of the aperture.

A second important improvement was the optimization of current spreading in the large-aperture devices. Our studies show that one of the most effective methods of spreading the current with a relatively thin current-spreading structure is to employ a partially reflective DBR. Implementation of our current-spreading ideas resulted in a three-times reduction of threshold current compared to what we had at the beginning of the project. Furthermore, we achieved average output powers $>300$ microwatts, which is notable given the $10 \%$ duty cycle.

We performed preliminary tests toward generating 430 $\mathrm{nm}$ E-VECSELs through integrating a $\mathrm{KNbO}_{3}$ crystal in the $860 \mathrm{~nm}$ cavity. These preliminary results demonstrated $>50$ microwatt average power of $430 \mathrm{~nm}$ output from a $50 \mathrm{~mm}$ diameter device operated at $170 \mathrm{~mA}$ and $10 \%$ duty cycle. We consider these results to be quite significant, in that they represent the first demonstration of electrically injected VECSEL technology in the important blue-violet region.
We performed preliminary tests toward generating $430 \mathrm{~nm} \mathrm{E-}$ VECSELs through integrating a $\mathrm{KNbO}_{3}$ crystal in the $860 \mathrm{~nm}$ cavity. These preliminary results demonstrated > 50-microwatt average power of $430 \mathrm{~nm}$ output from a $50 \mathrm{~mm}$-diameter device operated at $170 \mathrm{~mA}$ and $10 \%$ duty cycle. We consider these results to be quite significant, in that they represent the first demonstration of electrically injected VECSEL technology in the important blue-violet region. 
10745

\section{Photoresist Technology for $70 \mathrm{~nm}$}

\section{Lithography with $157 \mathrm{~nm}$ Radiation}

C. C. Henderson, D. R. Wheeler, C. L. Janssen

The proposed use of $157 \mathrm{~nm}$ radiation for microlithography for integrated circuit (IC) manufacturing poses a particularly difficult problem for the design of a suitable photoresist technology. All typical photoresist compounds absorb so strongly at $157 \mathrm{~nm}$ that the usable imaging depth is far below the thicknesses required for the material to act as either a single-layer resist material in subsequent IC processing or as the top imaging layer of a bilayer photoresist approach. Two possible avenues for addressing this problem include (1) the design and synthesis of nontraditional photoresist materials with a higher $157 \mathrm{~nm}$ transparency to be used in a normal bilayer scheme and (2) the novel pre-exposure processing of a single layer to produce a bilayer with an ultrathin imaging top layer for use at the highly attenuated $157 \mathrm{~nm}$ wavelength.

We completed the theoretical ab initio calculations of the $157 \mathrm{~nm}$ absorption coefficient for many model compounds and compared them to experimental vacuum ultraviolet (UV) absorption data in the literature to access the value of these $a b$ initio calculations in the prescreening of candidate $157 \mathrm{~nm}$ photoresist polymers prior to the actual time-consuming synthesis. As expected, the qualitative accuracy of the calculations appeared to increase with the level of sophistication of the $a b$ initio calculation, and the calculational results were overall very encouraging, if not extremely quantitative. The energy of $157 \mathrm{~nm}$ radiation is very close to the energy required to break covalent bonds in molecules and polymers, which makes accurate modeling of the excited state of these compounds difficult at best. Nevertheless, we observed certain qualitative trends that indicated which chemical groups or functionalities would contribute strongly to the $157 \mathrm{~nm}$ absorption coefficient and thus should be avoided in the design of a $157 \mathrm{~nm}$ transparent imaging layer. Indeed, reports of photoresists with measured $157 \mathrm{~nm}$ absorptions low enough to be useful as the top imaging layer of a bilayer format have begun to appear. At this point, the ab initio calculational approach developed here would be most useful in the design optimization of some of these new $157 \mathrm{~nm}$ photoresist candidates that we recently identified. ...we observed certain qualitative trends that indicated which chemical groups or functionalities would contribute strongly to the $157 \mathrm{~nm}$ absorption coefficient and thus should be avoided in the design of a $157 \mathrm{~nm}$ transparent imaging layer. 
A more novel approach to solving the $157 \mathrm{~nm}$ photoresist problem is to focus on producing an ultrathin, yet highly etch-resistant, top imaging layer in a bilayer format. If thin enough, this top layer would not have to be particularly $157 \mathrm{~nm}$ transparent. The ultrathin bilayer approach that we investigated for $157 \mathrm{~nm}$ lithography involved the design and synthesis of several new polymeric compounds that were positive tone photoresists, but were also reactive toward gasphase silanes to produce highly etch-resistant polymers. A single layer of these novel materials, when pretreated with silanes prior to exposure, did form a bilayer with the desired ultrathin, highly silylated top layer on top of the remaining unreacted material. However, the pre-exposure silylation process to form the bilayer appears to interfere with the normal imaging function of the photoresist. It is likely that the side products from the silylation interfere with the subsequent photochemical reactions or that some of the silicon-rich byproducts of the imaging process are themselves dissolution inhibitors. Design modifications of these polymers to solve these problems are possible, but more time-consuming polymer synthesis would be necessary at this point to complete the proof-of-principle imaging experiment for this $157 \mathrm{~nm}$ photoresist approach. Given the recent success of the photoresist suppliers in identifying somewhat more $157 \mathrm{~nm}$ transparent versions of their standard deep UV (DUV) photoresists, the chance that the IC industry would move to this higher-risk ultrathin resist format now seems more remote than one year ago.
The ultrathin bilayer approach that we investigated for $157 \mathrm{~nm}$ lithography involved the design and synthesis of several new polymeric compounds that were positive tone photoresists, but were also reactive toward gasphase silanes to produce highly etch-resistant polymers. 
10749

\section{Growth and Characterization of Quantum Dots and Quantum Dot Devices}

R. M. Biefeld, M. J. Hafich, K. D. Choquette, K. M. Geib, E. D. Jones, J. F. Klem, S. R. Kurtz, J. R. Wendt, W. W. Chow, A. J. Fischer, J. G. Cederberg

A clear systems need exists for the development of alternative short-wavelength (0.6 $\mu \mathrm{m}$, yellow) as well as 1.3 $\mu \mathrm{m}$ infrared (IR) lasers on gallium arsenide (GaAs) for use in vertical-cavity surface-emitting lasers (VCSELs). To meet this need, Sandia will develop efficient self-assembled quantum dot (QD) emitters. Confinement in three dimensions (3-D) will minimize the degradation of output power with increasing temperature and improve overall efficiency. Enhanced 3-D confinement should aid in the development of shorterwavelength visible VCSELs as well as produce ultralow threshold currents. The 3-D carrier confinement will decrease lateral carrier diffusion, thereby increasing the internal quantum efficiency. Additional benefits of QDs within VCSELs include polarization control and the ability to tailor the emission wavelength by accessing material compositions with greater strain than permitted in quantum wells (QWs). To maximize device performance, we will develop a microscopic theory that combines photonic and quantum kinetic aspects so that we can predict optical properties of the QD/extended-QW system and compare them to the observed characteristics. Using metal-organic chemical vapor deposition (MOCVD) and molecular beam epitaxy $(M B E)$, we will examine the effects of surface orientation and strain on the size and position distribution of self-assembled QDs. We will use a combination of surface orientation, strain effects, and substrate or mask patterning with electron beam lithography to grow QDs with controlled size, shape, and spatial distribution. We will explore development of stable QD formation by argon (Ar) ion beam etching of various substrates as an additional preparation technique. We will prepare QD light-emitting diodes (LEDs) and lasers using the techniques developed in this investigation to use in existing and future Sandia programs.

We used MOCVD and MBE to grow indium arsenide (InAs) and indium gallium arsenide (InGaAs) QDs on GaAs substrates for potential 1.1-1.3 $\mu$ m laser applications. We characterized the QDs by atomic force microscopy (AFM) and photoluminescence (PL). The QDs are relatively uniform in
...Sandia will develop efficient self-assembled quantum dot (QD) emitters. Confinement in three dimensions (3-D) will minimize the degradation of output power with increasing temperature and improve overall efficiency. Enhanced 3-D confinement should aid in the development of shorterwavelength visible VCSELs as well as produce ultralow threshold currents. 
size, varying from 15 to $30 \mathrm{~nm}$ in height and 50 to $100 \mathrm{~nm}$ in width. We obtained dot densities of $0.5^{-2} \times 1010 \mathrm{~cm}^{-2}$. The size and position uniformity varies, depending on the growth technique and conditions. The PL intensity and linewidth are also dependent on growth technique and conditions, including temperature and growth rate. We observed PL intensity at both low temperature $(4 \mathrm{~K})$ and room temperature. We also prepared gallium arsenide antimony (GaAsSb) QDs by MBE with larger dimensions.

We initiated QD formation by Ar ion beam etching on $\mathrm{GaSb}$ substrates. Ion beam divergence and initial GaSb surface conditions profoundly influence the size and regularity of quantum-scale bumps formed in the GaSb surface. These preliminary results show promise for development of this technique to prepare QDs of GaSb.

We initiated an investigation into the dynamics of excitation-energy (e. g., exciton) transport in multidot-well structures. We showed theoretically that the two dominant mechanisms by which an exciton hops between widely separated states are dipole-dipole and photon-exchange coupling. We found that for dot-to-dot coupling, the photonexchange interaction can compete with the dipole-dipole coupling only for long-distance (> $400 \mathrm{~nm}$ ) transfer. We are currently formulating multiphonon-assisted hopping transfer rates between dots.

We began computing gain spectra for a simplified QD model using a free-carrier approximation. At this level, the different localized QD and delocalized QW states show up as peaks in the optical gain spectrum and can be compared with experiment.

This project covers four main areas of investigation: (1) the growth of novel QDs by MOCVD, MBE, and ion beam etching using misoriented and patterned substrates, (2) characterization of these dots using PL, AFM, x-ray diffraction and transmission, and scanning electron microscopy (SEM), as well as in situ optical reflectance, (3) the fabrication of lasers and light-emitting diodes (LEDs), and (4) modeling and firstprinciples band-structure calculations to assist in the understanding of transport and light emission from the QDs and to aid in the design of the devices. Both MBE and MOCVD are capable of producing semiconductor nanostructures with dimensions on the order of 10 to $100 \mathrm{~nm}$ in all three directions (quantum dots). We demonstrated these types of structures at Sandia with emission in the range of 1.0 to $1.3 \mu \mathrm{m}$. We also started to explore a novel Ar ion beam
Both MBE and MOCVD are capable of producing semiconductor nanostructures with dimensions on the order of 10 to $100 \mathrm{~nm}$ in all three directions (quantum dots). We demonstrated these types of structures at Sandia with emission in the range of 1.0 to $1.3 \mu \mathrm{m}$. 
etching technique to produce dots. We will continue to explore all three of these techniques to produce more well-defined QDs in InAs and InGaAs on GaAs and start to explore the formation of Sb-based dots (GaAsSb, InGaSb) and phosphorus (P)-based dots (InGaP) on GaAs substrates. These new QDs will allow us to extend the emission to both longer $(1.55 \mu \mathrm{m})$ and shorter $(0.63 \mu \mathrm{m})$ wavelengths.
These new QDs will allow us to extend the emission to both longer (1.55 $\mathrm{mm}$ ) and shorter (0.63 $\mu \mathrm{m})$ wavelengths. 
10750

\section{THz Technologies for Ultrahigh-Data-Rate Communications}

J. A. Simmons, L. R. Sloan, S. Y. Lin, J. G. Fleming, J. P. Anthes

Future wireless data communications will inevitably move to higher and higher data rates, necessitating very high carrier frequencies. For data speeds of 10 to $100 \mathrm{Gbps}$, the ideal carrier frequencies range from 0.1 to $1 \mathrm{THz}\left(10^{12} \mathrm{hertz}\right)$.

This frequency range, while having almost limitless bandwidth for short-range wireless data links, is at present virtually unused due to technology limitations.

Sandia has several existing research activities that may offer breakthrough technologies at THz frequencies. First, the photonic bandgap (PBG) work could yield the necessary signal routing and signal processing needed for a $\mathrm{THz}$ transceiver. Secondly, the Double Electron Layer Tunneling Transistor (DELTT) may provide a THz power source and detector. Finally, microelectromechanical systems (MEMS) and associated technologies offer interesting possibilities for tuning structures and fabrication methods.

This project is a concerted effort to develop these technologies for high-speed wireless THz communications. We will design, fabricate, and test passive components and signal routes constructed from PBGs. We will fabricate PBG structures using micromachining techniques. We will reengineer DELTT-type structures so as to act as high-sensitivity detectors (double quantum-well [DQW] photon-assisted tunneling [PAT]). Additionally, we will explore the use of MEMS for novel tuning and/or resonant cavity structures as well as possible THz source technologies. This project offers a unique opportunity for Sandia to lead the future in wireless data communications.

We set up an organic gas far-infrared (FIR) laser (approximately 0.3 to $10 \mathrm{THz}$ ) to test the DQW PAT detectors. We completed numerous design, fabricate, and test iterations for the DQW PAT (DELTT-based) detector. We observed two types of photoresponse: a broad one consistent with electron heating, and a stronger one occurring when the twodimensional electron layers are almost completely depleted. We also developed a nonlinear response theory of photonassisted tunneling in multiple barrier structures. This theory predicts resonant absorption near a step potential, which may explain the second type of observed photoresponse. Although
Sandia has several existing research activities that may offer breakthrough technologies at $\mathrm{THz}$ frequencies... This project is a concerted effort to develop these technologies for highspeed wireless $\mathrm{THz}$ communications....This project offers a unique opportunity for Sandia to lead the future in wireless data communications. 
we have not yet observed a narrowband resonant detector response, we identified several antenna design and field coupling issues and expect a demonstration of narrowband resonant response shortly. We limited testing of photonic crystal structures to existing equipment frequencies around 100 $\mathrm{GHz}$. We fabricated mechanical samples of photonic crystals in the $100 \mathrm{GHz}$ range using a novel robocasting fabrication method. Initial testing of the crystals looks promising. We have started design and simulation of photonic crystal-based $\mathrm{THz}$ passive components.

\section{Refereed}

Lin, S. Y., J. G. Fleming, R. Lin, M. M. Sigalas, R. Biswas, and K. M. Ho. 2000. "Complete ThreeDimensional Photonic Bandgap in a Simple Cubic Structure." J. Opt. Soc. of Amer., accepted.

Lyo, S. K. 2000. "Photon-Assisted Tunneling in Double Barrier Structures." Appl. Phys. Lett.

(Woodbury, NY), submitted.

Smay, J. E., J. Cesarano, S. Y. Lin, J. N. Stuecker, and J. A. Lewis. 2000. "Solid Freeform Fabrication of Photonic Bandgap Structures in the $100 \mathrm{GHz}$ Frequency Range." Paper to be presented to the Annual Meeting of the American Ceramic Society (Boston, MA), submitted.

\section{Other Communications}

Simmons, J. A. 1999. “Terahertz Detection Based on Photon-Assisted Tunneling in Double Quantum Wells." Paper presented to the DARPA Terahertz Technology for Sensing and Satellite Communications Program Kick-off Meeting, Westlake Village, CA, 2-3 November.
We fabricated mechanical samples of photonic crystals in the $100 \mathrm{GHz}$ range using a novel robocasting fabrication method. Initial testing of the crystals looks promising. 
10756

\section{New Architectures for Micro-Total-Analytical} Systems

E. F. Hasselbrink, P. C. Galambos, T. W. Krygowski, S. Montague, $Y$. Fintschenko, M. Okandan

This project will leverage Sandia's unique capabilities in electrokinetic pumping (EKP) and microfabrication to establish a new technology, high-performance liquid chromatography (HPLC), in the field of micro-Total Analysis Systems ( $\mu$ TAS). While HPLC remains the preferred macroscale analytical technique, its adaptation to an integrated microscale device has proved elusive because of the lack of a miniaturizable high-pressure (> 3000 psi) pump.

Recently, Sandia demonstrated a capillary $\mu H P L C$ system that utilizes its proprietary EKPs, which are capillarysized pumps capable of delivering over 9000 psi pressure. Since these pumps can be integrated on-chip and several improvements to HPLC can be realized through microfabricated sample injectors and chip-based separations media, it is our goal to achieve better separations than possible with macroscale $\mu H P L C$ using an integrated device. The design will incorporate EKPs, sample injector, and separation media on a single chip. Besides EKPs, two other Sandia technologies are essential components of this goal: (1) microfabrication of high-aspect-ratio insulated microstructures, in order to engineer optimal separations media and EKPs using complementary metallic oxide semiconductor (CMOS)-compatible silicon/polysilicon/nitride materials, and (2) Sandia-proprietary sample injection technology, in order to prevent parasitic dispersion during sample injection. At the same time, this research will bolster Sandia's efforts in the national security mission area of chemical/biological weapons $(C W / B W)$ nonproliferation and its efforts in biotechnology.

All components of the proposed $\mu$ HPLC system are thus far performing as expected. We achieved incorporation of electrodes into microfluidic channels by etching conduit channels that intersect the microfluidic channel and depositing electrode metals selectively into these conduits. We constructed several packed-particle EKPs on glass chips; these designs use a deep-etched region containing the packed particles, which are held in place by a shallow-etched region on
This project will leverage Sandia's unique capabilities in electrokinetic pumping (EKP) and microfabrication to establish a new technology, high-performance liquid chromatography (HPLC), in the field of micro-Total Analysis Systems $(\mu T A S)$....this research will bolster Sandia's efforts in the national security mission area of chemical/biological weapons $(C W / B W)$ nonproliferation and its efforts in biotechnology. 
each side of the packed section. The pressure attained by these pumps is greater than 3000 psi.

We completed the SUMMiT V research and development technology design of an EKP, using additional silicon nitride ( $\mathrm{SiN}$ ) membranes along with the five layers of polysilicon standard in SUMMiT-V. Initial short-loop fabrication experiments demonstrated that the process for deposition and patterning of the $\mathrm{SiN}$ layers is feasible. We developed a theory of porous media and verified the predicted behavior of streaming currents and potentials across porous pump/chromatograph media using a newly constructed streaming-measurement instrument. Surface-coating procedures reduce the surface charge density of silica by as much as a factor of 50 .

Sample loading and injection experiments yielded simple design rules that result in well-defined sample injections that are free of diffusive leakage from the sample introduction channel. Use of these design rules eliminates a major source of dispersion and separation degradation present in microscale systems.

\section{Refereed}

Paul, P. H., D. W. Arnold, D. W. Neyer, and K. B. Smith. 2000. "Electrokinetic Pump Application to Micro-Total Analysis Systems: Mechanical Actuation to HPLC." Micro-Total Anal. Sys. 20004 (Enschede, The Netherlands, May): 583-590.
We developed a theory of porous media and verified the predicted behavior of streaming currents and potentials across porous pump/chromatograph media using a newly constructed streaming-measurement instrument. Surface-coating procedures reduce the surface charge density of silica by as much as a factor of 50. 
10766

Heterogeneous Integration of Optoelectronic Arrays and Microelectronics

K. M. Geib, D. J. Rieger, A. A. Allerman, S. A. Kemme

Integrated microsystems provide the benefits of small size, low power requirements, robustness, and potentially inexpensive manufacture. However, multifunctional advanced microsystems often require a combination of microelectronic and photonic technologies. For example, high-density twodimensional (2-D) integrated optoelectronic arrays are the basic components necessary to construct real-time electrooptical signal-processing and analog informationprocessing microsystems. In corresponding applications such as digital and neural computing, data-mining, and data communication switching, a critical need exists to provide local electronic processing in each optoelectronic pixel. Moreover, to achieve the required functionality in these systems, highly sophisticated devices with state-of-the-art performance are necessary. Thus, combinations of dense numbers of high-performance devices originating from disparate technologies are needed to achieve the next generation of information processing, interconnect, and intelligent sensing microsystems.

Sandia proposes to establish and/or optimize three heterogeneous integration technologies and to employ them for three sets of specific optoelectronic array demonstrations. The microsystem demonstrators will involve high-count 2-D arrays of vertical-cavity surface-emitting lasers (VCSELs), microoptic lenses, and III-V microelectronic circuitry. The three integration approaches we will pursue are flip-chip bonding, applique bonding, and wafer bonding. Flip-chip and waferbonding techniques can integrate VCSELs with appropriate microoptics, while all three approaches can integrate lensed VCSELs with III-V driver circuitry. Note that to fabricate the complete demonstrator microsystems described below, sequential combinations of the integration technologies are necessary. The two primary objectives of this research are to develop and/or optimize the fabrication infrastructure and to fabricate optoelectronic source arrays, receiver arrays, and transceiver arrays.

The Triquint Foundry Service designed and implemented high-speed gallium arsenide (GaAs) VCSEL drivers. The measured driver input/output characteristics are
Sandia proposes to establish and/or optimize three heterogeneous integration technologies and to employ them for three sets of specific optoelectronic array demonstrations...The two primary objectives of this research are to develop and/or optimize the fabrication infrastructure and to fabricate optoelectronic source arrays, receiver arrays, and transceiver arrays. 
almost identical to the design goals. We tested the drivers to $\sim 1 \mathrm{GHz}$ and showed rise times of $\sim 500 \mathrm{ps}$. We integrated the drivers and VCSELs into a multichip package and are currently evaluating them.

To reduce the wiring complexity and improve the reliability of the VCSEL/driver integration, we are developing a flip-chip capability. We developed a novel gold-coated, silver-post flip-chip technique that resulted in $100 \%$ bond yields on numerous test samples with 68 bondpads. We used heat and pressure to attach the die, then under-filled them with epoxy. The epoxy actually adheres and contracts slightly, enhancing the contact integrity. A new six-level mask set allows contact to both the anode and cathode of the $8 \times 8$ individually addressable VCSEL array from the top side of the wafer. Keeping both electrical contacts on the same side of the wafer is important for keeping the packaging as simple as possible. The novel construct of the mask set also allows both the cathode and the anode to be coplanar at the original wafer surface, significantly increasing the probability of good bonds. We fabricated $8 \times 8$ individually addressable $850 \mathrm{~nm}$ VCSEL arrays, tested them as top emitters, and flip-chipped them to test coupons. The flip-chipped die are awaiting the substrate removal step required for further testing.
To reduce the wiring complexity and improve the reliability of the

VCSEL/driver integration, we are developing a flip-chip capability. We developed a novel gold-coated, silver-post flip-chip technique that resulted in 100 percent bond yields on numerous test samples with 68 bondpads. 
10774

Biosensors Based on the Electrical Impedance of Tethered Lipid Bilayers on Planar Electrodes

R. C. Hughes, S. M. Brozik, D. Y. Sasaki

Impedance-based, planar chemical microsensors are the easiest sensors to integrate with electronics. The goal of this project is a several-order-of-magnitude increase in the sensitivity of this sensor type. Sandia recently developed novel chemical sensors based on planar interdigitated microelectrodes coated with thin films of polyelectrolytes. The ion conductivity of ions doped into these films is sensitive to a wide range of volatile organic compounds (VOCs), including nerve-agent simulants. We believe that the mechanism of detection involves reversible plasticization of the polymer chains, allowing faster ion motion that is detected by simple alternating current (ac) impedance at low frequencies (10 to $15 \mathrm{~Hz}$ ). We achieved parts-per-million (ppm)-level sensitivities with these new devices, but to achieve orders-of-magnitude increase in sensitivity, we seek here to explore ion motion (impedance) in very thin lipid bilayer capacitor membranes that are chemically tethered to our planar electrodes. The basic idea is to mimic biological chemical sensors that rely on changes in ion transport across very thin organic membranes for the sensing. The large increase in sensitivity over polyelectrolytes will come from molecular recognition elements like antibodies that bind the analyte molecule. The molecular recognition sites can be tied to the lipid bilayer capacitor membrane, and a number of mechanisms can be used to modulate the impedance of the lipid bilayers. These include coupled ion channels, pore modification, and double-layer capacitance modification by the analyte molecule.

We fabricated a flow-injection-analysis (FIA) cell with a transparent lid that allows fast turnover of liquid-phase samples. We designed and fabricated a new type of electrochemical analysis chip (the "lipid chip") for use in the FIA cell. It has an eight-electrode array with different-sized working electrodes and large counter and reference electrodes. An "O" ring seal prevents the liquid samples from reaching the electrode read-out pads, which are contacted with pogo-pin spring-loaded connectors. Each chip can then be removed for further processing steps on the electrodes and quickly retested without encapsulation or wirebonding. We performed
The goal of this project is a several-order-of-magnitude increase in the sensitivity of this sensor type. Sandia recently developed novel chemical sensors based on planar interdigitated microelectrodes coated with thin films of polyelectrolytes. The ion conductivity of ions doped into these films is sensitive to a wide range of volatile organic compounds (VOCs), including nerve-agent simulants. 
electrochemical experiments using both redox active and inactive solutions measuring electrode impedance and cyclic voltammagrams (CV) that establish the microscopic properties of the bare electrodes. We tested both gold ( $\mathrm{Au})$ and platinum (Pt) chips. We then performed the first measurements ever of templated sol-gel films covering the electrodes. We believe these types of films will serve as a framework for tethering lipid bilayers to the electrode surfaces. Fortunately, the templated glass films still allow good penetration of electrolyte and redox ions through pores to the electrode surface without poisoning the catalytic activity of the Pt. In parallel developments, we brought an apparatus for depositing Langmuir-Blodgett (LB) films online and deposited monolayer films. The first films were not compact enough to prevent transport of redox ions across them, and we are in the process of developing the technique to deposit better films. Vesicle fusion is another technique we are trying, and we are using atomic force microscopy (AFM) images to quantify the coverage of the LB films.
We tested both gold $(\mathrm{Au})$ and platinum (Pt) chips. We then performed the first measurements ever of templated sol-gel films covering the electrodes. We believe these types of films will serve as a framework for tethering lipid bilayers to the electrode surfaces. 
10775

Miniature Sensors for BW Agents Using FattyAcid Profiles

G. C. Frye-Mason, C. D. Mowry, R. P. Manginell, P. R. Lewis, K.

Wally, C. H. Morgan

Sandia is developing a microfabricated sensor for the rapid identification of bioorganisms. When fully developed, this device will provide a unique miniaturized capability for biological warfare $(B W)$ agent detection, an important national security mission. Tailored toward detection of pathogens and other BW agents such as anthrax, the device will also have significant spin-off applications.

The miniature $B W$ agent detector relies on an established technique of identification based on fatty-acid (FA) profiles. These biomarkers are used to distinguish bacteria at the gram-type, genera, and even species levels. Current commercial methods extract bacterial samples and chemically derivatize them to produce fatty-acid methyl esters (FAMEs). These methods are lengthy (over 1 hour) and labor-intensive, but have been used to differentiate BW agents, plant pathogens, foodborne pathogens, and other microorganisms. More recent work demonstrates that FAMEs can be obtained from whole bacterial cells in an in situ single-step pyrolysis/methylation/ mass spectrometry analysis. Derivatization and detection occur rapidly with little sample preparation. Bacteria have been differentiated. This method can also detect the causal agent of anthrax, amino acids, and oligopeptides.

We used microfabricated pyrolyzers to demonstrate the in situ pyrolysis of FAs to their volatile FAME derivatives and microfabricated gas chromatography $(G C)$ columns to separate the FAME peaks.

We demonstrated the ability of microfabricated hotplates to affect the pyrolysis/methylation reaction of lowvolatility FAs. Device membranes were more than capable of being heated adequately, both in magnitude $\left(>500^{\circ} \mathrm{C}\right)$ and in response time $(<5 \mathrm{sec}$ process time for a $1 \mu \mathrm{L}$ sample, including solvent evaporation, and $>70^{\circ} \mathrm{C} / \mathrm{ms}$ heating rate following liquid evaporation, producing a narrow pulse of volatile FAMEs). We also showed the ability of microfabricated hotplate membranes to process multiple consecutive samples. 
Using manual loading, the microfabricated pyrolyzer (micro-hotplate), and conventional separation and detection methods (commercial gas chromatograph/mass spectrometer [GC/MS]), we demonstrated successful pyrolysis/methylation of both simple and complex mixes of purified FAs. We identified as suitable for evaluation two types of coated microGC columns developed by the $\mu$ ChemLab ${ }^{\text {TM }}$ project and identified several other GC column coatings (polymer phases) available for purchase and future evaluation with FAMEs. Further, we showed successful separation of relevant FAMEs with both micro-GC columns, using both isothermal and temperature ramp profiles, and identified the need for increased temperature tolerance by column fixtures. We also identified moderate column bleed at elevated temperatures, verifying the need for modest improvements in phase stability, and delineated appropriate solutions. 
10776

\section{Enhanced-Sensitivity Acoustic-Wave Biosensor Arrays}

R. W. Cernosek, D. Y. Sasaki, S. M. Brozik, D. W. Branch

This project will develop enhanced-sensitivity biosensors utilizing acoustic platforms with biorecognition layers. The resulting sensors will detect both chemical and biological agents in aqueous media and have application in nonproliferation, military scenarios, environmental monitoring, intelligence, law enforcement, medical diagnostics, and food processing.

Resonant response enhancement occurs in acoustic sensors when the shear wavelength produced by the oscillating device is four times the mean thickness of the surface layer. In this case, the viscoelasticity of the layer can dominate the response mechanism and be significantly larger than that due to the bound mass. Recent collaborative investigations have demonstrated high-sensitivity detection for food pathogens (Salmonella and E. coli) and the anthrax simulant (Bacillus globigii). Sensors consisted of thickness shear mode (TSM) resonators with surface-immobilized monoclonal antibodies. We measured organism concentrations below the TSM resonator mass detection limits.

Sandia demonstrated a shear horizontal surface acoustic-wave (SH-SAW) sensor with an order-of-magnitudegreater sensitivity than the TSM resonator. As we continue this project, we will investigate several acoustic platforms (TSM resonators, SH-SAW devices, and flexural plate-wave [FPW] devices) that cover a wide range of operating frequencies. We will tune the sensor platform for optimum viscoelastic enhancement in the biolayer (probing bacterium size and modulus). Biorecognition results from antibody-antigen interactions (immunoassay) for target biological warfare $(B W)$ agents, including Bacillus anthracis, and the nonpathogenic Bacillus strains. We will also study viral pathogens to demonstrate the enhanced sensing technique for lowermolecular-weight analytes. A final aim of the project is to provide an integrated biosensor array microsystem based on Sandia's FPW technology.

We established collaborations with three universities to secure access to monoclonal antibodies for the acoustic immunosensors. The University of Alabama-Birmingham provided anti-anthracis and anti-subtilis antibodies and the
This project will develop enhanced-sensitivity biosensors utilizing acoustic platforms with biorecognition layers. The resulting sensors will detect both chemical and biological agents in aqueous media and have application in nonproliferation, military scenarios, environmental monitoring, intelligence, law enforcement, medical diagnostics, and food processing. 
associated spores for testing. We are working with the University of New Mexico to extract and purify Hantavirus antibodies and fragments from test samples used in past medical studies. Auburn University is providing antibodies raised against Salmonella typhymurium and E. coli as well as acoustic devices coated with Langmuir-Blodgett films incorporating these same antibodies.

We are exploring three different techniques for attaching or immobilizing antibodies to the acoustic device surfaces: lipid layers (monolayers, bilayers, and multilayers); self-assembled monolayers (SAMs); and direct linkers (covalent binding) to gold $(\mathrm{Au})$ or other metals, quartz, and polymer surfaces. All techniques yield high-quality films; maximum densities of accessible antibodies occur for SAMs or directly linked films.

We demonstrated a Love-wave biosensor consisting of a dual-delay line lithium tantalate $\left(\mathrm{LiTaO}_{3}\right) \mathrm{SH}-\mathrm{SAW}$ device with an optimized poly(methylmethacrylate) waveguide layer. The generic immunochemical reaction on the sensing line showed a detection limit of $1.7 \mathrm{ng} / \mathrm{cm}^{2}$, which is approximately an order-of-magnitude more sensitive than an equivalent sensor using a TSM resonator. Additionally, first tests using TSM resonators with anti-anthracis antibodies tethered to the surface showed good efficiency for binding spores from solution. Impedance studies (using network analysis) of the resonator surface interactions before and after the bacterial binding indicate a typical masslike response from the large, rigid spores with very small viscoelastic losses.

In addition, we created computational models for TSM quartz resonators with continuous, uniform surface layers of bound organisms operating in a liquid background. Model results show that resonant interactions with the organism layer can occur around $5 \mathrm{MHz}$, a common resonator operating frequency. Ongoing investigations show that antibody-coated TSM resonators can be insensitive to the viscosity and density changes produced by suspended cells (bacteria) in the liquid background. Larger organisms, not bound to the sensor surface, must be very near the resonator before entrainment occurs in the shallow-penetrating shear acoustic wave. We also demonstrated a technique in which Bacillus spores are collected on hydrated antibody surfaces directly from air samples moving over the coated TSM resonators. The process shows good reversibility.
We are exploring three different techniques for attaching or immobilizing antibodies to the acoustic device surfaces....All techniques yield high-quality films; maximum densities of accessible antibodies occur for SAMs or directly linked films. 


\section{Refereed}

Bailey, C. A., B. Fiebor, V. Vodyanoy, J. Barbaree, B. A. Chin, and R. W. Cernosek. 2000. "Thickness Shear Mode (TSM) Resonator Platform Used for Biosensing." Paper presented to the Acoustic WaveBased Sensors Symposium, 198th Meeting of the Electrochemical Society, Phoenix, AZ, October.

Bender, F., R. W. Cernosek, and F. J. Josse. 2000. "Love-Wave Biosensors Using Cross-Linked Polymer Waveguides on $\mathrm{LiTaO}_{3}$ Substrates." Electron. Lett. 36 (September): 1672-1673.

Bender, F., R. W. Cernosek, S. M. Brozik, and F. Josse. 2000. "Design of $\mathrm{LiTaO}_{3}$ Love-Wave Sensors for Chemical and Biological Detection in Liquids." Paper presented to the Acoustic Wave-Based Sensors Symposium, 198th Meeting of the Electrochemical Society, Phoenix, AZ, October.

Hillman, A. R., A. Jackson, S. J. Martin, and R. W. Cernosek. 2000. "A New Approach to Fitting of Crystal Impedance Data for Viscoelastic Films." Paper presented to the Acoustic Wave-Based Sensors Symposium, 198th Meeting of the Electrochemical Society, Phoenix, AZ, October.
Zinszer, K., R. Zhou, F. Josse, F. Bender, and R. W. Cernosek. 2000. "Optimization of SH-SAW Liquid Sensor Platforms for (Bio-)Chemical Detection Using Polymer Guiding Layers." Paper presented to the Acoustic Wave-Based Sensors Symposium, 198th Meeting of the Electrochemical Society, Phoenix, AZ, October.

\section{Other Communications}

Bender, F., R. W. Cernosek, S. M. Brozik, and F. J. Josse. 2000. "Receptor-Based Biosensors." Paper presented to the DOE Biomedical Engineering Contractors Meeting, Albuquerque, NM, May.

Cernosek, R. W. 2000. "Material Characterization and Sensing Using Thickness Shear Mode Quartz Resonators." (Invited) Paper presented to the Sensor Seminar at Auburn University, Auburn, AL, July. 
10782

High-Al-Content AIGalnN Devices for NextGeneration Electronic and Optoelectronic Applications

A. G. Baca, J. Han, R. J. Shul, M. H. Crawford, A. F. Wright, S. R. Lee

Recent breakthroughs in the technology of gallium nitride $(\mathrm{GaN})$ and related aluminum gallium indium nitride (AlGaInN) materials synthesis and device demonstrations sparked worldwide interest in these materials for lighting and other applications. By adding Al and In to GaN, researchers can tune the energy bandgap from the visible $(2.0 \mathrm{eV})$ to the ultraviolet $(U V)(6.1 \mathrm{eV})$. These advances will drive the development of multicolor light-emitting diode (LED) displays and white-light sources, with high reliability (> 10 years lifetime) and low-power consumption ( 10 watts). The large bandgap (2.0-6.1 eV), high breakdown field $(\sim 5 \times 106 \mathrm{~V} / \mathrm{cm})$ and saturation drift velocity $(2.7 \times 107 \mathrm{~cm} / \mathrm{s})$, and chemical inertness will enable a new generation of high-temperature and high-power electronics crucial for many commercial and defense applications.

Realizing efficient solid-state white-light illumination and high-power electronics will depend on successfully meeting the goals of this project in high-Al-content AlGaInN materials and devices. Finally, understanding of material growth led to the development of a new and superior method of material growth, termed cantilever epitaxy.

Sandia successfully demonstrated a 370-400 nm LED. We achieved output powers $>1 \mathrm{~mW}$ at $100 \mathrm{~mA}$ and emission peaked at $386 \mathrm{~nm}$. This is the first report of a UV LED based on AlGaInN alloys.

GaN p-type contacts performed better through experiments showing that the higher-temperature anneal conditions $\left(900^{\circ}-1000^{\circ} \mathrm{C}\right)$ enhanced LED performance.

We realized a new method of achieving reduced $\mathrm{GaN}$ defect density termed cantilever epitaxy. We demonstrated results as good as or better than epitaxial lateral overgrowth with a simpler process and showed a reduction in threading dislocation (TD) density in the cantilever region to a value below ten to the sixth power per square centimeter. We also demonstrated reduced TD densities over up to $1 \mathrm{~mm}$ from the edge regions of a $2.5 \mathrm{~mm}$ linear support design.

\section{Sandia successfully} demonstrated a 370-400 nm LED. We achieved output powers > $1 \mathrm{~mW}$ at $100 \mathrm{~mA}$ and emission peaked at $386 \mathrm{~nm}$. This is the first report of a UV LED based on AlGaInN alloys. 
We demonstrated an enhancement-mode high-electron mobility transistor (HEMT) from GaN/Si. We will improve its performance by implementing this new materials advance to produce crack-free GaN/Si material.

\section{Refereed}

Ashby, C. I. H., C. C. Willan, J. Han, N. A. Missert, P. P. Provencio, D. M. Follstaedt, G. M. Peake, and L. Griego. 2000. "Low-Dislocation-Density GaN from a Single Growth on a Textured Substrate." Appl. Phys. Lett., accepted.

Ashby, C. I. H., C. C. Willan, P. P. Provencio, N. A. Missert, D. M. Follstaedt, G. A. Peake, and J. Han. 2000. "Cantilever Epitaxy on Textured Substrates: A Simple Two-Step Approach to Low-Defect-Density GaN Substrates." Paper presented to the Electronics Materials Conference, Denver, CO, 21-23 June.

Han, J., J. J. Fiegel, G. A. Petersen, S. M. Myers, M. H. Crawford, and M. A. Banas. 2000. "Metal-Organic Vapor-Phase Epitaxial Growth and Characterization of Quaternary AlGaInN." Japanese J. Appl. Phys. 39: 2372-2375.

Han, J., K. E. Waldrip, S. R. Lee, J. J. Fiegel, S. J. Hearne, G. A. Petersen, and S. M. Myers. 2000. "Control and Elimination of Cracking of AlGaN Using Low-Temperature AlGaN Interlayers." Appl. Phys. Lett., accepted.

Zhou, H., M. Diagne, E. Makarona, A. V. Numikko, J. Han, K. E. Waldrip, and J. J. Fiegel. 2000. "A Near Ultraviolet Optically Pumped Vertical-Cavity Laser." Electron. Lett., accepted.
We demonstrated an enhancement-mode highelectron mobility transistor (HEMT) from GaN/Si. 
10783

\section{Microfabrication of Electromagnetic Devices}

T. R. Christenson, P. G. Clem, K. Eras, C. P. Tigges, W. G. Yelton

This project addresses monolithic electromagnetic

(EM) micro- and milliscale actuator fabrication for implementing a category of mechanical interface drives that require high-energy-density actuation at large gaps. The applications include devices needed on the micro-/milliscale for efficient robotic propulsion, weapon surety, high-reliability optical and electrical switching, and valves, pumps, and regulators for gas and liquid sampling and handling. This category, well suited to EMs, encompasses applications for which micromachined actuators are not available and that require pressures of 100 psi with millimeter travel. The implementation of EM actuators in a planar sense must address the two primary issues of magnetic materials and coils. Soft magnetic materials may be microelectroformed. Sandia recently achieved hard magnetic material (permanent magnet) integration via a project enabling low-power operation at small scale. Coils, however, remain most efficiently implemented with conventionally wound magnet wire forms, requiring that the total device rely on component assembly. We seek a technique combining direct-write thick-film materials and high-aspect-ratio electroforming as a means to duplicate magnet wire coils in a monolithic means. We expect further system integration to benefit from several features, including the low-driving-point impedance of a coil combined with integrated bipolar current drives, the possibility of magnetic coupling through hermetic or evacuated chambers, and the use of these devices driven in reverse-enabling generators capable of being on-chip power sources.

To accommodate the complex multiple-layer processing needed to realize three-dimensional (3-D) EM coil structures, we constructed several unique mechanical fixtures to allow tilted deep x-ray lithography (DXRL) exposures. Test masks used to define representative electromechanical geometry yielded results indicating that angled structures may be fabricated at angles as shallow as 17 degrees with the substrate. An additional technique using a batch wafer transfer approach was aided by a non-optical, entirely mechanical alignment fixture that makes use of precision-metal DXRL-fabricated mechanical alignment structures. To enable processing of two different metals on the same layer, we developed a procedure to
We seek a technique combining direct-write thick-film materials and high-aspect-ratio electroforming as a means to duplicate magnet wire coils in a monolithic means.

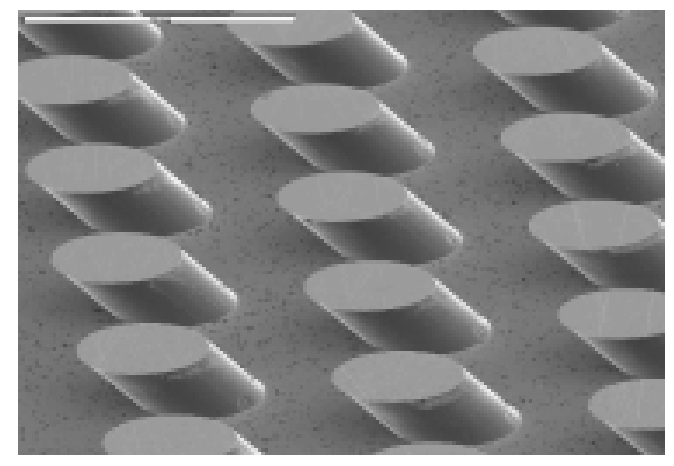

Electroformed copper post array with 200micron thickness at an angle of 45 degrees. 
isolate previously processed electroformed regions. This allows, for example, integration of copper $(\mathrm{Cu})$ with nickel (Ni)/iron (Fe) metals at substantial thicknesses. Combined with the angled exposure approach, the multiple material capability forms the basis for the realization of a completely integrated high-aspect-ratio, densely packed, integrated EM coil. We substantially completed an experimental design to determine the optimal electroplating parameters required to deposit highsaturation flux density $\mathrm{Ni} / \mathrm{Fe}$ and $\mathrm{Ni} / \mathrm{Fe} /$ cobalt $(\mathrm{Co})$ ferromagnetic materials from a sulfamate-based electrolyte. The sulfamate chemistry shows promise in the production of low-stress electrodeposits, which is desired for $\mathrm{Ni} / \mathrm{Fe}$ compositions that have significant magnetostriction associated with them. This is the situation with $45 / 55 \mathrm{Ni} / \mathrm{Fe}$, where a substantial increase in magnetic flux saturation is obtained over $78 / 22 \mathrm{Ni} / \mathrm{Fe}$, which is usually used due to its zero magnetostriction.
The sulfamate chemistry shows promise in the production of low-stress electrodeposits, which is desired for $\mathrm{Ni} / \mathrm{Fe}$ compositions that have significant magnetostriction associated with them. 
10784

\section{GaAs MOEMS Technology}

C. T. Sullivan, O. B. Spahn, E. J. Garcia, M. A. Polosky, J. L. Reno, A. A. Allerman

Many microelectromechanical systems (MEMS)-based components require optical monitoring techniques using optoelectronic devices for converting mechanical position information into useful electronic signals. While the constituent piece-parts of such hybrid microoptoelectromechanical systems (MOEMS) components can be separately optimized, the resulting component performance, size, ruggedness, and cost are substantially compromised due to assembly and packaging limitations. GaAs (gallium arsenide) MOEMS offers the possibility of monolithically integrating high-performance optoelectronics with simple mechanical structures built in very low stress epitaxial layers with a resulting component performance determined only by GaAs microfabrication technology limitations. For example, since submicron features can be etched into GaAs structures, submicron alignment and displacements can be sensed by a variety of approaches to provide dramatically improved sensitivity while eliminating packaging and alignment problems. More importantly, GaAs MOEMS implicitly integrates the capability for radiationhardened optical communications into the MEMS sensor or actuator component, a vital step toward rugged, integrated, autonomous microsystems that sense, act, and communicate.

This project will establish a new foundational technology that monolithically combines GaAs optoelectronics with simple mechanics. Using a single optoelectronic mechanical layer on AlAs (aluminum arsenide)-rich release layers, Sandia will develop designs and processes targeting the following devices important to Defense Program (DP) weapon surety systems: (1) an environmental sensing device using edge-emitting lasers and differential photodetection, (2) nonvolatile mechanical memory based on latching $1 \times 2$ optical waveguide switches, and (3) a surface-normal high-power optical/optoelectronic switch. We will demonstrate an integrated edge-emitting laser and photodetector in the MEMS layers that communicate via attached optical fibers to an external optoelectronic interface. Critical process issues that we plan to address include selectivity, electrochemical characteristics, anisotropy of the release chemistry, and postrelease drying and coating processes. We also plan to investigate the possibility of building multiple mechanical
This project will establish a new foundational technology that monolithically combines GaAs optoelectronics with simple mechanics. 
layers by epitaxial lateral overgrowth techniques or perhaps deposited materials and the use of multiple sacrificial materials and release chemistries.

We undertook considerable process development during this project. We concentrated on the release layer design, crystal orientation and layer doping effects, the release chemistry and its masking processes (in contrast to polysilicon [poly-Si] surface micromachines [SMMs], blanket etches are too restrictive), and post-release surface treatment. We investigated lateral etch rates and undercut profile for representative layer thicknesses and various over-etch conditions to determine the design modifications or process development required to build GaAs MOEMS. We studied release layers of about 2 microns thick ranging in AlAs mole fraction from $50 \%$ to $98 \%$ to permit the realization of the variety of interesting and proposed devices.

For high-reflectivity (tilting) micromirrors (HiRTM) for switches, filters, optical add-drop multiplexers (OADMs), and sensors, this device effort is focused on exploiting the ability of realized very high reflectivity distributed Bragg reflectors (DBRs) that are used in vertical-cavity surface-emitting lasers (VCSELs) and in reflectance modulators to realize tilting micromirrors that have reflectances much higher $(>99 \%)$ than post-deposited gold (Au) on poly-Si SMMs (95-97\%). The basic micromirror is either a single-axis torsional device for tilting, though this will be extended into two dimensions for tilt and tip, or a piston device. For tilting devices, we carried out 1-D simulations over tilt angle, wavelength, and with varied underlying gap (simulating reflectance variation over the micromirror aperture). We find that the reflectance is expected to be greater than $98.4 \%$ over all such varied conditions, better than the theoretical value of $\mathrm{Au}$ on poly-Si SMMs. We developed a general 2-D mechanical model for tilt and piston that shows that the switching voltage is expected to be less than about $14 \mathrm{~V}$, the undesired piston is less than about $0.65 \mathrm{~mm}$ before snap-down, and the tilt angle is about 1.4 degrees for a $100 \mathrm{~mm}^{2}$ micromirror suspended by a pair of $2 \mathrm{~mm}$-wide, 3 mm-thick, 100 mm-long GaAs/AlGaAs springs. Fabrication of our first devices is under way.

Another phase of the project addresses waveguide cantilevers for latching switches and optically read mechanical memory. Many of our waveguide devices switch state in the 4-6 V range before latch-up. We developed a model for this basic cantilever waveguide switch that indicates that for a hold
For tilting devices, we carried out 1-D simulations over tilt angle, wavelength, and with varied underlying gap (simulating reflectance variation over the micromirror aperture). We find that the reflectance is expected to be greater than 98.4 percent over all such varied conditions, better than the theoretical value of Au on polySi SMMs. 
voltage of $6 \mathrm{~V}$, one should expect switching times less than 10 ms. For a full $28 \mathrm{~V}$ drive with braking voltage (on the opposite electrode), we predict switching times in the few-microsecond range. We undertook optical insertion loss measurements using a wavelength-detuned Fabry-Perot transmittance technique that shows a considerable data scatter, but our best device has a mean insertion loss of $0.8 \mathrm{~dB}$ for a $5 \mathrm{~mm}$ length, a very good value. We have thoroughly characterized the effect of crossthroughs $(\sim 0.30 \mathrm{~dB})$, bends $(<0.05 \mathrm{~dB}$ in range investigated), and undercuts $(<0.05 \mathrm{~dB})$ on insertion loss, and are in the process of redesigning the basic switch that reduces the net insertion loss, particularly by reducing the number of crossthroughs.

The integration effort for laser-based MOEMS (LaMOEMS) is organized to combine recent developments in resonantly enhanced etched-facet edge-emitting lasers, edgecoupled photodetectors, and simple environmentally or electrostatically actuated devices (proof masses) in the (Al,Ga)As materials into a single die component. This will eliminate the very difficult problem of precision assembly and packaging of conventional poly-Si SMM with photonics. The basic LaMOEMS device design includes a doubly etched SQW GRINSCH (single quantum-well graded index separate confinement heterostructure [lasers]) laser at $840 \mathrm{~nm}$ with selfaligned and axially translatable mechanical reflectors, aligned normal to the laser axis. This permits the effective facet reflectance to be varied from below threshold $(<20 \%$ reflectance, giving laser threshold (lth) $>30 \mathrm{~mA}$ ) to much above threshold (> 55\%, giving lth $10 \mathrm{~mA}$ ) through either electrostatic control or environmental deflection. Beyond each movable reflector is a fixed-edge coupled photodetector that measures the light output for laser self-diagnostic purposes or for displacement readout, depending on the configuration. Very sensitive interferometric measurements are possible and can be combined together with threshold modulation tracking to construct a very small displacement sensor.

\section{Other Communications}

Spahn, O. B., C. Sullivan, J. Burkhart, C. P. Tigges, and E. Garcia., 2000. "GaAs-Based

Microelectromechanical Waveguide Switch." IEEE/ LEOS/OSA Optical MEMS 20001 (Kauai, Hawaii, August): 41-42.
We undertook optical insertion loss measurements using a wavelength-detuned Fabry-

Perot transmittance technique

that shows a considerable data scatter, but our best device has a mean insertion loss of $0.8 \mathrm{~dB}$ for a $5 \mathrm{~mm}$ length, a very good value. 
10785

Integrated Microsensors for Autonomous Microrobots

E. J. Heller, R. C. Hughes, R. H. Byrne, E. B. Duckett, S. A.

Casalnuovo, D. R. Adkins

The goal of this work is to develop a suite of new lowpower integrated microsensor systems that will enable the development of networks of 0.25-inch ${ }^{3}$ autonomous microrobots. The focus of this work is on two primary sensor classes. The first class consists of microsensors that can be used as navigational and communication aids. Several possible devices in this class include micro-global positioning systems (GPS), magnetometers, ultrasonic or optical detector systems (for proximity detection), and single-chip radio-frequency $(R F)$ transceivers. Although Sandia will consider a number of possibilities in this class, only those deemed most suitable to the microrobot application will undergo further development.

The second group of sensors consists of microsensors for environmental trace-chemical detection. There are a number of possible sensors to consider in this class, but microsensor systems based on chemiresistors and surfaceacoustic wave (SAW) devices have already undergone the most substantial development and will be given priority. The primary emphasis is to develop microsensors that can take advantage of modern semiconductor manufacturing techniques to efficiently produce large numbers of essentially disposable microsensor systems. As we complete devices, we will incorporate them into microrobots being constructed under separate funding. These microsensors will enable networks of autonomous microrobots. We will consider several methods of network organization, but the primary focus is expected to be on "marsupial" systems. These systems use a larger mother robot to deploy and support a large group of networked autonomous microrobots.

The overall size of the microrobot body represents a significant reduction in size. The electronics for the robot include a combination of devices in die form and standard offthe-shelf passive surface-mount components. We fabricated the substrates from both silicon $(\mathrm{Si})$ and Foturan and made them double-sided using conventional lithography methods. We mounted the control electronics on the front of the microrobot and made them accessible for in situ programming of the microprocessor. The dimensions of the substrate are $0.5^{\prime \prime} \mathrm{x}$
The goal of this work is to develop a suite of new lowpower integrated microsensor systems that will enable the development of networks of 0.25-inch ${ }^{3}$ autonomous microrobots....As we complete devices, we will incorporate them into microrobots being constructed under separate funding. These microsensors will enable networks of autonomous microrobots. 
0.5". A PIC 16C77 central processing unit (CPU) die drives Smoovy motors for mobility.

The robot design uses a commercially available DL1/3N rechargeable lithium $(\mathrm{Li})$ camera battery that is capable of powering the microrobot for about 15 to 20 minutes at maximum current. This prototype device measures approximately $0.75 "$ long x 0.5 " wide $x 0.5$ " tall. The body of the microrobot is fabricated from a lightweight epoxy resin that is resistant to chemicals that may be found in the environment where it may be used and is also strong enough to contain all the parts of the microrobot.

There has been substantial progress in the development of the chemiresistor microsensor arrays for the microrobot application. Chemiresistors are a particularly simple type of chemical sensor whose electrical resistance changes in the presence of chemical vapors. Chemiresistors are formed from standard insulating polymers turned into conducting composites by mixing with $20 \%-40 \%$ carbon by volume. These sensors are easy to fabricate, can be made very small, and can be measured by simple low-power circuits measuring dc resistance. By varying the polymer host, these sensors can be selected to sense a very wide variety of volatile organic chemicals. To improve the sensitivity, we built a preconcentrator/chemiresistor hybrid. The preconcentrator operates by sorbing molecules of analyte at ambient temperature in a highly porous sol-gel layer. A large number of molecules can be accumulated over time, even from low concentrations in the background air. At a selected time, the preconcentrator heater is heated to $150^{\circ} \mathrm{C}$ in a $1 \mathrm{msec}$ pulse using about $300 \mathrm{~mW}$ of power. The absorbed molecules are rapidly desorbed, creating an analyte pulse of much higher concentration of molecules than in the background. Because of the close proximity of the chemiresistor to the preconcentrator, a distance of about 5,000 $\mathrm{mm}$, the analyte pulse reaches the sensor very quickly. A preconcentrator/chemiresistor hybrid assembly loaded with methylsalicylate vapor and enclosed to form a sealed volume demonstrated resistance changes of about 300\%, which is easily measurable by simple analog/digital (A/D) converters on the microrobot. Chemical preconcentration was demonstrated, but optimization was clearly not achieved. Some preliminary fluid dynamic modeling shows that the preconcentrator does not have an optimal configuration to exploit the necessary buoyancy flow (convection) to aid in getting the hot chemical flow to the chemiresistor sensor. In addition, the preconcentrator absorbent resins do not concentrate all compounds with the same efficiency.
There has been substantial progress in the development of the chemiresistor microsensor arrays for the microrobot application. Chemiresistors are a particularly simple type of chemical sensor whose electrical resistance changes in the presence of chemical vapors. 
10786

\section{Silicon-Integrated Planar Microbatteries}

S. H. Kravitz

Sandia seeks a new microbattery concept that will be integrated into silicon (Si). By combining Si-micromachining with new cathode and anode materials, we will be able to build batteries directly into integrated circuit (IC) or microelectromechanical system (MEMS) devices. The concept is to micromachine onto a Si wafer the interdigitated reservoirs that will serve as containers for the battery materials. The reservoirs will be interconnected by microchannels, which provide a means to pass electrolyte between the interdigitated reservoirs without allowing particles through. We will use a thermally grown oxide to electrically isolate reservoirs from each other. We will then add a conductive liner to the reservoirs.

We will dispense the electroactive material into the reservoirs using Micropen, a computer-aided design (CAD)driven system consisting of a high-precision fluid-dispensing unit coupled with an X-Y table. To prevent clogging or migration between reservoirs, we will prepare spherical monodisperse powders with a narrow size distribution by coprecipitation of alkoxides or by flame pyrolysis of atomized chemical precursors.

We will design this device in two different ways. As a series-connected battery, it will supply $100 \mathrm{~V}$ to MEMS devices. In parallel connection, it will be a high-capacity/highpower $3 \mathrm{~V}$ battery capable of supplying high current needs for communication or mechanical motion. With some battery chemistries, it will be possible to recharge, using photovoltaic $(P V)$ cells. The approach of making an integrated and copackaged planar power source on a Si chip will reduce the size and cost of a battery-powered system.

We developed a planar microbattery concept, developed a fabrication process, developed a sealing design, developed a battery-material filling procedure, designed an eight-variation mask layout, ordered the masks, developed the Bosch-etch battery process, began device fabrication, decided on a singlebattery chemistry, and prepared monodisperse battery materials.

We conceived and laid out an eight-variation battery design with four different-size battery-material compartments and two post spacings. This design uses an array of 3-micron
Sandia seeks a new microbattery concept that will be integrated into silicon ( $\mathrm{Si})$. By combining Si-micromachining with new cathode and anode materials, we will be able to build batteries directly into integrated circuit (IC) or microelectromechanical system (MEMS) devices....The approach of making an integrated and co-packaged planar power source on a Si chip will reduce the size and cost of a battery-powered system. 
posts to separate the anode-cathode battery material but allow electrolyte to pass. We anodically bonded the Pyrex lid, which also contains the metal contacts. We then filled the sealed battery with a suspension of monodisperse battery materials, lithium (Li) carbonate (+), and microcarbon mesobeads (-). We are modifying the current package design to make anodic bonding seal a Pyrex lid to the Si substrate. To accomplish bonding, we are recessing the gold lid contacts into the Pyrex so that the lid surface is then flush with the Si. 
10796

High-Efficiency Optical MEMS by the Integration of Photonic Lattices with Surface MEMS

J. G. Fleming, S. Y. Lin, M. S. Rodgers, M. A. Polosky

Optical microelectromechanical systems (MEMS) are emerging as a key element of MEMS technology. However, despite the great interest in this area, the actual optical components employed are primitive: mirrors, simple lenses, and waveguides. In this project, Sandia seeks a revolutionary advance in both MEMS and photonics through a combination of surface MEMS and the newly emerging field of photonic lattices. Photonic lattices are the photonic analogues of semiconductors in which the photonic bandgap arises as the result of a periodic array of regions of high and low refractive index. As a general rule, the pitch of the lattice corresponds to roughly half the wavelength of light at midgap. The properties of these engineered materials rely only on the index of refraction of the materials and their arrangement. These engineered materials have a number of novel properties: They can act as extremely efficient mirrors, and they can be "doped" to form very high-Q (high-quality) optical cavities and highly efficient 90-degree-bend waveguides.

It is natural to try to combine surface MEMS with photonic lattices. Silicon (Si), the basic structural material of $M E M S$, can also be used to form the lattice. Since the lattice properties rely on the repeat unit, which is small, small changes in dimension can result in large changes in optical properties of the lattice. Such small, well-defined displacements are readily obtained using MEMS processing. Potential applications include high-reflectivity mirrors, switching mirror arrays, waveguide switches, and scanning spectrometers.

We completed and implemented a theoretical analysis and design scheme of a one-dimensional (1-D) high-efficiency mirror and a 2-D photonic lattice switch compatible with accepted MEMS fabrication technology.

We converted the theoretical model for a photonic lattice switch into a mechanical design and fabrication sequence, which has been executed, producing the first generation of photonic lattice switches. These switches consist of coupled stationary and movable 1-D photonic lattice stacks
Optical microelectromechanical systems (MEMS) are emerging as a key element of MEMS technology.... In this project, Sandia seeks a revolutionary advance in both MEMS and photonics through a combination of surface MEMS and the newly emerging field of photonic lattices.... We converted the theoretical model for a photonic lattice switch into a mechanical design and fabrication sequence, which has been executed, producing the first generation of photonic lattice switches. 
manufactured to allow mechanical modulation of light transmission through the lattice.

Measurement of the designed 1-D fixed lattice indicated the desired and calculated spectral response. The active 1-D switches are currently in test and evaluation. Testing of the fixed stacks indicates a performance very similar to that predicted by theory.

We rapidly adapted the design and process flow for the 1-D mirror and 1-D switch to be included in the SUMMiT-V process. The current SUMMiT-V technology has produced extremely sophisticated, steerable micromirror designs in polysilicon with demonstrated operation and application. The fabrication process for the above mirror and lattice switch is included in the SUMMiT process flow, thus providing highefficiency mirror surfaces as part of the SUMMiT-V polysilicon mirror platform. The 1-D lattice design targets high-efficiency reflection at $1.55 \mu \mathrm{m}$ wavelength, which is applicable to both internal and external Sandia needs.
We rapidly adapted the design and process flow for the 1-D mirror and 1-D switch to be included in the SUMMiT-V

process. The current SUMMiT-V technology has produced extremely sophisticated,

steerable micromirror designs in polysilicon with demonstrated operation and application. The fabrication process for the above mirror and lattice switch is included in the SUMMiT process flow, thus providing high-efficiency mirror surfaces as part of the SUMMiT-V polysilicon mirror platform. 


\section{Engineering Modeling and Simulation}

The Engineering Modeling and Simulation investment area is researching capabilities that utilize Teraflops-class (one trillion floatingoperations per second), massively parallel computers to support engineering decisions based primarily on experimentally validated computational simulations. These capabilities will greatly reduce the need for testing to certify that the nation's nuclear stockpile is safe, secure, and reliable.

Modeling and simulation advances also support Energy, Environment, and Critical Infrastructures-related issues. Research activities focus on three key areas: (1) development of highfidelity physics models and their computational algorithms, (2) improved numerical solutions for high-performance computing platforms, and (3) integrated multidisciplinary modeling and simulation of coupled systems.

The "Applied Microfluidic Physics" project is investigating advances in micron-size devices integral to the development of microfluidic microelectromechanical systems (MEMS). These systems make use of the chemical properties of liquids or gases and the electrical properties of semiconductors on a single chip or among nearby chips.

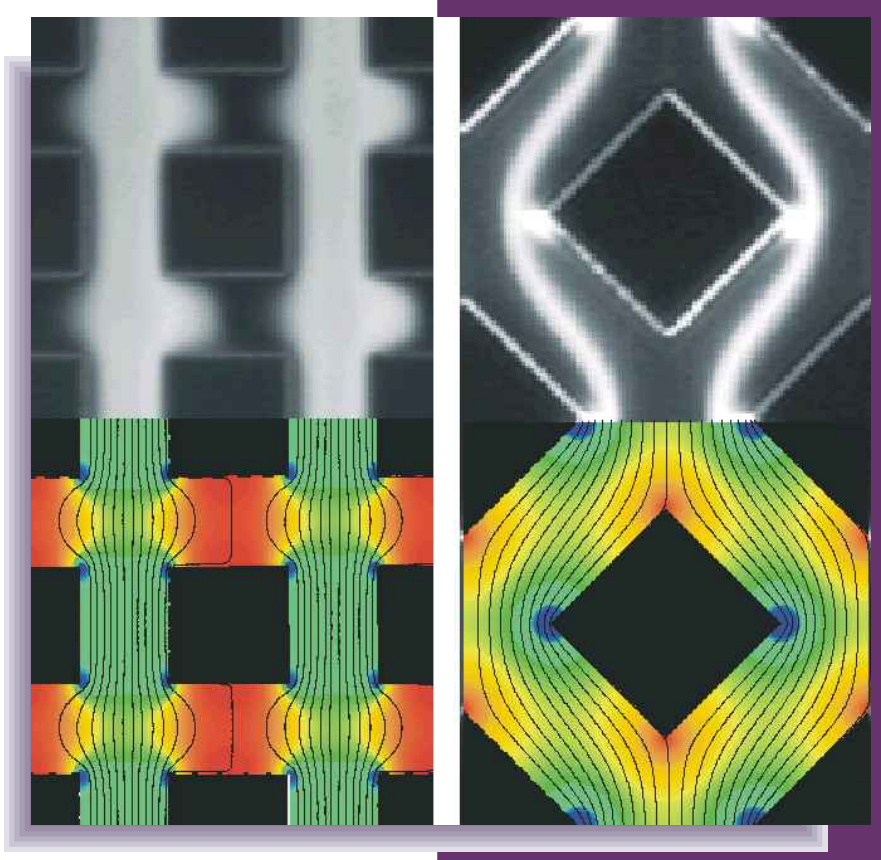

This project is developing, applying, and verifying mathematical models that accurately describe microfluidic processes. Results from this research have the potential to improve our understanding of currently nonexistent microfluidic design guidelines. This research has already produced optimization techniques and optimal device geometries for minimum dispersion in bends and junctions, for which a patent disclosure is filed. In addition, an advanced image-processing methodology has been developed to provide resolution down to a single pixel. Future applications of this research have the potential to improve airborne-toxin detection, to enable rapid DNA analysis for crime-scene investigators, and to produce new pharmaceutical testers for drug development. 
10335

\section{Development of In Situ Diagnostics for} Simultaneous Measurement of Transient Gas Species and Soot in Large Fires

\author{
C. R. Shaddix, D. K. Ottesen, S. W. Allendorf, L. A. Gritzo, P. J. \\ Santangelo, H. A. Johnsen, G. L. Hubbard, J. R. Ross
}

This project is motivated by the need for improvements in both the phenomenological understanding of heat transfer from aviation fuel fires and in developing advanced, predictive models of the hazard posed by these fires to a weapon or other engineered system. To address these needs, Sandia aimed to develop significant improvements in the temporal resolution and information content of advanced, in situ laser-optical fire diagnostics. In particular, we adapted near-infrared (NIR) tunable diode laser (TDL) technology to provide the capability of measuring transient gaseous species concentrations over short physical pathlengths in large fires. In addition, we refined the existing technology for making transient measurements of local soot concentrations and temperatures, to make these measurements possible using small lenses coupled to individual, commercial, multimode fiber-optic cables, and to improve the temporal resolution of this technique. Taken together, this new diagnostic capability should provide the experimental data required to understand the transient generation and transport of gaseous species and soot in large, fully turbulent fires.

We determined the optimal chemical species and their optimal optical absorption transitions for detection via tunable diode laser absorption spectroscopy (TDLAS). We developed novel techniques for multipassing the TDL light within a small, open sampling path and for separating the signals from several laser sources that have been combined together on a single fiber-optic cable (TDL demultiplexing). We incorporated a red diode laser absorption/emission measurement of soot concentration and temperature within the multipassing optics of the TDL measurement and shrunk the complete optics package to fit within a water-jacketed aluminum (Al) probe for measurements in large fires. We tested preliminary and final designs of the control hardware and optics package in laboratory buoyant flames with varying degrees of soot content. We performed measurements at several locations in 1-foot-diameter and 1-meter-diameter JP-8 pool fires. ...we adapted near-infrared (NIR) tunable diode laser (TDL) technology to provide the capability of measuring transient gaseous species concentrations over short physical pathlengths in large fires... [and] refined the existing technology for making transient measurements of local soot concentrations and temperatures, to make these measurements possible using small lenses coupled to individual, commercial, multimode fiber-optic cables, and to improve the temporal resolution of this technique.

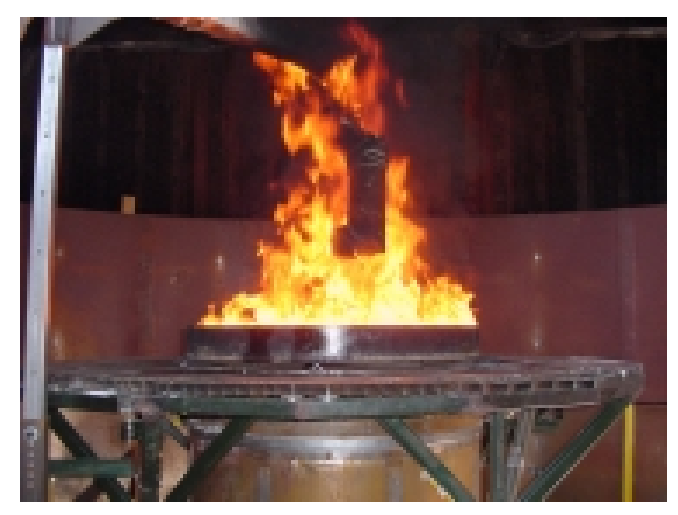

Insulated probe above the center of a 1-meter-diameter JP-8 pool fire in Sandia's FLAME facility. 
We fabricated and tested a water-cooled probe for performing TDL measurements of gas species and red diode measurements of soot in fires in both laboratory gas-fueled fires and in 1-meter JP-8 pool fires at Sandia's Lurance Canyon Burn Site. During the course of this work, final specification and acquisition of lasers for measuring carbon monoxide (CO) and methane $\left(\mathrm{CH}_{4}\right)$ occurred. In addition, we specified and fabricated a water-jacketed $\mathrm{Al}$ probe, constructed of standard 3.5-inch outside diameter (OD) and 2.5-inch OD standard square tubing. This probe provides a 2 -inch-diameter open square section for housing the various mirror, lens, and fiberoptic components and their mounts that constitute the fibercoupled optics package at the diagnostic end of the probe. We leak-tested this probe and made necessary modifications to the optical component mounts to allow for good transmission of both the NIR TDL beam and the red diode beam from a fiberlaunch back onto a receiving fiber. We tested the final optics package from within the manufactured fire probe in laboratory gas-fueled buoyant flames, with specific attention to evaluating the system for sensitivity to beam-steering optical losses from the narrow, wrinkled flame sheets.

Initial analysis of the measurement sensitivity of the final TDL/custom lock-in amplifier system indicates that it has a signal-to-noise ratio of approximately one $(\mathrm{S} / \mathrm{N}=1)$ in measuring a spectral absorption feature with a peak absorbence of $10^{-5}$ (10 parts per million [ppm]). This sensitivity is as good as has been regularly achieved in these types of systems and is required for this system to be sufficiently sensitive to measure the weak absorption features in the NIR region in hightemperature environments.

For the soot thermal-emission measurement, we acquired $\mathrm{Si}$ avalanche photodiodes and found them to have sufficient sensitivity in laboratory flames when used with 40 $\mathrm{nm}$ bandpass filters at 850 and $1000 \mathrm{~nm}$ center wavelengths. This type of detector has not previously been used for this measurement, but it has superior linearity and relatively flat spectral response to beyond $1000 \mathrm{~nm}$, in comparison to the photomultiplier tubes that have traditionally been used. We achieved the soot volume fraction measurement using an electronically modulated diode laser together with a lock-in amplifier, showing low noise and temporal resolution of greater than $1 \mathrm{kHz}$.

We took measurements at several locations in 1-footdiameter and 1-meter-diameter JP-8 pool fires. During this measurement campaign, it became apparent that the cooling

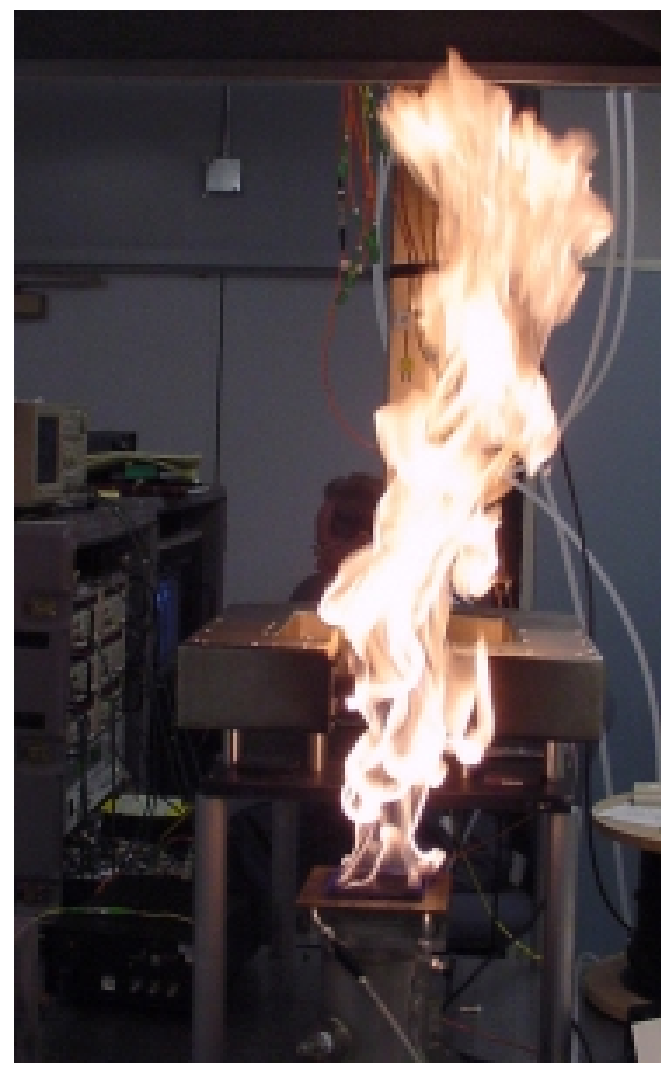

Laboratory testing of the probe in gas-fueled buoyant flames.

For the soot thermal-emission measurement, we acquired $\mathrm{Si}$ avalanche photodiodes and found them to have sufficient sensitivity in laboratory flames ....This type of detector has not previously been used for this measurement, but it has superior linearity and relatively flat spectral response to beyond $1000 \mathrm{~nm}$, in comparison to the photomultiplier tubes that have traditionally been used. 
water flow and the thin ceramic cloth insulation that we applied to the probe were insufficient to maintain the interior of the probe below $60^{\circ} \mathrm{C}$, as originally desired. The resultant heating of the optical package at the end of the probe led to severe TDL optical losses during the hottest portions of the 1-meter fires, presumably due to optical misalignment. The soot absorption/ emission diagnostic generally performed well, with the exception that the extreme temperature swing in the instrument trailer affected the performance of the emission detectors. Analysis of the data is ongoing.

\section{Other Communications}

Shaddix, C. R., P. J. Santangelo, P. D. Ludowise, S. W. Allendorf, and D. K. Ottesen. 1999. "Diagnostic for Measurement of Soot and Species Concentrations in Fires." Western States Section/Combustion Inst. (WSS/CI) Fall Mtg., Paper \#48 99F (Irvine, CA, 25-26 October).

Shaddix, C. R., S. W. Allendorf, G. L. Hubbard, and D. K. Ottesen. 2000. “A Rapid, Sensitive Diode Laser Diagnostic for Soot and Species Concentrations in Combustors and Fires." Proc. Amer. Flame Res. Committee (AFRC) Internat. Symp. (Newport Beach, CA, 17-21 September): 1-8.

Shaddix, C. R., S. W. Allendorf, G. L. Hubbard, and D. K. Ottesen. 2000. "Diode Laser Diagnostics for Soot and Species Measurements in Fires." Proc. 28th Internat. Symp. on Combustion (Edinburgh, Scotland, UK, 30 July-4 August).

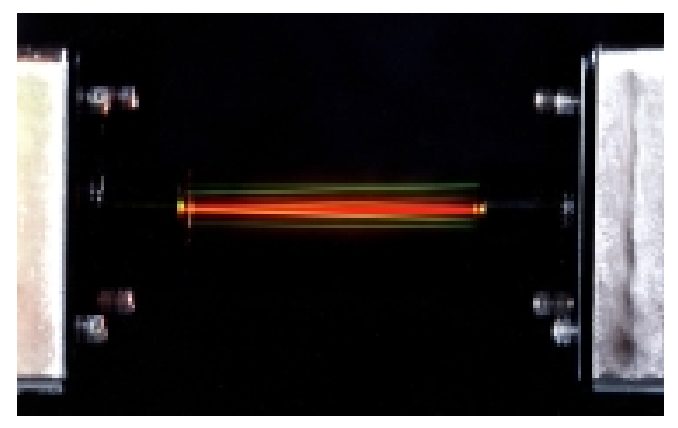

Close-up photograph of the optical paths traversed by the near-infrared tunable diode lasers (shown here by a green laser) and the red diode laser. 
10336

\section{Structural Simulations Using Multiresolution} Material Models

S. W. Key, D. C. Hammerand, R. S. Chambers, D. B. Adolf

The objective of this project is to develop computational methods for hierarchical modeling of homogeneous and heterogeneous materials. That is, starting with a simplified model, increasingly more comprehensive material representations are introduced into the simulation only to the extent required to meet a predefined error tolerance.

The success of this concept rests on the successful execution of four tasks: (1) the identification of a hierarchical sequence of material models, (2) the development of an error analysis that indicates when the next refinement in material representation is required as the simulation unfolds, (3) the design of a solution strategy and software architecture that will support the simultaneous refinements in mesh and material representation, and (4) the development of meshing technologies that automatically convert detailed experimental measurements of material inhomogeneities into practical analysis meshes suitable for adaptive refinement.

We performed this work in collaboration with the Texas Institute for Computational and Applied Mathematics (TICAM). We will make an assessment about the accuracy and effectiveness of material model adaptivity for both viscoelastic potting epoxies and heterogeneous elastic composites.

We performed complementary studies of unfilled viscoelastic potting epoxy and heterogeneous elastic materials. We identified for heterogeneous materials a nested family of constitutive models that span from a simple homogeneous characterization to a formulation representative of the microscale processes of interest. We also developed a family of approximations to the nonlinear viscoelastic constitutive law for potting epoxy.

We are developing error measures needed to describe the differences in the nonlinear continuum response of various members of a general material model family. In addition to investigating these general error indicators, we are also developing error indicators specific to the epoxy material model family. We created error measures for controlling the errors in user-defined quantities of interest in the linear analysis of heterogeneous materials.
The objective of this project is to develop computational methods for hierarchical modeling of homogeneous and heterogeneous materials. That $i s$, starting with a simplified model, increasingly more comprehensive material representations are introduced into the simulation only to the extent required to meet a predefined error tolerance. 
We are developing the criteria and process of switching for the adaptive modeling of potting epoxy. We developed and implemented the criteria and switching process necessary to adaptively select what level of fine-scale features must be included for the accurate determination of quantities of interest in heterogeneous elastic materials.

We wrote the computer codes necessary for the finiteelement analysis of structures made from either of the two material model classes under investigation.

We generated solutions using adaptive material model refinement for heterogeneous materials. We performed preliminary examples that demonstrate adaptive modeling for potting epoxies.

We produced a report describing the algorithms necessary to control the error in user-specified local quantities of interest for heterogeneous elastic materials.

We discussed the accuracy of the adaptive modeling process for heterogeneous materials for a preliminary numerical experiment. We are preparing a report describing modeling errors in nonlinear continuum mechanics. This report also documents the potting epoxy material model family and describes a numerical example in which adaptivity has been performed.

We developed the ability to incorporate item-specific material inhomogeneity data as generated from computerized x-ray tomography (CT).

We scanned several heterogeneous specimens in a CT machine, analyzed them, and tested them in the linear range of behavior.

\section{Refereed}

Mae, H. 2000. "Microstructural Characterization of Glass-Epoxy Specimens.” M. S. Thesis, University of Texas, Austin, TX.

Podnos, E. G. 2000. “Application of Fictitious Domain Method to the Analysis of Composite Materials." Ph.D. Dissertation, University of Texas, Austin, TX.
We wrote the computer codes necessary for the finite-element analysis of structures made from either of the two material model classes under investigation. 
10338

\section{Mechanisms of Adiabatic Shear Failure}

D. B. Dawson, S. H. Goods, J. J. Dike, R. A. Regueiro

High-rate loading of structural alloys can result in adiabatic shear, an extreme and usually catastrophic lowenergy form of localized deformation. Adiabatic shear failures are observed in impact, ballistic penetration, and high-rate deformation processing. Some aspects of the phenomenon are known, but accurate phenomenological and computational models do not exist. This leads to the current uncertain situation where, in a modeling and simulation-based environment, susceptibility to catastrophic adiabatic shear failure may be detected only in full-system testing or service. Current computational approaches to improved localization modeling do not address the complexity of adiabatic shear, with its inherently tight coupling of temperature and mechanical fields.

Sandia is conducting a systematic, coupled experimental and analytical study to establish deformation/ failure mechanism maps, to develop innovative, applicationsoriented scaling laws, and to design criteria relating critical physical aspects to adiabatic shear failure susceptibility.

We completed an experimental Phase I screening matrix of adiabatic shear susceptibility for a wide range of steels, aluminum ( $\mathrm{Al}$ ) alloys, and titanium (Ti) alloys used in Sandia defense programs. We used the results of the screening tests to select two of these alloys, $6061 \mathrm{Al}$ and Ti-6Al-4V Ti, for a more detailed Phase II examination of adiabatic shear band initiation characteristics. These alloys represent the two primary forms of adiabatic shear bands, the "deformation" type (6061) and the "transformation" type (Ti-6Al-4V). We tested specimens over a range of strain rates, imposed strains to determine shear band initiation criteria, and studied characteristics of incipient shear bands using metallographic sectioning of specimens.

We conducted screening tests and initial Phase II tests using the torsional Kolsky technique. While the technique has many advantages that led to its choice for the Phase I test matrix, results from those tests identified several shortcomings: inability to induce adiabatic shear in highly resistant alloys, premature failures at stress concentrations in highly susceptible alloys, and variability due to geometric effects. Therefore, we developed a novel specimen geometry that alleviates most of
Current computational approaches to improved localization modeling do not address the complexity of adiabatic shear, with its inherently tight coupling of temperature and mechanical fields. Sandia is conducting a systematic, coupled experimental and analytical study to establish deformation/ failure mechanism maps, to develop innovative, applications-oriented scaling laws, and to design criteria relating critical physical aspects to adiabatic shear failure susceptibility. 
these concerns. We evaluated the resultant dynamically loaded compression-shear specimen both experimentally and analytically and found it to provide a capability for large, highrate shear strains confined to a well-characterized gage section. Additional advantages include suppression of competing failure modes and low susceptibility to geometry-induced variability.

To facilitate modeling of adiabatic shear band initiation and growth, we determined material constants for Phase II alloys and implemented a modified adiabatic temperature evolution equation that more accurately represents conversion of plastic work to heat. Using this model, we completed simulations of torsional Kolsky and compression-shear specimens. We showed that we can achieve accurate simulation of uniform shear deformation, but no localization to form an incipient shear band is predicted without a geometric inhomogeneity. To facilitate reproducible triggering of adiabatic shear localization, we are investigating modification of the compression-shear specimen geometry to introduce a known geometric inhomogeneity both analytically and experimentally.
We evaluated the resultant dynamically loaded compression-shear specimen both experimentally and analytically and found it to provide a capability for large, high-rate shear strains confined to a well-characterized gage section. Additional advantages include suppression of competing failure modes and low susceptibility to geometryinduced variability. 
10339

\section{Evolvable Hardware}

\section{J. S. Jones, G. S. Davidson, D. J. Zimmerer}

We propose to continue research of the use of evolvable hardware (EHW) as a design tool with the potential to expand Sandia's capabilities in any area requiring dedicated complex circuitry. EHW refers to the innovative technique of using evolutionary algorithms (EAs) to design and synthesize circuits on field-programmable gate arrays (FPGAs), with the eventual goal of allowing these circuits to dynamically and autonomously reconfigure themselves to adapt to changing conditions. Because evolutionary design explores an unlimited design space, it can discover novel designs and create them without expert knowledge, thus reducing the requirement of a large staff of the highest-quality circuit designers. EHW does not require exact specification and thus can design complex systems that cannot be handled by conventional specificationbased design approaches. EHW can design circuits that might be too difficult or costly to design by human experts. EHW can evolve much simpler and more compact designs than traditional methods. This research has a number of applications, from on-satellite data processing to chemicalsensor devices to weapon components. Continuing to expand this capability will improve the speed and efficiency of the design and production phases, as well as make Sandia a leader in this next generation of design methodology.

A series of arithmetic circuits evolved, including a single-bit adder, a six-bit plus six-bit adder with carry, and a three-bit-by-three-bit multiplier. In addition to these small test problems, we conducted a study of digital signal-processing circuitry. We investigated the evolution of a 16th-order Hamming window low-bandpass filter (LPF). We used a training set of 20 white-noise sequences and 64 data samples for the evolution. We ran the simulation using 128 processors in a WinNT cluster. At various times in the simulation, we copied the best-evolved circuit and then examined it in a separate program by testing its performance against another 200 newly generated white-noise inputs. The evolved digital filter consisted of 1,307 gates. This circuit attenuated higher frequencies while continuing to pass the lower frequencies, which is the function of a low-pass filter. The second area of research concerned hardware evaluation of potential circuits. We designed a feed-forward multiplexor circuit with a
We propose to continue research of the use of evolvable hardware (EHW) as a design tool with the potential to expand Sandia's capabilities in any area requiring dedicated complex circuitry.... Continuing to expand this capability will improve the speed and efficiency of the design and production phases, as well as make Sandia a leader in this next generation of design methodology. 
maximum of 46 gates, then routed and loaded it into a Xilinx XC4000 FPGA, which we then mounted on a FAT HOTWorks PCI (peripheral component interconnect] board in a PC running Windows 95. We determined the break-even point for using the hardware evaluator over a software-only evaluator to be approximately 12 gates.

\section{Refereed}

Jones, J. S., and G. S. Davidson. 2000. "Synthesis of Logic Circuits with Evolutionary Algorithms." Paper presented to the GECCO-2000, Las Vegas, NV, 8-12 July. 
10340

Crack Nucleation and Growth: Combining Validated Atomistic and Continuum Modeling

J. E. Houston, J. C. Hamilton, A. K. Schmid, P. A. Klein, S. M.

Foiles, J. A. Zimmerman

Fracture and de-adhesion are critical material failure modes and impact areas ranging from advanced component development to the aging of stockpile components.

Consequently, computational models of these processes are essential to Sandia's programmatic interest. The wide range of length scales involved has dramatically hampered the computer modeling of fracture. For example, corrosion (i.e., molecular reactions at the crack tip) and stress (i.e., longrange elastic forces at a macroscopic scale) are the two fundamental processes involved in stress-corrosion cracking, a common failure mode for materials. Thus it is imperative to couple the atomistic understanding provided by solid-state physics with the stress analysis provided by theoretical mechanics. We have a coordinated project in which experimental investigation of prototypical fracture events will guide modeling using atomistic and continuum techniques. Our goals are to achieve a common working vocabulary that merges the ideas of atomistic and continuum analysis, to develop codes that combine the two methods, and to validate these codes by comparison with the experimental investigations of fracture situations.

We aimed our modeling work at qualitatively and quantitatively comparing atomistic and continuum methods for modeling mechanical behavior. We simulated quasi-statically the idealized two-dimensional (2-D) system of a steady-state crack using a standard interatomic potential (Lennard-Jones) to determine the parameters of cohesive strength and critical energy release rate to be used in finite-element (FE) calculations. Simulations at the continuum and atomistic scales showed identical response and the same traction distribution on the crack-plane, while dynamics simulations revealed other dissipation mechanisms not found in the continuum approach. Quasi-static atomistic simulations of a $\mathrm{Au}(111)$ (gold) surface with a step provided insight into the initiation of dislocation creation during nanoindentation. The results are similar to those seen in nanoindentation experiments, although lengthscale differences result in subtle contrasts. We developed a new simulation tool, the slip vector, to identify the Burgers vector
Fracture and de-adhesion are critical material failure modes and impact areas ranging from advanced component development to the aging of stockpile components.... Our goals are to achieve a common working vocabulary that merges the ideas of atomistic and continuum analysis, to develop codes that combine the two methods, and to validate these codes by comparison with the experimental investigations of fracture situations. 
content of the dislocation structures that form. Combining the precise direction of slip with a locally defined atomic stress tensor permits calculation of the resolved shear stress needed for dislocation nucleation. We compared stress fields in the atomistic simulation with those in an FE calculation using the Cauchy-Born constitutive model of the same interatomic potential. The fields agree well quantitatively, showing promise for a coupled atomistic-continuum calculation necessary for proper treatment of experimental length-scales. We continued our work investigating the effects of applied stress on $\mathrm{Au}(111)$ films grown on mica substrates using scanning tunneling microscopy (STM). In addition to the surface reconstructions reported previously, we discovered new phenomena that indicate that $\mathrm{Au}$ on mica is a prototype system for investigating interfacial fracture between the film and substrate. We believe that this emphasis change will greatly enhance the applicability of our work to understanding real fracture.

\section{Refereed}

Jarausch, K. F., J. D. Kiely, J. E. Houston, and P. E.

Russell. 2000. "Defect-Dependent Elasticity:

Nanoindentation as a Probe of Stress State." J. Mater.

Res. 15: 1693-1701.

Schaff, O., A. K. Schmid, N. C. Bartelt, J. de la Figuera, and R. Q. Hwang. 2000. "In Situ STM Studies of Strain-Stabilized Thin-Film Dislocation Networks Under Applied Stress." Mater. Sci. and Eng. A, in press.

Zimmerman, J. A., C. L. Kelchner, P. A. Klein, J. C. Hamilton, and S. M. Foiles. 2000. "Surface Step Effects on Nanoindentation." Phys. Rev. Lett., in press.
In addition to the surface reconstructions reported previously, we discovered new phenomena that indicate that $\mathrm{Au}$ on mica is a prototype system for investigating interfacial fracture between the film and substrate. We believe that this emphasis change will greatly enhance the applicability of our work to understanding real fracture. 
10341

\section{Applied Microfluidic Physics}

E. B. Cummings, S. K. Griffiths, R. H. Nilson

While microfluidic microelectromechanical systems (MEMS), such as Sandia's $\mu$ ChemLab ${ }^{T M}$, and electrokinetic pumps (EKPs) are believed to be capable of outperforming their macroscopic relatives, a sufficient understanding of the potential performance, design, and optimal operating protocols of these devices is lacking. The goal of this project is to provide this information for families of devices having an immediate and lasting impact on miniaturized chemical and biological analytical systems. Our approach is to couple analysis, numerical tools, and experiment to produce validated models of microfluidic processes from molecular to device scales and to apply these models to create high-performance designs.

This year, we focused on (1) developing electroosmotic sample-injection protocols, (2) quantifying hydrodynamic dispersion in turns, (3) quantifying electroosmotic and dielectrophoretic flow, dispersion, and mass transport in uniform arrays of posts, (4) developing user-friendly EK and dielectrophoretic flow simulation software suitable for analyzing flowfields and transport within planar microfabricated devices for design purposes, and (5) fundamental studies of electroosmosis in highly charged columns.

We accomplished the following:

- Completed development of optimized turn and junction geometries and operating protocols and transferred the information to designers,

- Completed experimental validation of our mathematical model of electroosmosis and performed experimental studies of flows with embedded electrodes,

- Experimentally, theoretically, and numerically studied flows in patterned posts arrays, obtained curves for pressure and electroosmotic permeability, and developed a theory linking unit-cell fields to dispersion and mass transport in arrays,

- Obtained fundamental solutions for electroosmotic flows in channels having nonnegligible zeta potential,

- Developed an analytical expression for dispersion in conventional turns,

\section{While microfluidic} microelectromechanical systems (MEMS ), such as Sandia's $\mu$ ChemLab ${ }^{T M}$, and electrokinetic pumps (EKPs) are believed to be capable of outperforming their macroscopic relatives, a sufficient understanding of the potential performance, design, and optimal operating protocols of these devices is lacking. The goal of this project is to provide this information for families of devices having an immediate and lasting impact on miniaturized chemical and biological analytical systems. 
- Created a new miniaturized standard microfluidic

fixture and a miniature, programmable, 16-channel highvoltage sequencer, and improved particle-image velocimetry hardware and analysis software for making reliable and repeatable experimental measurements,

- Began experimental and theoretical investigation of particle dielectrophoresis in uniform post arrays, and

- Created user-friendly design-analysis software to make our mathematical model of electroosmosis and dielectrophoresis widely available. This software provides accurate scalar-transport, velocity, and electric fields within a

This software provides accurate scalar-transport, velocity, and electric fields within a device given a bitmapped sketch, drawing, or photographic image of a device. device given a bitmapped sketch, drawing, or photographic image of a device.

We submitted a patent application.

\section{Refereed}

Cummings, E. B., and A. K. Singh. 2000.

"Dielectrophoretic Trapping Without Embedded Electrodes." Proc. SPIE Microfluidic Devices and Systems III, SPIE Symp. on Micromachining and Microfabrication 4177 (Santa Clara, CA, 19 September): 164-172.

Cummings, E. B., S. K. Griffiths, and R. H. Nilson. 2000. "Conditions for Similitude Between the Fluid Velocity and Electric Field in Electroosmotic Flow." Anal. Chem. 72: 2526-2532.

Griffiths, S. K., and R. H. Nilson. 2000. "Dispersion in Turns for Species Transport by Electrophoresis and Electroosmotic Flow." SPIE Microfluidic Devices and Systems III, SPIE Symp. on Micromachining and Microfabrication 4177 (Santa Clara, CA, 19 September): 229-240.

Griffiths, S. K., and R. H. Nilson. 2000.

"Electroosmotic Flow in a Tube or Channel at NonNegligible Zeta Potentials." MSM2000, Computational Publications, Proc. 3rd Internat. Conf. on Modeling and Simulation of Microsys. (San Diego, CA, 27 March): 532-535.

\section{Other Communications}

Griffiths, S. K., and R. H. Nilson. 2000. "Band Spreading in Two-Dimension Microchannel Turns for Electrophoretic and Electroosmotic Species Motion." Sandia Technical Report SAND2000-8230, Sandia National Laboratories, Albuquerque, NM (March).

Griffiths, S. K., and R. H. Nilson. 1999.

"Electroosmotic Fluid Motion and Late-Time Solute Transport at Non-Negligible Zeta Potentials." Sandia Technical Report SAND2000-8437, Sandia National Laboratories, Albuquerque, NM (December).

Griffiths, S. K., and R. H. Nilson. 2000. "Method and Apparatus for Reducing Sample Dispersion in Turns and Junctions of Microchannel Systems." PCT patent application (11 April).

Griffiths, S. K., and R. H. Nilson. 1999. "Method and Apparatus for Sample Injection in Microchannel

Devices.” Technical Advance SD-8263 (22

December). 
10343

\section{Innovative Measurement Diagnostics for Fluid/Solid and Fluid/Fluid Interactions in Rotating Flowfields}

\author{
V. A. Amatucci, J. F. Henfling, R. J. Erven, S. J. Beresh
}

The goal of this project is to develop and evaluate new, miniaturized, onboard instrumentation for a subscale rotating model in a wind tunnel. The project is motivated by the need to measure and understand the complex fluid interactions around and on a spinning body in a transonic flowfield, including the effects of jet impingement from the propulsive plumes that spin the vehicle. The interaction of the highly vortical plumes with structures on the vehicle, such as fins, causes modifications to spin-and-roll performance, which needs to be understood. The consequences for weapon performance and accuracy are significant, and better experiments will support ongoing efforts to model this rotating flowfield. The experiments for this project will incorporate miniaturized pressure instrumentation onto a scale model that will be mounted in Sandia's Trisonic Wind Tunnel (TWT) facility for measurements. The key to success of these types of measurements is a self-sustained, miniature data-acquisition and telemetry system that resides within the model and transmits acquired data to a receiver adjacent to the wind tunnel facility. The work includes (1) evaluation of the performance of the microelectromechanical systems (MEMS)-based pressure microsensors, (2) design and initial fabrication of a new wind tunnel model, and (3) selection and purchase of 16-channel data-acquisition modules miniaturized for installation into the new subscale model. In addition, we held technical discussions with an external vendor of pressure instrumentation to customize installation of timeresolved sensors onto the fins of the model for detailed pressure surveys of the surface interactions. The primary success of our work was to position the team for dataacquisition experiments once all hardware is acquired.

Our major accomplishments include hardware design and developments in the areas of instrumentation, telemetry systems, and model details. The driving goal is to assemble a system on a subscale model in Sandia's TWT facility that would measure pressure and rate information for a spinning model subjected to gas-jet-driven plumes impinging on the fins. The major work focused on (1) selection and evaluation of microscale pressure sensors, (2) design and fabrication of a
The goal of this project is to develop and evaluate new, miniaturized, onboard instrumentation for a subscale rotating model in a wind tunnel. The project is motivated by the need to measure and understand the complex fluid interactions around and on a spinning body in a transonic flowfield, including the effects of jet impingement from the propulsive plumes that spin the vehicle. 
new model with rotation and gas-jet supplies for use in the TWT facility, (3) evaluation, selection, and purchase of specially made 16-channel telemetry systems for data acquisition and transmission on extremely small geometries, and (4) technical discussions for development of pressure instrumentation that could be incorporated directly into the fin design of the new model to measure pressure fields due to impinging gas jets. Our work required integration of external companies into the Sandia team framework for the project to meet the subscale needs for an accurate model and effective data-acquisition and transmission hardware design.

We completed successful experiments with micronscale pressure transducers to determine their accuracy and response to input pressures under a variety of temperature conditions. Three transducers demonstrated similar accuracy, sensitivity, and repeatability. Certain transducers would require that temperature-compensation modules be included as part of the design. We will incorporate these micron-scale transducers into the sidewalls of the new wind tunnel model in large quantities (dozens) to obtain a detailed representation of the pressure contours on the surface of the model. The actual measured pressures during any one wind tunnel experiment will be limited by the capacity of the three 16-channel dataacquisition and telemetry units.

We received the new wind tunnel model designs; they include the capabilities for rotation, dozens of pressure ports, integrated instrumentation, interchangeable fins, and delivery of a jet supply to simulate the spin rocket motor exhaust plumes. The new model would contain a larger internal volume than existing models, allow better access to instrumentation, accommodate different types of sensors, provide a secondary gas source, and demonstrate an improved spin rig compared to current hardware.

Experiments with single-channel acquisition/telemetry systems show great promise for performance on the scale of the TWT, and expectations for the 16-channel units are high. The system requirements demand an extremely small size (less than one-inch diameter), multiple channel capability (about 50), and good dynamic response. Evaluations of the SRI Model ST-320 one-channel system yielded a system with low cost, adequate size and shape, good time response, and successful data retrieval. Issues such as antenna location, highly quantized data, vibrational difficulties, and roll positioning still need to be resolved for the design of the customized, multichannel system.
We completed successful experiments with micron-scale pressure transducers to determine their accuracy and response to input pressures under a variety of temperature conditions. Three transducers demonstrated similar accuracy, sensitivity, and repeatability....We will incorporate these micron-scale transducers into the sidewalls of the new wind tunnel model in large quantities (dozens) to obtain a detailed representation of the pressure contours on the surface of the model. 
The last elements of this work focused on the ability to embed pressure transducers on the fin surfaces via a die process and thus provide high spatial resolution with extremely thin transducers. Packaging issues may make the fabrication costs prohibitive. We are now positioned for wind tunnel experiments for both nonrotating and rotating cases with microsensors and telemetry package in place on the new subscale model.

\section{Other Communications}

Amatucci, V. A., and S. J. Beresh. 2000. "Jet/Fin Interactions on a Rotating Body in Transonic FlightExperimental Status." Paper presented to the Supersonic Tunnel Association International (STAI), Moffett Federal Airfield, CA, 30 April-2 May.
We are now positioned for wind tunnel experiments for both nonrotating and rotating cases with microsensors and telemetry package in place on the new subscale model. 
10344

\section{High-Resolution Modeling of Multiscale} Transient Phenomena in Turbulent Boundary

\section{Layers}

A. R. Kerstein, R. C. Schmidt, S. E. Wunsch, W. T. Ashurst

Modeling of transient multiphysics phenomena in turbulence is especially difficult near walls, where the enhanced role of molecular transport introduces length scales far smaller than scales resolvable by three-dimensional (3-D) computational models. Present-day empirical wall treatments are not applicable to strongly coupled multiphysics environments.

One-dimensional turbulence (ODT) is a new modeling approach that addresses this difficulty by capturing relevant length and time scales in an unsteady one-dimensional (1-D) simulation. In this project, Sandia incorporated the coupled effects of transient applied shear, buoyancy, exothermic chemical reactions, and heat and mass transfer, with attendant property variations, into ODT. This formulation provided the multiscale, multiphysics capabilities needed for near-wall closure of 3-D turbulent flow computations. We performed applications that demonstrate these capabilities of ODT.

The goal of this project is to develop and demonstrate a near-wall submodel for large-eddy simulation (LES) of multiphysics turbulent boundary layers. The strategy we adopted to accomplish this goal was to demonstrate the multiphysics capabilities of the ODT near-wall turbulence simulation model and to implement and demonstrate ODT as a near-wall submodel coupled to an LES code. This year's accomplishments address both elements of this strategy.

We generalized ODT to incorporate the energy exchange mechanisms needed to accomplish these objectives. The generalization includes conservative exchange between flow kinetic energy and gravitational potential energy and among the three components of the velocity vector. We demonstrated the performance of the generalized formulation by applying it to two free shear flows and two planar confined flows, one pressure-driven (channel flow) and one buoyancydriven (Rayleigh-Benard convection). We compared computed results to measurements and to the results of direct numerical simulations (DNS). We reproduced, with minimal parameter adjustment, many aspects of momentum and heat transfer in these flows, including fluctuation statistics.

\section{Modeling of transient} multiphysics phenomena in turbulence is especially difficult near walls, where the enhanced role of molecular transport introduces length scales far smaller than scales resolvable by three-dimensional (3-D) computational models....The goal of this project is to develop

and demonstrate a near-wall submodel for large-eddy simulation (LES) of multiphysics turbulent boundary layers. 
We completed the model formulation and algorithm development needed to couple ODT to an LES code and coupled an ODT near-wall submodel to an existing LES code. The focus of the initial demonstration effort was the use of ODT for near-wall momentum closure in incompressible flow. An iterative process of algorithm testing and refinement led to a formulation with good numerical properties. We tested the coupled model by simulating turbulent channel flow. We obtained good quantitative agreement with DNS results for mean flow properties and flow fluctuation statistics. We found the computational cost of the ODT near-wall closure to be a small fraction of the cost of the bulk-flow LES. Extrapolation to engineering applications indicates that ODT near-wall closure will have insignificant impact on the total computational cost.

We found the computational cost of the ODT near-wall closure to be a small fraction of the cost of the bulk-flow LES. Extrapolation to engineering applications indicates that ODT near-wall closure will have insignificant impact on the total computational cost.

\section{Refereed}

Dreeben, T. D., and A. R. Kerstein. 2000. "Simulation of Vertical Slot Convection Using 'One-Dimensional Turbulence." Internat. J. Heat and Mass Transfer 43: 3823-3834. 
10345

\section{Dispersive Measurements of Velocity in} Heterogeneous Materials

W. M. Trott, M. R. Baer, L. C. Chhabildas, M. D. Knudson, J. P. Davis

In order to provide real-time data for validation of three-dimensional (3-D) numerical simulations of heterogeneous materials subjected to impact loading, an optically recording velocity interferometer system (ORVIS) has been adapted to a line-imaging instrument capable of generating precise measurements of spatially resolved velocity variations during dynamic deformation. The demonstrated spatial resolution can be as fine as a few mm along a line segment together with a temporal resolution $<0.2 \mathrm{~ns}$. Simultaneous stop-motion imaging of the complete fringe field (using a fast-gating intensifier/charge-coupled device (CCD) system) was also developed; we can use records from this technique to generate a complete 2-D map showing velocity variations in a moving surface at one point in time. Firstgeneration analysis routines for processing the image data from both techniques have been developed and tested. A lineimaging velocity interferometer was packaged for use in wellcontrolled, well-characterized impact-loading experiments at a gas-gun facility. In these tests, we examined the mesoscopicscale dynamic response of several different classes of heterogeneous materials. Materials of programmatic interest include foams, glass-reinforced polyester composites, and pressed granular sugar as a nonreactive simulant of the explosive HMX (1,3,5,7-tetranitro-1,3,5,7-tetrazacyclooctane).

Sandia is using the evolving database to evaluate critically corresponding 3-D numerical simulations. We designed validation of numerical models at the mesoscopic scale to encourage development of predictive continuum models that will also be validated at the macroscopic level using conventional shock-wave profile techniques. A robust experimental/analytical methodology for validation of material response at the two different levels will provide an unprecedented capability for developing and validating physics-based models to achieve the goals of ASCI (Accelerated Strategic Computing Initiative) and Stockpile Stewardship programs directed at better predictive modeling capabilities as a replacement for extensive experimental testing of weapons systems and components.
A robust experimental/analytical methodology for validation of material response at the two different levels will provide an unprecedented capability for developing and validating physics-based models to achieve the goals of ASCI (Accelerated Strategic Computing Initiative) and Stockpile Stewardship programs directed at better predictive modeling capabilities as a replacement for extensive experimental testing of weapons systems and components. 
We implemented significant improvements to the lineimaging ORVIS assembly, completion of an analysis software package for processing fringe image data, execution and analysis of numerous experiments on sugar (simulant for HMX) under impact loading, and direct comparison of spatially resolved particle-velocity data to results of 3-D numerical simulations.

We designed and tested several modifications to the line-imaging ORVIS assembly. We assembled a robust, yet portable, rail system for mounting the input and collection optics of the line-imaging ORVIS, significantly decreasing the amount of time required for alignment, changes in image magnification, etc. We developed an in-line reticle projection system for facile measurements of target magnification in fixtures where physical access to the measurement plane is difficult or impossible (e.g., in measurements with interferometer windows). We developed a dichroic cube beamsplitter system to permit for the first time spatially overlapped, as well as simultaneous, single-point VISAR (velocity interferometry system for any reflector) and lineimaging ORVIS measurements. We successfully tested this innovation on tantalum (Ta) spall experiments.

The analysis software package developed under this project utilizes a rolling fringe analysis technique that makes use of the full field of image data. The complete package integrates this technique with the necessary image-processing methods and an existing VISAR analysis platform. Steps include image format conversion, image rotation, evaluation of average fringe spacing, evaluation of a series of fringe intensity-time data in a format suitable for export to the VISAR analysis platform, and export of velocity-time profiles to a 3-D plotting package. The software package also includes an optional regression solver to assist in the evaluation of fringe spacing for fringes of widely disparate intensities as well as numerous filters for smoothing the intensity-time data. With minor modifications, this package can also be used to generate an areal (2-D) velocity plot from stop-motion charge-coupled device (CCD) images of the full fringe field. We also evaluated several advanced approaches to data analysis, including fast Fourier transform (FFT) filtering and application of wavelet transforms.

In experiments on shock propagation in low-density sugar samples, we performed tests as a function of both impact velocity and sugar-particle-size distribution. Comparison of experimental results from the line-imaging ORVIS technique
We developed a dichroic cube beamsplitter system to permit for the first time spatially overlapped, as well as simultaneous, single-point VISAR (velocity interferometry system for any reflector) and line-imaging ORVIS measurements. We successfully tested this innovation on tantalum (Ta) spall experiments. 
and 3-D computations (performed using samples with matching particle-size distribution) indicate that the spatial and temporal resolution of the diagnostic is appropriate for discerning the most important of the longitudinal and transverse wave structures on meaningful length scales. In particular, the diagnostic provided validation data that include measurements of precursor motion and a definite velocity "overshoot" in the early part of the transmitted wave that settles down to a constant value. This work identified several productive avenues for follow-on studies, including variation of sample length (to test steady-wave behavior) and comparison of experiment and theory for sieved (narrowly defined particle size) samples. Tests on explosive materials such as HMX can be used to probe the additional effects of chemistry on the wave profiles.

We also explored application of the line-imaging ORVIS technique to several other materials (e.g., Ta spall, ALOX (alumina-filled epoxy), ALOX/PZT [lead zirconate titanate, a piezoelectric ceramic material]). We expect the data obtained in these experiments to provide useful tests for multidimensional computational models of wave propagation in these materials.

\section{Refereed}

Trott, W. M., J. N. Castaneda, J. J. O’Hare, M. D. Knudson, L. C. Chhabildas, M. R. Baer, and J. R. Asay. 2000. "Examination of the Mesoscopic Scale Response of Shock-Compressed Heterogeneous Materials Using a Line-Imaging Velocity

Interferometer." Proc. Explomet 2000, accepted.

Trott, W. M., J. N. Castaneda, M. D. Knudson, L. C. Chhabildas, and J. R. Asay. 2000. "Probing Shocked Microstructures." Proc. 50th Mtg. of the Aeroballistic Range Assoc., accepted.

\section{Other Communications}

Trott, W. M. 2000. "Probing Energetic Materials Chemistry at the Mesoscale Using Line-Imaging Velocity Interferometry." Paper presented to the Gordon Research Conference on Energetic Materials, Tilton, NH, 2-6 July.
This work identified several productive avenues for followon studies, including variation of sample length (to test steadywave behavior) and comparison of experiment and theory for sieved (narrowly defined particle size) samples. 
10346

\section{A Physically Based Computational Method for Predicting Generalized Fracture}

P. A. Klein, S. A. Wimmer, J. W. Foulk, III

The purpose of this project is to investigate and develop physically based numerical methods for simulating generalized fracture. Simulation of generalized fracture and fragmentation remains an ongoing challenge in computational fracture mechanics. There are difficulties associated not only with the formulation of physically based fracture criteria, but also with the numerical methods required to treat geometries that change in time. This work addresses the issue of fracture criteria through a cohesive view of material; that is, a finite material strength and work to fracture are included in the material description. We explored the capabilities of two approaches: (1) a cohesive surface approach and (2) a bulk cohesive approach.

This work provides a high-fidelity capability for predicting generalized fracture with application to a wide range of problems, including polymer aging, thermally induced cracking of encapsulants, glass fragmentation, and weapon slap-down.

Validation was a key component of this work. First, we studied the mesh-size resolution requirements associated with modeling fracture with cohesive approaches. Accurate resolution of the deformations in the near tip zone is required to capture the energy dissipation and therefore dictates the cohesive surface element dimensions. We investigated these mesh-size requirements using the double-cantilever beam (DCB) specimen geometry. For a slender DCB specimen, there is a closed-form solution of the crack length as a function of end-opening displacement that is derived by consideration of the variation in the strain energy release rate of the specimen as a function of crack length. In varying the cohesive parameters, we determined that approximately three to five elements are required in the cohesive zone to resolve the fracture energy with an error of less than $10 \%$, with linear displacement cohesive surface elements in two dimensions integrated using a two-point Gauss rule. The simulations showed that the crack tip jumped erratically when these resolution conditions were not satisfied. We also performed three-dimensional (3-D) simulations of peel testing in muscovite mica. Mica is a good
The purpose of this project is to investigate and develop physically based numerical methods for simulating generalized fracture. Simulation of generalized fracture and fragmentation remains an ongoing challenge in computational fracture mechanics. 
system for studying brittle fracture because it cleaves along well-defined planes. In agreement with experiments, the simulations showed that the crack front near the free edges trails behind the segments of the front toward the center of the specimen. Using elastic and fracture parameters for mica, our 3-D simulations reproduced the characteristic scale over which curvature of the crack front is observed.

We formulated a method to apply cohesive tractions across these cutting surfaces. We combined this method with the 2-D/3-D arbitrary fracture surface representation developed previously to allow adaptive insertion of cohesive surfaces in a mesh-free domain without introducing additional degrees of freedom (DOFs) as is produced with "nodal splitting" methods. We validated the mesh-free cohesive surface elements in 3-D simulations of uniaxial extension of a bar. We calculated the reaction force on the end of the bar as a function of end displacement for both regular and irregular particle arrangements. The curves of reaction force as a function of end displacement for all three cases agreed very well, indicating that the approach allows accurate resolution of the fracture energy regardless of the particle arrangement, as long as the particle spacing is sufficiently fine.

We modeled two additional fracture experiments as laboratory scale to determine the feasibility of simulating fracture in actual components using cohesive methods. We performed 2-D simulations of the Kalthoff-Winkler experiment using cohesive surfaces networks in a finite-element (FE) framework and using the virtual internal bond (VIB) model with mesh-free methods. The VIB model incorporates cohesive behavior in the constitutive relations for the bulk. The cohesive surface network approach proved successful in reproducing some dynamic crack behavior qualitatively, but had never been validated against an actual experiment. In these simulations of mixed-mode fracture in high-strength stainless steel, we varied mesh size, mesh design, and cohesive strength to determine the sensitivity of the predicted fracture path to these parameters. We were unable to produce reliable results with this approach because of the pathological mesh dependence described earlier. We found that no compromise exists between the mesh size needed to minimize the fictitious softening and the size dictated by resolution conditions. The fracture paths also varied with particle arrangement in the VIB simulations, though the fracture paths generally agreed with what we observed in the experiments. Finally, we performed 3-D simulations of dynamic fracture using the parallelized capabilities of the
We formulated a method to apply cohesive tractions across these cutting surfaces. We combined this method with the 2-D/3-D arbitrary fracture surface representation developed previously to allow adaptive insertion of cohesive surfaces in a mesh-free domain without introducing additional degrees of freedom (DOFs) as is produced with "nodal splitting" methods. 
fracture simulation code. We simulated dynamic crack propagation using 1.3 million particles distributed over 50 processors of the Computational Plant (CPlant) Alaska. The simulations produced complex fracture surface topologies exhibiting multiple branches. The mesh-free simulation code proved extremely robust, allowing the simulations to progress far beyond what could be attained using standard FEs over the same domain.
The mesh-free simulation code proved extremely robust, allowing the simulations to progress far beyond what could be attained using standard FEs over the same domain.

\section{Refereed}

Klein, P. A., and H. Gao. 2000. "Study of Crack Dynamics Using the Virtual Internal Bond Method." James R. Rice 60th Anniversary Volume of Solid Mechanics and Its Applications, accepted.

Klein, P. A., E. P. Chen, J. W. Foulk, and S. A. Wimmer. 2000. "Numerical Artifacts in Cohesive Fracture Simulation." Advances in Computational Eng. and Sci., Proc. Internat. Conf. on Computational Eng. Sci. 2 (Anaheim, CA, 24 August): 1790-1795.

Klein, P. A., E. P. Chen, J. W. Foulk, S. A. Wimmer, and J. A. Zimmerman. 2000. "Cohesive Modeling of Dynamic Fracture Using Meshfree Methods." Paper presented to the Workshop on Meshfree Methods, Chicago, IL, 11 June.

Klein, P. A., H. Gao, A. Vainchtein, H. Fujimoto, Q. Ma, and J. Lee. 1999. "Micromechanics-Based Modeling of Interfacial Debonding in Multilayered Structures." Proc. Mater. Res. Soc. Fall Mtg. (Boston, MA, 29 November). 
10694

\section{Micromechanical Failure Analyses for Finite-} Element Polymer Modeling

R. S. Chambers, T. R. Guess, D. B. Adolf, C. S. Lo, E. D. Reedy, Jr.

Polymer stresses around sharp corners and in constrained geometries of encapsulated components can generate cracks leading to system failures. Often, analysts use maximum stresses as a qualitative indicator for evaluating the strength of encapsulated component designs. Although this approach has been useful for making relative comparisons screening prospective design changes, it has not been tied quantitatively to failure. Accurate failure models are needed for analyses to predict whether encapsulated components meet life-cycle requirements. The purpose of this project is to investigate cohesive failure in thermosets with the intent to identify failure mechanisms and consider new failure models and analyses.

With Sandia's recently developed nonlinear viscoelastic polymer models, it is possible to examine more accurately the local stress-strain distributions in zones of likely polymer failure initiation looking for continuum metrics that correlate with the cohesive failure event.

This project has progressed along two paths, one extending fracture analyses to discontinuities other than cracks, and the other using nonlinear viscoelastic analyses of thermosets to study cohesive failure mechanisms in search of physically based, cohesive failure criteria.

In studying cohesive failure mechanisms for thermosets, we took four key steps: (1) conducted failure tests on three-point bending samples with nonsingular stress concentrations to collect the requisite failure data, (2) hypothesized failure mechanisms, (3) proposed failure metrics to relate local nonlinear viscoelastic finite-element $(\mathrm{FE})$ analysis results to failure events, and (4) analyzed failure tests to assess the validity of the proposed metrics and hypotheses.

We conducted failure tests in three-point bending. To initiate failure in a controlled way under variable conditions, we notched the test samples from the bottom at mid-span. We used two different nonsingular sample geometries: one with a narrow (200-micron) slot cut upward from the bottom surface and a second with a larger semicircular cutout made by centering the radius of the cutout on the bottom surface. We used four radii: $\mathrm{R}=0.0625 ", 0.125 ", 0.25 "$, and $0.345^{\prime \prime}$. We
Polymer stresses around sharp corners and in constrained geometries of encapsulated components can generate cracks leading to system failures....The purpose of this project is to investigate cohesive failure in thermosets with the intent to identify failure mechanisms and consider new failure models and analyses. With Sandia's recently developed nonlinear viscoelastic polymer models, it is possible to examine more accurately the local stress-strain distributions in zones of likely polymer failure initiation looking for continuum metrics that correlate with the cohesive failure event. 
conducted tests at temperatures of $-50^{\circ} \mathrm{C}, 18^{\circ} \mathrm{C}, 50^{\circ} \mathrm{C}$, and $120^{\circ} \mathrm{C}$ to collect data on both glassy and rubbery responses. We also considered variable loading rates.

The physical picture of thermoset failure is different for rubbery and glassy behavior. Rubbery failure seems to be controlled by the finite extensibility of the network chains, and glassy failure appears to involve a cavitation event. Based on these assumptions, we proposed local failure metrics. For the rubber, we found a local metric based on the maximum strain to correlate well with the observed failures. For the glass, a local metric based on the maximum hydrostatic tensile stress worked. Both criteria involve parameters readily attainable from nonlinear viscoelastic FE analyses. Hence, the methodology can be readily applied. However, this presumes that one is able to obtain sufficient solution accuracy to be able to quantitatively predict the maximum values of strain or hydrostatic tension to within the reasonable scatter of the experimental data. For this reason, we investigated nonsingular elastic test geometries (rounded notches).

In separate fracture analyses, we determined the asymptotically singular stress state found at the tip of a rigid, square inclusion embedded within a thin, linear elastic disk for uniform cooling. We characterized the singular stress field by a single stress intensity factor $\mathrm{K}_{\mathrm{a}}$ and determined the applicable $\mathrm{K}_{-\mathrm{a}}$ calibration relationship for both fully bonded and unbonded inclusions. A lack of interfacial bonding has a profound effect on inclusion-tip stress fields. A large radial compressive stress is generated in front of the inclusion tip when the inclusion is well bonded, whereas a large tensile hoop stress is generated when the inclusion is unbonded, and frictionless sliding is allowed. Consequently, an epoxy disk containing an unbonded inclusion appears more likely to crack when cooled than a disk containing a fully bonded inclusion. We carried out a limited number of tests to determine if encapsulant cracking can be induced by cooling a specimen fabricated by molding a square, steel insert within a thin, epoxy disk. Test results are in qualitative agreement with analysis. Cracks developed only in disks with mold-released inserts, and the tendency for cracking increased with inclusion size.

\section{Refereed}

Reedy, E. D., Jr., and T. R. Guess. 2000. "Rigid

Square Inclusion Embedded Within an Epoxy Disk: Asymptotic Stress Analysis." Internat. J. Solids and Structures, accepted.
A lack of interfacial bonding has a profound effect on inclusiontip stress fields. 


\section{Advanced Manufacturing}

The objective of the Advanced Manufacturing investment area is to support manufacturing technology advances with an emphasis on process-based quality. The goal of process-based quality is to generate manufactured goods (i.e., nuclear weapon components and subsystems) with little or no product inspection.

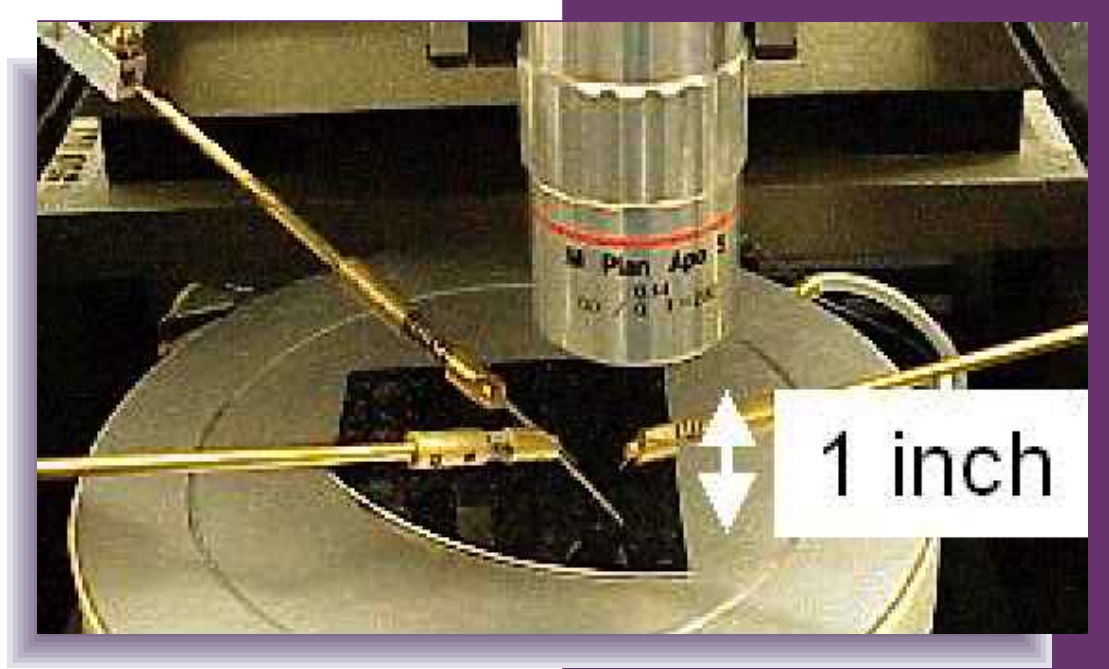

manufacturing lots. A small-area fracture test structure has been developed to monitor lots by electrostatic probing. The structure can be tested simultaneously with other structures to yield information on mechanical and surface properties. This information is essential to support improved MEMS designs, processes, and reliability for nuclear weapons surety and commercial applications. A patent application has been submitted based on this research. engineering design. The "Microdiagnostic MEMS Lab-on-a-Chip" project is exploring thin-film material properties for improving MEMS (microelectromechanical systems) structures. This is an important area of manufacturing research since the fracture strength of thin-film materials can vary with 
10347

\section{Microdiagnostic MEMS Lab-on-a-Chip}

M. P. De Boer, F. Bitsie, N. F. Smith, M. B. Sinclair

Although mechanical properties of

microelectromechanical systems (MEMS) structural materials

are critical to product design and performance, there exists no infrastructure for their routine measurement. Furthermore, when measurements are presently made on an interferometric microprobing station, resolution is very high, but accuracy is not well known. Sandia plans to overcome these deficiencies by developing a self-validating suite of small-area test structures for automated measurement. Statistical quality control can then become the basis for MEMS design, process improvement, and reliability. Because MEMS are radiation hard and demonstrate promise for nuclear weapons surety, this work will directly benefit the nuclear weapons program.

We modeled our approach for developing microdiagnostic MEMS devices after parametric devices in microelectronics; we believe a similar avenue is feasible and is indeed the best approach for MEMS. Relevant mechanical properties include residual stress, stress gradient, adhesion, friction, and fracture strength. Our project will have two main thrusts. The first is to optimize test structures, in conjunction with a determination of the accuracy of the mechanical tests from optoelectrical probing techniques. To validate test results, we will use measured device parameters to predict performance of a composite test structure. We will then compare predictions to actual performance. The second thrust is to implement the procedures on an automated prober.

The development of a standard suite of diagnostic device designs with properly validated measurement and analysis specifications will enable first-run success of designs and facilitate the production and reliability of MEMS, both in Sandia nuclear weapons surety applications and in the commercial world.

We submitted three technical advances (TAs) on new inventions: (1) a long-working-distance interferometer, (2) a new test structure for measuring fracture strength, and (3) a patent pending on the test system and methodology. We successfully modified a standard electrical probe station used for integrated circuit (IC) characterization to enable threedimensional (3-D) imaging of MEMS parts. This will not only enable success of our project on material property
The development of a standard suite of diagnostic device designs with properly validated measurement and analysis specifications will enable firstrun success of designs and facilitate the production and reliability of MEMS, both in Sandia nuclear weapons surety applications and in the commercial world. 
quantification, but also will have significant impact on characterization of MEMS devices. We are writing software to enable automatic testing while maintaining user verifiability of test results. We are negotiating a revenue-sharing agreement with a major MEMS design-and-simulation company to implement this test system and software. Four university students contributed to this project. We demonstrated a new test structure to measure fracture strength by electrostatic probing. Another test structure to measure friction was improved over previous work. Both of these test structures can be measured within the automatic test methodology that we are developing.

\section{Refereed}

Crozier, B. T., M. P. de Boer, J. M. Redmond, D. F. Bahr, and T. A. Michalske. 2000. "Friction Measurement in MEMS Using a New Test Structure." Mater. Sci. of MEMS Devices II, Proc. Mater. Res. Soc. Conf. 605 (September) (Boston, MA, November 1999): 129-134.

de Boer, M. P., and T. A. Michalske. 1999. "Accurate Method for Determining Adhesion of Cantilever Beams." J. Appl. Phys. 86 (2): 817-827.

de Boer, M. P., B. D. Jensen, and F. Bitsie. 1999. "A Small-Area In Situ MEMS Test Structure to Measure Fracture Strength by Electrostatic Probing." Proc. SPIE, Mater. and Device Characterization in Micromachining II 3512 (Santa Clara, CA, September): 97-103.
We demonstrated a new test

structure to measure fracture strength by electrostatic probing. de Boer, M. P., J. A. Knapp, T. A. Michalske, U. Srinivasan, and R. Maboudian. 2000. "Adhesion Hysteresis of Silane-Coated Microcantilevers." Acta Materialia, in press.

Jensen, B. D., M. P. de Boer, and F. Bitsie. 1999. "Interferometric Measurement for Improved Understanding of Boundary Effects in Micromachined Beams." Proc. SPIE, Mater. and Device Characterization in Micromachining II 3512 (Santa Clara, CA, September): 61-72.

Jensen, B. D., M. P. de Boer, F. Bitsie, and D. A. La Van. 2000. "Precise Measurement of Young's Modulus in the Presence of Test Structure NonIdealities." J. MEMS, submitted.

Sniegowski, J. J., and M. P. de Boer. 2000. "ICCompatible Polysilicon Surface Micromachining." Ann. Rev. Mater. Sci. 30: 297-333. 
10348

\section{Real-Time Error Correction Using Electromagnetic-Bearing Spindles}

The National Center for Manufacturing Science (NCMS) has identified electromagnetic (EM)-bearing spindles as a critical component needed to revolutionize manufacturing in the next century. Ultrahigh speeds are enabled by the noncontacting nature of the EM bearing, making it a candidate technology for machining very hard materials (such as ceramics) with reduced cutting forces, lower heating rates, and minimal coolant consumption as compared to conventional machining processes. Furthermore, high-bandwidth error correction can be achieved through manipulation of the magnetic fields in the bearing gaps, thus minimizing the potential for dimensional errors while producing superior surface finishes. As such, development of this technology is critical to the Defense Programs (DP) manufacturing infrastructure, which increasingly relies on rapid production of small-lot components.

Available research in this emerging technology has focused on simplistic control strategies for maintaining stable spindle levitation without regard to workpiece quality. In this project, Sandia will develop nonlinear control strategies to improve the spindles' dynamic stiffness while providing realtime correction for rotational and translational spindle errors. A central feature of this research project is the ability to leverage previous development of Sandia's EM-bearing grinder test-bed, thus minimizing the need for additional investments in capital equipment. An integrated project of process modeling, controller development, and experimental evaluation is envisioned through teaming of Sandia's manufacturing, experimental mechanics, and structural dynamics groups with the nonlinear control expertise at Michigan Technological University and magnetic-bearing researchers at the University of California-Santa Barbara.

We evaluated three adaptive controllers with the MBC 500 MagLev System. We based these controllers on least means square (LMS) algorithms and the "amplitude-phase adaptive control algorithm" (APACA). The adaptive controllers offered significant advantages over the standard nonadaptive feedback controller (proportional integral differential [PID]). The adaptive controllers could account for

\section{The National Center for}

Manufacturing Science (NCMS) has identified electromagnetic (EM)-bearing spindles as a critical component needed to revolutionize manufacturing in the next century....development of this technology is critical to the Defense Programs (DP) manufacturing infrastructure, which increasingly relies on rapid production of small-lot components....In this project, Sandia will develop nonlinear control strategies to improve the spindles' dynamic stiffness while providing real-time correction for rotational and translational spindle errors. 
disturbances to the motion of the spindle, such as imbalance or externally applied forces, and correct the position of the spindle in real time. This could be a significant advantage in some applications where the positional accuracy of the spindle is critical.

While the various adaptive controllers worked well, all of them had a variety of parameters governing their function. We had to carefully select these parameters to avoid instability or generally poor performance. The best choices of parameters required some trial and error to come up with acceptable combinations.

One goal of the project was to achieve very high revolutions per minute (rpm) spindle speeds, and we obtained speeds approaching 100,000 rpm with a special, shortened shaft controlled by the LMS algorithm.

\section{Refereed}

Barney, P., J. Lauffer, J. Redmond, and W. Sullivan. 2000. "Active Control of a Magnetically Levitated Spindle." Paper presented to the International Modal Analysis Conference (IMAC), Nashville, TN, 15 March 2000.

Barney, P., J. Lauffer, J. Redmond, W. Sullivan, and R. Pettys. 1999. "Adaptive Spindle Balancing Using Magnetically Levitated Bearings." Proc. ASME Internat. Mechanical Eng. Congress and Exposition Winter Ann. Mtg. 1 (New Orleans, LA, November): $1-10$.

Pettys, R., G. Parker, and J. Redmond. 2000.

"Disturbance Rejection Control of an Electromagnetic Bearing Spindle." Proc. ASME Internat. Mechanical Eng. Congress and Exposition 1 (New Orleans, LA, November).
One goal of the project was to achieve very high revolutions per minute (rpm) spindle speeds, and we obtained speeds approaching 100,000 rpm with a special, shortened shaft controlled by the LMS algorithm. 
10349

\section{Thin-Film Deposition Processes Incorporating} In Situ Monitoring Capabilities

D. P. Adams, J. A. Floro, R. S. Goeke, J. A. Romero, N. R. Moody

This project researches thin-film deposition processes that combine flexible growth techniques and in situ, rapidfeedback diagnostics. This impacts long-term film reliability by providing methods to manipulate and monitor important film material properties during fabrication and will potentially lead to new, robust production processes and parts having reduced numbers of defects.

Applications of metal hydride films are found in nuclear weapons and in industry. In general, simultaneous deposition of required species should allow for lower growth temperatures compared with processes that react singlecomponent films to form a desired phase. The higher temperatures required by other multistep processes often lead to large extrinsic stress. Development of co-deposition techniques that control film stress and crystal phase is challenging because growth can involve highly nonequilibrium atomistic processes. Phase and structure can be influenced by a number of thermodynamic and kinetic parameters, including strain energy and surface diffusion.

Our work also aims to determine the origin of intrinsic stress. As part of this work, we recently incorporated a multibeam stress sensor onto the deposition system. This allows accurate, real-time measurements of curvature during different stages of fabrication, including deposition, cooldown, and air exposure. We expect our research to increase the level of understanding of thin-film stress evolution. We have not performed detailed, in situ studies of stress evolution on metal hydride films. We will quantify other mechanical properties of thin films for the first time, including interfacial toughness.

We deposited stoichiometric erbium (Er) hydride thin films and analyzed them by $x$-ray diffraction, transmission electron microscopy (TEM), x-ray pole figure, and numerous ion-beam analysis techniques. This includes a full investigation of grain-size evolution, in-plane and out-of-plane crystal texture, composition, and phase. This study includes films grown at different temperatures and onto different substrate surfaces. In particular, we find that Er hydride films grown onto well-ordered substrate surfaces such as a-axis $\mathrm{Al}_{2} \mathrm{O}_{3}$ can form epitaxially. Strain energy appears to play a large role in
Our work also aims to determine the origin of intrinsic stress....We expect our research to increase the level of understanding of thin-film stress evolution...We will quantify other mechanical properties of thin films for the first time, including interfacial toughness. 
phase formation for this metal hydride system. We did not find a strong in-plane texture for growth onto amorphous surfaces. We also investigated the effects of growth parameters on film stress. Ion-beam sputtering at low pressures most often results in compressive stress. We are depositing other metal hydride materials, which includes reactive deposition of scandium $(\mathrm{Sc})$ hydride involving metal evaporation.

\section{Refereed}

Adams, D. P., J. A. Romero, M. A. Rodriguez, J. A.

Floro, and J. C. Banks. 2000. "Composition and

Structure of Sputter-Deposited Erbium Hydride Thin

Films." Proc. Mater. Res. Soc. Symp. (San Francisco,

CA, April).
Ion-beam sputtering at low pressures most often results in compressive stress. 
10351

Solid-State Neutron Generator for Use in Nuclear Weapons

J. C. Lund, N. R. Hilton, A. M. Morales, D. F. Clark, G. J. Miller, Jr., A. M. Romero

Traditional neutron generators (NGs) used in nuclear weapons systems employ a portable ion accelerator with tritium (T) target. Such neutron "tubes" work well, but they are difficult to make in small sizes, require high-voltage power systems, and have a limited shelf life. Another approach to neutron generation involves the use of an alpha-emitting radioisotope mixed with beryllium (Be). The traditional disadvantage of such an alpha-Be source is that they are always "on"; that is, they emit neutrons at a steady rate even when stored. Thus, it is extremely awkward to use a conventional alpha-Be source in a weapon because of the neutron shielding required to prevent exposure to personnel and sensitive electronics. However, with the advent of modern micromachining techniques (in particular, the LIGA [the German term Lithographie, Galvanoformung, und Abformung, for lithography, electroforming, and molding] process), it should be possible to build a "switchable" all-solid-state neutron source. The alpha particles from a typical radioisotope (e.g., 241-Am [americium]) have a very short range in dense materials (less than 50 microns). If an array of small isotopic sources is moved over an array of LIGA-fabricated "wells," with Be at the bottom of the wells, a fully switchable solid-state neutron source would result.

Sandia fabricated switchable solid-state NGs and developed a quantitative model describing their performance. We concluded that it would be very difficult to build a solidstate generator for use in nuclear weapons for two reasons: (1) gamma-ray dose from the daughters of the alpha emitters used in the device would cause a radiation hazard in the vicinity of the weapon, and (2) energy absorption by the alpha particles would dissipate too much power to remove adequately.

However, the technology developed in this project may be very attractive for use in other applications, particularly arms control and nondestructive testing, where an extremely portable NG would be an asset.

We made extensive progress in four general areas: (1) theory of operation of a solid-state NG and computer simulation of its performance, (2) selection of alpha-emitting
Sandia fabricated switchable solid-state NGs and developed a quantitative model describing their performance. 
radioisotopes for use in the solid-state neutron generator (SSNG), (3) design and construction of micromachined attenuator layers, and (4) validation of the computer simulation through experimentation with various alpha emitters and $\mathrm{Be}$ absorbers.

We developed a full three-dimensional transport code to predict the performance of NGs. The code computes the slowing down of alpha particles in the emitter and Be absorber and then computes the energy distribution and angular distribution of neutrons emitted from the Be. We selected alpha particle emitter candidates by examining all of the alpha emitters in a particular half-life range, computing the maximum neutron emission possible with these isotopes, and finally computing the gamma-ray dose (from daughter decay) for these isotopes. The trade-off between neutron emission rate and gamma-ray dose could be evaluated (without revealing classified information) by looking at the parametric plot between neutron output and gamma-ray dose. We fabricated micromachined gold ( $\mathrm{Au}$ ) layers that could be attached to both an alpha emitter and a Be absorber. Finally, we performed a series of experiments to validate the computer simulations. We coupled two relatively intense alpha-emitting sources (241-Am and 225-Ac [actinium]) with Be absorbers and evaluated the angular distribution of neutrons from these sources.
We developed a full threedimensional transport code to predict the performance of NGs. The code computes the slowing down of alpha particles in the emitter and Be absorber and then computes the energy distribution and angular distribution of neutrons emitted from the Be. 
10352

\section{Scripting for Video Inspection}

J. T. Feddema, C. W. Wilson

Sandia's primary mission of ensuring a safe, secure, and reliable nuclear deterrent depends critically on the reliability of deployed components. Visual inspection of these components serves as the last chance to prevent a defective part from entering the stockpile. Most of these parts are subjected to manual visual inspection, which raises questions of consistency and accuracy. Even state-of-the-art commercial visual inspection systems are inadequate because they require skilled engineers to artfully adjust camera parameters, lens settings, lighting, and processing, which is prohibitively expensive for small-lot manufacturing. In this project, we fill this research gap in visual inspection by developing technology to automatically inspect parts with a video camera. Using a computer-aided design (CAD) model of the part, our system will automatically determine camera locations, lens settings, and illumination to make good images. We refer to this combination of imaging parameters as a script, and it will result in a movie of the part, highlighting the sections that need to be inspected. These images will be compared automatically to $C A D$ renderings of the part, with differences marked as defects. We will enhance the system with specialized inspection routines that cannot be accomplished with the CAD model. The result of this work will be a system for automatically inspecting parts such as neutron generators (NGs), printed wiring boards $(P W B s)$, semiconductor wafers, and machined parts.

Sandia's primary mission of ensuring a safe, secure, and reliable nuclear deterrent depends on the reliability of deployed components. Most of these components are subjected to manual visual inspection. Because of the difficulty of standardizing visual inspection, this methodology is subject to inconsistencies and inaccuracies. This gap in science-based stewardship causes, at best, wasted work in part inspection and, at worst, uncertainties in the reliability of the stockpile.

We addressed this need for greater consistency and reliability in visual inspection by integrating a video camera and CAD simulation into the inspection process. The resulting technology realizes an automated visual inspection that is systematic, consistent, and easily documented. The NGs produced at Sandia served as the test materials for use of this methodology.
The result of this work will be a system for automatically inspecting parts such as neutron generators (NGs), printed wiring boards (PWBs), semiconductor wafers, and machined parts. 
The first challenge is to obtain real images of sufficient quality and reproducibility so that manual visual inspection is optimized. These need to be based on an automatic scripting system that will make a movie of a part showing the regions that need to be inspected. It should automatically adjust the camera position, lens parameters, and lighting. The second challenge is to automate the inspection by using computer comparisons of ideal parameters to images of actual parts, with a reproducible range of tolerances. Visual inspection exists as a subcategory of computer vision research. While it draws on various computer vision techniques (e.g., calibration, subpixel feature location), inspection comes with its own unique constraints and problems. An automated visual inspection faces different challenges in that the system must find defects it has never seen before (e.g., cracks, random solder splashes). We can ultimately use our research to automate the acquisition of good images and to constrain the inspection process by predicting all possible appearances of a good part, including acceptable tolerances.

We are concentrating on the inspection routines for the NG header and frame and have developed a suitable inspection station. Historically, the inspection path used has been generated manually; that is, the visual inspection system was not CAD-based, but taught images from real parts. This project developed an automatic scripting system that makes a movie of a part showing the regions that need to be inspected. It automatically adjusts the camera position, lens parameters, and lighting.

The inspection methodology is based on a CAD-based video scripting system that can make a movie or generate still pictures of an NG part at various stages of inspection. Within 3-D Studio Max, we are able to bring in ProEngineer CAD drawings of NG parts such as the header. The operator can position the simulated camera and lighting relative to the parts and view a photorealistic image of the part as seen by the camera. We can then download the resulting path to an actual positioning device that places the part underneath either a camera or a microscope. Currently, the operator selects the regions to be inspected, and the scripting system generates a movie or image file of the synthesized views. We use texture and lighting models to generate photorealistic images and have created initial path plans and lens control models.

We tested the imaging system in the inspection of the NG header. We combined these image inspection results with other information of the brazing process (e.g., the furnace
This project developed an automatic scripting system that makes a movie of a part showing the regions that need to be inspected. It automatically adjusts the camera position, lens parameters, and lighting. 
temperature profiles) to aid the engineers who are responsible for the design and manufacturing of the subassembly. We will archive the stored images for future comparison when the parts are tested.

The project did not detect anomalies by comparing CAD images with actual ones. Better imaging tools need to be developed for this. Differences in texture and lighting could not be distinguished from anomalies. We concluded that the best approach would be to compare current images with actual images of good parts and use the CAD interface to define the inspection process itself.
We concluded that the best approach would be to compare current images with actual images of good parts and use the $C A D$ interface to define the inspection process itself. 
10353

\section{Advanced Machining Processes for Microfabrication}

G. L. Benavides, D. P. Adams, P. Yang

An important product realization goal for Sandia is to reduce the volume of weapon components by 50\%. Sandia is a national leader in microfabrication technologies such as silicon (Si)-based microelectromechanical systems (MEMS) and electroformable metal-based LIGA (the German term Lithographie, Galvanoformung, und Abformung, for lithography, electroplating, and molding). However, there are micromachining technology voids that need to be filled to meet the volume reduction goal. Sandia must attain capabilities unavailable through LIGA, i.e., to fabricate subminiature parts from nonelectroformable metals or ceramics and to micromachine nonplanar features. We augmented our microfabrication technologies by researching novel applications of microelectrodischarge machining (microEDM), excimer laser, femtosecond laser, and focused ion beam (FIB). The process of micromachining by material removal is new to both Sandia and industry.

We used the Agie micro-sinker EDM to machine mesoscale features in a variety of conductive materials. We experimented with graphite electrodes that were ultrasonically ground and copper $(\mathrm{Cu})$ electrodes that were fabricated by the LIGA process. The micro-sinker EDM machined complex features into difficult-to-machine materials such as neodymium iron boron (NdFeB) and soft magnetic alloy (e.g., Hiperco).

We used the FIB to fabricate microsize features in materials such as tool steel and tungsten (W) carbide. The FIB fabricated steel microtools for use in conventional processes such as drilling, milling, and turning. The FIB width of 100 nanometers can create submicron features in high-strength/ high-temperature metals and ceramics. We partnered with Louisiana Tech University on FIB/micromachining research.

We used the excimer laser to fabricate microsize features in materials including ceramics, magnetic materials, and polymers. The ultraviolet (UV) laser ablation process removes material with minimal sample melting, providing the precision essential to fabricate high-aspect-ratio microstructures. We ablated channels into sheet materials by controlling the depth of material removal. We partnered with
An important product realization goal for Sandia is to reduce the volume of weapon components by $50 \%$. Sandia is a national leader in microfabrication technologies such as silicon ( $\mathrm{Si}$ )-based microelectromechanical systems (MEMS) and electroformable metal-based LIGA...Sandia must attain capabilities unavailable through LIGA, i.e., to fabricate subminiature parts from nonelectroformable metals or ceramics and to micromachine nonplanar features....The process of micromachining by material removal is new to both Sandia and industry. 
the pulsed-power center and the University of New Mexico to explore the micromachining capabilities of $\mathrm{Ti}$ (titanium)sapphire femtosecond lasers.

\section{Refereed}

Adams, D. P., M. J. Vasile, and A. S. M. Krishnan.

2000. "Microgrooving and Microthreading Tools for

Fabricating Curvilinear Features." J. Precision Eng., Elsevier Science Inc. (October).

Adams, D. P., M. J. Vasile, G. L. Benavides, and A. N. Campbell. 2000. "Micromilling of Metal Alloys with Focused Ion Beam Fabricated Tools." J. Precision Eng., Elsevier Science Inc.

\section{Other Communications}

Adams, D. P., G. L. Benavides, and M. J. Vasile. 1998. "Micrometer-Scale Machining of Metals and Polymers Enabled by Focused Ion Beam Sputtering." Mater. Sci. of MEMS Devices, Proc. 1998 MRS Fall Mtg. 546

(Boston, MA, 29 November-4 December): 201-205.

Benavides, G. L., D. P. Adams, and P. Yang. 2000.

"Mesoscale Machining Capabilities and Issues."

Nanotribology: Critical Assessments and Res. Needs

(October).

Yang, P., E. L. Venturini, and G. L. Benavides. 2000.

"Effects of Excimer Laser Irradiation on Micromachining of Glasses." Symp. on Laser-Solid

Interactions for Mater. Processing, Proc. 2000 Mater. Res. Soc. Spring Mtg. (San Francisco, CA, 24-28 April). 
10354

\section{Advanced Production Planning Models}

D. A. Jones, C. A. Phillips, W. E. Hart, G. F. List, M. A. Turnquist,

C. R. Lawton

The overall goal of this project is to provide an effective "suite" of solvers for advanced production planning at facilities in the nuclear weapons complex (NWC). Sandia focused effort in two areas - the development of an innovative modeling approach for solving resource-constrained, multiproject planning/scheduling problems, and producing technical papers, a SAND report, and a peer-reviewed article. It is also noteworthy that the formulation, developed and implemented at Pantex, was a finalist in the Edelman Award Competition, which included a recent press release. This type of formulation has direct application to the planning of stockpile evaluation activities at Pantex, as well as future stockpile life-extension program (SLEP) activities. Although we focused our development activities to date on data related to Pantex operations, these types of project scheduling problems appear in many contexts other than Pantex-both within the NWC (e.g., neutron generators [NGs]) and in other commercial manufacturing settings. Traditionally, due to their mathematical complexity, finding practical solutions to these large-scale task-scheduling problems is very difficult. We made significant progress toward providing an effective strategy for solving these types of problems and created a fundamentally different way of interpreting and formulating problems of this type. First, its time periods can have variable lengths; thus, calendar boundaries can be matched precisely. Second, the principal choice variables are the starting times for each task, rather than using a period index for a start time, as is done in integer programming approaches, thus allowing more efficient solution strategies. It appears that this approach to task-based production scheduling will be a major breakthrough for largescale, realistic scheduling problems in a variety of production settings. We believe that this approach will be the basis for a new generation of advanced production planning models.

We focused our effort in two areas: the development of an innovative modeling approach for solving resourceconstrained, multiproject planning/scheduling problems, including tests on Pantex and NG production data, and documentation of the formulation and a variety of solution
It appears that this approach to task-based production scheduling will be a major breakthrough for large-scale, realistic scheduling problems in a variety of production settings.

We believe that this approach will be the basis for a new generation of advanced production planning models. 
techniques.

Production planning in make-to-order operations

frequently must focus on the problems of assigning resources

for small production lots (often of size one), each of which has

specific requirements for a sequence of processing steps on

different machines, or by people from different crafts, etc. In

these environments, the production planning problem bears

considerable resemblance to resource-constrained project

scheduling, such as is practiced in construction operations or

other task-oriented situations.

We developed a method to plan production activities in a "project job shop" production environment. This new formulation has continuous variables that represent the start times for the individual tasks, rather than using discrete variables that represent whether or not a task begins (or terminates) in a given period. The continuous-variable formulation allows for time periods of variable lengths and for a natural mapping of the model's time periods to those that are meaningful in a specific application. This new formulation was motivated by a need to schedule disassembly, evaluation, and maintenance activities of nuclear weapons, but the general formulation and solution procedure can apply more broadly to resource-constrained project-scheduling problems.

The new formulation is a mathematical programming model with a linear objective function and mostly linear constraints, but it does contain complementarity constraints that make the feasible region nonconvex. We developed a heuristic method based on Generalized Benders' Decomposition to provide approximate solutions to this optimization problem. In the decomposition scheme, the subproblem is a linear program (LP) that optimizes the assignment of resources to tasks given the start times, while the master problem optimizes over the start times. The overall method is a heuristic because the master problem is not solved exactly.

The ideas developed in this work are transferable to other situations in both production planning and project scheduling. In situations where resource substitutions are excluded, for example, the subproblem formulation changes slightly, but it is still a LP. Thus, the details of the solution algorithm may vary, but the general procedure is the same.

An additional interesting extension is to include other measures of performance in the objective function. These additional measures might include makespan or terms that reflect "risk" in the schedule. In many instances of the

We developed a method to plan production activities in a “project job shop" production environment. This new formulation has continuous variables that represent the start times for the individual tasks, rather than using discrete variables that represent whether or not a task begins (or terminates) in a given period. 
resource-constrained project-scheduling problem, the task durations are uncertain, and the notion of identifying a "lowrisk" schedule is important. Given the structure of the solution procedure, it is likely that a measure of schedule risk can be incorporated.

\section{Refereed}

Hart, W. E., C. Phillips, and C. Lawton. 2000. “An

Evaluation of Optimization Formulations for

Production Planning at Pantex." Sandia Technical

Report SAND2000-1436, Sandia National

Laboratories, Albuquerque, NM (June).

Kjeldgaard, E. A., D. A. Jones, G. F. List, M. A.

Turnquist, J. W. Angelo, R. D. Hopson, J. Hudson, and

T. Holeman. 2000. "Swords into Plowshares: Nuclear

Weapons Dismantlement, Evaluation, and

Maintenance at Pantex." Interfaces 30 (January-

February): 57-82.

Nozick, L. K., C. R. Lawton, D. A. Jones, E. A. Kjeldgaard, M. A. Turnquist, G. F. List, and S. T. Wright. 2000. "Production Planning for a Project Job Shop, with Application to Disassembly, Evaluation, and Maintenance of Nuclear Weapons." Production Planning and Control, accepted.

\section{Other Communications}

Jones, D. A., C. R. Lawton, G. F. List, and M. A. Turnquist. 1999. "The Pantex Process Model: Formulations of the Evaluation Planning Module." Sandia Technical Report SAND99-2095, Sandia

National Laboratories, Albuquerque, NM (December). 
10721

\section{Volumetric Displacement Control (VDC) of Manufacturing Tools}

L. F. Bieg, M. T. Ensz, B. Jokiel, Jr.

Sandia's desire to eliminate or minimize independent product inspection must result in better process control. Statistical process control (SPC) has been exercised in the past by influencing the process based on trends. This method may be applied to high-volume manufacturing at low manufacturing tolerances. Enhancements in material/fabrication processes are driven by reducing cost and cycle time and by minimizing fabrication defects at increased accuracy and speed. Inprocess inspection has forced the requirement of periodically certifying the manufacturing process. Historically, methods as defined under American National Standard ASME B5.54 are applied to determine the performance of computer numerically controlled systems. A multitude of expensive and sensitive equipment has to be applied by trained experts to evaluate performance.

Today's feedback during the manufacturing process is limited to displacement (motion) sensing of the equipment, at great distances away from the actual point of operation. In most cases, rotating motion, produced by a motor, is translated into linear motion through the pitch or lead of a screw. External and self-induced thermal influences as well as geometric errors while in motion have never been detected and applied to control the process. Industry and university publications assign an $80 \%$ contribution of errors based on thermal and geometric issues. Our approach will measure and correct in real time process-induced errors on manufacturing tools of motion. The volumetric displacement control (VDC) system will provide total process control (TPC) to the fabrication floor.

We designed, developed, and modeled the VDC reference frame by applying state-of-the-art solid design and modeling software, allowing for all components to be assembled into subassemblies. Respectively, these subassemblies have been set into motion to check for accuracy, performance, interference, and functionality. Once we created a functional design in software, we calculated an error budget for the entire work volume. We conducted an independent conceptual design review and presented the design to Sandia internal and external experts in the field of manufacturing
Our approach will measure and correct in real time processinduced errors on manufacturing tools of motion. The volumetric displacement control (VDC) system will provide total process control (TPC) to the fabrication floor. 
control. We took the findings of this design review into consideration to revise the design prior to fabrication of hardware components. Therefore, our second task was to finalize the design and fabricate bearing joints, feedback mechanisms, and arm supports. We manufactured all subcomponents and assembled the system into a functional prototype. We tested the required electronics and performed data acquisition on all three joints of rotation, allowing for the VDC system to produce a 3-D coordinate system. We have generated software for data acquisition and testing of the prototype. The software has acquisitioned a full set of feedback analysis while in motion, and we archived the results for future use. Future data-acquisition needs may be subsidized by using off-the-shelf-type software, like LabView and Spatial Analyzer.

We are applying our VDC system to the earmarked machining test-bed. We retrofitted this three-axes milling machine with a PC-based control to assist in the integration of our VDC. By combining our system with the test-bed, we will demonstrate performance during motion and identify the application mode 1 of the VDC.

\section{Refereed}

Jokiel, Jr., B., L. F. X. Bieg, and M. Ensz. 2000.

"Development of an Independent Real-Time Position Feedback Device for CNC Machining Operations." Proc. Amer. Soc. of Precision Eng. Winter Mtg. (ASPE) 1 (Scottsdale, AZ, October): 4.
We manufactured all subcomponents and assembled the system into a functional prototype. We tested the required electronics and performed data acquisition on all three joints of rotation, allowing for the VDC system to produce a 3-D coordinate system. 
10722

\section{Process-Based Quality Tools to Verify Cleaning and Surface Preparation}

J. A. Emerson, D. P. Adams, P. A. Lemke, N. R. Moody, E. D. Reedy, $J r$.

Specifying material choices and the method for processing does not ensure the long-term durability of an adhesive bond. Manufacturing quality and control are crucial, and one must be able to fully characterize and certify the entire fabrication process. A recently completed cleaning study provides the knowledge needed to develop and move to the factory floor a tool to identify surface-preparation requirements and guarantee their effectiveness. The tool proposed in this project utilizes a method for measuring adhesion of dots in conjunction with combinatoric testing. Sandia is developing a small plate that contains many maskedoff regions. This plate runs through the processing line, and a new region is exposed during each step of the fabrication process. The plate contains the combined effects of all the processing steps, as well as any contamination added during storage and transit. This combinatoric process characterization plate $(P C P)$ is interrogated to determine if the desired adhesion level exists when the fabrication process is complete. We will use a method based on nanoindentation and contact mechanics testing to interrogate the PCP. We cast a thin layer of the adhesive over the plate, followed by a stressed overlayer. The radius of the blister generated during indentation can be directly related to interfacial fracture toughness. The ultimate aim of this work is a tool that could be used on the factory floor that would be viable for small-lot and rarely used processes. We are taking full advantage of current collaborative work with Honeywell/Federal Manufacturing and Technology (FM\&T) process engineers to further our effort.

We are developing small-area adhesion tests that are accurate, convenient, and scalable from conventional adhesion tests. To measure the adhesion of small areas, we are studying two techniques. We placed stressed layers on a polymer film to induce delamination. We completed studies for developing known and adjustable stress overlayers with $\mathrm{Ta}_{2} \mathrm{~N}$-superlayerstressed thin films under various vacuum deposition pressures. However, these stressed superlayers trigger delamination in weakly bonded films but not in strong adhering films. We
The tool proposed in this project utilizes a method for measuring adhesion of dots in conjunction with combinatoric testing. Sandia is developing a small plate that contains many masked-off regions....The ultimate aim of this work is a tool that could be used on the factory floor that would be viable for small-lot and rarely used processes. 
showed with the nanoindentation of thin epoxy films on aluminized glass that delamination occurs readily along the film-metal interface. To measure the interfacial toughness for 0.2 to $1 \mu \mathrm{m}$-thick epoxy films, we found that a critical indenter displacement is required to create stresses that drive interfacial crack growth. We are continuing work to measure the surface adhesion using contact mechanics and nanoindentation techniques for one polymer film system.

\section{Refereed}

Emerson, J. A., G. V. Miller, C. R. Sorensen, and R.

A. Pearson. 2000. "Assessment of Surface

To measure the interfacial Contamination with Contact Mechanics." Proc. 23rd Ann. Mtg. of the Adhesion Soc. 23 (Myrtle Beach, SC, February): 550-553.

Emerson, J. A., R. K. Giunta, G. V. Miller, C. R. Sorensen, and R. A. Pearson. 2000. "The Effect of Surface Contamination on Adhesive Forces as Measured by Contact Mechanics." Proc. Mater. Res. Soc. Symp. 629 (San Francisco, CA, April).

\section{Other Communications}

Woerdeman, D. L., and J. A. Emerson. 2000. "The Use of JKR Contact Mechanics for Probing Epoxy/ Aluminum Adhesion in the Presence of Hexadecane Contaminate." Paper presented to the Gordon Research Conference-Science of Adhesion, Tilton, NH, August. 
10723

\section{Fabrication of Three-Dimensional Microstructures Using Soft Lithography}

R. L. Myers, J. A. Emerson, D. P. Adams, A. D. Oliver

Microstructures are becoming increasingly important in technological advances in many fields, including the upgrade and maintenance of the stockpile of nuclear weapons. Microstructures are fabricated primarily by photolithography, a process that produces two-dimensional (2-D) parts; it has intrinsic limitations in making 3-D structures. The LIGA (the German term Lithographie, Galvanoformung, und Abformung, for lithography, electroplating, and molding) process uses $x$-ray lithography, making it costly and not readily accessible. The ability to fabricate 3-D microstructures by soft lithography using a wide variety of materials makes it possible to achieve unprecedented levels of system functionality for microelectromechanical systems (MEMS), microsensors, and microoptical devices.

Sandia seeks the ability to fabricate 3-D microstructures using soft lithography. This process could be used to create microelectromechanical devices such as those currently being developed for micro-arming, fuzing, and firing $(A F \& F)$ systems. A need exists for microelectromechanical devices in trajectory-safing subsystems, which provide enhanced safety in nuclear weapons. Two components in the trajectory-safing subsystem that could benefit from new microfabrication techniques are the environmental sensing device and the stronglink.

Soft lithography is a microcontact-printing method where a self-assembled monolayer (SAM) is patterned by transfer printing. A macroscopic analogy is linoleum-block printing, meaning low-cost and high-precision replication. The thin patterned layer can be an etch resist or a foundation on which to build new types of microstructures. Since an elastomeric stamp is flexible, it can be used to transfer a pattern to 2-D or 3-D substrates. For example, we have patterned metallized glass capillary tubes and then selectively etched them to form microcoils.

Current university research demonstrates that microcontact printing can achieve nanometer-level resolution on planar substrates; it can also pattern large areas. While this could be our lower limit, we will not focus on nanometer-scale patterning. We plan to fabricate 3-D microstructures with feature size in the tens of microns.
The ability to fabricate 3-D microstructures by soft lithography using a wide variety of materials makes it possible to achieve unprecedented levels of system functionality for microelectromechanical systems (MEMS), microsensors, and microoptical devices.

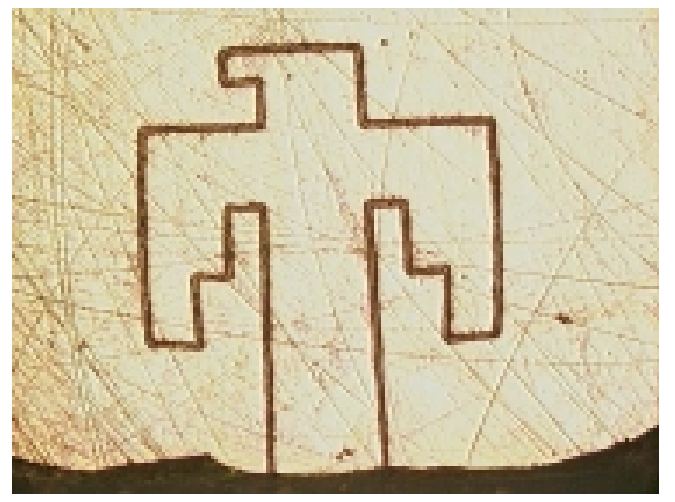

A photograph from an optical microscope of a brass master made with a 25-microndiameter micro-end-mill tool, making each line in the thunderbird pattern approximately 25 microns wide. 
We conducted a screening study to identify candidate materials for the elastomeric stamp to be used in microcontact printing. We looked at both silicone and polyurethane formulations in this study. All of the samples succeeded in transferring a silane to a glass surface. We decided to proceed using the Sylgard 184 silicone since this is the stamp material most often cited in the literature for microcontact printing; if we run into an application where the Sylgard 184 does not perform well, we will try one of the other materials from this study.

We made a Sylgard 184 stamp using a brass master that was made with a micro-end-mill tool. The Sylgard 184 stamp replicated the features of the brass master very well, including numerous small scratches on the surface. It is clear from this work that the quality of the master is critical to the integrity of the final parts. We successfully transferred the pattern from the stamp to a 2-D gold ( $\mathrm{Au}$ ) substrate by "inking" the stamp with hexadecanethiol and bringing it into contact with the $\mathrm{Au}$ surface.

We used the microcontact-printing process to transfer a variety of patterns to flat substrates of $\mathrm{Au}$, silver (Ag), and copper $(\mathrm{Cu})$. We examined the uniformity of the printed SAMs with an atomic-force microscope (AFM) and a scanningelectron microscope (SEM). The presence of contaminants and stamp defects can cause debris and/or holes in a monolayer. In addition, uneven contact of the elastomeric stamp with the substrate can lead to incomplete patterns. We are currently optimizing our microcontact-printing process based on our observations of the uniformity of the monolayers. In addition, we have initiated the fabrication of the master for the microchain (our first 3-D part).
We conducted a screening study to identify candidate materials for the elastomeric stamp to be used in microcontact printing. We looked at both silicone and polyurethane formulations in this study. All of the samples succeeded in transferring a silane to a glass surface. 
10751

\section{Automatic Design of Practical Fixtures}

J. C. Trinkle, R. J. Gross, M. C. Sloane, E. Gottlieb, P. G. Xavier

The cost of designing and manufacturing fixtures has driven significant research in the automatic design of fixtures at Sandia, in academia, and in industry. Nonetheless, automated fixture design systems are not widely used. The two primary problems are (1) lack of generality (i.e., arbitrary three-dimensional (3-D) parts and fixturing element geometries cannot be handled), and (2) inability to accurately predict important performance criteria (i.e., tolerance stack-up, loading difficulty, and thermal expansion mismatches). These problems lead to failures. For example, in the Neutron Generator Facility, fixture failures cause significant costs and delays.

Fixture design systems have fallen short because there have been no underlying theory or analysis tools to efficiently predict loading difficulty and problems associated with dimensional errors and changes in parts and fixtures. We will develop new fixture design techniques based on recent results developed by Sandia and a new idea for mitigating the effects of dimensional errors. The new results allow more general analysis of stability, realistic simulation of fixture loading, including sliding and rolling, and sticking contacts. Our work will yield a principled way to include unstructured and systematic dimensional variations in the design process.

This approach can be broadly classified as simulationbased design and, as such, will require the simulation of multiple scenarios, each corresponding to possible unknown loading conditions and manufacturing errors. To maximize the effectiveness of this approach, we will apply variants of bundle methods to minimize the number of scenarios to be analyzed, while still guaranteeing the achievement of fixture design specifications and on experiments to validate our simulations. Also, since the approach is simulation-based, any phenomenon that can be simulated can be included without changing our approach.

We formulated the thermal problem as a multi-objective optimization problem, the loading problem as a tractable parameter estimation problem, and the problem of tolerance stack-up as a minimax problem.

We embedded the analytical results on the thermal design problem in a prototype, interactive fixture design
The cost of designing and manufacturing fixtures has driven significant research in the automatic design of fixtures at Sandia, in academia, and in industry.... We will develop new fixture design techniques based on recent results developed by Sandia and a new idea for mitigating the effects of dimensional errors. The new results allow more general analysis of stability, realistic simulation of fixture loading, including sliding and rolling, and sticking contacts. 
algorithm. The results also provided a way to determine sets of high-temperature operations that can be performed simultaneously and within tolerance. Our success with the 2-D thermal design problem allowed us to begin building the infrastructure needed for the extension to 3-D problems.

We designed objective function for loading problem test on deterministic 2-D examples. This task required an algorithm, which we have tested, to simulate rigid-body systems with intermittent contact. We designed and tested a minimax algorithm for uncertainty on 2-D thermal/loading problems.

We are preparing a technical advance on the design method for thermally compliant fixtures.

\section{Refereed}

Pang, J. S., and J. C. Trinkle. 2000. "Stability Characterizations of Fixtured Rigid Bodies with Coulomb Friction." Proc. IEEE Internat. Conf. on Robotics and Automation 1 (San Francisco, CA, April): 361-368.

Pang, J. S., and J. C. Trinkle. 2000. "Stability Characterizations of Rigid-Body Contact Problems with Coulomb Friction." Zeitschrift für Angewandte Mathematik und Mechanik 80 (October): 643-663.

Stewart, D. E., and J. C. Trinkle. 2000. "An Implicit Time-Stepping Scheme for Rigid-Body Dynamics with Coulomb Friction." Proc. IEEE Internat. Conf. on Robotics and Automation 1 (San Francisco, CA, April): 162-169.

\section{Other Communications}

Pang, J. S., and J. C. Trinkle. 2000. "Stability Characterizations of Fixtured Rigid Bodies with Coulomb Friction." Paper presented to the 1st SIAM Conference on Computational Science and Engineering, Washington, DC, September.
Our success with the 2-D thermal design problem allowed us to begin building the infrastructure needed for the extension to 3-D problems. 
10770

\section{IMEMS Packaging and Interconnection}

\section{Technology}

K. A. Peterson, S. E. Garrett, C. A. Reber

Microelectromechanical systems (MEMS) and

integrated MEMS (IMEMS) offer the opportunity to miniaturize key components in nuclear weapons at low cost. Many advantages from this miniaturization are subsequently sacrificed, however, by packaging MEMS structures with the present understanding of conventional microelectronic packaging processes. The resulting low yields of MEMS packaging are a "show-stopper" for the eventual success of MEMS.

This project undertakes multiple micropackaging issues to efficiently and reliably take advanced MEMS structures from functionality in a laboratory setting to fielded applications. Sandia has employed concurrent engineering to develop initial bondpad definition and metallization with gold (Au) or aluminum (Al) that will be effective for conventional packaging as well as plastic encapsulation and wafer-scale packaging concepts. We are studying and improving packaging materials and processes so they do not "kill" released, functional MEMS. Package sealing has been the most challenging of these, and we are improving seam-sealing and furnace-sealing approaches to address this. We are investigating a new processing sequence utilizing high-capacity commercial polymer encapsulation to afford complete protection to conventional wirebonds and die-attach prior to the release of the MEMS devices. This approach protects MEMS devices through some potentially damaging micropackaging such as backside preparation, die-separation, handling, die-attach, interconnection, and possibly transfer molding. This project will complete these evaluations with the goal of ensuring advanced manufacturing at yield. We addressed the fundamentals to enable our work on the "package now, release later" methods. The success of this project will be a major step in the advanced manufacturing of reliable MEMS structures and will make them more available to Sandia weapon applications.

We have a vision wherein problems in conventional packaging of MEMS are solved and innovative packaging techniques are made feasible. Our primary new approaches involve a "package now, release later" processing order. 
Several of these approaches are interrelated. In the face of a late start, we established this work and advanced the infrastructure needed to accomplish these goals. We established a good understanding of the MEMS packaging open literature. We procured a MEMS driver system and implemented it with both a new device-probing capability and packaged parts. We procured materials and devices for evaluations, including modules that have microengines and diagnostic features. We recently obtained devices that will help us solve etchant spreading issues during release. We have used these samples for both die-scale and waferlike processing. Our die-scale work primarily supports conventional packaging work, and our waferlike work supports post-processing and extensions to wafer-scale packaging.

We developed an understanding of the release rates and control required for bondpad definition and release of MEMS structures. We successfully performed a photoresist lift-off technique for bondpad metallization with sputtered titanium (Ti)/gold (Au) and sputtered Al. We demonstrated bondability and adhesion to metallized and nonmetallized bondpads. We successfully released devices and tracked their functionality through conventional packaging steps, including known-gooddie verification, die-attach, wirebonding, and sealing. We packaged unreleased, post-processed MEMS devices in ceramic packages. We are implementing a prototyping technique for MEMS packaging in support of tasks for transfermolding plastic packages. This consists of a package that looks like the ready-to-release packaged device described in the original plan. We implemented fixtures for post-packaging release and performed preliminary evaluations of packaging materials compatibility with release processing. We identified additional challenges in the release of packaged parts, and we have two new ideas for ensuring metallization integrity through the release step. We demonstrated a new capability in our lab to perform field-assisted bonding of glass to silicon, which will enable longer-term goals in MEMS packaging. We are ready to prove the "package now, release later" concepts.
We demonstrated a new capability in our lab to perform field-assisted bonding of glass to silicon, which will enable longer-term goals in MEMS packaging. We are ready to prove the "package now, release later" concepts. 
10755

\section{Microreplication: Precision Metal Parts from Electroformed Master Molds}

R. P. Janek

Batch fabrication of weapons-related microsystems has been successfully demonstrated using deep x-ray lithography and subsequent electroforming through plastic molds (LIGA [the German term Lithographie, Galvanoformung, und Abformung, for lithography, electroplating, and molding]). While this process is well-characterized and well-understood, low cost and quick turnaround of precision microscale parts have not yet been realized, due primarily to the high cost of $x$-ray exposure and the inherent single-use nature of the LIGAgenerated plastic mold. Replication of metal microcomponents from an electroformed, multiple-use metal master mold would represent a significant technological breakthrough and would enable a production process that delivers metal parts with unparalleled precision, reproducibility, and reliability at a much lower cost than currently possible.

Replication is accomplished through a sequence of processing steps. The metal master mold is first fabricated using standard LIGA techniques. A conformal dielectric film (e.g., polystyrene) is then electrochemically deposited over the surface of the master, followed by selective etching of the dielectric at the base of each molded feature. A conductive layer of amorphous carbon is sputtered over the master, creating electroactive sites at the base of each molded feature. Metal parts are then electrodeposited through the mold onto the conductive seed layer. The insulating sidewalls of the master ensure high-fidelity replication of the part as the metal fills the molded features. Metal parts are released from the mold by simultaneous thermal decomposition of the sacrificial dielectric and carbon seed layers. The master mold is then cleaned and reused.

This project utilizes existing Sandia expertise in LIGA, electrodeposition, and electro-initiated polymerization that can be applied directly to fabrication of integrated microsystems for Defense Program (DP) applications. In addition to DP needs, low-cost and time-saving replication of metal microparts would impact microelectronics, telecommunication, and precision automotive and aerospace components technologies.
This project utilizes existing Sandia expertise in LIGA, electrodeposition, and electroinitiated polymerization that can be applied directly to fabrication of integrated microsystems for Defense Program (DP) applications. In addition to DP needs, low-cost and time-saving replication of metal microparts would impact microelectronics, telecommunication, and precision automotive and aerospace components technologies. 
We developed a small-scale test cell to determine test plating conditions and current densities. The three-electrode electrochemical cell uses $0.1 \mathrm{M}$ tetrabutyl ammonium perchlorate/propylene carbonate solution as the electrolyte and solvent. We position the working electrode (Pt [platinum] or $\mathrm{Ni}$ [nickel] plate) in the cell, and the counter electrode (Pt plate of equal area) and the reference electrode ( $\mathrm{Ag}$ [silver]/Ag+) in the cell, such that none of the electrodes are touching. The cell is driven by a potentiostat, which controls the voltage and the voltage waveform. We selected the driving potential for these experiments (+1.8 versus $\mathrm{Ag} / \mathrm{Ag}+)$ because of previous work in the literature. We also built a larger cell of similar design to accommodate 4"-diameter wafers.

Vinyl monomers can be initiated by electrochemical methods. As the molecular weight of the polystyrene increases, it precipitates from solution evenly over the electrode. Since polystyrene is an insulator, it passivates the electrode. The initial tests in the small-scale cell used rolled Ni plate and Niplated glass slides. The rolled Ni plate appeared to be passivating after five minutes in the plating bath, as determined by the decreasing activity of the cell. When we repeated the experiment on the Ni-coated glass, the Ni was etched off the surface. We repeated the experiments using a thin layer (\#000A) of Pt on the Ni-plated glass, and the surface was soon passivated; thus, a short Pt sputter step protects the Ni master mold. The styrene radical cation appears to be reacting with the Ni surface. We are currently evaluating other solvent/ electrolyte systems and metal surfaces.

Glass carbon thin films were dc and ac sputterdeposited on glass substrates from a pressed graphite target in an Ar (argon) plasma. We then etched these films in an oxygen (O) plasma to test the removal rate.
We developed a small-scale test cell to determine test plating conditions and current densities. 
10773

\section{Assembly of LIGA Using Electric Fields}

J. T. Feddema, T. M. Weber, L. K. Warne, W. A. Johnson, D. L. Armour

The goal of this project is to develop a device that uses electric fields to levitate and grasp LIGA (the German term Lithographie, Galvanoformung, und Abformung, for lithography, electroplating, and molding) parts. This noncontact form of grasping would solve many of the problems associated with grasping parts that are only a few microns in dimensions. Scaling laws show that for parts this size, electrostatic and electromagnetic (EM) forces are dominant over gravitational forces. This is why microparts often stick to mechanical tweezers. If these forces can be controlled under feedback control, the parts could be levitated, even rotated, in air. In this project, Sandia will use capacitive and optical sensing techniques to track the part and feedback control to regulate the applied field of an array of electrodes. This tool would be used to assemble metal and nonmetal LIGA parts being designed for weapons surety devices.

We demonstrated that we can pick up and release a 4 mm-diameter copper plate using electric fields. We mounted a $4 \mathrm{~mm}$-diameter, four-electrode stator from the microgyro project in our microassembly workcell in an inverted position, and we tested picking up the part while varying the applied voltage and gap between the part and stator. We also tested the stiction effects of different rotor materials and coatings. Integrated on the stator is a capacitance sensor that measures the gap between the part and the stator. We also performed a theoretical analysis that predicts when the parts will be picked up and released. So far, the experimental and theoretical results compare favorably. In addition, we designed two new stators with EM coils that we will use to create a repulsive force between the part and the stator. We will use this repulsive force to overcome stiction effects (such as an attractive surfacetension force caused by humidity and van der Waals forces that result from smooth surfaces) while releasing the part. We will also combine this repulsive EM force with the attractive electrostatic force to levitate the part. This will be the first time that Sandia has combined electrostatics and EMs to levitate parts. We used a Sandia-developed boundary-element electrodynamics code to simulate the performance of these new stator designs. We are now fabricating these stators for testing.
This will be the first time that Sandia has combined electrostatics and EMs to levitate parts. We used a Sandiadeveloped boundary-element electrodynamics code to simulate the performance of these new stator designs.

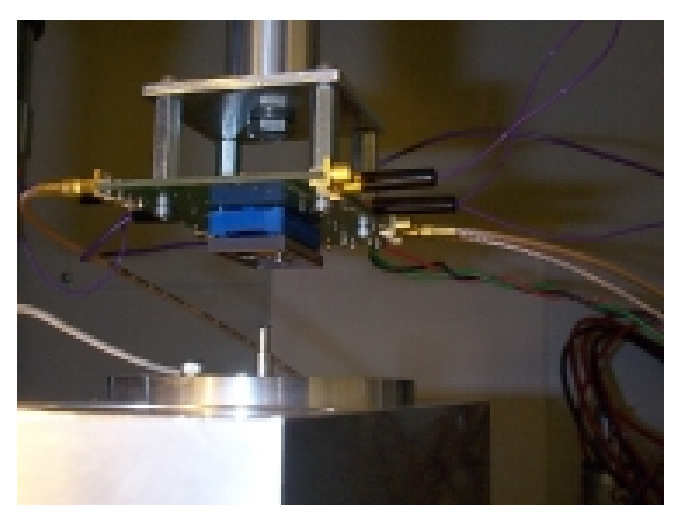

Picking up a $4 \mathrm{~mm}$ copper rotor using applied electrostatic force. 
10793

\section{Computer Numerically Controlled} Micromachines

G. L. Benavides, J. J. Allen, L. F. Bieg, D. P. Adams, B. Jokiel, Jr.

Historically, microelectromechanical systems (MEMS)type micromachines are fabricated to function in twodimensional (2-D) workspace. Sandia will design, manufacture, package, and apply computer numerically controlled (CNC) silicon ( $\mathrm{Si}$ ) micromachines. These multipledegree-of-freedom (DOF) machines (including 3-D) would serve as the basic building block for a variety of applications. We contemplate the use of this building block in micromilling, microcoordinate measuring, microalignment, laser pointing, rastering, and a device that performs surgery on living cells. A micromilling machine, capable of machining nanosized features, can be built by employing a high-speed motor that holds a cutting-tool all on one chip. Some of the innovative technologies that we need to enable CNC micromachines include the following:

(1) A parallel mechanism is the only method of fabricating a microstage on a chip since serial mechanisms require the stacking of actuators.

(2) A focused ion beam (FIB) can fabricate microsized cutting tools.

(3) A micromachine stepper motor can provide openloop positional control.

(4) Nonsilicon-based microfabrication can provide packaging of Si micromachines.

CNC micromachines will have war reserve (WR) and commercial applications. CNC micromachines can be used to machine or inspect nonplanar parts to dimensions previously unheard of. A mirrored platform can couple optical energy to multiple sensors or detonators. Safety critical parts can be micromilled immediately before detonation. Medical applications include micromanipulation of biological specimens using a dexterous CNC manipulator.

We performed kinematic analysis of a parallel mechanism restricted to planar motion. We Si-surfacemicromachined a functional planar parallel mechanism to demonstrate feasibility. We successfully tested and demonstrated the feasibility of a Sandia-fabricated planar parallel mechanism. We designed and are in the process of building the next generation of a planar parallel mechanism in
Sandia will design, manufacture, package, and apply computer numerically controlled (CNC) silicon (Si) micromachines. These multiple-degree-offreedom (DOF) machines (including 3-D) would serve as the basic building block for a variety of applications.... We designed and are in the process of building the next generation of a planar parallel mechanism in Si. 
Si. A conceptual design of the CAD/CAM (computer-aided design/computer-aided manufacturing) interface needed to drive the CNC micromachine device is near completion.

We will perform detailed design definition of the Sibased MEMS devices. The design definition entails the design of the masks necessary for the photolithography process. The costly portion of developing a MEMS device is the device design. The actual fabrication cost is a smaller fraction of the overall cost.

We performed kinematic analysis of the planar parallel mechanism in two ways. We used the Dynamic Analysis and Design Systems (DADS) software package to perform kinematic analysis and simulation of the planar parallel mechanism. In addition to the analysis performed on the computer, we mathematically derived a kinematic solution. We used this analysis as a design tool to design linkage lengths and linkage joints.

The feasibility device that we fabricated in $\mathrm{Si}$ confirmed the validity of the kinematic analysis. The feasibility model also allowed us to exercise the fabrication process as applied to a planar parallel mechanism. The device is operational, although the range of displacement is limited. We made a short video of the feasibility device in operation.

We designed and are fabricating two variations of the next generation of planar parallel mechanism. The mechanism is the same in both the variations; however, the actuation scheme is different. One design uses a nanostepping actuator called the shuffle motor that has been fabricated and demonstrated at Sandia. The advantage of the shuffle motordriven mechanism is that it has nanometer resolution and high displacement. The disadvantage of the shuffle motor is that it requires a position feedback sensor to perform closed-loop position control. The second design uses an electrostatic combdriven stepping linear actuator. The advantage of this actuation scheme is that it can be operated open loop and does not require a position sensor for feedback. The disadvantage of this second design is that the step size is much coarser.

We are investigating several applications for $\mathrm{CNC}$ micromachines. We can attach a nonsilicon probe that is somewhat perpendicular to the platform to make a microsize coordinate measuring machine (CMM). Similarly, a CNC micromachine with an attached probe can be used in atomic force microscopes (AFMs). We can mirror the platform surface to make an optical metrology device that relies on the principle of interferometry. The CNC micromachine can be used as a
We performed kinematic analysis of the planar parallel mechanism in two ways. We used the Dynamic Analysis and Design Systems (DADS) software package to perform kinematic analysis and simulation of the planar parallel mechanism. In addition to the analysis performed on the computer, we mathematically derived a kinematic solution. We used this analysis as a design tool to design linkage lengths and linkage joints. 
stage for nanomachining applications. For example, this nanopositioning stage could be placed inside the vacuum chamber of a FIB machine for nanomachining material. Optical switching is also an application that could benefit from a CNC micromachine. A single optical input could be switched to multiple outputs. 
10794

\section{Electromicrofluidic Packaging}

G. L. Benavides, P. C. Galambos, D. Gutierrez, C. A. Reber, K. A.

Peterson

The number of surface-micromachined (SMM) microfluidic devices is likely to increase as issues of manufacturing efficiency and integration of microfluidics with electronics become important. SMM microfluidic devices are manufactured with the same tools as integrated circuits (ICs), and their fabrication can be incorporated into the IC fabrication process.

To realize applications for SMM microfluidic devices, an efficient method for getting fluid into these devices must be developed. This project will focus on packaging for electromicrofluidic devices-specifically on an efficient method for delivering fluids of interest to SMM microfluidic channels that are integrated with electronics. Sandia will develop a microfluidic/microelectronic package (MMP) that allows for electrical connections using standard techniques and microfluidic connection from either a substrate or a test fixture.

We will manufacture SMM microfluidic channels in the SUMMiT process; they require both electrical and fluidic connection. We will introduce the fluid into the microfluidic devices from the back of the wafer through Bosch-etched via. These via will align with passages in the MMP, allowing fluid delivery to each individual channel in the wafer.

We will also investigate the next level of packaging that takes the fluid from the MMP to standard connectors. In production devices, the next level of packaging will probably be a fluidic printed wiring board (FPWB) with embedded fluid channels. For fluidic IC testing, the intermediate packaging level will consist of a test fixture that allows connection of fluidic devices for pressure and flow-rate measurements. The $M M P$, test fixture, and FPWB will require microfabrication in nonsilicon materials. Our investigation will primarily focus on the fabrication and packaging of an MMP made from either ceramic or plastic material.

We selected and fabricated an electromicrofluidic IC. The electromicrofluidic IC has multiple pumps for pumping liquid and is electrically driven. We investigated candidate materials for the MMP and selected plastic. Plastic is advantageous mostly because of cost considerations for a mass- 
production scenario. A plastic MMP can most likely be molded with the proper channels and holes already in place. Also, plastic has better options for making fluidic seals and connections. We conceptualized a standardized MMP design that has the fluidic connections along a known pattern, which is analogous to the electrical connections, found on a dual-inline pin (DIP). We developed a manufacturing and assembly process that should enable the electromicrofluidic architecture that we originally proposed. This manufacturing and assembly process is probably patentable, and we are pursuing a technical advance.

We designed and fabricated an MMP test fixture. This test fixture will allow us to demonstrate the feasibility of the assembly and bonding processes. We will accomplish the critical alignment of the electromicrofluidic IC to the MMP by using a flip-chip bonder. The bonding material is basically an adhesive film. We have made progress toward fabricating the MMP and are searching for vendors to supply plastic DIPs that can be easily modified to be an MMP.

\section{Other Communications}

Galambos, P. C., and G. L. Benavides. 2000.

"Electrical and Fluidic Packaging of Surface-

Micromachined Electromicrofluidic Devices." Proc.

SPIE Microfluidic Devices and Sys. III,

Micromachining and Microfabrication 4177 (Santa

Clara, CA, 18-20 September): 200-207.
We developed a manufacturing and assembly process that should enable the electromicrofluidic architecture that we originally proposed. This manufacturing and assembly process is probably patentable, and we are pursuing a technical advance. 


\section{Information Systems and Technology}

The Information Systems and Technology investment area is charged with positioning Sandia for national leadership in the development of information systems, networks, and technologies. Secure, safe, integrated information systems are key enabling technologies for the Laboratories' strategic goals. Areas of research emphases include (1) high-speed, secure networks, (2) location-insensitive computing, (3) conversion of data to information, and (4) development of the science of information.

The "Ten-to-One-HundredGigabit/Second Network-Enabling R\&D” project is investigating an end-to-end architecture that supports 10 to 100 Gbps computer networking. High-speed, secure networking is critical to supporting national security objectives. For example, data visualization, highperformance network storage, and massively parallel distributed processing for national security applications all demand these (and higher) communication rates.

Currently, critical gaps in standards and technology impede collaborative

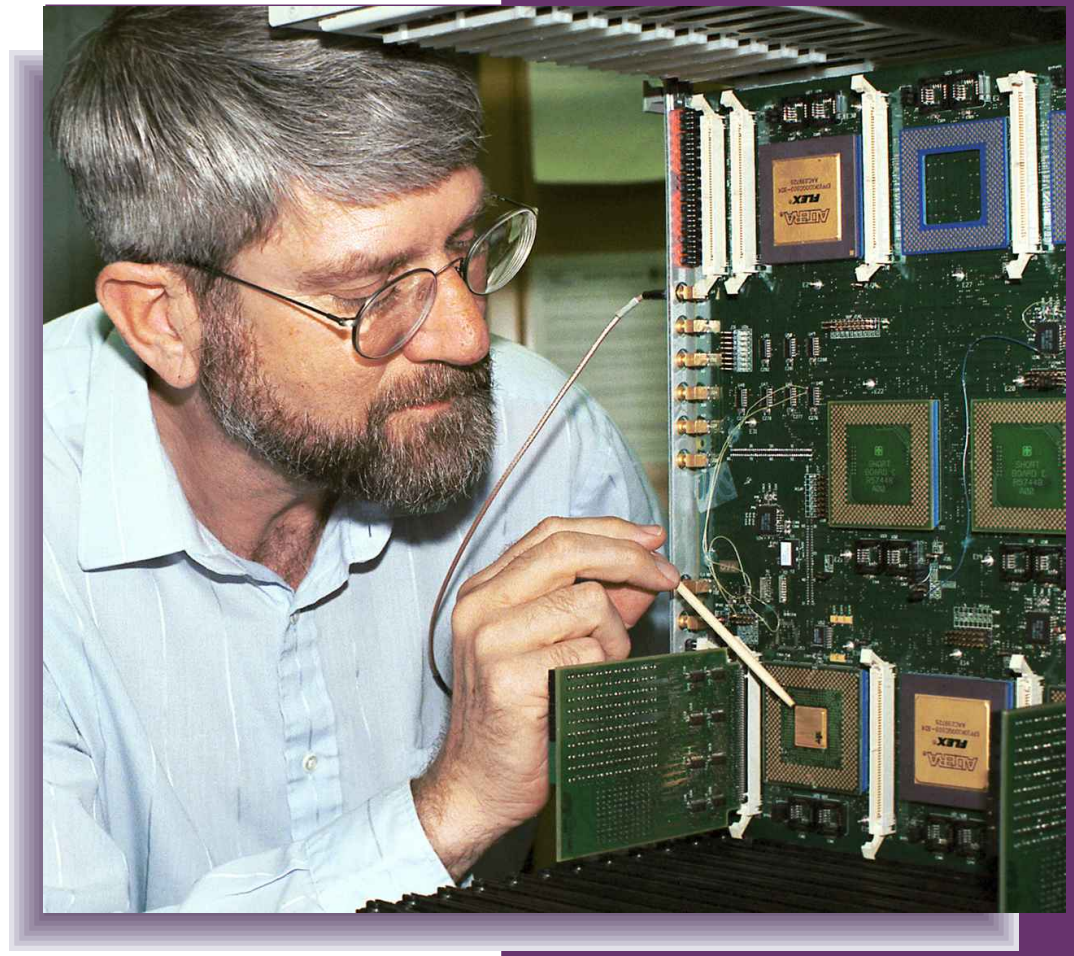

development and information delivery in a secure fashion. To address these problems, this project is researching methods to efficiently process communication protocol functions at extremely high speed. 
10355

\section{Weighted-Nearest-Neighbor (WNN) Decision Making for Data Mining}

Data mining involves the discovery of information sources that are in some way related to observed phenomena and the determination of models that accurately define the relationships. Current efforts involve application of statistical techniques, fuzzy logic, neural networks, and expert systems. These efforts generally have been unsuccessful because there is no validated theory for adaptively discovering, selecting, and fusing distinguishing features in terms of uncertainties caused by measurement errors, randomness, ambiguities, and information warfare tactics. The result is the development of a large number of highly specialized algorithms that work only in very limited conditions.

A major thrust of this research involves the development of a theoretical and practical data-mining methodology. Our approach is to develop a methodology for discovering and fusing features to achieve near-minimum probability of error (MPE) decision and estimation algorithms. Our approach combines a weighted-nearest-neighbor (WNN) decision model for classification and estimation with a genetic algorithm (GA) for feature discovery and model optimization. The WNN model will provide the mathematical framework for adaptively discovering and fusing features into near-MPE algorithms. We will use the GA to discover and select inputs and to determine the weights in the WNN decision model that will optimize performance. A major advantage of the WNN model over other approaches is that the WNN model requires estimation of significantly fewer parameters (therefore, significantly fewer training samples) to accurately partition the feature space. This is of paramount importance as information sources are often incomplete or sparse in many dimensions. We expect to demonstrate that the WNN model will allow robust and practical application to a wide variety of data-mining problems.

We extended our research using the WNN fusion model to handle problems in unsupervised learning. The unsupervised learning problem involves the identification or detection of pattern classes contained within a dataset. We investigated and developed several approaches with varied levels of success and applicability to data mining. The most promising approach was
A major thrust of this research involves the development of a theoretical and practical datamining methodology. Our approach is to develop a methodology for discovering and fusing features to achieve near-minimum probability of error (MPE) decision and estimation algorithms. 
based on the K-nearest-neighbor (KNN) concept. The KNN clustering model uses similarity measures based on mutually shared KNNs to combine two samples into a common cluster. The model requires no assumptions on underlying probability density functions characterizing datasets. The method also works well with either large or small datasets. The similarity measures used in the KNN model result in clusters that are visually appealing. Another advantage of the KNN clustering model over other approaches is that it shares the same computational operations as the WNN fusion model and, therefore, can leverage parallel decompositions developed previously for the WNN model. We applied the clustering methods developed to several unsupervised data-mining problems. Success varied, depending on the problem and the statistical characteristics of the dataset. The KNN clustering model generally outperformed other approaches.
The model requires no assumptions on underlying probability density functions characterizing datasets. The method also works well with either large or small datasets. 
10356

\section{Varying QoS for Fixed and Mobile Networks}

J. T. Michalski, T. D. Tarman, J. P. Brenkosh, J. D. Dillinger, J. E. Stamp

Sandia is building a prototype system that will provide a network reservations interface (NRI) to allow a policy server to dynamically request bandwidth from the underlying network system. Currently an application with quality of service $(Q o S)$ requirements would require a network administrator to statically establish a channel.

The system we are building is essentially a bandwidth broker system that will provide signaling for distributed resource management applications that require Internet Protocol (IP) network QoS (quality of service). A bandwidth broker gathers and monitors the state of QoS resources. This information, along with its corresponding policy information, will be used to provide admission control to QoS flows to the network, as well as to aid en route computation for connectionoriented flows.

Our bandwidth broker system is an essential component for allowing distributed applications to share network resources while receiving a predictable level of network QoS.

For networks with insufficient capacity to support requested QoS, we are developing methods of integrating hierarchical encoding of data. We are using multicast trees to transport base and enhancement layers of information. The base layer is encoded with the minimum of sufficient information, while each enhancement layer is encoded with an incremental improvement in information. Unsatisfied QoS requests will trigger incremental rejection of enhancement layers. This approach will ensure efficient use of available network resources.

- Implemented queuing mechanisms that facilitate the QoS attributes in a packet switched network. We developed scripting code, using a differential service model (Diffserv) to define classification and scheduling for the Linux router. This code defines two functions; the first is the definition of a route classifier that is used as a filter to identify and mark a flow with a unique identifier. The second function maps the marked data flows into a defined queuing discipline. We are extending the class-based mechanisms to incorporate individual flow classification.
Sandia is building a prototype system that will provide a network reservations interface (NRI) to allow a policy server to dynamically request bandwidth from the underlying network system....The system we are building is essentially a bandwidth broker system that will provide signaling for distributed resource management applications that require Internet Protocol (IP) network QoS (quality of service). 
- Developed interface protocol to communicate QoS control information among network resources. We created a communication interface protocol based on the Common Open Policy Service (COPS) static model. This model comprises a policy decision point (PDP) and a policy enforcement point (PEP). The PDP is hosted on a server and communicates with the PEP located on the ingress Linux router. The PDP relays policy information such as application or host priority. This information is presented by the PEP to the Linux router in the form of a packet marking and queuing script.

- Developed enhancements to the Portable Batch System (PBS) resource management package to allow network resource management. The $\mathrm{PBS}$ provides a distributive advance scheduler function for a multiple processor environment. Jobs are scheduled based on priority and processor availability. We successfully enhanced the source code for this utility to allow the scheduler to communicate with a resource management function and reserve bandwidth resources requested by an application. Advanced network resource reservation will be possible once we complete the Linux router resource availability code.

- Completed system analysis of a hierarchical encoding technique for the efficient distribution of data. We completed the analysis of the hierarchically encoded data. We demonstrated a video conferencing (VIC) application in a multicast environment using the hierarchical encoding scheme. We created three hierarchical streams on a source host and distributed them to multiple target hosts located on different networks. Each target hosts subscribed to multiple layered streams to demonstrate the QoS technique of this approach.
We successfully enhanced the source code for this utility to allow the scheduler to communicate with a resource management function and reserve bandwidth resources requested by an application. Advanced network resource reservation will be possible once we complete the Linux router resource availability code. 
10357

\section{A Real-Time Decision-Support Framework to Guide Facility Response to Abnormal Events}

J. E. Brockmann, D. M. Edwards, F. Gelbard, K. K. Murata, L. D. Brandt, S. P. Gordon

Adverse consequences to facilities that are subject to abnormal environments can be dramatically reduced if timely and appropriate mitigation actions are taken. Success in damage limitation requires the real-time fusion of data from diverse sources. An information and decision framework is required to evaluate these data, assess the probable impacts, and develop a decision and control strategy. The framework must develop graded facility responses appropriate to the quality of information available and the predicted consequences of the event and prospective mitigation options.

This project will provide the technical basis and prototype information tools for guiding facility response in threatening environments. Sandia will develop a decision framework that propagates prediction uncertainty, addresses decision risk measures, incorporates temporal updating, and links disparate information. We will also develop robust predictive models able to utilize sparse sensor data to predict transport and consequences. We will construct an integrated decision tool and evaluate it initially against a realistic reference facility. The initial prototype will provide a proof of concept and indication of the performance of the new technologies. As the final step in the research, we will refine the tools and evaluate them against more diverse facilities and more general environments.

Successful completion of this research will provide key results supporting development of information and decision systems that improve safety and security of virtually all of the key facilities in the nation. The work will provide a technical foundation for several emerging areas of importance to Sandia, including a broad range of infrastructure protection programs. The work is particularly applicable to the development of protection systems for chem/bio attacks on civilian infrastructure targets, a growing area of national interest. The research is structured specifically to provide Sandia with a demonstrated technological base and fundamental tools to address these problems.

We developed the sensor placement algorithm (SPA) to complement the sensor analysis algorithm (SAA) that we
Successful completion of this research will provide key results supporting development of information and decision systems that improve safety and security of virtually all of the key facilities in the nation. The work will provide a technical foundation for several emerging areas of importance to Sandia, including a broad range of infrastructure protection programs. The work is particularly applicable to the development of protection systems for chem/bio attacks on civilian infrastructure targets, a growing area of national interest. 
developed previously. The algorithms, SAA and SPA, form the technical basis that allows us to consider the possibility of a real-time decision-support framework. They allow us to solve the inverse problem; without this capability, no reasonable predictions of agent transport could be made. With the algorithms as a backbone, we can define the path for the functional design of the decision-support system (DSS).

We completed the functional design of the DSS. We defined the form and function of the decision support framework. We determined that a priori flow matrices calculated with $\mathrm{K} 2$ can be used by the SAA code to calculate the inverse problem and to predict future transport. This code can be interfaced with $\mathrm{K} 2$ to display the results.

We made a preliminary demonstration of the DSS. This demonstration exemplifies how all of the pieces of a functional real-time DSS will fit together. This demonstration also shows a floor plan map with icons at the locations of every chemical sensor, telephone call box, and closed-circuit television (cctv) camera; an indication of the predicted concentrations; and the predicted results of HVAC (heating, ventilation, and air conditioning) options.

\section{Other Communications}

Gelbard, F., J. E. Brockmann, and K. K. Murata. 1999. "Sensor Analysis Software (SAS) and an Algorithm for Determining Airborne Agent Concentrations Throughout a Large Multi-Room Building." Sandia Technical Report SAND99-1945, Sandia National Laboratories, Albuquerque, NM (August).

Gelbard, F., J. E. Brockmann, K. K. Murata, and W. E. Hart. 2000. "An Algorithm for Locating Sensors in a Large Multi-Room Building." Sandia Technical Report SAND2000-0851, Sandia National Laboratories, Albuquerque, NM (May).
The algorithms, SAA and SPA, form the technical basis that allows us to consider the possibility of a real-time decision-support framework. They allow us to solve the inverse problem; without this capability, no reasonable predictions of agent transport could be made. With the algorithms as a backbone, we can define the path for the functional design of the decision-support system (DSS). 
10358

\section{Physical Model-Based Fusion of Sensor}

\section{Array Data}

\author{
I. R. Shokair, D. C. Roe, N. M. Berry, R. G. Hillaire, C. F. Melius, \\ C. Springer
}

Two motivations for this work are particularly exciting:

discovering the wide variety of bioregulators that affect so much our existence and well-being, yet are so poorly understood, and uncovering the many harmful substances that have been either carelessly polluted or intentionally engineered for our harm.

The purpose of this project is to develop computational models, analysis methods, and algorithms for detecting unknown compounds using array separations. These separations exploit a variety of chemical characteristics, including molecular weight, charge, hydrophobicity, and many other properties to induce differential migration in capillaries. The differential migration results in chemical separation. For the present devices, detection is accomplished using laserinduced fluorescence ( $\mathrm{LiF})$, and chemical identification is achieved by comparing retention times with known standards.

With arrays of separations, multiple separation properties can be measured that potentially allow highcertainty identification of known substances and good insight into the likely make-up and properties of an unknown agent.

We analyzed several datasets for separation of nitrogencontaining aromatic compounds, explosives components, and proteins using various separation methods and investigated the crucial issue of data reproducibility and how to realistically deal with it in the analysis algorithms.

We significantly improved our Adaptive Background Suppression-Peak Detection (ABS-PD) algorithm for better convergence and will submit a technical advance for it.

We developed a graphical user interface (GUI)-driven analysis code to facilitate effective generation of database information from laboratory-measured data. The code uses the ABS-PD algorithm applied to laboratory-measured data of known samples and fits the detected peaks to the appropriate shapes. We also developed a multivariate analysis code that uses the generated database information to identify the presence of species and to calculate concentrations and error estimates.
The purpose of this project is to develop computational models, analysis methods, and algorithms for detecting

unknown compounds using array separations.... With arrays of separations, multiple separation properties can be measured that potentially allow high-certainty identification of known substances and good insight into the likely make-up and properties of an unknown agent. 
For the modeling effort we focused on separating pools of amino acids, peptides, and proteins. We implemented computational models for predicting separation properties of peptides. 
10359

\section{Large-Scale Distributed Information System Modeling and Simulation}

A. S. Yoshimura, T. D. Plantenga, M. E. Goldsby

Large enterprises are evermore dependent on their large-scale information systems (LSIS): computer systems that are distinguished architecturally by distributed componentsdata sources, networks, computing engines, simulations, human-in-the-loop control, and remote access stations. These systems provide such capabilities as workflow, data fusion, and distributed database access. The Nuclear Weapons Complex (NWC) contains many examples of LSIS components, a fact that motivates this research.

However, most LSIS in use grew up from collections of separate subsystems that were not designed to be components of an integrated system. For this reason, they are often difficult to analyze and control. The problem is made more difficult by the size of a typical system, its diversity of information sources, and the institutional complexities associated with its geographic distribution across the enterprise. Moreover, there is no integrated approach for analyzing or managing such systems. Indeed, integrated development of LSIS is an active area of academic research.

This work developed such an approach by simulating the various components of the LSIS and allowing the simulated components to interact with real LSIS subsystems. This research demonstrated two benefits. First, applying it to a particular LSIS provided a thorough understanding of the interfaces between the system's components. Second, it demonstrated how more rapid and detailed answers could be obtained to questions significant to the enterprise by interacting with the relevant LSIS subsystems through simulated components designed with those questions in mind.

Our work showed that reduced-fidelity models (RFMs) of a significant portion of an actual LSIS (and one actual model) could be combined in a coordinated simulation environment with human-in-the-loop control. We constructed a simple, unclassified simulation of SecureNet. We simulated this communication system in parallel in Dartmouth SSF (Scalable Simulation Framework) and IDES (Infrastructure for Distributed Enterprise Simulation). We did this to test the flexibility with which the workload in the various parts of a
Our work showed that reducedfidelity models (RFMs) of a significant portion of an actual LSIS (and one actual model) could be combined in a coordinated simulation environment with human-in-theloop control. 
network could be varied to allow comparison of low- and highfidelity models.

We wrote JAVA interfaces for Microsoft Excel. We made external model interface improvements to enhance interaction with the commercial off-the-shelf (COTS) iThink application. We wrote interfaces to allow communication between IDES, written in the JAVA programming language, and external information systems, written in Extend, Microsoft Access, and Microsoft Excel. These wrappers allow IDES to drive models written in these COTS software packages during a simulation. We wrote methods for converting data formats from each software package to JAVA.

We undertook work in modeling information as a fluid in collaboration with Dartmouth College. We wrote a pair of simulations of interconnected communications switches, one using a fluid model and the other a packet model, to explore fluid techniques and compare a nontrivial fluid simulation to its more detailed counterpart.

We used the CoMPASS system as a platform for integrating LSIS components. We integrated RFMs of the Transportation System Division's Transportation Planning Model (TPM), the Pantex Planning Model (PPM), and the Y-12 CSA Planning Model. The real Tritium Production Model was released to the CoMPASS team and has been integrated with CoMPASS. Use of the RFMs provides an example of faster-than-real-time simulation; for instance, the real PPM can require 8 hours to produce a schedule, whereas the RFM PPM produces one in seconds. Various Defense Program Requirements Planning Model (DPRPM) models were integrated into CoMPASS.

We modeled and simulated traffic source and sink devices common to SecureNet in a simplified, unclassified form. We parameterized the simulation to run at different resolutions. We used the intermixing of submodels of different fidelities to investigate communication mechanisms between high- and low-level simulation entities. To compare full-detail and reduced-fidelity models on a large scale, it was necessary to simulate very large traffic flows at the packet level. We gave IDES the ability to handle larger simulations by applying recently developed techniques (Fujimoto, et al.). These techniques dramatically improved IDES memory usage, enabling it to handle correspondingly larger models on given hardware.

We applied fluid-based communication system simulation techniques to wireless telemetry systems. We
We gave IDES the ability to handle larger simulations by applying recently developed techniques. These techniques dramatically improved IDES memory usage, enabling it to handle correspondingly larger models on given hardware. 
developed prototype hardware and software to demonstrate intercommunication between wireless components and to investigate communication between remote sensor model simulations.

\section{Refereed}

Ammerlahn, H. R., M. E. Goldsby, M. M. Johnson, and D. M. Nicol. 2000. "A Geographically Distributed Enterprise Modeling System.” Future-Generation Computer Sys., accepted.

Nicol, D. M., M. E. Goldsby, and M. M. Johnson. 1999. "Fluid-Based Simulation of Communication Networks Using SSF." Proc. 11th European

Simulation Symp. and Exhibition, Simulation in Industry (Erlängen-Nuremberg, Germany, 26-28 October). 
10360

\section{High-Surety SCADA for the Critical Energy Infrastructures}

R. E. Carlson, C. L. Beaver, R. J. Granfield, J. E. Stamp, A. E. Bentley

The domestic critical energy infrastructures are a complex and interdependent network that is vital to the national security and social well being of our nation. The control mechanisms for these infrastructures are often referred to as SCADA (Supervisory Control and Data Acquisition) systems. The President's Commission on Critical Infrastructure Protection has identified that the widespread and increasing use of SCADA for control of energy systems provides an increasing ability for an adversary to cause serious damage to the energy infrastructures. To meet the new infrastructure threats and requirements, not only must contemporary SCADA systems be able to report the infrastructure state with confidence, but they need to measure the entire infrastructure, be robust in their communication, and be timely and optimal in their control. In addition, the SCADA systems must provide these services under adverse conditions. These are attributes that a SCADA system needs to enhance the surety (security, safety, and reliability) of the underlying infrastructure. The difficult aspect of this $S C A D A$ research has been separating the role of standards from proprietary systems and discerning stable architectures from those that are likely to change significantly. With this understanding, Sandia developed an intuition for how surety will influence the composite system that is SCADA. The fundamental problems in upcoming work will be mapping SCADA into the high-surety SCADA specification (HSSS) and validating this architecture on the Sandia SCADA Test-bed (SST). The HSSS will ensure that Sandia becomes the "provider of choice" in this domain for technologically enhancing the surety of our national energy infrastructures. Validation of the HSSS on the Sandia SST will provide a differentiating aspect of this research that promises to be valued by both government and industry.

We developed an intuition for how surety will influence the composite system that is SCADA. In addition, we developed alliances and relationships that expedited the understanding and acquisition of this information. Our effort went further than expectations of both government and industry. A collection of collaborations evidences the evolving
The President's Commission on Critical Infrastructure Protection has identified that the widespread and increasing use of SCADA for control of energy systems provides an increasing ability for an adversary to cause serious damage to the energy infrastructures....The HSSS will ensure that Sandia becomes the "provider of choice" in this domain for technologically enhancing the surety of our national energy infrastructures. Validation of the HSSS on the Sandia SST will provide a differentiating aspect of this research that promises to be valued by both government and industry. 
recognition by industry that Sandia is a leader in SCADA. Our accomplishments include the following:

(1) Received an invitation to participate in a number of security assessments involving SCADA.

(2) Completed the second phase of the SST, focusing on the control and security of infrastructure assets at Sandia.

(3) Completed the logical, functional, and dynamic SCADA model views based on standards IEEE C37.1-1994 and IEEE 999-1992.

(4) Completed the prototype real-time controller for SCADA.

(5) Completed a draft information security model for SCADA systems.

\section{Other Communications}

Carlson, R. E. 1999. "Developing a High-Surety

SCADA System." Paper presented to the Seminario Internacional de Automatizacion Y Geoprocesamiento, Punta del Este, Uruguay, 5-7 December.

Carlson, R. E. 2000. "Security Systems in Substations." Paper presented to the Distribution/ Substation Automation Workshop, Western Electric Power Institute, San Francisco, CA, 3-5 May.

Carlson, R. E., and C. L. Beaver. 2000. "A Protection Profile for TASE.2." Sandia Technical Report SAND2000-0922, Sandia National Laboratories, Albuquerque, NM (May).

\section{A collection of collaborations evidences the evolving recognition by industry that Sandia is a leader in SCADA.}


10361

\title{
Agent-Based Mediation and Cooperative Information Systems
}

\author{
L. R. Phillips, S. Y. Goldsmith, S. V. Spires, H. E. Link
}

Agent-mediated information management is currently the most promising solution to the problem of integrating and accessing large legacy data stores and for utilizing networked information sources such as the Internet. Collections of agents can assist human users and other agents with cooperative information processing and time-consuming search tasks. Sandia developed the Standard Agent Framework (SAF), a general-purpose development environment for constructing multiagent systems that assist a diverse set of users with knowledge-intensive tasks in a network environment. The RETSINA framework under development at Carnegie-Mellon University (CMU) is being used to develop distributed collections of intelligent software agents that cooperate asynchronously to perform goal-directed information retrieval and information integration in support of performing a variety of decision-making tasks. A collection of RETSINA agents forms an open society of reusable agents that self-organize and cooperate in response to task requirements. We will develop agent-based mediation functions that enable interoperation and cooperative problem-solving across both agent systems. By combining the two technologies, we will achieve a superior system capable of complex real-world information processing in an information environment such as the Internet.

Our agents can share knowledge with other agents, including CMU agents, and can be instructed to be mediation agents to share ontologies. We evaluated some cooperative capacities, although we have not defined or executed more complex methods in the context of this problem. We executed significantly complicated cooperative behaviors in the context of other work. We understand what is required to share tasks with a non-SAF agent and have begun preliminary construction of more complex processes for our own agents.

Our work identified new requirements: (1) agents need to deal with all inputs and act appropriately for each message, regardless of type, (2) agents need to accommodate new kinds of inputs dynamically, and (3) agents need a declarative form of goal achievement procedure, not the static process descriptions we used earlier. We achieved all these capabilities. Furthermore, objectification of ontologies for transmission to
Sandia developed the Standard Agent Framework (SAF), a general-purpose development environment for constructing multiagent systems that assist a diverse set of users with knowledge-intensive tasks in a network environment.... We will develop agent-based mediation functions that enable interoperation and cooperative

problem-solving across both agent systems. By combining the two technologies, we will achieve a superior system capable of complex real-world information processing in an information environment such as the Internet. 
another (possibly non-SAF) agent surfaced as a challenging research issue.

Our SAF agents can have conversations; we implemented and executed some examples. SAF agents can formulate and pursue collaborative goals. Each SAF agent can contain models of information states and the actions to transition among them, determine which action is appropriate and execute it, and update its state model to reflect what it thinks should happen. We are currently working on the Our SAF agents can have conversations; we implemented and executed some examples. SAF agents can formulate and pursue collaborative goals. formation and encoding of ontologies to be communicated among groups of agents potentially ignorant of this information. 
10362

Dynamically Self-Configurable Network Protocol

B. P. Van Leeuwen, J. Espinoza, L. B. Dean, D. L. Harris, W. F.

Young

This project addresses the problem of adaptive message routing in a decentralized packet communication network with nodal mobility. Nodal mobility leads to frequent network topological changes, thus requiring routes to be reestablished. The communication medium is a radio channel shared by many contending users. These ad hoc networks are of importance in the context of reliable information systems, tactical battlefield communications, antipersonnel landmine replacement communications, mobile robotic communications, and other distributed communication networks.

The objectives of this research are to investigate current routing, media access algorithms, and security approaches, and to develop a wireless ad hoc network that will permit networks to self-configure their communication topology dynamically. A major product of this research will be the development of a self-configuring ad hoc network. This secure network will be applicable to systems having an initial random node distribution and/or containing mobile nodes.

Sandia's research will take advantage of existing research in network protocols and components of protocols used in networking technologies, with a focus on minimizing the amount of control necessary since these nodes may be mobile and have limited life spans (e.g., battery-operated). The research will develop a secure network that is applicable to communication systems with the described attributes. Efforts will include simulating a model of the developed network with the latest version of the OPNET (SMDPS [Strategic Mission Data Preparation System] Phase III Operational Network) network simulation tool. Network performance, such as throughput and packet delay, will be characterized, evaluated, and optimized for the developed network. Finally, our research will present a demonstration simulation and possibly demonstrate the developed protocol on an actual communication network.

Our accomplishments include incorporating a hybrid approach to ad hoc routing. With the rapid advances taking place in this area, the Sandia approach is to incorporate the necessary attributes of current research in protocols into a 
hybrid approach to ad hoc routing. This hybrid approach offers the greatest amount of flexibility by applying varying degrees of a link-state approach in combination with an on-demand approach to ad hoc routing. This approach also contributes to our need of a power-efficient tri-state protocol that allows for a trade-off on the amount of power availability versus the amount of message latency permitted.

We collaborated with Cornell University in building OPNET simulator modeling blocks to simulate the hybrid approach. We tested and extended several custom OPNET models that are critical to simulating this approach. These simulation models are linked with models from the OPNET library representing the transport layer, data link layer, and physical layer to simulate the performance implications of their mutual interactions.

In addition, our research simulated the proposed Dynamic-Source Routing (DSR) protocol on the NS-2 simulator with the Carnegie-Mellon University (CMU) wireless/mobility extensions. We documented these simulation results and will compare them to actual data that are being obtained from a DSR implementation coded for the FreeBSD operating system. We are currently addressing DSR implementation issues with CMU. We examined the proposed Temporally-Ordered Routing Algorithm (TORA) approach to ad hoc routing and progressing in our hardware implementation for testing on a Linux platform with the Lucent WaveLAN radios.

Our research efforts also included investigations of security approaches for distributed networks. The objective is to tailor or extend an accepted cryptographic technique to effectively secure the control-plane data of a wireless ad hoc network. Current approaches of distributed network security focus on wired networks. Our research identified the key characteristics that differ in a wireless ad hoc network when compared to its wired counterpart. We intend to tailor or extend the cryptographic approach to operate under the set of network characteristics.
Our research identified the key characteristics that differ in a wireless ad hoc network when compared to its wired counterpart. We intend to tailor or extend the cryptographic approach to operate under the set of network characteristics. 
10363

\section{Optical Backplane/Interconnect for Super-} High-Speed Communication

P. J. Robertson, L. G. Pierson, L. B. Dean, E. L. Witzke, T. D.

Tarman, C. T. Sullivan, H. Y. Chen

Current copper backplane technology has reached the technical limits of clock speed and width for systems requiring multiple boards. Currently, bus technology such as versa module Eurocard (VME) and peripheral component interconnect (PCI) (types of buses) will face severe limitations as the bus speed approaches $100 \mathrm{MHz}$. At this speed, the physical limit of an unterminated bus is barely 3 inches. Terminating the bus enables much higher clock rates but at drastically higher power cost. Sandia has developed highbandwidth parallel optical interconnects that can provide over 40 Gbps throughput between circuit boards in a system. Based on Sandia's unique vertical-cavity surface-emitting laser (VCSEL) technology, these devices are compatible with complementary metallic oxide semiconductor (CMOS) chips and have single-channel bandwidth in excess of $20 \mathrm{GHz}$. In this project, we are researching the use of this interconnect scheme as the physical layer of a greater asynchronous transfer mode (ATM)-based backplane. There are several advantages to this technology, including small board space, lower power, and noncontact communication. This technology is also easily expandable to meet future bandwidth requirements in excess of $160 \mathrm{Gbps}$, sometimes referred to as UTOPIA 6. ATM over optical backplane will enable automatic switching of wide high-speed circuits between boards in a system. The goal of this project is to develop a parallel optical bus technology utilizing integrated VCSELs. We used a PCI card to implement the physical layer of the optical bus.

We developed operational concepts and target technologies that will realize a massively parallel (MP) optical backplane. At the hardware level, we identified commercially available fiber-optic connectors that can contain as many as 160 optical fibers. The fibers are made up of 12 fiber ribbon cables. A new generation of VCSELs and receivers is being designed and initial devices will be fabricated within Sandia's Compound Semiconductor Research Laboratory. A concept that we will try is an integrated transmitter/receiver (tx/rx) pair that can either be used over separate fibers or possibly operate bidirectionally over a single fiber.
Sandia has developed highbandwidth parallel optical interconnects that can provide over 40 Gbps throughput between circuit boards in a system....In this project, we are researching the use of this interconnect scheme as the physical layer of a greater asynchronous transfer mode (ATM)-based backplane. 
We developed three optical interconnect architectures. Utilizing a star interconnect architecture, the most promising of the three is a computer interconnect with distributed reservation and scheduling. We will perform simulations of this protocol later and implement the protocol on the prototype hardware.

We developed ATM and datagram operational models. We are now targeting UTOPIA 6 (our definition) of $160 \mathrm{Gbps}$ as the standard data rate. This work is years ahead of the ATM Forum Standards Committee.

We are currently designing prototype hardware on a PCI bus card. We will fabricate prototype hardware that consists of a PCI-based card with two optical connections. We will plug multiple cards into a host system and send data over the optical bus to exercise the architectural concepts. The cards will be interconnected with the optical fibers.

We made substantial progress in the development of the integrated VCSEL and detector. The development of a fully integrated $\mathrm{rx} / \mathrm{tx}$ awaits the completion of a planarization process development activity. We progressed to a test-bed implementation using commercially available VCSEL array components. This led to the design of a PCI card containing 12-channel VCSEL-based interconnect $r x$ and tx modules. The board contains a single programmable logic device (PLD) that interconnects the electrooptical modules and the PCI control bus. We examined several architectures and undertook at least three separate board designs. Sandia's membership in the Optical Internetworking Forum (OIF) gave us access to information on upcoming 10-Gigabit Ethernet (GBE) standards using parallel optical channels. In addition, introduction of true low-voltage differential signal (LVDS)-compatible devices from Altera enabled the design of a board that used a PLD to directly drive the optics modules. These two developments led to a design of the PCI card.

\section{Other Communications}

Chen, H. Y., J. M. Brandt, C. T. Sullivan, E. L.

Witzke, J. G. Burns, and L. G. Pierson. 2000. "Optical

Backplane/Interconnect for Super High-Speed

Communication." Sandia Technical Report, in review.
We developed ATM and datagram operational models. We are now targeting UTOPIA 6 (our definition) of 160 Gbps as the standard data rate. This work is years ahead of the ATM Forum Standards Committee. 
10364

\section{PUSH Technology Demonstration}

W. E. Boebert, D. A. Rieb, T. Q. Thai, J. T. Gardner, M. G. Wilde, R. E. Curry, D. E. Farrell, D. S. O'Bannon

This project evaluated the Web technology referred to as PUSH (also known as Web Casting), with respect to its built-in security features and their efforts on an already secure Windows NT system or network architecture. A secure Windows NT system is defined in this context as one in which all underlying architecture facilitates zero-based administration. BackWeb servers are the leading developers in this technology area. These servers are being implemented in various DOE and DoD sites. PUSH technology is being incorporated into numerous software applications and systems to allow for the software, firmware, and associated systems settings to be remotely and automatically updated, thus reducing system administration and personnel costs. However, testing shows that there is no realistic way to secure systems that utilize this technology. Software and system configuration changes may be implemented without user knowledge, removing or altering security policies, and thus exposing or granting access to classified or sensitive information. The technology implementation defeats most security architecture the minute it is installed.

Sandia evaluated this technology using various configurations of firewall(s), router, intrusion-detection system(s), and a wide range of security policies. We conducted these tests under controlled conditions, with network and systems files being monitored and evaluated for system and network security impacts. In almost every configuration, we were able to modify the system of interest. These modifications altered the security settings of the systems and impacted the security of the operational network.

We chose a sample PUSH technology vendor (BackWeb) and server base (Windows NT).

We built a functional NT4/2000/Linux-based test-bed including router, firewalls, intrusion-detection systems, network servers, and clients.

We studied networking protocols used by BackWeb servers and clients to communicate.

We learned various methods of building BackWeb clients and installing them via the BackWeb server. We were 
especially looking for security-critical elements of clients and client-server interaction.

We found and demonstrated to interested Sandia security personnel and customers several surprising or securitycritical results. Surprises were related to BackWeb features that might be considered to enhance system and network security vulnerabilities. Security-critical effects could be introduced intentionally at the server and without the possibility of repair by the client. We exceeded expectations by finding modules that allowed these effects even when protected by a personal intrusion-detection system, personal firewall, network firewall, and intrusion-detection systems.

We delivered software to the BackWeb client that ran independently of the BackWeb system that impacted system and network security.

We demonstrated the ability to SPOOF (emulate) a BackWeb server and take over selected data transactions between the client and server, with and without a firewall in the We found and demonstrated to interested Sandia security personnel and customers several surprising or security-critical results....We exceeded expectations by finding modules that allowed these effects even when protected by a personal intrusion-detection system, personal firewall, network firewall, and network. 
10365

\section{Controlling Information: Its Flow, Fusion, and Coordination}

T. M. Berg, R. G. Hillaire, N. M. Berry

Sandia is creating theory, algorithms, and analysis tools for controlling information in self-organizing cooperative computer systems requiring no central control. Removing reliance on any central facility (e.g., blackboard memory, fusion center, communications bus, etc.) increases survivability, robustness, resistance to attack and compromise, and autonomy of the individual sensor nodes. These networks are self-organizing to the extent that each agent/actor/node is self-aware of its distribution properties and can communicate them. We are focusing on sensor-based information-gathering networks.

We expect to apply our results in two technology demonstrations and/or simulations: a simulation and flight test of a unoccupied aeronautical vehicle (UAV) squadron in coordinated flight (in collaboration with researchers at the University of California-Los Angeles [UCLA]), and an agentbased simulation of cooperative robotics. Our technologies are also useful for controlling information in high-performance computing and Internet routing.

We produced two simulations to test and improve our results. We found that our algorithm improves information fusion by simplifying interconnected system design, minimizing communication, speeding up fusion, and reducing estimation latency (latency refers to how out of date the fused information is compared to when the sensor collected the data).

We simulated our decentralized Fisher information filter using MATLAB to compare two small, distributed information filters with the corresponding centralized and larger Kalman (optimal) filter. These simulations showed that the decentralized Fisher information filter produces estimates identical to the virtual optimal filter for the tracking application chosen. The MATLAB code has subsequently been converted to JAVA code and is being integrated with agent technologies to implement the filters.

Systems composed of collectives of cooperating components are inherently complex, and their design trade-offs are fundamentally difficult to describe. We demonstrated that the decentralized Fisher information filter minimizes the increased communication of a decentralized system in two
Sandia is creating theory, algorithms, and analysis tools for controlling information in self-organizing cooperative computer systems requiring no central control....We produced two simulations to test and improve our results. We found that our algorithm improves information fusion by simplifying interconnected system design, minimizing communication, speeding up fusion, and reducing estimation latency (latency refers to how out of date the fused information is compared to when the sensor collected the data). 
ways. First, communication occurs only when it increases the information state of the receiving node. This means that a design tool can automatically eliminate unnecessary communication links. Secondly, when communication does occur, sufficient statistics of information are communicated. With sufficient statistics, fusion is accomplished efficiently. With sufficient statistics of information, irrelevant state information is filtered out before communication.

We found that minimizing the communication did not result in a computational increase trade-off, but instead simplified and sped up the fusion process. Part of the speedup is free because information fusion is more direct than the state space fusion methods. If information is relevant to a cooperating node, it is immediately communicated to it; otherwise, it is just used locally. In hierarchical state estimation, a local processor typically makes a local update first, only to have to subtract out this globally suboptimal computation when preparing its sensor data for communication. The decentralized information filter prevents this waste of time and compute power.

Our decentralized filter reduces latency by moving the required compute-intensive matrix inversions to the prediction and reporting portions of the estimation procedure, where latency is typically less costly. The high-speed, machineautomated functions require the freshest access to the updated data for their stability (e.g., milliseconds). The data prediction and information analysis stages (especially human analysis) take relatively much longer, so small delays in formatting the fused data are more tolerable. Moving the latency-producing operations to the more delay-tolerant filter stages produces a better overall system.

Another benefit of the information fusion approach we discovered is that the information space is a mathematical abstraction of the state space. Thus, even without encryption, intercepted data are less intelligible, particularly if the information matrix and information vectors are sent on separate channels.

Our colleagues at UCLA performed simulations that focus on filters distributed with overlapping information spaces. This led to a paper describing the bounds on distributed filter performance under failures as compared to centralized filters. This result uses Lyapunov theory to assess the stability of decentralized systems under attack, with the analysis also providing a measure of the performance of a decentralized
We found that minimizing the communication did not result in a computational increase tradeoff, but instead simplified and sped up the fusion process. 
network as compared to a virtual "optimal" central processor.

The virtual optimal processor concept helps overcome the limitations of a systems theory that currently does not acknowledge or enable the analysis of decentralized systems concerning redundancy, survivability, coordinated action, etc. The Lyapunov analysis tool enables us to analyze cooperative processors as "stable and within what percentage of optimal" under a wide range of failure scenarios (under which a centralized system may likely have catastrophically failed).
The Lyapunov analysis tool enables us to analyze cooperative processors as "stable and within what percentage of optimal" under a wide range of failure scenarios (under which a centralized system may likely have catastrophically failed). 
10366

Low-Power, Reduced-Computation, PublicKey Protocols

C. L. Beaver, T. J. Draelos, R. A. Gonzales, R. D. Miller, R. C. Schroeppel, E. V. Thomas

Public-key cryptography can be used to provide privacy, data authentication, and nonrepudiation. The advantage of public-key cryptography is the ease of key management in terms of the amount of data that must be protected (i.e., private keys). For instance, a single authenticated message can be verified by multiple parties, each of whom only require access to the appropriate public key. This is a very advantageous feature in remote monitoring applications in support of multilateral treaties. However, many practical applications of public-key mechanisms may require a reduction in the computational intensity. One or more parties may be computationally limited. No solutions to this problem of computational intensity exist, and while key management issues are minimized in public-key applications, they are not inconsequential. System developers will often use symmetric or private-key techniques where power or computational capabilities are limited and then re-encrypt or reauthenticate at some intermediate system. Unfortunately, this mechanism requires that the intermediate system be unconditionally trusted. In addition, the data are authenticated (in the case of authentication) only from the intermediate system, not from the data originator. Therefore, source-to-sink data authentication, while desirable, is often difficult to achieve. Sandia can enhance its current cryptographic capabilities and leadership in defense and industry by developing an innovative solution(s) to these problems. This project proposes to research and develop mechanisms that will enable the use of public-key cryptography in low-power and/or reduced-computation environments by investigations in several technical areas, thereby enhancing the chances of project success.

Our criteria for choosing a suitable signature algorithm for the low-powered environment included signature size and computation and storage required for computing the signature. We continued to look at various algorithms, including the hidden field equations (HFE) algorithm based on the difficulty of solving a system of quadratic polynomials over finite fields. However, we focused this year on our best candidate: an elliptic curve implementation of the El Gamal digital signature. 
There were several optimizations made to the algorithm based on an innovative elliptic-curve point-halving technique created earlier. In addition there were optimizations in the underlying finite field arithmetic — by choosing our field appropriately, we could reduce computation efforts considerably.

We tested the feasibility of some of our candidate algorithms by implementing them in software and considering the computation time required. We concentrated on the implementation of our elliptic-curve version of the optimal El Gamal. We developed the primitives in software, and this was instrumental in testing our hardware design of the same algorithm.

Our primary choice candidate (elliptic curve El Gamal) was developed for hardware applications. Taking into consideration low-power design techniques, we used VHDL (VHSIC [very high speed integrated circuit] Hardware Description Language) to capture the functionality of the public-key cryptography operations and algorithms. We developed a set of basic mathematical functions in VHDL, which we used to build the elliptic-curve algorithms. We developed the operations for both 89-bit and 178-bit keys. The chip was not fabricated since it did not seem appropriate to do so, but the design and code are available upon request and ready for integration.
There were several optimizations made to the algorithm based on an innovative elliptic-curve pointhalving technique created earlier. In addition there were optimizations in the underlying finite field arithmetic - by choosing our field appropriately, we could reduce computation efforts considerably. 
10367

Ten-to-One-Hundred-Gigabit/Second Network-Enabling R\&D

L. B. Dean, L. G. Pierson, P. J. Robertson, E. L. Witzke

The next major performance plateau for high-speed, long-haul networks is at $10 \mathrm{~Gb} / \mathrm{s}$ (OC-192). Data visualization, high-performance network storage, and distributed massively parallel processing (MPP) demand these (and higher) communication rates. Previous research has shown that MPPto-MPP distributed processing applications and MPP-toNetwork File Store applications already require singleconversation communication rates in the range of 10-to-100 Gb/s. MPP-to-Visualization Station applications can already utilize communication rates in the 1-to-10-Gb/s range. Standards are only now emerging to meet the needs of distance-insensitive, very high bandwidth, secure computer communications. There are critical technology gaps in the components and systems required to collaboratively develop and deliver such information in a secure fashion. Some of these are (1) framing asynchronous transfer mode (ATM) cells into synchronous optical network (SONET) payload envelopes, (2) segmentation and re-assembly at different speed regimes, (3) inverse multiplexing of ATM and its parallelization features, (4) assured scalability of ATM/SONET architectures, and (5) maturation of standards to these ends. Implementations of communication systems that carry 10 to $100 \mathrm{~Gb} / \mathrm{s}$ exist only as laboratory curiosities. Expensive $10 \mathrm{~Gb} / \mathrm{s}$ (SONET OC-192) optical multiplexers are now on the market, but these do not support "single-conversation" computer-to-computer communication. To enable practical "single-conversation" communications in the range of 10 to $100 \mathrm{~Gb} / \mathrm{s}$, the manufacturing cost of 2.5, 10, 40, etc., Gb/s electrooptics and dense wavelength division multiplexing (DWDM) equipment costs must be greatly reduced, and methods of efficiently processing communication protocol functions in parallel must be developed. Toward this end, this project will concentrate on the development of the 10 and $40 \mathrm{~Gb} / \mathrm{s} \mathrm{I/O}$ (input/output) interfaces required to mate physical-layer (PHY) integrated circuits (ICs) to higher-layer protocols.

The activities of this research project focused on extending a UTOPIA-like interface to perform variable-length transfers as well as fixed-length cell transfers, and the scaling of this extended interface to achieve data throughputs
The next major performance plateau for high-speed, longhaul networks is at $10 \mathrm{~Gb} / \mathrm{s}$ (OC-192). Data visualization, high-performance network storage, and distributed massively parallel processing (MPP) demand these (and higher) communication rates....this project will concentrate on the development of the 10 and $40 \mathrm{~Gb} / \mathrm{s} \mathrm{I/O}$ (input) output) interfaces required to mate physical-layer (PHY) integrated circuits (ICs) to higher-layer protocols. 
equivalent to SONET OC-768 and higher. Cost and complexity trade-offs for high-speed network interfaces were studied, presented, and discussed in the Technical Committees of both the Asynchronous Transfer Mode (ATM) Forum and the Optical Internetworking Forum (OIF). We developed innovative "in-channel" signaling for flow control, thus reducing the complexity of the interface. A central issue is the cost and reliability trade-offs for the number of parallel pins/ conductors required to keep the data transfer clock rate at a feasible level. Advances in low-voltage differential signaling (LVDS) electrical I/O technology have made greatly increased clock rates feasible, thereby reducing the cost (number of parallel connections) and increasing reliability. Yet, generating and communicating these LVDS signals through relatively large circuit board distances and connectors with sufficiently low data skew and reflections push the state of the art. We developed prototype designs using these innovations for scalable $2.5 \mathrm{~Gb} / \mathrm{s}, 10 \mathrm{~Gb} / \mathrm{s}$, and $40 \mathrm{~Gb} / \mathrm{s}$ parallel interfaces. We presented one of these designs to the ATM Forum for standardization as the UTOPIA Level $410 \mathrm{~Gb} / \mathrm{s}$ Interface Specification; after deliberation, it became an approved standard. A similar interface scaled for operation at $40 \mathrm{~Gb} / \mathrm{s}$ was presented and is under consideration within the ATM Forum and also within the OIF. Rapid maturation and industrial adoption of these standards will facilitate early deployment of ultrafast communication and encryption technology needed for the high-speed secure network infrastructure required by the Department of Energy, other government agencies, and the burgeoning network industry.

Rapid maturation and industrial adoption of these standards will facilitate early deployment of ultrafast communication and encryption technology required by the Department of Energy, other government agencies, and the burgeoning network industry.

\section{Other Communications}

Campbell, P. L. 1999. "An Implementation of the Berlekamp-Massey Linear Feedback Shift-Register Synthesis Algorithm in the C Programming Language." Sandia Technical Report SAND99-2033, Sandia National Laboratories, Albuquerque, NM (August).

Campbell, P. L., and L. G. Pierson. 1999. "LFSRs Do Not Provide Compression." Paper proposed to the Data Compression Conference, Snowbird, UT, 20-22 March 2000. SAND99-2892 (December).
Pierson, L. G. 2000. "Tip Sheet for UTOPIA Level 4: Table of Contents." Paper presented to the ATM Forum Technical Committee (Vienna, VA, 17 October). SAND2000-2399.

Pierson, L. G. 2000. "Ultra-High-Speed Encryption Research at Sandia National Laboratories." (Invited) Paper presented to Seminar at IBM Watson Research Center (Hawthorne, NY, 10 October). SAND20002478C.

Witzke, E. L. 1999. "Super Gigabit-Per-Second Secure, Compressed Communications." Paper presented to the Supercomputing '99 Conference, Portland, OR, 15-18 November. SAND99-2909C. 
Witzke, E. L., and T. D. Tarman. 2000. "Proposed

Foreword to the ATM Security Specification Version

1.1." Paper presented to the ATM Forum Technical

Committee (San Francisco, CA, 7-12 May).

SAND2000-1053C. 
10767

\section{Intrusion Detection for Asynchronous} Transfer Mode (ATM) Networks

T. D. Tarman, R. L. Hutchinson, K. C. Bauer, W. F. Young, E. L. Witzke

Currently, network intrusion-detection (NID) systems are in place in networks at Sandia and its customers. NID systems allow site security personnel to monitor network devices and hosts for activities that indicate a possible attack, misuse, or operational anomaly in the information system. Since NID systems protect against attacks from inside and outside users, they provide a complementary service to devices that protect internal networks against outside attacks (e.g., encryptors and firewalls). However, current NID systems work only down to the Internet Protocol (IP) level. Data and control flows below IP are not covered by the current technology. This represents a risk to Asynchronous Transfer Mode (ATM) network backbones, which are found inside many enterprises (including Sandia), public carriers, and the Internet. This makes ATM networks vulnerable to a variety of denial-ofservice attacks, routing infrastructure attacks, and other attacks.

This project is developing an ATM intrusion-detection system that monitors the signaling flows associated with a variety of ATM protocols (including virtual circuit setup, call routing, local area network [LAN] emulation, etc.) to determine if insider or outsider attacks are being conducted against the ATM network. This project will research and document issues such as analysis of ATM protocol attacks, sensor performance on high-bandwidth links and switch fabrics, and scalability. We will develop prototype ATM intrusion-detection systems that incorporate methods that address these specific concerns.

Sandia is uniquely qualified to make significant contributions to this area due to its recognized leadership in ATM security and high-speed encryption, and in-depth experience with ATM protocols and IP NID. Results from this work will lead to key technologies that can be incorporated into the ATM intrusion-detection devices for Sandia and DOE technologies. This work will also enhance Sandia's leadership in ATM security.

We made much progress in the development of a proofof-concept software-based ATM intrusion-detection system.
This project is developing an ATM intrusion-detection system that monitors the signaling flows associated with a variety of ATM protocols...to determine if insider or outsider attacks are being conducted against the ATM network.

Sandia is uniquely qualified to make significant contributions to this area due to its recognized leadership in ATM security and high-speed encryption, and indepth experience with ATM protocols and IP NID.

Results from this work will lead to key technologies that can be incorporated into the ATM intrusion-detection devices for Sandia and DOE technologies. 
We selected and documented an architecture for the intrusion-detection system. This architecture uses a number of ATM-specific sensors that monitor routing message flows, virtual circuit signaling flows, and Simple Network Management Protocol (SNMP) trap information. To accommodate this variety of sensors, we developed a common communication protocol, sensor message formats, and communication library functions. This protocol will allow the addition of new sensor types (e.g., hardware-based sensors) in the future.

We selected an assessment engine to correlate intrusion sensor events and to determine whether an attack is occurring in the network. This assessment engine provides a flexible rule specification language, which allows the end user to specify custom attack templates and responses to verified attacks. These rules are specified in a text file, which is generated manually or via a JAVA graphical user interface (GUI).

We developed a template for a simple but interesting attack scenario. While researching attack scenarios, we met with a researcher from Arizona State University to discuss his findings. We used this attack scenario to show successful detection of an ATM network attack. The prototype intrusiondetection system used software-based network event sensors, along with the assessment engine and rule generator described above.

To accommodate this variety of sensors, we developed a common communication protocol, sensor message formats, and communication library functions. This protocol will allow the addition of new sensor types (e.g., hardwarebased sensors) in the future.

\section{Refereed}

Tarman, T. D., and E. L. Witzke. 2000. "IntrusionDetection Considerations for Switched Networks." Proc. SPIE Photonics East 2000, accepted.

\section{Other Communications}

Kellogg, B. R. 2000. "Intrusion-Detection Systems for Asynchronous Transfer Mode (ATM) Networks." Paper presented to the Student Intern Program Symposium, 10 August, Sandia National Laboratories, Albuquerque, NM. SAND2000-1866.

Tarman, T. D. 2000. "Asynchronous Transfer Mode Intrusion Detection and Prevention." (Invited) Paper presented to the Banking Industry Technical Symposium, Santa Fe, NM, 9 August. SAND990518C.
Tarman, T. D. 2000. "Sandia's Straw Ballot Comments on the Security Version 1.1 Specification." Paper presented to the ATM Forum Technical Committee, San Francisco, CA, May.

Tarman, T. D., and M. Laurent (ENST, Bretagne, France). 2000. "Security Services Negotiation Through Operations and Management (OAM) Cells." Paper presented to the ATM Forum Technical Committee, San Francisco, CA, May.

Witzke, E. L., and T. D. Tarman. 2000. "Proposed Foreword to the ATM Security Specification, Version 1.1." Paper presented to the ATM Forum Technical Committee, San Francisco, CA, May. 
10768

Conversational Policies for Multiagent Systems

N. M. Berry, C. M. Pancerella

The field of software multiagent systems is considered to be one of the most important and exciting areas of research and development in computer science today. This technology is applicable to a diverse number of problem domains, including information retrieval and filtering, enterprise integration, and manufacturing systems. The current popularity and growth of multiagent systems is due to its diverse application and the widespread use of distributed and Internet-based computing. The success of these systems is based on their ability to use communication to successfully achieve interoperability, to exchange knowledge, and to establish social order between the systems. However, their continued success is being hampered by the inability of multiagent systems to deliver on their promise of real interoperability. The problem resides in the agent's ability to concisely understand messages consisting of verbs, nouns, adjectives, and conversation policies. A conversation consists of a collection of ordered messages between two or more agents designed to accomplish some goal. A conversation policy governs how an agent understands and responds to the messages in the conversation.

Conversation policies are key in managing communication, and communication is the foundation for achieving interoperability within and between multiagent systems. Sandia believes that the influence of a conversation policy extends beyond communication and also includes the agent's internal goal-driven behavior. These behaviors influence an agent's decision-making process by constraining or scoping the exchange of knowledge and the reasoning process within the agent.

This project focuses on the development and evaluation of conversation policies, to address issues concerning (1) the selection of conversation policies, (2) conversation policies and system communication behavior, and (3) the effects of conversation policies on agent reasoning. By addressing these issues we will uncover important principles, criteria, and concepts that are applicable to agent-based system development both at Sandia and elsewhere.

We hypothesized that there is a direct link between agent conversation and the autonomy of the agent, which is
This project focuses on the development and evaluation of conversation policies, to address issues concerning (1) the selection of conversation policies, (2) conversation policies and system communication behavior, and (3) the effects of conversation policies on agent reasoning. By addressing these issues we will uncover important principles, criteria, and concepts that are applicable to agent-based system development both at Sandia and elsewhere. 
implemented in the control/reasoning structure of the agent. To understand these influences, we must detect and isolate certain control factors and criteria in an agent for experimentation. We are employing an agent model based on an agent template design because it permits isolation of different agent functionality into different orthogonal layers (particularly conversation, control, and application layers) and allows us to manipulate each different functionality. The conversation protocol layer determines the order of the performatives within a given conversation based on predefined state machines, and also allows agents to put policies and constraints on conversations. The application layer contains the implementation of the domain-dependent agent functionality.

The control layer contains the planning library, knowledge bases, and proactive behavior library used in the decision-making processes of the agent. Within the planning and proactive behavior libraries reside heuristics (rules) that are clustered within predefined plans. These heuristics permit the agent to maintain a certain level of autonomy over its reactions to both requests and environmental changes.

We made significant progress in understanding the limitations and directions of conversation policy research. We also pursued research into the area of agent autonomy, which has proven to be somewhat less understood by the overall community.

We completed the preliminary agent model with control
We made significant progress in understanding the limitations and directions of conversation policy research. We also pursued research into the area of agent autonomy, which has proven to be somewhat less understood by the overall community. factors. 
10791

\section{Fuzzy Data Mining}

S. M. Deland, C. L. Jenkin, L. A. Kennicott

The use of remote and/or unattended sensor-based monitoring systems offers considerable benefits in terms of the information they can provide as well as possible cost benefits. However, these systems present a classic information overload problem to the user: The data are voluminous and at such a low level that the significance of any given event (say a temperature reading) is not obvious. Sophisticated techniques are needed to extract "normal" patterns in the data and to isolate and characterize "abnormal" patterns. Such deviation detection would be very useful in identifying changes in operational status of the buried or hardened targets as well as for damage assessment after an attack on such a facility. In addition, this work will significantly increase our ability to detect undeclared activities at monitored nuclear facilities.

Data mining is an emerging technology that uses artificial intelligence (AI) techniques to identify patterns in large data streams and thus is clearly applicable to identifying patterns in large sensor datasets. Fuzzy set theory permits membership in more than one set; it is useful for quantifying uncertainty or allowing alternative interpretations. This type of mathematical formulation is promising for addressing two key challenges in applying data mining techniques to a raw sensor data stream: deciding when two events are similar enough to be treated as arising from the same source and separating interspersed events from many simultaneous processes. We will combine these two approaches into fuzzy data mining to develop new pattern identification algorithms that are well suited to the data streams from sensor-based monitoring systems.

We obtained and mined datasets from the unattended monitoring systems fielded to guide our thinking and to test ideas. One such dataset comprises some 11,000 events from five radiation sensors being used to monitor the transfer of spent fuel from a cooling pond to long-term dry storage at a reactor facility. Another dataset is approximately 6 months of data taken from a simulated storage bunker at Sandia. The bunker is instrumented with a door switch, two motion detectors, two video cameras, and 50 "T-1" multisensor item monitors that can be attached to items of interest with a fiberoptic seal and that report seal status, motion, and temperature
Data mining is an emerging technology that uses artificial intelligence (AI) techniques to identify patterns in large data streams and thus is clearly applicable to identifying patterns in large sensor datasets....We will combine these two approaches into fuzzy data mining to develop new pattern identification algorithms that are well suited to the data streams from sensor-based monitoring systems. 
of the item. They also report state-of-health information including battery voltage. The bunker data differ from the radiation data in that they contain a mixture of discrete events and continuous measurements. These two datasets have been analyzed manually by others, providing us with a good understanding of what patterns to expect in the data.

We explored different approaches (several data-mining techniques, fuzzy logic, and Asynchronous Transfer Modes [ATMs]) as we familiarized ourselves with these datasets. We are emphasizing unsupervised learning methods, applicable to broad classes of sensors. Our approach is to make use of pattern recognition (PR) techniques, specifically to identify significant patterns ("motifs") in the sequential data and to extract attributes from these motifs that can be mined. These motifs can then be aggregated to higher levels of abstraction to automatically recognize repeating patterns. Fuzzy logic will assist in determining closely similar but not identical patterns to account for variations in physical processes that are commonly found in sensor data.

We wrote pattern-detection techniques for time-ordered data to extract the significant "events" or "features." We explored Hidden Markov Models and two different dynamic time warping (DTW) algorithms in some detail. We are focusing our efforts on a DTW algorithm, which shows promising results thus far. DTW is an algorithm for removing timescale variations in time series data and identifying which features contribute most toward deciding if curves are similar. We obtained an implementation of this algorithm and are exploring how to modify it to apply to our datasets.

Our approach is to make use of pattern recognition $(P R)$ techniques, specifically to identify significant patterns ("motifs") in the sequential data and to extract attributes from these motifs that can be mined. These motifs can then be aggregated to higher levels of abstraction to automatically recognize repeating patterns. 


\section{Directed Energy}

The Directed Energy investment area researches concepts and technologies for advanced directedenergy systems and applications. In the broadest sense, directed energy deals with the active projection of energy over a distance for weapons, sensors, and materials-processing applications.

Research in this investment area addresses novel solutions for the (1) destruction, disruption, or defeat of adversary systems, (2) active imaging or other sensing applications, (3) processing methods such as chemical/biological decontamination or materials processing/ inspection, and (4) technology for protection against adversarial use of directed energy against U.S. systems.

Sandia is developing a method for decontaminating biological materials to improve our nation's capabilities for countering biological weapons hazards. The "Surface Decontamination of Bacterial Protein Toxins by RF Power" project is

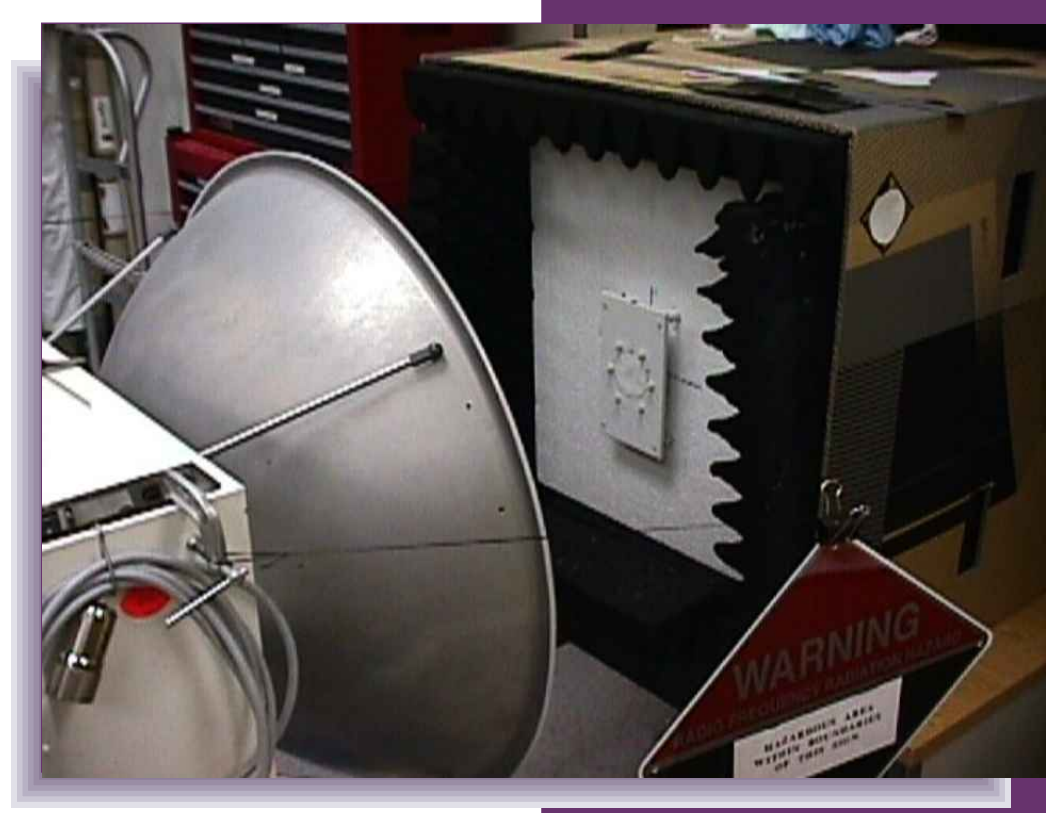

exploiting radio frequency electromagnetic radiation as a method of decontamination. This method requires no water, no cleanup, and no drying, and offers very fast inactivation of bacterial protein toxins. In addition, this method penetrates joints, bearings, and similar complex surfaces. In addition, the method is energyefficient, leaves no toxic residue, and may be applied either by hand-held antenna, with a cartmounted power source, or by a transportable multimode cavity. A patent disclosure has been filed on this process. 
10368

\section{Surface Decontamination of Bacterial Protein} Toxins by RF Power

R. P. Toth

This project is based on a radio-frequency $(R F)$

electromagnetic (EM) (nonionizing radiation) method of decontamination. The method requires no water, no drying, and no cleanup, and offers very fast inactivation of bacterial protein toxins. Further, it penetrates joints, bearings, etc. The photon energy is so low that neither photocurrents nor electrostatic discharge effects are produced; thus, sensitive equipment is not at risk.

The method operates by irreversible alteration of the tertiary (conformational) state of biomacromolecules. It is energy efficient, leaves no residue, and may be applied either by scanning (as was the case here) or all at once in a multimode cavity.

We developed and tested the RF system, which comprises the RF power source, near-field focused antenna, scanner, and test cell.

We developed assay methods for quantification and test of inactivation using a toxin surrogate, acetylcholine esterase. We verified inactivation by enzyme kinetic analysis. Procedural controls included inoculation onto and recovery from surfaces, drying/rehydration of enzyme solutions, and RF exposure. Positive controls for inactivation included denaturation and conjugation with organophosphate. We also selected the actual toxin-Kanagawa toxin, a product of Vibrio parahaemolyticus - and assay method — colorometric based on $\mathrm{HGb}$ catalysis of an oxidation reaction that absorbs $600 \mathrm{~nm}$ light - to be used in the next task.

We found that the drying of enzyme solutions reduced activity by only $50 \%$ rather than the $99 \%$ expected. The RF exposures further inactivated the dried enzyme by about another factor of ten. The organophosphate inactivated the enzyme less than the RF exposure. This is in spite of the short dwell time (100 $\mathrm{msec}$ ) of the RF as it was scanned over the surface. Denaturation with exposure to dry heat of $150^{\circ} \mathrm{C}$ for 10 minutes produced two-log inactivation.
This project is based on a radiofrequency $(R F)$ electromagnetic (EM) (nonionizing radiation) method of decontamination. The method requires no water, no drying, and no cleanup, and offers very fast inactivation of bacterial protein toxins. Further, it penetrates joints, bearings, etc. The photon energy is so low that neither photocurrents nor electrostatic discharge effects are produced; thus, sensitive equipment is not at risk. 
10369

Intense White-Light Pulse Propagation in Air Using Self-Guided Optical Filamentation: Applications to Remote Sensing and Countermeasures

S. M. Cameron, A. C. Bernstein, A. L. Sobel, D. E. Bliss, T. S. Luk

Anomalous laser channeling launched by high-intensity femtosecond lasers above the self-focusing critical power is a new phenomenon that will dramatically shift conventional paradigms by eliminating fundamental limitations of diffractive imaging on resolution and projected spectral brightness. Dynamic stabilization of the laser channel is attributed to a balance between the Kerr self-focusing nonlinearity in air and the combined effects of normal diffraction and refractive defocusing from the induced ionization column, which act synergistically to produce a longitudinal waveguide or moving focus structure for robust power transmission. Self-phase modulation of the transmitted optical pulse produces a conical white-light emission signature with a divergence angle and spatio-spectral pattern dependent on the evolving chirp and bandwidth as a function of propagation distance of the trapped self-focusing state. By using adaptive pulse shaping and dispersion compensation to manipulate onset and stability of the self-focused beamforming process, optical energy can be concentrated in a prescribed focal volume at predetermined range for remote sensing measurements and high-field material interactions. When the self-guiding light filament interacts with a targeted material, the electric field in the photoconductive channel can induce a transient chargedisplacement dipole and intense electromagnetic pulse (EMP). Power compression and impulse loading can be accomplished in a time significantly shorter than the hydrodynamic expansion or thermal diffusion response time of materials leading to unique shock, spallation, and dynamic fracture mechanisms. Strong enhancement of light intensity and associated properties in the channel affords new classes of applications for DOE and DoD missions that are inaccessible with conventional laser technology, including EM disrupt of power lines feeding underground facilities, remote optical tagging, active multispectral sensing, and non-attributable countersensor interdiction.

We diagnosed effects of nonlinear dispersion and ionization on channel self-phase modulation and continuum
Strong enhancement of light intensity and associated properties in the channel affords new classes of applications for DOE and DoD missions that are inaccessible with conventional laser technology, including EM disrupt of power lines feeding underground facilities, remote

optical tagging, active multispectral sensing, and nonattributable countersensor interdiction. 
generation as a function of initial laser conditions to establish operational limitations for projection and remote sensing. We performed systematic laboratory experiments to characterize and visualize the evolution of critical constitutive parameters underlying self-focusing phenomenology and to benchmark and validate predictive models. We also performed experiments investigating the relationship between anomalous group velocity dispersion in air and channel conductance and conical emission characteristics (spatio-spectral content, divergence) at distance. We accomplished extended atmospheric propagation experiments to $\sim 1 \mathrm{~km}$ using an upgraded terawattt laser and rooftop laser radar facility. We applied open-loop deformable optics and fixed-aperture masks to the transverse spatial intensity profile of the laser to improve laser-filament energy coupling and exercise spatial control, including dual-filament cross-modulation. We measured x-ray yield, shock impulse, ablation, and EMP production resulting from laser-filament interaction with materials for a variety of operating conditions and lethality scenarios. We tested new white-light multispectral remote sensing and optical tagging concepts using the unique properties of short-pulse lasers.
We accomplished extended atmospheric propagation experiments to $\sim 1 \mathrm{~km}$ using an upgraded terawatt laser and rooftop laser radar facility.

\section{Refereed}

Bernstein, A. C., T. S. Luk, T. R. Nelson, J. C. Diels, and S. M. Cameron. 2000. "Experimentally Observed Spatio-Temporal Dynamics of a Self-Focusing Ultrashort Pulse in Air." CLEO/QELS 2001, submitted.

Borisov, A. B., S. M. Cameron, T. S. Luk, T. R. Nelson, A. J. Van Tassle, J. Santoro, W. A. Schroeder, Y. Dai, J. W. Longworth, K. Boyer, and C. K. Rhodes. 2000. "Bifurcation Mode of Relativistic and ChargeDisplacement Self-Channeling." J. Phys. B, submitted.
Nelson, T. R., A. B. Borisov, K. Boyer, S. M. Cameron, J. W. Longworth, T. S. Luk, A. McPherson, W. A. Schroeder, J. Santoro, A. J. Van Tassle, and C. K. Rhodes. 2000. "L-Shell Emission from High-Z Solid Targets by Selective Inner-Shell Collisional Electron Ejection." J. Phys. B, submitted.

Nelson, T. R., T. S. Luk, A. C. Bernstein, and S. M. Cameron. 2000. "Laser Filamentation of a Femtosecond Pulse in Air at 400 nm." CLEO/QELS 2001, submitted.

Luk, T. S., T. R. Nelson, and S. M. Cameron. 2000.

"Conical Emission Properties Associated with Atmospheric Self-Focusing Femtosecond Pulse Propagation." CLEO/QELS 2001, submitted. 
10370

\section{HPM Vulnerability Assessment and Tests}

G. J. Denison, L. D. Bacon, G. M. Loubriel

High-power microwave (HPM) technology has matured to the point where practical electromagnetic (EM) weapons are becoming technically feasible. One opportunity for this type of directed energy $(D E)$ is the attack on our facilities resulting in upset and/or damage of electrical and electronic equipment, particularly computers, cellular phones, and radio systems. In this project, Sandia concentrated on one type of system: handheld radios. The major initial challenges are modeling and simulation and the development of a source designed for closerange, in-chamber effects research on target electronics. Further, we will assess electronic system susceptibilities to HPM attack causing system upset for one specific system with the view that this process can be applied to other, more complex, systems and scenarios. We will examine possible entry path(s) of EM energy into a critical system, its interactions with electronic circuits that may cause upset or latch-up leading to damage, and the EM parameter set (frequency, power level, pulsewidth, repetition rate, etc.) that exacerbates these tendencies. To understand this system's vulnerability at the component level, we tested specific circuit elements for damage. Once we determine HPM system requirements, we will carry out validation tests and work on critical components of an HPM system. Thus, our work consists of three aspects: (1) vulnerability assessment to determine HPM transmitter parameters, (2) development of an HPM system, and (3) modeling and simulation.

We made three major efforts to meet our objectives and to ensure success in the final tests, where we expect to test and understand the effects observed. Those efforts were (1) computer modeling and simulation of effects, (2) an experimental effort that included testing targets for effects using the systems that we previously designed and built, and (3) designing and building a new narrowband test system to better understand the observed effects. Finally, we added new systems to our test matrix, which now includes radios (spread spectrum), pagers, image intensifiers, and cellular phones.

We developed data analysis algorithm/software and performed initial tests. We modeled the system as a complete system and carried out computer simulations and compared them to some direct-injection experiments. We also modeled
In this project, Sandia concentrated on one type of system: hand-held radios. The major initial challenges are modeling and simulation and the development of a source designed for close-range, inchamber effects research on target electronics. Further, we will assess electronic system susceptibilities to HPM attack causing system upset for one specific system with the view that this process can be applied to other, more complex systems and scenarios. 
subsystems at higher frequencies for understanding new

effects. We developed a new narrowband cell for effects testing and purchased and tested image intensifiers, cell phones, and pagers. 
10720

\section{Proton Beam Directed-Energy Weapon}

M. G. Mazarakis, T. J. Renk

One of the most pressing U. S. emerging threats is the MACH-2 sea-skimming missiles that are expected to be widely available to opposing naval and shore offensive forces. The time available from detection to destruction is only 10-20 seconds. Terminal defense of high-value aircraft carriers against such sea-skimming missile attacks is presently nonexistent. A rapid-response, total-lethality, flexible-aiming, and firing defensive system is essential for continued protection of the U.S. naval fleets, and carrier battle groups in particular.

Sandia is working on a new type of directed-energy weapon based on high-energy proton beams. Not only could it be capable of destroying the missile, but it could also neutralize any biological or chemical agent before destruction.

Our research concentrated mainly on the feasibility of proton beam propagation in the atmosphere. We carried out analytical work and simulations with the codes IPROP and SPL to study the stability of the beam against resistive hose instabilities. The results are very promising. We discovered that a 3:1-radius tailored beam can propagate stably without appreciable radius growth. We introduced perturbations up to $30 \mathrm{~cm}$ wavelength to the beam. The perturbation amplitude increased initially but quickly saturated (stopped growing).

We propagated the beam numerically 30-50 meters in full pressure air. We observed an enhanced beam front erosion several times the classical inductive limit. We suspect that this may be due to the nonradius tailored beam utilized for the erosion study simulations.

We studied the multiple scattering of the beam protons with the air molecules (Nordsieking) analytically for very long propagation lengths. For the beam parameters chosen, 300 $\mathrm{MeV}$ energy, $10 \mathrm{kA}$ current, and $1 \mathrm{~cm}$ radius, the beam radius growth is not serious. After $1 \mathrm{~km}$ of propagation in fullpressure air, the beam radius increased only by a factor of two. However, the range of $300 \mathrm{MeV}$ beams is only $300 \mathrm{~m}$, so by the time the $100 \mathrm{~ns}$ train of pulses strikes the target in a holeboring mode, the beam radius increase due to multiple scattering should not be larger than one-third of the original
One of the most pressing $U$. S. emerging threats is the MACH-2 sea-skimming missiles that are expected to be widely available

to opposing naval and shore offensive forces....Sandia is working on a new type of directed-energy weapon based on high-energy proton beams. Not only could it be capable of destroying the missile, but it could also neutralize any biological or chemical agent before destruction. 
launched radius. In addition to beam propagation studies, we carried out further beam target interaction studies utilizing the simulation code TRIM with more realistic missile designs.

Given the high-energy and high-current density of the beam, we can deeply penetrate into missile structures and deposit enough energy in the high explosives (HE) to exceed the thermal initiation explosion threshold.
Given the high-energy and highcurrent density of the beam, we can deeply penetrate into missile structures and deposit enough energy in the high explosives $(H E)$ to exceed the thermal initiation explosion threshold. 
10772

\section{Intense Directed-Energy Theory Initiative}

J. S. Wagner, P. C. Gray, M. W. Trahan, C. L. Olson, M. G. Mazarakis

Sandia has previously achieved national recognition for leadership in end-to-end design, modeling, and simulation of directed-energy (DE) systems, including charged-particle beams (CPBs), high-power radio-frequency $(R F)$ and microwaves (HPMs), and hybrid laser-assisted CPB systems. This project will advance and leverage these never-superseded simulation methods to new national security applications. Applications will include difficult targets such as exoatmospheric ICBM (intercontinental ballistic missile) warhead-decoy discrimination and kill, endoatmospheric antimissile systems, difficult-target electromagnetic (EM) sources and signatures and their exploitation, and end-to-end accelerator designs supporting all DE applications.

Sandia's nearly abandoned simulation legacy includes a full suite of 1-D (one-dimensional), 2-D, and 3-D simulation codes with a variety of physics-based approximations ranging from simple macroscopic envelope models of beams to fully self-consistent microscopic electrostatic, magnetostatic, molecular hydrodynamics (MHD), and EM particle-in-cell (PIC) plasma codes.

To date, two especially relevant codes have been updated: a 3-D grid-optional magnetostatic code, "Buckshot," and a 2-1/2-D EM PIC code. Buckshot is Sandia's, and arguably the nation's, only 3-D particle code capable of studying both complex accelerator systems and long-range CPB systems end-to-end. We modified the fully EM PIC code to simulate EM sources and signatures that might result from DE interactions with plasmas and environments formed from membranes and surfaces for warhead-decoy intercept problems. We have begun a parallelization of "Buckshot" that will enable very large problems that were inaccessible in the 1980s. This concurrency will also enable the use of heuristic tools such as genetic algorithms to provide globally optimal accelerator designs and globally optimal signature exploitation for the first time.

We will direct future technical work toward (1) spaceenvironment ICBM discrimination and kill applications, (2) new unexplored ion-beam endoatmospheric standoff weapons in collaboration with a related project, (3) globally optimal
Sandia has previously achieved national recognition for leadership in end-to-end design, modeling, and simulation of directed-energy $(D E)$ systems, including charged-particle beams (CPBs), high-power radio-frequency $(R F)$ and microwaves (HPMs), and hybrid laser-assisted CPB systems. This project will advance and leverage these never-superseded simulation methods to new national security applications. 
end-to-end accelerator designs for all DE applications, and (4) active and passive EM signature generation and exploitation.

We met our simulation milestone by reconstructing two highly relevant simulation codes and their support and analysis diagnostics, and configured them for demonstration purposes.

The first, "Buckshot," is a fully 3-D particle code. Buckshot includes the features of (1) a multicomponent background of electrons and multiple ion species, (2) a beam of arbitrary charge, mass, energy, and current, (3) an applied magnetic field (could be but is not limited to geomagnetic), (4) an optional laser-ionized channel for studying laser-assisted or laser-guided CPB problems, and (5) inertial forces related to curvilinear beam lines.

We rebuilt the second, a 2-1/2-D EM plasma (particlein-cell) PIC code, with (1) multiple species (electrons and ions), (2) applied magnetic fields, (3) applied incident EM or laser pulses, and (4) pre-ionized surfaces and membranes.

We conducted demonstration simulations for both codes.

We used Buckshot to simulate a relativistic electron beam in the ionosphere. The beam is focused and guided by a laser pre-ionized xenon channel. Such a heavy-ion channel could be achieved in the ionosphere by deliberately prereleasing xenon $(\mathrm{Xe})$ gas to enhance beam transport for antisatellite and warhead discrimination/kill scenarios.

Historically, Sandia planned to ionize naturally occurring atomic oxygen.

We used the EM plasma code to simulate the interaction of an intense pulse of radiation incident on a thin membrane of pre-ionized plasma. We simulated the spectral (wavelength, phase, and direction) content of the incident, reflected, and transmitted fields. Spectral signatures may contain discriminating information that could be exploited in differentiating decoys from warheads.

\section{Other Communications}

Wagner, J. S. 2000. "Directed-Energy Research at Sandia National Labs." Paper presented to Phillips Lab management at Sandia National Labs, Albuquerque, NM, 7 June.
We used the EM plasma code to simulate the interaction of an intense pulse of radiation incident on a thin membrane of pre-ionized plasma. We simulated the spectral (wavelength, phase, and direction) content of the incident, reflected, and transmitted fields. Spectral signatures may contain discriminating information that could be exploited in differentiating decoys from warheads. 


\section{Sensing and Intelligent Controls}

The Sensing and Intelligent

Controls investment area investigates advanced sensor and algorithm technologies to address a variety of national security problems. Advances in sensors and algorithms are key to creating systems that can reason as well as navigate and manipulate complex environments. Potential applications of the results of this research include battlefield applications, nonproliferation issues, landmine detection, and environmental cleanup activities.

Sensing and Intelligent Controls research responds to the Laboratories' needs in the following areas: (1) physical imaging of difficult targets, (2) distributed sensor systems, (3) distributed command and control of distributed military assets, (4) algorithms and sensing for autonomous navigation in complex, unstructured environments, (5) dexterous manipulation and dexterous robotics, and (6) chemical and biochemical sensing of complex mixtures.

The "Dispersible Granular Sensor (Smart Sand) for Landmine Detection Based on TNT Immunoassay" project is investigating remote sensor technology capable of rapidly screening large areas for the presence of mines or other unex-

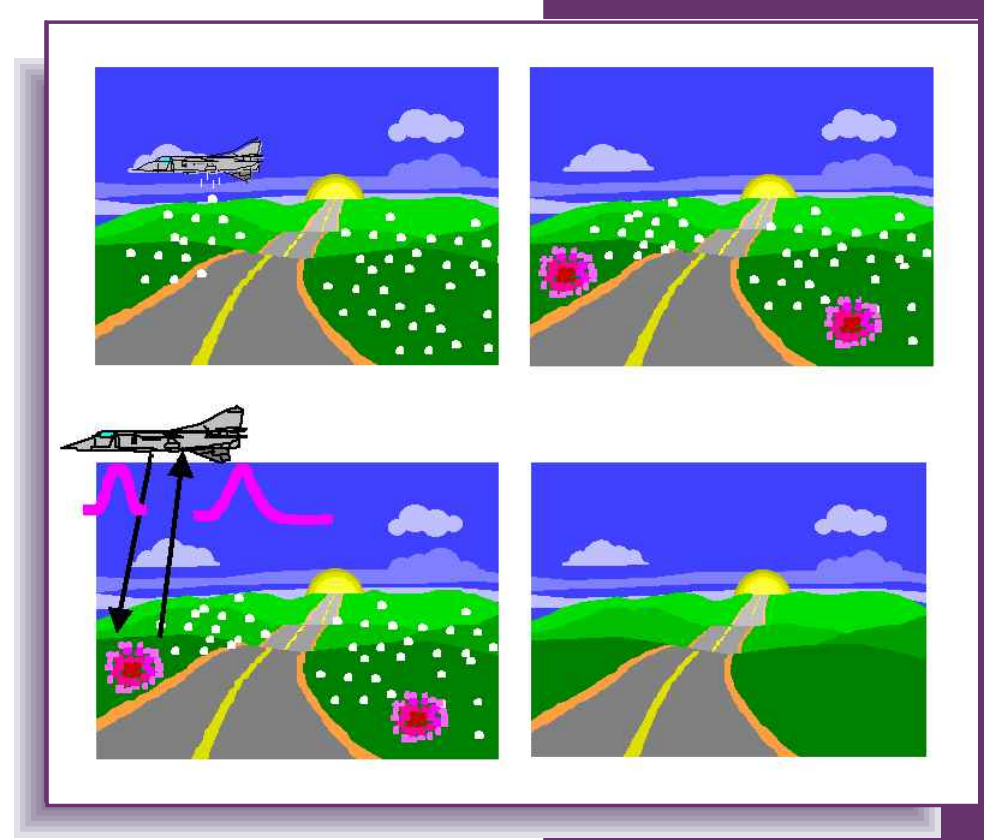

ploded ordnance (UXO). The results of this research have the potential to address the approximately 100 million unexploded landmines in the world today. An average of 2,000 people each month are killed or maimed by these landmines. This research effort is developing enzyme-based granular sensors that combine sample preconcentration, molecular recognition, amplification, and optical transduction in a single grain of "sand." Each "sand" sensor is dispersed by air and is capable of performing an immunoassay of its local environment. A luminescent signature is included to allow remote imaging of locations. A patent disclosure has been filed on this research. 
10371

\section{Miniature Bioaerosol Concentrator}

K. Wally, R. F. Renzi, D. J. Rader, R. P. Janek, R. W. Crocker, H. S. J. Lau, S. M. Ferko

The goal of this project is to develop a miniature bioaerosol concentrator capable of collecting biological warfare $(B W)$ agents from bioaerosols as diffuse as one pathogen particle per liter of air. To be effective with microseparations-based detectors, a minimum of 10 to 20 of these pathogen particles must be concentrated into as little as one microliter of collection solvent. Sandia is developing a concentrator device that employs a two-stage geometry to achieve its goals. The first stage is a microseparator that uses geometry to collect and concentrate the bioaerosol (particles in the 1- to 5-micron-size range) via inertial separation principles. We discard particles smaller or larger than this size range, as well as the bulk of the collected air. The second stage is a microimpinger that collects a directed, concentrated stream of size-segregated particles into a tiny amount of fluid within collection microchannels. We use electrokinetic (EK) flow phenomena to move the aqueous collection solvent through the open microchannels in the microimpinger. The microseparator that we are developing achieves inertial separation and concentration by employing the Opposed-Flow Virtual Cyclone (OFVC) geometry invented by Sandia, in part in response to the performance requirements of this project.

We designed and experimentally verified devices that focus and collect particles with diameters larger than 1 micron with a pressure drop of less than 1 inch of water, preferably lower. We performed numerical simulations and experimental tests to demonstrate two such particle focusing/collecting designs: the OFVC and the focusing impactor (FI).

The FI accelerates the air stream and suspended particles into a two-dimensional (2-D) converging wedgeshaped slit inlet. The particles tend to align with flow streamlines and are aimed toward the wedge vertex. At the exit of the converging section, inertia prevents larger particles from turning, so the air can be turned while particles continue toward the vertex. An impinger placed near the vertex can collect particles. We examined analytically and numerically the particle flow in the converging wedge region. We developed a closed-form analytical approximation based on Stokes drag law. We performed numerical simulations using the
The goal of this project is to develop a miniature bioaerosol concentrator capable of collecting biological warfare (BW) agent from bioaerosols as diffuse as one pathogen particle per liter of air....The

microseparator that we are developing achieves inertial separation and concentration by employing the Opposed-Flow Virtual Cyclone (OFVC) geometry invented by Sandia, in part in response to the performance requirements of this project. 
commercial finite-element (FE) code FIDAP and modeled a geometry. Numerical simulation also predicts pressure drop through the system. Simulations clearly show the potential for particle focusing and collection. Lower pressure drops require smaller throat dimensions than the 400-micron throat analyzed, which was selected to match how small we could accurately make parts with conventional machining. Micromachining of smaller slits would allow lower pressure drops.

We fabricated and tested a prototype FI with 400micron throat. We made pressure drop measurements for volumetric air flow rates between 3 and 15 Lpm [liters per minute]. The experimental data could be well approximated by $\mathrm{D} P=\mathrm{r}^{*} \mathrm{~V} 2$ where rho (r) is the gas density and V is the gas mean velocity through the throat. Numerical simulation is shown to be in excellent agreement with experimental data. Particle characterization tests using monodisperse drops of oleic acid conclusively demonstrated focusing. We made a complete characterization of particle collection efficiency for two flow rates, 5.37 and $7.35 \mathrm{Lpm}$.

We developed a design for a microfluid impinger in which fluid is moved by EK principles. Microchannels etched into a glass wafer are located at the vertices of the aerodynamic concentrators. Windows and slits micromachined into the two wafers that comprise the microimpinger allow a collection fan to pull air and particles into and through the microimpinger, turning the airflow symmetrically to either side of each microchannel. Development of the microimpinger wafer sandwiches have been hampered by problems in two areas. The first is wafer warpage resulting from laser micromachining. We are pursuing alternate fabrication techniques to avoid warpage. The second problem involves excessive evaporation with aqueous collection fluids. This has hampered our ability to perform EK testing in the open-top microchannels and has so far prevented any particle collection testing with prototype microchannels. We are examining use of alternate collection fluids, less volatile but still electrokinetically pumpable. Development to resolve these problems continues.
Numerical simulation is shown to be in excellent agreement with experimental data. Particle characterization tests using monodisperse drops of oleic acid conclusively demonstrated focusing. We made a complete characterization of particle collection efficiency for two flow rates, 5.37 and 7.35 Lpm. 
10372

\section{Recognizing Partially Obscured Targets by Combining Multiple Data Sources Using Evidential Reasoning}

M. W. Koch, D. A. Yocky, M. M. Moya, J. S. Salazar

Any sensor has limited detection capability and inherent sensitivities to false targets. Multiple sensors can provide complementary target information and improve detection and false alarm performance. Multiple sensors also can provide overwhelming amounts of data covering large geographical regions or many looks at a single area and quickly overwhelm a human analyst. Automating the target recognition process by using pattern-recognition $(P R)$ algorithms to combine the data from multiple sensors can assist analysts in identifying significant threats.

Sandia's overall goal is to exploit multiple sensors to reduce the false alarm and missed detection errors in recognizing obscured targets. We chose to investigate evidential reasoning (ER) as a method for combining the uncertain and disparate information from these multiple sensors. ER can handle missing and conflicting information and allows for fusion at the feature and target identification levels. Based on certain restrictions, we showed that ER's weight-of-evidence and combination rules are equivalent to the sequential probability ratio test (SPRT). This equivalence allows us to use the SPRT theoretical results for determining error rates, handling partial obscuration, and acquiring dependent data for ER.

Since SPRT fixes the error rates, we use the number of target and non-target no-calls to measure the fusion performance. Using actual synthetic aperture radar (SAR) and multispectral (MS) image data, we show no-call performance before and after fusion.

We developed algorithms for fusing information from multiple sensors at the feature level and the identification level. The algorithms can handle partially obscured targets and dependent knowledge sources. We initially investigated using ER for performing the fusion processes. For the two-class problem with consonant evidence, we discovered that the conversion to belief and evidence combination is equivalent to the SPRT. This equivalence allowed us to introduce many of the SPRT theoretical results for determining error rates, handling partial obscuration, and sending dependent data to ER.
We developed algorithms for fusing information from multiple sensors at the feature level and the identification level. The algorithms can handle partially obscured targets and dependent knowledge sources. 
The problem with some fusion systems is the possibility of getting worse results after fusion than before fusion. This issue does not occur in the SPRT since the SPRT keeps gathering observations only as long as the test is between the upper stopping boundary and the lower stopping boundary. Once the test goes above the upper boundary or below the lower boundary, a decision is made and no more observations are used. The upper and lower stopping boundaries are determined by the desired false alarm error rate and the desired missed detection error rate. Therefore, once a decision has been made, the addition of more observations will not change the outcome of the test or the desired error rates.

The focus-of-attention algorithm (FOA) forms the glue for holding the fusion process together. An FOA processes each image and identifies possible target locations. In general, FOAs are fast algorithms, but they tend to produce a large number of false alarms. Initially, the FOA hit locations indicate where to apply sensor-specific processing. In the final stage, the FOA hit locations determine which evidence in one sensor to associate with evidence from another sensor. We accomplish this by transforming the hit locations from each sensor into a common coordinate system.

There are three key ingredients necessary for developing a successful fusion system. The first ingredient is a set of knowledge sources (KSs) that represent image processing and $\mathrm{PR}$ algorithms tailored to a specific sensor and target. Based on an FOA's hit locations, we applied KSs to identify target subparts. For the SAR sensor, we used templates to describe the target at a specific aspect and depression. For the MS sensor we use endmembers or the pure signatures of the target materials to describe a target. By linearly mixing different amounts of the endmembers together, we can get the many different spectral pixels that may appear on the target. We use the residue or the amount of a pixel that cannot be described with the endmembers as evidence for a possible target pixel.

The second ingredient is the probabilistic modeling of the KSs. Here we need to determine the conditional probability density functions of evidence from the KSs given a target or non-target. For the SAR data, we modeled the target template data with a Gaussian distribution with mean and standard deviation from the template chips. For the SAR non-target data, we used a log normal distribution to model the extremely heterogeneous pixels coming from a non-target signature. For the MS residues we used a beta distribution.
The focus-of-attention algorithm (FOA) forms the glue for holding the fusion process together. An FOA processes each image and identifies possible target locations. In general, FOAs are fast algorithms, but they tend to produce a large number of false alarms. 
The third ingredient is many examples of target and non-target data for developing the probabilistic models and testing the fusion algorithms. The data are essential for comparing the actual and desired error rates.

Since we can fix the error rates in SPRT, we use the number of no-calls to determine the fusion performance. The no-call rate measures the fraction of targets or non-targets that still have a test value between the upper and lower decision boundaries after all the sensor data are processed. Using actual SAR and MS data, we show the no-call rates before and after fusing the two datasets together for partially obscured targets.

\section{Other Communications}

Koch, M. W., J. S. Salazar, and D. A. Yocky. 2000.

"Recognizing Partially Obscured Targets Using Sensor Fusion (OUO)." Paper presented to Sensor Fusion Meeting, Livermore, CA, 11 April.

Koch, M. W., J. S. Salazar, and D. A. Yocky. 2000. "Recognizing Partially Obscured Targets Using Sensor Fusion (OUO)." Presentation to Brown Bag Meeting, Sandia National Laboratories, Albuquerque, NM, 17 October.

Koch, M. W., J. S. Salazar, and D. A. Yocky. 2000. "Recognizing Partially Obscured Targets Using Sensor Fusion." Presentation to Fusion Integration Methods Meeting, Sandia National Laboratories, Albuquerque, NM, 12 October.

Koch, M. W., J. S. Salazar, and D. A. Yocky. 2000. "SAR and Multispectral Fusion for Recognizing Partially Obscured Targets." Paper presented to the Automatic Target Recognition Working Group Meeting, Albuquerque, NM, 24 October.
Since we can fix the error rates in SPRT, we use the number of no-calls to determine the fusion performance. The no-call rate measures the fraction of targets or non-targets that still have a test value between the upper and lower decision boundaries after all the sensor data are processed. Using actual SAR and MS data, we show the nocall rates before and after fusing the two datasets together for partially obscured targets. 
10373

\section{Computational Engineering of Sensor Materials and Integration with a Novel Biological Weapon Detection System}

D. C. Roe, S. Istrail, J. S. Schoeniger

The goal of this project is to design combinatorial peptides (CPs) for use as sensor materials in the detection of bioagents. A necessary step in sensor design is to discover a material that binds and molecularly "recognizes" a target biomolecule. CPs are a new class of molecular recognition materials (MRMs) based on small molecules that can be engineered to bind tightly and specifically to a target analyte.

They have a significant advantage over antibodies and enzymes in that they are much more rugged, cheaper, and more versatile. However, identifying highly selective CPs can be time-consuming since potentially $10^{12} \mathrm{CPs}$ may be possible, but only $10^{5}$ compounds can be tested at a time. Sandia will use computational modeling of molecular recognition to greatly enhance the efficiency of the sorting process to discover highly select CPs. In our approach, we will generate CP MRMs in a simplified three-step process. First, we use bead synthesis to create and evaluate an initial set of CPs specific for a target analyte. Second, we apply computer modeling to abstract the key interaction properties of the most promising candidates of the initial bead synthesis, and search a "virtual library" of all possible CPs for ones that contain those recognition features and thus may have improved detection properties. Third, we generate and test these designed CPs in a second bead assay. The final result will be CPs that are highly selective for a target analyte. The successful completion of this project will have several important impacts. The methodology developed will enable rapid generation for a broad range of other biological warfare $(B W)$ targets. The computational methods developed will be generally useful tools for all areas of molecular recognition.

We continued a collaboration with North Carolina State University (NCSU) to (1) synthesize and test a secondgeneration library that we designed to bind to Staphylococcal enterotoxin $B$ (SEB), and (2) perform selectivity and sequencing tests of top candidates from the results of this screen. NCSU completed the generation and testing of the second-generation CP library, which we designed using a reduced amino acid set. From this set we found that there were
Sandia will use computational modeling of molecular recognition to greatly enhance the efficiency of the sorting process to discover highly select CPs....The final result will be CPs that are highly selective for a target analyte. ...The methodology developed will enable rapid generation for a broad range of other biological warfare $(B W)$ targets. The computational methods developed will be generally useful tools for all areas of molecular recognition. 
35 positive beads that presumably bind to SEB. This is threefold more than the number of positive beads found in screening the first-generation CP library against SEB, demonstrating a success with our designed library. We sequenced these beads; 27 out of 35 sequences are known, but there are some continuing ambiguities with the remaining sequences, which we are currently re-analyzing. Once this is done, our collaborators at NCSU will resynthesize several sequences and verify binding affinity to SEB. ...we found that there were 35 positive beads that presumably

bind to SEB. This is threefold more than the number of positive beads found in screening the first-generation CP library against $S E B$, demonstrating a success with our designed library. 
10374

\section{Biological Weapon Detector Using Bioaffinity} Array Impedance Analysis with Chemical Amplification Through Redox Recycling

A. W. Flounders, R. P. Janek, J. S. Schoeniger, K. Wally, R. C. Hughes, S. H. Kravitz, S. M. Brozik

Sandia investigated a novel structure for ultrasensitive detection (one particle per liter of air) of biological warfare (BW) agents, e.g., anthrax. We based the structure on surfaceattached specific affinity components (antibodies, combinatorial peptides [CPs], or glycolipids) that capture a particle on one element of a microelectrode array. We monitored the microelectrode array via impedance analysis, chemically amplified through disruption of redox recycling. We monitored Faradaic impedance by using a reversible redox couple (e.g., [Fe(CN)6]-3/-4) recycled between its oxidation states by each microelectrode and a shared counter electrode. Capture of a single pathogen blocks one microelectrode, disrupting the redox cycle at that electrode. An array of pathogen-sized, individually monitored, small-area $\left(2 \times 2 \mu m^{2}\right)$ microelectrodes enables single-particle detection. Many identically coated array elements (100s-1000) covering one surface of the flow cell enhances the probability of singleparticle capture and provides interferant discrimination by spatio-temporal tracking of the capture/release of each particle traversing the cell. Small capture area is key to single-particle detection; a multi-element array is key to rapid response, realistic sample volume, and improved discrimination.

This project demonstrated the merit of several unique sensor strategies, specifically matching length scale of the detector to that of the analyte, disrupting an established redox current, and integrating microelectrodes with an array of charge-storage devices. However, the project reinforced the need for significant improvements in mass transfer of analyte to sensor surface for any sensor attempting to capture extremely dilute samples from a flowing sample stream.

Discriminatory capture of a specific particle against a background of interferant particles is key to sensor success. The proposed sensor was dependent on the recognition ability of antibodies developed against specific species of Bacillus spores. We obtained antibodies specific for a Bacillus anthracis surrogate organism, Bacillus subtillis (Bs), in collaboration with the University of Alabama-Birmingham.
This project demonstrated the merit of several unique sensor strategies, specifically matching

length scale of the detector to that of the analyte, disrupting an established redox current, and integrating microelectrodes with an array of charge-storage devices. However, the project reinforced the need for significant improvements in mass transfer of analyte to sensor surface for any sensor attempting to capture extremely dilute samples from a flowing sample stream. 
We also obtained Bacillus subtillis var. niger $(\mathrm{Bg})$ specific antibodies. We did not call into question the ability of antibodies to recognize and interact with specific membrane components of a pathogenic spore. However, we demonstrated as infeasible the ability of immobilized antibodies to capture 1-5 $\mu \mathrm{m}$ particles from a flowing fluid stream. Capture, not discrimination, was the show-stopping issue. It should be emphasized that spore capture after a lengthy incubation time or with forced interaction of antibody and spore, a filtering approach, is feasible; capture directly from a flowing stream over a planar surface is the condition considered impractical.

The issues raised by this investigation are not limited only to this sensor strategy. Numerous other sensor approaches should be reevaluated with a better consideration of the mass transport of large bacterial particles to a sensor surface and physical parameters associated with capture at that sensor surface. We attempted to measure surface charge (zeta potential) of model polystyrene spheres and Bacillus globigii spores under a range of fluid conditions and to correlate particle adhesion with particle and sensor surface charge. Fundamental investigations of this nature are critical to BW sensor development. The limited number of articles in the biological sciences literature suggests that this is a growing but recent awareness. However, a significant literature related to this topic exists in the paint and coatings arena, and we highly recommend mining of this field for cross-over information.

Our second goal was the more technically challenging and resulted in a technical advance; we are also preparing a patent application. We defined a process flow that would enable integration of read-out integrated circuit (ROIC) with platinum $(\mathrm{Pt})$ deposition and patterning via liftoff and would result in the desired charge-coupled device (CCD)microelectrode integration. The major obstacle to this integration is the cost of the underlying ROICs, which are specialized devices that sell for approximately $\$ 25 \mathrm{~K}$ per wafer. Troubleshooting integration of the Pt layer with the underlying circuitry would require multiple wafers for success. Therefore, we proposed a process based on modification of single die. We purchased a single wafer and control circuitry for testing. This hardware investment will serve as the basis for additional research. The microelectrode-CCD integration has applications other than the particle detection system originally proposed.
The issues raised by this investigation are not limited only to this sensor strategy. Numerous other sensor approaches should be reevaluated with a better consideration of the mass transport of large bacterial particles to a sensor surface and physical parameters associated with capture at that sensor surface. 
10375

\section{ATR / Exploitation Utilizing Ultrahigh- Resolution, Complex SAR Imaging}

D. W. Harmony, W. J. Bow, K. M. Simonson, D. C. Carlson, B. K. Bray

The goal of this research is to develop novel automatic target recognition (ATR) and exploitation techniques utilizing ultrahigh resolution as well as complex (magnitude, phase) synthetic aperture radar (SAR) imagery. Current ATR techniques utilize classification metrics applied to highresolution magnitude images. Sandia will research and develop new classification metrics and algorithms designed to take advantage of the increased information content of complex and ultrahigh-resolution SAR imagery. Analyses show that significant increases in the number of resolved scatters as well as improvements in the ability to localize them are necessary before extended operating conditions of partial obscuration, articulation, camouflage, etc., can be successfully handled by the ATR. This is particularly true for small tactical targets where the number of image-resolution elements on target is limited. Our research will provide these needed improvements and then some. Not only will we take advantage of the enhanced information content coming from increased signature bandwidth, but the improved resolution will now separate the underlying scatters enough to allow us to utilize the additional information carried in the phase of the complex image pixels. Other DoD efforts are under way, struggling to improve performance by modifying their current algorithms without upgrading sensors. We believe, from a system perspective, that our proposed combination of sensor-resolution enhancements coupled with new complex-image ATR algorithms will significantly outperform these other approaches.

The primary effort this year focused on developing a template-based identification algorithm using coherent change detection (CoCD) metrics. We created the algorithm templates from target design chips containing both magnitude and phase information. We identified or rejected test chips as targets, depending on how well they compared with the template through a complex correlation. We declared a target if enough pixels in the chip correlated with the template above a specified threshold. Most of the work concentrated on the template design process and techniques for aligning and combining complex, magnitude, and phase target chips. Methods
The goal of this research is to develop novel automatic target recognition (ATR) and exploitation techniques utilizing ultrahigh resolution as well as complex (magnitude, phase) synthetic aperture radar (SAR) imagery.... Not only will we take advantage of the enhanced information content coming from increased signature bandwidth, but the improved resolution will now separate the underlying scatters enough to allow us to utilize the additional information carried in the phase of the complex image pixels. 
developed for image-level CoCD were not directly applicable to target chips. A major obstacle to using CoCD for target identification is its extreme sensitivity to subtle target differences. We explored several methods to decrease the sensitivity while still maintaining good algorithm performance.

We also investigated other phase exploitation techniques. One method examined subaperture correlations as a means for eliminating false alarms. The approach was not fully developed, but preliminary results are promising. We also developed a physics-based model that relies on inverse scattering theory in an effort to extract target shape information. There was not sufficient time to implement and test the model, and consequently its viability is presently unknown.
A major obstacle to using CoCD for target identification is its extreme sensitivity to subtle target differences. We explored several methods to decrease the sensitivity while still maintaining good algorithm performance. 
10376

\section{Thin-Skin Deployable Mirrors for Remote Sensing Systems}

T. D. Henson, J. M. Redmond, J. C. Wehlburg, J. W. Martin

Meeting the long-term needs of the DOE nonproliferation mission as well as future space exploration initiatives requires the development of large-aperture spacebased optical systems for dramatic improvements in resolution and sensitivity. While many researchers are considering onorbit assembly of conventional rigid optical mirrors to circumvent geometric limitations imposed by launch vehicles, their volumetric and weight constraints limit the aperture diameter to less than $\sim 10$ meters. Therefore, ultralarge apertures will likely be obtained using only deployable thinskin mirror technology. Ultralarge deployable thin-skin mirrors may offer orders-of-magnitude improvement in resolution and sensitivity over what is achievable today, yet many technological barriers must be overcome to make this approach a viable alternative for future system designs. Of primary concern is the development of control methodologies for achieving and maintaining optical tolerances from a highly flexible surface. Our initial research focused on the development of piezoelectric thin-skin mirrors. A thin-skin bimorph mirror will bend in response to an applied electric field and can therefore be deformed into desirable shapes using a scanning electron gun. The key areas of our work included mirror figure sensing methods, shape-control algorithm development, and electron-gun excitation.

We made significant progress toward the development of fundamental technology that would enable deployable thinskin mirrors for remote sensing systems. The key areas of accomplishments are mirror figure sensing methods, shapecontrol algorithm development, and electron-gun excitation.

Achieving an optical-quality surface from a deployable mirror hinges on the ability to accurately assess mirror figure on orbit. To satisfy the immediate need for continued laboratory-based development of the electron gun while working toward stringent specifications of future flight hardware, we developed both coarse $(\sim 50 \mathrm{~mm})$ and fine $(\sim 25$ $\mathrm{nm})$ measurement systems. We obtained coarse surface profile measurements with the Keyence LK-2500 series chargecoupled device (CCD) laser-displacement sensor. We obtained
Our initial research focused on the development of piezoelectric thin-skin mirrors.... We made significant progress toward the development of fundamental technology that would enable deployable thin-skin mirrors for remote sensing systems. The key areas of accomplishments are mirror figure sensing methods, shape-control algorithm development, and electron-gun excitation. 
fine surface profile measurements with electronic speckle pattern interferometry (ESPI) developed at Sandia.

We investigated quasi-static open-loop control algorithms suitable for reshaping bimorph mirrors by using finite-element modeling (FEM) techniques. We adapted temperature-sensitive layered-shell elements in ABAQUS to mimic the behavior of piezoelectric bimorphs. We accomplished two-dimensional curvature correction on a bimorph plate using modeling-derived curvature-voltage sensitivity matrices and allowing for electron-gun excitation on both sides of the bimorph. However, the impracticality of twosided control in a space application led to the improvisation of a one-sided control algorithm based on modeling-derived displacement-voltage sensitivity matrices. We developed and demonstrated each of these algorithms on a FEM of PZT-5A bimorph subjected to arbitrary disturbances. We filed technical advances for the two-sided and the one-sided control schemes.

We used the ESPI system to interrogate a number of bimorph mirror samples. First, we measured the bimorph mirror with the ESPI system after 30 seconds of electron-gun excitation with an $800 \mathrm{eV}$ uniform beam and a $2 \mathrm{~V}$ backpressure voltage. We measured it with the ESPI system again after an additional 30 seconds of electron-gun excitation. The change in the interferograms indicated a change in the shape of the mirror on the order of $150 \mathrm{~nm}$. This result verifies the ability to effect minuscule surface changes with the electron gun as needed to achieve optical quality.

We used the Keyence LK-2500 sensor to calibrate the change in the mirror shape as a function of electron-gun energy and bimorph mirror back-pressure voltage. We also used it to provide mirror-position feedback to the electron gun in a closed-loop mirror-tip control experiment. We took complete sets of data for bimorph mirrors with various electrode materials.

When we excited a bimorph mirror with a uniform electron beam at $800 \mathrm{eV}$ energy and a $20 \mathrm{~V}$ back pressure, the tip of the mirror displaced approximately $1 \mathrm{~mm}$ at a radius of $40 \mathrm{~mm}$, causing a net change in the radius of curvature of the surface of approximately $800 \mathrm{~mm}$. This demonstrates that large shape changes may be initiated with electron-gun excitation when the bimorph mirror is unconstrained.

We developed and tested the control algorithms and electron-gun control methods in a closed-loop feedback system. After we calibrated the bimorph mirror shape changes as a function of electron-gun energy and back-pressure voltage,
We accomplished twodimensional curvature correction on a bimorph plate using modeling-derived curvature-voltage sensitivity matrices and allowing for electron-gun excitation on both sides of the bimorph. However, the impracticality of two-sided control in a space application led to the improvisation of a one-sided control algorithm based on modeling-derived displacement-voltage sensitivity matrices. We developed and demonstrated each of these algorithms on a FEM of PZT-5A bimorph subjected to arbitrary disturbances. We filed technical advances for the two-sided and the one-sided control schemes. 
we developed a simple control algorithm to control the deflection of the bimorph mirror at its tip position. We used the Keyence sensor to measure the initial position of the tip of the mirror, then changed the tip position through electron-gun excitation. We continually monitored the position of the mirror tip and adjusted the control voltage until the mirror tip was in the new desired position. Then we monitored and maintained that position through electron-gun excitation until we commanded a new desired position. This confirmed our ability to control the location of the mirror tip in a closed-loop fashion and also validated that we calibrated the materials correctly.

\author{
This confirmed our ability to \\ control the location of the \\ mirror tip in a closed-loop \\ fashion and also validated that \\ we calibrated the materials \\ correctly.
}

\section{Refereed}

Martin, J. W., J. A. Main, and G. C. Nelson. 1999. "Development of a Test-Bed for Evaluating ElectronGun Shape Correction of Distributed Structures." Proc. 1999 AMSE Internat. Mechanical Eng. Congress and Exposition AD 59 (Nashville, TN, November): 253-256.

Redmond, J. M., P. S. Barney, T. D. Henson, J. A. Main, and J. W. Martin. 1999. "Distributed Sensing and Shape Control of Piezoelectric Bimorph Mirrors." Proc. 1999 ASME Internat. Mechanical Eng. Congress and Exposition AD 59 (Nashville, TN, November): 245-252.

\section{Other Communications}

Henson, T. D. 2000. "Thin-Skin Deployable Mirrors for Remote Sensing Applications." Presentation to the DOE Annual LDRD Review, Albuquerque, NM, 14 September.

Henson, T. D., J. M. Redmond, J. C. Wehlburg, J. W. Martin, and J. A. Main. 2000. "Electron-GunControlled Thin-Film Mirrors for Remote Sensing Applications." Paper presented to the SPIE OptoSouthwest Conference, Albuquerque, NM, April.

Henson, T. D., J. M. Redmond, J. W. Martin, and J. A. Main. 2000. "Current Sandia Work with ElectronGun Excitation of Thin-Film Mirrors." Presentation to a joint meeting between Sandia and AFRL, Albuquerque, NM, 28 July. 
10377

Dispersible Granular Sensor (Smart Sand) for Landmine Detection Based on TNT Immunoassay

C. J. Brinker, B. G. Hance, A. K. Singh, J. S. Schoeniger, C. S. Ashley

Remote detection of specific trace chemicals has application to a range of intelligence, counterproliferation, treaty verification, and military missions. To date, Sandia has concentrated on developing a sensor technology capable of rapidly screening large areas for the presence of mines or other unexploded ordnance (UOX) by detecting trinitrotoluene (TNT). We will continue to develop enzyme-based granular chemical sensors that combine sample preconcentration, molecular recognition, signal amplification, and optical transduction-all in a grain of sand. Goals are to improve the spectral characteristics of the sensor particles for field use and to broaden the detection capability to analytes other than TNT. In operation, the sensor particles would be dispersed by air, and each granule would perform an immunoassay of its local environment. A fluorescent signature would develop, allowing remote detection by LIDAR (light detection and ranging). To produce a fieldable sensor based on these results, we will develop a custom amplification enzyme for incorporation into the sensor particles. This will enable production of a spectrally advantageous fluorescent reporter dye as the product of the enzyme-amplified assay. Further, this will enable adaptation of the sensor system for detection of analytes other than TNT. We will select new candidate analyte( $s$ ) for counterproliferation, deeply buried targets, and intelligence applications.

We directed our granular sensor development toward the detection of low concentrations of TNT for the purpose of detecting buried landmines. Our team, including collaborators at UCLA, accomplished the following. First, we combined a TNT substitutional immunoassay with enzyme-amplified dye production within the pores of a silica sol-gel matrix. This key result indicates that the necessary biochemical reagents can be immobilized in inorganic host particles without loss of the required specific reactivity. Thus, we validated our basic approach to developing granular sensors. The enzyme available for this assay, G6PDH (glucose-6-phosphate dehydrogenase), catalyzes the production of NADH (protonated nicotine adenine dinucleotide). Since NADH is not an ideal candidate
To produce a fieldable sensor based on these results, we will develop a custom amplification enzyme for incorporation into the sensor particles. This will enable production of a spectrally advantageous fluorescent reporter dye as the product of the enzyme-amplified assay. Further, this will enable adaptation of the sensor system for detection of analytes other than TNT. We will select new candidate analyte(s) for counterproliferation, deeply buried targets, and intelligence applications. 
dye for field detection of sensor particles, we attempted to link NADH production to production of fluorescein in the host matrix. The NADH-fluorescein reaction results are not promising; thus, we concluded that development of a different enzyme is necessary. We therefore identified a strategy to produce a new enzyme. The development of such a "custom" enzyme will also allow adaptation of the "smart sand" sensors to detect analytes other than TNT. In addition to the biochemically based sensor particles, we produced prototype TNT-sensing granular materials based on the quenching of a fluorescent polymer. We used these prototype materials to measure spectroscopic signal-to-noise ratios (SNRs) for $\sim 1$ $\mathrm{mg} / \mathrm{cm}^{2}$ coverage of the sensor particles on natural soil. We conducted these measurements using Sandia's fluorescence LIDAR apparatus at a standoff range of $0.5 \mathrm{~km}$. This result indicates that the proposed sensor particles will be detectable with existing LIDAR technology. We also used these field measurements to validate calculations of fluorescent signal/ noise for the granular sensor particles as a function of several variables, including particle and receiver characteristics, standoff range, pump laser characteristics, and coverage. This allows us to predict the "detectability," in terms of fluorescence SNR, of the dispersed sensor particles under a range of possible deployment conditions. Finally, we used the polymer-based prototype TNT-sensing particles to demonstrate the detection of 1 part per million (ppm) (1 mg TNT/kg soil) of TNT in natural soil at $0.5 \mathrm{~km}$ standoff range. The $1 \mathrm{ppm}$ concentration level is comparable to the concentration of nitroaromatic explosives compounds detected in soils sampled from above actual buried landmines. This result demonstrates that the sensitivity of the prototype distributable granular sensors is potentially adequate for the detection of landmines under field conditions. Our final efforts will be directed primarily toward extending the granular sensor concept to analytes other than explosives.
We therefore identified a strategy to produce a new enzyme. The development of such a "custom" enzyme will also allow adaptation of the "smart sand" sensors to detect analytes other than TNT. In addition to the biochemically based sensor particles, we produced prototype TNT-sensing granular materials based on the quenching of a fluorescent polymer. 
10378

Characterization of Underground Facilities in an Urban Environment

M. D. Ladd, D. L. Faucett, G. J. Elbring, H. D. Garbin, T. S. McDonald, C. W. Sicking, M. W. Holzrichter

It is well known that rogue nations may conceal facilities for the manufacture and storage of weapons of mass destruction (WMD) by placing them in urban areas. The ability to characterize and monitor these facilities is recognized as an important national concern. Because of the multisource and high-noise nature of urban environments, such sites pose special challenges for unattended sensors. The intent of this project is to develop and apply novel adaptive signalprocessing methods and sensor deployment configurations to permit the characterization of concealed facilities in an urban environment. In particular, Sandia will explore seismic monitoring from adjacent building basements of a suspect facility. We will require characterization of the source vibrations and the use of advanced signal-processing techniques, such as the application of adaptive array techniques, to cancel out noise signals based on source location. In addition to algorithmic development, we will establish factors affecting sensor fidelity, such as timing accuracy and separation tolerances in deployment layouts. We will study novel deployment strategies, including the use of three-dimensional (3-D) seismic arrays in neighboring buildings. To validate the algorithms, we will perform simulations and process recorder data collected at a site. The system being developed in this project provides a totally new capability for the urban environment. This project directly addresses the nonproliferation and materials control need to collect information that can characterize proliferation activities in a covert manner. It also addresses the emerging threats need to characterize difficult targets.

Recent efforts focused on developing and performing a field test to obtain "real-world" data. We will use these data to validate the array-processing techniques. The field test allowed us to work through the logistics of deploying a practical array. We completed a rather extensive test plan and conducted data collections. We developed a $1 \mathrm{C}$ sensor and a $3 \mathrm{C}$ sensor array design under the constraint of fitting inside an existing small basement and of using only 30 sensors. Based on the array designs and operating frequencies, we completed a parameter
The system being developed in this project provides a totally new capability for the urban environment. This project directly addresses the nonproliferation and materials control need to collect information that can characterize proliferation activities in a covert manner. It also addresses the emerging threats need to characterize difficult targets. 
sensitivity study. We tested the baseline version of the adaptive beamforming algorithm with field data and are making improvements. We developed techniques to characterize equipment operations on the beamformed data and will submit a technical advance. We completed field collections; preliminary analysis of the data using the beamformer code yielded very positive results. We demonstrated the ability to find the source using a search algorithm and to steer the beamformer toward the source while suppressing an interfering signal of roughly equal power. The beamformer realized a signal-to-interferer ratio (SIR) of $19 \mathrm{~dB}$ that compares favorably to $9 \mathrm{~dB}$ from the traditional delay-and-sum method. We characterized urban cultural noise by comparing data collected locally with data collected at the Nevada Test Site (NTS), a remote area without urban cultural noise.

\section{Refereed}

Ladd, M. D., M. K. Alam, G. E. Sleefe, D. E.

Gallegos, and H. D. Nguen. 2000. "Seismic and

Acoustic Signal Identification Algorithms." Proc.

SPIE, Unattended Ground Sensor Technol. and Applic.

4040 (Orlando, FL, 26 April): 106-120.
We demonstrated the ability to find the source using a search algorithm and to steer the beamformer toward the source while suppressing an interfering signal of roughly equal power. The beamformer realized a signal-to-interferer ratio (SIR) of $19 \mathrm{~dB}$ that compares favorably to $9 \mathrm{~dB}$ from the traditional delay-and-sum method. 
10379

\section{Dexterous Robotic Manipulation of Hazardous Materials in Unstructured Environments}

P. C. Bennett, D. M. Kozlowski, R. J. Anderson

Sandia will develop the science and technology base required to revolutionize the remote manipulation of hazardous materials in difficult, unstructured environments. DOE and others require dexterous remote manipulation and other organizations to safely and quickly respond to increasing numbers of encounters with unexploded ordnance (UXO), such as munitions and mines, and improvised devices left by terrorists. The science base created here includes advances in geometric reasoning, sensor fusion and model-building, automated planning, and task-based reasoning. These advances are motivated by the need to reduce risk to explosive ordnance disposal (EOD) and other field technicians, who are frustrated by the slow, unsafe primitive remote manipulation capabilities available on the market today.

The engineering motivations for constructing such systems are to determine how laboratory technologies such as sensing and control perform when actually fielded and to determine how they are best integrated. The first advances the science of component technologies by establishing limits on their practical use and flaws in accepted problem definitions. The second also establishes limits on component technologies and allows them to successfully coexist with one another. This knowledge increases successful transition of technologies to the field.

We originally envisioned the system to be built as teleoperated, but it now includes significant autonomy to lessen operator burden. Autonomy will be in the form of automatic planning of manipulator motions required to manipulate objects or to perform operations at locations designated by the operator. Autonomy requires high-bandwidth force-control responses that enable safe contact operations. With the manipulator mounted on a mobile vehicle, the system will be capable of performing tasks such as opening unlocked doors and going through them, drilling holes, inserting inspection devices, and unscrewing fuses autonomously under task-level direction of an operator.

We sought increased autonomous operation and less teleoperation in this project in response to user community input, which stressed systems that were easy to use and that
Sandia will develop the science and technology base required to revolutionize the remote manipulation of hazardous materials in difficult, unstructured environments. DOE and others require dexterous remote manipulation and other organizations to safely and quickly respond to increasing numbers of encounters with unexploded ordnance (UXO), such as munitions and mines, and improvised devices left by terrorists. 
significantly reduced the operator burden. Thus, we developed closed-loop force-control algorithms to allow such operations as door opening to be performed automatically. This eliminated the need to provide high-bandwidth force feedback to the operator as originally planned. We demonstrated automated force-control door opening. We achieved grasping of objects using a three-fingered hand developed in collaboration with the University of New Mexico.

We made significant advances in improving operator interfaces for manipulation systems. Increased incorporation of autonomy enabled by sensor-based control (e.g., force control), vision-based modeling (e.g., visual targeting), and motion planning enables an interface in which operators indicate an object in the video domain, and the robot automatically executes a task such as retrieval. Initial tests show an increase of four times in task execution speed.

The above technologies enabled us to demonstrate a broad range of robotic operations in unstructured environments, including retrieval of randomly placed objects, insertion of inspection probes through confined spaces, forcecontrolled opening of doors, rapid definition of trajectories such as those used by water-jet cutters, placement of explosive charges on walls under force control to open passageways, and others.
We made significant advances in improving operator interfaces for manipulation systems. Increased incorporation of autonomy enabled by sensorbased control (e.g., force control), vision-based modeling (e.g., visual targeting), and motion planning enables an interface in which operators indicate an object in the video domain, and the robot automatically executes a task such as retrieval. Initial tests show an increase of four times in task execution speed. 
10380

\section{Autonomous Dynamic Soaring Platform for Distributed Mobile Sensor Arrays}

M. B. E. Boslough, B. L. Spletzer, L. C. Marron, K. M. Jensen, K. W. Boyack

Among the greatest practical challenges to the realization of robotic swarms is the relative lack of rapid mobility and the limited power supply associated with currently available autonomous devices. Unmanned airborne vehicles $(U A V s)$ are particularly constrained because they must consume significant power just to stay aloft. This limits the potential feasibility of UAV swarms for most applications unless the individual vehicles can derive energy of locomotion from their local environment. It is theoretically possible, and has now been demonstrated with support from flight data collected under this project, for a robotic glider to remain aloft and to navigate by dynamic soaring in the shear boundary layer near the earth's surface or in separation flow on the leeward side of a topographic high. Dynamic soaring was first described by Lord Rayleigh in the nineteenth century and is the means by which many species of albatross cover vast distances of the ocean without flapping their wings. Moreover, the flight cycle required to derive energy from the wind-velocity gradient is a circuitous pattern of climbing upwind, turning and diving downwind, and turning back upwind, allowing vast areas of the sea to be scanned with no net expenditure of flight energy. The same method that optimizes foraging for seabirds can be applied to search, tracking, sentry, and monitoring problems; the wandering albatross, Diomedia exulans, has been observed to follow ships for several days in a row. With communications links, collective intelligence can be designed to improve on nature.

Our primary progress was on two fronts: (1) hardware development, test flights, demonstrations, and data collection, and (2) software development and flight simulation. We designed and built three 8-foot expanded polypropylene (EPP) foam test gliders and outfitted them with instrumentation and data loggers. The data logger hardware and software that we designed and built include three-axis accelerometers, three-axis gyros, magnetometers, dynamic and static pressure sensors, global positioning system (GPS), and control surface data commands, with 4 MBytes of static random access memory (SRAM) for collection of about 25 minutes of flight-test data. 
Logged data can be converted to airspeed, altitude, and total energy, and six-degree-of-freedom (6 DOF) position and attitude histories can be extracted.

We collected data for several test flights in dynamic soaring conditions and analyzed the data to show unambiguously that dynamic soaring can be used to sustain flight in a fundamentally different way than static soaring. For the simulation part of the project, we developed a genetic programming model to provide a flight behavior for a glider or albatross in static soaring conditions. This behavior allowed the plane to find an area of lift and gain altitude. When it gained sufficient altitude, the model also instructed the glider to investigate the surrounding area and return to the lift area before losing too much altitude and striking the ground. We also developed a flight simulator for the genetic program for debugging purposes. The simulator will run on any Macintosh computer. We also installed two aerodynamic flight simulation programs, LaRCsim, and FlightGear, on our Silicon Graphics computers. These are full 6 DOF codes that implement rigidbody equations of motion and full aerodynamics. We will couple the LaRCsim routines to the genetic program on the Computational Plant (CPlant) and will use FlightGear graphics to display data from both simulations and actual flights.
We collected data for several test flights in dynamic soaring conditions and analyzed the data to show unambiguously that dynamic soaring can be used to sustain flight in a fundamentally different way than static soaring. 
10381

\section{Miniature UV Fluorescence-Based Biological} Agent Sensor

K. L. Schroder, K. S. Potter, M. H. Crawford, P. J. Hargis, Jr., A. M. Morales

This project will develop a miniaturized ultraviolet $(U V)$ fluorescence biosensor based on UV-LED (light-emitting diode) and miniature spectrometer technology developed at Sandia. We will evaluate the performance of an LED operating at $360 \mathrm{~nm}$ in biofluorescence measurements. The LED is based on the AlGaN (aluminum gallium nitride) family, which has direct optical bandgaps from $364 \mathrm{~nm}(\mathrm{GaN})$ to $200 \mathrm{~nm}$ (AlN). The wide wavelength range of these materials makes them attractive as compact UV light sources. To date, efforts in developing nitride-based emitters have focused mostly on visible (blue and green) light emission using InGaN as the active region; $U V$ applications using GaN and AlGaN remain relatively unexplored. We will fabricate the UV-LED in a recently established metalorganic chemical vapor deposition (MOCVD) facility that has demonstrated the growth of GaN materials with structural, optical, and electrical qualities comparable to the best reported thus far. The project will (1) develop novel UV-LED light sources, (2) demonstrate their usefulness for the detection of bio materials, (3) develop a miniature spectrometer for biofluorescence measurements, and (4) culminate with the design for a prototype instrument that can be used for demonstration measurements.

The performance of $~ 360 \mathrm{~nm}$ LEDs improved significantly through development of a new contact geometry that improves current spreading. For shorter-wavelength LEDs, due to difficulty in obtaining good photoluminescence (PL) efficiency in AlGaN layers at wavelengths below $350 \mathrm{~nm}$, we developed a new AlInGaN (aluminum indium gallium nitride) alloy with much higher PL efficiency. We fabricated and characterized $386 \mathrm{~nm}$ LEDs using the new alloy and obtained an output power of $500 \mathrm{~mW}$.

We fabricated and characterized Fiber Bragg gratings (FBGs) as a candidate for use in a miniature spectrometer. Although we demonstrated our ability to produce band-filtering elements that may be used to perform spectroscopic analysis of biofluorescence emission, it does not appear that the geometry will provide the most compact solution to this problem. Therefore, we made a decision to pursue the modification of a
We will fabricate the UV-LED in a recently established metalorganic chemical vapor deposition (MOCVD) facility that has demonstrated the growth of GaN materials with structural, optical, and electrical qualities comparable to the best reported thus far.

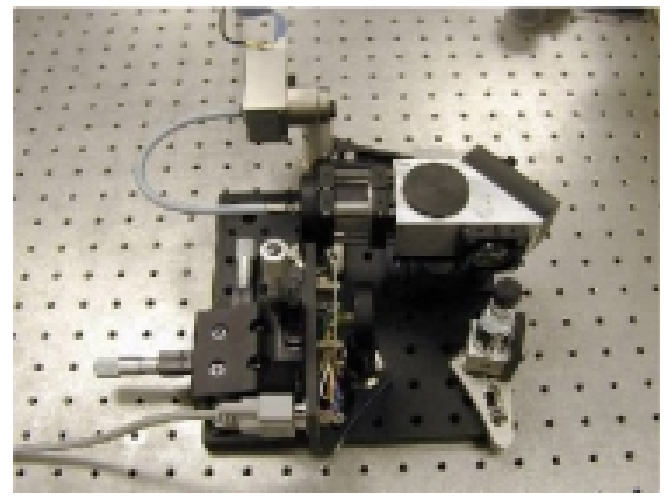

Photo of experimental setup for acquiring fluorescence data from Zeiss miniature spectrometer and Sandia-developed UV LED. The UV LED is mounted on a three-axis stage for convenience in alignment. 
commercially available miniature spectrometer for incorporation into a prototype sensor system.

We conducted some experiments to evaluate both the newest LEDs fabricated at Sandia and commercial (off-theshelf) miniature spectrometer technology applied to the biological agent sensor problem. We built a small biofluorescence detector using a Sandia-developed UV-LED as the excitation source and a Zeiss miniature spectrometer as the detector. Overall, the data show that the UV-LED/ minispectrometer combination has sufficient sensitivity to detect useful concentrations of biological material with reasonably short sampling times (on the order of seconds to minutes).

The UV-LED selected was device NE 1208-B, a wedge/wave device. At room temperature $\left(\sim 20^{\circ} \mathrm{C}\right)$ and at a forward current of $\sim 47 \mathrm{~mA}$, the LED produces about $310 \mu \mathrm{W}$ of optical power. We chose this particular LED because of its relatively narrow emission bandwidth and lack of a longwavelength tail that was present on most of the previous LED devices tested for this project. The long-wavelength emission tail on the previous devices was a concern because it overlapped with the fluorescent emission band from the biological material and could limit the ultimate sensitivity of the sensor.

In the experimental setup for measuring fluorescence spectra, some of the UV light emitted from the LED is collected by a short-focal-length lens and re-imaged into a quartz cuvette containing the sample under study. We collect fluorescence from the sample using a lens system that matches the $\mathrm{f} / \#$ of the spectrometer. The fluorescence is collected at $90^{\circ}$ to the excitation to minimize the amount of elastically scattered light detected. The collected fluorescent light is imaged into the fiber-optic input of a Zeiss miniature spectrometer.

The overall size of this experimental apparatus is not indicative of the potential small size for an optimized biological agent sensor. We used a three-axis stage to hold the LED and facilitate alignment. A miniaturized sensor does not require this stage. Also, in a fully optimized system, a ball lens could be designed into the LED package, greatly reducing the overall size of the excitation source. Likewise, the fluorescence collection optics could be miniaturized and integrated into the end of the fiber bundle of the spectrometer. ...the data show that the $U V$ LED/minispectrometer combination has sufficient sensitivity to detect useful concentrations of biological material with reasonably short sampling times (on the order of seconds to minutes). 
10382

\section{Enabling Human Skills with Cooperative} Automation

D. J. Schmitt, D. E. Small

This project produced, for the first time, the ability of humans and automated systems to cooperate to perform tasks within the same workspace. The intent was to enable efficient task execution by simultaneously utilizing the best skills of the human and machine to perform a task. Cooperative automation frees the human from mundane and hazardous tasks and allows the human to perform the more insightful operations such as task supervision. The developed technology provides the potential for reducing personnel requirements in battlefield and battleship operations by providing cooperative automated assistants and by allowing multiple cooperative systems to be directed by a single individual.

This project focused on developing new methods for human-machine interaction and produced the ability of a human to direct the motion of a pedestal robot by using arm gestures. We originally planned to extend the indoor cooperative automation environment to an outdoor environment with mobile robots. However, we modified our plans to continue our work in the established indoor lab and focused our attention on developing the new gesture-based control environment.

Our research focused on developing the technologies required to capture and interpret human-made, machine-control gestures made by an operator working within the monitored workspace. For a gesture language, we chose crane-control signals commonly used in construction operations. The technologies for gesture recognition included gesture data capture, data interpretation, and a machine-control interface. We based the data capture system on Sandia's ThreeDimensional Video Motion Detection (3DVMD) system. The 3DVMD system is a vision-based system that monitors a working volume and that can detect the presence of moving objects by the subvolumes (voxels) occupied within that space. We enhanced this system to customize it for data capture of human gestures.

Data interpretation involved algorithm development to analyze the captured voxels to determine the gesture. We developed, implemented, and tested two algorithms. The first used a more simplified approach of analyzing the voxel content
This project produced, for the first time, the ability of humans and automated systems to cooperate to perform tasks within the same workspace....The developed technology provides the potential for reducing personnel requirements in battlefield and battleship operations by providing cooperative automated assistants and by allowing multiple cooperative systems to be directed by a single individual. 
to determine a centroid and an outermost voxel relative to that centroid. We then connected these voxels to produce a vector that could be interpreted for gesture recognition. We limited this technique to recognizing 16 gestures. We developed a more sophisticated approach that analyzed voxel connectivity to fit skeletonlike shapes to the upper-body appendages. We could then analyze these skeleton representations for angular relationships, resulting in a much wider variety of gestures that could be recognized.

We defined and implemented a demonstration that involved gesture-based control of a pedestal robot. We restricted the robot's motions to simulate those of a boom-type crane. We defined a set of gestures for controlling the robot's motion and included raise/lower load, raise/lower boom, and swing right/left. The operator stands in the $12^{\prime} \mathrm{x} 12^{\prime}$ volume in front of the robot that is monitored by the 3DVMD system. The system detects the operator's gestures, and a supervisory control system commands the robot to perform the corresponding operations.

The developed system successfully demonstrated that a human operator can control a machine using a standard gesture control language.
The developed system successfully demonstrated that a human operator can control a machine using a standard gesture control language. 
10383

\section{Cloud to CAD}

A. L. Ames, C. Q. Little, P. G. Xavier

A fundamental requirement of sensing and intelligent controls is the ability to see unexpected objects and to construct models of them to support reasoning. A typical mechanism for seeing objects is to perform range scanning with structured lighting techniques. Many of the interesting devices are engineered and exhibit significant regularity. Surfaces are parallel and perpendicular, and surface equations are quadric rather than fractal. These regularities permit a variety of advantages in understanding and dealing with the unknown.

Sandia is developing algorithms that recognize engineering shapes from scanned data. The algorithms partition points into groups that represent engineering surfaces, trim and extend surfaces, and adjust representations to achieve model closure. The algorithms will integrate geometry from multiple views and suggest surfaces based on engineering expectations to account for occlusions. The result of this processing is a computer-aided design (CAD) solid model. This representation is suitable for a wider variety of applications than any other geometric representation.

The benefits of generating engineering representations of scanned objects include tremendous data compression and object reasoning. The shape of an object can be stored, transmitted, and reasoned about in an appropriate language (e.g., radius and height of a cylinder) rather than in the verbose terms of thousands of points. An object in the field can be compared to known designs in engineering terms. This reduces communication requirements for battlefield reconnaissance and surveillance.

All of our automatic engineering algorithms can be directly applied to the engineering representation of an acquired object in the following applications: identifying targets, analyzing a competitor's products, designing fixtures, planning operations for demilitarizing old ordnance, and archiving the as-built geometry of a stockpile component.

Our work focused on those activities required to prepare the algorithm for fielding in a mobile robot. We enhanced surface recognition algorithms to provide more robustness in the face of sensor noise. We also enhanced solidmodel construction algorithms to provide faces having
Sandia is developing algorithms that recognize engineering shapes from scanned data....All of our automatic engineering algorithms can be directly applied to the engineering representation of an acquired object in the following applications: identifying targets, analyzing a competitor's products, designing fixtures, planning operations for demilitarizing old ordnance, and archiving the as-built geometry of a stockpile component. 
piecewise linear boundaries approximated from scan information. We began work on an algorithm for finding adjacency between scanned patches so that exact curve equations can be computed by surface intersection.

We took scans of a number of real-world objects of interest to the Contact Operations Clump, including a door handle, a car door, and a simulated pipe bomb. Surface construction for those geometries was appropriate.

We performed work in determining operational requirements for providing good surface reconstruction. We investigated scanning resolution, signal-to-noise ratio (SNR), viewing orientation, and scanner limitations as to their effect on the quality of CAD models produced. We must adequately address each consideration before fielding in a mobile robot will be sufficiently successful.
We began work on an algorithm for finding adjacency between scanned patches so that exact curve equations can be computed by surface intersection. 
10697

\section{Sparse Geophysical Networks for Monitoring} Deep Targets

G. E. Sleefe, G. J. Elbring, J. P. Claassen, H. D. Garbin, M. D.

Ladd, C. W. Sicking, L. C. Bartel, A. A. Yee

Facilities for the manufacture and storage of weapons of mass destruction (WMD) are often contained within deeply buried structures or tunnels. The ability to detect, characterize, and monitor these deep underground production and storage sites is critical to the defeat of these threats to our national defense. The use of a sparse seismic network is very desirable for this application since it represents a practical fielddeployable configuration. However, the application of sparse networks for accurately determining the three-dimensional (3-D) location of underground activities has heretofore never been addressed. As such, the objective of this project is to design, develop, and test an advanced algorithm that exploits sparse seismic networks to accurately determine the 3-D location of underground activity. The proposed algorithm incorporates several novel elements to address the sparse network problem: (1) the use of enhanced 3-D geophysical modeling to optimize sensor layout and distribution, (2) the isolation of local soil effects by using both velocity inversion and spectral ratio methods, (3) the use of modified array processing methods to handle the spatially aliased network dataset, (4) exploitation of advanced signal-processing techniques such as multisensor covariance and threecomponent polarization analysis, (5) and the incorporation of an active calibration source to remove geophysical biases. To validate these advanced geophysical and signal-processing methods, we processed data previously acquired at the Nevada Test Site (NTS) P-Tunnel using the novel algorithms. Additionally, we collected novel datasets at a representative tunnel site to confirm the optimized sparse network approach.

This project made significant progress previously in developing and testing a novel algorithm for locating underground target activity using sparse seismic networks. We integrated the complete model-based algorithm into a MATLAB environment. The complete algorithm consists of signal preprocessing, adaptive parameter estimation (crosscorrelations, covariance matrices, times-of-arrival, and threecomponent estimators), model-based coherent beam-forming, and model-based location estimation. We fully tested and ...the application of sparse networks for accurately determining the threedimensional (3-D) location of underground activities has heretofore never been addressed. ... the objective of this project is to design, develop, and test an advanced algorithm that exploits sparse seismic networks to accurately determine the 3-D location of underground activity. 
validated the algorithm using both synthetic seismograms and data from the P-Tunnel. The algorithm testing involved the study of phenomenological processes in the datasets as well as enhancing the algorithms to provide the required performance. Using the novel 3-D finite-difference (FD) seismic propagation model developed under this project, we successfully designed an optimized sensor layout to perform a novel sparse network data collection. Henceforth, we fielded a sparse seismic network at an NTS tunnel and collected a unique dataset on underground activity. We applied the novel algorithm to portions of the NTS dataset, and localization performance on the underground activity is very promising.

We successfully assessed the absolute location accuracy of the algorithms. Furthermore, we catalogued and studied several active seismic datasets from controlled sources. Finally, we successfully used the active source data to improve location accuracy performance.

\section{Refereed}

Elbring, G. J., H. D. Garbin, and M. D. Ladd. 2000. “A Comparison of Methods for 3-D Target Localization from Seismic and Acoustic Signatures." Proc. SPIE Unattended Ground Sensor Technol. and Applic. II, SPIE Aerosense 4040 (Orlando, FL, April): 154-164.

Sleefe, G. E., M. D. Ladd, T. S. McDonald, and G. J. Elbring. 1999. "Acoustic and Seismic Modalities for Unattended Ground Sensors." Proc. SPIE, Unattended Ground Sensor Technol. and Applic., SPIE Aerosense 3713 (Orlando, FL, April): 2-9. ...we successfully designed an optimized sensor layout to perform a novel sparse network data collection...successfully assessed the absolute location accuracy of the algorithms.... catalogued and studied several active seismic datasets from controlled sources.... [and] successfully used the active source data to improve location accuracy performance. 
10724

High-Speed 2-D Hadamard Transform Spectral Imager for IR Applications

J. C. Wehlburg, L. J. Krumel, J. W. Daniels, D. M. Haaland, D. A.

Smith, C. M. Wehlburg, C. L. Grotbeck

There is a need for staring infrared (IR) imaging systems capable of real-time high-speed imagery in a large number of spectral bands. Existing spectral imaging technologies do not support these rates where mechanical scanning mechanisms are too slow. Techniques that employ electronic scanning methods and two-dimensional (2-D) focal planes are required. In addition, techniques that support changing spectral sampling on-the-fly are able to match changing target and background conditions, particularly when optimization of signal-to-noise ratio (SNR) is desired. Finally, under certain noise conditions, spectral multiplexing techniques have the potential of increasing the SNR and decreasing the sample rate for a given measurement.

Two-dimensional Hadamard transform spectral imaging approaches have the potential to achieve all of the above requirements. Recent advances in micromirror array technology allowed the possibility of realizing these designs. The micromirrors allow electronic switching of light between optical systems necessary to realize the Hadamard encoding matrices, without significant loss of light. Since these devices are electronically programmable, they allow the system to be spectrally reconfigured in microseconds. Under nonphoton noise-limited conditions, Hadamard techniques achieve a $\left(N^{0.5}\right)$ SNR advantage over nonmultiplexed approaches, where $N$ is the number of spectral samples.

This project will develop a prototype staring Hadamard Transform Spectral Imager operating in the 1.25-2.5 $\mu \mathrm{m}$ spectral region using commercially available micromirror arrays. Successful completion of the project would require demonstration of the technique at high scan rates and the electronically tunable features required.

We created a system model that allows us to evaluate the effect of system parameters such as detector resolution, detector noise, micromirror resolution, and spectrometer parameters. The model also allows the evaluation of the effect of the multiple transitions from analog to digital signals performed by the system.
This project will develop a prototype staring Hadamard Transform Spectral Imager operating in the 1.25-2.5 $\mu \mathrm{m}$ spectral region using commercially available micromirror arrays. ...We created a system model that allows us to evaluate the effect of system parameters such as detector resolution, detector noise, micromirror resolution, and spectrometer parameters. The model also allows the evaluation of the effect of the multiple transitions from analog to digital signals performed by the system. 
We identified a need for spectral coverage from $\sim 1$ to $2.5 \mu \mathrm{m}$ at between 25 and $50 \mathrm{~nm}$ resolution (< 60 samples). In addition, net spectral frame rates of $10 \mathrm{~Hz}$ or more were required, driving the camera frame rate up to as much as 600 frames per second. Using this information, we identified a camera that could not only meet the spectral response, but also was able to frame at $400 \mathrm{~Hz}$ in $256 \times 256$ mode and at $1 \mathrm{KHz}$ in $128 \times 128$ mode.

Independent review by appropriate Sandia personnel confirmed the technical validity and applicability of this approach to proposed future sensor systems. In this analysis, we outlined a comparison and contrast to existing approaches and found significant advantages and flexibility that are not represented in other transient spectral imaging approaches.

We completed an optical design that proved that the necessary interfaces to the Texas Instruments Digital Mirror could be satisfied and that the appropriate optical specifications could be met. We are continuing work on a nondiffractive design using aplanatic prisms to simplify the required optics, improve efficiency, and reduce the potential for stray light.

We prepared several technical advances.

\section{Refereed}

Wehlburg, C. M., J. C. Wehlburg, S. M. Gentry, and C. L. Grotbeck. 2000. "Optimization and Characterization of an Imaging Hadamard Spectrometer." Algorithms for Multispectral, Hyperspectral, and Ultraspectral Imagery VII, SPIE 2001, accepted. ...we identified a camera that could not only meet the

spectral response, but also was able to frame at $400 \mathrm{~Hz}$ in $256 \times 256$ mode and at $1 \mathrm{KHz}$ in $128 \times 128$ mode. 
10727

\section{Micro-High-G Acceleration Recorder}

T. A. Rohwer, V. I. Bateman, R. G. Lundgren, R. R. Lockhart, C. W. Dyck, D. J. Savignon

All attempts to collect projectile acceleration data at impact velocities greater than 3500 feet per second have failed. These data are becoming increasingly more important to the hard and deeply buried target community to determine highvelocity penetration dynamics, multilayered target characteristics, and survivability of the fuse, explosives, and penetrator. The micro-acceleration recorder concept will integrate Sandia microelectronics and a microelectromechanical systems (MEMS) accelerometer into a miniature package, approximately $1 \mathrm{~cm}^{3}$ compared to the 24 $\mathrm{cm}^{3}$ of the smallest existing system that uses commercially available parts. The electronics will implement an analog and digital mixed-signal application-specific integrated circuit (ASIC) that integrates a MEMS accelerometer, signal conditioning, analog-to-digital conversion, and memory. An existing microcontroller core will provide system functionality and interfacing. The electronics will be assembled at Sandia using hybrid chip-on-board techniques and packaged in a miniature housing machined at Sandia. Integration at this unprecedented level involves high risk, but the resulting small size and weight will allow this acceleration recorder to survive in environments where data have never been collected. Sandia's Reentry Vehicle and Penetrator Technology department, which has extensive experience in gun-launched penetrator testing, will test prototypes in these severe environments. The highly integrated microelectronics/MEMS technology could be used in an ultrahigh-shock fuse or in nonintrusive instrumentation systems that do not exist because of the size limitations of current technology.

Our work focused on the design of the data-acquisition application-specific integrated circuit (ASIC). We split this design process into two efforts: the MEMS accelerometer design and the microelectronics design. We designed three MEMS accelerometer architectures for evaluation and laid out various iterations of the three architectures for fabrication. The iterations (a total of 50) include various spring constants, geometries, references, and interfaces. Fabrication of the MEMS accelerometers is near completion. After fabrication, we will package the accelerometers and evaluate them for
The micro-acceleration recorder concept will integrate Sandia microelectronics and a MEMS

(microelectromechanical systems) accelerometer into a miniature package, approximately $1 \mathrm{~cm}^{3}$ compared to the $24 \mathrm{~cm}^{3}$ of the smallest existing system that uses commercially available parts.

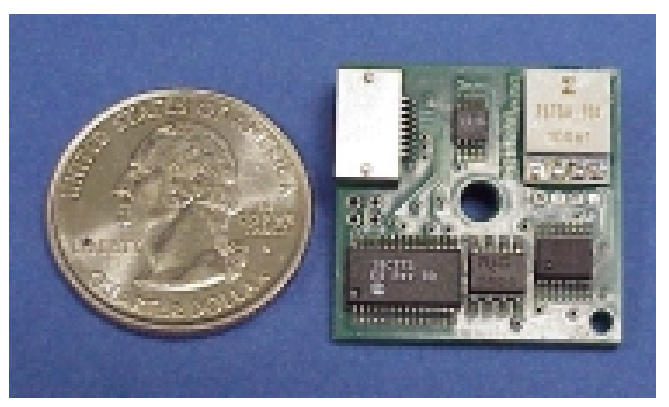

This photo shows the micro-acceleration recorder system realized with commercially available parts for system validation. 
functionality. We have completed the conceptual microelectronics ASIC design, with the detailed design to be completed shortly thereafter. The microelectronics will interface the MEMS accelerometer to the microcontroller. It will provide the gain and filtering necessary to directly digitize the acceleration signal within the microcontroller.

We also completed the design for the microacceleration recorder. A microcontroller core provides system functionality and interfacing to the data-acquisition ASIC and the outside world. We realized this design with commercially available parts for system validation. We performed validation testing of the recorder system in the shock lab. The entire recorder system is contained on a four-layer one-inch-square printed circuit board. We have begun work to reduce this size even further to achieve our design goals by designing a multichip module (MCM) containing the same electronics in bare die form.

We fabricated and evaluated the MEMS accelerometer designs. In addition, we designed and shock-tested the recorder system and are ready to interface to the data-acquisition ASIC and MEMS accelerometer.
We also completed the design for the micro-acceleration recorder. A microcontroller core provides system functionality and interfacing to the dataacquisition ASIC and the outside world. 
10736

Dynamic Range Imaging for Terrain Mapping, Position Determination, and Obstacle Avoidance in Autonomous Navigation

C. L. Smithpeter, S. M. Lebien, R. O. Nellums, J. T. Feddema, S. K. Dunlap

This project addresses the need in autonomous navigation for sensing position, generating a terrain map, and obstacle assessment/avoidance from a moving platform. The three-dimensional (3-D) image data from a light amplification for detection and ranging (LADAR) is a possible solution for these sensing needs; however, current LADAR sensors are limited by cost, complexity, required laser power, and sensitivity to motion. Other LADAR applications include weapon guidance, physical security, target surveillance, and docking of spacecraft.

To address the limitations of LADAR sensors, Sandia is seeking to develop a miniature LADAR architecture that is not sensitive to platform motion. The ultimate embodiment of this goal is reducing the LADAR receiver to a focal plane array, similar to a charge-coupled device (CCD) camera. In addition, we seek innovative techniques to reduce the required laser illumination power, which currently dominates system cost and power requirements.

Our initial approach was to develop reflective optical modulators based on semiconductor multiple-quantum-well (MQW) structures to miniaturize our scannerless range imager (SRI). The primary metric was adapting the MQW modulators to the SRI requirements of an $855 \mathrm{~nm}$ center wavelength, a diameter greater than $5 \mathrm{~mm}$, and a contrast ratio greater than five. We designed the MQW structure by computer modeling and fabricated prototypes. Reflectance data from the prototype demonstrated an acceptable $852 \mathrm{~nm}$ center wavelength and a contrast ratio exceeding the $5 \mathrm{x}$ goal across the $5 \mathrm{~mm}$ diameter. To address the fundamental issue of $10 \mathrm{MHz}$ modulation across a $5 \mathrm{~mm}$ diameter, we developed an innovative current spreading grid contact for the prototype. We expect the contrast ratio to exceed the desired factor of five based on initial results. The final metric was demonstrating a LADAR image with the MQW modulator in a bench-top system. The design and fabrication of the imaging system and the demonstration image are complete. We achieved a number of innovations in the
To address the limitations of LADAR sensors, Sandia is seeking to develop a miniature LADAR architecture that is not sensitive to platform motion....To address the fundamental issue of $10 \mathrm{MHz}$ modulation across a 5 mm diameter, we developed an innovative current spreading grid contact for the prototype. We expect the contrast ratio to exceed the desired factor of five based on initial results. 
MQW technology that demonstrate its feasibility for the LADAR application.

Another development is a concept to move the parallel channel receiver closer to the project goal: a single focal plane array (FPA) of detectors. A new feature of the FPA approach is a reduction in the cost, size, and power of the laser illuminator through electronic filtering within each pixel. Currently, the required laser power is determined by the need to compete with sunlight. The sunlight background and much of the inherent noise can be rejected using the filtering concept.

We will develop a feasibility demonstration of an FPA LADAR receiver within pixel filtering. The challenges of placing a filter within the pixel include limited area for components and maintaining the operating point of the amplifier transistors. To reduce the number of components and pixel area, we will explore using the inherent capacitance of the amplifier transistors for the filter. To control the operating Another development is a concept to move the parallel channel receiver closer to the project goal: a single focal plane array (FPA) of detectors. A new feature of the FPA approach is a reduction in the cost, size, and power of the laser illuminator through electronic filtering within each pixel. point of the circuit across the array, we will explore a number of bias schemes, including feedback mechanisms. We will design and fabricate several circuit topologies addressing the above issues in a standard integrated circuit (IC) process. We also will demonstrate the circuit operation and quantify filter rejection efficiency and cutoff frequency. 
10747

Induced Molecular Markers for Pathogen Detection: Microincubators for Rapid Toxin

\section{Expression}

J. S. Schoeniger, V. A. Vandernoot, R. Behrens, Jr., J. V. Volponi, S. M. Ngola

Existing fielded technologies for detection of biological warfare $(B W)$ agents rely primarily on antibody-based affinity assays in order to sensitively detect and identify agents. Programs such as the DOE Chemical and Biological Nonproliferation Program (CBNP) have identified development of synthetic ligands that bind to $B W$ agents in a manner suitable for affinity assays as a high priority for improving BW detection systems. The work pursued in this project involves development of technologies to enable new approaches to ligand development that, if successful, will expand our ability to deliver synthetic ligands for BW agent detection. The project focuses on new technologies for mapping the binding site of ligands on target proteins and for the synthesis and rapid measurement of affinity constants of small-molecule ligands for $B W$ agent proteins. Ligand binding sites are mapped by covalently crosslinking the ligand to the target protein, chemically cutting the target protein into short peptides, and using mass spectrometry (MS) to identify the peptides that have been modified by adding the crosslinked ligand. Sandia chose Ricin as an initial target for ligand development and developed a versatile small-molecule ligand synthesis strategy based on carbohydrate derivatives in order to bring it into line with the needs of potential sponsors for small-molecule replacements for antibodies in detectors.

We determined the binding site by covalently crosslinking the ligand to the target protein, cutting the protein into short peptides, and using MS to identify peptides modified by addition of the crosslinked ligand. We met two major milestones: to establish the necessary reactions for protein crosslinking and cleavage and to perform MS of peptides formed by protein cleavage. We produced spectra on the recently acquired Fourier-transform ion cyclotron resonance (FT/ICR) with state-of-the-art mass range and resolution. We are preparing to demonstrate liquid chromatography/mass spectrometry (LC/MS) of the crosslinked peptide fragments.

We added a small-molecule ligand synthesis effort to the project for synthesis of carbohydrate ligands to Ricin. Our
The work pursued in this project involves development of technologies to enable new approaches to ligand development that, if successful, will expand our ability to deliver synthetic ligands for BW agent detection. The project focuses on new technologies for mapping the binding site of ligands on target proteins and for the synthesis and rapid measurement of affinity constants of small-molecule ligands for BW agent proteins. 
synthetic strategy is to react the reducing end of lactose with an excess of a nitrogen-containing linker and reduce the resulting Schiff base to form a stable amide-bonded adduct. We confirmed the success of this strategy using MS and showed previously that boronate affinity chromatography can be used for product purification.

We plan to use MS to measure ligand-binding affinities but have determined that an additional high-throughput ligandbinding technique is desirable in a nearer time frame. Changes in fluorescence polarization (FP) that occur upon binding of fluorescently labeled small ligands to larger proteins can be used to measure the binding isotherm of the ligand to the protein. We therefore constructed an FP instrument and are presently optimizing the optics and planning synthesis of fluorescent-labeled galactose-based ligands, which will allow their binding affinities to Ricin to be measured.
We added a small-molecule ligand synthesis effort to the project for synthesis of carbohydrate ligands to Ricin. Our synthetic strategy is to react the reducing end of lactose with an excess of a nitrogencontaining linker and reduce the resulting Schiff base to form a stable amide-bonded adduct. We confirmed the success of this strategy using MS and showed previously that boronate affinity chromatography can be used for product purification. 
10752

\section{Distributed Reconfigurable Homogeneous Microrobotic Systems}

\section{T. L. Calton, J. T. Feddema, S. E. Eskridge, D. M. Hensinger}

A distributed reconfigurable microrobotic system is a collection of unlimited numbers of distributed small, homogeneous robots designed to autonomously organize and reorganize in order to achieve mission-specified geometric shapes and functions. The concept of a homogeneous system, which is similar to the cell structure of biological organisms, has fundamental applications and advantages (massproducible/low-cost, fault-tolerant, self-maintainable/repairable) in the design of multimodal, complex artifacts. Such systems can evolve into many different shapes (e.g., manipulators, antennas, buildings, and even animals) and perform a variety of functions (e.g., crawl, walk, swim). If some part of the system malfunctions, it can still maintain total function through automatic reconfiguration. For example, a system could piggyback into space on the Space Shuttle in some contorted form to maximize the shuttle's payload. Upon arrival, it could reconfigure itself from its traveling position and form a tether for shuttle docking, reconfigure itself again and form a lasso to rope a damaged satellite, and then reconfigure once again to form a satellite-repair tool. Even better would be to have the satellite built from a collection of homogeneous reconfigurable robots and have it repair itself. For difficult terrain maneuverability, a homogeneous system could become snakelike for narrow passages, grow legs for walking or climbing, or roll down a slope as a ball. Conceptual designs for both 2-D (two-dimensional) and 3-D reconfigurable homogeneous robots exist. Sandia is investigating design, control, and planning issues for selfconfiguring and self-organizing for both systems. The ultimate goals are (1) the building and testing of a suite of miniature, homogeneous robots designed for self-reconfiguration systems, (2) the development of control electronics that will guide and dock multiple robots that form a larger robot, and (3) the development of planning algorithms to achieve selected shapes and motion-planning algorithms to move the resulting shapes.

Research focused on hardware-interconnect technologies between homogeneous robotic modules that can achieve motion in 2-D space and on shape and motion-
Conceptual designs for both 2-D and 3-D reconfigurable homogeneous robots exist. Sandia is investigating design, control, and planning issues for self-configuring and selforganizing for both systems. 
planning algorithms for task reconfiguration spaces using homogeneous modules that can achieve motion in 3-D space.

For 2-D, we adopted the Gemini robotic platform. We designed and built a quick-disconnect mechanism utilizing rare-earth (RE) magnets to attach modules, an articulating joint allowing three types of joint conditions, and a release mechanism allowing Gemini bodies to intentionally disconnect and provide automatic separation. The quick-disconnect mechanism is designed for a two-stage hook-up. During stage one, male and female quick-disconnect couple, allowing a ring of RE magnets on the male end to attach to a metal flange on the female end. Approximately 200 pounds of maximum force is attainable. During stage two, the female flange rotates passively on bearing, allowing spring-loaded alignment pins on the male end to snap into place. The articulating joint allows for three types of joint conditions between Gemini bodies. The first is a controlled articulation, which can potentially optimize body configuration to terrain. The second is a rigid connection in a straight configuration, which has been shown to be beneficial in certain types of terrain. The last is a free mode in which there is no contact between the pushrods and the interface plate. The release mechanism works by storing approximately 250 pounds of force in the main springs. This force is held by a sear, which is in turn held by a solenoid. Turning the power off releases the spring force, and the release pin forces the male and female ends of the quick-detach mechanism to separate.

For 3-D, we conducted a feasibility study to help guide the initial design for a self-reconfigurable system composed of cube-shaped modules. For this study, we prototyped a constraint-based self-reconfiguration planning infrastructure that couples the geometry, kinematics, and dynamics of the robotic system and generates the space of all possible (geometrically feasible and dynamically stable) reconfigurations. The approach taken in the selfreconfiguration planning allows randomized motion of the cubes to take place within the influence of a potential energy field, which encourages them to move toward a minimal energy position (target configuration). The energy function need not be analytic and can be evaluated from a look-up table or from using a geometric representation. The movement of cubes is determined by (1) selecting a random cube, (2) selecting a random direction for the cube to move, (3) determining whether a move is possible based on the constraints, and (4) determining whether a move will lower the
The approach taken in the selfreconfiguration planning allows randomized motion of the cubes to take place within the influence of a potential energy field, which encourages them to move toward a minimal energy position (target configuration). 
total energy of the system. Constraints imposed within the system are that (1) the cubes cannot occupy the same space as each other, (2) the cubes cannot occupy the same space as an obstacle, (3) the cubes must always remain linked to the face of at least one other cube, and (4) the collection of cubes must be stable (will not topple over) before, during, and after moves. Analysis capabilities that were integrated into the planning infrastructure include (1) ensuring that before and after each move the collection of cubes will be statically stable as a rigid body, (2) determining at the beginning and end of each change in configuration the maximum acceleration rate that can be applied to the moving cubes without causing the collection of cubes to topple about one of its supporting edges, and (3) generating a finite-element $(\mathrm{FE})$ geometry input deck in the EXODUS II format after each move. Most of Sandia's internally developed structural, dynamic, and thermal analysis codes use this format. 
10753

\section{Robust Planning for Autonomous Navigation of Mobile Robots in Unstructured, Dynamic} Environments

\section{G. R. Eisler}

Collections of autonomous mobile robots have the advantages of stealth, compactness, redundancy, and longduration operation. They can provide effective communication networks, manifest collective intelligence via distributed algorithms, and carry a variety of sensors.

This project takes the first step toward this goal by developing a single vehicle characterized by an algorithmicdriven, multispectral approach to point-to-point navigation. Ultimately the vehicle will contain on-board trajectory planning, global positioning system (GPS) satellite position fixes with inertial measurement backup, a mix of sensors to determine obstacle size, configuration, and density, the ability to replan a path in the face of impenetrable terrain, and Internet communication for receipt of mission objectives.

Sandia will use a phased approach for long-range path planning incorporating digital maps. We will characterize the mission terrain from various standpoints: slope, obstacle density, covertness, line-of-sight (LOS), etc.

We made accomplishments in three areas: path planning, vehicle development, and obstacle sensing.

We developed a weighted graph search method for path planning on actual terrain elevation data and operated it via a graphical user interface (GUI). The interface provides inputs for map detailing as well as weight adjustment for terrain slope, path length, radio contact path (LOS), path width, and a userdesignated item.

The "AutoNav" test-bed developed is a secondgeneration robotic all-terrain lunar exploration rover (RATLER ${ }^{\mathrm{TM}}$ ) mechanical chassis incorporating an improved all-carbon central pivot joint and improved wheel motor mounts. We updated on-board computing, sensing, telemetry, and control systems to the level of the autonomous SWARM vehicle systems. Each body half is equipped with a Faraday cage to house all electronics and eliminate emitted radiofrequency $(\mathrm{RF})$ noise. We configured an on-board odometry sensor suite with a compass, pitch and roll inclinometers, optical encoder wheel odometers, differential GPS, and a sixaxis inertial rate sensor.
Sandia will use a phased approach for long-range path planning incorporating digital maps. We will characterize the mission terrain from various standpoints: slope, obstacle density, covertness, line-of-sight (LOS), etc. 
This obstacle detection system for HAGAR consists of two different types of sensors. There is an ultrasound sensor that can detect targets up to 10 feet and a camera and visible laser combination. The ultrasonic sensor will be placed in front of the vehicle and look out approximately 10 feet ahead. The vision detection system has a line projector with a 30-degree fan angle placed in front of it. The camera will see the entire laser line in its field of view. Positive and negative obstacles can be identified and characterized by the deflection of the line as it appears in different pixels in the image collected by the We made a successful traversal
over a 1.5-mile gravel road via
differential GPS navigation and
course heading feedback using a
small SWARM vehicle. camera.

We made a successful traversal over a 1.5-mile gravel road via differential GPS navigation and course heading feedback using a small SWARM vehicle. 


\section{Energy and Power Systems}

The Energy and Power Systems investment area investigates technical advances in such areas as energy efficiency, clean energy, and carbon sequestration to address critical issues facing the nation's energy infrastructure. New energy sources that provide compact power (i.e., highly efficient and mobile) are being researched to address a wide variety of national security applications. In addition, advanced technologies are being investigated that have the potential to provide sustainable, affordable energy and power sources with a high degree of surety.

The "Designed Ionophores for Liquid-Membrane Separation and Extraction of Metal Ions" project is investigating new materials and inexpensive processes to promote metal-ion separation and extraction, based on selective transport of ions across membranes. These materials and processes address the reprocessing and treatment of fuel and waste associated with nuclear fuel. In this research, molecular simulation was used to design highperformance ionophores specifically for the extraction of heavy metals and radionuclides. Ion-extraction technologies using designed ionophores and photoreducers have

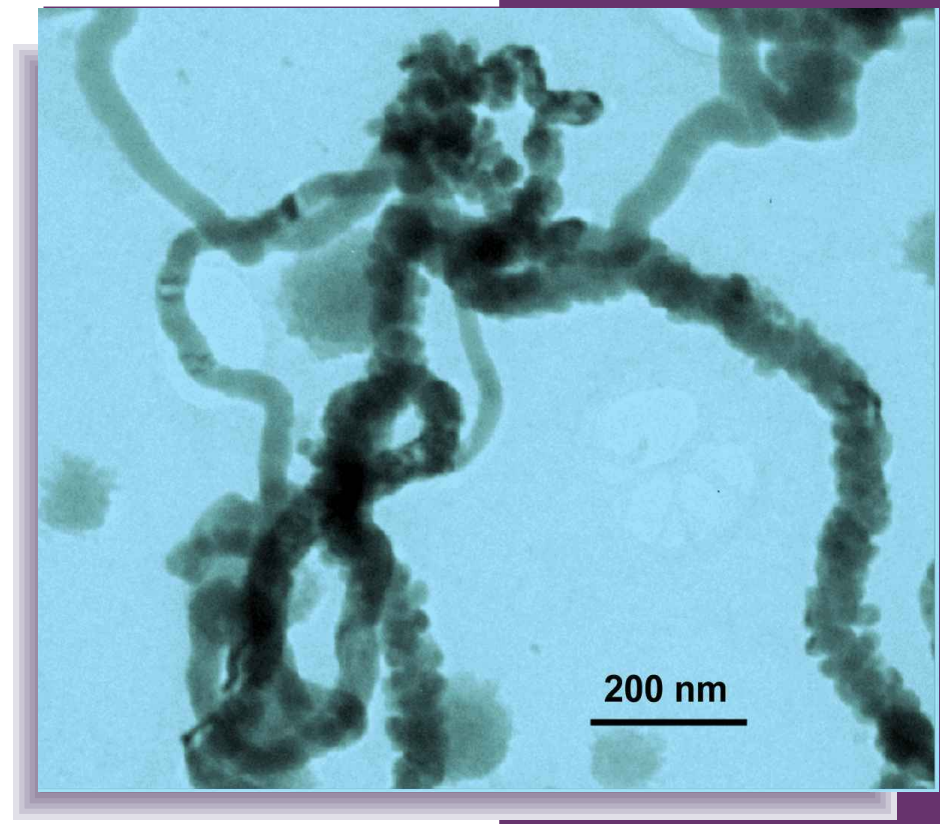

demonstrated increased sustainability and lower cost and complexity of manufacturing processes. These extraction technologies offer convenient new means for decontamination and decommissioning, for radioactive-waste remediation, and for heavy-metal waste stream and in situ treatment applications. 
10384

\section{Adaptive 3-D Sensing}

C. Q. Little, J. J. Carlson, D. J. Schmitt, D. E. Small

The purpose of this project is to create sensing, reasoning, and control technologies that will allow the safe and effective cooperation of humans and intelligent machines in a variety of operations. This will rely heavily on real-time three-dimensional (3-D) sensing and interpretation of the 3-D data. The results of this research will include a new adaptive 3-D sensing system as well as new research into dynamic object modeling, behavior recognition, and sensor-based control. The 3-D sensing system will combine two existing sensing technologies in a new way to create a site-monitoring system that can continuously maintain an accurate 3-D model of the site and real-time estimates of the shape, location, and motion of humans and machines. A key strength of this system will be the ability to monitor simultaneous activities, such as several robots and humans working independently, while also detecting unexpected entry into the workspace. Sandia will use data from this sensing system to allow automated systems such as robots to not only safely co-exist with humans within the workspace but also to cooperate in performing tasks. This will improve the practicality and efficiency of many operations by simultaneously using the best skills of both humans and machines.

We demonstrated human-machine cooperation with technology-related applications. A human operator can engage an active pedestal robot that uses human location data to control its movement to track and hand off objects to and from its gripper. The operator can additionally give direction to the robot while in the workspace through voice and body gestures. This has opened a new area of research for human-machine interface work with an active test-bed. Incorporated technologies include voice input, audio feedback, firstgeneration gesture recognition, motion detection and tracking, 3-D scene construction, object recognition, and a wireless "live-man" switch for greater flexibility of human interaction while maintaining strict safety standards.

We enhanced the dynamic sensing capabilities through increased resolution of the 3-D volume, which means we can measure greater detail. We achieved a level of human tracking that includes tracking overall body position and the position of arms in four degrees. That is, we track the position of both
Sandia will use data from this sensing system to allow automated systems such as robots to not only safely co-exist with humans within the workspace but also to cooperate in performing tasks. This will improve the practicality and efficiency of many operations by simultaneously using the best skills of both humans and machines. 
elbows and wrists in motions both forward and back and up and down. We incorporated this into a robot control to prototype human-machine interaction within an environmental workspace. The application is to drive a crane. Gestures are given using both arms to raise and lower a load, raise and lower a boom, and move the crane to the left and right. 
10385

\section{Designed lonophores for Liquid-Membrane Separation and Extraction of Metal lons}

J. A. Shelnutt, J. E. Miller

Sandia is deveoping new materials and processes for metal-ion separation and extraction based on selective transport of ions across membranes. Using molecular simulations, we are designing a new group of porphyrin-based ionophores (molecular ion-carriers) for selectivity in complexing and transporting metal ions by controlling basicity, shape, and size of the ionophores. Using the initial design results, we are designing ionophores specifically for extraction of heavy metals and radionuclides. Promising ionophores are synthesized and tested for application in two different membrane systems. The first is a supported liquid membrane that separates two liquid phases-one containing waste and the other a liquid to which the specified metal ions are extracted. The ionophores selectively transport a specified metal ion through the intervening liquid layer to give a pure solution of the metal ion that is easily concentrated. In the second, we are developing metal-ion transport and entrapment in surfactant vesicles using the new ionophores. Separation of the vesicles leaves a pure metal-ion solution. These extraction technologies offer convenient new means of separation and extraction for processing ${ }^{99} \mathrm{Mo}$ (molybdenum), decontamination and decommissioning, radioactive-waste remediation, and heavy-metal waste-stream treatment. The initial focus area is high-level-waste tank remediation of heat and gamma emitters $\left({ }^{137} \mathrm{Cs}\right.$ [cesium] and ${ }^{90} \mathrm{Sr}$ [strontium]), long-lived radionuclides ( ${ }^{99}$ Tc [technetium]), and other metal ions contained in highand low-level waste. Ion-extraction technologies using designed ionophores lead to increased sustainability and lower cost and complexity of manufacturing processes. We also consider adaptation to pump/treat methods. This technology is differentiated from other efforts by the use of molecular simulation to engineer high-performance ionophores, the application of Sandia's recognized expertise in computer-aided molecular design (CAMD) of unexplored ionophore types, and the use of two new membrane systems.

While using molecular simulations to design new ionophores for improved selectivity and efficiency for metalion transport activity, in collaboration with the University of New Mexico (UNM) we discovered a new, efficient reductive
Sandia is deveoping new materials and processes for metal-ion separation and extraction based on selective transport of ions across membranes.

This technology is differentiated from other efforts by the use of molecular simulation to engineer high-performance ionophores, the application of Sandia's recognized expertise in computer-aided molecular design (CAMD) of unexplored ionophore types, and the use of two new membrane systems. 
method for extracting a wide variety of heavy-metal ions from contaminated water. This new deployable extraction process is highly preferable to the extraction into detergent vesicles and liquid-membrane extraction into aqueous solutions that were being developed. We thus invested most of our effort into evaluating these new metal-ion extraction techniques.

Nevertheless, we made significant progress in understanding the use of a new type of ionophore, one that eliminates the need for added picric acid as a coagent. The latter allows a great improvement in the design, efficiency, and safety of liquidmembrane metal-ion extraction processes. By varying the $\mathrm{pH}$ of the source and receiving phases, the equilibrium between free-base and anionic forms can be adjusted to optimize transport by H2Br8TPP and its analogs. We designed new ionophores with the required electronic and steric properties to further enhance selectivity for this new extraction and transport process. We obtained x-ray crystal structures of the ionic porphyrin complexes and some picrate-porphyrin complexes that have greatly increased our understanding of porphyrins as ionophores.

For actual heavy-metal remediation studies, we turned to the more efficient newly discovered reductive method for in situ treatment. The method is based on a "wagon wheel" treatment system used at UNM for nitrate-contaminated sites, but it utilizes the ability of proteins such as cytochrome c 3 to reduce $U+6$ to $U+2$, which then precipitates as the very weakly soluble mineral uraninite. Many other metals are subject to this reductive precipitation method, including Mo and chromium (Cr). Many of the metal ions are reduced to the zero-valent metal, including lead $(\mathrm{Pb})$, mercury $(\mathrm{Hg})$, copper $(\mathrm{Cu})$, silver $(\mathrm{Ag})$, gold $(\mathrm{Au})$, selenium $(\mathrm{Se})$, and platinum $(\mathrm{Pt})$. In most cases, the process lowers the solubility to Environmental Protection Agency (EPA)-acceptable limits, providing a longterm solution to the contamination problem. The method is environmentally benign since only proteins from naturally occurring nitrate- and sulfate-reducing bacteria are utilized along with $\mathrm{H}_{2}$ (hydrogen) for regenerating the reduced cytochrome $\mathrm{c} 3$. A version of this novel approach may also be used to extract plutonium $(\mathrm{Pu})$ from sites where the contamination is above the groundwater saturation zone.

We subsequently found that a tin ( $\mathrm{Sn}$ )-porphyrin analog of the cytochrome was just as successful in reducing most of these metals. The $\mathrm{Sn}$ porphyrin has the added advantage of using light as the source of the strong reductant needed. The ultimate source of the electrons in our model studies was
While using molecular simulations to design new ionophores for improved selectivity and efficiency for metal-ion transport activity, in collaboration with the University of New Mexico (UNM) we discovered a new, efficient reductive method for extracting a wide variety of heavy-metal ions from contaminated water. 
environmentally benign triethanolamine. This weak reductant could be replaced by other reductants, including water itself. The Sn-porphyrin photocycle has a quantum efficiency of approximately 0.5 and utilizes the entire ultraviolet (UV)visible solar spectrum for metal reduction. We also showed that the $\mathrm{Sn}$ porphyrin could be incorporated into mesoporous silica while retaining its photochemical activity. Ag nanoparticles could be precipitated and trapped within the calcined silica matrix at low concentrations of Sn porphyrin or as more densely packed metal conglomerates for porphyrin at higher concentrations.

Besides the waste-treatment aspects of the Sn-porphyrin photochemistry and protein-mediated reduction, we found

Besides the waste-treatment aspects of the Sn-porphyrin photochemistry and proteinmediated reduction, we found these methods useful in the processing of new nanostructured materials such as nanoparticles and nanowires. these methods useful in the processing of new nanostructured materials such as nanoparticles and nanowires. For example, cytochrome c3 reduction of Se ions in aqueous solution at room temperature gives self-assembled nanowires of Se metal. The Sn-porphyrin photosensitizer for metal reduction has proven suitable for photolithographic processing of nanostructured metals within structured nanoscale environments of inorganic materials.

\section{Refereed}

Abdelouas, A., W. L. Gong, W. Lutze, and J. A. Shelnutt. 2000. "Reductive Precipitation of Metals Photosensitized by Tin Protoporphyrin." Water Res., accepted.

Abdelouas, A., W. L. Gong, W. Lutze, E. H. Nuttall, F. Sprague, J. A. Shelnutt, B. A. Strietelmeier, R. Franco, I. Moura, and J. J. G. Moura. 2000.

"Reduction of Heavy Metals by Cytochrome c3." Water Res., accepted

Abdelouas, A., W. L. Gong, W. Lutze, J. A. Shelnutt, R. Franco, and I. Moura. 2000. "Using Cytochrome c3 to Make Selenium Nanowires." Chem. Mater. 12: 1510-1512.

Cullen, D. L., L. V. Desai, M. Zimmer, and J. A. Shelnutt. 2000. "Conformational Analysis of the NonPlanar Deformations of Cobalt Porphyrin Complexes in the Cambridge Structural Database." Struct. Chem.
Kiefl, C., R. W. Woody, Y. Lu, Y. Qiu, and J. A. Shelnutt. 2000. "Analysis of Heme Distortions in Molecular-Dynamics-Generated Structures of Both Rotational Isomers of Sperm Whale Carbonmonoxy Myoglobin. Correlations Between Intrinsic Rotational Strength and Heme Deformations." J. Amer. Chem. Soc.

Lemke, C., R. Schweitzer-Stenner, J. A. Shelnutt, J. M. E. Quirke, and W. Dreybrodt. 2000. "Conformational Distortions of Metalloporphyrins with Electron Withdrawing $\mathrm{NO}_{2}$ Substituents at Different Meso Positions. I. Vibrational Analysis." J. Amer. Chem. Soc.

Muzzi, C. M., C. J. Medforth, K. M. Smith, S. L. Jia, and J. A. Shelnutt. 2000. "Synthesis and Characterization of a Chiral Nonplanar Porphyrin." Chem. Commun.: 131-132. 
Pauleta, S. R., Y. Lu, C. F. Goodhew, I. Moura, G. W.

Pettigrew, and J. A. Shelnutt. 2000. "Calcium-

Dependent Conformation of a Heme and Fingerprint

Peptide of the Di-Heme Cytochrome c Peroxidase

from Paracoccus Pantotrophus." Biochem.

Schweitzer-Stenner, R., C. Lemke, J. A. Shelnutt, J.

M. E. Quirke, and W. Dreybrodt. 2000. "Electronic

and Structural Distortions of the Porphyrin Macrocycle

by Meso Substituents with Strong Electron

Withdrawing Capacity." J. Amer. Chem. Soc.

Shelnutt, J. A. 2000. "Molecular Simulations of

Porphyrins and Heme Proteins." J. Porphyrins

Phthalocyanines 4: 386-389.

Shelnutt, J. A. 2000. "Normal-Coordinate Structural

Decomposition and the Vibronic Spectra of

Porphyrin.” J. Porphyrins Phthalocyanines, accepted.

Shelnutt, J. A., R. Haddad, Y. Lu, and J. M. E. Quirke. 2000. "Steric Bulkiness of Pyrrole Substituents on Out-of-Plane Deformations of Porphyrins: Nickel(II) Octa(isopropyl)porphyrin and Its Meso-Nitro Derivative." J. Amer. Chem. Soc.

Woody, R. W., C. Kiefl, N. Sreerama, Y. Lu, Y. Qiu, and J. A. Shelnutt. 2000. "Molecular Dynamics Simulations of Carbonmonoxy Myoglobin and Calculations of Heme Circular Dichroism." Insulin and Rel. Proteins from Structure to Func. and Pharmacology, edited by M. Federwisch and M. L. Dieken. Dordrecht, The Netherlands: Kluwer Academic Publishers, accepted 
10386

\section{An Electromagnetic Imaging System for Environmental Site Reconnaissance}

G. M. Loubriel, G. J. Denison

The advantages of time-domain, ultra-wideband transmitters for ground-penetrating radar (GPR) are many and well known: GPR has the potential for high-resolution subsurface imaging unmatched by any other subsurfacesensing method. With GPR there are large contrasts between contaminated and noncontaminated regions. Thus GPR may detect man-made buried objects and detect and define the extent of contaminated soil. This project seeks to develop a GPR system that can be utilized for environmental site reconnaissance and, in particular, for (1) detecting disrupted soil layers where there is a potential for buried waste, (2) finding buried metallic objects such as 55-gallon drums at depths of up to 10 meters (and other man-made objects at various depths), and (3) detecting contaminated soil. Because of the simplicity of the time-domain technique, the system will be fast enough to evaluate extensive land areas that may be contaminated. The primary disadvantages of GPR have been low penetration depth and low signal to clutter. This project is designed to minimize both of these problems. The penetration depth will be augmented by using (1) high-peak-power, highrepetition-rate operation for high average power, (2) low center frequencies that better penetrate the ground, and (3) short-duration impulses. The latter allow for the use of low platforms that increase the power on target relative to a highflying platform (due to the $R 4$ term in the radar equation, where $R$ is the range to the target). Clutter is minimized by time-gating the surface-clutter return and using low frequencies (since natural objects are smaller than the wavelengths used here and their cross-section is smaller than for higher-frequency systems). The factor that makes this work unique is that Sandia will utilize direct time-domain radar at low frequency and high average power.

We modeled the problem, built an impulse transmitter for GPR field tests, and designed and are building a variablecenter-frequency transmitter. We added a new task: measure switch jitter and longevity with the original pulser design. We met with success in the field tests because of three major efforts: (1) theoretical model of ground penetration to guide the transmitter development in collaboration with Duke University,
This project seeks to develop a GPR system that can be utilized for environmental site reconnaissance.... The factor that makes this work unique is that Sandia will utilize direct timedomain radar at low frequency and high average power. 
(2) pulser construction at fixed frequency (but higher than attempted previously) and design of variable-frequency system, and (3) switch development. We started tests of the system in transmit/receive mode and of reflection from targets in air. Our biggest challenge was to increase the operating frequency of the transmitter; this forced us to design and test different pulser designs that were variants of the original design. Because of the introduction of the new pulser designs, we wanted to assess the effect of the different designs on switch longevity. We modeled the problem and developed a transmitter. 
10387

\section{Advanced Geosphere Transport Simulation}

M. J. Martinez, P. L. Hopkins, P. C. Reeves, J. L. Ramsey, Jr.

During the past decade, the need for predictive tools in areas ranging from environmental remediation to oil and gas extraction have prompted the development of subsurface, multiphase-flow, and contaminant-transport simulators. Numerical simulation is a key technology in system design and decision analysis for radioactive-waste disposal, subsurface environmental remediation, and petroleum reservoir management. Present limitations in computational technology drive model simplifications in the range of physical processes simulated, site geometry, and geologic realism, with significant negative impacts: (1) simplifications become regulatory points of contention, 2) critical details about fast-path transport are lost, and (3) turnaround times for assessing uncertainty are too long to drive project decisions. A massively parallel (MP) geosphere transport model will overcome these limitations and place Sandia in an advanced position with respect to repository simulation capabilities. The Waste Legacy, Weapon Safety, and National Energy Security programs have a unique opportunity to leverage major Defense Program (DP) investments in computational simulation and to develop a fast, flexible geosphere transport simulation capability, running on MP computers and networked workstations. This project effort is being built on a recently completed MP research code. This code will be a key component of the Nuclear Waste Management Program's (NWMP's) long-term strategic initiative to significantly enhance performance assessment capability. This code will also have the potential to enhance research and development in other technologies whose performance hinges on understanding and controlling geosphere transport, including exploitation and management of oil and gas and geothermal resources.

New developments on the species transport model included the completion of a full-tensor hydrodynamic dispersion model. We included a general distribution coefficient provision that allows various general models of adsorption of species by the porous matrix. We verified the transport model against known solutions for both isotropic and anisotropic hydrodynamic dispersion. We also demonstrated the model on a high-resolution simulation (> 1 million grid
This project effort is being built on a recently completed massively parallel (MP) research code. This code will [have the potential to support] the Nuclear Waste Management Program's (NWMP's) long-term strategic initiative to significantly enhance performance assessment capability. This code will also have the potential to enhance ... other technologies whose performance hinges on understanding and controlling geosphere transport.... 
points) of flow and species transport through highly heterogeneous fractured granite.

We improved capabilities for computing phase evolution problems (i.e., dynamically evolving regions of single phase adjacent to two phase states) as part of applications. We applied PorSalsa to study the subsurface transport of JP8 jet fuel during fuel-spill fires. PorSalsa computed the dynamics of liquid fuel dry-out due to a $10 \mathrm{~kW} / \mathrm{m}^{2}$ heat-flux simulation of a fire and predicted the associated subsurface-to-surface mass flux of fuel. This study also required the development of a capability to model twophase liquid/liquid incompressible flows. This enhances the ability to also model a variety of contamination problems. We also conducted verification of phase evolution for thermal convection from a heated subsurface tunnel. We demonstrated convection in liquid-saturated media and thermal convection in two-phase systems. The latter problem was driven to dryout to demonstrate the phase-evolution capabilities.

Well-bores are common in many problems involving injection and withdrawal in subsurface transport. There is also a large variation in the complexity of well-bore behavior, ranging from a source/sink description to models that involve separate multiphase flow equations for describing the coupled transport along the length of the well-bore and with surface conditions. We implemented a general multiphase well-bore model for unstructured grids, thereby allowing very general geometric configurations such as horizontal and deviated wells. Implementation of this feature for parallel processing was challenging because the model requires nonprocessor local data. We developed a careful formulation to maintain ease of use while allowing a very general well-bore model description.

We ported PorSalsa for use by VP 6000 personnel on the DEC8400 cluster and on the Aranae Linux cluster. The Linux cluster implementation in particular makes the massively parallel aspects of PorSalsa conveniently available. This implementation includes a set of complete test problems, including mesh files, load-balance files, and input and output files, for the convenience of the user. These problems can be used to verify the implementation of the code on this system, and more importantly they allow a rapid tutorial on the use of PorSalsa.
We improved capabilities for computing phase evolution problems (i.e., dynamically evolving regions of single phase adjacent to two phase states) as part of applications. 


\section{Refereed}

Martinez, M. J. 1999. "Slug Flow Model for

Infiltration into Fractured Porous Media." Proc. ASME

Heat Transfer Div.-1999, ASME Internat.

Mechanical Eng. Congress and Exposition HTD 364-2

(Nashville, TN, 14-19 November 1999): 125-132.

Martinez, M. J., and P. L. Hopkins. 2000. "Modeling

Subsurface Multiphase Transport of JP8 During a Fuel

Spill Fire." Sandia Technical Report SAND2000-2464, Sandia National Laboratories, Albuquerque, NM

(October).

Martinez, M. J., and R. H. Nilson, 1999. "Estimates of Baropumping of Moisture Through Unsaturated

Fractured Rock." Transport in Porous Media 36: 85119.

\section{Other Communications}

Martinez, M. J. 1999. "Notes on Implementing Well

Bore Models in PorSalsa." Sandia internal memo to distribution, Sandia National Laboratories, Albuquerque, NM (October).

Martinez, M. J., P. L. Hopkins, and J. N. Shadid. 2000. "PorSalsa-A MP Geosphere Two-Phase Thermal Subsurface Simulator." DOE/MICS (Mathematical, Information, and Computational Sciences Division, Office of Computational and Technology Research, DOE) Newsnote, June.

Reeves, P. C. 2000. "Geosphere Specific Upgrades to PorSalsa." Sandia internal memo to distribution, Sandia National Laboratories, Albuquerque, NM (5 January). 
10718

A Novel Microcombustor for Sensor and Thermal-Energy Management Applications in Microsystems

T. J. Gardner, R. J. Kottenstette, R. P. Manginell, G. C. FryeMason, D. L. Mowery, S. B. Margolis

The development of a small and stable on-chip flamel combustor (" $\mu$ Combustor") would permit the adaptation and translation of several very useful macroscopic devices into the microsystem domain: on-chip flame ionization detectors ( $\mu$ FIDs), microreactors, micropropulsion, energy conversion, and, importantly, heating and thermal management. The argument for the use of on-chip combustive heating is compelling. The energy density of butane (including storage cylinder mass) is 50 times that of the best high-output batteries (LiMnO ${ }_{2}$ nonrechargeable). Thus, a tiny fuel tank could replace several bulky batteries in hand-held microanalytical systems like the $\mu$ ChemLab ${ }^{T M}$ and would supply a Combustor for efficient heating of essential components.

Sandia will develop a $\mu$ Combustor as a basis for thermal-energy management in microsystems as well as in the development of new $\mu F I D$ devices for use for intelligent process control of energy-intensive processes (e.g., reactors, distillation columns, and dryers in the petrochemical industry). Stable flames are difficult to achieve on a small scale due to enhanced heat loss arising from large surface-to-volume ratios. Catalytic materials provide a natural surface-based method for flame ignition and/or stabilization of the reaction zone. Further stabilization can be achieved by the use of a thermally isolating membrane as a support for microfabricated fuel injectors. One key enabling technology for the $\mu$ Combustor will be the development of active and stable (deactivation-resistant) catalyst materials (likely supported precious-metal catalysts) for the combustion of different feedstocks, ranging from hydrocarbons (e.g., butane or propane) to hydrogen (H). Deposition of these catalysts in a high-surface-area form on relevant platforms (i.e., resistively heated filaments) to facilitate rapid combustion may also be required for device fabrication.

We achieved our most significant milestone by successfully designing, fabricating, and testing $\mu$ Combustor prototypes with $\mathrm{H}$ fuel. We deposited evaporated platinum $(\mathrm{Pt})$ films onto free-standing, micromachined silicon/silicon nitride
Sandia will develop a $\mu$ Combustor as a basis for thermal-energy management in microsystems as well as in the development of new $\mu F I D$ devices for use for intelligent process control of energyintensive processes (e.g., reactors, distillation columns, and dryers in the petrochemical industry)....We achieved our most significant milestone by successfully designing, fabricating, and testing $\mu$ Combustor prototypes with $H$ fuel. 
( $\mathrm{Si} / \mathrm{SiN}$ ) membrane devices. We used the Pt films to define integrated heaters and resistive temperature sensors, in addition to serving as a catalytic film to initiate the combustion process. We mounted the $\mu$ Combustor wafer in a dual-inline package (DIP), using Pyrex lids to seal the device and provide a flow channel to mix and direct gas flow in and out of the $\mu$ Combustor device. This first-generation $\mu$ Combustor prototype demonstrated stable, self-sustaining oxyhydrogen flames. This successful demonstration supports our original hypothesis that $\mathrm{SiN}$ thermal isolation membranes could help solve the problem of enhanced heat loss in microsystems that otherwise would prevent stable flames on this scale. An important aspect of demonstrating successful $\mu$ Combustor prototype performance was extensive gas-flow and temperature-sensing testing and calibration. Plans are in place to evaluate alternate catalytic materials, forms (supported versus unsupported), and preparation methods.

Progress toward our second major milestone, adapting the $\mu$ Combustor design for use as a $\mu$ FID for hydrocarbonsensing applications in microsystems such as the $\mu \mathrm{ChemLab}^{\mathrm{TM}}$, involved two separate efforts. First, we are fabricating a new common fixture to support both the $\mu$ Combustor and the $\mu$ FID efforts, which will improve our testing throughput and analysis capabilities by having interchangeable lids tailored to each application. Second, we modified our control circuitry design for better $\mu$ Combustor operational control as well as the inclusion of high-sensitivity $\mu$ FID detection circuitry. We will continue work related to the modeling of oxyhydrogen flames to support FID designs.
This first-generation $\mu$ Combustor prototype demonstrated stable, selfsustaining oxyhydrogen flames.

This successful demonstration supports our original hypothesis

that SiN thermal isolation membranes could help solve the problem of enhanced heat loss in microsystems that otherwise would prevent stable flames on this scale. 
10725

A Microgas Chromatograph (mGC)-Based
Controller for Energy-Intensive Processes

M. D. Ivey, D. A. Loy, G. C. Frye-Mason, K. N. Groom

Intelligent sensors are not widely used to control drying and distillation processes in U.S. chemical and petroleum sectors. A reluctance to implement new, energy-saving technologies can be attributed to a lack of proven sensors that can adapt to a variety of complex processes and detect a wide variety of compounds within a single process. Currently, pilotscale processes are empirically scaled to full-scale processes with safety factors added to meet quality-control standards. Quantitative analytical testing is time-consuming and not currently possible in real time. Drying and distillation in the U.S. chemical and petroleum sectors consume about 12 Quads of energy annually. Intelligent real-time control, based on robust, configurable sensors with a wide dynamic range, is needed to implement a science-based solution that can improve the process efficiency and decrease energy consumption. Research and development are required to meet this goal.

The focus of this project is to develop a viable industry solution for science-based, real-time control of chemical and petroleum processes to increase process efficiency and decrease energy consumption. Research and development are required for sensor development, on-line calibration, data fusion, and system-integration architectures. Current research efforts on the $\mu$ ChemLab ${ }^{T M}$ sensors will provide the fundamental platform for the microsensor-based system. Key sensor research elements will focus on identifying new absorbing materials and methods for coating the microgas chromatograph ( $m G C$ ) column, on-line delivery of reference samples, investigating and implementing new front ends (such as valve matrices), identifying and implementing carrier-gas options and detectors, and developing a quantitative rather than qualitative output.

We will integrate these elements into a workable prototype that can be used for intelligent real-time process control. We will use existing industry ties to ensure viability of the directions taken during the execution of this project.

We made significant technical progress toward defining the mGC controller and developing new components for the controller. We also took major steps toward developing formal
The focus of this project is to develop a viable industry solution for science-based, realtime control of chemical and petroleum processes to increase process efficiency and decrease energy consumption....We made significant technical progress toward defining the $m G C$ controller and developing new components for the controller. 
relationships with partners in the pharmaceutical, petroleum, and micro-instrumentation industrial sectors.

A project goal is to demonstrate quantitative capability for the solvents commonly used in pharmaceutical manufacturing. These are methanol, ethanol, isopropanol, acetone, methyl tert butyl ether, methylene chloride, hexane, and toluene. We tested on-chip microchannel columns with our current coatings for this full set of analytes. With the exception of toluene, which is cleanly separated, the other analytes are poorly retained due to their high volatility. We also tested a micropacked GC column and found that these analytes are retained too strongly on this type of column. A microchannel column with a thicker stationary phase is needed. We developed techniques for fabricating thicker coatings of both nonpolar (e.g., poly dimethyl siloxane) and polar (e.g., polyethylene glycol) stationary phases and demonstrated effective separation of these analytes using these phases.

Our work included microencapsulation as a means to release known quantities of reference solvents. We investigated the use of co-polymers that release hydrocarbon standard compounds when thermally decomposed. This method of releasing a reference solvent appears to offer greater control than microencapsulation. We tested co-polymers fabricated for use as a reference internal standard and investigated a microdiffusion source for internal standard.

At present, a controlled-volume loop appears to be the best method to address target application. One potential industrial partner has front-end sampling loops that appear to be suitable for use with Sandia's mGC.
We developed techniques for fabricating thicker coatings of both nonpolar (e.g., poly dimethyl siloxane) and polar (e.g., polyethylene glycol) stationary phases and demonstrated effective separation of these analytes using these phases. 
10732

\section{A MEMS Microelectric Generator}

A. D. Oliver, R. G. Jungst, T. R. Christenson, A. A. Jojola

Sandia will design a microelectromechanical system (MEMS)-based electromagnetic (EM) generator to convert mechanical energy to electrical energy. Although generator technology is not new, the opportunities presented by the miniaturization of mechanical components are new and should be exploited. Specifically, we refer to the new developments in LIGA (the German term Lithographie, Galvanoformung, und Abformung, for lithography, electroplating, and molding)fabricated permanent magnets, in the development of mesoscale machinery based on LIGA (LIGA is a MEMS process in which $x$-ray radiation and thick photoresist are used to create very precise, high-aspect-ratio, and electroplated metal parts), and in the miniaturization of coils. The advantage of an EM energy-conversion method in comparison to batteries or fuel-based schemes is that it has higher reliability and a long lifetime, requires less maintenance, and provides more energy output over its lifetime than current devices. The generator could be used as a replacement for batteries in weapons. The generator has a predicted peak output of $30 \mathrm{~mA}$ at $3.3 \mathrm{~V}$. The design goal for the size of the generator is less than $2 \mathrm{~cm} \times 2 \mathrm{~cm} \times 2 \mathrm{~cm}$.

We made substantial progress in the areas of defining the system power requirements, coupled mechanical and EM design of the generator system, and battery selection and testing. Once we complete the mechanical design of the generator, we will begin fabrication.

We summarized the power requirements for potential electrical loads and selected the proper size of the generator and battery because there is a trade-off between energy output and volume. In addition, the generator/battery system had to be small ( $2 \mathrm{~cm} \times 2 \mathrm{~cm} \times 2 \mathrm{~cm}$ or smaller) and lightweight. We noted that no currently available batteries could supply the high-current applications and still be capable of being recharged. We decided to use the largest-capacity battery that could fit in the desired volume and still leave room for a recharging circuit.

The generator operates by a permanent magnet moving back and forth in a cylindrical enclosure surrounded by coils. Under the influence of vibration, the magnet moves back and forth, causing the amount of flux through the coils to vary. The 
time-varying flux through coils produces an electrical voltage that charges the battery. We maintain the position of the magnet by balancing the magnetic force and the spring force. We chose neodymium (Nd)-iron (Fe)-boro (B) as the permanent magnetic material because of its superior magnetic properties $\left(\mathrm{Hc}=8.99 \times 10^{5} \mathrm{~A} / \mathrm{m}, \mathrm{Br}=1.23 \mathrm{~T}\right)$.

The main task was to select and test the best available commercial technology that can meet the projected requirements for the overall generator system. We established a list of the power requirements at the beginning of the project. Because of the expected intermittent nature of the power output from the microgenerator, the overall mode of operation for the device will be to power the application from the battery with supplementation or opportunity charging from the generator whenever power is available from it. A rechargeable battery using lithium ( $\mathrm{Li}$ ) chemistry appears to come closest to satisfying the collective set of requirements.

Our survey of commercial rechargeable battery products confirmed that no cylindrical or prismatic cells could be found that would fit within the desired size envelope. The only choices that come close to meeting the size constraint are button cells, and only a few of these are rechargeable. The best choices for this application are the MC621, the ML 1216 and the ML 2024 manufactured by Varta.

To investigate the actual capacity and rate capability of these small button cells, we obtained three samples for the Varta products. Prior to cycling, we measured the impedance of the cells at the cell voltage received.

We began cycling the Varta cells with the determination of the proper charge profile to obtain stable capacity values at low charge/discharge rates. Preliminary observations are that the 8 and $30 \mathrm{mAh}$ cells are delivering discharge capacities above the nominal rating, even with an incompletely optimized charge profile. This testing will be followed by measurements of deliverable capacity at increasing rates and evaluations of how the cells respond to charging from the simulated output of the microgenerator.
We began cycling the Varta cells with the determination of the proper charge profile to obtain stable capacity values at low charge/discharge rates. Preliminary observations are that the 8 and $30 \mathrm{mAh}$ cells are delivering discharge capacities above the nominal rating, even with an incompletely optimized charge profile. 
10748

\section{Nanostructured Silicon Surfaces for Cost- Effective Photovoltaic Efficiency}

\section{Improvements}

\author{
D. S. Ruby, R. J. Buss, S. A. Kemme, S. H. Zaidi
}

The goal of this project is to evaluate a new regime of plasma surface texturing by using silicon (Si) solar cells as an experimental vehicle. Sandia will develop plasma models to explain, predict, and guide the plasma texturization process. These models will then have usefulness in future applications whenever surface texture evolution needs to be better understood and utilized. We will also develop optical modeling to optimize the surface texture required for maximum performance. The optical models developed here should be extremely useful in many of the nanoscale optoelectrical applications currently under development. Additionally, the development of textured surfaces on low-cost multicrystalline silicon ( $\mathrm{mc}$-Si) solar cells could significantly advance photovoltaic technology and potentially enable new thin-film crystalline-Si approaches that may produce high-power, lightweight, mobile power supplies.

This interdisciplinary project utilizes Sandia's expertise in photovoltaics, plasma processing, sensor-based controls, and process modeling to develop environmentally benign processes that can reduce costs and improve product performance. We will use large-area $\left(130 \mathrm{~cm}^{2}\right)$ commercial mc-Si solar cells as the test vehicle in this project so that improvements in performance can be immediately adopted into mass production. Successful completion of this project will also provide validated process models that can be used in a variety of applications for surface texturing, such as to promote adhesion for neutron-generator (NG) assembly and to optimize new optoelectronic devices.

We examined reactive ion etching (RIE) for texturing large-area mc-Si wafers. By using a systematic approach to adjusting both the variables of the RIE-texturing process and the subsequent damage removal etches, we obtained a combination of processing parameters that resulted in very dark, highly absorptive cells with little or no surface damage and excellent performance. We developed several metalcatalyst-assisted RIE-texturing techniques using $\mathrm{SF}_{6} / \mathrm{O}_{2}$ plasma chemistry in a Plasma-Therm 790 reactor. We investigated a large-parameter space of power, pressure, gas ratio, flow rate,
This interdisciplinary project utilizes Sandia's expertise in photovoltaics, plasma processing, sensor-based controls, and process modeling to develop environmentally benign processes that can reduce costs and improve product performance.... Successful completion of this project will also provide validated process models that can be used in a variety of applications for surface texturing, such as to promote adhesion for neutron-generator $(N G)$ assembly and to optimize new optoelectronic devices. 
and etch time. We found a narrow parameter range to be useful for texturing Si wafers up to 6" in diameter and mc-Si wafers of $130 \mathrm{~cm}^{2}$. The textured surfaces exhibit reflectance of between $1 \%$ and $5 \%$ for wavelengths below $1 \mu \mathrm{m}$ without the benefit of antireflection films.

The challenge involved in incorporating the lowreflectance surfaces obtained from RIE-texturing into a complete solar cell has been to remove the plasma-induced contamination and surface damage without removing too much of the texture. With the selection of the proper cleaning, damage-removal etches, and RIE-texture process, we fabricated cells whose internal quantum efficiencies (IQE) were increased to levels as high as those of our best wettextured cells on monocrystalline $\mathrm{Si}$ with reflectances almost as low. We used single-crystal wafers to compare the RIEtexturing process both to planar cells, which represent today's untextured mc-Si cells, and to high-efficiency wet-textured cells. The best RIE-textured cells had current densities of over $34.3 \mathrm{~mA} / \mathrm{cm}^{2}$ and efficiencies up to $16.5 \%$, which far exceeded the $31 \mathrm{~mA} / \mathrm{cm}^{2}$ and $14.75 \%$ typical of the planar control cells. These results, in fact, slightly exceeded the high currents and efficiencies of our best high-efficiency wet-textured, singlecrystal cells. We obtained IQE higher than those on planar and wet-textured cells, boosting cell currents and efficiencies by up to $11 \%$ on mc-Si and $2.5 \%$ on multicrystalline Si cells.

The first goal for plasma modeling was to develop a texture etch model by introducing the chemistry of oxygen $(\mathrm{O})$ plasmas to a pure $\mathrm{SF}_{6}$ model. We added four $\mathrm{O}$ radical species to the gas phase. We added the electron impact processes, three body recombinations, and some charge-exchange reactions of $\mathrm{O}$ species to the $\mathrm{SF}_{6}$ model. We altered the surface mechanism to include the surface neutralization of $\mathrm{O}$ ions and surface recombination of atomic $\mathrm{O}$. We designed a matrix study of etch rates versus the major process parameters and completed the first segment of this study. We refined the model to produce good agreement with the experimental results for etch rate versus gas mixture ratio $\mathrm{O}_{2} / \mathrm{SF}_{6}$.

We used a rigorous coupled-wave analysis code, GSOLVER, to predict the amount of reflected light from structured Si surfaces and compared these results to measured values. These structures were one-dimensional rectangular profile gratings, all with $50 \%$ duty cycles. We set the etch depth and pitch of the gratings to match experimental measurements. We are in the process of developing a numerical modeling code to predict experimental reflectance measurements.
These results, in fact, slightly exceeded the high currents and efficiencies of our best highefficiency wet-textured, singlecrystal cells. We obtained IQE higher than those on planar and wet-textured cells, boosting cell currents and efficiencies by up to $11 \%$ on $\mathrm{mc}$-Si and $2.5 \%$ on multicrystalline Si cells. 


\section{Refereed}

Damiani, B. M., R. Lüdemann, D. S. Ruby, S. H.

Zaidi, and A. Rohatgi. 2000. "Development of RIE-

Textured Silicon Solar Cells." Proc. 28th IEEE

Photovoltaic Specialists Conf. 1 (Anchorage, AK, September).

\section{Other Communications}

Ruby, D. S., S. H. Zaidi, and S. Narayanan. 2000.

"Plasma-Texturization for Multicrystalline Silicon Solar Cells." Proc. 28th IEEE Photovoltaic Specialists

Conf. 1 (Anchorage, AK, September). 
10763

\section{Control Strategies for Homogeneous Charge- Compression Ignition $(\mathrm{HCCl})$ Engines}

J. H. Chen, A. R. Kerstein, J. E. Dec, C. A. Kennedy, J. C. Hewson

Sandia will implement novel computational and modeling tools to develop active control strategies for incylinder fuel/air/residual mixture composition and temperature in homogeneous charge-compression ignition (HCCI) engines. HCCI engines are an attractive alternative to diesel engines, offering the potential for high diesel-like efficiencies (> 50\%) while concurrently producing extremely low emissions (superULEV [ultralow-emissions vehicle] standards) without expensive aftertreatment. An HCCI engine operates on the principle of compressing a dilute premixed charge until it autoignites and burns volumetrically. This is a relatively new realm of combustion, wherein the mixture is often sufficiently dilute so as to prevent flame propagation; hence, combustion occurs volumetrically. For appropriate mixture and fuel type, engines have been successfully operated in an HCCI mode. However, achieving appropriate combustion across the loadspeed map of the engine and slowing the combustion to achieve a smooth heat-release time-history to prevent engine damage and noise remains a formidable challenge. Recent zerodimensional (0-D) computational studies show that it is difficult to achieve a smooth heat-release history with a fully homogeneous mixture. A key technical challenge, then, is how to actively control the intake protocol and in-cylinder fluid motion to obtain the needed heat-release time profile across the operating conditions of the engine.

We will develop the proposed computational tools and use them in parametric studies to actively control the incylinder fuel/airlexhaust gas residual mixture composition and temperature space/time history to obtain a smooth heat-release time profile across the load-speed map of the engine. If successful control strategies are developed from this project, the numerical tools would be invaluable for providing guidance to engine designers, providing a significant jumpstart in HCCI design efforts.

We developed a stand-alone 0-D turbulence model coupled with CHEMKIN (chemical kinetics) for treating autoignition in HCCI engines and used the model to perform studies of autoignition of hydrogen/air mixtures at conditions representative of HCCI operation. We formulated reduced
We will develop the proposed computational tools and use them in parametric studies to actively control the in-cylinder fuel/air/exhaust gas residual mixture composition and temperature space/time history to obtain a smooth heat-release time profile across the loadspeed map of the engine. If successful control strategies are developed from this project, the numerical tools would be invaluable for providing guidance to engine designers, providing a significant jumpstart in HCCI design efforts. 
chemistry for a gasoline surrogate fuel, n-heptane, and validated it against experimentally measured ignition delay times for conditions representative of HCCI engines. We conducted studies with reduced and skeletal chemistry for nheptane in unsteady, homogeneous, constant-volume configurations to simulate truly homogeneous HCCI conditions. We developed a linear-eddy model (LEM)/direct numerical simulation (DNS) mapping procedure to provide realistic mixture statistics during the initial radical build-up stages of autoignition for DNS. We performed DNS of hydrogen/air autoignition for different turbulence conditions. We developed accurate partitioned Runge-Kutta methods for numerically integrating large systems of stiff ordinary differential equations and are implementing them in a 3-D massively parallel processing (MPP) DNS code. We implemented an autocode generator and a CHEMKIN librarycompatible application-programming interface (API) into Sandia's 3-D MPP DNS code, resulting in significant savings in computational expense for the evaluation of the chemical source terms.

As an alternative to direct evaluation of the chemical source terms, we implemented an in situ adaptive tabulation scheme in the MPP DNS code. The scheme uses a $\mathrm{k}-\mathrm{D}$ tree database for rapid search and retrieval of table data, as the table is dynamically filled during the course of a simulation. In principal, calculated values and table look-ups for repeated accesses to a given region in state space are more efficient than direct calculation, although the present implementation requires further improvements in error monitoring and efficiency. Finally, we developed initial control and design strategies for HCCI engines. We identified two couplings: (1) the thermokinetic coupling and (2) the fluids mixing coupling.
We implemented an autocode generator and a CHEMKIN library-compatible applicationprogramming interface (API) into Sandia's 3-D MPP DNS code, resulting in significant savings in computational expense for the evaluation of the chemical source terms. 
10771

Silicon/Carbon Nanocomposites for Rechargeable Battery Applications

D. Ingersoll, K. J. Gross, M. A. Rodriguez

The utility of nanomaterials has been determined to be the next arena of advanced materials development, where entering this nanosize domain may drastically alter material behavior, at least compared to their larger-sized brethren. The limitations of energy-storage devices (e.g., batteries), such as runtime, power, and voltages, are imposed by the intrinsic characteristics of the materials used, including the anode and cathode materials. Consequently, by improving the performance of these materials, either alone or together, the overall performance of the battery can be improved, thereby enabling new missions or expanding old ones. Toward this end, Sandia has initiated development of new composite silicon (Si)/ carbon $(C)$ nanomaterials for use as the anode material in reversible energy-storage systems that have theoretical capacities an order of magnitude greater than the current state-of-the-art materials. We can combine these new materials with existing cathode and electrolyte materials to create energy-storage devices with vastly improved volumetric and gravimetric energy densities, i.e., smaller and lighter batteries.

We developed and investigated three methods for preparation of Si nanoparticles. The first method involved selective oxidation and removal of an active metal center from a Si alloy, leaving the Si nanocrystalline framework intact. Beginning with $\mathrm{Li}_{13} \mathrm{Si}_{4}$, we selectively removed the lithium (Li) by chemical oxidation to form a soluble alkoxide that is easily removed from the starting material. We used a secondary alcohol in the initial phase of the reaction, followed by a primary alcohol, and then water. In this way we successfully prepared nanometer-size Si crystallites. Furthermore, this approach offers the possibility of general applicability to formation of other nanocrystalline materials.

The second approach used is a synthetic route based on chemical condensation. Our studies focus on reductive coupling of $\mathrm{SiCl}_{4}$ (or $\mathrm{SiBr}_{4}$ ) without any additives. We identified three critical issues: (1) reducing agents, (2) particle sizes, and (3) the dispersity or size distribution. We focused on evaluating three reducing agents, which is ongoing. The final method used for preparation of the Si nanomaterials employs a brute-force approach and consists of mechanical milling. In this
The utility of nanomaterials has been determined to be the next arena of advanced materials development, where entering this nanosize domain may drastically alter material behavior...

Sandia has initiated development of new composite silicon $(\mathrm{Si}) /$ carbon $(\mathrm{C})$ nanomaterials for use as the anode material in reversible energy-storage systems that have theoretical capacities an order of magnitude greater than the current state-of-the-art materials...[and lead to] smaller and lighter batteries. 
approach, we used high-purity single-crystal Si wafers as starting material. We crushed and sealed these into a milling pot under an argon (Ar) atmosphere and then milled them for 3 hours using a high-energy mixer/mill. The initial work demonstrates that this approach is suitable for preparation of oxygen $(\mathrm{O})$ free-Si nanoparticles. It is also clear that the process must be modified to produce materials with a discrete nanosize distribution. We believe that we can achieve this by reducing the quantity of material used in the milling process. We have begun electrochemical characterization of the materials obtained.
The initial work demonstrates that this approach is suitable for preparation of oxygen $(O)$ freeSi nanoparticles. It is also clear that the process must be modified to produce materials with a discrete nanosize distribution. We believe that we can achieve this by reducing the quantity of material used in the milling process. 


\section{Surety Sciences}

The Surety Sciences investment area focuses on researching and developing technologies that ensure reliable performance, safe environments, and security against malevolent attacks. Surety is defined as confidence that a system will perform in acceptable ways in both intended and unintended circumstances. Surety is a key component of the strategy to address safety and reliability needs of the Labs' national security missions.

The Surety Sciences investment area invests in research with the potential to impact national security applications through (1) studies in integrated methodologies and technologies, (2) the development of robust surety principles, (3) examinations of how systems fail, (4) the creation of validation models for sure system designs, and (5) improved simulation capabilities that lead to predictive techniques.

The "Optically Triggered Semiconductor Switch for Firing Systems" project is developing an optically triggered switch that is useful in environments where high levels of radiation are present. Compared to sprytrons (i.e., electrically triggered, handassembled vacuum switches), the new switches have the added advantages of semiconductor batch manufacture, compactness, optical isolation, and low jitter. The main

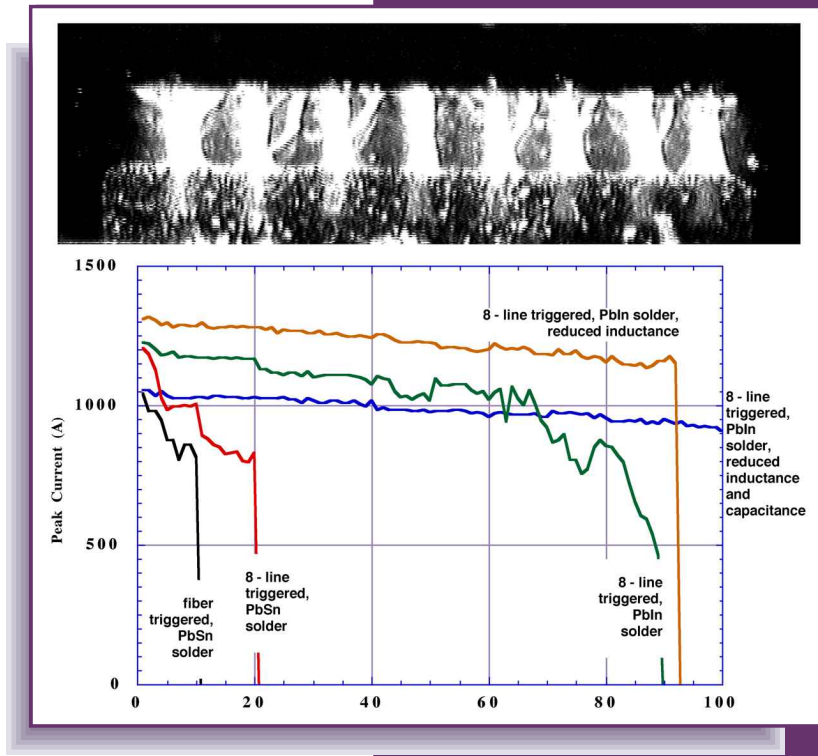

challenges in making this device a reality are switch longevity and ease of use. Such a switch would result in dramatically reduced size, reduced part count, and enhanced surety of firing sets for nuclear weapons, including stockpile retrofit. Surety may be enhanced with this device because its reduced size frees up space for joint test assemblies, is optically isolated, and allows for state-of-health monitoring. Other potential applications for the switch include direct optical ignition, ground-penetrating radar for environmental site reconnaissance, and high-power microwave generation, among others. 
10388

Hybrid Processing of Measurable and Subjective Information in Surety Analysis

\section{J. A. Cooper}

Conventional systems analysis generally depends strictly on measurable or physical-model-derived data. However, high-consequence system-surety analysis must also use subjectivity. This project will develop surety analysis tools that combine subjective information along with conventional objective metrics in a hybrid methodology facilitating system assessment and specifying the amount of subjectivity in the analysis. This is an important contribution to decision-makers because it more accurately represents the information available. Included are such essential contributions as the amount of dependency that necessarily exists among inputs as well as metrics giving the importance and sensitivity of the inputs. The approach is to first identify situations that were not well modeled by existing methodologies, then to research methods for improving the modeling and analysis, then to seek ways to bring the various approaches developed together in a hybrid analytical structure, and finally to implement the approaches in a coordinated software tool.

We are continuing our methodology development, specifically adding and refining surety analysis tools. Examples of tools developed are a contest tool (to assess competing component or system failures), a fuzzy-logic tool (to assess failures dominated by the weakest or strongest components in a series of failures), and a dependency tool (to assess system relationships that transcend correlation). The constrained mathematics work is complete, with the addition of a formal mathematical proof that the probabilistic bounds of logical variables are necessary and sufficient to determine the bounds on their Boolean functions. This results in improved computational performance because no values within the bounds need be considered. Since this methodology overcame an approximation that was commonly used in prior analyses, we worked to publicize the various aspects of it through workshops, journal articles, and conference papers.

We took a major step in developing and implementing the basic structure for a "latent effects" analysis, based on a "soft-aggregation" of pertinent information. We named this tool the "Markov Latent Effects Tool" because of the storage of previous information to help explain current occurrences.
This project will develop surety analysis tools that combine subjective information along with conventional objective metrics in a hybrid methodology facilitating system assessment and specifying the amount of subjectivity in the analysis. This is an important contribution to decision-makers because it more accurately represents the information available. 
Contributing to the need for such an approach is a growing body of literature indicating the importance of early root causes for improper actions based on surety culture, management attitudes about surety, communication (including training) style and precision, and employee attitudes and morale. We introduced soft aggregation to more realistically model how operational actions build in a nonlinear manner on foundation effects that can result in significant influence on the actions taken. To test these ideas, we asked for and received evaluation from a select number of FAA (Federal Aviation Administration) and airline personnel, generally very positive. We presented key aspects of the approach at conferences and published a peer-reviewed paper.

We developed software to an extent that allows distribution of research and development trial copies, enabling us to get feedback from "beta" users.

In most cases, the methodology developed previously exceeded our expectations, and we derived some important unscheduled developments. Most of the software was implemented as the methodology was developed. Our work will further develop the tool and methodology in applicationspecific areas and ultimately help decision-makers to make better and more defensible decisions in managing their highconsequence operations. ...the methodology developed previously exceeded our expectations, and we derived some important unscheduled developments. Most of the software was implemented as the methodology was developed. Our work will further develop the tool and methodology in application-specific areas and ultimately help decision-makers to make better and more defensible decisions in managing their highconsequence operations.

\section{Refereed}

Cooper, J. A. 2000. "Markov Modeling with Soft Aggregation for Safety and Decision Analysis."

Foresight and Precaution-Proc. European Safety and Reliability (ESREL) 2000, Europe Ann. Conf. 2 (Edinburgh, Scotland, UK, 15-17 May): 1015-1021.

Cooper, J. A. 2000. "Model-Based Derivation of Safety Performance Measures." Paper presented to the Society of Automotive Engineers Aviation Safety Conference, Daytona Beach, FL, 11-13 April.
Cooper, J. A., A. J. Johnson, and P. Werner. 2000. "Hybrid Safety Analysis Using Functional and Risk Decompositions." Proc. 2000 Internat. Sys. Safety Conf. 1 (Ft. Worth, TX, 11-16 September).

Cooper, J. A., S. Ferson, and D. K. Cooper. 2000. "Constrained Mathematics for Calculating Logical Safety and Reliability Probabilities with Uncertain Inputs." J. Sys. Safety 36 (1) (First Quarter, 2000): 23-29. 
10389

\section{Approximate Public-Key Authentication with Information Hiding}

T. J. Draelos, E. V. Thomas, A. M. Johnston, D. R. Waymire, J. M. Cochran

This project seeks a solution to the problem of authenticating the shapes of statistically variant gamma spectra while simultaneously concealing the shapes and magnitudes of the sensitive spectra. The shape of a spectrum is given by the relative magnitudes and positions of the individual spectral elements. Class-specific linear orthonormal transformations of the measured spectra are used to produce output that meet both the authentication and the concealment requirements. For purposes of concealment, the n-dimensional gamma spectra are transformed into n-dimensional output spectra that are effectively indistinguishable from Gaussian white noise (independent of the class). In addition, the proposed transformations are such that statistical authentication metrics computed on the transformed spectra are identical to those computed on the original spectra. The approach taken to authenticate and hide information, called the permutation-transformation-permutation (PTP) algorithm, is applicable to other data sources as well, such as those used in biometric authentication (e.g., fingerprints, retinal scans, hand geometry, etc.).

We selected the PTP algorithm as the candidate of choice for approximate authentication and information hiding. The reason for choosing the PTP algorithm is primarily its provable security. Also, an interesting feature of the algorithm is that the operations (permutations and a linear orthonormal transformation) are such that statistical authentication metrics computed on the transformed signal are identical to those computed on the original signal.

Several Sandia mathematicians reviewed the PTP algorithm to evaluate its security and to identify practical attacks. We developed two attacks that revealed an upper bound on the security of the algorithm, but a very high and extendable level of security remains achievable by the algorithm. This review and analysis process helped enhance the security and utility of the PTP algorithm.

We implemented the PTP algorithm in LISP, MATLAB, and C. A C library is available for use by

This project seeks a solution to the problem of authenticating the shapes of statistically variant gamma spectra while simultaneously concealing the shapes and magnitudes of the sensitive spectra.... The approach taken to authenticate and hide information, called the permutation-transformationpermutation (PTP) algorithm, is applicable to other data sources as well, such as those used in biometric authentication (e.g., fingerprints, retinal scans, hand geometry, etc.). 
application programmers. The attacks were also implemented in MATLAB and LISP.

Weapons physicists used the C library of the PTP algorithm for evaluation in gamma spectrum authentication and hiding. Although the algorithm performed as designed, it was not considered to be an advantage over competing techniques. 
10390

New Network Analysis Approaches to Evaluate Infrastructure Risk and Reliability

G. D. Wyss, F. A. Duran

The objective of a risk assessment is to identify the scenarios and mechanisms that can push an engineered system away from its intended behavior into a region of unintended consequences. Traditional risk-assessment methods have failed when applied to infrastructure systems for a variety of reasons, including the nondeterministic ordering of failure events and the tight coupling of failure effects between elements of a networked infrastructure. This project developed a new ObjectBased Event Scenario Tree (OBEST) methodology that can be used to track the probabilistic behavior of systems and efficiently predict the existence of pathological scenarios characterized by very low probabilities but potentially extreme consequences. The method is an extremely versatile replacement for the traditional event-tree analysis methodology and can be linked with traditional network analysis methods to provide enhanced fidelity for infrastructure assessment problems.

The original objective of this research was to explore the probabilistic scenario space created when failing infrastructures interact with one another. For example, electric power failures can affect telecommunications, water, and other infrastructures, while failures in those infrastructures can also inhibit the restoration process for electric power. After examining a wide variety of existing methodologies, we identified a methodological void. Traditional event-tree analysis of such problems quickly becomes intractable because these problems are characterized by variable event ordering. Traditional simulation-based analyses are unable to explore the scenario space probabilistically because they are designed to give a deterministic prediction of system performance based on specified input parameters. To obtain a probabilistic exploration of the scenario space, simulation tools might be driven by a Monte Carlo sampling algorithm. However, such a probabilistic analysis is often computationally intractable and highly inefficient because probabilistically dominant scenarios are discovered repeatedly while highly unlikely scenarios are missed because of their statistical insignificance.

Our objective was to develop a method that could quickly and efficiently explore a large probabilistic scenario
This project developed a new Object-Based Event Scenario Tree (OBEST) methodology that can be used to track the probabilistic behavior of systems and efficiently predict the existence of pathological scenarios characterized by very low probabilities but potentially extreme consequences. The method is an extremely versatile replacement for the traditional event-tree analysis methodology and can be linked with traditional network analysis methods to provide enhanced fidelity for infrastructure assessment problems. 
space (as is characteristic of event tree analysis) while allowing for self-ordering of the probabilistic events and without requiring the analyst to "distill" the "important" events and phenomena from a behavioral system model. We selected object-oriented analysis as a point of departure for this method because it enables an analyst to develop a purely behavioral model of the system that can be reused for a variety of analyses simply by changing the attributes and boundary conditions within the model. We modified the action-description language of the Shlaer and Mellor object-oriented analysis method to include probabilistic branching on two different time scales: "immediate" responses and "delayed" responses. Our action language derives from the fact that many infrastructure failure scenarios are characterized by relatively long periods of quasiequilibrium punctuated by short periods of intense activity (at times when perhaps a set of backup batteries becomes depleted). The OBEST methodology captures this behavior for probabilistic systems in a computationally efficient manner.

We developed and refined the OBEST risk-assessment methodology and demonstrated its applicability to a wide variety of classes of problems, including infrastructure analysis, contingency planning, aviation safety, and the response of electrical systems under cable fire conditions. We developed a computationally efficient model solution scheme and implemented the methodology and solution scheme in demonstration software. The recursive nature of the solution scheme, as well as the treelike nature of the analysis results, indicates that dramatic computationally efficient gains are possible through parallel computation. Also, the method is amenable to uncertainty analysis through direct extension of methods already in common use for event-tree analysis. It may be possible in the future to move from object-based models to intelligent agents to embody the behavior of components within a system. This could provide an answer for one of the chief deficiencies in the OBEST methodology (the laborintensive nature of object model creation) as well as one of the chief deficiencies in agent-based simulation models as applied to risk assessment (the difficulty of characterizing a large probabilistic scenario space). ...we developed and refined the OBEST risk-assessment methodology and demonstrated its applicability to a wide variety of classes of problems, including infrastructure analysis, contingency planning, aviation safety, and the response of electrical systems under cable fire conditions. We developed a computationally efficient model solution scheme and implemented the methodology and solution scheme in demonstration software. 


\section{Other Communications}

Wyss, G. D. 2000. "An Overview of the Object-Based Event Scenario Tree (OBEST) Method." Paper presented to the System Safety and Simulation Modeling Workshop, San Jose, CA, 4-5 April.

Wyss, G. D. 2000. "Object-Based Approaches to Aviation Risk Assessment." (Invited) Paper presented to the NASA Design for Safety 2000 Conference, San Jose, CA, 10-12 October.

Wyss, G. D. 2000. "Sandia National Laboratories Activities in Support of the NASA Aviation Safety Program." Paper presented to the NASA Aviation Safety Program System-Wide Modeling \& Simulation Conference, San Jose, CA, 5 October. 
10391

Improved Tools for Identifying and Quantifying Potentially Dangerous Human Actions

J. A. Forester, H. O. Whitehurst, D. P. Miller, V. J. Dandini

Human reliability analysis (HRA) is a critical component of probabilistic risk assessment (PRA) and is an important tool for understanding human errors in complex adaptive systems. HRA is needed to assess the potential for human error in high-consequence arenas such as nuclear power, medicine, military command and control, and aviation. Sandia developed a new HRA method called Aviation Safety Human Reliability Analysis Method (ASHRAM) that will allow us to identify, understand, and quantify (if necessary) potentially dangerous human actions and accident scenarios. The dangerous human actions include errors of commission, e.g., Three Mile Island 2, friendly fire in military operations, and numerous aircraft accidents, that result from the situations people are put in. In other words, unsafe actions occur because of an error-forcing context that makes the action seem logical at the time. The work builds on our expertise in developing HRA methods for the nuclear power industry. The result is a product that Sandia can use to reduce human error in many different high-consequence domains, with special application to aviation. Given the expected increase in the number of aviation-related accidents over the next 30 years, human performance in aviation is a critical application domain. The work has developed and documented systematic retrospective and prospective search processes for identifying potential unsafe actions and their error-forcing contexts. The process will allow users to identify potentially dangerous aviationrelated accident scenarios (and their causes), and the results can be used as a basis for implementing improvements in aircraft-human interface design, flight operations manuals, and pilot training. In addition, the likelihood of the identified events and scenarios can be quantified and the results can be incorporated into PRAs.

We completed a trial application of the new method (ASHRAM) to a loss-of-engine scenario. A commercial airline pilot volunteered his time, provided appropriate flight documents (procedures, checklists, etc.), and assisted in applying an initial version of the method. The goal was to gain
Sandia developed a new HRA method called Aviation Safety Human Reliability Analysis Method (ASHRAM) that will allow us to identify, understand, and quantify (if necessary) potentially dangerous human actions and accident scenarios. ...The work builds on our expertise in developing HRA methods for the nuclear power industry. The [objective of this research] is a [tool] that Sandia can use to reduce human error in many different highconsequence domains, with special application to aviation. 
experience in applying the initial method and to gain insights to serve as a basis for developing the final method.

A draft of the final method is complete. The document provides a model and systematic retrospective and prospective search processes for identifying potentially dangerous actions and aviation-related accident scenarios and their causes. The results can be used as a basis for implementing improvements in aircraft-human interface design, flight operations manuals, and pilot training. In addition, the likelihood of the identified events and scenarios can be quantified, and the results can be incorporated into PRAs.

\section{Other Communications}

Miller, D. P., and J. A. Forester. 2000. "Aviation Safety Human Reliability Analysis Method." Sandia Technical Report SAND2000-2955, Sandia National Laboratories, Albuquerque, NM.
The results can be used as a basis for implementing improvements in aircraft-human interface design, flight operations manuals, and pilot training. In addition, the likelihood of the identified events and scenarios can be quantified, and the results can be incorporated into PRAs. 
10392

\section{Advanced Signal Processing for Thermal} Flaw Detection

M. T. Valley, B. D. Hansche, T. L. Paez, D. M. Ashbaugh, A. Urbina

Dynamic thermography is a promising technology for inspecting metallic and composite structures used in highconsequence industries. This technology has demonstrated the ability to detect, identify, and quantify hidden flaws at levels that in many applications dramatically exceed the capabilities of competing nondestructive testing (NDT) technologies. However, the reliability of this emerging technology is limited by the need for extensive operator experience and the use of human judgment and visual acuity to detect flaws in the large volume of infrared (IR) image data collected. The inspection speed, reliability, sensitivity, and cost effectiveness of dynamic thermography can be significantly improved with advanced signal-processing algorithms.

This project developed new automated data analysis algorithms and software that rely on the spatial and temporal physics of the thermal detection process and automated characterization of system noise to highlight the presence of hidden flaws in metallic structures. Operationally, this technology flash heats an inspection surface and monitors the surface temperature over time with an IR camera. Internal anomalies perturb heat-conduction processes by increasing the local storage or transmission of energy as heat. These perturbations induce local spatial temperature gradients on the inspection surface that are imaged by the IR camera. However, since surface features (e.g., emissivity variations, chalk lines, etc.) can also create apparent spatial gradients, the temporal image characteristics (e.g., latency, arrival time) must be used to distinguish between true internal flaws and surface artifacts. The developed software package, ATAC (Automated Thermal Analysis Code), performs these functions and produces a composite image highlighting suspected internal anomalies.

Research efforts focused on three areas: evaluation and development of signal-processing operators, development of flaw visualization tools, and collection of experimental data.

Signal-processing algorithm development began with an investigation of analytical solutions to the transient heat diffusion equation with impulsive heating. Analytical solutions can be derived in many forms with differing convergence properties, but each relies on series expansions to describe the
This project developed new automated data analysis algorithms and software that rely on the spatial and temporal physics of the thermal detection process and automated characterization of system noise to highlight the presence of hidden flaws in metallic structures. 
surface temperature as a function of position and time. We briefly investigated causal predictive filters relying on analytical solutions as a class of potential flaw detection filters. However, preliminary results demonstrated that in addition to being numerically intensive, causal filters are not sufficiently robust in the presence of the level of spatial and temporal noise and inhomogeneities typically encountered with dynamic thermography. Hence, causal filters were discounted, and the algorithm development focused on variants of nonpredictive digital signal-processing (DSP) operators such as the Laplacian, Roberts' cross gradient, Sobel, Kirsch, Prewitt, Differential Hysteresis, Marr-Hildreth, Frei and Chen, Homomorphic, Relief, and Sequential operators implemented with differing applications of equalization.

The governing differential equation for transient diffusion shows that the time rate of change of internal energy is balanced by the net lateral conduction heat flux along the surface. Since the IR camera rapidly (up to 1000 images per second) measures the surface temperature over time, it is possible to estimate both the lateral conduction (image spatial Laplacian) and the change in internal energy (first derivative with respect to time) near the surface. Internal flaws locally perturb both of these parameters and can be used to digitally filter the data for the presence of flaws.

The application of DSP operators developed to detect hidden flaws relies on a statistical method for the estimation of parameters in a model to characterize measured temperature data. The parameters in the model and the general model form are used to estimate surface temperature and partial derivatives as a function of $(x, y, t)$ along the inspection surface. The method used is a weighted least-squares identification of the parameters in a polynomial model with temporal weighting. The parametric model is an 18-parameter spatio-temporal polynomial that simultaneously fits the data to a trivariate quadratic polynomial. The parameter output directly feeds the developed DSP flaw detection operators.

Currently thermography users manually adjust the contrast in each image to visualize a flaw. Typically there is a large dynamic range in each image, and "hot spots" often dominate the contrast-enhanced image and hide flaws. Further, since the acquired images are 12-bit and the display is 8-bit, the system operator must select which 8 bits to display.

Considering that more than 1000 images are often taken during a single data-acquisition cycle, this is a labor-intensive and often unreliable process. To address this issue, we developed a
The governing differential equation for transient diffusion shows that the time rate of change of internal energy is balanced by the net lateral conduction heat flux along the surface. Since the IR camera rapidly (up to 1000 images per second) measures the surface temperature over time, it is possible to estimate both the lateral conduction (image spatial Laplacian) and the change in internal energy (first derivative with respect to time) near the surface. Internal flaws

locally perturb both of these parameters and can be used to digitally filter the data for the presence of flaws. 
utility in MATLAB to read and display thermal datacubes as 3-D interactive colored images. This utility, together with a user-friendly graphical interface, was converted to a standalone package and integrated into the ATAC software that we developed to analyze the thermal data. Further, we developed an algorithm for the ATAC software package to generate a single composite image highlighting the presence of suspected anomalies. This software uses the temporal and spatial filter outputs to generate an image that highlights image locations that have a high probability of containing a flaw.

We used a TWI (thermal wave imaging) system to collect experimental data on metallic plates (painted and unpainted) with machined flaws. Flaw aspect ratios (ratio of lateral size to depth from surface) varied from 0.5 to 5 , and data-acquisition speeds varied from $30 \mathrm{~Hz}$ to $1000 \mathrm{~Hz}$. We drew chalk lines on the specimen surface to provide a significant noise source and to act as registration points.
Considering that more than 1000 images are often taken during a single data-acquisition cycle.... 
10393

\section{Production Surety and Disruption} Vulnerability Analysis

D. A. Jones, C. A. Phillips, W. E. Hart, G. F. List, M. A. Turnquist,

C. R. Lawton

The goal of this project is to develop new capabilities for improving planning under uncertainty, focusing on production and transportation surety in the Nuclear Weapons Complex (NWC). Sandia's focus is on optimization models for resource-constrained planning that focuses on both production and transportation operations. Explicit recognition of uncertainties in the solutions to these models leads to creation of plans that are less vulnerable to disruption and more robust under a variety of unknown future conditions. Furthermore, the expected degree of compliance with specified performance standards can be quantified. Early in the project, we researched promising mathematical formulations of resourceconstrained planning under uncertainty, and we created prototype models for neutron-generator $(N G)$ production planning and Transportation Safeguards Division (TSD) resource planning. We will develop mathematical formulations that allow explicit evaluation of trade-offs between the expected performance of a resource-constrained system and the degree of confidence that the solution will satisfy specified minimum criteria (solution robustness). In addition, we plan to create prototype software applications based on robust model formulations for the NG and TSD environments. We will stress the development of performance metrics directed to vulnerability assessment.

A key advance is the development of an improved method for exploring the trade-offs between risk and expected value performance in evaluating solutions from stochastic optimization models. Previous work in this general topic area (e.g., for evaluation of risk in financial portfolios) focused on a trade-off between mean performance and variance (e.g., the trade-off between average return on investment and the variance of that return). However, variance is not always the most useful measure of risk, and because it is symmetric (i.e., values above and below the mean are considered identically), mean-variance trade-off analysis can be misleading. In NWC applications of planning under uncertainty (as well as in evaluations of critical infrastructure, etc.), it is natural to be much more concerned with "downside" risk than with "upside"

\author{
A key advance is the \\ development of an improved \\ method for exploring the trade- \\ offs between risk and expected \\ value performance in evaluating \\ solutions from stochastic \\ optimization models.
}


possibilities. We created a method for integrating a measure of one-sided tail probability along with the standard expected value criterion in stochastic optimization, and we demonstrated the insights provided by such an approach in the context of transportation fleet sizing.

Our research using a variation on chance constraint programming for $\mathrm{NG}$ production and stochastic decomposition for TSD vehicle planning indicates that explicit treatment of uncertainty allows much more effective planning. Increased effectiveness must be measured with respect to the criteria established for a given application, but in general, we can achieve greater output with a given set of resources, or require fewer resources to achieve a given output level, if we plan with explicit recognition of uncertainty.

\section{Refereed}

Turnquist, M. A., D. A. Jones, E. A. Kjeldgaard, L. K.

Nozick, G. F. List, and B. Wood. 2000. "Robust Optimization for Fleet Planning Under Uncertainty: Models and Experimentation." J. Trans. Res., to be submitted.
We created a method for integrating a measure of onesided tail probability along with the standard expected value criterion in stochastic optimization, and we demonstrated the insights provided by such an approach in the context of transportation fleet sizing. 
10394

\section{An Optically Triggered Semiconductor Switch for Firing Systems}

A. Mar, G. M. Loubriel, A. G. Baca, H. P. Hjalmarson, S. C. Holswade

Conventional high-current firing sets are complicated, demanding systems that require special switches. For Defense Program (DP) applications we use a sprytron: an electrically triggered, hand-assembled vacuum switch $(3 \mathrm{kV} / 3 \mathrm{kA})$. This project will develop an optically triggered gallium-arsenide (GaAs) switch that performs as a sprytron with the added advantages of semiconductor batch manufacture, compactness, optical isolation, and low jitter. The main challenges in making this device a reality are switch longevity (> 100 pulses) and ease of use. The results of this low-current study and improvements in longevity at even lower currents $(\sim 10 \mathrm{~A})$ show that it is feasible to meet the demanding requirements of the firing-set application. The techniques that Sandia will use to improve longevity at kiloamp currents rely on spreading the current by either using state-of-the-art semiconductor processes such as epitaxial growth, regrowth, and diffusion or using improved switch geometries. Such a switch would result in dramatically reduced size, reduced part count, and enhanced surety of firing sets for nuclear weapons (including stockpile retrofit). Surety is enhanced because it enables new architectures, has small size to increase its high-g tolerance and free volume for features such as joint test assemblies, is optically isolated, and allows for state-of-health monitoring. Other switch applications are direct optical ignition, $Q$ switching lasers for the X-1 accelerator, ground-penetrating radar (GPR) for environmental site reconnaissance, sensors in low-visibility environments, and high-power microwave generation.

We achieved this key result by using a diode stack laser array for multiline triggering to distribute the current, optimized optics for anamorphic imaging of this array onto the switch, minimization of stray circuit inductance, and substitution of either gold ribbon wirebonds or lead/indium solder for lead/tin solder to bond the device contacts.

We demonstrated $>20$ shots at $1 \mathrm{kV} / 1 \mathrm{kA}$, limited by damage about contact apertures used to observe filament formation. Improved performance is expected from devices without such apertures.
This project will develop an optically triggered galliumarsenide (GaAs) switch that performs as a sprytron with the added advantages of semiconductor batch manufacture, compactness, optical isolation, and low jitter.

Such a switch [has the potential to] dramatically reduce size, reduce part count, and enhance surety of firing sets for nuclear weapons (including stockpile retrofit)....[it] is optically isolated, and allows for state-ofhealth monitoring. 
We tested switches in pulsed reactor facilities. The switches demonstrated the ability to fire in as little as $50 \mathrm{~ms}$ after and were not (falsely) triggered into their on-state from the rad pulse. A low-level conduction during the rad pulse occurs.

We demonstrated as many as 120 shots at $1 \mathrm{kV} / 1 \mathrm{kA}$. So far, we have obtained $\sim 20$ shots at $3 \mathrm{kV} / 3 \mathrm{kA}$ in similar configurations.

\section{Refereed}

Mar, A., G. M. Loubriel, F. J. Zutavern, M. W. O’Malley, W. D. Helgeson, D. J. Brown, H. P. Hjalmarson, and A. G. Baca. 1999. "Doped Contacts for High-Longevity, Optically Activated, High-Gain GaAs Photoconductive Semiconductor Switches."

Proc. 12th IEEE Pulsed Power Conf. 1: 303.
The switches demonstrated the ability to fire in as little as $50 \mathrm{~ms}$ after and were not (falsely) triggered into their on-state from the rad pulse. 
10395

\section{Source Code Assurance Tool}

J. Espinoza, P. L. Campbell

While significant effort has gone into delivering into the hands of programmers the ability to develop software systems correctly from the start, human inspection and testing of code remains a key element in ensuring the code's correctness. Unfortunately, manual inspection methods are time-intensive, and existing tools do little to alleviate the problem. Given this, safety-and security-critical software projects are often forced into one of two unacceptable outcomes: to slip delivery dates to finish manual inspections or to deliver code that has not been fully assessed. The quality of the assessment is also highly dependent on the analyst. Analyst biases and the sheer volume of things to be considered in an assessment can lead to critical problems being overlooked. For these reasons, a tool that increases the human analyst's level of performance in software assessment, both in terms of time invested and of accuracy, would be of significant benefit.

To this end Sandia plans to develop a software tool that assists the human analyst by:

- automatically parsing a program's source code to map the causal relationships that exist between the source code elements,

- permitting the analyst to model the interactions between a system's software and its nonsoftware elements, and

- enabling the analyst to quickly gain an understanding, for any given system variable in question, of the causal dependencies associated with that variable.

Using a special browser, an analyst will rapidly explore the full range of software/system interdependencies that exist within any system that is built around software. The initial release of this tool will target the Clanguage and will be implemented as a JAVA application. Analysis of nonsoftware components will be based on the analysis framework developed at Sandia.

We developed a prototype of a system analysis tool using commercial off-the-shelf (COTS) software: a slicing engine, CodeSurfer from GrammaTech, and a custom graph editor created with Graph Editor Toolkit (GET) from Tom Sawyer Software (TSS). A user of our tool constructs a model using GET and analyzes it using CodeSurfer.
... a tool that increases the human analyst's level of performance in software assessment, both in terms of time invested and of accuracy, would be of significant benefit....We developed a prototype of a system analysis tool using commercial off-the-shelf (COTS) software: a slicing engine, CodeSurfer from GrammaTech, and a custom graph editor created with Graph Editor Toolkit (GET) from Tom Sawyer Software (TSS). 
We submitted a technical advance (TA): "System Analysis Tool (SAT): A Tool for Analyzing Systems of Software." We generated an idea, "set value propagation," and are considering it as a second TA. Currently, each arc in a program is either in a slice or not, depending on whether or not both the arc source is in the slice and we are slicing forward (or, if we are slicing backwards, if the target of the arc is in the slice). This approach tells us nothing about the data traveling on the arc. There is a finer-grained approach that would be cost-effective: enable each arc to carry a set of values. The user could provide a function for each software component. The function would generate an output set given an input set.

We are exploring the idea of visual programming. A tool is designed for "systems"; the components are no smaller than functions but are complete stand-alone programs. However, if the components are now program primitives, i.e., (1) arithmetic operations, (2) control primitives, such as conditional and looping statements, and (3) a primitive to group statements into functions, we have visual programming.

We submitted a technical advance (TA): "System Analysis Tool (SAT): A Tool for Analyzing Systems of Software.” We generated an idea, "set value propagation," and are considering it as a second TA.

\section{Other Communications}

Campbell, P. L., and J. Espinoza, Jr. 2000. “A System Analysis Tool." Sandia Technical Report SAND20001645, Sandia National Laboratories, Albuquerque, NM (June).

Campbell, P. L., and J. Espinoza, Jr. 2000. "System Analysis Tool (SAT): A Tool for Analyzing Systems of Software." Technical Advance Disclosure SD-6688, Albuquerque, NM (May). 
10396

\section{Physical Models for Predicting the Effect of Atmospheric Corrosion on Microelectronic Reliability}

J. W. Braithwaite, N. R. Sorensen, J. N. Sweet, D. G. Robinson, J. R. Michael, K. L. Erickson, C. C. Battaile

Atmospheric corrosion is a stockpile age-related degradation mode that has a high likelihood of occurrence and the potential for significant consequence. As such, our sciencebased stockpile stewardship mission requires that we develop and use an analytical capability to predict any effect that corrosion can have on weapon reliability. Presently, the stockpile contains numerous microelectronic devices contained in ceramic hermetic packages (CHP). However, the unavailability of CHP devices is already driving the insertion of plastic-encapsulated microelectronic (PEM) devices into stockpile electrical components. A significant concern with the use of PEM devices is the uncharacterized effect that corrosion can have on device reliability during the long-term dormant storage conditions associated with our systems. Effective assessments of microelectronic corrosion damage must be based on a physical understanding of the relevant metallization degradation phenomena. The main objective of this project is to provide this needed foundation specifically for the corrosion of aluminum ( $A l)$, the prime vulnerability. We initially defined and followed throughout this project a phased approach that has culminated in a predictive toolset that includes a constitutive model for the controlling corrosion processes combined with advanced computational techniques that allow process and environmental uncertainties to be explicitly addressed.

Although the complexity of the corrosion process (e.g., influence of intermetallic compounds) that was identified during this work inhibited some of our desired advancements, the final product has clearly satisfied the motivation and basic intent of this work. That is, the knowledge and analytical capabilities developed in this project are already functioning as a basis for a number of important decisions, including PEM insertion and several SFIs (significant finding investigations), that recently emerged. We completed a preliminary quantitative assessment of the validity of the PEM storage concern and demonstrated the effectiveness of using computational reliability to model materials-degradation processes within the context of system-level performance.

...the knowledge and analytical capabilities developed in this project are already functioning as a basis for a number of important decisions, including PEM insertion and several SFIs (significant finding investigations), that recently emerged. 
We developed a number of novel experimental techniques to quantify and characterize corrosion in microelectronic devices. These techniques are of interest to and are applicable to users both internal and external to Sandia.

Although $\mathrm{Al}$ corrosion in microelectronics is a very complex phenomenon involving a number of coupled processes, we now know what factors and parameters are the most important (e.g., Al-gold [Au] intermetallics, galvanic couples, passivation defects). This information will be critical during the upcoming formulation of valid tests for qualifying and accepting PEM devices for use in weapon systems.

Development of a rigorous mathematical model to quantify expected service life for all devices under a wide range of environments and manufacturing conditions is very difficult to complete. Nevertheless, simplified models, such as the one developed in this project, are still very useful tools for performing assessments of general behavior (e.g., PEM insertion) and as a basis for analyzing specific situations (e.g., op amps sealed with a wide range of internal atmospheres).

Analytical capabilities that are truly predictive must integrate the following components: (1) mechanistic understanding of the corrosion degradation phenomena into a deterministic mathematical model, (2) uncertainty analysis to address the stochastic nature of corrosion and environmental conditions, and (3) electrical system performance models.

The long-term reliability (corrosion-related) of both PEM and CHP microelectronic parts manufactured with bestcommercial practices is expected to be very good.

We made a number of specific advancements. The primary thrust was devoted to characterizing the effect that the intermetallic $\mathrm{Au}-\mathrm{Al}$ compounds have on the corrosion process. This topic was identified earlier as the probable cause of the apparent stochastic behavior of Al corrosion. Our new backthinning/scanning electron microscopy (SEM) technique allowed us to noninvasively examine and profile the $\mathrm{Au}-\mathrm{Al}$ wirebond structure. The resulting SEM images showed a substantial variation in the quantity and type of intermetallic compounds formed that were significantly affected by various heat-treating processes. We then used focused ion-beam machining to provide unambiguous cross-sectional and corrosion morphology information. Importantly, these data provided a qualitative correlation between the measured resistance change and the extent of corrosion. Furthermore, the stepped resistance response suggested that parallel conductive paths existed through the wirebond. We formulated three-
Our new back-thinning/scanning electron microscopy (SEM) technique allowed us to noninvasively examine and profile the $A u$-Al wirebond structure. The resulting SEM images showed a substantial variation in the quantity and type of intermetallic compounds formed that were significantly affected by various heat-treating processes. We then used focused ion-beam machining to provide unambiguous cross-sectional and corrosion morphology information. Importantly, these data provided a qualitative correlation between the measured resistance change and the extent of corrosion. 
dimensional (3-D) intermetallic maps based on the SEM images. We then developed a 3-D electrical conductance model (based on Poisson's equation) and used it in conjunction with the maps. The modeling results predicted the stepped resistance, thus further refining our understanding of bondpad corrosion. We believe that this refinement will enable us to remove (describe) much of the observed stochastic nature of bondpad corrosion.

Other studies and analyses that were completed this year involved (1) developing a procedure to uniformly distribute a mixture of solid-phase contaminants over the PEM surface, (2) characterizing the effects of these contaminants along with other possible gas-phase contaminants (bromine, chlorotoluene), (3) showing that daily fluctuations in relative humidity are damped out by the presence of plastic encapsulant (temperature gradients are also not a factor), (4) characterizing the known distributions of physical defects in plastic (prime susceptibility is bondpad delamination), and (5) performing a simulation of the effect of corrosion on the reliability of a fourdevice comparator circuit. In this latter activity, the corrosion model was directly coupled for the first time with the ChileSpice electrical system model that permitted subsystem-level reliability to be directly calculated.

\author{
The modeling results predicted \\ the stepped resistance, thus \\ further refining our \\ understanding of bondpad \\ corrosion. We believe that this \\ refinement will enable us to \\ remove (describe) much of the \\ observed stochastic nature of \\ bondpad corrosion.
}

\section{Refereed}

Sorensen, N. R., J. W. Braithwaite, D. W. Peterson, J. R. Michael, D. G. Robinson, and M. P. Strizich. 1999. "Characterization of the Effect of Au/Al Bondpad Corrosion on Microelectronic Device Reliability." (Invited) Symp. on Corrosion and Reliability of Electron. Mater.-Proc. 196th Mtg. Electrochem. Soc. (Honolulu, HI, October).

Sorensen, N. R., J. W. Braithwaite, J. R. Michael, D. G. Robinson, and C. C. Battaile. 2000. "Predicting the Effects of Corrosion on Device-Level Reliability Under Dormant-Storage Conditions." Symp. on Atmospheric Corrosion and Reliability of Electron. Mater.-Electrochem. Soc. (San Francisco, CA, September 2001).

\section{Other Communications}

Braithwaite, J. W. 1999. "Characterization of the Effect of Corrosion on Microelectronic Device Reliability." Paper presented to the Materials Science Seminar, NM Institute of Mining and Technology, Socorro, NM, October.

Braithwaite, J. W. 2000. "The Effect of Corrosion on the Long-Term Reliability of Microelectronic Devices." (Invited) Paper presented to the National Symposium on Developments in Advanced Packaging Reliability, Binghamton, NY, July.

Braithwaite, J. W., N. R. Sorensen, K. S. Chen, and H. K. Moffat. 2000. "Predicting the Effect of Corrosion on the Reliability of Electronic Devices." (Invited) Paper to be presented to the Lifetime Prediction Symposium-NACE 2001, Houston, TX, March 2001. 
10397

\section{Security of Bulk Power Systems}

D. G. Robinson

The electrical grids of North America are an extremely large and complex set of interconnected networks vital to the economic lifeblood and safety of over 380 million people. These networks are dynamic, constantly changing systems whose surety (safety, security, and reliability) is vulnerable to significant disruptions due to evolving energy policies as well as to natural and man-made sources. The President's Commission on Critical Infrastructure Protection has identified electric power as a critical infrastructure sector. The 1996 breakups of the Western power system demonstrated the weaknesses of the current power-grid vulnerability analyses and highlighted the need for improved techniques to deal with the unexpected. An alternative approach involves probabilistic load flow characterization and is closely related to the analysis methods being developed as part of the nuclear weapon system stockpile surveillance program. Integration of the new probabilistic load flow analysis techniques and sensitivity analysis methods will provide the tools necessary to statistically characterize the load shedding at each major bus in a very large bulk power system. By probabilistically characterizing the amount of load shed at each network node and then relating this measure to the sensitivity of the grid to failure of this node, the vulnerability of the grid can be more thoroughly understood. A particular benefit of this approach is the ability to statistically characterize the dependency between generation and load. This capability will be an extremely important tool for network reliability analysis in a competitive, open-market environment. When completed, these risk-based analytical tools can be used for short-term (daily) vulnerability assessment and long-term (yearly) planning for improved network security.

We previously developed a contingency-based reliability analysis capability and performed preliminary analyses on the Institute of Electrical and Electronics Engineers (IEEE) Reliability Test System (RTS) and a 400-node model of the Arizona-New Mexico-Texas region within the WSCC (Western States Coordinating Council). In addition to considering the random failure of various power-grid elements, we expanded the software to permit the load at each system bus to be random variable, possible as a function of local
The electrical grids of North America are an extremely large and complex set of interconnected networks vital to the economic lifeblood and safety of over 380 million people. These networks are dynamic, constantly changing systems whose surety (safety, security, and reliability) is vulnerable to significant disruptions due to evolving energy policies as well as to natural and man-made sources.... When completed, these risk-based analytical tools can be used for short-term (daily) vulnerability assessment and long-term (yearly) planning for improved network security. 
environmental conditions. Three additional goals were to expand the size of the bulk power systems that could be analyzed, to build a user interface, and to host the software on the massively parallel (MP) Teraflops computer at Sandia. The size of the networks increased dramatically this year, and the types of uncertainty analyses were expanded to include new simulation methods being developed at Sandia. We hosted the software on a number of MP machines at Sandia to explore the unique interface and operational requirements of each platform. We developed and implemented an interactive user interface.

The size of the networks increased dramatically this year, and the types of uncertainty analyses were expanded to include new simulation methods being developed at Sandia. This permitted the user to dynamically change the network configuration and view the impact of the changes on the reliability of the bulk power system. 
10687

\section{A Massively Parallel Microsimulation Model of Infrastructure Interdependency}

D. C. Barton, R. J. Pryor, K. L. Stamber, E. D. Eidson, D. A.

Schoenwald

The U.S. infrastructure is a complex system of interdependent elements whose continuous and stable operation is vital to national security. These interdependencies create tremendous vulnerabilities that must be understood and then managed to minimize the impact of deliberate disruptions, human error, or natural disasters to the surety of the infrastructure system.

To understand these interdependencies and the results of unexpected events, we developed a model of critical infrastructure interdependencies utilizing Sandia's parallel microsimulation approach. This simulation technology capitalizes on recent technological advances in evolutionary learning algorithms and massively parallel (MP) computing. Interactions among infrastructure elements are modeled individually by intelligent software agents representative of real-world decision-makers. This modeling protocol can utilize thousands of agents to model very complex systems and offers several advantages over traditional modeling techniques for modeling infrastructure interdependencies. For example, unlike analytic models, functional forms of the model's endogenous relationships are not required. For problems where macroscale information is sparse or nonexistent, as is the case for infrastructure interdependencies, microsimulation models are differentiated in their capacity to utilize extensive sources of microlevel data to develop interaction forecasts.

We completed a parallel agent-based model, Aspen-EE, of critical U.S. infrastructures using time-dependent Monte Carlo methods and a genetic algorithm learning classifier system to control decision making. The new model extends Sandia's previously validated agent-based model, i.e., Aspen, to include agents for electric power, a power market, and fuel infrastructures as well as an industrial sector that supplies the power-generation market. The model also implicitly contains a communication infrastructure that can be disrupted by the user. Some special general agents that were added to the model include:
To understand these interdependencies and the results of unexpected events, we developed a model of critical infrastructure interdependencies utilizing Sandia's parallel microsimulation approach.... Interactions among infrastructure elements are modeled individually by intelligent software agents representative of real-world decision-makers. 
- Weather: used by the industrial sector to calculate power need and by the power companies to forecast demand for static customers;

- Bulletin board: used as a communication method between agents, where agents can obtain information like product prices or weather through direct reading without message passing. This agent has the special property that there is one present on every process when running on a multiprocess system; and

- Disaster: an agent that is specified by the user to schedule and execute power outages.

Existing Aspen agents (e.g., industries, households, etc.) have been retrofitted in several ways. Each of the agents developed in the original Aspen framework is now dependent on electric power for the production of goods, conduct of business, and satisfaction of household needs. As such, electric demand portfolios have been to define each agent's characteristics.

Electric-power agent concepts were derived from an analysis of the regulated market, the different varieties of restructuring, and the energy consumption and forecasting decision-making processes of agents. We implemented and tested a basic-energy trading model. We tested it with single and multiple power markets, where markets can choose to set price caps on power trading. We also tested the trading model on scenarios of power outages on one- and two-market structures. Results of these tests indicate how market structure and outages can affect the economic conditions of all other agents in the model.

Agents have behaviors and interact through message passing. The model is event-driven with the explicit assumption that the external state of an agent, which is of interest to other agents, changes only at events. The internal state of an agent may be continuous in nature but is manifest to other agents only through the changes in external state that it causes. Processing an event can invoke various agent behaviors, generate messages between agents, and schedule additional events on the calendar.

Message passing in Aspen-EE is very similar to the original Aspen system but with several important changes. In Aspen-EE, scheduling is "driven" by the events on the calendar, and different kinds of events have different priority. Priority is determined by the event's time, its type, and any secondary priority. Parallelism comes from our observation that events with equal priority can be processed at the same
Results of these tests indicate how market structure and outages can affect the economic conditions of all other agents in the model. 
time. As a consequence, messages are now events, and the whole idea of "message passing" has been subsumed into a more general "event passing" mechanism. Thus, the priority scheme implicitly replicates the original Aspen's timestep "stages," but with some added capabilities.

Conceptually, there is only one calendar, but because communications overhead makes it impractical for the calendar to be centralized, we instead distribute "the" calendar among all processes. That is, each process has its own calendar with events that involve agents belonging to that process. When running, the current priority is determined by a collective operation that finds the priority of the highest-priority event(s) on the calendar. Then all processes process events with that priority from their local calendars. Once each process has completed processing all local events that have the current priority, the transfer queues are exchanged and the events are scheduled into the correct local calendars. The process repeats until the current priority indicates that the current time is beyond the scheduled stop time.

\section{Other Communications}

Barton, D. C. 2000. "Analysis of Complexity in Infrastructure Systems Using Agent-Based Microsimulation." Proc. Complex Sys. and Policy Analysis: New Tools for a New Millennium (Arlington, VA, September).

Barton, D. C., and K. L. Stamber. 2000. “An AgentBased Microsimulation of Critical Infrastructure Systems." Energex 2000: Proc. 8th Internat. Energy Forum 8 (Las Vegas, NV, July): 709-714.

Barton, D. C., E. D. Eidson, D. A. Schoenwald, K. L. Stamber, and R. Reinert. 2000. "Aspen-EE-An Agent-Based Model of Infrastructure Interdependency." Sandia Technical Report SAND2000-2925, Sandia National Laboratories, Albuquerque, NM (December). 
10758

\section{Network Intrusion Detection Using Adaptive Critic Neural Networks}

T. J. Draelos, J. T. Michalski, M. J. Collins, J. M. Cochran

Current network intrusion-detection systems (IDSs) have many shortcomings, including the inability to analyze large amounts of network traffic, the propensity to generate huge quantities of false alarms, and the inability to identify new or evolving adversarial behaviors.

Adaptive critic designs (ACDs) are architectures that make use of multiple neural networks (a model network, an action network, and a critic network) to solve difficult optimization problems. In the intrusion-detection problem, an ACD could be used to continually model a network environment, adapt to changes as they are presented, and provide intrusion-detection decisions based on these changes.

This project will focus on the following technical advancements to meet the challenges presented by the intrusion-detection problem:

(1) The development of an ACD to dynamically adjust/ control a standard IDS such that detection errors are minimized, and

(2) The development of a complete ACD-based IDS to detect unknown adversarial behaviors. In addition to identifying abnormal events, ACDs might also detect a richer variety of known adversarial behaviors, provided suitable training data were available.

This project intends to use the intrusion-detection training dataset from the Massachusetts Institute of Technology (MIT) Lincoln Labs to train an ACD to filter false alarms from a commercial intrusion-detection sensor in an effort to better characterize and identify true adversarial behavior. These data are a result of an ongoing effort in the development of a comprehensive, independent, repeatable evaluation process for IDSs.

This project will leverage Sandia's experience in many disciplines: intelligent software, vulnerability assessment, adversary modeling, network security and modeling, and cyber red-teaming. Intrusion detection is a new and important application of ACDs that has been very successful in real-time optimal control.
This project will leverage Sandia's experience in many disciplines: intelligent software, vulnerability assessment, adversary modeling, network security and modeling, and cyber red-teaming. Intrusion detection is a new and important application of ACDs that has been very successful in real-time optimal control. 
We made significant progress this year conducting research in intrusion detection using ACDs.

We acquired IDS evaluation data that are available from Defense Advanced Research Projects Agency (DARPA) via MIT/Lincoln Labs. We are currently utilizing Solaris Basic Security Module (BSM) audit data to assess intrusions to computer systems. Principles developed using this data will be usable for intrusion detection based on other data, such as network packets and Windows NT audit files.

Our team acquired and installed the Emerald eXpertBSM from the Stanford Research Institute (SRI), which is designed to detect intrusions by examining BSM audit files. In collaboration with University of Missouri at Rolla (UMR) researchers, we investigated the use of a multilayer perceptron (MLP) neural network as the IDS.

We identified several controllable parameters of the eXpert-BSM IDS to dynamically adjust based on the state of the environment and conclusions drawn by the ACD. These parameters are part of the eXpert-BSM configuration files. We demonstrated that manually adjusting the configuration does indeed result in different detection results of eXpert-BSM. The next step is to automate the dynamic reconfiguration of eXpert-BSM. UMR developed an ACD as the IDS for use with BSM data. UMR has also developed important BSM dataparsing tools.

At this time an ACD has not been developed to control the eXpert-BSM IDS. When the controller is developed, since eXpert-BSM is capable of accepting the DARPA BSM evaluation data directly, its intrusion-detection performance will be measured with and without ACD control in order to gauge the effectiveness of this approach to IDS optimization. The ACD-based IDS developed by UMR is not performing well at this time.
We identified several controllable parameters of the eXpert-BSM IDS to dynamically adjust based on the state of the environment and conclusions drawn by the ACD. These parameters are part of the eXpert-BSM configuration files. We demonstrated that manually adjusting the configuration does indeed result in different detection results of eXpert-BSM. 
10759

Key Management Techniques for Authenticating Highly Secure SCADA Systems in the Electric Power Industry

D. R. Gallup, A. M. Johnston, R. E. Carlson, M. D. Torgerson, W.

D. Neumann

Supervisory control and data-acquisition (SCADA) systems are the control mechanisms for the infrastructures of the electric power, gas, and oil industries. In recent years, awareness of potential dangers that could arise through cyber infiltration of SCADA networks has caused concern in the industry and a movement at Sandia toward development of high-surety SCADA systems. A high-surety SCADA system proposes to enhance the security, safety, and reliability of the underlying infrastructure of the system. Achieving these goals necessarily requires cryptographic techniques. All cryptographic techniques, whether used for encryption, identification, or digital signatures, ultimately rely on some form of cryptographic key. Management and maintenance of these keys for efficient and secure use is an ongoing problem. One aspect of a highly secure SCADA system is key management for the component members of the system. Key management of a SCADA system, which may have 30,00050,000 nodes, can be a problem for real-time operation. In an emergency, many of the nodes may be simultaneously reporting exceptions. In order to mount an effective control-response strategy, these messages must be authenticated and decoded immediately. Key management and control systems must be designed to work with the real-time computational abilities of the system. In support of a design for highly secure SCADA systems, this project will develop techniques for managing cryptographic keys and inserting authenticated trust into the infrastructure of the SCADA system. Results toward this goal will also impact future inter-SCADA communications. A major challenge of the future power grid is managing such communications; the ability to provide authentication and trust in intra-SCADA information will be critical to the successful extension of local SCADA network control to regional control and ultimately wide-area monitoring, control, and crisis management.

\author{
In support of a design for highly \\ secure SCADA systems, this \\ project will develop techniques \\ for managing cryptographic \\ keys and inserting authenticated \\ trust into the infrastructure of \\ the SCADA system. Results \\ toward this goal will also impact \\ future inter-SCADA \\ communications.
}


After extensive research, we developed an accurate model of SCADA systems and their various communication links. We evaluated this model to determine threats to the system.

We developed our security goals to mitigate the threats, and the candidate solutions gave us a suite of algorithms that could potentially fulfill these security goals.

Based on our SCADA model, we identified three different types of communication links that should be secured in different ways. Hence, we have suggested "first-try solutions" for each of these three types of links. At the same time, we are in the process of researching and documenting key management techniques in general. For our solution, we will follow government standards so there can be no doubt that our techniques are reliable. We specified particular key management techniques for our first-try solution and will make sure they comply with these regulations.

Based on our SCADA model, we identified three different types of communication links that should be secured in different ways. 
10762

\section{Self-Assembled Tamper-Detection Seals}

W. R. Even, Jr., P. M. Dentinger, E. B. Cummings

Attempting to protect assets with the use of tamperindicating seals is a well-established practice. The practical use and effectiveness of various seal technologies remains an engineering challenge. Specifically, rapid, real-time, quantitative seal validation in an inexpensive package is an illusive commodity. Sandia divided work on an advanced series of self-assembled tamper-indicating seals into two main thrust areas: (1) related to means of providing the unique signature, and (2) a robust methodology for quickly and conveniently reading and assessing the seal. Identifying and implementing the chiral aspect of the signature has been more difficult than anticipated. Species solubility and compatibility have been a challenge for the select group of materials with high specific optical rotation. Other means of providing unique signatures are also being pursued and include fluorescent labels and holographic media. The desirability and therefore the utility of a tamper-indicating seal technology is, in large part, dictated by the logistics of actual field use. Hand-held barcode scanning is our design goal, i.e., to produce a comparable level of convenience or reliability in seal assessment and validation. Embedding key aspects that control seal acquisition fidelity into the actual seal itself will allow for more robust signature collection, geometric correction, and correlation/ authentication with stored identifiers.

From an original set of thirty, we selected three chiral compounds as materials to test for their adaptability to the seal technology. These materials are (1) griseofulvin (highest specific rotation @ 376 $)$, (2) methyl alpha-D glucopyranoside (low-cost/nontoxic, specific rotation @ 158 ), and quinine (specific rotation @ $165^{\circ}$ ). These materials are soluble in polar solvent systems, and routes have been established for producing prototypical, high-viscosity fluid seals and solidphase reversible seals. The fluid seals are particularly valuable for use during code method development.

To date, examination of materials for seal media possessing secondary phase changes, i.e., gelation or glass transitions, has resulted in questions of practicality. The kinetics related to these state changes are too slow to provide much utility in an actual field application. We are evaluating two candidates for reversible, primary, solid-phase seal media.
Attempting to protect assets with the use of tamper-indicating seals is a well-established practice. The practical use and effectiveness of various seal technologies remains an engineering challenge....Handheld barcode scanning is our design goal, i.e., to produce a comparable level of convenience or reliability in seal assessment and validation. Embedding key aspects that control seal acquisition fidelity into the actual seal itself will allow for more robust signature collection, geometric correction, and correlation/authentication with stored identifiers. 
These materials are polymeric oligomers, which are of sufficiently low molecular weight as to have "reportably" true melting points. Polyethylene phthalate and polymethylstyrenevinyltoluene are proving acceptable as prototype seal media. Their melting points are within a reasonable range, and the materials are transparent over their complete phase domain. The polymethylstyrene-vinyltoluene is rather brittle as a solid, and, as yet, it is not clear whether this is a problem (long-term aging compatibility) or an advantage (a sensitive indicator of tampering)! The bulk mechanical properties can be tailored somewhat with the addition of plasticizers.

Multiphase fluid seals have been prototyped, combining one of the chiral species dissolved in glycerin and the potential seal media, and polymethylstyrene-vinyltoluene dissolved in dodecane. We are using these experimental seals in data acquisition to evaluate sensitivity and spatial resolution of the chiral/matrix interface.

For the seals under development to be practical, the inspection system should tolerate hand-held operation. The inspection system must be able to evaluate tags with viewing distortions, including tilt, perspective, and surface curvature. The system should be insensitive to illumination nonuniformity and illumination differences between tag-recording and tagevaluation steps. We will realize these system features by placing a cooperative imaging target or "barcode" under the semitransparent tag. This barcode both embeds information to assist with tag identification and provides high-precision fiducials across the tag. These fiducials allow the inspection system to infer the viewing distortions in the tag image. The information and fiducials embedded in the barcode must be readily recovered in spite of significant distortions and obscuration from the tag.

We developed a gray-scale spread-spectrum barcode to meet these requirements. The autocorrelation of this barcode is a prescribed pattern that allows the inspection system to recognize a tag and quantify viewing distortions. Data are also encoded in the cross-correlations of the barcode with pseudorandom codes saved in the inspection system. Different data can be multiplexed on the same barcode using different codes. Data can be recovered from the barcode only if the corresponding code is known, facilitating various levels of data secrecy.

Distortions of the autocorrelation pattern provide estimates of the imaging tilt and zoom parameters. The imaging pan and curvature parameters can be estimated by
Multiphase fluid seals have been prototyped, combining one of the chiral species dissolved in glycerin and the potential seal media, and polymethylstyrenevinyltoluene dissolved in dodecane. We are using these experimental seals in data acquisition to evaluate sensitivity and spatial resolution of the chiral/matrix interface. 
autocorrelating subsets of the image. These inferred parameters are then refined by the use of an optimal nonlinear filter based on a least-squares fit between the recorded barcode image and the distortion-corrected measure image. The product of this processing and filtering operation is an optimally corrected image that allows a comparison to the originally recorded images via cross-correlation and differencing techniques.

We wrote software to generate these barcodes and process unskewed experimental barcode images. This software successfully recovers embedded data in spite of significant added noise, blur, defocus, and aperturing. The software to perform the automated image correction is being adapted from existing particle-image velocimetry software developed within Sandia.
We wrote software to generate these barcodes and process unskewed experimental barcode images. This software successfully recovers embedded data in spite of significant added noise, blur, defocus, and aperturing. 
10781

Virtual Reliability Realization System

C. L. Henderson, E. J. O'Toole

Current approaches to reliability are not adequate to keep pace with the need for faster, better, and cheaper products and systems. This is especially true in highconsequence-of-failure applications.

Sandia proposed to look at this challenge and see if there was a new paradigm that could make reliability predictions, along with a quantitative estimate of the risk in that prediction, in a way that was faster, better, and cheaper. We would base such an approach on the underlying science models that are the backbone of reliability predictions. The new paradigm would be implemented in two software tools: the Virtual Reliability Realization System (VRRS) and the Reliability Expert System (REX). Our concept is designed to advance the state of the art in reliability prediction and modeling. We designed the software to have two major components. The first component is a reliability prediction package that incorporates model quality and uncertainty to help better predict reliability based on science, rather than relying solely on statistical methods. The second component is an expertise tool that can serve as a knowledge repository for reliability information. This repository is designed to be easily accessed through the Web and updated using standard Microsoft Office applications (Word, Excel, PowerPoint).

Initially, we explored the option of using Microsoft FrontPage 2000 and Access 2000 to create the Reliability Expertise Site. Although we were able to create a database model and link it to FrontPage, we decided that an approach using an E-commerce server might be in order. Using an E-commerce server, various pieces of content or combinations could be accessed for certain fees. This would enable more control for other similar applications in the future. The REX Site using Microsoft SiteServer Commerce Edition and SQL Server was developed.

The basic development and content are complete; the results can be viewed at http://www.suretyinstitutes.sandia.gov/ mtrs.

We performed work on developing the knowledge content for REX. The initial application considered is microelectronics reliability. We used the commercial program MathCAD to create an interactive ability to calculate a large set
Current approaches to reliability are not adequate to keep pace with the need for faster, better, and cheaper products and systems. This is especially true in highconsequence-of-failure applications.... Our concept is designed to advance the state of the art in reliability prediction and modeling. 
of basic semiconductor properties that would be used in reliability analysis. The user can click on any of the equations and/or graphs and change the variables used in the quantity being calculated (e.g., use a different doping level or a different temperature). 
10792

\section{Ultrahigh-Resolution Radiography for Detailed Inspection of Weapon Components and Systems}

R. B. James, B. A. Brunett, W. P. Ballard, E. E. Tarver, III, A. J. Antolak, J. J. Cashen, J. C. Erickson

For more than 60 years, $x$-ray film, in combination with phosphor screens, has been the standard medium for displaying $x$-ray images of objects. Despite technical advances in displays, data storage systems, and high-speed communication networks, there has been no method, until now, to effectively obtain high-resolution $x$-ray imaging data in a fully digital format. Recent successes at Sandia have led to the emergence of solid-state $x$-ray detectors produced from widebandgap semiconductors (e.g., cadmium zinc telluride [CZT]) that are capable of efficient capture and direct conversion of $x$-ray energy into current. By constructing a detector array, this digital technology is capable of providing full-view imaging for most objects, and the system would be able to read out images electronically with better image quality. Operation of the devices is based on the generation of electron-hole pairs in the detector elements due to absorption of incident $x$-rays. The charge carriers move under the influence of an applied voltage, and customized electronics read out each detector element for determination of the $x$-ray flux and energies of the $x$-rays transmitted through an object (i.e., spatial and spectral information). Modern Silicon Valley manufacturing techniques enable the production of small, position-sensitive detector elements for ultrahigh-resolution images of systems and components, while the energy information allows one to precisely determine the specific elements under study. Together, these features can provide a new and powerful tool for certification of weapon components. A digital x-ray system can also be used for monitoring the state of health of components to allow warnings of impending failure due to aging and/or defects during manufacturing. In addition, it can be deployed to address issues of reliability and safety of nuclear weapons in the stockpile in the absence of underground testing. The project goal is to demonstrate an ultrahigh-resolution $x$-ray imaging system using solid-state technology.

We concentrated on developing semiconductor detector modules of both thick-film and hybrid type for use in advanced
Recent successes at Sandia have led to the emergence of solidstate $x$-ray detectors produced from wide-bandgap semiconductors (e.g., cadmium zinc telluride [CZT]) that are capable of efficient capture and direct conversion of $x$-ray energy into current....The project goal is to demonstrate an ultrahigh-resolution $x$-ray imaging system using solid-state technology. 
$\mathrm{x}$-ray imaging systems. Recent successes at Sandia have led to a new class of solid-state $\mathrm{x}$-ray detectors based on widebandgap semiconductors, particularly CZT and mercuric iodide $\left(\mathrm{HgI}_{2}\right)$. These detectors operate at room temperature, detect $\mathrm{x}$ rays with high efficiency, are compact, and require little power; furthermore, they can be read out electronically, directly, and with better spatial information. We constructed a characterization system to map the $\mathrm{x}$-ray response of semiconductor wafers to screen their uniformity, charge transport properties, and energy resolution. Areas of good response were harvested for fabrication of monolithic pixilated CZT detector arrays. We deposited gold ( $\mathrm{Au}$ ) electrodes to produce two-dimensional arrays, followed by surface passivation and mounting onto a ceramic substrate for testing. We measured the response of each pixel using a tightly collimated cobalt-57 gamma-ray source scanned across the array and obtained energy spectra. We also investigated the use of vapor deposition of thick $\mathrm{HgI}_{2}$ films directly onto an amorphous silicon ( $\mathrm{Si}$ ) transistor array. The array had a pixel size of $60 \times 90$ microns and a $256 \times 256$ format. The array was wire-bonded to external electronics, which consisted of shift registers for gate lines and amplifier chips, A/D (analog/digital) converters, memory, and display modules for data collection. We conducted imaging studies using the $\mathrm{HgI}_{2}$ semiconductor detector array for a line-pair phantom and some specific weapon components. We prepared specific components to more objectively assess image quality (spatial resolution and contrast) using this new technique and to compare the results with conventional $\mathrm{x}$-ray and neutron radiographic techniques. We submitted one technical advance and one patent application.
Recent successes at Sandia have led to a new class of solid-state $x$-ray detectors based on widebandgap semiconductors, particularly CZT and mercuric iodide $\left(\mathrm{HgI}_{2}\right)$. These detectors operate at room temperature, detect $x$-rays with high efficiency, are compact, and require little power; furthermore, they can be read out electronically, directly, and with better spatial information.

\section{Refereed}

Chattopadhyay, K., M. Hayes, J. O. Ndap, A. Burger, W. J. Lu, H. G. McWhinney, T. Grady, and R. B. James. 2000. "Surface Passivation of CdZnTe Radiation Detectors by the Use of Potassium Hydroxide Solution.” J. Electron. Mater. 29: 708.

Erickson, J. C., H. W. Yao, R. B. James, H. Hermon, and M. Greaves. 2000. "Time-of-Flight Experimental Studies of CdZnTe Radiation Detectors." J. Electron. Mater. 29: 699.
Pastuovic, Z., M. Jaksic, R. B. James, K. Chattopadhyay, X. Ma, and A. Burger. 2000. "Influence of Electrical Contacts on the ChargeCollection Profiles in CdZnTe Studied by Focused Proton Beams." Nucl. Instru. and Meth., in press.

Van Scyoc, J. M., B. A. Brunett, T. E. Schlesinger, and R. B. James. 2000. "Further Investigation of the Operation of Cadmium Zinc Telluride Pixel Array Detectors." Nucl. Instru. and Meth., in press. 


\section{Other Communications}

Cirignano, L. J., K. S. Shah, P. R. Bennett, L. Li, F.

Lu, J. Buturlia, W. Yao, G. Wright, and R. B. James. 2000. "Characterization of Multi-Element CZT Arrays." Paper presented to Hard X-Ray, GammaRay, and Neutron Detector Physics II, San Diego, CA, 31 July.

Tepper, G. C., R. Kessick, and R. B. James. 2000. "Contactless Measurements of Charge Traps and Carrier Lifetimes in Detector-Grade Cadmium Zinc Telluride and Mercuric Iodide." Paper presented to Hard X-Ray, Gamma-Ray, and Neutron Detector Physics II, San Diego, CA, 1 August.

Vizkelethy, G., B. L. Doyle, D. S. Marsh, and R. B. James. 2000. "Nuclear Microprobe Studies of the Electronic Transport Properties of Cadmium Zinc Telluride Radiation Detectors." (Invited) Proc. Hard X-Ray, Gamma-Ray, and Neutron Detector Physics II 4141, edited by R. B. James and R. C. Schirato (SPIE, Bellingham, WA) (San Diego, CA, 1 August).

Wright, G. W., R. B. James, D. Chinn, B. A. Brunett, R. W. Olsen, J. M. Van Scyoc, II, I. M. Clift, A. Burger, K. Chattopadhyay, D. Shi, and R. Wingfield. 2000. "Evaluation of Ammonium Fluoride/Hydrogen Peroxide Effectiveness as a Surface Passivation Agent for Cadmium Zinc Telluride Crystals." Hard X-Ray, Gamma-Ray, and Neutron Detector Phys. II 4141, edited by R. B. James and R. C. Schirato (SPIE, Bellingham, WA), in press. 


\section{Grand Challenges}

Grand Challenges projects investigate innovative ideas with the potential for dramatically impacting national-level concerns and for creating technologies and processes required for the twenty-first century. Grand Challenges engage Lab-wide multidisciplinary research teams that establish critical mass in participation and funding to focus on world-class research. High-risk, potentially outrageous ideas are considered for investigation. In addition, all Grand Challenges research efforts must identify linkage to achieving Sandia's business goals.

The "Engineering Complex Distributed Systems" project is researching the use of software agents to create a revolutionary approach for improving computer security. The research utilizes intelligent software agents that function in a multiagent collectivea distributed program that runs on multiple computers in a network. The "agent" collective constantly compares notes to determine what unusual requests or commands have been received from external or internal sources. Because of this, the system response is not limited to waiting until someone has figured

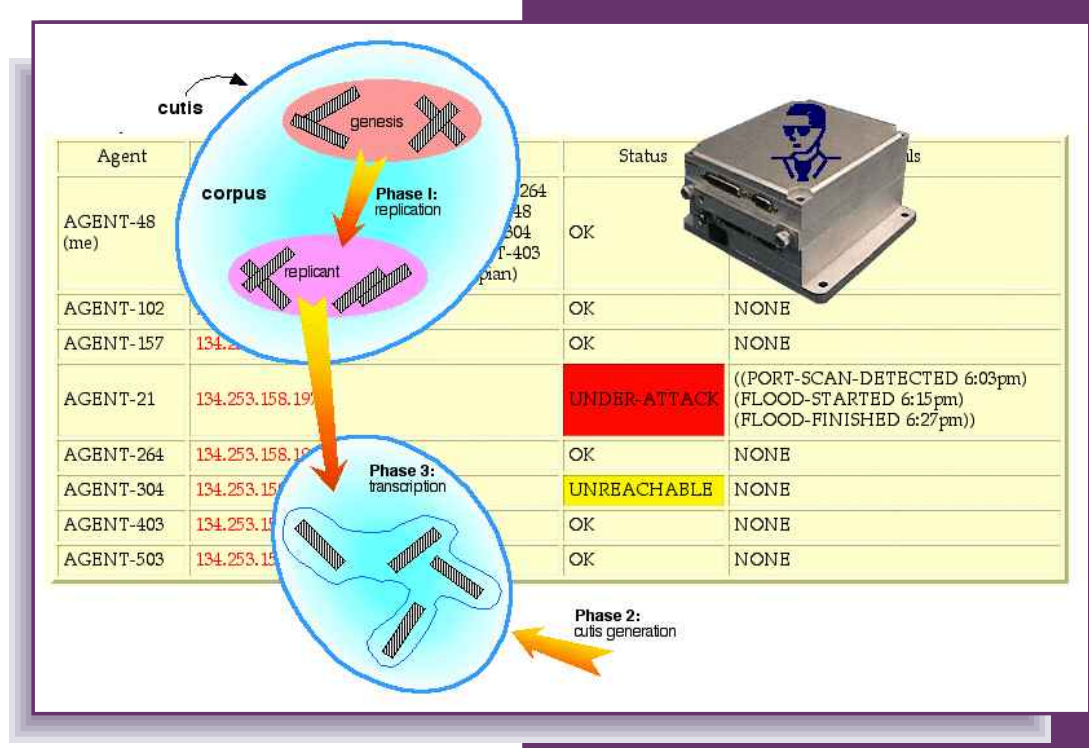

out a defense and put it into a virus checker. "If every node on the Internet was run by one of these agents, the 'I-Love-You virus' would not have got beyond the first machine," says Steve Goldsmith, lead scientist on the project. Several patents are in process, as well as a copyright, on this project. 
10398

\section{Engineering Complex Distributed Systems}

S. Y. Goldsmith, H. E. Link, S. V. Spires, L. R. Phillips, B. D.

Murphy-Dye

In this project, Sandia will demonstrate a collective composed of intelligent software agents operating in an open, Internetlike environment with a high degree of both security of operations and capability of operations. The Secure Agent Collective will be configured as a purely cyber collective (a cooperating team of software agents on computers) and, if time and resources allow, as a collective with one of the computers replaced by an agent-equipped robot participating in the collective via wireless communication. The principal focus of the demonstration will be to exhibit the enhanced level of reliability and integrity provided by the collective when under attack by malevolent or defective insiders. The insider threat constitutes one of the toughest challenges in information system security.

We based our selection of this proof-of-concept demonstration on a focus provided by examining two applications of significant direct importance to national security: the DOE requirement to establish and operate within secure (i.e., need-to-know) enclaves in a less-secure information environment, and the DoD need to securely manage information provided by mobile and fixed sensors in the battlefield information environment. The Secure Agent Collective is a major milestone on the critical path for both of these applications. The demonstration utilizes a number of the core technical capabilities developed previously in this project, including invention of the secure agent architecture (SAA), development of agent components of that architecture, and the exploration of collective security concepts. Our metric of success, to be provided by Red-team analysis, will be to exhibit the level of improvement in reliability and integrity achieved during the course of the autonomous performance of a set of information-processing tasks.

At the conclusion of this project, we will have demonstrated a collective composed of intelligent software agents operating in an open (Internetlike) environment with a high degree of both security of operations and of capability of operations. The Secure Agent Collective will be configured as a purely cyber collective (a cooperating team of software agents on computers) and, if time and resources allow, as a collective with one of the computers replaced by an agent-
The demonstration utilizes a number of the core technical capabilities developed previously in this project, including invention of the secure agent architecture (SAA), development of agent components of that architecture, and the exploration of collective security concepts....At the conclusion of this project, we will have demonstrated a collective composed of intelligent software agents operating in an open (Internetlike) environment with a high degree of both security of operations and of capability of operations. 
equipped robot participating in the collective via wireless communication. The principal focus of the demonstration will be to exhibit the enhanced level of reliability and integrity provided by the collective when under attack by malevolent or defective insiders. The insider threat constitutes one of the toughest challenges in information-system security.

Our team's selection of this proof-of-concept demonstration was based on a focus provided by examining two applications of significant direct importance to national security: the DOE requirement to establish and operate within secure (i.e., need-to-know) enclaves in a less secure information environment and the need to securely manage information provided by mobile and fixed sensors in the battlefield information environment. The Secure Agent Collective is a major milestone on the critical path for both these applications. The demonstration utilizes a number of the core technical capabilities developed in the first two years of the project, including invention of the SAA, development of agent components of that architecture, and the exploration of collective security concepts. Our metric of success, to be provided by red-team analysis, will be to exhibit the level of improvement in reliability and integrity achieved during the course of the autonomous performance of a set of informationprocessing tasks.
Our metric of success ... will be to exhibit the level of improvement in reliability and integrity achieved during the course of the autonomous performance of a set of information-processing tasks. 
10399

\section{Information Collection}

D. D. Spencer, J. P. Anthes, F. T. Mendenhall, T. S. Prevender, R. M. Axline, W. C. Sweatt, M. B. Murphy, K. O, Wessendorf, M. W.

Scott, M. R. Daily, G. J. Elbring, M. C. Walck, R. J. Simonson, L. C. Bartel, K. T. Stalker, C. J. Brinker, K. L. Black, W. R. Bolton, Jr., D. L. Keese, G. A. Thomas, R. W. Crocker, L. R. Thorne, R. D.

Robinett, III, H. M. Poteet, J. T. Gardner, A. K. Miller, J. S. Pfohl, D. M. Shawver, S. A. Dupree, L. A. Boye, M. R. Prasad, S. A. Stansfield, G. J. Fischer, B. L. Spletzer

This is a classified project, and no summary is available. 
10693

\section{Open Access (Nanosat)}

J. L. Schoeneman, P. J. Robertson, D. Ingersoll, A. A. Allerman, D. J. Johnson, C. T. Sullivan, W. C. Sweatt, H. C. Shen, J. R.

Klarkowski, F. Bitsie, S. H. Kravitz, C. E. Lanes, D. L. Armour, T. J. Kim, R. J. Shul, J. A. Nevers, W. T. Wheelis, K. R. Lanes, R. M. Axline, J. C. Chavez

A paradigm shift in satellite systems is occurring based on the use of many small, inexpensive satellites to replace functions performed by large and expensive satellites. These small satellites weigh less than $10 \mathrm{Kg}$ and are referred to as nanosatellites or nanosats. They would be used in a variety of applications including constellations of several hundred nanosats covering the globe; or closely knit "clusters" of nanosats flying in formation and acting cooperatively for imagery, surveillance, intelligence collection, or threat detection; or single nanosats used for satellite inspection and anomaly resolution.

Numerous government agencies, including the Department of Energy, have documented the need for smallsatellite technology to meet future requirements for surveillance of potential proliferants of chemical/biological weapons $(\mathrm{CW} / \mathrm{BW})$, threat/missile detection, tracking/impact prediction, etc.

In order for nanosat systems to be affordable and reliable, satellite and launch costs must be drastically reduced. Nanosat development requires a wide range of enabling technologies, for example, miniaturization and multifunctional integration of satellite systems. Successful implementation will bring the microsystems revolution to space.

In this project, Sandia concentrated on researching, designing, and prototyping critical nanosat satellite systems using microcomponents and fabrication technologies. These systems included high-efficiency solar cells, structural battery, laser communications, attitude- and position-determination system elements, a sparse aperture study, and a nanosat deployment study. Although the systems identified above do not represent all nanosat systems, they do represent those that are critical to the success of a nanosat.

In advanced photovoltaics, our goal is to develop a four-junction solar cell with an efficiency of $35 \%$, compared to normal efficiencies of $25 \%$. We are working on a fourthjunction indium/gallium/arsenide/nitride (InGaAsN) single-
In this project, Sandia [is concentrating] on researching, designing, and prototyping critical nanosat satellite systems using microcomponents and fabrication technologies. These systems included high-efficiency solar cells, structural battery, laser communications, attitudeand position-determination

system elements, a sparse aperture study, and a nanosat deployment study. 
crystal growth process. We grew pure InGaAsN crystals by varying parameters (e.g., temperature, material quality, pressure, flow rate). Quantum efficiency of InGaAsN material continues to hover around 70\%; however, that is still the best quantum efficiency achieved by any organization. The verticalcavity surface-emitting laser (VCSEL) group at Sandia used their materials knowledge to successfully develop a commercially viable 1.3-micron VCSEL.

In the laser communications system, our goal is to create a detailed design of a gimballess, high-speed intersatellite laser communications link that supports a 45degree field of view (FOV), $3 \mathrm{~km}$ range, and data rate of 1 gigabit/second. We built the prototype hardware and conducted a demonstration. The prototype hardware used a separate transmitter/receiver and achieved a transmission distance of 3 feet and a +500 megabits/second data rate. Our two-axis translation stage demonstrated system tracking within the specified 45-degree FOV at relatively slow speeds. We fabricated and characterized optical phased-array steering system (OPASS) photonic integrated circuits (PICs); however, high side lobes severely limited their possible application.

With the integrated power supply, our goal is to design and demonstrate a lightweight and rigid structural lithium (Li) ion battery based on silicon ( $\mathrm{Si}$ ) wafer processing techniques. Our completed design consists of four Si wafers. The two outer wafers provide structural support; the two inner wafers are etched with a honeycomb pattern to hold Li-ion battery material. To enhance battery efficiency, we included a 1micron separator between positive/negative battery materials as part of the design. Normal separators are 25 microns thick. Theoretical battery performance is 3.6 volts and $1.2 \mathrm{amp} /$ hour. We successfully deposited Li-ion material in honeycombpatterned wafers with minimal breakage. We assembled a nonfunctional structural battery unit. Because we were not able to successfully etch a 1-micron film separator as part of the etching process, we used a standard separator. We are in the process of assembling a functional structural battery.

We achieved our goal for attitude control of designing a prototype mini three-axis reaction wheel. Because of the small size of the motors (diameter of a pencil, 3/4-inch long), we used open-loop control, which required extensive software control algorithm development. We also designed and fabricated the resolver table to allow one-axis testing. We assembled a prototype reaction wheel unit of approximately 30 cubic inches. We successfully demonstrated software control of
We grew pure InGaAsN crystals by varying parameters (e.g., temperature, material quality, pressure, flow rate). Quantum efficiency of InGaAsN material continues to be around $70 \%$; however, that is still the best quantum efficiency achieved by any organization. The verticalcavity surface-emitting laser (VCSEL) group at Sandia used their materials knowledge to successfully develop a commercially viable 1.3-micron VCSEL. 
reaction wheels from stop to approximately 14,000 revolutions per minute (rpm). We are in the process of characterizing the attitude capability of the reaction wheel, with a goal of 0.1 degree on the resolver table.

Our position and attitude determination goal is the design and demonstration of a differential carrier-phase global positioning system (GPS) attitude and relative position determination system, where attitude knowledge is $\sim 0.2$ degree and relative position knowledge is $\sim 2 \mathrm{~cm}$. We developed detailed system architecture that incorporates radiation-induced single-event upset (SEU) and latch-up recovery methods. We completed breadboarding of GPS frontend with no lightweight/low-power radiation-hardened GPS receivers currently available, as well as preparing the processing hardware. The board size is $2 \times 21 / 2$ inches. We also completed the software to interpret and display position information.

In the sparse aperture study, our goal was to determine the best radio-frequency (RF) mission that a sparse cluster of nanosats could support. We found that the best application for a nanosat cluster would be as a geolocation system for transmitting ground systems, i.e., the nanosat sparse array could be used to determine the location of ground systems.

\section{Refereed}

Allerman, A. A., S. R. Kurtz, E. D. Jones, J. M. Gee, J. C. Banks, and A. Clement-font. 1999. "The Growth of InGaAsN for High-Efficiency Solar Cells by Metalorganic Chemical Vapor Deposition." (Invited) Photovoltaics for the 21st Century.

Allerman, A. A., S. R. Kurtz, E. D. Jones, N. A. Modine, J. M. Gee, and D. M. Follstaedt. 1999. "InGaAsN: A Novel Material for High-Efficiency Cells and Advanced Photonic Devices." Sandia Technical Report SAND99-0922, Sandia National Laboratories, Albuquerque, NM (July).

Allerman, A. A., S. R. Kurtz, E. D. Jones, N. A. Modine, R. M. Sieg, S. A. Ringel, D. Kwon, and R. J. Kaplar. 1999. "InGaAsN for High-Efficiency Solar Cells." (Invited) Paper presented to the Fall Meeting of the Materials Research Society, Symposium OO, Boston, MA, 29 November-3 December.
We found that the best application for a nanosat cluster would be as a geolocation system for transmitting ground systems, i.e., the nanosat sparse array could be used to determine the location of ground systems.
Allerman, A. A., S. R. Kurtz, E. D. Jones, R. M. Sieg, D. J. Aiken, and N. A. Modine 2000. "MOCVD Growth of InGaAsN for High-Efficiency Solar Cells." Paper presented to the 12th American Conference on Crystal Growth, Vail, CO, 13-18 August.

Hoe, H. Q., K. C. Reinhardt, S. R. Kurtz, J. M. Gee, A. A. Allerman, B. E. Hammons, P. C. Chang, and E. D. Jones. 1998. "Novel InGaAsN pn Junction for High-Efficiency Multiple-Junction Solar Cells." Proc. World Conf. and Exhibition on Photovoltaic Energy Conversion (Vienna, Austria, 6-10 July).

Jones, E. D., A. A. Allerman, I. J. Fritz, S. R. Kurtz, and N. A. Modine. 1999. "Effective Masses for InGaAsN Alloys Lattice-Matched to GaAs." Paper presented to the Fall Meeting of the Materials Research Society, Symposium OO, Boston, MA, 29 November-3 December. 
Jones, E. D., A. A. Allerman, I. J. Fritz, S. R. Kurtz, J. F. Klem, R. M. Sieg, O. B. Spahn, and C. P. Tigges. 2000. "Low-Temperature Photoluminescence Characterization of GaAsSb Quantum Wells with InGaAs and AlGaAs Barriers on GaAs." Paper presented to Photonics West: Optoelectronics '00, San Jose, CA, 23-28 January.

Jones, E. D., A. A. Allerman, S. R. Kurtz, I. J. Fritz, N. A. Modine, K. K. Bajaj, S. T. Tozer, and X. Wei. 1999. "Effective Masses for $1 \%$ and $2 \%$ Nitrogen in InGaAsN Alloys." Paper presented to the 1999 Four Corners Section Fall Meeting, Tucson, AZ, 1-2 October.

Jones, E. D., N. A. Modine, A. A. Allerman, I. J. Fritz, S. R. Kurtz, A. F. Wright, S. T. Tozer, and X. Wei. 1999. "Optical Properties of InGaAsN: A New 1 eV Bandgap Material System." Light-Emitting Diodes: Res., Manuf., and Applic. III, Proc. SPIE Conf. 3621.

Jones, E. D., N. A. Modine, A. A. Allerman, S. R. Kurtz, A. F. Wright, S. T. Tozer, and X. Wei. 1999. "Band Structure of InGaAsN Alloys and Effects of Pressure." Phys. Rev. B 60: 4430-4433.

Jones, E. D., N. A. Modine, A. A. Allerman, S. R. Kurtz, A. F. Wright, S. T. Tozer, and X. Wei. 1999. "Photoluminescence Studies of InGaAsN Alloys Lattice-Matched to GaAs." (Invited) Photovoltaics for the 21st Century.

Kaplar, R. J., A. R. Arehart, D. Kwon, S. A. Ringel, A. A. Allerman, S. R. Kurtz, E. D. Jones, and R. M. Sieg. 2000. "Influence of Nitrogen Content and Dopant Type on Deep-Level Spectra of MOCVD-Grown InGaAsN." Paper presented to the Electronic Materials Conference, Denver, CO, 21-23 June.

Kurtz, S. R., A. A. Allerman, and E. D. Jones. 1999. "InGaAsN Solar Cells and Minority Carrier Properties." Paper presented to the 1999 Four Corners Section Fall Meeting, Tucson, AZ, 1-2 October.

Kurtz, S. R., A. A. Allerman, E. D. Jones, and C. H. Seager. 1999. "Minority Carrier Lifetimes, Defects, and Solar Cells of InGaAsN, Latticed-Matched to GaAs." (Invited) Photovoltaics for the 21 st Century.
Kurtz, S. R., A. A. Allerman, E. D. Jones, J. M. Gee, J. J. Banas, E. D. Jones, and B. E. Hammons. 1998. "InGaAsN Solar Cells with 1.0 eV Bandgap, LatticeMatched to GaAs." Appl. Phys. Lett. 74: 729-731.

Kurtz, S. R., A. A. Allerman, R. M. Sieg, E. D. Jones, and C. H. Seager. 2000. "Minority Carrier Diffusion, Defects and Localization in InGaAsN, with $2 \%$ Nitrogen." Appl. Phys. Lett. 77: 1-3.

Kurtz, S. R., A. A. Allerman, R. M. Sieg, E. D. Jones, and C. H. Seager. 2000. "Transport Studies of Compensated, InGaAsN Solar Cell Materials." Paper presented to the Electronic Materials Conference, Denver, CO, 21-23 June.

Kwon, D., R. J. Kaplar, J. J. Boeckl, S. A. Ringel, A. A. Allerman, S. R. Kurtz, and E. D. Jones. 1999. "Deep-Level Defect Studies in MOCVD-Grown InGaAsN Films Lattice-Matched to GaAs." Integration of Dissimilar Mater. in Micro- and Optoelectronics, Proc. Mater. Res. Soc. Symp. (Boston, MA).

Kwon, D., R. J. Kaplar, S. A. Ringel, A. A. Allerman, S. R. Kurtz, and E. D. Jones. 1999. "Deep Levels in pand n-Type InGaAsN for High-Efficiency Solar Cells." Special Issue of Solar Energy Mater. \& Solar Cells, Proc. 11th Internat. Photovoltaic Sci. and Eng. Conf. (PVSEC-11) 1 (Hokkaido, Japan, 20-24 September).

Kwon, D., R. J. Kaplar, S. A. Ringel, A. A. Allerman, S. R. Kurtz, and E. D. Jones. 1999. "Deep Levels in pType InGaAsN Lattice-Matched to GaAs.” Appl. Phys. Lett. 74: 2830-2832.

Modine, N. A., E. D. Jones, A. A. Allerman, and S. R. Kurtz. 1999. "Band Structure Calculations for Dilute Alloys with Application to GaAsN." Paper presented to the Fall Meeting of the Materials Research Society, Symposium D, Boston, MA, 29 November-3 December.

Modine, N. A., E. D. Jones, A. A. Allerman, and S. R. Kurtz. 2000. "Optical and Electronic Properties of InGaAsN Photocell Material." Paper presented to the DoD High-Performance Computer Users Meeting 2000, Albuquerque, NM, 6-9 June. 
Modine, N. A., E. D. Jones, A. A. Allerman, and S. R. Kurtz. 1999. "Theory of the Pressure-Dependent Band Structure of GaAsN Alloys." Paper presented to the 1999 Four Corners Section Fall Meeting, Tucson, AZ, 1-2 October.

Sieg, R. M., A. A. Allerman, S. R. Kurtz, E. D. Jones, and O. B. Spahn. 2000 "Effect of (1,1)-DMHy Purity on MOCVD-Grown InGaAsN Performance and on Post-Growth Annealing Behavior." Paper presented to the Electronic Materials Conference, Denver, CO, 2123 June.

\section{Other Communications}

Reynolds, D. A. 2000. "Nanosat Activities." Briefing presented to meeting with Dr. Paul Kolodzy, DARPA Advanced Technology Office. Sandia National Laboratories, Albuquerque, NM, 25 July.

Wheelis, W. T. 2000. "Nanosat Activities at Sandia National Laboratories." Briefing presented to meeting with Bart Johnson, Director, Lockheed Martin Corporation Technology Services, Sandia National Laboratories, Albuquerque, NM, 18 May.
Wheelis, W. T. 2000. "Nanosat Activities at Sandia National Laboratories." Briefing presented to meeting with Dr. Gerald Witt, Air Force Office of Scientific Research (AFOSR), Sandia National Laboratories, Albuquerque, NM, 9 May.

Wheelis, W. T. 2000. "Nanosat Activities at Sandia National Laboratories." (Invited) Presentation to the DARPA/JPL/AFRL Workshop on Pico-Satellites for In-Space Testing of MEMS Technologies, Pasadena, CA, 24 July.

Wheelis, W. T. 2000. "Satellite Activities at Sandia National Laboratories." Presentation to meeting with Ian Moyle, Lockheed Martin Space System Company, Product Technology Group, and Mike Meier and Al Sherman, Lockheed Martin Advanced Technology Center (ATC), Sandia National Laboratories, Albuquerque, NM, 26 June.

Wheelis, W. T. 2000. "Satellite Laser Communications Activities at Sandia National Laboratories." Paper presented to the AIAA Workshop on Reducing the Cost of Space Operations, San Jose, CA, 11-12 April. 
10698

\section{Autonomous MicroChem Laboratory} ( $\mu$ ChemLab ${ }^{\mathrm{TM}}$ )

G. C. Frye-Mason, D. J. Rakestraw, S. H. Kravitz, R. J. Kottenstette, C. G. Bailey, P. R. Lewis, D. W. Arnold, R. P. Manginell, M. E. Warren, E. J. Heller, C. M. Matzke, M. R. Daily, J. Wu, D. L. Lindner, D. R. Adkins, R. F. Renzi, G. R. Dulleck, Jr., J. F. Stamps

This project will lead to a new generation of field-ready and compact chemical-analysis devices that have unprecedented capabilities for measuring and deciphering detailed chemical signatures. The project includes development of both gas- and liquid-phase analysis capabilities. The gasphase module has demonstrated sensitive and selective detection of chemical warfare $(\mathrm{CW})$ agent simulants using three cascaded components: (1) a microfabricated sample collector/concentrator, (2) a miniature gas chromatograph (GC) column, and (3) a sensitive and chemically discriminating detector based on an array of surface acousticwave (SAW) devices. The liquid-phase module has demonstrated effective separation and detection of explosives using electrokinetic (EK) separations with laser-induced fluorescence ( $\mathrm{LiF}$ ) and/or electrochemical detection. The separations are conducted in complex microfabricated structures that are interfaced and integrated with miniaturized fluidic and electronic components. The project also involves developing the system architecture and supporting electronic hardware and software to produce complete autonomous systems.

We will demonstrate an optimized autonomous microscale chemical laboratory ( $\mu$ ChemLab $\left.{ }^{T M}\right)$ that will establish Sandia as a leader in this field. This project will position Sandia to support increasing demands for detailed chemical information by current national security customers and is essential for developing new industrial partnerships as well as new customers in nonproliferation, counterterrorism, energy, and critical infrastructures.

We made dramatic progress toward developing an autonomous, hand-held chemical analyzer. A highlight was the demonstration of both gas- and liquid-phase separations modules. While we identified several issues needing to be addressed, this demonstrated that we had gone from a technical concept to working hardware.
We will demonstrate an optimized autonomous microscale chemical laboratory $\left(\mu C_{\text {ChemLab }}^{T M}\right)$ that will ....support increasing demands for detailed chemical information by current national security customers and is essential for developing new industrial partnerships as well as new customers....

We made dramatic progress toward developing an autonomous, hand-held chemical analyzer. A highlight was the demonstration of both gas- and liquid-phase separations modules. 
Selected accomplishments for the gas module focused on detection of CW agents and include the following: (1) demonstrated microfabricated sample preconcentrators with concentration factors $>100,(2)$ demonstrated effective separation of Sarin, Soman, and mustard simulants using miniature GC chips, (3) demonstrated sensitive and selective detection using an acoustic array with miniature GaAs (gallium arsenide) drive electronics, (4) demonstrated collection, separation, and detection of trace concentrations of CW simulants in 2 minutes using these three components together, (4) designed and fabricated a novel PC board that provides both electrical and fluidic interconnections for dual analysis channels, (5) designed and fabricated a PC board for supporting electronics, including temperature control and dataacquisition circuitry, (6) wrote software for system operation, data acquisition, data processing, and pattern recognition (PR), and (7) assembled a complete autonomous hand-held system that could collect a sample, separate and detect the species present, and display the results on the liquid crystal display (LCD) (e.g., detected CW simulant and its concentration).

To help demonstrate the capabilities for other potential applications, we performed tests demonstrating (1) collection of volatiles using the microfabricated preconcentrator with carbon adsorbents, (2) separation of gas such as methane, ethane, and carbon dioxide $\left(\mathrm{CO}_{2}\right)$ using novel packed GC columns, and (3) separation of explosives such as dinitrotoluene (DNT) and trinitrotoluene (TNT) using microfabricated GC columns.

Selected accomplishments for the liquid module include (1) demonstrated LiF detection in a microchannel separation using a microoptical detection system, (2) demonstrated onchip separations of 10 explosives in one minute using micellar EK chromatography, (3) achieved detection limits of $\sim 1$ part per million (ppm) in on-chip separations of explosives, (4) demonstrated $\sim 1 \mathrm{ppm}$ detection limits for explosives using electrochemical detection, (5) developed capability to fabricate complex fluidic architectures required for three independent on-chip separations, (6) demonstrated ability to generate 490 $\mathrm{nm}$ laser emission by frequency doubling a vertical-cavity surface-emitting laser (VCSEL), (7) demonstrated $350 \mathrm{~nm}$ emission for $\mathrm{GaN}$ (gallium nitride) multiple quantum-well (QW) light-emitting diodes (LEDs), (8) developed compact, high-sensitivity photodiode-based optical detector, (9) demonstrated EK pumping as a means to generate high
[We] demonstrated LiF detection in a microchannel separation using a microoptical detection system, demonstrated on-chip separations of 10 explosives in one minute using micellar EK chromatography,... 
pressures, and (10) demonstrated the use of these high pressures to perform high-performance liquid chromatography (HPLC).

For the system and supporting electronics, we (1) designed and built an integrated, programmable high-voltage supply to drive EK separations, (2) developed a control system that allows manipulation of up to 80 input/output (I/O) lines for system operation, (3) wrote code for system operation of both gas- and liquid-phase modules using menu-driven options and a four-button keypad, (4) fabricated a robust fluidic system to interface the "macro outside world" with the microseparation chip for liquid-phase analyses, and (5) designed an anodized aluminum housing to fit all the components for both gas- and liquid-phase analysis modules.

\section{Other Communications}

Frye-Mason, G. C., et al. 2000. "Hand-Held Miniature Chemical Analysis Systems ( $\mu$ ChemLab $\left.{ }^{\mathrm{TM}}\right)$ for Detection of Trace Concentrations of Gas-Phase Analytes." Micro-Total Anal. Sys. 2000 (Enschede, The Netherlands, 14-19 May): 229-232. Dordrecht, The Netherlands: Kluwer Academic Publishers.

Manginell, P. R., G. C. Frye-Mason, R. J. Kottenstette, and C. C. Wong. 2000. "Microfabricated Planar Preconcentrator." Proc. Solid-State Sensors (Hilton Head, SC, June): 179-182. Transducers Research Foundation, Inc.

Paul, P., D. Arnold, D. Neyer, and K. Smith. 2000. "Electrokinetic Pump Application in Micro-Total Analysis Systems Mechanical Actuation to HPLC." Proc. m-TAS 2000 Workshop (Enschede, The Netherlands, 14-18 May): 583-590.
Wallenborg, S., C. Bailey, and P. Paul. 2000. “OnChip Separation of Explosive Compounds—Divided Reservoirs to Improve Reproducibility and Minimize Buffer Depletion." Proc. m-TAS 2000 Workshop (Enschede, The Netherlands, 14-18 May): 355-358.

Wong, C. C., J. H. Fleming, R. P. Manginell, R. J. Kottenstette, and G. C. Frye-Mason. 2000. "Design and Analysis of a Preconcentrator for the $\mu C h e m L a b{ }^{\mathrm{TM}}$." ASME MEMS 2 (Hilton Head Island, SC, June).

Wong, C. C., T. L. Zoeller, D. R. Adkins, and J. N. Shadid. 2000. "Investigation of Gas Flow in Long and Narrow Channels." Proc. ASME FED 2000 Fluids Eng. Div. Summer Mtg. 251 (Boston, MA, 11-15 June). 


\section{0}

\section{Molecular Integrated Microsystems (MIMS)}

L. M. Napolitano, T. J. Shepodd, J. S. Schoeniger, D. Y. Sasaki, D. R. Wheeler, K. S. Potter, S. A. Casalnuovo, B. G. Potter, Jr., S. M. Brozik, C. J. Brinker, A. P. Thompson, D. A. Loy, A. K. Singh, E. F. Hasselbrink, A. R. Burns, B. C. Bunker, S. A. Kemme

Microfabrication tools and processes developed for the microelectronics industry make it possible to miniaturize devices such as chemical analysis systems, navigational components, and mechanical locks. Sandia's experience with microsystems shows that direct miniaturization of conventional architectures can provide significant benefit through increased portability, low power consumption, and improved speed of operation. We believe that the full benefit of miniaturization will be achieved only when microscale addressability is used to control materials properties and molecular interactions. We seek a revolutionary approach to microsystems in which microscale patterning of functional materials is combined with local addressing (electrical, optical, thermal fields) to provide fully programmable systems and architectures.

In this new approach, we can dynamically control the function of the microsystem in real time to optimize performance, compensate for defects or damage, or adapt to changing environmental conditions. Advances in biotechnology and materials science provide new approaches to control materials and manipulate molecules at the nanometer scale. The ability to manipulate nanoscale structures and molecular interactions is at the heart of the next revolution in programmable microsystems. We fully expect that molecular integrated microsystems (MIMS) will provide new means to encode information and safeguard weapons, open completely new routes to attack important problems in chemistry and biology, and lead to the development of a new class of materials that combine functions found in biological and molecular systems with robust and manufacturable materials. The overall goal of this project is to combine molecularly tailored nanomaterials with addressable microdevice architectures to demonstrate the first-ever programmable MIMS devices for protein and peptide analysis and encoded optical interconnects.

We explored a broad range of functional materials that have the potential to provide new approaches needed to achieve our project goals. We refined our goals and identified technical
We seek a revolutionary approach to microsystems in which microscale patterning of functional materials is combined with local addressing (electrical, optical, thermal fields) to provide fully programmable systems and architectures.

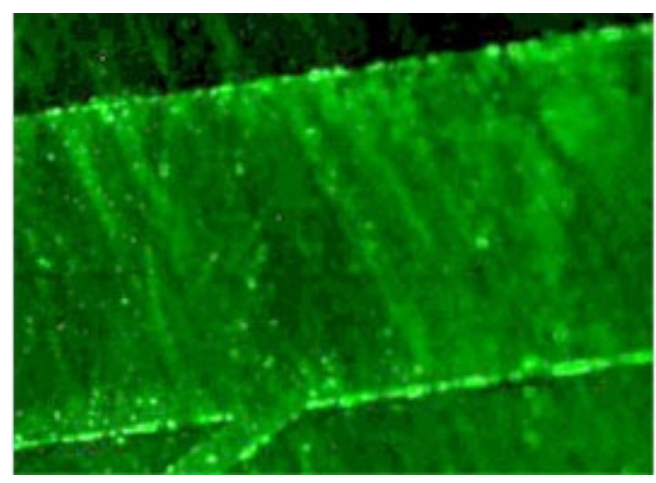

Fluorescence image of an encapsulated flow channel (top view). 
approaches through a detailed technical assessment. Our subsequent research uncovered a number of exciting new materials and phenomena that will provide important cornerstones to our development of programmable microsystems for biomolecular sorting and detection. The development of electrokinetic (EK) trapping could lead to a totally new means of preconcentrating and sorting biomolecules. We demonstrated thin films that allow us to switch the hydrophobic/hydrophilic nature of surfaces and to control protein adsorption. We learned to self-assemble materials for molecular sorting that can be cast in place and photodefined. These new materials could also lead to new concepts for valving fluid channels in microchips. Our work with membranes demonstrated new approaches for molecular recognition that rely on principles of self-assembly. We developed completely new approaches for fabricating fluid channels that take direct advantage of standard microelectronics processing. This new manufacturing approach has the potential to allow for the integration of a much broader range of materials and devices in chip-based chemical analysis systems. Other work focused on the development of programmable optical interconnect architectures. We identified two families of photosensitive materials that provide extremely large optical index changes when exposed to moderate optical signals. Preliminary optical structures such as planar waveguides and grating structures have yielded promising results.

\section{Refereed}

Doshi, D. A., et al. 2000. "Optically Defined Multifunctional Patterning of Photosensitive ThinFilm Silica Mesophases.” Science 290: 107-111.

Fan, H., et al. 2000. "Rapid Prototyping of Patterned Functional Nanostructures.” Nature 405: 364-368.

\section{Other Communications}

Last, J. A., T. A. Waggoner, and D. Y. Sasaki. 2000. "AFM Imaging of Molecular Reorganization in Lipid Bilayers Induced Through Ion Recognition." Paper presented to the American Chemical Society 2000 Spring National Meeting, San Francisco, CA, 26-30 March.
Lu, Y., et al. 2000. "Self-Assembly of Mesoscopically Ordered Chromatic Polydiacetylene/Silica Nanocomposites Using Polymerizable Surfactants as Structure-Directing Monomers." Nature 1: 1.

Sasaki, D. Y. 2000. "Functionalized Lipid Membranes: Self-Assembled Systems and Optical Sensing." (Invited) Lecture given at North Dakota State University, Fargo, ND, 29 September.

Waggoner, T. A., and D. Y. Sasaki. 2000. "SurfaceCompatible Sol-Gel Materials for Immobilized Lipid Bilayers." Paper presented to the American Chemical Society 2000 Spring National Meeting, San Francisco, CA, 26-30 March. 
Waggoner, T. A., J. A. Last, P. G. Kotula, and D. Y. Sasaki. 2000. "Self-Assembled Columns of Stacked Lipid Bilayers Mediated by Metal Ion Recognition.” J. Amer. Chem. Soc. (March): 1-10.

Yu, K., et al. 2000. "PS-b-PEO Films with Regular and Reverse Mesostructures of Large Characteristic Length Scales Prepared by Solvent EvaporationInduced Self-Assembly." Polymer Preprints 1: 1. 


\section{Corporate Objectives}

The Corporate Objectives investment area researches novel concepts and technologies directed to the specific needs of the

Laboratories' program areas.

Sophisticated research and development efforts target the goals of Sandia National Laboratories' strategic business and management units and are primarily focused on applied research goals rather than basic research.

There are six main thrusts in the Corporate Objectives area: (1) the Nuclear Weapons area explores innovative ideas relevant to meet future weapons' requirements; (2) the Nonproliferation and Material Control area examines advanced technologies for improving the physical security of weapons and special nuclear materials; (3) the Energy and Critical Infrastructure area focuses on technologies that enhance the safety, security, and reliability of the nation's critical infrastructures; (4) the Emerging Threats area examines technologies and concepts enabling high-impact responses to emerging national security threats; (5) the Science and Technology area explores novel concepts addressing the interfaces between various disciplines; and (6) the Partnerships area engages in strategic partnerships with industry and universities, collaborates with other government laboratories, and develops strategic international collaborations. 
10401

\section{Chemiresistors Based on Metal-Loaded Polymers for Solvent Spill Detection}

R. C. Hughes

Sandia discovered that small, planar microsensors for solvents can be fabricated by mixing common commercial polymers with metal powders such as graphite. Thin films of these composites can be deposited on interdigitated electrodes on an insulating substrate. An example of such a film is fabricated from polyisobutylene mixed with $40 \%$ by weight of carbon $(C)$ powder. The resistance of such a film appears to be dominated by percolation of charge through the conducting $C$ particles. The resistance depends on the number of such contacts and thus on the volume fraction of the $C$ particles. Vapors of some solvents can cause the polymer to swell and thus decrease the volume fraction of conducting particles. The electrical resistance thus increases with increasing solvent vapor pressure. We are particularly interested in the reproducibility of the sensor signals, as well as long-term drift in the base (dry) resistance. Temperature dependence of the signals is also important for an understanding of the thermodynamics of the polymer swelling. We seek to understand the role of entropy and heat of mixing of the solvent vapors in the sensor output. It may turn out that different polymers will have a sufficiently different response to various vapors and that arrays of sensors can be used to identify an unknown vapor by pattern recognition $(P R)$.

We demonstrated several improvements in detecting solvent vapors. We fabricated many array chips that have four different chemiresistors on them, along with a platinum $(\mathrm{Pt})$ temperature sensor and two Pt heaters at each end. These small chips can be bonded out into a 16- or 24-pin dual-inline pin (DIP) package and are thus available for different configurations for vapor sampling. They can be put into a small header (< one cubic inch) and attached by ribbon cable to distant electronic packages that control the chip temperature and take the sensor reading. The selection of the four chemiresistor polymer inks leads us to believe that all normal solvents will be detected by at least one of the chemiresistors if the vapor pressure (concentration) is greater than about one percent of the saturated vapor pressure at the ambient temperature. PR techniques will enable us to identify most solvents if they are in our training database.
Sandia discovered that small, planar microsensors for solvents can be fabricated by mixing common commercial polymers with metal powders such as graphite.... We seek to understand the role of entropy and heat of mixing of the solvent vapors in the sensor output. 
We also succeeded in fabricating a hybrid DIP that includes a micropreconcentrator along with the sensor array. The preconcentrator can accumulate vapors at concentrations too low to be readily detected by the chemiresistor in real time, and on command it can be heated to $200^{\circ} \mathrm{C}$, thus producing a transient high concentration on the chemiresistor that can be seen as a spike signal. The height of the spike is proportional to the quantity of vapor adsorbed on the preconcentrator between heat pulses.

In a collaborative project employing our idea of using magnetic field-structured conducting powders, we achieved direct current (dc) electrical conductivity at loading fractions lower than $10 \%$ by volume, as predicted by theory. The field structure was frozen in by polymerizing the matrix, and the resulting samples had huge positive temperature coefficients of resistance, again as predicted by theory. We have not yet cast the samples into a form (thin film) that can test their chemical response. 
10402

\section{Advance Neutron-Tube Design and Producibility}

J. P. Brainard, R. S. Goeke, D. P. Adams

Some neutron tubes use $94 \%$ alumina insulators for the high-voltage insulators that are coated with a fired-on mixture of manganese (Mn) and titanium (Ti). The Mn reduces the secondary electron emission on the insulator, and the Ti helps bleed off the wall charge that builds up during tube operation. Both materials and their properties help reduce high-voltage breakdown. The Ti, however, reacts with the alumina surface in a very nonuniform manner. It appears very blotchy and in places very porous, even though the original coating was uniform. If we could eliminate the Ti, our insulators would be much more reproducible.

We are currently using an in situ optical stress sensor to monitor changes in wafer surfaces during film processing. Wafer curvature can be related to stress if thin wafers are used. This work studied scandium (Sc) hydride films on molyalumina cermet substrates used in some neutron tubes. We found the Sc film goes into compression when hydrogen (H)-loaded and goes into tension when cooled back to room temperature. These films periodically flake, and this work will help us minimize film stress by modifying our manufacturing processes.

A commercially available 1 percent moly-doped insulator looked like a promising candidate to replace the neutron-tube high-voltage insulator and to eliminate the uncontrolled process of Ti coating used to bleed off the insulator wall charge. This new insulator has sufficient bulk conductivity to bleed off wall charge between tube operations. Of 12 neutron tubes built by production, we made 4 using our standard processed Ti/Mn-coated insulators for control, 4 from moly-doped insulators, and $4 \mathrm{Mn}$-coated moly-doped insulators. We measured insulator wall charge and tube performance during tube operation. Above a certain wall charge level, high-voltage breakdown rates increased substantially. The moly-doped insulator without Mn coatings charged to higher levels and had higher breakdown rates. This is a condition we would not recommend. However, the molydoped insulators with Mn coatings appear to be equivalent to our standard processed insulators, and the Ti coating can be eliminated.
We are currently using an in situ optical stress sensor to monitor changes in wafer surfaces during film processing....this work will help us minimize film stress by modifying our manufacturing processes. 
The challenge in measuring film stress was the fabrication of thin cermet wafers. We tried to simulate the moly-alumina cermet substrate used in some neutron tubes to deposit Sc films. Thin wafers were required so that we could measure wafer curvature and relate it to film stress. We used multilaser beam reflection to determine the wafer curvature. We made these wafers 400 microns thick with a sufficiently smooth and reflective backside for the wafer curvature measurements. We deposited the Sc film with the substrate held at elevated temperatures in vacuum and observed little stress when Sc was deposited. When the film was hydrided, however, the film went into compression. When the film was cooled back to room temperature, the film went into tension. In the near future, we will determine the effect of stress on the process variables. We believe that this will lead to nonflaking films.
In the near future we will

determine the effect of stress on the process variables. We believe that this will lead to nonflaking films. 
10403

Surface Hardening by Nanoparticle Precipitation and Atomic Clustering in $\mathrm{Ni}(\mathrm{Al}, \mathrm{O})$

S. M. Myers, Jr.

This project explores strengthening of the near-surface of nickel $(\mathrm{Ni})$ by dense dispersions of hard oxide particles and atomic clusters in the nanometer-size range. Ion implantation and thermal diffusion of oxygen $(O)$ and aluminum ( $A l)$ form these dispersions into $\mathrm{Ni}$ in conjunction with heat treatments to manipulate particle size. Mechanical properties are quantified by nanoindentation coupled with finite-element modeling (FEM), and microstructures are characterized by transmission electron microscopy (TEM). Theoretical considerations suggest that the extremely small sizes and separations of the hard particles in such metal matrices may give rise to new highs of strength with retention of ductility. Specific objectives of the project are (1) to probe and understand the limits of strength that can be reached at the extremes of small particle size and high particle density, and (2) to extend such hardening to the technologically important Fe-Ni (iron-nickel) class of materials, and in particular to Ni because of its anticipated use in defense-related microelectromechanical systems (MEMS).

Oxygen-ion implantation to a concentration of 8 at.\%, in conjunction with annealing treatments, produced a series of nickel oxide $(\mathrm{NiO})$ particle dispersions with a volume fraction of $15 \%$ and average particle sizes ranging from 2 to $14 \mathrm{~nm}$, far below the size range of conventional dispersion-hardened alloys. Implantation of both $\mathrm{O}$ and $\mathrm{Al}$, each to a concentration of 8 at.\%, yielded $\mathrm{Al}_{2} \mathrm{O}_{3}$ particles with a volume fraction of $11 \%$ and average size of $1.5 \mathrm{~nm}$. Nanoindentation of these materials, with FEM to extract the mechanical properties from force-displacement data, indicated intrinsic yield strengths extending to the exceptionally large value of $5 \mathrm{GPa}$. These measured strengths conform quantitatively to theoretical predictions based on pinning of dislocations by the hard particles, exhibiting in particular the predicted proportionality of strength to inverse particle spacing. Alternative synthesis by high-temperature diffusion of $\mathrm{O}$ into $\mathrm{Ni}$ and $\mathrm{Ni}-\mathrm{Al}$ alloys was less successful, producing less refined and less homogeneous microstructures with lower strengths. The $\mathrm{O}$ implantation treatment also reduced friction and wear. Unlubricated pin-on- 
disc testing with a hard steel pin showed a reduction in the friction coefficient from 0.9 to 0.2 .

This project established that we can extend dispersion hardening of Ni substantially beyond previously achieved levels through refinement of the particle size to the nanometer scale. Further, we established that standard dispersionhardening theory, based on continuum-model analysis of dislocation pinning, remains applicable even at scales as small as several lattice spacings. Lastly, the implantation hardening of Ni examined in this work appears promising for application to micromachine components constructed of $\mathrm{Ni}$.

This project established that we can extend dispersion hardening of Ni substantially beyond previously achieved levels through refinement of the particle size to the nanometer scale. 
10404

\section{Dynamical Properties of Polymers:} Computational Modeling

\section{J. G. Curro}

The Polymer Reference Interaction Site Model (PRISM) theory of Curro and Schweizer is extremely successful in modeling the equilibrium structure and physical properties of bulk amorphous polymers. In this project, Sandia used the PRISM theory to extract information regarding nonequilibrium properties. In the past, dynamical properties such as the glass transition and viscoelasticity were correlated with the static equilibrium structure of the polymer through the free-volume distribution. Although the free-volume concept has long been useful from a qualitative viewpoint, it has not had a precise statistical mechanical interpretation. PRISM theory not only provides this fundamental interpretation, but also provides a computationally tractable modeling tool to compute the freevolume distribution for polymers at the atomistic level. In this investigation, we also performed molecular dynamics (MD) simulations on coarse-grained and atomistic models to obtain diffusion constants and the velocity autocorrelation function.

We extracted the free-volume distribution from a series of coarse-grained models of varying chain stiffness and compared them to the free-volume distribution obtained from exact MD calculations. We found good agreement between theory and simulation. We also carried out free-volume computations on an atomistic model of polyethylene. We found the results to have similar features to the free-volume distribution of the coarse-grained models. We correlated these free-volume distributions and the diffusion constants of various penetrants diffusing through the polymers.

We completed MD calculations on atomistic models of polyisobutylene to obtain the velocity autocorrelation function with a nitrogen penetrant. We found the tail of the velocity autocorrelation function of the diffusing molecule to have a power law form with an exponent of $5 / 2$ in agreement with earlier studies on coarse-grained models. In addition, we obtained the dynamic structure factor of the penetrant diffusing in polyisobutylene from MD simulations. We interpreted these results using liquid-state ideas; they will form the basis of a molecular theory for penetrant diffusion based on modecoupling theory.
Sandia used the PRISM theory

to extract information regarding nonequilibrium properties....In this investigation, we also performed molecular dynamics (MD) simulations on coarsegrained and atomistic models to obtain diffusion constants and the velocity autocorrelation function. 
10405

\section{Broadening Mechanism in 2-D Excitonic and Electron Gases}

E. D. Jones

This project investigates the origins for the broadening mechanisms leading to the Gaussian photoluminescence (PL) lineshapes observed in doped and undoped quantum-well $(Q W)$ structures. All homogeneous broadening mechanisms predict a Lorentzian (lifetime)-broadened lineshape, while inhomogeneous effects lead to Gaussian lineshapes. Experimentally, Sandia observed Gaussian PL lineshapes for most samples, ranging from nearly free exciton (undoped) systems to doped structures exhibiting band-to-band transitions at high magnetic fields. The latter result is surprising because it is generally believed that electron screening in doped systems will smooth out the effects of varying internal electric fields arising from $Q W$ fluctuations. Because of the well-studied material properties of the alloy system aluminum gallium arsenide (AlGaAs), we are investigating this system to test our current understanding of exciton alloy broadening mechanisms.

We studied the linewidth broadening mechanisms in AlGaAs alloys. The initial experiments concentrated on double heterostructures of AlAs/AlGaAs (barrier/alloy) with different $\mathrm{Al}$ concentrations, varying between $5 \%$ and $30 \% \mathrm{Al}$. We found that existing theories for the lineshape and linewidth do not correctly account for the observed behavior.

To study the differences between theoretical predictions and experimental observation, we made magnetic fielddependent linewidth studies at $1.4 \mathrm{~K}$. We used two types of magnets to study the PL lineshape: (1) a superconducting magnet operating between 0 and 13.5 tesla, and (2) a pulsed magnet capable of generating 50 tesla fields. We found that the lineshape changed from a Voigt profile to a Gaussian at high magnetic fields.

An analysis of the Voigt profile yielded enough information to extract the Gaussian contribution at all magnetic fields. Using the newly determined Gaussian linewidths for all magnetic fields, we obtained good agreement between theory and experiment.

\section{Because of the well-studied} material properties of the alloy system aluminum gallium arsenide (AlGaAs), we are investigating this system to test our current understanding of exciton alloy broadening mechanisms. 
Refereed

Jones, E. D., A. A. Allerman, S. R. Kurtz, N. A.

Modine, K. K. Bajaj, S. W. Tozer, and X. Wei. 2000.

"Photoluminescence-Linewidth-Derived Reduced

Exciton Mass for InGaAsN Alloys." Phys. Rev. 62

(September): 7144-7149. 
10406

Direct Fabrication of Multifunctional Nanocomposites Via Supramolecular SelfAssembly

\section{J. Brinker}

The ability to efficiently organize molecular components at the nanometer scale will greatly influence the future of advanced materials in applications for electronics, catalysis, photonics, and sensors and their integration into microsystems. This stems from the fact that interplay of structure, organization, and dynamics at the molecular level is vital in determining a functional response. Based on this premise, Sandia successfully developed an efficient, continuous process to form ordered nanocomposite thin films and particles via evaporation-induced self-assembly (EISA). Thin films are prepared by spin- or dip-coating a homogeneous sol containing alcohol, silica precursors, organic monomers, initiators, and surfactant (prepared with an initial surfactant concentration [co] below the critical micelle concentration [cmc]). In a process not so unlike washing dishes, we use micelle formation to spatially separate and organize organic precursors (sequestered within the hydrophobic micellar interiors) and inorganic precursors (organized around the hydrophilic micellar exteriors). Further self-organization of micelles into periodic hexagonal, cubic, or lamellar mesophases simultaneously positions both the organic and inorganic precursors into precise three-dimensional (3-D) arrangements. Combined organic/inorganic polymerization locks in the nanocomposite architecture and covalently bonds the organic/inorganic interface. The beauty of this approach is its simplicity and efficiency - if we seek to prepare laminated organic/inorganic composites, many hundreds of alternating organic/inorganic layers can be assembled in seconds in a single step. We can extend the process to particles with ordered (vesicular) structures via aerosol processing of related sols.

This project enabled patterning of the mesostructure within a thin film. Our procedure uses evaporation-induced self-assembly to prepare a photosensitive thin-film mesophase containing a PAG (a diaryliodonium salt). We then exploited the $\mathrm{pH}$-sensitivity of both the siloxane condensation rate and the silica-surfactant self-assembly process to define optically film location, mesostructure, and properties. The procedure 
begins with a homogeneous solution of silica, surfactant, PAG, and hydrochloric acid $(\mathrm{HCl})$ with initial acid concentration designed to minimize the siloxane condensation rate. Preferential ethanol evaporation during dip- or spin-coating concentrates the depositing solution in water and nonvolatile constituents, thereby promoting self-assembly into a photosensitive, one-dimensional hexagonal (1-dH) silica/ surfactant mesophase. Because it bears a long-chain hydrocarbon, the PAG serves as a co-surfactant during the assembly process, which promotes its uniform incorporation within the mesostructured channels of the 1-dH film.

Irradiation of the PAG at $1_{\max }=256 \mathrm{~nm}$ results in homolytic or heterolytic photodecomposition to yield the Brønsted super-acid, H+SbF6-, plus an iodoaromatic compound and organic by-products. Thus, ultraviolet (UV) exposure of the photosensitive mesophase through a mask creates patterned regions of differing acid concentrations compartmentalized within the silica mesophase.

Suppression of the siloxane condensation rate during film deposition enables several modes of optically mediated patterning. Because acid generation promotes siloxane condensation, selective UV exposure results in patterned regions of more and less highly condensed silica. Differential extents of siloxane condensation result in differential solubility, allowing selective etching of more weakly condensed regions in aqueous base. This process could potentially eliminate the need for a photoresist in the patterning of mesoporous low dielectric constant films needed by the microelectronics industry.

Organic by-products of the (patterned) PAG photodecomposition process render UV-exposed regions of the film hydrophobic (contact angle $=40^{\circ}$ ) relative to adjoining unexposed regions (contact angle $<10^{\circ}$ ). This differential wetting behavior can be used to selectively derivatize hydrophilic regions with aqueous-based reagents and/or biomolecules.

A second aspect of our approach is the optical definition of film mesostructure and associated structurerelated properties like pore size, pore connectivity, and refractive index. Depending principally on the type and concentration of surfactant, we realize two contrasting types of behavior. If we heat the patterned film, we obtain adjoining regions of differing refractive index where the sign of the refractive index contrast may be either positive (n[irradiated] $>\mathrm{n}$ [unirradiated]) or negative. Both types of behavior result
This process could potentially eliminate the need for $a$ photoresist in the patterning of mesoporous low dielectric constant films needed by the microelectronics industry. 
from a greater extent of siloxane condensation in the UVexposed regions. We used a cantilever beam technique to measure the development of condensation-induced, thin-film biaxial tensile stress and found that the differing extent of siloxane condensation was UV-dose dependent. Therefore, by spatially varying the UV exposure using grayscale lithography, we can pattern continuous variations in the film refractive index and thickness, as well as the pore size, surface area, and pore volume. This is extremely important for applications in membranes, sensors, and separations.

We used our ability to pattern refractive index to create optical diffraction gratings. Exposure of a photosensitive film with an excimer laser $(1=248 \mathrm{~nm})$ through a phase mask resulted in a $543 \mathrm{~nm}$ grating structure with delta $\mathrm{n} \sim 0.025$ after calcination to remove the surfactant templates. The measured Littrow angle of $34^{\circ}$ is consistent with the mask period of 564 nm.

Using a higher initial surfactant concentration, near that required for the 1-dH to cubic transformation without added acid, we observed a negative refractive index contrast that results from a hexagonal-to-tetragonal mesophase transformation. We obtained evidence for the optically defined phase transformation by $\mathrm{x}$-ray diffraction (XRD) and transmission electron microscopy (TEM).

\section{Refereed}

Doshi, D. A., C. J. Brinker, et al. 2000. "Optically

Defined Multifunctional Patterning of Photosensitive Thin-Film Mesophases." Science 290 (6 October): 107-111. ...by spatially varying the $U V$ exposure using grayscale lithography, we can pattern continuous variations in the film refractive index and thickness, as well as the pore size, surface area, and pore volume. This is extremely important for applications in membranes, sensors, and separations. 
10407

\section{Information Extraction from Hyperspectral} Images Obtained from Satellites

\section{M. Haaland, C. M. Wehlburg}

Sandia completed a comparison of several new multivariate algorithms that have significant potential for hyperspectral image (HSI) analysis from satellites. In addition, we applied a variety of multivariate curve resolution methods to HSI data of a desert scene with military targets collected using the HYDICE (Hyperspectral Digital Imagery Collection Experiment) airborne imaging system. This project will apply a variety of newly developed multivariate analysis tools to the analysis of multispectral image and HSI data generated from imaging spectrometer systems to be flown on satellites for target recognition applications. Sandia's newest proprietary concentration-residual-augmented classical least-squares (CRACLS) and spectral-residual-augmented classical leastsquares (SRACLS) algorithms have the potential to more efficiently extract quantitative and target recognition information from multispectral images and HSIs. Another method (score-augmented classical least-squares, or SACLS) that we recently conceived involves the use of scores calculated from the spectral residuals of the CLS model to augment the CLS calibration. In combination, SRACLS and SACLS may be able to iterate better models for spectral unmixing of pixels in the HSIs. These new information-extraction methods can be tested with spectral data from either Sandia's InformationEfficient Spectral Imaging Sensor (ISIS) system or the Multispectral Thermal Imager (MTI) system. In addition, these newest algorithms should reduce the detrimental effects of atmospheric variations in viewing angle, elevation, and atmospheric interferences such as water vapor and carbon dioxide. We will test these potential benefits of the new algorithms with HSIs with unmodeled atmospheric variations present in the data.

We performed multiple simulations on spectral data to compare the performance of our proprietary hybrid algorithm with our newest algorithms, called CRACLS, SRACLS, and a CLS variant where orthogonal concentrations are added to the CLS calibration to fit unmodeled sources of spectral variation. The simulations used three-component systems of highly overlapped pure-component spectra and included the addition of noise to reference concentrations and calibration and
This project will apply a variety of newly developed multivariate analysis tools to the analysis of multispectral image and HSI data generated from imaging spectrometer systems to be flown on satellites for target recognition applications.

Sandia's newest proprietary concentration-residualaugmented classical leastsquares (CRACLS) and spectralresidual-augmented classical least-squares (SRACLS) algorithms have the potential to more efficiently extract quantitative and target recognition information from multispectral images and HSIs. 
prediction spectra. The results demonstrate that CRACLS and SRACLS equal the performance of the hybrid when the prediction spectra are consistent with the calibration spectra. The CLS variant provides a less efficient model, but can be used with existing commercial CLS software. When prediction spectra are generated with spectral variation not contained in the calibration spectra, then the CRACLS and SRACLS algorithms are found to yield improved predictions relative to partial least squares (PLS) and the hybrid when updated during the prediction phase of the algorithm. Since prediction updating is fast, the CRACLS and SRACLS algorithms represent dramatic improvements over the traditional CLS and PLS algorithms and our proprietary hybrid algorithm. We expect these new algorithms to have important applications in HSI analysis, and we anticipate significant commercial interest in these new algorithms.

We also applied a variety of multivariate curve resolution methods to HSI data of a desert scene with military targets collected using the HYDICE airborne imaging system. We applied a self-modeling mixture analysis method called Simplisma and chemometric alternating least-squares methods Since prediction updating is fast, the CRACLS and SRACLS algorithms represent dramatic improvements over the traditional CLS and PLS algorithms and our proprietary hybrid algorithm. We expect these new algorithms to have important applications in HSI analysis, and we anticipate significant commercial interest in these new algorithms. to the analysis of the HSI. The initial results identified calibration errors in some of the pixels in the linear array detector that generate the push-broom image.

\section{Refereed}

Haaland, D. M. 2000. "New Augmented Classical Least Squares Methods for Improved Quantitative Spectral Analyses." (Invited) Paper to be presented to the 1st International Conference on Advanced Vibrational Spectroscopy, Turku, Finland, 19-24 August 2001. 
10408

\section{Advanced Radiation Sources: Rayleigh-Taylor Mitigation Via Perturbation Reduction}

M. R. Douglas

Sandia proposes to increase the overall efficiency of zpinch plasma radiation sources by reducing the effect of the dominant magnetohydrodynamic instability, the RayleighTaylor (RT) instability. In particular, we plan to investigate the source and magnitude of the density perturbations in the initial plasma sheath both experimentally and computationally. These perturbations seed the RT instability. Calculations suggest that modest reductions in the level of the initial perturbations $12.5 \%$ to $1.25 \%$ ) could increase the radiated $x$-ray power from z-pinches by two times or equivalently allow the present level of performance from longer implosion times, thereby reducing pulsed-power driver requirements. In collaboration with the University of Nevada-Reno (UNR), we will explore the evolution of z-pinch plasmas.

Sandia is developing the computational capability to model the initial formation of z-pinch plasmas. This has involved state-of-the-art research in the fields of plasma resistivity and plasma equation-of-state (EOS). We are incorporating these new data into Sandia codes and will use them to model Sandia and UNR data.

Precise power flow measurements are on hand, using calibrated state-of-the-art current and voltage diagnostics and detailed SCREAMER computer modeling. We took up to seven z-pinch shots in one day (9 A.M.-5 P.M.). We made significant progress in organizing all Zebra z-pinch experimental data in a user-friendly database, to facilitate detailed comparisons between theory and experiment.

We investigated z-pinch physics. Two experiments are yielding new data on wires and wire arrays driven by current prepulses similar to those at $\mathrm{Z}$. Analytic and numerical studies are complementing these experiments. We made significant progress in computer-modeling z-pinch plasmas with the Los Alamos National Laboratory (LANL) computer code MHRDR (magnetohydroradiative dynamics research). An experiment on single wires driven by current prepulses similar to, and faster than, those at $\mathrm{Z}$ is finding that the characteristics of wire explosions depend dramatically on the rate of rise of the current, on the heat of vaporization of the wire material, and on the diameter of the wire. For substances with high vaporization
Sandia is developing the computational capability to model the initial formation of $z$ pinch plasmas. This has involved state-of-the-art research in the fields of plasma resistivity and plasma equationof-state (EOS). 
energy (W [tungsten], Mo [molybdenum], Ti [titanium], Ni [nickel], Fe [iron], Au [gold]-plated W), faster-rising pulses are much more effective at exploding single wires than $\mathrm{z}$-like pulses. Faster-rising pulses may lead to a plasma less divided into cold core and unstable corona than has typically been produced. We are comparing the measurements with MHRDR simulations at UNR. Improved experiments with full Sandia single- and nested-wire-arrays are being developed for Zebra. We made an initial measurement of the division of current between the inner and outer wire arrays. We initiated a detailed analytical and numerical study of z-pinch linear instability. The calculations aim to understand the growth of fluctuations observed in experiment and modeling. X-pinches are being driven at $900 \mathrm{kA}$ and investigated with novel diagnostics.

We developed a variety of optical, extreme ultraviolet (EUV), x-ray, and laser diagnostics and are developing numerous more. We built and are testing a two-dimensional (2-D) X-ray imaging spectrometer. X-ray diagnostics on Zebra include a five-channel tunable $\mathrm{x}$-ray spectrometer ("Polychromator") with nanosecond time resolution, two 1-D $\mathrm{x}$-ray imaging polarimeters/spectrometers, two universal survey convex crystal spectrometers/pinhole cameras, and a multihole $x$-ray pinhole camera. We are developing x-ray diagnostics for $Z$ (Sandia), including a multichannel 1-9 keV $\mathrm{x}$-ray spectrometer to characterize the x-ray flux being used to certify stockpile components. We developed time-resolved laser absorption imaging and interferometry and are using it to study the expansion of Z (Sandia) wires exploded by zprepulse-like currents. Laser polarimetry, collective Thomson scattering, and induced fluorescence diagnostics are under development.

We refined and are refining atomic and radiation physics calculations and data to meet the challenges of plasma EUV and x-ray diagnostic development. We will calculate the polarization of x-ray spectra and compare it with experiment, with the aim of determining the magnetic field and the energetic non-Maxwellian component of the electron distribution function. We are improving K-shell diagnostics of Ti plasmas.
We developed a variety of optical, extreme ultraviolet (EUV), $x$-ray, and laser diagnostics and are developing numerous more. We built and are testing a 2-D x-ray imaging spectrometer. 


\section{Refereed}

Amiranoff, F., et al. 2000. "Experimental Evidence of the Effect of Heat Flux on Thomson Scattering Off Ion Acoustic Waves." Phys. Rev. E 61: 1949-1953.

Safronova, U. I., et al. 1999. "Inner-Shell Excitation Energies and Autoionization Rates for C-, N-, O-, and F-Like Ions with Z=6-54." Physica Scripta 60: 36-45.

Shlyaptseva, A. S., et al. 1999. "Polarization Properties of Dielectronic Satellite Lines in the K-Shell X-Ray Spectra of B-Like FeXXII." J. Phys. B 32: 1041-1051. 
10409

\section{Capillary Elastohydrodynamics in Manufacturing Processes}

\section{P. R. Schunk}

Many practical problems involve wetting of a fluid onto a deformable substrate. Numerical techniques for solving wetting problems on rigid solids are well established. However, these techniques are not readily applied to wetting on a deformable substrate. A goal of this project is to develop a new finite-element method (FEM) to predict the interfacial deformation of solids near static and dynamic wetting lines. Static wetting is a balance of elastic deformation in the solid and wetting-line forces with no fluid flow. The boundary conditions $(B C s)$ at the wetting line can be applied in several ways by replacing different components of the elastic momentum equation with a contact-angle constraint or a lineforce condition. The results show that Young's equation is not valid for wetting on deformable solids because the angles between the interfaces at the wetting line are functions of the elastic modulus. This discrepancy is due to a singularity in the elastic stresses at the contact line. Dynamic wetting is a balance between fluid flow, elastic deformation, and wetting forces. To predict dynamic wetting on a deformable substrate while accounting for the local deformation of the contact line requires a reformulation of the standard methods for solving solid mechanics problems.

In static wetting problems, we tested several methods for resolving the surface tension forces exerted at the contact line on an elastic substrate. At the contact line, three line forces are exerted due to the surface-free energies of the liquid-gas, liquid-solid, and solid-gas interfaces. The surface forces cause a crease, or kink, in the solid at the contact line. The crease angle can be applied as a BC on the elastic deformation. Alternatively, the line force associated with the liquid-vapor interface can be applied directly to the solid at the contact line, taking advantage of the weak formulation of the FEM. The predicted deformations using the two BCs (imposed crease angle and line force) differ by several orders of magnitude when the crease angle is calculated from Young's equation and the surface-free energies. Hence, Young's equation is not valid for wetting on deformable solids because the angles between the interfaces at the wetting line depend on the elastic deformation and the elastic modulus. This discrepancy is due to
A goal of this project is to develop a new finite-element method (FEM) to predict the interfacial deformation of solids near static and dynamic wetting lines. Static wetting is a balance of elastic deformation in the solid and wetting-line forces with no fluid flow. 
a singularity in the elastic stresses at the contact line. In dynamic wetting, the singularity in the fluid stress near the contact line will also influence the force balance at the contact line.

We developed an FE formulation for dynamic wetting on flexible substrates, which solves for conservation of momentum in both the fluid and solid phases. In both phases, we chose to use an arbitrary-Lagrangian-Eulerian (ALE) mesh motion scheme in which the motion of the mesh is not linked to the motion of the solid or liquid phases, except at the boundaries. The advantage of the ALE formulation for this problem is that the dynamic contact line can move across the solid surface without having the mesh elements in the liquid slide past the mesh elements in the solid.

The test problem for the new dynamic wetting formulation is the upstream gap of a slot coater with a deformable substrate. This test problem is one of the simplest configurations of steady-state dynamic wetting on flexible substrates. The deformable solid is a planar substrate that continually passes under the slot die. The liquid domain is a recirculation zone between the die and the substrate. The vapor-liquid free surface is pinned to the upstream corner of the die and can move freely on the substrate to balance fluid mechanical, elastic, and surface tension forces. At the downstream end of the domain, the fluid is assumed to be in fully developed flow.

The primary challenge of designing an FEM for dynamic wetting on flexible substrates is formulation of BCs along the fluid-solid interface and at the dynamic wetting line. Along the fluid-solid interface, the velocity of the fluid is equal to the velocity of the solid (no slip and no penetration), and the tractions in the fluid and solid phases must satisfy an interfacial momentum balance. The mesh along the fluid-solid interface is constrained to have the same vertical displacement as the solid, but can slide freely in the tangential direction; however, the node associated with the dynamic contact line is also constrained to move with the liquid-vapor interface. At the dynamic contact line, there are several potential formulations for the BCs (as in the static wetting case) that we will test for robustness, numerical stability, and accuracy. The most likely set of BCs at the dynamic contact line is imposed contact angle and crease angle, zero fluid velocity at the contact node, and Navier slip between the fluid and solid for a short distance near the contact line.
The advantage of the ALE formulation for this problem is that the dynamic contact line can move across the solid surface without having the mesh elements in the liquid slide past the mesh elements in the solid. 


\section{Refereed}

Baer, T. A., R. A. Cairncross, P. R. Schunk, R. R. Rao, and P. A. Sackinger. 2000. "A Finite Element Method for Free-Surface Flows of Incompressible Fluids in Three Dimensions, Part II: Dynamic Wetting Lines." Internat. J. Numer. Meth. in Fluids 33: 405-427.

Cairncross, R. A., P. R. Schunk, T. A. Baer, R. R. Rao, and P. A. Sackinger. 2000. "A Finite Element Method for Free-Surface Flows of Incompressible Fluids in Three Dimensions, Part I: BoundaryFitted Mesh Motion." Internat. J. Numer. Meth. in Fluids 33: 375403.

Madasu, S., and R. A. Cairncross. 2000. "A Finite Element Procedure for Modeling Wetting on Flexible Substrates." Internat. Soc. for Coating Science \& Technol. Symp. 1 (Scottsdale, AZ, 25 September): 1 . 
10410

\section{Real-Time Design of Improved Powder- Pressing Dies Using Finite-Element Method Modeling}

K. G. Ewsuk, A. F. Fossum, J. G. Arguello, Jr., D. H. Zeuch

Sandia is developing scientific and technological expertise in characterizing and modeling powder compaction and is compiling it into a user-friendly software package that incorporates unprecedented flexibility to design powder-press tooling and pressing processes faster and more economically. This advanced computational technology, in a unique, userfriendly, software package, will provide considerable advantages in manufacturing pressed-powder compacts by mechanical and isostatic compaction, including powder compacts made of advanced ceramics and metals. The technology will contribute significantly to advanced materials and design by (1) reducing manufacturing costs, cycle time, and waste, (2) enabling more cost-effective manufacturing of specialty components and small-lot sizes, (3) reducing tooling and component design, development, and prototype time, and (4) maximizing design and manufacturing flexibility and agility. A successful project will significantly impact ongoing Sandia Defense Program (DP) efforts to design and manufacture advanced ceramic components with improved performance and reliability for weapons systems, including cermets and PZT (lead zirconate titanate) voltage bars for neutron generators (NGs).

We devised a unique concept to design a variable dimension and parameter template to build and design dies on a computer in real time and to complete compaction simulations with granulated ceramic powders in a time-effective manner. We validated this concept and developed a user-friendly interface (i.e., Unipack) that was integrated into Sandia's SEACAS engineering software to simulate powder compaction. We successfully ported the compaction software to an IBM ThinkPad (laptop) computer, which significantly enhanced our ability to demonstrate and test the software in a manufacturing environment. We identified affordable desktop computers with Pentium II or III chips that, using the Linux operating system, allow simulations to be completed as fast as those on a SUN workstation. Three-dimensional (3-D) compaction simulations with up to 50,000 elements can be run on the PC platform. A standard 30,000-element simulation
Sandia is developing scientific and technological expertise in characterizing and modeling powder compaction and is compiling it into a user-friendly software package that incorporates unprecedented flexibility to design powderpress tooling and pressing processes faster and more economically....A successful project will significantly impact ongoing Sandia Defense Program (DP) efforts to design and manufacture advanced ceramic components with improved performance and reliability for weapons systems, including cermets and PZT (lead zirconate titanate) voltage bars for neutron generators (NGs). 
takes about 30 minutes of central processing unit (CPU) time using a $450 \mathrm{MHz}$ Pentium III chip.

Five ceramic component manufacturers invested in computers to test and evaluate the compaction modeling software. Using the Linux operating system and the newly developed interface software, the model was successfully applied to simulate the compaction of 3-D ceramic compacts. We tested, refined, and validated the compaction model by comparing model predictions of density gradients after pressing to density gradients measured in actual ceramic powder compacts. For experimental validation, we evaluated four different geometry compacts, including a right circular cylinder, a tube, a bushing, and a commercial ceramic nozzle insert. In all cases, the model predictions showed excellent qualitative and quantitative agreement with experimental results. Quantitatively for a simple shape, model and experiment agree within $1 \%$ relative density. For a complex shape, model and experiment agree within $3 \%$ relative density. We refined the model to enhance its usability and applicability and filed a technical advance for the Unipack software and the concept behind the software at Sandia. We successfully used the compaction model to enhance fundamental understanding of ceramic powder processing; it provided guidance to achieve better process reproducibility, reliability, and control. Additionally, we identified some of the critical powder properties and characteristics required for reproducible and reliable press powder manufacturing.

To obtain the materials parameters required as input into the finite-element (FE) compaction model, we characterized ceramic powders and powder compacts using hydrostatic and triaxial compression tests adapted from the soil mechanics field. These tests provided the data necessary to define the shear failure and hardening cap surfaces that make up the cap-plasticity constitutive model used within the FE framework. Additionally, these same tests allowed us to measure or calculate the remaining bulk properties (i.e., powder compact bulk modulus, shear modulus, and Poisson's ratio) required to model ceramic powder compaction. Initially, we characterized a nonproprietary spray-dried $94 \mathrm{wt} . \%$ alumina powder containing $3 \mathrm{wt}$.\% of a cellulose binder and developed techniques to reduce the data generated to obtain the material parameters required as input for the FE compaction model. We completed most of the compaction simulations using the $94 \%$ alumina powder properties, including the validation tests. We characterized seven additional proprietary ceramic powders, as
Using the Linux operating system and the newly developed interface software, the model was successfully applied to simulate the compaction of 3-D ceramic compacts. We tested, refined, and validated the compaction model by comparing model predictions of density gradients after pressing to density gradients measured in actual ceramic powder compacts. 
well, including four alumina powders and three zirconia powders. In total, we obtained the materials parameters required for FE compaction modeling for three granular materials - the 94\% alumina body, a second alumina body, and a zirconia body. We incorporated these data into the Unipack software to afford comparisons of compaction simulations with powders that have different degrees of pressability.

\section{Refereed}

Zeuch, D. H., J. M. Grazier, J. G. Arguello, and K. G.

Ewsuk. 2000. "Mechanical Properties and Shear

Failure Surfaces of Two Alumina Powders in Triaxial

Compression." J. Mater. Sci., submitted.

\section{Other Communications}

Arguello, J. G., A. Fossum, D. Zeuch, and K. Ewsuk. 2000. "Continuum-Based FEM Modeling of Ceramic Powder Compaction Using a Cap-Plasticity Constitutive Model." KONA Powder and Particle, accepted.

Ewsuk, K. G. 2000. "Powder Granulation and Compaction." Encyclopedia of Mater.: Sci. and Technol., accepted.

Ewsuk, K. G., J. G. Arguello, D. H. Zeuch, L. Carinci, B. Farber, J. Kaniuk, C. Cloutier, B. Gold, R. B. Cass, J. D. French, B. Dinger, and W. Blumenthal. 2000. "Industry Teams with National Laboratories to Develop a Predictive Model for Powder Pressing and Die Design." Bulletin of the Amer. Ceram. Soc., accepted. 
10689

\section{Calculation and Interpretation of the Energies that Underlie Transition-Metal Surface Structure}

\section{P. J. Feibelman}

Recent advances in computer power and in algorithms for first-principles, electronic structure calculation, make it possible to evaluate energy versus atomic arrangement for essentially any bonding geometry of any combination of atomic species. Sandia plans to use this power in a systematic effort to uncover basic laws of surface energetics. Using state-of-the-art electronic-structure methods, we will determine the energies and geometries of fundamental atomic configurations at transition-metal surfaces to a well-defined level of numerical convergence. The results will serve as a standard, systematic foundation for the discovery and explanation of trends in adatom and vacancy formation energetics, diffusion mechanisms, vibration spectra, molecular-fragment stability, surface stress effects, work functions, etc. They will also provide a reference by which to judge the range of accuracy of the basic physical approximations used to represent the effects of electron exchange and correlation, i.e., the local-density and generalized gradient approximations.

A systematic ab initio search encompassing the energetics of sulfur $(\mathrm{S})$-decorated, adsorbed copper $(\mathrm{Cu})$ monomers through tetramers implies that the best candidate to explain S-enhanced $\mathrm{Cu}$ transport on $\mathrm{Cu}(111)$ is the $\mathrm{Cu}$ trimer decorated with three $\mathrm{S}$ atoms. Its formation energy is $\sim 0.5 \mathrm{eV}$ lower than that of a $\mathrm{Cu}$ adatom and, corresponding to tight internal bonding, its diffusion barrier is $\leq 0.35 \mathrm{eV}$. A problem with this potential explanation is the entropic price of extracting three $\mathrm{S}$ atoms from the low-coverage adsorbed "sea" of $S$, which considerably reduces the expected population of $S$ decorated clusters. With this countervailing result in mind, quench experiments that directly reveal $\mathrm{Cu}_{3} \mathrm{~S}_{3}$ on terraces would obviously be desirable. A suggestive positive result in this direction is that scanning-tunneling microscopy (STM) of a low-T (low-temperature) "honeycomb phase" of $\mathrm{S} / \mathrm{Cu}(111)$ exhibits one surface protrusion per deposited $S$, separated by $\sim 4.25 \AA$. We find an S-S separation of $4.4 \AA$ for ad- $\mathrm{Cu}_{3} \mathrm{~S}_{3}$. If $\mathrm{S}$-decorated $\mathrm{Cu}$ clusters do prove to be the explanation of rapid $\mathrm{Cu}$ island decay on $\mathrm{Cu}(111)$, this would show that despite its divalency, S can act as a "skyhook,"
Using state-of-the-art electronic-structure methods, we will determine the energies and geometries of fundamental atomic configurations at transition-metal surfaces to a well-defined level of numerical convergence. The results will serve as a standard, systematic foundation for the discovery and explanation of trends in adatom and vacancy formation energetics, diffusion mechanisms, vibration spectra, molecular-fragment stability, surface stress effects, work functions, etc. 
weakening the bonds of a $\mathrm{Cu}$ adatom cluster to the substrate and promoting its transport. Such an effect has been proposed for hydrogen $(\mathrm{H})$ on metals $(\mathrm{M})$, through formation of $\mathrm{HM}$ dimers, but how divalent S might act as a "skyhook" has not been known until now.

\section{Refereed}

Feibelman, P. J. 2000. "Formation and Diffusion of SDecorated $\mathrm{Cu}$ Clusters on $\mathrm{Cu}(111)$." Phys. Rev. Lett. 85 (17 July): 606-609. 
10692

\section{Interfacial Reactions in Ceramic Systems}

R. E. Loehman

Transition element additions to otherwise inert braze alloys tend to promote their wetting and bonding to ceramics. Titanium (Ti) is most widely used as an added element, but zirconium ( $\mathrm{Zr}$ ) and vanadium (V) have also been considered. Sandia investigated interfacial reactions and bonding of $V$ containing braze alloys on sapphire, alumina, mullite, and fused silica and compared their behavior with that of Ti-, hafnium (Hf)-, and Zr-containing alloys on similar substrates. Gold ( $\mathrm{Au}$ )-nickel (Ni)-molybdenum (Mo )-V alloys form initially obtuse contact angles on sapphire at $1,000^{\circ} \mathrm{C}$, which decrease slowly over time at temperature. There is a rough trend of decreasing molten alloy contact angles on the ceramic substrates in the order of $96 \%$ alumina $>94 \%$ alumina $>$ fused silica. The extent of reaction zones between those substrates and the Au-Ni-Mo-V alloys increases with the silicate content of the ceramic. The sapphire substrates are roughened by contact with the metal, but any reaction layer must be considerably thinner than $1 \mu \mathrm{m}$. We also identified small, discrete $\mathrm{AlV}_{2} \mathrm{O}_{4}$ and $\mathrm{AlVO}_{3}$ particles at some sapphire interfaces by transmission electron microscopy (TEM), but our scanning electron microscopy (SEM) investigations did not find them. There is some evidence from prior work that bonding occurs preferentially to the $\mathrm{SiO}_{2}$ (silicon dioxide) grain-boundary phases in alumina. Our reaction study provides a mechanistic explanation for those bonding results.

Transition element additions to otherwise inert braze alloys tend to promote their wetting and bonding to ceramics. Ti is most widely used as an added element, but $\mathrm{Zr}$ and $\mathrm{V}$ have also been considered. We investigated interfacial reactions and bonding of $\mathrm{V}$-containing braze alloys on sapphire, alumina, mullite, and fused silica and compared their behavior with that of Ti-, Hf-, and Zr-containing alloys on similar substrates. The $\mathrm{V}$-containing alloy results are perhaps the most detailed. Specifically, the Au-Ni-Mo-V alloy (82.0 wt.\% Au, 15.5 wt.\% $\mathrm{Ni}, 0.75$ wt.\% Mo, $1.75 \mathrm{wt} \% \mathrm{~V}$ ) exhibits sluggish wetting of the substrates, with slowly diminishing contact angles over time at $1000^{\circ} \mathrm{C}$. The extent of reaction between those substrates and the Au-Ni-Mo-V alloy depends on the silica content of the substrate ceramic. Cross-sections of alloy-sapphire interfaces formed by heating 5 to 30 minutes at $1000^{\circ}-1050^{\circ} \mathrm{C}$ show that
Sandia investigated interfacial reactions and bonding of $V$ containing braze alloys on sapphire, alumina, mullite, and fused silica and compared their behavior with that of Ti-, hafnium (Hf)-, and $\mathrm{Zr}$ containing alloys on similar substrates. Gold (Au)-nickel (Ni)-molybdenum (Mo)- $V$ alloys form initially obtuse contact angles on sapphire at $1,000^{\circ} \mathrm{C}$, which decrease slowly over time at temperature. 
the originally smooth sapphire surface is roughened by contact with the molten metal, presumably because of slight solubility of the ceramic in the molten metal. However, there is no evidence of reaction products with sapphire under SEM examination, and any product layer must be thinner than $1 \mu \mathrm{m}$. SEM/EDS (energy dispersive spectroscopy) analysis shows some micrometer-scale segregation of $\mathrm{V}$ to those same interfaces. Reaction interfaces between the $\mathrm{V}$-containing alloy and $96 \%$ alumina (balance a silicate glass) show more extensive reaction than with sapphire, with 5-10 $\mu \mathrm{m}$ particles at the interface that contain $\mathrm{Al}, \mathrm{V}$, and oxygen $(\mathrm{O})$. The alloy reacts most extensively with fused-silica substrates and forms a reaction zone at the interface that is a very fine-grained composite of $\mathrm{V}, \mathrm{Si}, \mathrm{O}$, and the base $\mathrm{Au}-\mathrm{Ni}$ alloy. The fine-scale features of the reaction zone have a microstructure that resembles a cermet. Work by others obtained some evidence that bonding between the Au-Ni-Mo-V alloy and different alumina ceramics occurs preferentially to the $\mathrm{SiO}_{2}$ grainboundary phases in the alumina. The present work shows that bonding is likely controlled by reactivity between the $\mathrm{V}$ in the braze alloy and silicate phases in the alumina. The higher the silicate content, the greater the reaction. This result suggests that the $\mathrm{V}$-containing braze alloys will be more suitable for joining $96 \%$ and $94 \%$ aluminas.
The present work shows that bonding is likely controlled by reactivity between the $V$ in the braze alloy and silicate phases in the alumina. The higher the silicate content, the greater the reaction. This result suggests that the V-containing braze alloys will be more suitable for joining $96 \%$ and $94 \%$ aluminas. 
10695

\section{Poco Switch Tubes}

G. E. Boettcher

Sandia explored various poco sprytron design variations for feasibility in providing improved reliability, performance, and ease of fabrication in new applications. Poco sprytrons are radiation-hard triggerable vacuum switches used for current amplification and pulse-shaping operations in nuclear weapons. Typical applications include main switch and trigger-signal pulse-shaping in weapon firing sets, neutron generator $(N G)$ electronic timing applications, and Department of Defense (DoD) applications, i.e., flight-test destruct firing sets, missile stage separation, and impact fuze amplification circuits.

We showed that graphite electrodes are feasible for poco sprytrons operated from $100 \mathrm{~V}$ dc to $2500 \mathrm{~V}$ dc and offer significant advantages over solid-metal electrodes. For example, they are easy to machine, various shapes and sizes are more readily obtained, and the increased resistance of the carbon erosion products provides longer tube shot life. Using graphite electrodes also allowed us to reduce the number of parts in a tube, effect improved miniaturization, and process yields. By totally eliminating any metal in active anodecathode discharge area, we have no evaporated metal deposits on the tube's side walls, which tend to produce anode-cathode leakage currents and in some cases partial breakdown gaps, a source of tube temporary hold-off voltage failures. We previously showed that in cathodes with both niobium $(\mathrm{Nb})$ and graphite inserts, some of the carbon evaporated deposits as diamondlike carbon. Graphite electrodes lower the tube's short circuit peak current by approximately $8 \%-10 \%$, due to the higher resistivity of graphite. This does not appear to be a significant disadvantage when designing very small optically triggered tubes.

Further experimentation on the best material for our pressed trigger wires in probes showed that $\mathrm{Nb}$ is superior to nickel ( $\mathrm{Ni}$ ). The $\mathrm{Nb}$ does not harden as readily as $\mathrm{Ni}$, and therefore forms more easily into a probe and makes improved contact with chemical vapor-deposited carbon (CVD) on the probe. Both visually and electrically we found $\mathrm{Nb}$ superior to Ni-pressed wires.
Using graphite electrodes also allowed us to reduce the number of parts in a tube, effect improved miniaturization, and process yields. By totally eliminating any metal in active anode-cathode discharge area, we have no evaporated metal deposits on the tube's side walls, which tend to produce anodecathode leakage currents and in some cases partial breakdown gaps, a source of tube temporary hold-off voltage failures. 
10729

\section{Ultraminiaturization of RF Circuitry}

G. R. Sloan, L. R. Sloan, C. E. Sandoval, C. W. Ottesen, R. T.

Knudson, K. W. Plummer

Many synthetic aperture radar (SAR) and radarresponsive tag applications now require microwave electronic miniaturization that is well beyond Sandia's current state of the art. However, recent advances that Lockheed Martin (LM) has made in high-density interconnect (HDI) technology, in conjunction with Sandia's research in microwave circuit efficiency and RF MEMS (radio-frequency microelectromechanical systems), position us for another breakthrough. This project proposes to develop a revolutionary microwave capability by adapting HDI to advanced Sandia technology and applying the result to SAR, tag, and fuze applications.

Potential new applications of this advancement are legion. Sandia is developing several tags that must be reduced in size to be truly fieldable. This project makes the needed level of miniaturization possible. The project also facilitates the development of an affordable active phased array (APA) for miniaturized radar applications.

We prepared state-of-the-art software and developed a microwave, full-wave, three-dimensional (3-D) modeling capability and applied it to HDI geometries/structures.

We designed and modeled novel HDI structures, including an innovative broadside coupler, a Ku-band filter, and a stripline hybrid coupler. We designed HDI groundsignal-ground and coplanar launches as well as stripline, buried microstrip, and coplanar test structures. We also designed a mixed Ku/X-band transmit/receive chain, consisting of commercial die: amplifiers, switches, and a 5-bit phase shifter. We laid out this integrated test structure on a four-layer HDI stackup using 1 and 2 mil Kapton. We submitted the drawings for fabrication, and they passed the LM design qualification process.

We obtained a selection of state-of-the-art indium phosphate (InP) high-electron mobility transistors (HEMTs). We tested these devices (power/noise load pull, etc.) to verify their amenability to novel, ultra-efficient power amplifier design; a prototype is partially complete. We completed a preliminary study of the feasibility of HDI packaging of Sandia microwave/RF MEMS devices. Since LM HDI is a "chips- 
first" technology, this offers a special challenge for MEMStype devices. The initial results look promising, and we plan a test layout. We also began developing an internal HDI-type capability.

We are designing a modified prototype module that will combine most of the original prototype circuitry as well as new circuitry that benefits other projects (microtransmitter tags and RF MEMs). This approach will maximize the utility of the first processing run without increasing cost.
We are designing a modified prototype module that will combine most of the original prototype circuitry as well as new circuitry that benefits other projects (microtransmitter tags and RF MEMs). This approach will maximize the utility of the first processing run without increasing cost. 
10731

\section{A Stronglink Prime Mover Based on an Electrokinetic Pump}

A. D. Oliver, P. H. Paul, C. W. Vanecek, A. M. Morales, E. F. Hasselbrink

This project seeks to enable miniature stronglinks through the integration of advanced actuator technology into both macro- and microscale devices. Ongoing efforts at Sandia to miniaturize conventional systems have created a requirement to develop a small-format mechanical driver capable of producing approximately one Newton (N) of force. We plan to meet this need by using a new type of drive based on a unique Sandia patent-pending technology, the electrokinetic pump (EKP). The EKP is a microfluidic device that converts electrical power directly to high-pressure fluid hydraulic power. In the laboratory we have demonstrated EKP-generated pressures to over 9,000 psi at a performance exceeding 10 psi per volt applied. We established the physical basis for how this process works and how it depends on the media and validated the theory experimentally.

A 2 mm-long EKP operating at 25 volts will provide $250 \mathrm{psi}$; therefore, if a $1 \mathrm{~mm}$ bellows is used as an actuator, the output will be $1.3 \mathrm{~N}$ of force. We can simply obtain much higher forces by increasing the bellows diameter or the voltage (e.g., at 100 volts, the $1 \mathrm{~mm}$-diameter bellows will yield $5.4 \mathrm{~N}$, or over 1.2 pounds force). In this application the EKP offers another unique advantage: Since the force is hydraulic, the EKP can be remotely located from the actuator. We can use a small-diameter, nonconducting fused-silica capillary to carry the hydraulic power over tens of centimeters to transmit the mechanical force to a bellows actuator.

We will use an EKP to drive a miniature stronglink. A 2 mm-long EKP operating at 10 volts and connected to a 100 mm-diameter actuator will provide $10 \mathrm{mN}$ of force to the device. The actuator will convert hydraulic force to mechanical movement by means of a bellows at the end of the capillary.

The bulk of our effort focused on defining the technical approach for demonstrating the EKP technology for specific applications. This device is a candidate for application in future weapons systems. This design approach consists of two key elements required to advance the countermeshing gear discriminator (CMG): the EKP assembly and a linkage
Ongoing efforts at Sandia to miniaturize conventional systems have created a requirement to develop a smallformat mechanical driver capable of producing approximately one Newton $(N)$ of force. We plan to meet this need by using a new type of drive based on a unique Sandia patent-pending technology, the electrokinetic pump (EKP). 
assembly that converts linear motion to rotary motion for gear rotation.

We constructed a first-generation mesoscale highflowrate pump and assessed its performance. This $1 \mathrm{~cm}$ diameter, $3 \mathrm{~mm}$-thick polymer EKP, fixed between stainlesssteel electrodes, operates at $30 \mathrm{~V}$. We achieved steady-state flowrates (at near-zero back pressure) of approximately 0.15 $\mathrm{mL} / \mathrm{min}$ with this pump, using acetonitrile as the working fluid. We completed a design of a scaled-up version of this pump. Four constraints determine the pump design: the applied force of $1 \mathrm{~N}$, the stroke length of $1 \mathrm{~mm}$, an actuation cycle time of 60

We constructed a firstgeneration mesoscale highflowrate pump and assessed its performance. msec, and a maximum operating voltage of $100 \mathrm{~V}$. The first three determine the power output of the pump (33 $\mathrm{mW})$, which, due to the relatively low efficiency of EKPs (about 1\%), places minimum bounds on the size of the pump to prevent overheating. The resulting design is a pump whose active medium is a disk approximately $4 \mathrm{~cm}$ in diameter and $3 \mathrm{~mm}$ thick, which is clearly too large to be integrated within the stronglink housing, necessitating external hydraulic actuation.

We designed the pump to be remotely coupled to the load via a stainless-steel tube. This tube is brazed to a commercial $3 \mathrm{~mm}$ bellows assembly that is located inside the load housing. This bellows operates a mechanically leveraged linkage that advances the gears. We plan to demonstrate motion of the CMG with an EKP. 
10739

\section{Laser-Assisted Microgas Metal Arc Welding}

P. W. Fuerschbach, C. E. Heath, R. A. Anderson, L. A. Bertram

Laser beam welding is the principal welding process for the joining of nuclear weapons $(N W)$ components because it can provide a small fusion zone with low overall heating. Improved process robustness is needed since laser-energy absorption is extremely sensitive to variations in surface condition and dimensional tolerances. The current widespread use of robotic welding in U.S. manufacturing demonstrates the reliability of gas metal arc welding (GMAW) for assembly of more loosely toleranced components. Despite the robustness and metallurgical advantages of a filler metal process, GMAW has not been suitable for Sandia components because the scale of the resulting welds is too large.

Sandia has been a leading participant in a multi-DOE laboratory Office of Industrial Technology project that has found it advantageous to combine laser and autogenous arc welding heat sources. We intend to leverage our newly discovered knowledge of the interactions between laser beams and arcs into developing a hybrid filler metal-based welding process. We will advance a wholly new welding process by combining a fiber-optic-delivered Nd:YAG laser with a miniaturized GMAW system. The laser-assisted micro-GMAW process will couple the process advantages of these two unique heat sources and will also enable process capabilities never before obtained with arcs and only alluded to with lasers. Problems with smooth melt-in of the miniature wire in microGMAW will be overcome with the assistance of a fiberdelivered laser beam. Additional workpiece heating and fusion zone dimensions can be selected and independently controlled with the laser assistance. This project will investigate this technology as a robust method for weapon component joining with metallurgical control. The project is a team effort combining the skills of several in-house experts who have many years of experience in analyzing electric arcs.

We are attempting to develop a laser-assisted microGMAW process using $250 \mathrm{~mm}$-diameter wire to produce miniature welds with the inherent advantages of filler metal joint augmentation and low heat input. We completed successful welding with the hybrid process and constructed an instrumented experimental test-bed employing several analytical techniques. We assembled a high-speed imaging
Sandia has been a leading participant in a multi-DOE laboratory Office of Industrial Technology project that has found it advantageous to combine laser and autogenous arc welding heat sources. We intend to leverage our newly discovered knowledge of the interactions between laser beams and arcs into developing a hybrid filler metal-based welding process. We will advance a wholly new welding process by combining a fiberoptic-delivered Nd:YAG laser with a miniaturized GMAW system. 
technique using a helium neon (He-Ne) laser for shadow backlight illumination and featuring a 2000 frames/second video camera with $632 \mathrm{~nm}$ notch filter. This imaging system enabled clear observation of droplet detachment in the metal arc with sufficient resolution to observe transfer mode and to calculate droplet acceleration times. Additional features of the test-bed include instrumentation of the welding power supply to record arc current and voltage waveforms, a high-resolution encoder on the wire drive motor, and a math-processing oscilloscope to record real-time wire-feed velocity changes. Our experiments increased our understanding of droplet detachment in micro-GMAW with an exceptionally smalldiameter metal wire. We photographed several distinct microGMAW transfer modes and analyzed them with the experimental test-bed using 0.25-meter-diameter ultrahighstrength (UHS) steel wire. Previous researchers have observed similar modes but not using the miniature wire that is unique to our experiments. We have completed the hybrid laser/arc welds in all of the modes. Base metal heating was lowest in hot wire mode as evidenced by heat-affected zone extent. We made promising narrow $1.8 \mathrm{~mm}$-wide welds in this mode with a smooth bead surface and full penetration into the $1.8 \mathrm{~mm}$ steel workpiece.
Our experiments increased our understanding of droplet detachment in micro-GMAW with an exceptionally smalldiameter metal wire....Previous researchers have observed similar modes but not using the miniature wire that is unique to our experiments. We have completed the hybrid laser/arc welds in all of the modes. 
10795

Surface-Micromachined Mechanical Timer

L. L. Lukens, G. E. Vernon

Solid-state oscillators have been widely used and are well suited for many applications. However, solid-state timing devices possess two distinct soft spots: they are inherently sensitive to radiation, and they require uninterrupted external power. Surface-micromachined (SMM) mechanical timers offer an elegant solution for applications where solid-state oscillators are not ideal. Mechanical timers are insensitive to radiation and can store elastic energy for long periods of time. In addition, micromachine devices offer a suite of attributes that are quite attractive, e.g., small, lightweight systems that are insensitive to mechanical shock and vibration environments due to their nearly massless part sizes. Also, work is ongoing at Sandia to integrate electronics and optics with microelectromechanical system (MEMS) devices; this work will eventually provide devices with even greater utility. Recent proof-of-concept MEMS devices demonstrate the feasibility of developing realistic weapon components.

This project will develop two mechanical timing mechanisms using Sandia's surface-micromachining process. Our vision for each device is based on a wound spiral spring that stores energy and is connected to a gear train. Terminated at the end of the gear train is an escapement mechanism that we use to provide mechanical damping. The escapement mechanism retards the unwind speed of the spiral spring and regulates the device so that timing information can be measured as a function of gear position. Our first timing device will operate like a wind-down timer and, at the end of a prescribed period of time, close an optical switch. The second device serves a mechanical clock function. This device will run for one hour and possess millisecond resolution. We will use an optical circuit consisting of vertical-cavity surface-emitting lasers (VCSELs) and photodetectors positioned above and below the clock gears, respectively, to read coded information embedded in the rotating gears.

- Mechanical timer development. We designed and fabricated three MEMS wind-down mechanical timers in the SUMMiT 5-Level SMM process. The focus of our design efforts was to investigate the ability to wind a polysilicon spiral spring and store potential energy, to demonstrate mechanical ...work is ongoing at Sandia to integrate electronics and optics with microelectromechanical system (MEMS) devices; this work will eventually provide devices with even greater utility. Recent proof-of-concept MEMS devices demonstrate the feasibility of developing realistic weapon components...This project will develop two mechanical timing mechanisms using Sandia's surfacemicromachining process. 
damping by the use of a runaway escapement mechanism, and to develop a step-up transmission where angular velocity is increased in each successive step in the gearing.

Each timer design contains six common elements: a rotary actuator, gearing for a step-up transmission, counterrotation pawls, a spiral spring, mechanical damping mechanism (runaway escapement), and a locking mechanism for start/stop operation. We use the rotary actuator in conjunction with a step-down transmission, which increases the available output torque, to wind the spiral spring. We attach one end of the spring to a winding gear engaged with the final gear in the step-down transmission. We attach the other end of the spring to a second gear concentrically mounted above the winding gear. This second gear engages a step-up transmission that interfaces with a mechanical damping mechanism (commonly known as a runaway escapement) positioned at its distal end.

We developed performance models for all three timers using a commercial rigid-body dynamics code. The first timer features a step-up transmission with a ratio of 260 to 1 . This timer features an earlier version of the runaway escapement mechanism and has a predicted runtime of about one second. The second and third timers feature step-up transmissions with a ratio of 89 to 1 . The timers feature runaway escapement mechanisms with significantly higher mass moments of inertia (increased time per beat). One timer features a single escapement mechanism, and the other features two escapement mechanisms. These timers have predicted runtimes of about 0.04 second and 0.07 second.

Since the mid-90s, this film has been $500 \mathrm{~nm}$ thick, resulting in an equivalent amount of hub play or lateral uncertainty of a gear about its hub. This amount of movement is highly undesirable in spur gear systems with teeth less than $10 \mathrm{~mm}$ tall. Two gears meshing under load could move apart by up to twice the amount of the hub play, significantly changing the contact point between the gears and increasing the associated pressure angle. The increased pressure directs more of the drive energy radially into the hub, thus increasing friction and wear while reducing the amount of energy transmitted to the load. The more the hub play, the more significant the impact. In support of this project, we developed fabrication processes that reduce the hub play in the SUMMiT technology to just $300 \mathrm{~nm}$, cutting the amount by $40 \%$.

Spur gears fabricated in the SUMMiT technology have typically employed the 200 pressure angle that is the industry standard in the macro world. Smaller pressure angles should be
We developed performance models for all three timers using a commercial rigid-body dynamics code. 
less sensitive to hub play, but they are generally avoided due to reduced strength at the base of the tooth. Since gear strength is not an issue for many of the mechanical timer gears, we designed speed-multiplying gear assemblies with pressure angles of 10, 14.5, 20, and 25 degrees and are now fabricating them. We wrote custom software to generate these gear teeth to within $3 \mathrm{~nm}$ of their ideal profiles.

It is generally beneficial to minimize the contact area between rubbing surfaces in silicon SMM devices. The adhesion energy between surfaces is almost directly related to the amount of area in contact, so significantly reducing this area will similarly reduce the energy required to overcome the adhesive force. This produces a more robust system that can be reliably driven with less energy. We implemented a new processing sequence that reduces the lower vertical constraint contact area between a gear and its hub by more than $75 \%$.
Since gear strength is not an issue for many of the mechanical timer gears, we designed speedmultiplying gear assemblies with pressure angles of 10, 14.5, 20, and 25 degrees and are now fabricating them. We wrote custom software to generate these gear teeth to within $3 \mathrm{~nm}$ of their ideal profiles. 
16167

\section{Pseudostationary Separation Materials for Highly Parallel Microseparations \\ A. K. Singh}

Sandia is developing new materials for performing separations in highly parallel microseparation systems. The materials synthesis and initial testing are being performed in collaboration with New Mexico Tech. The materials being developed are specialized chromatographic materials known as pseudostationary phases that are easily introduced into microfabricated channels, enable high-performance chemical separations, and can be tailored to provide a high degree of chemical diversity. These developments support a number of Sandia efforts in microanalytical devices based on highly parallel separations. This project will develop and apply microscale techniques to the analysis of biomolecules that carry the "signals" that control life processes such as growth, aging, memory, and emotion. The development and implementation of new separation methodologies and materials are central to the Molecular Integrated Microsystems (MIMS) project.

We made significant progress in the development and characterization of copolymer pseudostationary phases for electrokinetic (EK) chromatography. In addition to the polyacrylamide chemistry originally proposed, we studied siloxane-based pseudostationary phases. We developed a solvent system that permits reproducible and controlled synthesis of AMPS (2-acryamido-2-methyl-1-propanesulfonic acid) copolymers in selected mole ratios of AMPS to acrylamide, acrylate, or methacrylate comonomers. Using this solvent system, we synthesized and characterized eight AMPS copolymers with varied monomer ratio, backbone structure, and side chain length. We observed some significant differences in chemical selectivity between these polymers. The nature of the chemical interactions and the source of the differences in chemical selectivity are currently under investigation. We also synthesized and characterized a series of five siloxane-based polymers with varied ionic-group and sidegroup chemistry. These siloxane polymers provide better control and knowledge of molecular weight and allow the development and investigation of many chemistries for selective chemical separations. The five siloxane polymers synthesized to date provide unique chemical selectivity, and
Sandia is developing new materials for performing separations in highly parallel microseparation systems....These developments support a number of Sandia efforts in microanalytical devices based on highly parallel separations.

This project will develop and apply microscale techniques to the analysis of biomolecules that carry the "signals" that control life processes such as growth, aging, memory, and emotion. 
structures with sufficient hydrophobic character provide highefficiency separations. In addition to the development of pseudostationary-phase chemistry, we constructed and evaluated a capillary electrophoresis instrument with fluorescence detector. We are constructing an electrochemical detector for the same instrument.

\section{Refereed}

Chen, T., and C. P. Palmer. 1999. "Evaluation of Polymers Based on a Silicone Backbone as Pseudostationary Phases for Electrokinetic Chromatography." Electrophoresis 20: 2412-2419.

Palmer, C. P. 2000. "Polymeric and PolymerSupported Pseudostationary Phases in Micellar Electrokinetic Chromatography: Performance and Selectivity." Electrophoresis, accepted.

Palmer, C. P., and K. T. Tellman. 1999. "Poly(sodium 10-undecylenate) as a Pseudostationary Phase for Micellar Electrokinetic Chromatography: Effects of Polymerization Initiator." J. Microcolumn Separations 11: $185-191$.

Palmer, C. P., W. Shi, and D. S. Peterson. 2000.

"Performance and Selectivity of Polymeric Pseudostationary Phases in Electrokinetic Chromatography." Paper presented to the Materials Research Scientist, San Francisco, CA, May.

Peterson, D. S., and C. P. Palmer. 2000. "An Anionic Siloxane Polymer as a Pseudostationary Phase for Electrokinetic Chromatography." Electrophoresis 21: 3174-3180. ...we constructed and evaluated a capillary electrophoresis instrument with fluorescence detector.
Peterson, D. S., and C. P. Palmer. 2000. "Ionic Silicone Polymers as Pseudostationary Phases for Micellary Electrokinetic Chromatography." Paper presented to the 23rd International Symposium on Capillary Chromatography, Riva del Garda, Italy, June.

Peterson, D. S., and C. P. Palmer. 2000. "Synthesis and Characterization of Novel Anionic Siloxane Polymers as Pseudostationary Phases for Electrokinetic Chromatography." Electrophoresis, accepted.

Shi, W., C. J. Watson, and C. P. Palmer. 2000.

"Sulfonated Acrylamide Copolymers as

Pseudostationary Phases in Electrokinetic

Chromatography." J. Chromatography A, accepted.

Tellman, K. T., and C. P. Palmer. 1999. "Polymers of Sodium-N-undec-10-en-1-oyl taurate and Sodium-Nundec-10-en-1-oyl aminoethyl-2-phosphonate as Pseudostationary Phases for Electrokinetic Chromatography." Electrophoresis 20: 152-161. 
17230

\section{Novel Coatings for Microelectromechanical (MEM) Devices}

M. P. De Boer

Sandia aims to integrate new materials (in particular as thin films) into polysilicon-based microfabrication technology. Specifically, we are pursuing two classes of new materials: superhard materials and metals. We are making a special effort to develop processes that integrate materials at the microstructure release step because such processes would afford a wider class of materials and a greater degree of flexibility to device engineers. Consequently, processes have to allow conformal deposition.

We chose superhard materials with the following properties: wear resistance, hydrophobicity, lubricity, and chemical and thermal inertness. Our initial efforts focused on developing a process to coat polysilicon micromachines with a conformal layer of silicon carbide (SiC) — a tough, radiationhard, corrosion-resistant material, second only to diamond in wear resistance. Furthermore, in contrast to self-assembled monolayers (SAMs), which do not withstand temperatures employed in packaging technologies, we expected $\mathrm{SiC}$ to be much more thermally stable. We explored the use of C60 and its interaction with Si to realize a thin conformal SiC layer. Carbon nitride is predicted theoretically to be even harder. We also explored ways of integrating it into micromechanical structures.

Integration of metals offers special opportunities for realizing devices such as microrelays. Standard metaldeposition techniques suffer from being line-of-sight (LOS). One way of circumventing this problem is to use electroless plating techniques, based on coating the structures with a selfassembled organic monolayer with a special end-group functionality that catalyzes the growth of a continuous metallic layer from solution.

In the aforementioned coating processes, little is known about the tribological properties of the resulting materials in thin-film forms. Therefore, we also characterized these films using surface analytical techniques in our laboratory and specially designed test microstructures. Metal surfaces may be hydrophilic, and hence additional surface modification may be necessary to alleviate stiction.
Sandia aims to integrate new materials (in particular as thin films) into polysilicon-based microfabrication technology. Specifically, we are pursuing

two classes of new materials: superhard materials and metals. 
We originally envisioned using freeze sublimation as a way of achieving released structures with a conformal film of C60 (since C60 is the only soluble form of carbon). However, we found that this process is not reproducible and did not perform sufficiently well to meet our needs. Therefore, we studied and optimized the freeze sublimation process as a first step toward this goal.

In parallel, we demonstrated complementary strategies for realizing $\mathrm{SiC}$ microstructures by an alkene monolayer pretreatment that is similar to the SAM release already in use at Sandia, but also exhibits improved process margin.

Furthermore, it is compatible with $\mathrm{C} 60$ chemistry in that it can serve as a nucleation layer for selective $\mathrm{SiC}$ growth.

We also explored the deposition of $\mathrm{SiC}$ by an alternative route. Namely, we heated a Si mold to about $850^{\circ} \mathrm{C}$ in ultrahigh vacuum under a flux of C60 for 10 minutes. C60 reacted with the exposed $\mathrm{Si}$ at the bottom of the mold to make $\mathrm{SiC}$, but not with oxide-coated surfaces. We believe the SiC thickness is on the order of microns, sufficient to transform the microelectromechanical system (MEMS) structural material from $\mathrm{Si}$ to SiC. This opens up the possibility of developing a process in which both a stiffer structural material and a hard coating simultaneously result. An issue that must be addressed is the residual stress that develops in this transformed material.

We decided to initially work with copper $(\mathrm{Cu})$ rather than gold $(\mathrm{Au})$ because it is cheaper. In this process, we gained significant insight into the galvanic deposition on Si that we expect to aid us with Au and refractory metal deposition.

We developed a novel $\mathrm{Cu}$-deposition method to plate $\mathrm{Si}$ surfaces. We obtained continuous $\mathrm{Cu}$ films galvanically on $\mathrm{p}$ or n-type, single- or polycrystalline $\mathrm{Si}$. The films possess homogeneous structure, smooth surface, and improved adhesion to the substrate. The plating bath comprises an aqueous solution containing a $\mathrm{Cu}$ compound, ascorbic acid, ammonium fluoride, and an antistress agent. With this process, we avoid using seed layers to improve adhesion between metal and semiconductor. We used the coating process to plate polycrystalline $\mathrm{Si}$-micromachined devices after the release step. We observed excellent sidewall coverage, indicating that the coating process is conformal, as expected. ...we demonstrated

complementary strategies for realizing SiC microstructures by an alkene monolayer pretreatment that is similar to the SAM release already in use at Sandia, but also exhibits improved process margin. Furthermore, it is compatible with C60 chemistry in that it can serve as a nucleation layer for selective SiC growth. 


\section{Refereed}

Ashurst, W. R., C. Yau, C. Carraro, and R. T. Howe. 2000. "Alkene-Based Monolayer Films as AntiStiction Coatings for Polysilicon MEMS." Proc. Solid-State Sensor and Actuator Workshop 1 (Hilton Head Island, SC, June): 320-323.

Ashurst, W. R., C. Yau, C. Carraro, and R. T. Howe. 2000. "Alkene-Based Monolayer Films as AntiStiction Coatings for Polysilicon MEMS." Sensors and Actuators, submitted.

Ashurst, W. R., C. Yau, C. Carraro, R. Maboudian, and M. T. Dugger. 2000. "Dimethyl-dichlorosilane as Anti-Stiction Coating for MEMS: A Quantitative Comparison to Octadecyltrichlorosilane." J. Microelectromechanical Sys., in press.

Maboudian, R., W. R. Ashurst, and C. Carraro. 2000. "Self-Assembled Monolayers as Anti-Stiction Coating for MEMS: Characteristics and Recent Progress."

Sensors and Actuators A 82: 219-223.

Magagnin, L., R. Maboudian, and C. Carraro. 2000. "Deposition of Thin Copper Films onto Silicon with Improved Adhesion." Electrochem. and Solid State Lett., submitted. 
19989

Investigation of the Feasibility of RTL Concept for Fusion Energy

S. A. Slutz, G. E. Rochau, M. S. Derzon, R. B. Spielman, W. A. Stygar

The $Z$ machine at Sandia is the most powerful multimodule synchronized pulsed-power accelerator in the world. We need a rep-rated fusion capability for inertial fusion energy (IFE). Recent developments have led to a viable conceptual approach for a rep-rated z-pinch power plant for IFE. This concept exploits the advantages of going to high yield $(\sim$ few GJ) at low rep-rate $(\sim 0.1 \mathrm{~Hz})$ and using a recyclable transmission line $(R T L)$ to provide the necessary standoff between the fusion target and the power-plant chamber. We will investigate the feasibility of the RTL concept for fusion energy.

Suggested candidate materials for the RTL include solid lithium (Li), fluorine-lithium-beryllium (FLiBe), lead $(\mathrm{Pb})$, tin (Sn), aluminum (Al), and various combinations. We must determine if transmission lines constructed from these materials using rapid, inexpensive fabrication processes (casting, extrusion, forming) will efficiently transport electrical power at the large currents required of a fusion driver. We are investigating candidate RTL materials in experiments on Saturn (and possibly Z) to demonstrate feasibility of the RTL concept. This research supports all fusion energy research in DOE.

We successfully completed experiments on the Saturn accelerator to test several candidate RTL materials for the reprated z-pinch power plant concept. We performed these experiments with a model RTL coaxial transmission line of diameter $8 \mathrm{~cm}$, length $30 \mathrm{~cm}$, and gap width $3 \mathrm{~mm}$. Materials tested include stainless steel (SS), Al, and a Sn coating on SS. The purpose of the experiments was to investigate the uniformity of the electrical turn-on of the RTLs. We used three sets of three azimuthally placed B-dots to measure the current. The azimuthal current readings were essentially identical, and there was no indication of current loss in any of these cases. These initial results are important indicators of potential success for the RTL rep-rated, z-pinch power plant concept.

Our three university collaborators also performed supporting work. The University of California-Berkeley performed initial studies of candidate RTL materials. We

\author{
The Z machine at Sandia is the \\ most powerful multimodule \\ synchronized pulsed-power \\ accelerator in the world....We \\ will investigate the feasibility of \\ the RTL concept for fusion \\ energy.
}


investigated the power plant coolant material FLiBe (containing $\mathrm{F}, \mathrm{Li}$, and $\mathrm{Be}$ ) and cast small samples to examine their structural characteristics and compatibility of expansion characteristics with coatings of other materials (such as $\mathrm{Pb}$ ).

The University of California-Davis performed initial studies of direct molecular hydrodynamics (MHD) conversion. They found that a competitive power plant with a 1.6 GJ target yield could be built using a z-pinch driver with a compact blanket of Li hydride for direct MHD conversion.

The University of Wisconsin performed initial studies of the rep-rated z-pinch power plant concept, including analysis of the tritium-breeding ratio, energy-conversion ratio, neutronshielding properties, and activation analysis. All of these results are very encouraging and are already demonstrating several of the advantages of the RTL rep-rated z-pinch power plant concept over other existing IFE concepts (heavy ions and lasers).

\section{Refereed}

Olson, C. L. 2000. "Z-Pinch for High Yield and IFE." (Invited) Article in the American Nuclear Society Newsletter (ANS Newsletter) (Madison, WI, June).

Slutz, S. A., C. L. Olson, G. E. Rochau, M. S. Derzon, P. F. Peterson, J. S. De Groot, N. Jensen, and G. Miller. 2000. "Z-Pinch-Driven Fusion Energy." Proc. ICENES 2000, The 10th Internat. Conf. on Emerging Nucl. Energy Sys. (Petten, The Netherlands, 25-28 September).

\section{Other Communications}

De Groot, J. S., M. S. Derzon, C. L. Olson, G. E. Rochau, S. A. Slutz, and R. B. Spielman. 2000. "Conceptual Design of ZPMHD Rep-Rated Z-Pinch Power Plant." Paper presented to the American Physical Society-Division of Plasma Physics Meeting, Montreal, Quebec, Canada, 23-27 October.
Olson, C. L. 2000. "Rep-Rated Z-Pinch Power Plant." (Invited) Paper presented to the Fusion Power Associates Symposium, San Diego, CA, 17 July.

Olson, C. L., G. E. Rochau, M. S. Derzon, S. A. Slutz, R. B. Spielman, R. E. Olson, M. K. Matzen, M. E. Cuneo, D. H. McDaniel, J. P. Quintenz, P. F. Peterson, J. S. De Groot, G. A. Rochau, and R. R. Peterson. 2000. "Inertial Fusion Energy with Pulsed Power." Paper presented to the 13th International Conference on High-Power Particle Beams, Nagaoka, Japan, 25-30 June.

Olson, C. L., the Z Team, and the Z-Pinch IFE Team. 2000. "Recent Results Related to the Z Machine and Their Implications for Fusion Energy." (Invited) Paper presented to the 14th ANS Topical Meeting on Technology of Fusion Energy, Park City, UT, 15-19 October. 
19990

\section{Rapid Discovery of Materials for Energy} Systems

D. A. Loy

Sandia will demonstrate the successful application of combinatorial or rapid discovery methodologies to discovering new materials for energy-saving chemical separations systems. This proof-of-principle project will demonstrate that materials discovery can be accelerated using rapid discovery techniques in complement to traditional research processes. Rapid discovery refers to the design and implementation of highly parallel materials synthesis of large numbers of new materials, their rapid evaluation for some desired properties, and data mining for identifying new directions or trends. The result is the manyfold increase in productivity in screening materials with a manyfold decrease in laboratory waste. Traditional methods can be used to confirm and extend the studies.

We will evaluate the materials targeted in this project for construction of facilitated transport membranes for separating olefins from other hydrocarbons in chemical processes. If successful, these materials would save the U.S. chemical industry quads of energy per year that is presently used in steam-driven distillative separations.

We invented several key combinatorial technologies. Industrial concerns demonstrated intense interest in these inventions. The inventions arose from our interests in developing more rapid techniques for discovering and testing membrane materials. The first invention is a rapid combinatorial screen for materials to be used for facilitated transport of olefins. We synthesized and characterized the materials, prepared and characterized model systems, and performed preliminary thermal screening. Final quantitative screening is the only task not completed in the project. Once complete, the project will result in one patent application.

The second invention is the concept of screening membrane materials using arrays of quartz microbalances (QMBs). This invention is based on the application of QMBs to measure gas permeation in thin polymer films. Since membranes are essentially thin polymer films, this method is perfect for screening membrane materials for different chemical separations. This technology can readily be applied by building arrays of commercially available QMBs and surveying both composition and processing of the polymeric
This proof-of-principle project will demonstrate that materials discovery can be accelerated using rapid discovery techniques in complement to traditional research processes....We invented several key combinatorial technologies. 
materials. The same technology can monitor polymer curing and aging. We already have significant industrial interest in the latter applications.

The next invention is a colorimetric dye for screening catalysts for fuel-cell membranes. The colorless viologen dye is easily reduced to form an intense purple compound. The new dye is designed to be covalently incorporated into the membrane material to prevent migration or leaching. The dye would be doped into a ceramic material that would then be treated with an array of different catalysts through highly parallel techniques. We will characterize active catalysts that would cause the material to change color by surface analysis techniques for composition and structure. The dye would also be useful for screening materials for arsenic uptake.

The last invention permits rapid screening of membranes based on photocatalysts used for cleaning air of organic and biological contaminants. These metallic oxide semiconductors (MOS) are prepared and processed using gradient techniques to afford combinatorial libraries of photocatalysts. The activity of the photocatalysts is assayed using organic fluorescent dyes that degrade only with metal/ metal-oxide-mediated photocatalyzed oxidation.

\author{
We already have significant \\ industrial interest in ... \\ applications.
}


19991

\section{In Situ Formation of Reactive Barriers for Radionuclide, Heavy Metal, and Organic Stabilization}

R. C. Moore

It is well documented that actinides and heavy metals such as plutonium (Pt), uranium (U), americium (Am), lead $(\mathrm{Pb})$, and chromium $(\mathrm{Cr})$ bind onto the surface of apatites and become almost totally immobile. Apatite has been proposed as a very effective barrier material to immobilize and contain radionuclides around contaminated sites and leaking storage containers. The current methods to place apatite to form a reactive barrier are to dig trenches around the waste and backfill with apatite or pump apatite into the soil as slurry of very fine particles. The latter method requires drilling many boreholes around the waste to form a continuous overlapping barrier. Both of these methods require extensive excavation. An alternative method to construct an apatite barrier is to synthesize apatite in situ by mixing chemicals in the soil. An example is mixing highly soluble calcium chloride and sodium phosphate in the soil. Chemical conditions can be controlled to maximize the efficiency of hydroxyapatite formation.

Preliminary results from laboratory experiments indicate hydroxyapatite can be formed in soil at near neutral $\mathrm{pH}$. The in situ method would provide an economical, fast, and environmentally safe method to contain radioactive materials and heavy metals and would not involve extensive excavation.

We proved that apatite can be formed in situ in soil and will sequester $U$ and strontium ( $\mathrm{Sr}$ ). We completed batch kinetic experiments on $\mathrm{U}$ and $\mathrm{Sr}$ uptake by apatite. We synthesized new compounds for sequestering radionuclides, and we proved that substituted apatites can be used to sequester $\mathrm{U}$ and technetium (Tc) in solution.

This work led to the development of a new method for constructing in situ reactive barriers for radionuclides. The method does not require extensive excavation, thus resulting in a more economical, safe process. The method can be used at various DOE sites, including Hanford, Savannah River, and Sandia.

This work also led to the development of new materials that can be used in in situ barriers or in waste tanks to sequester Tc, actinides, and other radionuclides and heavy metals.
We proved that apatite can be formed in situ in soil and will sequester $U$ and strontium (Sr).

We completed batch kinetic experiments on $U$ and $S r$ uptake by apatite. We synthesized new compounds for sequestering radionuclides, and we proved that substituted apatites can be used to sequester $U$ and technetium (Tc) in solution. 
This work has resulted in two patent applications and five technical advances.

\section{Refereed}

Moore, R. C. 2000. "Formation of In Situ Apatite

Barriers for Sequestering Strontium and Uranium." ACS Mtg.: PACIFIC Internat. Mtg. (December), accepted.

Moore, R. C. 2000. "In Situ Apatite Barriers for Containing Radionuclides and Heavy Metals." Paper presented to the TACS International Meeting, Honolulu, HI, December. 
19993

\section{MPP Direct Numerical Simulation of Diesel}

\section{Autoignition}

H. N. Najm, R. L. Clay, J. H. Chen, J. Ray, B. A. Allan, W. S.

Koegler

The major obstacle to reliable modeling of diesel engine combustion processes is also fundamental to simulation of many other problems involving turbulence, phase change, or chemistry, i.e., our inability to resolve all relevant scales, typically ten decades or more, using numerical simulation of the physical and chemical processes involved. The future predictive design tools for combustion systems will rely not on direct simulation, but on conceptualization of the complex interdisciplinary phenomena that underlie turbulent combustion and the solution of model equations describing turbulent combustion that involve some form of averaging or approximation.

This project enables three-dimensional (3-D) highfidelity simulations on massively parallel processor (MPP) computers of processes critical to applications such as gas turbines and chemical processing systems, thus implementing a revolutionary new approach to the development of predictive models for device-scale simulation.

This project will establish critical research that positions Sandia as an innovator and leader in high-fidelity MPP simulations of reacting flow. The capabilities developed will revolutionize the way combustion systems are designed in the future, allowing for faster iteration and more highly optimized design to meet increasingly stringent government emissions regulations while maintaining or improving efficiency. These design approaches will enable new technologies that could save up to $\$ 33$ billion and 267 tons of carbon dioxide $\left(\mathrm{CO}_{2}\right)$ per year while meeting the increasingly stringent emissions regulations anticipated over the next decade.

We constructed, demonstrated, and profiled a 3-D MP compressible fixed-mesh reacting-flow direct numerical simulation (DNS) code on various computational platforms. This code is now being used for large-scale 3-D DNS of turbulent flow with detailed kinetics, greatly enhancing prior capabilities, now limited by the availability of compute nodehours. 
We evaluated and tested two potential object-oriented (OO) frameworks for adaptive mesh refinement (AMR) DNS of reacting flow (OVERTURE and GRACE) for their overall efficiency, overhead costs, and scalability. We concluded that the GRACE framework is more efficient, lightweight, scalable, and more readily available for distributed parallel computing. As a result of this investigation, GRACE is now being used as the basis of new common component architecture (CCA) code development at Sandia.

We developed a set of tools for data mining/feature extraction targeted at general unstructured/structured grids. We demonstrated these tools on specific test problems, and they are being targeted at large-scale AMR or fixed-mesh reacting-flow datasets.

We have started ongoing discussions with National Energy Technology Laboratories (NETL) regarding collaborative work on efficient large-scale MP reacting-flow code development based on their Multiphase Flow with Interphase eXchanges (MFIX) code. This will leverage our expertise in numerical integration of stiff chemical systems in the context of multidimensional reacting-flow codes.

The execution of this project has brought us significantly further along in the formulation and development of advanced efficient and scalable next-generation MP codes for resolved reacting-flow computations with detailed kinetics and transport in large-scale flow.
The execution of this project has brought us significantly further along in the formulation and development of advanced efficient and scalable nextgeneration MP codes for resolved reacting-flow computations with detailed kinetics and transport in largescale flow. 
20407

\section{A Functional Parallel Discrete-Event Simulation of a Snoopy Coprocessor Software Monitor}

\section{L. Winter}

The prevalent use of computers in our society is creating a reliance that requires high-assurance systems. Because verification and validation $(V \& V)$ techniques are typically not sufficient to identify the existence of software faults in complex systems, use of a runtime softwaremonitoring system can be critical for providing assurance of correct program behavior. The high-level research goal of this project is to define and develop a comprehensive, knowledgebased system that complements existing V\&V methods, supporting production of high-quality systems. The DynaMICs system uses integrity constraints to capture knowledge about real-world objects, maintains the constraints in a repository, automates compile-time application-code instrumentation, and initiates the execution of constraint-checking code at runtime. Performance issues have long plagued promotion of softwaremonitoring approaches; to address this, Sandia is investigating the development of a DynaMICs snoopy-coprocessor system (COPS) that employs coprocessor and bus-monitoring hardware to facilitate concurrent execution of application and constraint-checking code. In this approach, the coprocessor executes constraint-checking code while the main processor executes the application code. The bus-monitoring hardware is similar to the snoopy hardware utilized in bus-based, sharedmemory multiprocessors to maintain cache coherence. The work proposed for the project was the development of a parallel discrete-event simulation of COPS.

Because development of a prototype of COPS is not feasible, a system simulator is under development. This simulator will provide an experimental platform that can be used for proof of concept of the DynaMICs approach, simulator validation, and systematic exploration of the design space. The simulator is a parallel discrete-event simulator that is composed of five logical processes (LPs): the main processor, Snoop, COP, bus, and main memory, which communicate and synchronize their actions using sharedmemory queues. The simulator is written in $\mathrm{C}++$, and the threads (one for each LP) are created and managed using the
The high-level research goal of this project is to define and develop a comprehensive, knowledge-based system that complements existing $V \& V$ methods, supporting production of high-quality systems....The work proposed for the project was the development of a parallel discrete-event simulation of COPS. 
POSIX Threads library. The execution platform is an eightprocessor SGI Origin 2000.

Execution of a test suite indicates that a base simulator that creates LP threads, implements communication and synchronization among the threads via shared queues, and outputs performance data functioned according to specifications. In this simulator, the main processor, COP, and main-memory LPs are simplified. The main processor LP is trace-driven and does not model a cache hierarchy. Instead of simulating the execution of a program, it emits the addresses that the program would generate during its execution, assuming a fixed delay for instruction execution. An address trace, generated a priori, is input to the main processor. The main processor LP updates its local virtual time (LVT) according to the instruction execution time and corresponding memory delays. The COP removes elements from the CSQueue, a queue containing memory access information, and updates the variables of interest (VOI) status cache, essentially counters used to indicate which VOIs are awaiting constraint checking. In this version of the simulator, the COP does not update VOI memory, nor does it actually execute constraint-monitoring or knowledge-producing code. Instead of executing this code, the COP models its execution by busy-waiting for a random amount of time whenever it receives an update to the special path-tag variable, updating its LVT accordingly. The main memory is simplified in the sense that instead of actually simulating memory accesses, it updates its LVT in accordance with specified memory read/write time input parameters. Instead of satisfying memory requests with data, it satisfies them only with respect to associating time delays with the requests. 
20409

New Complexing Agent for Co(II) Analysis

S. E. Klassen

This work was performed in collaboration with the Energetic Materials Research and Testing Center at the New Mexico Institute of Mining and Technology (NMIMT) and continued evaluation of a modification to an existing ultraviolet/visible (UV/Vis) spectrophotometric method for cobalt (II) (Co [II]) analysis of the explosive 2-(5cyanotetrazolato)pentaammine cobalt (III) perchlorate (CP). Co (II) is an indicator of CP decomposition. The current methods use a complexing agent with absorption bands that overlap with those of $C P$, thus requiring a blank, and so a new complexing agent that is expected to have less overlap was synthesized. Sandia and NMIMT previously evaluated the new complexing agent with Co (II) standards and focused our work on comparing the new and standard complexing agents with a variety of CP samples.

The current method of Co (II) analysis in the explosive CP uses 2,2'-dipyridyl-2-pyridylhydrazone (DPPH) to complex with Co (II), which is formed during the initial stage of CP decomposition. The maximum absorption wavelength for the DPPH-Co (II) complex occurs at a wavelength where CP itself contributes absorbence, and so a CP blank must also be run and its absorbence subtracted. Previously we investigated a new complexing agent based on the quinoline analog, 2,2'dipyridyl-2-quinolylhydrazone (DPPQH) using Co (II) standards and showed it to have a maximum wavelength in an area with less CP absorbence. We showed DPPH and DPPQH to be similar with respect to lower limits of detection, reproducibility, and linearity in the standard use range of 0.1 to 0.75 parts per million (ppm) of Co (II). We also made comparisons with mixed results using actual CP samples.

We reassessed linearity of both complexing agents and found them to be good for Co (II) standards of 0 to $4.5 \mathrm{ppm}$. This range is more than adequate for the concentrations of Co (II) that are expected to be found in CP taken from devices. In taking a closer look at the lower limits of detection, we discovered that concentrations of $0.05 \mathrm{ppm}$ could be seen with DPPH, but not with DPPQH. The lower limit of detection is $0.125 \mathrm{ppm}$ for DPPQH. We also discovered that the maximum wavelength varied with concentration at low levels for both complexing agents, but the problem was more severe with
This work ...continued evaluation of a modification to an existing ultraviolet/visible (UV/Vis) spectrophotometric method for cobalt (II) (Co [II]) analysis of the explosive 2-(5cyanotetrazolato)pentaammine cobalt (III) perchlorate (CP). Co (II) is an indicator of CP decomposition. The current methods use a complexing agent with absorption bands that overlap with those of $C P$, thus requiring a blank, and so a new complexing agent that is expected to have less overlap was synthesized. 
DPPQH than DPPH. At low concentrations, the maximum wavelength of DPPQH is similar to DPPH and offers little advantage.

We spiked a variety of CP samples with known concentrations of $\mathrm{Co}$ (II) to determine recovery and reproducibility. In general, the matrix does not appear to be interfering because the recoveries are good. The average recoveries ranged from $94 \%$ to $100 \%$ with standard deviations of 3-6. One sample set, however, did have an average recovery of only $81 \%$, but the reason for this is not known. Even though DPPH can be used for detection of low levels of Co (II), the reproducibility is worse at these levels. In another comparative study on a variety of CP samples, it appears that DPPQH has better reproducibility than DPPH, but we also discovered that the instrument response had degraded during the course of these runs, so these reproducibility data are suspect.

DPPQH is not recommended as a replacement for DPPH in the Co (II) analysis of CP. DPPH has a lower limit of detection and less variation in maximum wavelength for the concentrations of Co (II) typically desired.
We also discovered that the maximum wavelength varied with concentration at low levels for both complexing agents, but the problem was more severe with DPPQH than DPPH. 
20410

\section{Infrared Polarization for Target Identification}

W. C. Sweatt

Sandia is researching the value of passive polarimetry in the mid-infrared (MIR) spectral region for distinguishing man-made objects and targets from their environments. Passive means that the polarimetry system will not illuminate target scenes, but will only analyze radiation emitted by the scene or reflected from sources already present. We are using a complete imaging polarimeter in a series of laboratory and field experiments. These experiments are intended to characterize the degree to which polarization information can augment remote detection of objects' targets.

We are carrying out laboratory measurements of polarization to characterize the behavior of various bare metal and painted surfaces with respect to material, finish, and viewing angle. We are also conducting field measurements from horizontal and downward viewing angles with targets with known reflectivities and with military vehicles to characterize the usefulness of polarization information in the detection process. Outdoor tests with known targets are critical since the reflected background will have a major impact on the polarimetric signatures of tanks and trucks. We are giving particular consideration to the open question of whether a significant component of circular polarization should be expected in naturally illuminated scenes. We will cast results in terms of such figures as degree of polarization and contrast.

We plan to develop a quantitative assessment of the usefulness of IR polarimetry for target identification. This research will support DOE in its efforts to monitor compliance with the nuclear nonproliferation treaties.

In collaboration with the University of Arizona's Optical Sciences Department, we built an instrument to measure the polarization properties of IR light. More specifically, this instrument and the accompanying electronics measure several variables that define the Stokes parameters of IR light in the $3 \mathrm{~mm}$ to $5 \mathrm{~mm}$ waveband. We developed a suite of software that turns the measured data into the Stokes parameters, which describe the characteristics of the polarization (i.e., circular, linear, unpolarized, etc.). The instrument has been largely debugged and calibrated. We used to study the polarization of several in-the-lab surfaces, including smooth copper $(\mathrm{Cu})$ surfaces, roughened $\mathrm{Cu}$, thick
These experiments are intended to characterize the degree to which polarization information can augment remote detection of objects' targets.... We plan to develop a quantitative assessment of the usefulness of IR polarimetry for target identification. This research will support DOE in its efforts to monitor compliance with the nuclear nonproliferation treaties. 
acrylic paint, and glass that is opaque in the $3 \mathrm{~mm}$ to $5 \mathrm{~mm}$ band.

We were unable to acquire scans of various man-made objects like airplanes, cars, buildings, etc., to see if polarization might be useful for identifying man-made things (target discrimination).

\section{Refereed}

Sabatke, D., M. Descour, E. Dereniak, W. Sweatt, S.

Kemme, and G. Phipps. 2000. "Optimization of

Retardance for a Complete Stokes Polarimeter." Opt.

Lett. 25 (March): 802-804. 
20411

Characterization of Polymer-Metal Interface Properties for LIGA-Based Microsystem Technologies

B. P. Somerday, S. H. Goods

Sandia's goal for this project is the design and execution of experiments characterizing the adhesion of polymer films to metallized wafers. These polymer/metal interfaces are a critical feature in the multilayer composite structure used to fabricate LIGA (the German term Lithographie, Galvanoformung, und Abformung, for lithography, electroplating, and molding)-based microsystem components. Debonding of polymethylmethacrylate (PMMA) micromolds from the metallized silicon $(\mathrm{Si})$ substrate limits the yield of LIGA components. This debonding results from the combined effects of residual stress and poor adhesion. The project was conducted in collaboration with Stanford University. This report summarizes efforts to measure the stress that evolves from bonding PMMA to metallized Si wafers and to quantify the adhesion between PMMA films and metallized surfaces.

This research focused on the measurement of stress and adhesion in polymers bonded to metallized Si surfaces. The material system of interest was PMMA bonded to Si wafers having sputtered titanium (Ti) films. This material system replicates a critical feature of the composite structure used to fabricate LIGA-based microsystem components. We measured residual stress and adhesion on PMMA films that were spincast on Ti-coated surfaces of Si wafers. In addition, we conducted stress measurements on a thick PMMA sheet that was solvent-bonded to the spin-cast PMMA.

We measured stresses in spin-cast PMMA films and solvent-bonded PMMA sheet using the wafer curvature technique developed at Stanford. PMMA, with a molecular weight of 2.2 million, was spin-cast on $\mathrm{Si}+\mathrm{Ti}$ wafers to a thickness of 1 micrometer. These PMMA films were annealed at $180^{\circ} \mathrm{C}$ for 1 hour. We measured the residual tensile stress in this spin-cast and annealed PMMA film as 10 to $25 \mathrm{MPa}$. Calculations show that this relatively low stress is not a severe driving force for interface debonding. We bonded thick (1.6 millimeter) PMMA sheet to the spin-cast PMMA at $25^{\circ} \mathrm{C}$ using methylmethacrylate solvent. Immediately after solventbonding, we conducted wafer curvature measurements to
Sandia's goal for this project is the design and execution of experiments characterizing the adhesion of polymer films to metallized wafers. These polymer/metal interfaces are a critical feature in the multilayer composite structure used to fabricate LIGA-based microsystem components. 
characterize the evolution of stress in the thick-sheet PMMA. These measurements showed that compressive stress in the PMMA reaches $25 \mathrm{MPa}$ near the interface at short times, but abates to levels near zero as the solvent diffuses through the PMMA over a period of 40 hours. The stress measurements indicate that neither residual stress in the spin-cast PMMA nor solvent-induced stress in the thick-sheet PMMA contributes to debonding of the LIGA micromolds.

We identified two techniques for quantifying adhesion between PMMA and metallized Si. The first technique was the blister test, which involves applying pressure to a thin-film membrane through an aperture in the substrate. We committed significant effort to producing specimens for the blister test using lithography and nanofabrication methods. Specimens suitable for testing were not produced by the end of the fiscal year because of several significant difficulties in the fabrication procedure. We pursued an alternate technique for measuring adhesion that involves fracture mechanics testing of millimeterscale beams. We produced these beams by diffusion-bonding 1-micrometer-thick PMMA films $\left(170^{\circ} \mathrm{C} / 30\right.$ minutes $)$ that were spin-cast on $\mathrm{Si}+\mathrm{Ti}$ wafers. One side of the composite beam was notched, and the beam was loaded in four-point bending. The strain energy release rate required to propagate a crack along the PMMA/Ti interface was measured as $14 \mathrm{~J} / \mathrm{m}^{2}$. While the significance of this value is uncertain, these experiments demonstrate that adhesion can be quantified so that efforts to improve the interface properties can be assessed.
We identified two techniques for quantifying adhesion between PMMA and metallized Si.... While the significance of this value is uncertain, these experiments demonstrate that adhesion can be quantified so that efforts to improve the interface properties can be assessed. 


\section{2}

\section{Fault-Tolerant Computing Services for} Heterogeneous Clusters

\section{P. D. Hough, E. J. Walsh, M. E. Goldsby}

As the size and complexity of current and nextgeneration parallel computers continue to increase, the issue of fault tolerance becomes more prominent. Even though fault tolerance on homogeneous massively parallel (MP) systems is essentially a solved problem, the issues arising on very large heterogeneous platforms such as Sandia's Computational Plant (CPlant) are just now being explored. There are two primary components of fault tolerance: (1) fault detection and (2) fault recovery. Collaborations with the University of Florida focused on the first component. The initial stages of the project involved developing and testing efficient and scalable fault-detection algorithms in a simulative environment. This work will focus not only on continuing to develop improvements to these protocols, but also on implementing and testing them on CPlant-like systems. Testing will include evaluations of the effectiveness of the protocols and of their impact on the efficiency of the distributed system. Furthermore, we will combine these protocols with algorithm-dependent fault-recovery techniques developed at Sandia to form faulttolerant applications. This work is a valuable part of developing robust distributed computing applications for large-scale heterogeneous systems like CPlant, and it contributes to Sandia's presence at the forefront of distributed computing research.

We performed a number of computational studies to evaluate scalability and resource utilization. As expected, the variants of the protocol performed differently. We made the following observations:

- Flat gossiping exhibits limited scalability in consensus times and poor scalability in resource utilization.

- Network bandwidth and central processing unit (CPU) utilization place a limit on the maximum system size that can be supported by flat gossiping (for example, about 200 nodes requires 100 percent CPU utilization).

- Layered gossiping with broadcast provides a scalable alternative to flat gossiping. For a 96-node system, resource utilization of a two-layered system is about $10 \%$ of flat gossiping. For a layered system of a given size, there exists an optimum group size that minimizes network bandwidth and
Even though fault tolerance on homogeneous massively parallel (MP) systems is essentially a solved problem, the issues arising on very large heterogeneous platforms such as Sandia's Computational Plant (CPlant) are just now being explored....This work is a valuable part of developing robust distributed computing applications for large-scale heterogeneous systems like CPlant, and it contributes to Sandia's presence at the forefront of distributed computing research. 
CPU utilization. In terms of consensus time, layered gossip without broadcast is the least scalable of the alternatives considered. In systems where true broadcast is not supported, layered gossip without broadcast provides a trade-off between failure-detection time and resource utilization versus flat gossip. For low CPU utilization, time between gossip messages should be greater than or equal to the operating system time slice (for example, 10 milliseconds for Linux). Analytical modeling of gossiping is a promising direction for future

Analytical modeling of gossiping is a promising direction for future research as a means to project performance research as a means to project performance characteristics on a large scale.

\section{Refereed}

Ranganathan, S., A. George, R. Todd, and M. Chidester. 2000. "Gossip-Style Failure Detection and Distributed Consensus for Scalable Heterogeneous Clusters." Cluster Computing J., submitted. 
20413

\section{DNA Microarray Technology}

G. S. Davidson

Deoxyribonucleic acid (DNA) microarrays are revolutionizing the field of genetics and medical research because they allow one to follow the expression of all, or a significant fraction, of the entire genome in experimental organisms. This new tool is exciting because it offers the potential to see the simultaneous interactions of all active genes in a cell and may eventually reveal a great deal of emergent phenomena that could not be detected with smaller experiments. However, because microarray laboratory methods are new and are still relatively uncharacterized, knowledge about their uses, including sensitivity, reproducibility, and other limitations, are still being discovered and reported. Further, Sandia researchers have the choice of at least three radically different DNA microarray technologies: (1) DNA synthesized directly on chips, (2) DNA oligonucleotides printed on glass slides, or (3) DNA oligonucleotides printed on nylon membranes. To choose the best technology for an experiment, researchers should have access to comparable assessments of sensitivity, reproducibility, and appropriate protocols for these technologies.

We previously studied gene expression in the yeast Saccharomyces cerevisae with genome microarrays printed on nylon membranes. We completed construction of an arrayer to produce microarrays on glass slides, allowing us to compare glass-based and membrane-based arrays. With that experience and equipment, we are now investigating the sources of error in the array printing process and in the mRNA (messenger RNA) sample preparation process. Successful completion of this work will enable microarrays to be a more reliable and more sensitive tool to investigate gene expression and regulation.

We completed building a microarrayer, enclosed it in a Plexiglas cabinet, and successfully engineered it to maintain constant temperature and humidity. We then added a camera to monitor printing pin contact with the slides and are now characterizing SSC (sodium, chloride, and citrate, an aqueous buffer that contains SDS, a detergent) versus various Trisbased buffers. We are currently determining how to maintain constant amounts of DNA in the spots during hybridization.
Deoxyribonucleic acid (DNA) microarrays are revolutionizing the field of genetics and medical research because they allow one to follow the expression of all, or a significant fraction, of the entire genome in experimental organisms. This new tool is exciting because it offers the potential to see the simultaneous interactions of all active genes in a cell and may eventually reveal a great deal of emergent phenomena that could not be detected with smaller experiments....Successful completion of this work will enable microarrays to be a more reliable and more sensitive tool to investigate gene expression and regulation. 
Even in commercial production facilities, approximately $70 \%$ of the spotted DNA is known to be lost in solution during or prior to hybridization. Since the spot DNA must always be in excess, loss of DNA from the spot will significantly affect reproducibility. We are also determining which labeling procedures, e.g., incorporation of fluorescent tags into cDNA (complementary DNA) result in the greatest efficiency and reproducibility.

We currently do not have the primers for a yeast gene microarray, but we are developing an array for another related fungus, Neurospora crassa. We will then make a yeast array once we obtain the primers. The milestone involving yeast primers is near completion.

To date, we have printed slides on four different slide surfaces (two aldehyde-based surfaces, one aniline-based surface, and a polylysine-based surface), determined which software we will use for preliminary data acquisition (Quantarray) and analysis (GeneSpring), and developed tools for matching spots with samples in microtiter dishes.

With respect to membrane arrays, we conducted an experiment with five time points for examining changes in mRNA levels during exit from stationary phase. We put this data into VxInsight ${ }^{\mathrm{TM}}$, which allowed us to quickly identify the genes induced in stationary phase. We examined mutants in two genes induced in stationary phase and one gene that appeared from our preliminary experiments to be induced in stationary phase, but that was not clustered with stationaryphase genes by VxInsight ${ }^{\mathrm{TM}}$. Mutants in the two genes clustered as stationary-phase genes by VxInsight ${ }^{\mathrm{TM}}$ have phenotypes associated with stationary phase, i.e., they die after 7 days at $37^{\circ} \mathrm{C}$. However, the mutant that was not clustered with stationary phase by VxInsight ${ }^{\mathrm{TM}}$ appears to be essentially a wild type, contrary to our preliminary analysis, that was conducted using Pathways ${ }^{\mathrm{TM}}$ software.

\section{Refereed}

Davidson, G. S. 2000. "VxInsight" ${ }^{\mathrm{TM}}$ and Microarray

Analysis." Paper presented to the SWARM 2000

(Regional AAAS Meeting), Las Cruces, NM, 11 April.

Werner-Washburne, M. 2000. "Genomics: A Revolution Before Our Eyes." Paper presented to the AAAS SWARM Meeting 2000, Las Cruces, NM, 10 April. ...approximately $70 \%$ of the spotted DNA is known to be lost in solution during or prior to hybridization. Since the spot DNA must always be in excess, loss of DNA from the spot will significantly affect reproducibility. We are also determining which labeling procedures, e.g., incorporation of fluorescent tags into cDNA (complementary DNA) result in the greatest efficiency and reproducibility.
Werner-Washburne, M., E. Fuge, V. Peck, and G. S. Davidson. 2000. "Exit from Stationary Phase in Yeast: Gene Expression Analysis." Proc. Yeast Genetics and Molecular Biology Mtg. 2000 (Seattle, WA, 25-30 July). 


\section{Other Communications}

Werner-Washburne, M., G. S. Davidson, and E. Fuge. 2000. "Clustering and Visualizing Yeast Microarray

Expression Data Using VxInsight ${ }^{\mathrm{TM}}$." Paper presented to the Southwest Genomics and Biotechnology

Alliance-Day of Talks I, Albuquerque, NM, 22

March.

Werner-Washburne, M., G. S. Davidson, and E. Fuge. 2000. "VxInsight ${ }^{\mathrm{TM}}$ and Microarray Gene Expression Data Mining." Proc. DOE Genome Contractor-

Grantee Workshop Talks and Poster Abstracts (28

February-2 March, Santa Fe, NM). 
20414

\section{Shock Response of Diamond Crystals}

M. D. Knudson

Sandia is investigating the shock response of singlecrystal diamond up to several Mbar pressure in a collaborative effort with the Institute for Shock Physics (ISP) at Washington State University (WSU). This project will determine (1) the usefulness of diamond as a window material for high-pressure velocity interferometry measurements, (2) the maximum stress level at which diamond remains transparent, (3) the Hugoniot elastic limit (HEL) for the [110] orientation of diamond, and (4) if a two-wave structure can be detected and analyzed.

We designed targets to produce instantaneous elastic stresses up to $\sim 3$ Mbar in the [110]-oriented diamond crystals using the Sandia two-stage light-gas gun. The principal measurement technique for these experiments was the conventional velocity interferometer (velocity interferometry system for any reflector [VISAR]). The basic issues addressed in this experimental run were whether a two-wave structure could be observed in the thin diamond samples and, if so, what is the HEL under shock compression for [110] diamond. We performed three experiments using this experimental configuration. We achieved pressures of $2 \mathrm{Mbar}$ and 2.5 Mbar in the diamond. In all cases, we observed a two-wave structure in the recorded wave profile. Based on the measurements, we determined that the HEL of [110] diamond crystals is approximately $900 \mathrm{kbar}$ to $1 \mathrm{Mbar}$. This is the first experimentally determined HEL measurement for singlecrystal diamond.

To improve the resolution of these measurements, which is necessary because of the high shock speeds and small diamond samples, we designed and partially assembled a lineimaging velocity interferometry diagnostic. We also developed an experimental design, utilizing the line-imaging VISAR diagnostic, that will maximize the data obtained from each experiment performed. These experimental targets are currently in fabrication.
Based on the measurements, we determined that the HEL of [110] diamond crystals is approximately 900 kbar to 1 Mbar. This is the first experimentally determined HEL measurement for single-crystal diamond. 


\section{5}

\section{The Use of Active Fiber Composites for the Health Monitoring of Wind Turbine Blades}

T. W. Simmermacher

Evaluating the structural integrity of composite structures, such as wind turbine blades, and metallic structures, such as aging aircraft, can reduce the cost of operation and extend the life of these structures. The use of active composite $(A C)$ material and laser-distributed sensors to assess the condition of operating structures potentially can provide advantages of large area coverage, conformability/ scanning, and ease of integration/testing. In this project, Sandia performs a combination of modeling and testing to allow the ac sensor to measure acoustic emissions and strains. In particular, the distributed-sensor concept will simplify damage detection by allowing monitoring of large structural areas. The distributed-sensor concept proposed here does not have as good sensitivity as conventional sensors, but the sensor is located over the whole structure and therefore will probably be close to damage. Being close to the damage site is an important factor in detecting damage.

We tested a sensor with nine nodes (small PZT [lead zirconate titanate] patches) for vibration sensing on a fiber glass panel that measures 36 x 48 x 0.125 inches. We used an external excitation (by hand) to vibrate the panel and recorded the response of the continuous sensor with an oscilloscope. We are investigating methods of interpretation of the data and applications for this sensor type.

We are also developing a model-independent boundary effect detection (BED) method for locating cracks and boundaries of nonuniform material distribution. Moreover, we are developing a method based on fracture mechanics for estimating crack depth using an energy approach.

We conducted a study on detecting the delamination of a graphite-epoxy laminate. The composite laminate is made of 3501-6 pre-preg tape and is impacted at the center with 2.48 pound-feet impact energy with a maximum load of 344.8 pounds. We use a random signal of $30 \mathrm{kHz}-50 \mathrm{kHz}$ to excite the laminate through an integrated 2" x 1" x 0.015 " PZT patch. A method of locating the edges of a delaminated area using boundary-layer solutions obtained from the BED method and stress intensity factors from fracture mechanics is under current
In this project, Sandia performs a combination of modeling and testing to allow the ac sensor to measure acoustic emissions and strains. In particular, the distributed-sensor concept will simplify damage detection by allowing monitoring of large structural areas....Being close to the damage site is an important factor in detecting damage. 
study, and we will perform more detailed tests and numerical analyses on this composite laminate.

In addition, we conducted a study on an electronic component using a laser-doppler velocimeter to determine the mass loading effects of sensors used to identify vibrational characteristics of the unit. We determined that the sensors significantly affect the vibration of the unit and that a model that simulates the test data would have to include the mass of the instrumentation.
We determined that the sensors significantly affect the vibration of the unit and that a model that simulates the test data would have to include the mass of the instrumentation. 
20416

\section{Information-Sharing Security Module}

L. A. Snyder, C. L. Beaver, L. M. Chavez, R. C. Schroeppel, W. D.

Neumann

This project researched security platform concepts for tools such as the Fast Analysis Infrastructure Tool (FAIT). Sandia concentrated on studying anonymous, authenticated communications and took an approach to the protocol design not previously considered. By protocol, we mean the procedures used to communicate securely. We offer an innovative combination of truly anonymous and revocable anonymous communications. The anonymous communications are authenticated to protect against unauthorized use of the system. However, anonymous communications open the door for undetectable system abuse. This can be dealt with by using revocable anonymity features, which may be unacceptable for competitive communicating parties. We employ a unique multilevel communication structure that mitigates system abuse, yet retains true anonymity at the highest level. Our scheme is modular and can accommodate different architecture designs as well as different levels of anonymity. We support authentication without anonymity, with anonymity, and with revocable anonymity. In addition to the research, we implemented an initial prototype of our design in software and provided a foundation for future research and development on this project. Providing anonymous authentication is the first step in support of a design for a full security platform for models such as the FAIT.

We developed a unique protocol for authenticated, yet anonymous, domain-to-domain communication. For the basic design of domain-to-domain anonymous communications, we first "anonymize" the message by a preprocessing technique that sanitizes the message content. Next, we create an encryption key and encrypt the message using a symmetric key algorithm. We create the key by using a combination of a random number from a compact disc (CD) and a special token that is issued daily by a trusted third party. We encrypt the message and send it to another domain. We use an onion router to obscure the electronic path of the message. In an onionrouting network, rather than connecting directly to a socket on the machine with which a user wants to communicate, the user will communicate by passing messages through a sequence of machines called "onion routers" that are situated between the
We developed a unique protocol for authenticated, yet anonymous, domain-to-domain communication. 
two communication endpoints. When a user wishes to initiate a conversation with another machine, the software randomly selects the sequence of machines through which a message will pass. All identifying information is removed from the data stream, and the onion-routing network provides end-to-end anonymity. Finally, when the receiving domain gets the message, it checks to see that a valid key was used to encrypt the message and, if so, the message is decrypted and authenticated.

We also described intradomain communications. We gave protocols for nonanonymous authenticated communication, revocable-anonymous communication, and truly anonymous communication. Revocable anonymous communication enforces anonymity unless compelling evidence requires that a user be identified. In that case, the anonymity can be revoked. In each case, malicious users inside a domain can be detected and reported.

Our designs allow for many configurations of a communication network and offer a variety of anonymity/ authentication options. We implemented an initial prototype of the domain-to-domain anonymous communication described, as well as the onion router in software.
Our designs allow for many configurations of a communication network and offer a variety of anonymity/ authentication options. We implemented an initial prototype of the domain-to-domain anonymous communication described, as well as the onion router in software. 


\section{0}

\section{Adaptive Sensor Optimization and Cognitive Image Processing Using Autonomous Optical} Neuroprocessors

S. M. Stewart, D. V. Wick, M. R. Ackermann, D. E. Bliss

Integration of on-board autonomous learning and adaptive control structures within a remote-sensing architecture would substantially improve the utility of intelligence collection by facilitating real-time optimization of measurement parameters for variable filed conditions. A problem faced by conventional digital implementations of intelligent systems is the conflict between a distributed parallel structure on a sequential serial interface that functionally degrades bandwidth and response time. In contrast, optically designed networks exhibit the massive parallelism and interconnect density needed to perform complex brainlike functions within a dynamic asynchronous environment. Recently, all-optical self-organizing neural nets exhibiting emergent collective behavior that mimics perception, recognition, association, and contemplative learning have been realized using photorefractive holography in combination with sensory systems for feature maps, threshold decomposition, image enhancement, and nonlinear matched filters. Such hybrid information processors depart from the classical computational paradigm based on analytic rules-based algorithms and instead utilize unsupervised generalization and perceptronlike exploratory or improvisational behaviors to evolve toward optimized solutions. These systems are robust to instrumental systematics or corrupting noise and can enrich knowledge structures by allowing competition between hypotheses. This feature enables them to rapidly adapt or selfcompensate for dynamic imprecise conditions that would be unstable using conventional linear control models. By incorporating an intelligent optical neural processor in the backplane of an imaging sensor, a broad class of high-level cognitive image-analysis problems, including geometric change detection, pattern recognition $(P R)$, and correlated feature extraction, can be realized in an inherently parallel fashion without information bottlenecking or external supervision. Using this approach, autonomous control systems embodied with basic adaptive decision-theoretic capabilities can be developed for imaging and surveillance sensors to improve discrimination in stressing operational environments.
[In this research], autonomous control systems embodied with basic adaptive decisiontheoretic capabilities [are being investigated] for imaging and surveillance sensors to improve discrimination in stressing operational environments. 
We successfully demonstrated a visual PR system using a joint transform correlator and cascaded novelty filter as a precursor to a fully parallel optical reconfigurable learning structure. Our design uses degenerate four-wave mixing in a photorefractive crystal located in the transform plane to obtain a motion-induced signature and subsequent correlation between test and reference images. We tested this basic system in the laboratory to baseline applications to autonomous geometric change detection, feature extraction, and defocusing compensation. The real-time correlation process is limited only temporally by the response time of the crystal and does not require external electronics or filters for efficient operation. We acquired optically addressable spatial light modulators to implement neural net patterns, and designed and used a phase conjugate resonator to provide feedback (gain) for associative memory and learning in the photorefractive material. We will evaluate learning and taxonomy with various network protocols as part of an on-going remote-sensing study for convergence, latency, and noise tolerance for a range of measurement parameters to complete this study. First demonstrations will involve focusing and light-level variables in the glint-detection process.
We successfully demonstrated a visual PR system using a joint transform correlator and cascaded novelty filter as a precursor to a fully parallel optical reconfigurable learning structure. 


\section{1}

Integration of Sandia Microsensors to the Internet for Logistics Applications

\author{
K. B. Pfeifer, R. W. Cernosek
}

Rapidly evolving technology coupled with new standards makes it possible to connect microsensors directly to the Internet. Connecting sensors to the Internet may be particularly useful in the military domain in a number of areas including logistics.

There are many applications for microsensors, which could be used to assess the readiness of a resource before shipping, during transport, and during use. Sensors range from viscosity monitors, battery condition indicators, indirect liquidlevel monitors, pressure sensors, gas and liquid monitors/ identifiers and many others. A number of these sensors could be applied to resource readiness applications. The applications might include items like monitoring the readiness condition of vehicle subsystems (viscosity of oil or battery condition), monitoring parameters of stored chemicals/foods to determine usability (gas or liquid analysis), tracking the location of a resource either at the local or global level (micronavigator), monitoring transport stresses (sensor suite), or various security applications (sensor suite).

Almost as important as having the right sensor is the ability to acquire the sensor information in real time. In today's environment, the Internet, by virtue of its presence, is the easiest means of providing information to an authorized user anywhere in the world - thus the motivation for Internetattached sensors.

Another benefit of attaching sensors to the Internet is the ability to analyze and compensate for a large number of sensors in real time. This project will develop hardware interface circuits and software objects consistent with industry standards to enable the operation of a Sandia microsensor on the Web. The health-monitoring technology demonstrated in this project can be applied to remote surveillance of critical nuclear components.

Sandia investigated three distinct types of Web architectures that allow the transfer of sensor data from a measurement point to a central location via the Web: (1) the simple Web server/client system using a commercial browser software, (2) developing a proprietary browser that has features such as the ability to save input data to disk, and (3) using the
This project will develop hardware interface circuits and software objects consistent with industry standards to enable the operation of a Sandia microsensor on the Web. The health-monitoring technology demonstrated in this project can be applied to remote surveillance of critical nuclear components. 
Web as a distributed serial cable. We implemented and tested each of these techniques and described their various advantages and limitations. In general, proprietary software must be written in order for data logging and storage to be accomplished. This is possible with the proprietary browser technique and by using the network-capable applications processor as a distributed serial cable interface in conjunction with a serial-based data-acquisition program such as HP-Vee. The simple client/server approach is not useful for this application.

\section{Other Communications}

Pfeifer, K. B., R. W. Cernosek, S. J. Martin, R. L.

Waldschmidt, and A. N. Rumpf. 2000. "An

Examination of System Architectures for Distributing

Sensor Data Via Ethernet Networks." Sandia

Technical Report SAND2000-3228, Sandia National

Laboratories, Albuquerque, NM.
In general, proprietary software must be written in order for data logging and storage to be accomplished. This is possible with the proprietary browser technique and by using the network-capable applications processor as a distributed serial cable interface in conjunction with a serial-based dataacquisition program such as HP-Vee. 
21272

\section{Automatic Target Recognition in Three} Dimensions

W. J. Bow, M. W. Koch, C. L. Smithpeter, R. O. Nellums, K. K. Green

Over the past decade, the DoD community has invested substantially in the development of automatic target recognition (ATR) technology, and the field has advanced rapidly as a result. While some military programs utilize onedimensional (1-D) range profiles for target recognition, the majority of research funding to date has supported ATR development for 2-D imaging sensors, like synthetic aperture radar (SAR) and infrared (IR). The current generation of ATR systems (including Sandia's Joint STARS SAR/ATR) enhances battlespace awareness in a variety of realistic scenarios. However, there is a growing consensus among ATR practitioners that solutions to more difficult problems, including the identification of vehicles that are significantly obscured from the sensor, will require 3-D data.

Sandia has developed the scannerless range imager (SRI), which is a prototype of next-generation LADAR (light amplification for detection and ranging) sensors. We will collect data with the SRI on various objects, including vehicles, with the goal of using these data to derive ATR algorithms that might exploit the kinds of 3-D information that the SRI sensor provides.

Utilizing the SRI sensor built under this project, we performed several data collections on test objects, people, and vehicles. The data collected will be useful in presentation materials that will be prepared for future opportunities to market the SRI sensor for applications in ATR and human presence detection in Safeguards and Security.

- We constructed the Sandia version of a 3-D camera for 3-D algorithm feasibility demonstration.

- We developed innovative 3-D sensor concepts for Safeguards and Security applications.

- We analyzed benefits of 3-D image data for ATR and developed new operating scenarios.

- We collected a baseline dataset to demonstrate new concepts and started an infrastructure to work with 3-D datasets.

\section{Utilizing the SRI sensor built} under this project, we performed several data collections on test objects, people, and vehicles. The data collected will be useful in presentation materials that will be prepared for future opportunities to market the SRI sensor for applications in ATR and human presence detection in Safeguards and Security. 
- We wrote code to convert raw SRI sensor data (angle, angle, range) to standard three-space "XYZ" coordinates for 3-D data visualization purposes.

- We wrote code to convert the raw SRI range data to a virtual reality modeling language (VRML) format.

- We demonstrated a derivation of a volume feature that we can use to develop pattern-recognition software to identify human presence. A future goal is to develop ATR classifiers that use feature sets (e.g., volume, width, height) to determine whether the object that has entered the scene is human or nonhuman.
A future goal is to develop ATR classifiers that use feature sets (e.g., volume, width, height) to determine whether the object that has entered the scene is human or nonhuman. 


\section{3}

\section{Modeling the Jini Infrastructure Protocol}

\section{L. Harris, M. E. Senglaub}

Command, control, and communication $\left(C^{3}\right)$ are evolving into more distributed, dynamic, and complex systems. These systems require advanced architectures to facilitate their implementation, and advanced modeling and simulation tools to capture these characteristics. A foundational work determining the basic simulation approach is essential prior to defining extensive model development programs. The goal of the research is to investigate the capabilities of a representative state-of-the-art modeling tool and a distributed computing architecture. We selected the OPNET (SMDPS [Strategic Mission Data Preparation System] Phase III Operational Network) model simulation tool, which is the principal network model simulation tool currently used at Sandia, and the Jini architecture, which currently is used by advanced military $C^{3}$ systems.

The approach to evaluating the techniques and requirements for modeling ad hoc, dynamically reconfigurable networks was to select a specific example of an emerging technique for implementing these networks. We selected a specific example of a state-of-the-art network model to determine the fundamental limitations to expanding the scope of the modeling approach. The network protocol and analysis tools selected for the study are the Jini networking architecture and the OPNET model simulation tool, respectively. The Jini architecture is an emerging standard beginning to see employment in military contexts. The OPNET model simulation tool represents the current state of the-art for Sandia's network modeling capabilities. The evaluation determined the utility of emerging simulation techniques for representing the detailed dynamics by which network services and clients identify each other, form ad hoc relationships, and evolve to a federation while other elements of the network evolve.

We successfully installed the Jini infrastructure and a software test system on a network of Linux machines. The test system generated network traffic that we subsequently monitored and analyzed to determine the packet load on the network. This analysis provided the basis for implementing an OPNET simulation model that could be used to perform

\author{
The goal of the research is to \\ investigate the capabilities of a \\ representative state-of-the-art \\ modeling tool and a distributed \\ computing architecture.
}


experiments on arbitrary configurations of networked Jini services and clients.

With the analysis and validation of each of the specific Jini element models, we can perform a range of "what-if" scenarios by changing attributes, network configurations, and any of the model's inputs. OPNET provides the capability to change network technologies and protocols and client/server attributes.

The Jini simulation components provide us a means to simulate countless scenarios that may benefit from the Jini infrastructure protocol. In addition, we can introduce elements that model the additional loads and stresses on a network.
OPNET provides the capability to change network technologies and protocols and client/server attributes. The Jini simulation components provide us a means to simulate countless scenarios that may benefit from the Jini infrastructure protocol. 


\section{5}

\section{Intelligent Targeting for Remote Weapons}

W. D. Morse, K. M. Jensen, J. J. Carlson, D. E. Small

There currently exists a diametric requirement to reduce the number of members of the armed forces while at the same time increasing overall warfighting capabilities. One technology that directly addresses this need is remotely operated weapons. Remotely operated weapons have the potential to offer significant force multiplication and enhancement by enabling near instantaneous response to attackers in many locations, increasing target accuracy, removing personnel from direct weapon fire, offering immunity to suppressive fire, and reducing the force size necessary to effectively fight and win. Sandia conducted a series of evaluation tests on commercially available remote weapon systems and identified key technology areas for improvement, one of which is intelligent targeting. To target and command the weapon, present systems are enslaved to a remote operator's joystick input. Intelligent targeting would enable the remote operator to select multiple desired targets within a cluttered scene containing miscellaneous objects and personnel, friend and foe. The system would be capable of autonomously tracking and monitoring target movements while computing real-time firing solutions. The low-level tracking and targeting details are thereby offloaded from the operator. This enables the operator to focus on high-level tactical and firing decisions.

The robots to be demonstrated have direct applicability to maintaining the security of the nuclear stockpile. Further, the control algorithms apply directly to the requirements for maintaining positive control of nuclear materials and infrastructure.

We successfully developed and demonstrated an intelligent targeting system (ITS) using Three-Dimensional Video Motion Detection (3DVMD). The ITS demonstrates how low-level, tedious, and difficult targeting details can be offloaded from the operator. This allows the operator to focus on high-level threat information and decision-making. A human operator remains in the loop to authorize weapon discharge, i.e., teleauthorization. The ITS monitors the scene from several points of view, triangulates to locate motion in the 3-D scene, and automatically target the weapon(s) on the intruder. ITS video and targeting solutions are fed back to the
Sandia conducted a series of evaluation tests on commercially available remote weapon systems and identified key technology areas for improvement, one of which is intelligent targeting....The robots to be demonstrated have direct applicability to maintaining the security of the nuclear stockpile. Further, the control algorithms apply directly to the requirements for maintaining positive control of nuclear materials and infrastructure. 
operator, enabling immediate situational assessment and determination of appropriate response(s) commanded by the operator.

The laboratory demonstration presents a 3DVMD The ITS successfully graphical portrayal of intruders to the operator as detected by the ITS in a hypothetical interior application. The resolution of the ITS 3DVMD is 1.5 inches per voxel, or volume element, which is presented to the operator as colored cubes. Each voxel corresponds to a unique 3-D position in the world coordinate system. We use different colors in the display to represent different heights. Using developed closed-loop 3-D machinevision tracking and targeting algorithms, the ITS 3DVMD calculates a firing solution for single-volume clusters within the monitored volume. We simulated physical targeting of generic weapons (lethal and nonlethal) using eye-safe targeting lasers mounted on pan-tilt units with firing authorization provided by the operator. The ITS successfully demonstrated how the operator can be offloaded from low-level targeting tasks and can focus on high-level decision-making while providing weapon targeting responses equal to or faster than human responses. 
21276

\section{Microfuel Reformer: Hydrogen for Microfuel} Cells

K. Wally, B. C. Wu, R. W. Crocker, S. F. Rice, C. M. Herdman

Portable power supplies are critical to the prolonged operation of miniature field devices in applications such as unattended ground sensors, man-portable sensor packages, small robot vehicles, even soldier-power systems for minimally manned warfare. Existing electrochemical battery technology, even the best state-of-the-art rechargeable batteries (e.g., energy density of $480 \mathrm{~W}$ hr/liter), simply can not meet the projected needs of most future applications. Sandia seeks a radically different approach to traditional batteries by using liquid organic fuels for energy. The small power systems we propose to develop would use common logistical fuels such as kerosene, methanol, or other alcohols or hydrocarbons. The concept is readily scalable from man-portable power $(<100$ We) to micropower $(<250 \mathrm{mWe}$ ) size. The systems would consist of a small-scale hydrogen (H)-gas generator coupled to a commensurately small proton exchange membrane (PEM) fuel cell producing electrical power. The H-gas generator would be a supercritical water microreactor functioning as a universal fuel reformer. This supercritical water reformer $(S C W R)$ is an innovation that we recently studied and plan to develop. It would reform logistical fuels and water into $H$-rich gas to fuel state-of-the-art PEM H fuel cells. Such cells are easily capable of direct-conversion efficiencies greater than 50\%. Energy for the endothermic reformer comes from product Hor system electrical output.

In the SCWR, liquid organic fuel and water are fed into the supercritical water $\left(\mathrm{T}=400^{\circ}\right.$ to $700^{\circ} \mathrm{C}, \mathrm{P}=3600 \mathrm{psig}$ ) where it is readily reformed to $\mathrm{H}_{2}$ and carbon dioxide $\left(\mathrm{CO}_{2}\right)$. An illustrative chemistry for methanol fuel is shown here:

$$
\mathrm{CH}_{3} \mathrm{OH}+\mathrm{H}_{2} \mathrm{O} \rightarrow \mathrm{CO}_{2}+3 \mathrm{H}_{2}
$$

The deficiency of other reformer processes is that carbon monoxide (CO) is not fully converted via the water-gas shift:

$$
\mathrm{CO}+\mathrm{H}_{2} \mathrm{O} \rightarrow \mathrm{CO}_{2}+\mathrm{H}_{2}
$$

Sandia seeks a radically different approach to traditional batteries by using liquid organic fuels for energy. The small power systems we propose to develop would use common logistical fuels such as kerosene, methanol, or other alcohols or hydrocarbons. 
The result is excessive $\mathrm{CO}$ in the product gas that poisons the platinum (Pt)-based electrocatalyst in the PEM fuel cell. With SCWR, the supercritical water appears to enhance the water-gas shift, and CO concentrations drop significantly.

We conducted a series of experiments to examine the feasibility of generating high $\mathrm{H}$ yields from simple fuels in supercritical water. We used a $400 \mathrm{~cm}, 1.06 \mathrm{~mm}$ inner diameter (ID) Inconel 625 coiled capillary as a flow reactor inside a tube furnace. We fed a $5 \mathrm{wt}$ \% solution of methanol through the reactor at variable flow rates with pressure maintained at 4100 psi. We analyzed gas samples by a residual gas analyzer (RGA) that we calibrated for the various gases of interest. We reported the fractions of the $\mathrm{C}$ gases relative to a normalized $\mathrm{H}$ yield of 1.00. We estimate conversion based on the concentration of methanol in the condensed and cooled aqueous liquid phase.

Although preliminary, the evidence seems clear that $\mathrm{H}_{2}$ / $\mathrm{CO}$ ratios greater than 50:1 can be achieved in less than one minute at a temperature perhaps as low as $540^{\circ} \mathrm{C}$. At lower temperatures, much of the $\mathrm{C}$ appears as $\mathrm{CH}_{4}$ (methane) and $\mathrm{CO}$. The high-temperature data agree very well with calculation, but the low- and intermediate-temperature data do not. It appears that the reforming process at intermediate temperatures (e.g., $550^{\circ} \mathrm{C}$ ) in supercritical water does not reach equilibrium for modest reaction times. The experiment shows formation of $\mathrm{CH}_{4}$ at low temperatures. These data suggest that an SCWR system can be operated to produce a product spectrum that has much more $\mathrm{H}_{2}$ and $\mathrm{CO}_{2}$ and less $\mathrm{CH}_{4}$ and $\mathrm{CO}$ than is predicted by equilibrium calculations. It is clear that reforming in supercritical water is controlled by three main processes: (1) conversion of fuel to simple gases, (2) competitive pathways to either $\mathrm{CO}$ or $\mathrm{CH}_{4}$ at low temperature, and (3) subsequent conversion of $\mathrm{CO}$ to $\mathrm{CO}_{2}$ by the water-gas shift reaction.

We also completed the thermodynamic efficiency calculations for the reformation methanol in supercritical water. The efficiency is normalized to the ideal reaction: $\mathrm{CH}_{3} \mathrm{OH}+2 \mathrm{H}_{2} \mathrm{O}=4 \mathrm{H}_{2}+\mathrm{CO}_{2}$ at room temperature. Two inefficiencies are estimated herein. The first is the thermal efficiency loss from heating the reactants up to the reaction temperature of $720^{\circ} \mathrm{C}$. We assumed no recovery of this heat nor heat loss in this analysis. The second is the chemical efficiency loss from the production of $\mathrm{CH}_{4}$ instead of $\mathrm{H}_{2}$, and the incomplete water-gas shift of $\mathrm{CO}+\mathrm{H}_{2} \mathrm{O}=\mathrm{CO}_{2}+\mathrm{H}_{2}$. We calculated the final chemical efficiency through a chemical equilibrium at the appropriate conditions. These results indicate
Although preliminary, the evidence seems clear that $\mathrm{H}_{2} / \mathrm{CO}$ ratios greater than 50:1 can be achieved in less than one minute at a temperature perhaps as low as $540^{\circ} \mathrm{C}$. At lower temperatures, much of the $C$ appears as $\mathrm{CH}_{4}$ (methane) and CO. 
that for our simple experimental setup, a maximum reformation efficiency of nominally $35 \%$ at a methanol concentration of 10 wt.\% was achieved. This relatively low efficiency is for an unoptimized continuous flow process. In future testing, we hope to focus on improving the reformation efficiency considerably. 


\section{7}

Advanced Techniques for Real-Time Visualization of Data-Intensive Missions

M. R. Platzbecker, T. E. Owen, B. R. Sturgis, G. W. Ashcraft, J. W.

Giron

A real-time, field-deployable, advanced visualization system is needed to enhance vital mission monitoring during missile launch and target deployment windows. Real-time visualization of deployed-instrumentation data would allow mission controllers, range safety, and system engineers to quickly see, comprehend, and react to mission events in an interactive three-dimensional (3-D) environment that is compatible with very short decision timetables.

Software tools are currently under development for post-flight data visualization, and their usefulness and reusability have been demonstrated on numerous space-based programs within Sandia. Unfortunately, with currently available processes, technologies, and software tools, data manipulation and visualization can begin only after data tapes are recovered post-flight for each specific mission. A number of research and development issues must be addressed before this capability can be fully defined and implemented. This project focuses on the first of these issues-how to display meaningful information in a real world of conflicting data input.

Outcomes will provide a greatly enhanced capability to perform the experiments necessary to validate the computer simulations used to pursue DOE's Science-Based Stockpile Stewardship.

Since real-time data interpretation can be computationally intensive, we completed work to verify that sufficient resources could be made available to support the real-time visualization. These initial applications visualized the motions of exoatmospheric reentry vehicles and used a simple rigid-body dynamic equation to bridge telemetry dropouts. These results demonstrated that the data-correction algorithm took only a few milliseconds of the tens of milliseconds available between data points. The applications made strong use of multiple processors, and they demonstrated the feasibility of more robust real-time data processing for visualization.

We decided to use generated data to act as the input to the Kalman filter and to the rigid-body dynamics equations.
This project focuses on ...how to display meaningful information in a real world of conflicting data input. 
This will allow us to concentrate on the filter algorithm and to compare results with known truth data.

The data consisted of the of the following sequence:

(1) a 10-second segment of clean data,

(2) a 30-second segment containing dropouts,

(3) a 30-second segment containing spikes,

(4) a 30-second segment of scale and bias errors (not activated at this point), and

(5) a 20 -second segment containing dropouts and spikes.

We generated these data using a simulation of a reentry vehicle in exoatmospheric flight. We corrupted a "perfect" data stream from the simulation by including dropouts and spikes according to the schedule above. The uncorrupted data stream was also provided to show the actual orientation of the vehicle in space (truth data).

We integrated the results from the Kalman filter, the rigid-body dynamics equations, and the truth data into an existing visualization tool in which reentry vehicle deployments are displayed for data interpretation. We believe that the Kalman-filtered data were a more accurate representation than the rigid-body dynamics data. By integrating these results with the visualization tool and adding in the truth model, visual depiction of the reentry-vehicle motion indicates that the Kalman model was much closer to the truth data than that of the previous rigid-body equation.

The above efforts will form a firm foundation for moving forward with real-time visualization of data-intensive scenarios. he above efforts will form a firm foundation for moving forward with real-time visualization of data-intensive scenarios. 


\section{8}

\section{Early Diagnosis of Human Exposure to Biological Agents}

J. S. Schoeniger, V. A. Vandernoot

This project is investigating a new way to detect very early human exposure to pathogens. Currently, methods to detect the biological agents primarily focus on detection of the agent in the environment. In many biodefense scenarios, there is a critical need to determine rapidly whether individuals have been exposed or not in order to determine treatment needs, extent of exposure, or even whether an otherwise undetected covert attack has taken place (using the humans as a back-up monitoring system). Diagnostic methods presently focus on identifying a specific causative agent. This approach requires that sufficient numbers of the organisms (or DNA [deoxyribonucleic acid] copies) be obtainable from an infected individual for growth in culture or tests such as polymerase chain reaction (PCR). For many organisms, this condition is not met until the organism has proliferated sufficiently to ensure substantial morbidity and mortality.

Rather than waiting for the organism to overwhelm the host and make itself apparent, Sandia is determining whether it is possible to detect signal molecules responsible for ramping up the host immune response. Our targets are phophotyrosylated proteins that are the earliest markers that signify activation of immune cells by antigens. The experimental plan was to construct antiphosphotyrosine (anti$P Y)$ affinity microcolumns through which we pass samples extracted from immune cells. The capillary microcolumns concentrate PY-proteins from several 100x column volumes of sample, achieving $>100 x$ concentration. We elute the concentrated proteins from samples of activated (i.e., by lipopolysaccharide or cytokine exposure) or unactivated cells from the column and further analyze them (i.e., by capillary zonal electrophoresis [CZE] /liquid chromatography [LC]/ mass spectrometry [MS]) to determine if their levels and the fingerprints of different proteins present differ significantly before and after activation.

This project increases Sandia's technology base to pursue the objective of microminiaturizing sensors and monitoring hardware for sustaining the nuclear stockpile and preventing the proliferation of nuclear technology and weapons of mass destruction (WMD).
This project is investigating a new way to detect very early human exposure to pathogens....Rather than waiting for the organism to overwhelm the host and make itself apparent, Sandia is determining whether it is possible to detect signal molecules responsible for ramping up the host immune response....This project increases Sandia's technology base to pursue the objective of microminiaturizing sensors and monitoring hardware for sustaining the nuclear stockpile and preventing the proliferation of nuclear technology and weapons of mass destruction (WMD). 
We obtained and evaluated two commercially available monoclonal antibodies (clone PY69 and PY20) as affinity matrices. Each was immobilized onto either $5 \mu \mathrm{m}$ nonporous or $7 \mu \mathrm{m}$ porous silica particles using standard silanization and cross-linking chemistry.

We prepared affinity columns (15 cm packed length) by slurry packing derivatized silica particles into $100 \mu \mathrm{m}$ inside diameter (ID) capillaries followed by equilibration with $10 \mathrm{mM}$ (millimolar) sodium phosphate, $\mathrm{pH} 7.4$ (running buffer). We blocked nonspecific protein binding sites by flushing with bovine serum albumin (BSA). We detected Cy5-labeled proteins with a portable laser-induced fluorescence $(\mathrm{LiF})$ detection system built in-house. Flow rates were typically $0.1-$ $0.15 \mu \mathrm{min}^{-1}$ (500 psi). Samples of either BSA or PY-modified BSA (typically $0.05 \mathrm{mg} \mathrm{ml}^{-1}$ ) were injected onto the column from a $20 \mathrm{nl}$ internal loop, and the bound protein was eluted with $1 \mu \mathrm{l}$ of $330 \mathrm{mM}$ sodium chloride $(\mathrm{NaCl})$ in running buffer. While more PY-containing protein appeared to be bound and eluted over the control protein (BSA), the high degree of nonspecific binding makes it unacceptable for this application.

Comparable experiments to those above were carried out using porous silica particles that have significantly greater surface area (and, consequently, bound protein). Studies showed it was much more difficult to elute protein quantitatively from these matrices, requiring as many as five injections of elution buffer.

In addition to high ionic strength, it is possible to elute specifically with phosphotyrosine; we found $100 \mathrm{mM}$ PY to be even more effective than $330 \mathrm{mM} \mathrm{NaCl}$ solutions. Elution peaks were larger, and there was little or no protein observed in a second elution peak, indicating full removal of bound protein from the column.

We obtained a commercially available cell lysate of human epidermoid carcinoma cells, stimulated with epidermal growth factor (EGF) for 5 minutes, to demonstrate the collection of PY proteins from cell systems and the rapid increase of PY proteins upon stimulation. Prior to use, we replaced the buffer as supplied with $10 \mathrm{mM}$ sodium phosphate, $\mathrm{pH} 7.5$ centrifugation and reconstitution. For work involving the cell extract, we carried out $\mathrm{LiF}$ using fluorescamine, a fluorogenic dye that reacts very rapidly with proteins, and a Krypton ion laser-based system.

The preliminary experiments showed very little specifically bound protein. Several possibilities exist. It may be that residual sodium dodecyl sulfate (SDS, used in gel ...we found $100 \mathrm{mM}$ PY to be even more effective than 330 mM NaCl solutions. Elution

peaks were larger, and there was little or no protein observed in a second elution peak, indicating full removal of bound protein from the column. 
electrophoresis of proteins) detergent from the commercial

preparation denatured the antibody, reducing its ability to bind PY-containing protein. Alternatively, the fluorescamine tagging may have interfered with the antibody-PY interaction. The most likely possibility is that the amount of phosphorylated protein in the sample is very low relative to the total amount of protein present in the cell lysate. It is possible that with a $20 \mathrm{nl}$ sample size, the bound protein is at concentrations below the detection limit. This would indicate that larger injection sizes will be more successful for the quantitation of PY-containing proteins. 


\section{9 \\ Multisensor Fusion for Characterizing Underground Facilities (UGFs)}

M. L. Yee, M. W. Koch, K. M. Simonson

The characterization and defeat of high-valued underground facility (UGF) targets require that their openings (adits) and umbilicals are addressed. A multiplicity of sensor modalities is required to characterize these adits. Appropriate sensor modalities include electrooptic imagers (EOs), synthetic aperture radar (SAR), hyperspectral imagers (HSIs), and a diversity of unattended ground sensors (UGSs). This diverse set of sensor modalities offers complementary information that can best be exploited through a multisensor data-fusion process. Such multisensor fusion, however, represents significant technical challenges due to the drastic differences in information content in the various modalities and potential temporal/spatial registration differences. As such, Sandia will investigate various approaches to fusing these critical disparate datasets.

Three novel multisensor data-fusion methods are candidate approaches for this problem of national importance. The candidate approaches are the following: (1) image/sensor overlay fusion, (2) probabilistic ATR fusion, and (3) evidential reasoning ATR data fusion. The first fusion method fuses sensor and imagery data by registering the spatial and temporal aspects of each modality and presenting the multidimensional information to the analyst. The resulting information includes time-histories of relevant adit activity, colocation of critical equipment, and spatial/temporal correlations across different modalities. Alternatively, evidential reasoning and probabilistic ATR fusion fuse information after ATR algorithms have been applied to each of the sensor and imaging modalities. These fusion methods allow disparate sensors to be fused to determine the adit activity, location, and relative confidence of the fused results.

In this project, we plan to investigate which of the three novel fusion algorithms, or combination of the three, is most appropriate for the UGF application. We will evaluate each of the candidate algorithms by applying them to available representative UGF datasets, assess the merits of each of the approaches, and demonstrate the value-added aspects of fusion.
In this project, we plan to investigate which of the three novel fusion algorithms, or combination of the three, is most appropriate for the UGF application. We will evaluate each of the candidate algorithms by applying them to available representative UGF datasets, assess the merits of each of the approaches, and demonstrate the value-added aspects of fusion. 
To demonstrate the merits of multisensor fusion, we searched available datasets for scenarios where coincident data from multiple sensors exist and where fusion could be expected to provide added value over the individual sensors. The datasets were taken during exercises at the Nevada Test Site (NTS) and consist of seismic, acoustic, and SAR data. There were three specific scenarios found to have enough data for examination in detail: equipment identification and tracking, position estimation, and UGF configuration characterization. Unfortunately, the principal candidate fusion methods could not be applied due to lack of quantitative results computed from the individual sensor data. In particular, the SAR data were not processed using any ATR algorithms and thus were unavailable for quantitative fusion, which prevented us from evaluating which fusion method is superior. However, we examined the SAR data visually in conjunction with processed seismic and acoustic results. This gave us a qualitative evaluation indicating that multisensor fusion could be useful for specific situations and applications.

Additionally, we investigated the possibility of using an analysis technique developed at Sandia, known as Knowledge Generation, for processing a sequence of multisensor data. The existing Knowledge Generation tool must be reworked to be applied to these particular datasets and scenarios. This again ruled out quantitative analysis. However, this approach shows promise for future work. We also examined theoretical methods for applying the other statistical fusion methods (evidential reasoning and probabilistic fusion) to sequence data. ...we investigated the possibility

of using an analysis technique

developed at Sandia, known as

Knowledge Generation, for processing a sequence of multisensor data. ....this

approach shows promise for future work. 
21280

Developing Land Surface-Temperature Maps from Multispectral Thermal Imager (MTI) Data

B. R. Stallard, L. A. Boye, J. R. Yoder, J. G. Taylor, D. D. Spencer, M. Hoffman, J. L. Smith

The multispectral thermal imaging satellite, MTI, developed by Sandia and Los Alamos National Laboratory, was launched in March 2000. MTI images in 15 accurately coregistered bands from 0.5 to 11 micrometers, with $5 \mathrm{~m}$ ground sample distance (GSD) in the four short-wavelength bands and $20 \mathrm{~m}$ GSD in the rest. A thrust of this development was the radiometric calibration of these bands. We are developing algorithms specific to MTI for producing land surface-temperature maps with unprecedented accuracy from a spaceborne sensor. In the thermal infrared (IR), the photons arriving at the sensor carry convoluted temperature and emissivity information that must be unraveled. We are producing pixel materials maps from the spectra in the 0.5 to $2.5 \mu \mathrm{m}$ region. We can then estimate the emissivity and calculate the surface temperature and heat capacity. Many targets of interest are subpixel in the thermal bands. Lack of data has limited demonstration of this approach with accurate pixel coregistration over a wide spectral range. We plan to improve the accuracy of the temperature map by using the spatial content of the small GSD bands of MTI and higherresolution images from other platforms to enhance the effective spatial resolution of the thermal bands. We are including collections of data from test sites where mock targets are imaged and ground truth is collected. Once the approach is verified, we will image other targets of interest. Accurate temperature maps can benefit many applications. Temperature maps are important in detecting and characterizing exit ports and other features of underground facilities. Maps may be useful to discriminate cloud cover from ice and snow. Temperature maps will provide information useful for DOE nonproliferation programs, military intelligence, surveillance, crop monitoring, meteorological and climate monitoring, forestry, and ecology.

We made considerable progress in our capability to exploit multithermal imager (MTI) imagery. We solved some of the preprocessing issues, especially in the area of band-toband co-registration. Usable, but still imperfect, data became available rather late, at which point we were able to obtain
The multispectral thermal imaging satellite, MTI, developed by Sandia and Los Alamos National Laboratory, was launched in March 2000....We are developing algorithms specific to MTI for producing land surfacetemperature maps with unprecedented accuracy from a spaceborne sensor...Accurate temperature maps can benefit many applications. 
some results in the areas of anomaly detection, target detection, and change detection. However, progress on thermal mapping We also were pioneers in doing was limited because we have not yet accomplished adequate radiometric calibration of the data.

We also were pioneers in doing classified tasking and classified tasking and processing. processing. 


\section{1}

\section{Enterprise Modeling of Medical Surveillance}

\section{Network}

D. E. Kataoka, M. M. Johnson, T. D. Plantenga, T. J. Sa, J. A.

Snavely, E. L. Hoffman, A. S. Yoshimura

Just as an integrated data sharing network has revolutionized investing, it is clear that such a network will revolutionize medical surveillance and epidemiology. Sandia's ongoing work on behalf of DOE and DoD has identified the broad requirements and quantified the potential benefits of more rapid indications and warning from an improved medical surveillance system for defense of citizens from biological attacks.

We seek an initial examination of requirements and utility for a secure, integrated communication network and ancillary components to improve medical surveillance. We are addressing two key issues: first, to qualitatively model a disease outbreak (from a chemical/biological [C/B] attack or natural epidemic) and its effect on the medical infrastructure, and second, to assess the requirements and functions of such a system.

This project will unify and extend Sandia's expertise in enterprise modeling and $C / B$ defense by providing the foundation for a quantitative tool for assessing improvements in medical surveillance.

We developed an application to demonstrate the functionality of an improved surveillance system. The application consists of two pieces: a database utility and a graphical display system. We wrote all software in JAVA to create a platform-independent application. Our first step was to acquire real data. We acquired a 3-year hospital discharge database, which consists of several million patient records. One drawback of this dataset was the lack of refinement in space and time; location was specified by 3-digit zip code and county and time by month.

We developed the database application in several stages. First, we wrote custom parsers to ensure that each record was composed of valid codes. Second, we built a database construction utility to enable queries. We used the JAVA Database Connectivity (JDBC) applicationprogramming interface (API) to create and populate a Microsoft Access database. Using the JDBC API facilitates rapid development of new databases as additional data become
Sandia's ongoing work on behalf of DOE and DoD has identified the broad requirements and quantified the potential benefits of more rapid indications and warning from an improved medical surveillance system for defense of citizens from biological attacks....This project will unify and extend Sandia's expertise in enterprise modeling and $C / B$ defense by providing the foundation for a quantitative tool for assessing improvements in medical surveillance. 
available. Third, we cross-linked data field codes with their descriptions to make the application user-friendly. The utility therefore provided an efficient and user-friendly tool to extract the desired data and display it in tabular form.

The graphical display system consists of two parts: a graphical geographic information system (GIS) that displays spatial distribution and a charting capability to produce graphs that display the temporal distribution of data.
The utility therefore provided an efficient and user-friendly tool to extract the desired data and display it in tabular form. 


\section{2}

\section{The Thermal History of Charred Materials by Raman Spectroscopy}

\section{R. Tallant, R. L. Simpson}

In a typical fire, organic materials are only partially converted to combustion gases. The solid material remaining is a form of graphite known as char. While its molecular structure is generally the same as graphite, the long-range order of char crystallites increases as a function of the temperature to which they are exposed. Raman spectroscopy, an inelastic scattering technique using optical photons, is sensitive to the variation in char structure with temperature. The goal of this project is to determine if a set or sets of Raman spectra can be used to determine exposure temperatures of char from diverse materials at a fire scene. Such a capability is expected to be useful in forensic analysis and research into fire causes and the dynamics of fires.

In consultation with experts at the National Institute of Standards and Technology (NIST) and the National Center for Forensic Science at the University of Central Florida, we selected a set of materials and exposure temperatures of greatest interest for forensic and fire research applications. The materials that we used in this study are a pine 2-by-4, plywood, OSB (oriented stand board, a high-tech plywoodlike material used in the construction industry), polyvinylchloride (PVC) pipe, nylon carpet, and Formica. The range of temperatures generally encountered in structure fires is from about $200^{\circ} \mathrm{C}$ to about $1200^{\circ} \mathrm{C}$. We charred pieces of the listed materials at temperatures from $235^{\circ} \mathrm{C}$ to $1140^{\circ} \mathrm{C}$ (the maximum temperature of the tube furnace available for these studies).

We obtained Raman spectra of the charred materials using $458 \mathrm{~nm}$ and $514 \mathrm{~nm}$ laser wavelengths for excitation. The results using $514 \mathrm{~nm}$ excitation generally provided superior differentiation with respect to exposure (charring) temperatures, and we used these spectra in the subsequent analysis. We also obtained Raman spectra using a near-infrared (NIR) $(785 \mathrm{~nm})$ wavelength for excitation and found interesting variations in luminescence and Raman bands. These variations are too complex to analyze adequately within the limited scope of this project, but they suggest an area for subsequent work.

We analyzed the spectra using multivariate partial leastsquares (PLS) techniques. In PLS analysis, the set of Raman
The goal of this project is to determine if a set or sets of Raman spectra can be used to determine exposure temperatures of char from diverse materials at a fire scene. Such a capability is expected to be useful in forensic analysis and research into fire causes and the dynamics of fires. 
spectra are decomposed into a set of component spectra. The component spectra can be scaled and summed to reproduce any spectrum in the original set. The scaling factors are different for each spectrum and are regressed against some parameter, in this case exposure temperature, to see if there is a correlation between the differences in the spectra and the parameter (exposure temperature). We found a strong correlation between the Raman spectra of char from five of the six materials and exposure temperatures when the spectra from each material were treated as a set and analyzed separately by PLS. The spectra from charred pieces of the pine 2-by-4, for example, form a calibration set capable of determining exposure temperatures of other charred pieces of pine 2-by-4 over the temperature range of $300^{\circ}-1140^{\circ} \mathrm{C}$ with an average error of $50^{\circ} \mathrm{C}$. Only the Raman spectra from charred Formica, which is a composite heavily loaded with inorganic materials, did not show a strong correlation with exposure temperature.

We then combined all the Raman spectra from the five materials (pine 2-by-4, plywood, OSB, PVC pipe, and nylon carpet) whose char correlated strongly to exposure temperature and analyzed the combined set by PLS. This combined set also correlated strongly with exposure temperature and provided a calibration that will determine the exposure temperature of char from any of these materials with an average error of about $60^{\circ} \mathrm{C}$. The strong correlation of the combined set of Raman spectra provides encouragement that we may be able to develop a single calibration set of Raman spectra that can be used to profile the exposure temperatures of nearly all the charred materials at a fire scene.
The strong correlation of the combined set of Raman spectra provides encouragement that we may be able to develop a single calibration set of Raman spectra that can be used to profile the exposure temperatures of nearly all the charred materials at a fire scene. 
21283

\section{Optical Interconnections to Focal Plane} Arrays

J. L. Rienstra, M. K. Hinckley

Sandia is investigating the feasibility of optical interconnections for the output signals of cryogenically cooled focal plane arrays (FPAs). We used an optical source and receiver on the warm side of the interface where power dissipation is not as critical as on the cryogenic side. A very low power optical modulation device on the cold side of the interface imparts the output signal to the free space optical beam.

As FPAs for satellite sensor systems become larger and faster, the power required for output-signal drivers becomes a limiting factor. When large-format, fast-framing arrays need to be cooled to cryogenic temperature, the power required to drive multiple output signals across low-thermal-conductance cryogenic cables becomes a significant fraction of the total cooling load. While advances in microelectronics technology promise further reductions in device size and electrical power dissipation, the power required to drive off-chip capacitive loads will remain nearly constant. In next-generation sensorsystem concepts, nearly one-third of the electrical-power dissipation of the FPA is due to output-signal drivers. This fraction will most likely continue to increase with larger and faster FPAs. By using optical interconnections for the signal outputs, we can reduce the active power dissipation of the FPA.

The objective of this project was to identify several concepts for focal plane optical interconnection and to demonstrate one of those concepts using an actual focal plane array (FPA) output. With both a preliminary demonstration setup and an optical interconnect breadboard, we successfully demonstrated transmission of a FPA output waveform over an optical link. The remainder of the project focused on characterizing the performance of the optical interconnect breadboard and analyzing the results to understand the key performance drivers.

The optical interconnect approach that we selected and demonstrated made use of a reflectance modulator fabricated with multiple quantum-well (QW) technology. We aimed a continuous-wave $(\mathrm{CW})$ laser source at the modulator and impressed the focal plane output analog signal on the reflected
Sandia is investigating the feasibility of optical interconnections for the output signals of cryogenically cooled focal plane arrays (FPAs)....The objective of this project was to identify several concepts for focal plane optical interconnection and to demonstrate one of those concepts using an actual focal plane array (FPA) output. With both a preliminary demonstration setup and an optical interconnect breadboard, we successfully demonstrated transmission of a FPA output waveform over an optical link. 
beam due to the modulator's voltage-dependent reflectance. The laser source was a temperature-controlled diode laser operating at $849 \mathrm{~nm}$. We fed the received signal into the same data-acquisition and -analysis system that we use for FPA characterization.

This project was very useful for

We performed a number of measurements to assess the characteristics of the optical link output signal relative to the focal plane array output signal. The signal-to-noise ratio was degraded a factor of 10 or more by the optical link, and the maximum output signal level was limited due to photocurrent generation in the reflectance modulator and the limited current drive capability of the focal plane output amplifier.

We analyzed the noise components of the optical interconnect breadboard and identified laser-intensity noise and photodetector noise as the limiting factors. The noise in the measured output signal that behaved as laser-intensity noise was higher than the direct laser noise that measurements would indicate. This discrepancy should be investigated further. Our making us aware of the design challenges involved with optical data links. By observing the performance of actual devices and analyzing their behavior, we are better able to refine this optical interconnection approach and to investigate alternative approaches. analysis of noise sources established performance goals for laser sources and photodetectors for future efforts.

This project was very useful for making us aware of the design challenges involved with optical data links. By observing the performance of actual devices and analyzing their behavior, we are better able to refine this optical interconnection approach and to investigate alternative approaches. 
21285

\section{Change Detection in Flash Memory}

T. Q. Thai, T. D. McConnell, K. L. Harrison, M. J. Jacobus

Flash memory is now used extensively in personal computers and network components to store critical software and system configuration. Currently, the process of detecting change in the flash memory content is time-consuming. The initial flash must first be obtained using special software. To detect any change, we must then compare the initial memory image with the current memory image. Every time the software of configuration stored in the flash memory is updated, the entire process is repeated.

Sandia originally planned to explore two different techniques to detect change to the flash memory content without modifying the existing hardware or relying on special software. The first technique is to use simple coil configuration to monitor the level of Eddy current on the write. The second technique is to deposit a thin layer of magnetic material that will respond to high current when the device is in write mode. We found both techniques to be unrealizable at this time.

We modified our approach to build a hardware prototype that connects to a few pins on the flash device to detect content change. The design allows the hardware to be used across a large number of flash memory devices. We have been able to verify this feature.

The objective of the project is to develop a method to monitor changes in a flash memory device. To successfully work with the many different types of flash memories on the market, we developed a general-purpose hardware device that can monitor and detect when the contents of a flash memory device are modified. We tested the hardware prototype on a computer BIOS flash memory in dual-inline pin (DIP) configuration for an example demonstration device. The hardware prototype consists of a custom-designed circuit board using a Microtek PIC that connects to selected pins on the flash memory device to monitor changes to the memory contents. The size of the circuit board is 0.555 " by 0.300 ", occupying less than $0.17 \mathrm{inch}^{2}$. When the circuit detects a change to the contents of the flash memory, the light-emitting diode (LED) on the board illuminates. The status of the change is saved in nonvolatile memory so that it is maintained through power cycling. A switch allows users to reset the hardware prototype.
The objective of the project is to develop a method to monitor changes in a flash memory device. To successfully work with the many different types of flash memories on the market, we developed a general-purpose hardware device that can monitor and detect when the contents of a flash memory device are modified. 
We developed the hardware prototype and completed the testing with a computer BIOS flash memory in DIP configuration. 
21287

Study of Effectiveness of SpectroPolarization Parameters in Military Target Detection

S. M. Gentry, M. R. Keenan

Polarization is perceived in the national community to be a vital component of the signature information for manmade targets of interest related to national security. The extent to which the spectro-polarimetric signatures are more indicative of man-made materials than simple polarimetric attributes is unclear. Is there more information in the spectropolarimetric image than in the traditional broadband polarization image? Sandia seeks to answer this question in the context of one of the most difficult target-detection environments-finding man-made materials under foliage cover. We believe that a systematic study would allow definitive suggestions regarding the impact of polarization parameters on target identification.

Sandia already has the basic instrumentation necessary, requiring minimal modification, to collect the vital spectro-polarimetric data needed for the study. The Information-Efficient Spectral Imaging Sensor (ISIS) instrument can be easily modified to become a precisionimaging spectro-polarimeter in the 0.45-1.0 $\mu \mathrm{m}$ spectral regime. Since the ISIS instrument covers the spectral region over which many materials and vegetation have important features, it is ideal for the task at hand.

Particularly useful to this effort are previous results from others that determined that circular polarization is almost nonexistent in natural and typical man-made scenes. While circular polarization is important for an absolutely general answer, it proves to be virtually superfluous for the question of detecting military targets in natural backgrounds. Therefore, measurement of two orthogonal linear polarization states is sufficient to capture the required information. The ISIS instrument with the proposed modifications measures these parameters directly.

We retrofitted the ISIS for spectro-polarimetric operation by modifying the Lyot depolarizer from $12 \mathrm{~mm}$ thick to $6 \mathrm{~mm}$ thick. This modification changed the spectral modulation of the signal to a level detectable with the $5 \mathrm{~nm}$ spectral resolution of the instrument. We made cursory
We believe that a systematic study would allow definitive suggestions regarding the impact of polarization parameters on target identification. Sandia already has the basic instrumentation necessary, requiring minimal modification, to collect the vital spectro-polarimetric data needed for the study. 
instrument calibration in preparation for the field test, including channel spectral responsivity and wavelength calibration. We participated in the PETSS (Polarization Effectiveness Test of Spectral Sensors) Spectral Polarization Imaging Test. We performed detailed instrument characterization, including verification of the spectro-polarimetric response as a function of linear polarization angle. This testing revealed that the hypothesized spectral modulation did not change character as a function of polarization angle as expected, making analysis of field-test data difficult. We modified a spectral-data

workstation to process classified data. We acquired preliminary data analysis indicating that the spectral modulation of the Lyot depolarizer needs further study. 


\section{Damaged Chip}

D. K. Kramer, T. J. Drummond, E. I. Cole, Jr.

This project addresses the need to be able to power up and operate microelectronic devices that have ceased to operate due to catastrophic events. Just as the recovery of the "black box" from downed aircraft can assist in determining why the airplane crashed, recovery, repair (if necessary), and subsequent operation of microelectronic components and avionics systems can be of equal importance in such an investigation. In such an accident scenario, a microelectronic part may be damaged in one of several ways, including impact and fire. Physical damage to the microelectronic devices can include damage to the package, burned or vaporized conductor lines, and breakage of the silicon (Si) chip.

Sandia has considerable expertise in the analysis of microelectronic operation and microelectronic failure analysis. The failure-analysis team demonstrated the capability to repair microelectronic devices on a localized scale using their in-house capabilities, including the focused ion beam (FIB) system (for localized deposition and removal of both insulator and conductor materials) and laser cutters (for quick localized removal of material). Such localized repairs are used to make design changes during the verification of new integrated circuit (IC) designs, and the modified chip is electrically characterized to ensure that the design changes accomplish the required modification in performance. These tools are also used in failure analysis, for example, to "create" a failure in a known good part and determine the effects on performance. We also successfully repackaged ICs when the original package was damaged. The purpose of this project is to begin to address the much more ambitious goal of repairing an IC that has sustained significant physical damage to the point that it cannot be operated. We will use intentionally damaged chips to address the specific problems of repairing melted/vaporized interconnect lines and "patching” cracked chips.

The damaged chip project assesses the ability to recover functionality in a damaged circuit. The two most likely damage mechanisms during a catastrophic event (fire, airplane crash, etc.) are thermal (burning) and mechanical damage. In the former, thermal damage harms the metal interconnects and possibly destroys transistor function in either a limited area or
This project addresses the need to be able to power up and operate microelectronic devices that have ceased to operate due to catastrophic events....Sandia has considerable expertise in the analysis of microelectronic operation and microelectronic failure analysis.... The purpose of this project is to begin to address the much more ambitious goal of repairing an IC that has sustained significant physical damage to the point that it cannot be operated. 
across the chip. In the latter, the die is cracked or broken, resulting in the severing of metal interconnects along the length of the crack and loss of transistor function for any transistor bisected by the crack. This study examined repair strategies for the case of mechanical damage.

It is known that damage to metal interconnects can be repaired if the damage is not too extensive. Damaged transistors can sometimes be recovered by thermal annealing if they have sustained electrical or perhaps moderate mechanical stressing. However, a transistor with extensive thermal damage or one that has been cleaved through will be very difficult, if not impossible, to repair. Thus, the likelihood of success of the repair of a particular damaged chip will depend on the details of how and where the chip was broken. Certain classes of chips are functionally generic and derive specific functionality from a program stored on-chip in nonvolatile electrically programmable read-only memory (EPROM). Restoring a damaged chip of this type would require only the recovery of the memory contents from the damaged chip and the availability of an unprogrammed, undamaged duplicate part for programming. This project assesses the constraints on the recovery of program memory from a damaged microcontroller as a test case.

The microcontroller chosen for the study was an ATMEL AT89C51 8-bit microcontroller with $4 \mathrm{kB}$ of flash memory. The benchmark test was to program an undamaged part and determine whether or not we could verify that the device had been programmed by providing only power and ground paths to the device. We were able to do so using microelectronics analysis techniques.

We prepared the "damaged" chip samples by (1) programming the chip, (2) removing the chip entirely from its package, and (3) cleaving the die through the memory block to simulate a crack. The separate pieces were repackaged into standard 40-pin packages. The larger of the two pieces contained the bulk of the microcontroller circuitry and the power and ground pads for the die. The smaller of the two pieces contained half of the memory and some of the addressing circuitry. For the larger piece, we bonded the power and ground leads to the package. For the smaller piece, we used the FIB system to create contact pads to the peripheral power and ground rails on the die; we used these contact pads for wirebonding to the package. Our analysis of these pieces indicated that the memory contents remained in the smaller piece containing only memory cells and some addressing
The benchmark test was to program an undamaged part and determine whether or not we could verify that the device had been programmed by providing only power and ground paths to the device. We were able to do so using microelectronics analysis techniques. 
circuitry. We were not able to determine whether data remained in the larger of the two pieces.

We drew several conclusions from this work. If the memory block of a damaged chip is intact, it is probable that the program data can be obtained in its entirety and its functionality instated in a duplicate chip. If the memory block is damaged, the situation is more difficult. It is highly unlikely that cells in which the transistors are damaged are recoverable. Blocks of memory for which the addressing circuitry is intact and can be powered can probably be recovered. Blocks of memory for which the addressing circuitry is not intact will likely be recovered only if damage in the memory circuitry is sufficiently limited that it can be repaired by conventional techniques. This implies the use of a tool such as an FIB system.
Blocks of memory for which the addressing circuitry is not intact will likely be recovered only if damage in the memory circuitry is sufficiently limited that it can be repaired by conventional techniques. 
21289

Engineered Window Glass for Architectural Surety Applications

S. J. Glass, C. S. Newton, R. G. Stone, E. K. Beauchamp

Currently there is wide interest in applications of glass in which control of the fragmentation behavior (smaller and controlled fragment sizes) and a significant reduction in the strength variability are required. These include architectural glazing to protect building occupants against terrorist bombings, removable valves, and Defense Program (DP) applications. A recent development in the processing of glass produces a glass with both high strength and reliability (i.e., narrow strength distribution). The glass is called engineered stress profile (ESP) glass. The stress profile in the glass is produced using a double-ion-exchanged process that requires high temperatures and long times. Additionally, Sandia made the initial developments on a special glass composition that is not commercially available. The major objective of the current project is to determine whether similar stress profiles and similar glass properties could be obtained using a more manufacturing-friendly process and using a commercially available soda lime silicate (SLS) glass.

The major accomplishment in this project was identifying the double-ion-exchanged process parameters that could be used to produce the desired ESP in SLS glass. We identified several sets of processing parameters that produce both high-strength and low-strength variability in SLS glass. They use a two-step ion-exchange process in which the first step produces a relatively deep ( 20-30 microns) layer with a higher concentration of potassium (K) ions than in the bulk glass and a second shorter exchange step in which some of the $\mathrm{K}$ ions are removed from the near surface area. We measured stress profiles for each of the sets of processing conditions, and they matched the desired profile, with the surface compressive maximum being located approximately 10 to 20 microns below the surface. The strengths were approximately 2.5 times higher than those for annealed SLS glass, and the coefficient of variation (one standard dev/mean strength) was $\sim 4 \%$, as opposed to $17 \%$ for the annealed glass and $27 \%$ for single-ionexchanged glass. The strength variability of the double-ionexchanged glass corresponds to a Weibull modulus of 45, significantly higher than the values typically found for glasses and ceramics (5-10). The sets of optimized conditions also
...Sandia made the initial developments on a special glass composition that is not commercially available. The major objective of the current project is to determine whether similar stress profiles and similar glass properties could be obtained using a more manufacturing-friendly process and using a commercially available soda lime silicate (SLS) glass. The major accomplishment in this project was identifying the double-ionexchanged process parameters that could be used to produce the desired ESP in SLS glass. 
produce the noncatastrophic multiple surface cracking prior to failure and insensitivity to surface damage as measured by tests in which the glass surface was abraded in a controlled manner prior to strength testing. Surface cracking begins at approximately $50 \%$ of the final failure stress.

\section{Refereed}

Abrams, M., and S. J. Glass. 2000. "Engineered Stress

Profiles in Soda Lime Silica Glass." Paper presented

for Sandia's Annual Student Symposium, Sandia

National Laboratories, Albuquerque, NM, August.

SAND2000-2299A.

Beauchamp, E. K. 2000. "Fracture Behavior of Double-Ion-Exchanged Glass." Proc. IV Conf. on the Fractography of Glass and Ceram. (Alfred, NY, July).

\section{Other Communications}

Glass, S. J. 2000. "Reliability of Ceramics and Other Brittle Materials: The Way It Is and Unique Opportunities." (Invited) Paper presented to New Mexico Tech, Socorro, NM, October.

Newton, C. S., S. J. Glass, E. K. Beauchamp, D. J. Green, M. Abrams, and V. Sglavo. 2000. "Engineered Stress Profile (ESP) Glasses: Breaking the Ceramic Reliability Paradigm." Paper presented to the 12th Annual Rio Grande Regional Symposium on Advanced Materials, Albuquerque, NM, October. 
21290

Layered Pyrotechnic Smoke Materials for Barriers

M. C. Grubelich, C. J. Greenholt, B. M. Melof

Security threats to Department of Energy facilities are escalating. These sites frequently identify the need for more advanced barriers that can provide additional delay against these escalating threats.

Pyrotechnically generated smokes can effectively obscure a large area very rapidly, with one volume of smoke mix yielding as many as 100,000 volumes of smoke. Pyrotechnic smokes are very persistent, especially if released in an enclosed area. The temperature of burning smoke mix ranges from $400^{\circ} \mathrm{C}$ to over $1,000^{\circ} \mathrm{C}$. Generation of hot obscurant precludes any further adversary attack, as well as preventing passage through any breached barriers still producing smoke. Sensory irritants such as CS (ochlorobenzylidene malonitrile) may be added to pyrotechnic smoke formulations to enhance their delay capability, but high concentrations of any pyrotechnic smoke are irritating or injurious to someone unprotected.

As seen above, pyrotechnic smokes possess a combination of favorable properties, allowing them to significantly increase the delay time of an adversary attack when used in conjunction with other physical-security measures. The primary disadvantage of current smoke systems is that they are activated systems. An activated system requires an input signal to function. The equipment used to evaluate a threat and send this signal is sophisticated, expensive, and subject to false alarms. A passive system is simpler, safer, and maintenance-free compared to an activated one. Passive smoke barrier systems will be adversary-activated by high-energy attack, i.e., an attack using explosives, thermal tools, or aggressive mechanical tools.

Of the many pyrotechnic smoke formulations considered, we chose the following mixture for field evaluation:

$51.8 \%$ ammonium perchlorate

$34.2 \%$ zinc powder

$12.72 \%$ HTPB (hydroxy-terminated polybutadiene)

$1.28 \%$ IPDI (isophorone diisocyanate)

Security threats to Department of Energy facilities are escalating....pyrotechnic smokes possess a combination of favorable properties, allowing them to significantly increase the delay time of an adversary attack when used in conjunction with other physical-security measures. The primary disadvantage of current smoke systems is that they are activated systems. 
This formulation is simple to mix and may be cast into many configurations. Due to the high binder content, the mixture is relatively insensitive to mechanical stimuli and thus is safe to process. None of the components is particularly toxic or expensive, and similar compositions have a long history of use as solid rocket propellants. Upon ignition, the mixture burns vigorously and liberates copious amounts of dense white smoke composed of zinc chloride, zinc oxide, and carbon.

We constructed steel test fixtures for field evaluation of smoke mixtures. The fixtures were made of square tubing 12 inches long and 1.5 inches wide. We welded a steel plate onto one end of the tubing to create a seal. We poured approximately 500 grams of smoke mix to a depth of 10 inches inside each tube and allowed it to cure overnight. We poured silica-filled epoxy into the remaining 2 inches of tube. We attacked the filled tubes by a chop saw with abrasive blade, 35 grains per foot linear-shaped charge, and detasheet explosive. All insults successfully initiated the smoke composition, which proceeded to liberate large amounts of smoke.

We constructed and tested a prototype smoke barrier panel. The panel was a square steel configuration of 2 feet on a side and 2.125 inches thick. We cast 7 kilograms of smoke mix into the panel and allowed it to harden. We poured a layer of silica-filled epoxy over the smoke composition to slow surface flame spread and increase the burn time of the panel. To simplify testing, the panel was center-initiated using an electric igniter. The panel generated an enormous amount of white smoke for over 3 minutes. The smoke cloud remained close to the ground and would definitely delay any further adversary attack, especially if deployed in an enclosed area.
This formulation is simple to mix and may be cast into many configurations. Due to the high binder content, the mixture is relatively insensitive to mechanical stimuli and thus is safe to process. None of the components is particularly toxic or expensive, and similar compositions have a long history of use as solid rocket propellants. 


\section{1}

\section{Miniaturized Flying Penetrator}

W. T. Gutierrez, K. T. Stalker, L. R. Rollstin, J. R. Phelan, S. M. Kohler, M. S. Howard, A. D. Foster

A need and opportunity exist for the time-urgent characterization and defeat of hard targets. Both conventional and nonconventional air-/sea-/ground-delivered attack options rely on improved understanding of the threat structure as well as optimal placement of defeat warheads. There are currently no missile systems in the inventory that deliver both sensors and warheads to accomplish target characterization and defeat.

A candidate to meet this integrated need is based on the Army Tactical Missile System (ATACMS). ATACMS is a ground-launched, fin-guided missile. In the conceptual engagement scenario, the ATACMS booster would seed the target area with a contingent of penetrating sensors released from ATACMS. Separation would be similar in nature to submunitions or multiwarhead reentry vehicles. During penetration and upon coming to rest, target characteristic information from delivered sensors would be relayed to targeting forces. This information would be utilized for warhead selection, fuzing, and targeting (position, depth of penetration). A second missile launch would then carry a warhead load of autonomous, guided penetrators for target defeat. Both the sensing and warhead penetrators would benefit from miniaturized airframes' inclusion of microdevice technology. This technology supports Sandia's mission to maintain the technical capability to sustain, upgrade, redesign, and, if required by national security policy, to design nuclear weapons.

The primary goal of the project was to examine the feasibility of developing a miniaturized maneuvering vehicle. In concept, we envisioned the vehicle system as approximately one-third the size (characteristic length) of the Sandia Technology Demonstration 2 airframe.

This preliminary study of a potential miniaturized flying penetrator (MFP) configuration supports an aerodynamic approach of no body lift enhancement (no strakes-control fins only). Such a configuration provides simple vehicle construction, relatively high impact speed, and no strakecontrol fin aerodynamic interference concerns, and eliminates potential strake-shroud mechanical interference in the missile

\section{This technology supports}

Sandia's mission to maintain the technical capability to sustain, upgrade, redesign, and, if required by national security policy, to design nuclear weapons. The primary goal of the project was to examine the feasibility of developing a miniaturized maneuvering vehicle. 
carriage configuration. A preliminary assessment of fin loads (bending and hinge) indicates that they are of reasonable magnitude and compatible with the potential mechanical design and the proposed miniaturized actuator control system.

We scoped a guidance and autopilot concept that meets the requirements. We identified mature technologies that could result in navigation, guidance, and control components consistent with the tail-kit volume. Specific components addressed included the inertial measurement unit, flight computer, digital actuator controller, global positioning system (GPS) receiver, fin actuator motor drives, a missile interface box, antennas, and batteries.

We conducted a mechanical layout, and the resulting dimensions and mass properties were consistent with the primary MFP goal for size reduction.

We identified several classes of penetrators, but did not scope them in detail as this was not a focus of this project.
We scoped a guidance and autopilot concept that meets the requirements. We identified mature technologies that could result in navigation, guidance, and control components consistent with the tail-kit volume.

\section{Other Communications}

Kohler, S. M., L. R. Rollstin, J. R. Phelan, M. S. Howard, D. L. Keese, and W. T. Gutierrez. 2000. "Miniaturized Flying Penetrator (MFP)." Sandia Technical Report SAND2000-2928, Sandia National Laboratories, Albuquerque, NM (November). 


\section{2 \\ Preconcentration of Volatile Chemical Agents and Explosives}

\section{L. Rhykerd, Jr., R. J. Kottenstette}

The Sandia preconcentrator functions by adsorbing the heavy organics from a large volume flow of air at room temperature and then, by heating the metal screen to $200^{\circ} \mathrm{C}$, desorbing those explosives into a much smaller flow of air. For high explosives, the collection efficiency has been measured at 40\%-70\%, and the preconcentration factor is about 1000x. However, the current metal screen preconcentration method is much less efficient at collecting volatile vapors like chemical agents (Sarin, VX, etc.), high-vapor-pressure explosives (nitroglycerin [NG], dinitrotoluene, and ethylene glycol dinitrate), and taggants. Several potential customers are interested in detection of these vapors at the part per billion level and lower (levels where preconcentration is necessary for detection), especially for preventing terrorism and force protection applications. Coating the metal screen in the current preconcentrator with a more strongly adsorbing material (perhaps Tenax or buckyballs) could greatly enhance the efficiency of collection and result in better detection systems with expanded capabilities. Such coatings must adhere robustly to the metal screen, be thermally stable during temperature cycles from $25^{\circ}-300^{\circ} \mathrm{C}$ several times a minute, and strongly adsorb volatile materials at $25^{\circ} \mathrm{C}$.

This is an experimental study to investigate coatings for improving adsorption of higher-vapor-pressure chemicals with Sandia's screen preconcentrator.

We coated the following materials onto stainless-steel screens: a sol-gel, OV-225, and Tenax. We conducted experiments with dimethylmethylphosphonate (DMMP) and nitroglycerin (NG) to investigate the adsorption/desorption properties of these coatings. This method for testing screen coatings appears sound, and the sol-gel coating shows promise to enhance collection of volatiles. Further investigation of these coatings is required before we can recommend specific coating and operating parameters.

We deposited the sol-gel onto 0.5-inch-diameter screens using liquid drop and spray deposition methods. Electron micrographs of the spray depositions look much more uniform compared to the drop depositions; thus the spray deposition method is preferred. We installed the 0.5 -inch screens in a
This is an experimental study to investigate coatings for improving adsorption of highervapor-pressure chemicals with Sandia's screen preconcentrator....We conducted experiments with dimethylmethylphosphonate $(D M M P)$ and nitroglycerin (NG) to investigate the adsorption/ desorption properties of these coatings. This method for testing screen coatings appears sound, and the sol-gel coating shows promise to enhance collection of volatiles. 
preconcentrator connected to an ion-mobility spectrometry (IMS) detector. We placed drops of acetonitrile doped with DMMP, a simulant for chemical agents, directly onto the screen, allowed the solvent to evaporate, and heated the screen $\left(100^{\circ}-200^{\circ} \mathrm{C}\right)$ to desorb the DMMP into the IMS detector. We identified peaks in the mobility spectra for DMMP (17.5 ms drift time) and acetonitrile (14.2 ms drift time). We detected 50 ng and 100 ng of DMMP, with smaller masses blending with the background noise. The sol-gel coating requires higher screen temperatures than the uncoated screen to desorb the analyte. Heating rate and maximum temperature of the screen appear to be critical variables, but there was insufficient time to systematically investigate these parameters. While this coating shows promise for enhancing preconcentrator performance, more experimentation is needed to determine the optimal parameters (screen temperature for desorption, coating thickness, and pore size).

We coated these materials onto 6-inch-diameter screens. We tested both for desorption behavior, placing drops of acetonitrile doped with NG onto the coated screen, evaporating the solvent, then heating the screen to desorb the NG into an IMS detector. While we identified the NG peak, even moderate heating $\left(100^{\circ} \mathrm{C}\right)$ of these coatings causes the coating to form drops on the metal, presumably due to poor wetting of the metal. Higher temperatures $\left(200^{\circ} \mathrm{C}\right)$ cause these drops to detach from the screen and create unwanted peaks in the detector. Thus, OV-225 and Tenax are not robust enough when coated onto stainless-steel screens to survive repeated high-temperature cycles necessary for a detection system.
While this coating shows promise for enhancing preconcentrator performance, more experimentation is needed to determine the optimal parameters (screen temperature for desorption, coating thickness, and pore size). 
21545

\section{Accelerated Beta Decay for the Reduction of Legacy Wastes}

M. S. Derzon, F. J. Zutavern

High-level waste (HLW) comprises over 60\% (500,000 cubic meters) of the DOE current inventory and projected generation of mixed waste over the next 5 years. HLW is generated and stored at several DOE sites. The majority of HLW is composed of radioactive materials that have forbidden beta (b) decays, like ${ }^{137} \mathrm{Cs}$ (cesium) and ${ }^{90} \mathrm{Sr}$ (strontium). The lowest order transition probability for beta decay in these materials is rigorously zero due to selection rules. These rules are not valid in a strong electromagnetic (EM) radio-frequency $(R F)$ field, which penetrates to the nucleus. Thus, the isotopes can be induced to beta decay if they are placed in an $R F$ field. The magnitude of the RF effect has been calculated $(H . R$. Reiss, Phys. Rev., pp. 1199-1243, 1983). The theory predicts that we may reduce the half-life of radioactive materials to allow for treatment of HLW on site. In particular, the theory shows that the strength of the effect depends on an intensity parameter, $z$, that is proportional to $(E / f)$ squared where $E$ is the electric field and $f$ is the frequency of the $R F$ field. We expect a maximum in the decay rate in the range $10 \mathrm{kV} / \mathrm{cm}$ $\mathrm{MHz}<\mathrm{E} / \mathrm{f}<1000 \mathrm{kV} / \mathrm{cm}-\mathrm{MHz}$. We designed an experiment to sweep through this range by independently varying $E$ and $f$. Some experiments have already been performed that show promising results, but are not definitive because they did not have sufficiently strong RF fields at sufficiently low frequency. Sandia performed a key experiment that did not verify the existence of the effect of $R F$-accelerated beta decay. We carried out experiments over a large range of field characteristics consistent with and more expansive than the fields and frequencies identified in the theoretical work.

We performed experiments that subjected a $\mathrm{CsCl}$ sample to RF fields that range from $1 \mathrm{kHz}$ to $90 \mathrm{kHz}$ at fields that were on the order of $13.2 \mathrm{kV} / \mathrm{cm}$. We used loads of 8 ohms, $400 \mathrm{ohms}$, and 10 mega-ohms to examine the effect of varying the ratio of electric to magnetic field. We measured for about 3 minutes, accumulating about 100,000 counts, then turned on the RF field for 15 minutes and counted for the last 3 minutes. The reason for this is to allow the metastable state (3 minutes) to be fully depopulated from the off state. The 3-minute measurement time was such that we were able to
High-level waste (HLW) comprises over $60 \%$ (500,000 cubic meters) of the DOE current inventory and projected generation of mixed waste over the next 5 years. HLW is generated and stored at several DOE sites.... We performed experiments that subjected a $\mathrm{CsCl}$ sample to RF fields that range from $1 \mathrm{kHz}$ to $90 \mathrm{kHz}$ at fields that were on the order of $13.2 \mathrm{kV} / \mathrm{cm}$. 
ascertain only if the RF field would cause $\sim 1 \%$ increase in the decay rate. We observed no such increase. In all cases, the observed radioactivity was the same (within statistical errors) whether the RF field was on or off. Testing is continuing to ascertain if there is any increase $>0.1 \%$. 
21548

\section{Kinetic-Energy Lightning Generator}

R. J. Kaye, M. S. Derzon, B. M. Marder

The concept of this project is to develop a kineticenergy zapper that can be used to deliver high-intensity directed-energy pulses into military targets. The potential advantage of this concept is that it can deliver highly concentrated energy focused in the target of interest. Sandia intends to model the relevant physics of the concept and to determine the credibility and feasibility of the concept. This project supports the following Sandia initiatives: pulsed power, Defense Programs (DP), energy and the environment, and high-power microwaves (HPMs).

At the onset of this work, the benefits of a kineticenergy-based lightning generator were easy to visualize, but the method of generating the high-energy pulse and coupling to a load were unknown. Our work focused on the development of this source and on a concept to couple the generated pulse to a load of interest. We have not analyzed a response to a broad range of loads, but we can easily follow from the circuit models developed to analyze the concept.

We conducted a survey of alternative pulse-generating systems to establish a reference for potential performance of the concept. Integration of the alternative systems with the kinetic generator concept appears to be a viable option for certain cases.

We created a detailed analytic model for two specific geometries for estimation of performance of the generator concept that fits a fixed volume. This model defines the elements of the electrical circuit that generates the high-energy pulse and makes analytic estimates of that circuit's performance. We consistently defined the components within a bounding geometry and included definition of size and weight. We estimated response range of the circuit components from analytic analysis or performance of published systems.

We developed time-dependent circuit models for the energy conversion stage and the switching stage of the concept to verify the efficiency factors used in the analytic system model. We performed analysis for one concept. Results show that the fundamental performance is correct, but very specific details of the component must be included to get an accurate estimate of conversion efficiency. Validation of these models with existing data must also be performed.
The concept of this project is to develop a kinetic-energy zapper that can be used to deliver highintensity directed-energy pulses into military targets....Sandia intends to model the relevant physics of the concept and to determine the credibility and feasibility of the concept. This project supports the following Sandia initiatives: pulsed power, Defense Programs (DP), energy and the environment, and highpower microwaves (HPMs). 
The system model identifies the complexity of the concept, particularly when attempting to self-consistently package the components in a fixed space. The model identified limits of performance that must be considered in the detailed design of the components. Overall system performance is governed by estimates of efficiency factors for conversion and switching stages.

Circuit simulations based on assumed behavior of source circuit elements yield a performance that may be of use.

Circuit simulations based on assumed behavior of source circuit elements yield a performance that may be of use. However, the construction details of the components must be included to get a more accurate determination of functionality. These circuit simulations also identify the need to optimize the electrical characteristics required for the application. Inclusion of the time-dependent geometry details in the circuit model is a necessary next step to evaluate the proposed concept. 
21550

Comprehensive Representation of Human Decision Process: Sensor Shooter Problem

E. M. Raybourn, S. M. Cameron, R. J. Pryor, R. L. Rinne, J. C. Forsythe

This project provides the basis for improved realism in simulating human behavior. There has been extensive effort devoted to developing computational models mimicking human behavior. Primarily, these efforts have employed either purely psychological models, low-level neural models, or task-based physical models. One of the key problems is how to realistically quantify, in a computer code, the underlying organic factors that influence behavior, i.e., combined psychological and physiological factors, such as arousal, fatigue, and stress. Consequently, existing computer models suffer from lack of correspondence to real human behavior and have limited applicability when off-normal, non-optimal conditions are expected or desired. This project developed an approach for quantifying the organic factors in ways that account for the different effects on decision-making and thus behavior. The resulting computational behavior model will be much more realistic than current models.

Human naturalistic decision-making has been conceptually modeled as a process wherein pattern recognition $(P R)$ based on environmental cues and knowledge leads to recognition of known situations, with appropriate behavior being implicit in this recognition. This project maps the processing of environmental cues and knowledge to quantified semantic processes, and situation recognition to quantified episodic processes. In the brain, these are two distinct processes with different electrophysiological properties. The model uses a semantic network to represent semantic processes and a PR template to represent episodic processes. Underlying each node in the semantic network, there is a neural assembly (i.e., a collection of individual neural units) that serves as a basis for an algorithm for cognitive processing. Similarly, underlying the PR template, there is a single neural assembly that serves as a basis for an algorithm for its processing. The computational model functions as neural assemblies oscillate at frequencies determined by arousal and by intrinsic and extrinsic stimulation. Cognitive processes are a function of the interplay between separately oscillating neural assemblies.
This project provides the basis for improved realism in simulating human behavior....[and] developed an approach for quantifying the organic factors in ways that account for the different effects on decision-making and thus behavior. The resulting computational behavior model will be much more realistic than current models. 
We made the following accomplishments:

- We identified key concepts for the simulation of the example problem via expert elicitation. We thus specified scenario events and event sequences.

- We identified and mapped key processes - the perceptual, semantic, and episodic processes.

-We developed a model that mapped physiological processes into the naturalistic decision-making psychological model. Supporting the model development was an extensive literature review.

- We used the comprehensive model to develop a computational model. We used the computational model for comparing results to published data.

- We defined organic factors for the demonstration. These included emotion, surprise, arousal, metabolism, expertise, stress, personality, fatigue, and culture.

- Using commercial software (MicroSaint), we performed a demonstration simulation. The original intent was to develop new software using genetic programming. However, for the purposes of a demonstration simulation, the commercial software was adequate and helped define future needs.

-We proposed a methodology for future applications.

- We identified long-term development needs, including representations of knowledge, learning, perceptual processes, group processes, and the need for specialized computational capabilities.

\author{
We identified key concepts for \\ the simulation of the example \\ problem via expert elicitation. \\ We thus specified scenario \\ events and event sequences.
}


21553

\section{Spectroscopic Detection of Pathogens}

M. K. Alam, D. M. Haaland, L. E. Martin, J. A. Timlin

The threat of biological terrorism has introduced an entirely new aspect to weapons of mass destruction (WMD). In the event of an exposure to biological agents, it will be important to rapidly assess that exposure. Current tests that detect exposure to agents rely on pathogen replication or host responses using tests that can take from days to weeks. Rapid detection of those infected is essential to triage treatment, to prevent death, and to limit the spread of the agent throughout the population. Sandia seeks to develop a rapid, highly sensitive screening mechanism that would detect exposure and provide a significant tool against bioterrorism. We conducted initial studies to demonstrate the potential of mid-infrared (mid-IR) spectroscopy to detect the initial cellular responses to infectious agents.

Initial data collected suffered from low signal to noise, primarily due to the poor purge and low cell counts. We resolved surge problems and reduced resolution, increasing signal to noise. We increased cell counts; however, the initial cell line that we examined, J774, suffered repeated culture death, resulting in several weeks without cells to examine. We introduced the RAW cell line as a substitute. We collected several weeks of data using this cell line from the unactivated cells, cells activated in situ using lipopolysaccharide (LPS), and cells pre-activated using a combination of LPS and gamma-interferon. Data collected from the cells activated using just LPS show little change from the unactivated cells.

However, transmission spectra collected from unactivated cells and pre-activated cells show distinct differences in their mid-IR signature.
The threat of biological terrorism has introduced an entirely new aspect to weapons of mass destruction (WMD).... Sandia seeks to develop a rapid, highly sensitive screening mechanism that would detect exposure and provide a significant tool against bioterrorism. We conducted initial studies to demonstrate the potential of mid-infrared (midIR) spectroscopy to detect the initial cellular responses to infectious agents. 
21554

Technical Solutions to National Security Issues Based on Limited Natural Resources

M. E. Tadros, J. E. Miller, M. D. Tucker, V. P. Gupta, M. S. Derzon, J. Paul

Systems-level solutions are needed to ease freshwater shortages in many regions throughout the world that could lead to conflicts and increased tension. The Department of Energy's (DOE's) Environmental Quality R\&D Portfolio FY 1999-2001 states that one of the three primary areas of responsibility of the environmental quality business line is to provide the technologies and institutions to solve domestic and international environmental problems. Scarce and contaminated water resources are a major environmental problem, both in many parts of the U.S. and in areas of the world that are central to U.S. national security concerns such as the Middle East, Mexico, and China. The DOE is one of the lead agencies with responsibility to help create and maintain the scientific and technological infrastructure that supports the nation's security and environmental integrity. This project focuses on the development of two new methods for water desalination: a method for water evaporation suppression from reservoirs, and a method for improving protected agriculture to reduce water consumption.

We submitted two technical advance disclosures:

(1) Cover materials to moderate the atmosphere in greenhouses and reduce water requirements. We built two greenhouses and are growing a fall tomato plant in them to obtain quantitative information on the cost and return on investment. We also developed a new approach for cooling the greenhouses to replace evaporative coolers that use significant amounts of water by solar energy-driven refrigeration units using variable-speed direct-current (dc) compressors.

(2) Surfactant formulation with improved watersuppression effectiveness under wind and wave conditions. We accomplished this by optimizing the ratio of long-, moderate-, and short-chain fatty alcohols to enable mobility and selfhealing of the monolayer.

We will continue to pursue the concept of using hollow-fiber membrane modules for desalination and purification of water based on the good initial results obtained.
Scarce and contaminated water resources are a major environmental problem, both in many parts of the U.S. and in areas of the world that are central to U.S. national security....

This project focuses on the development of two new methods for water desalination: a method for water evaporation suppression from reservoirs, and a method for improving protected agriculture to reduce water consumption. 
21557

Sustainable Bioelectronic Microsystems: Life on a Chip

S. M. Brozik, R. C. Hughes, C. J. Brinker

The sequencing of entire genomes is fueling a revolution in biological sciences and computational biology. The completion of numerous microbial genomes and the mapping and sequencing efforts of the Human Genome Project are launching a number of post-genomic challenges. Although there are very effective techniques to study gene expression, the same high-throughput systems do not exist in proteomics (the study of protein structure and function). Sandia will develop a whole-cell biosensor that serves not only as a highly sensitive, selective, and flexible detector, but also as a technology that will have a major impact in the area of proteomics. Our device will allow the simultaneous measurement of protein expression in cells, providing high-throughput insight into protein function.

Utilizing yeast, whose complete genome is known, we are using microarray analysis to identify genes that are regulated in response to specific stimuli. We will then fuse the promoters of these genes with green fluorescent protein (GFP) and introduce them into yeast cells to create multiple cell lines, each carrying a novel promoter:reporter fusion. Upon exposure to targeted compounds (toxins), the cellular response will occur at the genetic level, leading to gene expression, i.e., protein/reporter synthesis. Essentially, the analyte initiates a response in the cell such that the fused genes are expressed, and proteins that generate a fluorescent signal are synthesized. Using technologies developed in yeast, we will eventually construct a reporter system that is resettable and will adapt cell-cell signaling pathways to effect amplification in the system. We will utilize pattern-recognition $(P R)$ algorithms to determine multiple analytes simultaneously from each distinct cell line. Finally, we will construct a cell-based array on a microfluidic substrate. We will incorporate porous nanostructures currently being investigated into the microfluidic network to serve as cell immobilization matrices and to act as control microvalves to regulate liquid and gas flow.

Initially, we planned to plate neurons onto an electrode array and measure their electrical response to an external stimulus. Instead, we are utilizing yeast cells as a model system
The sequencing of entire genomes is fueling a revolution in biological sciences and computational biology....Sandia will develop a whole-cell biosensor that serves not only as a highly sensitive, selective, and flexible detector, but also as a technology that will have a major impact in the area of proteomics. Our device will allow the simultaneous measurement of protein expression in cells, providing high-throughput insight into protein function. 
and using an optical detection scheme to record the cellular response to specific stimuli. Based on the availability of the complete genome sequence of the yeast Saccharomyces cerevisiae, we plan to extend existing array-based approaches to study Saccharomyces cerevisiae RNA (ribonucleic acid) expression levels in response to a wide variety of analytes. Then we will (1) identify subsets of up- or down-regulated genes using novel gene expression analysis techniques, (2) fuse their promoters with GFP, (3) and transform them into yeast cells. We will incorporate these genetically modified yeast cells into microfluidic arrays where their unique spatial/temporal and optical/electronic responses can be used to identify a wide range of target analytes. Using arrays of whole cells, we expect to endow the sensor with a richer, more complex responsive behavior than can be derived currently from arrays of individual tethered oligonucleotides.

(1) We developed an optical detection scheme to detect GFP. Our University of Nnewn Mexico collaborator developed a compact fluorescence-detection instrumentation that allows simultaneous, multichannel, intensity, and lifetime-based detection of fluorescence-based biosensors. We are evaluating this fluorescence-detection system and calibrating the response of genetically modified yeast to a well-known system, the galactose/glucose system.

(2) We showed that stationary-phase yeast respond to external stimuli (specifically heat shock) and quantitatively measured gene expression levels in these cells, justifying the use of stationary-phase cells for our future studies with biotoxins. Prior to these experiments, it was not known what level of expression, if any, that cells in stationary phase would exhibit.

(3) We evaluated several immobilization schemes and successfully patterned yeast cells on quartz using polylysineand polyorithine-patterned surfaces.

(4) We trapped cells within porous silica nanostructures that we will use as materials in our microfluidic array. We are currently investigating materials that are biocompatible to fabricate the porous network since our initial attempts were successful in trapping the cells, but the viability count of the cells was low.
We showed that stationaryphase yeast respond to external stimuli (specifically heat shock) and quantitatively measured gene expression levels in these cells, justifying the use of stationary-phase cells for our future studies with biotoxins. Prior to these experiments, it was not known what level of expression, if any, that cells in stationary phase would exhibit. 
21558

Near-Real-Time Surveillance Against Bioterror Attack

M. W. Koch, S. A. McKenna, T. H. Karas

This project will open a major new avenue by which Sandia can address the national security problem of bioterrorism. The objective of this project is a distributed information system for gathering and analyzing public health data in near real time so that monitors can detect and characterize emerging diseases-including bioterror attacksmore rapidly than ever before. The central technical issue for this project is whether Sandia-developed signal-processing and statistical methods for identifying patterns rapidly in massive datasets can be fruitfully applied to these epidemiological missions. The control mechanism is a hypothesis-test approach. We are modeling, drawing on health records, the normal fluctuations found in the fields of interest. Based on this model we can scan current records and look for any anomalies. If any anomalous activity is found, we hypothesize a disease for this activity and use geographic, time, and environmental conditions to predict how the disease and symptoms will progress. If our predictions match future activity, we then assume that our hypothesis is correct. If our hypothesis is incorrect, we then need to generate a new hypothesis or sound the alarm of a potential biological attack. This methodology of hypothesis testing should be applicable to many other epidemiological research problems as well.

We focused on developing a discrete spatial-temporal SEIR simulator. We extended the concept of the SEIR model that was applied to populations, or somewhat loosely coupled subpopulations, to work on a population of discrete individuals located on a two-dimensional grid. We controlled the propagation of the disease across this grid and through time by an exposure likelihood function developed for this project from spatial covariance functions used in other fields. The exposure likelihood function controls the spread of the disease from one individual to surrounding individuals. We developed the connection between the exposure likelihood function and the reproductive ratio, $\mathrm{R}_{\mathrm{o}}$, used in the traditional SEIR model. We completed a series of numerical experiments using the discrete spatial-temporal SEIR simulator to determine the theoretical value of $\mathrm{R}_{\mathrm{o}}$ corresponding to different parameterizations of the exposure likelihood function. The current version of the
The objective of this project is a distributed information system for gathering and analyzing public health data in near real time so that monitors can detect and characterize emerging diseases-including bioterror attacks-more rapidly than ever before. [This project is researching] signal-processing and statistical methods for identifying patterns rapidly in massive datasets can be fruitfully applied to these epidemiological missions.... [and to] many other epidemiological research problems as well. 
discrete spatial-temporal SEIR simulator is capable of producing time histories of the progression of the disease for every discrete individual. We will use these time histories as the basis for assigning the proper syndrome-symptom-sign $\left(\mathrm{S}^{3}\right)$ data to each individual. To provide the $S^{3}$ data, we will link the simulator to a probabilistic $\mathrm{S}^{3}$ estimation technique. The addition of spatial variation in the initial levels of susceptibility can drastically alter the spread of the disease. Spatial variation in the susceptibility pattern can also allow for the spread of the disease, even when the theoretical $\mathrm{R}_{\mathrm{o}}$ value is zero.

\section{Other Communications}

Koch, M. W., S. A. McKenna, K. K. Green, and D. D. Boozer. 2000. "Near-Real-Time Surveillance Against

Bioterror Attack." Paper presented to the Sandia National Laboratories Disease Surveillance

Workshop, Albuquerque, NM, 6 June.

McKenna, S. A. 2000. "Spatial-Temporal Modeling of Infectious Disease." Paper presented to the Sandia National Laboratories Disease Surveillance Workshop, Albuquerque, NM, 6 June.
The addition of spatial variation in the initial levels of susceptibility can drastically alter the spread of the disease. Spatial variation in the susceptibility pattern can also allow for the spread of the disease, even when the theoretical $R_{o}$ value is zero. 
21559

Investigation of Modeling Approaches for Nuclear Futures

A. B. Baker, S. H. Conrad, L. A. Malczynski, G. A. Mann, W. E. Beyeler, D. L. Harris, P. E. Rexroth

The results of this project will be an integrated framework and a dynamic simulation model of key aspects of nuclear energy, nuclear materials storage and disposition, global nuclear materials management (GNMM), defense nuclear materials, and nuclear proliferation risk, and will link nuclear energy dynamically to global energy and greenhousegas emissions. Specifically, this project will "reduced-form" model the "back end" of the nuclear fuel cycle (waste storage and disposition), the "front end" of the nuclear fuel cycle (nuclear power generation), and the defense materials system (nuclear materials from weapons).

To provide for integrated energy and environmental systems integration, this project also will "reduced-form" model global energy demand by sector (industrial, residential/ commercial, transportation, and electricity), by fuel (oil, natural gas, coal, nuclear, renewables), and for global carbon emissions. The Defense Materials Module will "reduced-form" model the nuclear materials flow from weapons to the "back end" of the fuel cycle and will be benchmarked to credible sources as far as possible. Research will proceed in parallel for these main modules and for dynamic characterization of both proliferation risk and environment. Thus, this project will produce an integrated model of nuclear energy, energy-based carbon $(C)$ emissions, and GNMM of defense and civiliangenerated waste streams that will permit strategic assessment of integrated research needs and provide a tool for complex policy analysis.

There is a need for such an integrated model to explore policy and programmatic decisions for worldwide defense program nuclear materials, assurance of nuclear materials safety and security, and development and use of environmentally acceptable, reliable, and cost-effective energy. The interrelationships among these areas are complex and involve different decision-making metrics that must be integrated for sound U.S. national and international security, environmental, and energy strategies. This model can help identify where the largest systemic benefits can be achieved, with research dollars invested for simultaneously meeting national security and energy policy goals and objectives. ...this project will produce an integrated model of nuclear energy, energy-based carbon (C) emissions, and GNMM of defense and civilian-generated waste streams that will permit strategic assessment of integrated research needs and provide a tool for complex policy analysis.... This model can help identify where the largest systemic benefits can be achieved, with research dollars invested for simultaneously meeting national security and energy policy goals and objectives. 
We used an overall modeling approach and choice of dynamic simulation tool and "common currencies" for the model. We conducted a workshop to establish use cases for the dynamic simulation model. In the next phase, we designed a modular program architecture that will accommodate future revisions and improvements. We conducted literature searches of relevant models and data sources. Next, we developed conceptual models for each module, including energy production and generation of greenhouse gases, back end of fuel cycle, weapons production, potential for nuclear proliferation, and generation of materials that impact the environment. Finally, we constructed working versions of each module, populated the models with globally averaged data from valid sources, and integrated the modules into a working code that could be assessed and demonstrated. We demonstrated the model. These demonstrations resulted in generally favorable reactions to the model and also in a set of suggestions/recommendations. ...we designed a modular program architecture that will accommodate future revisions and improvements. 
22126

Understanding and Managing Threats to the Social Fabric of the United States

D. Engi, J. Espinoza

Terrorism is the use of force or violence against persons or property in violation of the criminal laws of the U.S. for purposes of intimidation, coercion, or ransom. One way that governments attempt to reduce our vulnerability to terrorist incidents is by increasing security at airports and other public facilities. However, there is apparently little attention given to a more subtle, yet potentially far more consequential, form of terrorism than bombing facilities and airlines. This form of terrorism would be based on creating an acute, accentuated, invidious sentiment among major segments of the citizenry of the U.S. This has the potential to have farreaching, profound effects and-if properly orchestratedcould essentially cause the U.S. to be unable to provide for the common good of its citizenry.

This project will develop an understanding of the meaning of undermining the social fabric of our society, the potential threats, the warning signals, and the viable countermeasures. It should be emphasized that this work is distinct from but complementary to Sandia's Critical Infrastructure Surety activity. The focus is not on what a malevolent agent might do to our infrastructures but rather what might be done to cause major segments of the citizenry of the U.S. to violently interact with each other.

The innovative features of this work are (1) the synthesis of an integrated picture of the potential threats to our society as seen through the eyes of myriad historically disparate perspectives, and (2) the development of a dynamic simulation that can provide valuable insights into the complex phenomena that serve as the "glue" for our social fabric.

We created a set of definitions and terms to identify and label the malevolent actors and ultraterrorists (UT) that would employ asymmetric warfare (AW) and other means to undermine the social fabric of the U.S.

We identified six states/groups as potential

ultraterrorists. We identified and assembled a 12-member panel to discuss these groups and to identify the groups' motivations, goals, likelihood of conflict, and timeframe for executing an ultraterrorist event.
This project will develop an understanding of the meaning of undermining the social fabric of our society, the potential threats, the warning signals, and the viable countermeasures. It should be emphasized that this work is distinct from but complementary to Sandia's Critical Infrastructure Surety activity. The focus is not on what a malevolent agent might do to our infrastructures but rather what might be done to cause major segments of the citizenry of the U.S. to violently interact with each other. 
Participants in the panel were a mix of national security experts, country/target group experts, experts on terrorism and disadvantaged populations, and so forth. The panel group determined the following ideological parameters with respect to the six identified terrorist groups: motivation, goals, likelihood of conflict, and timeframe. They also identified themes that the various terrorist groups had in common or that were considered significant and noteworthy. They constructed several "UTAW" scenarios and critiqued them for their feasibility and likelihood.

We produced a textual report documenting the discussions and outcomes of the panel meeting. It summarizes the panel discussions, the various themes that arose, and possible UTAW scenarios. The report identified the six terrorist groups, along with their motivations, goals, likelihood of conflict, and timeframe.

We also developed a dynamic model in real time. Using Powersim Constructor, the simulation model was developed during the meeting, capturing the gist of the various panelists' discussions, arguments, and counterarguments. The model has since been updated and modified to properly reflect the outcome of the panel meeting. Additional versions of the model were also created to test several UTAW hypotheses.

\section{Other Communications}

Advanced Concepts Group, Sandia National Laboratories. 2000. "Ultra-Terrorism and Asymmetric Warfare: Report of a Panel Discussion." Internal report, Sandia National Laboratories, Albuquerque, NM (September).

\author{
We also developed a dynamic \\ model in real time....Additional \\ versions of the model were also \\ created to test several UTAW \\ hypotheses.
}


22127

Physical and Functional Modeling of a Bioelectronic Microsystem

J. S. Wagner

Hybrid bioelectronic computational arrays offer a novel information-processing approach with the potential for superior cognitive, learning, and intuitive capabilities over conventional silicon ( $\mathrm{Si}$ )-based compute engines.

A key requirement in the development and utilization of such a bioelectronic system is the ability to control and optimize the processes of information storage, retrieval, and processing, as well as learning and reasoning, while maintaining the array in an optimal state of health. Issues associated with supervised versus unsupervised learning, life cycle and aging, and robustness against errors and failure modes also need to be addressed.

To understand and utilize these arrays, we need a modeling and simulation effort. This modeling effort could have at least two levels of complexity: (1) a physics-based model of the time-dependent electrochemistry and signal transmission of the array, and (2) a functional model of the array to explore information processing, reasoning, and complexity scaling.

We conducted a short Web-based survey on hybrid neural-Si computer chips. We now understand MEG-EEG (magnetoencephalography-electroencephalography) systems and analysis methods somewhat, which plays into the understanding of these systems as they get more complex. We explored computational methods of training recurrent neural nets with the hope of developing methods suitable for hybrid systems. This looks very promising. These time-dependent biomimetic artificial neural nets offer great promise. 
22128

\section{Analytic Models for Cooperative Utilization of Critical Resources}

M. L. Abate

This project will develop a model for cooperative utilization and allocation of critical resources. We will expand the previously developed model for verifying compliance with international treaties to a model that evaluates the risks, interactions, and trade-offs in cooperatively utilizing resources. The model for cooperative utilization of critical resources will be demonstrated by selecting a shared resource. The shared resource by members of the powerpool-both domestic and foreign-for this application will be both energy and reserve capacity. Although this activity will identify and demonstrate concepts via a specific case study, we will develop the methodology with a broader view toward synthesizing the science of utilizing shared resources. The methodology will be developed jointly by Sandia and Purdue University.

Developing analytic models for cooperative utilization of electricity addresses the Sandia strategic objective of advancing the surety of critical infrastructures by (1) identifying critical resources and infrastructures that are of national interest, (2) providing a method to evaluate the impact of shared resources on a nation, and (3) expanding Sandia's capability to provide significant input into future treaties, agreements, and protocols.

In collaboration with Purdue University, we developed electricity and gas trade models to determine patterns of gas and electricity shipments and capacity expansion plans. We developed these models to explore and explain the impact of trade and to develop equitable methods based on economic impact for cooperatively sharing electricity and natural-gas resources.

The developed electricity modeling methodology captures the dynamic interactions between customer demand, the utility's operating and investment decisions, and customer rates by cycling through the various submodels until an equilibrium is attained. The developed natural-gas model is a version of the standard dynamic production/transportation/ consumption model found in the mathematical programming literature.

\section{Although this activity will} identify and demonstrate concepts via a specific case study, we will develop the methodology with a broader view toward synthesizing the science of utilizing shared

resources.....we developed electricity and gas trade models to determine patterns of gas and electricity shipments and capacity expansion plans. We developed these models to explore and explain the impact of trade and to develop equitable methods based on economic impact for cooperatively sharing electricity and natural-gas resources. 


\section{Other Communications}

Sparrow, F. T., B. Bowen, and F. Smardo. 2000.

"Overview of Purdue Modeling Methodology and

Update of Efforts." Presentation by Purdue University to Sandia National Laboratories, Albuquerque, NM, 8 August.

Sparrow, F. T., B. Bowen, and F. Smardo. 2000.

"Overview of Purdue Modeling Methodology and Update of Efforts." Presentation by Purdue University to Sandia National Laboratories, Albuquerque, NM, 18 September. 
22129

\section{Cryopreservation of Biological Tissue}

R. C. Dykhuizen

Tissue engineering is the development of biological substitutes incorporating living cells and synthetic or natural materials and the fostering of tissue regeneration and/or remodeling for the purpose of repair, replacement, or enhancement of tissue function. Sandia will develop a computer model-based simulation of the thermal response of living tissues. We anticipate that the engineered tissues will have to be stored and transported in a cryogenic state.

In collaboration with Georgia Tech University, we will focus on the freezing of multicelluar tissue samples. The freezing of living tissues is complicated by the cellular structural and the complex chemical makeup of the material. Current models of the freezing of living tissue concentrate on single-cell models. In these models, both mass and heat transfer must be considered. As the liquid outside the cell freezes, pure ice forms, resulting in concentration of salts in the remaining liquid. Osmotic flows then develop that extract liquid from the cell in an attempt to equalize the chemical potential. This process continues until either the cell dehydrates or the cell's internal liquid solidifies.

The development of these models will allow Sandia to gain experience with complex chemical systems and also will identify the types of flexibility that will be required in developing a truly general system code for simulation of complex systems.

We identified that the appropriate type of data to be collected include (a) the size of cells and (b) the concentration of cryoprotective agent (CPA) as a function of radial position in spherical encapsulated cell constructs and following freezing/thawing procedures.

We performed a thorough literature search, which yielded no publications with experiments of the same type. However, we identified articles describing other useful studies, including the freezing of hepatocytes in different types of extracellular matrix.

We verified that an appropriate model system would be cells entrapped in spherical alginate beads and identified the appropriate experimental techniques for collecting the data mentioned above. We confirmed that calcium alginate gels can withstand freezing down to $-20^{\circ} \mathrm{C},-70^{\circ} \mathrm{C}$, or $-196^{\circ} \mathrm{C}$ (boiling
Sandia will develop a computer model-based simulation of the thermal response of living tissues....The development of these models will allow Sandia to gain experience with complex chemical systems and also will identify the types of flexibility that will be required in developing a truly general system code for simulation of complex systems. 
point of liquid nitrogen), followed by thawing to room temperature, without collapsing. It should be noted that, although no structural changes of the gels could be observed microscopically when freezing occurred to $-20^{\circ} \mathrm{C}$ or $-70^{\circ} \mathrm{C}$, such changes were noticed when beads were frozen to $-196^{\circ} \mathrm{C}$ and then thawed to room temperature. In no case, however, did the gels collapse.

To measure cell-size distribution in beads, we decided to use confocal microscopy. To measure the distribution of CPA, we decided it would be best to section frozen beads with a cryotome, then thaw the sections and assay the CPA amount in each.

We developed a realistic model that incorporates a number of changes and improvements relative to initial model version.

\section{Refereed}

Dykhuizen, R. C., and A. Sambanis. 2000. "Cryogenic Simulations of Tissue Freezing." Cryobiology, submitted.
We developed a realistic model

that incorporates a number of changes and improvements relative to initial model version. 


\section{1}

\section{Investigation of Nanoscience Technologies}

A. R. Burns

In collaboration with Harvard University, Sandia is conducting research in the general area of nanoscale structures, biomolecular materials, and their application in support of Sandia's microelectromechanical system (MEMS) technology. The expertise at Harvard is crucial in fostering these fundamentally interdisciplinary developments. Specific areas of research include the following:

(1) Nanofabrication, both exploiting traditional methods (mostly from silicon [Si] technology) and developing new methods (such as stamping or optical methods),

(2) Self-assembly, of both organic and inorganic systems,

(3) The dynamic response on the nanoscale: assembly and dynamics of membranes and microfluidics,

(4) Study of the hierarchy of scales in assembly,

(5) Innovative imaging methods, in particular, those based on scanning probes, and

(6) Hard (engineering)/soft (biological) interfaces.

These include actual physical interfaces (e.g., for manipulating cells), but also conceptual interfaces between the two disciplines.

In collaboration with Harvard University, we designed an experimental test station that Sandia will use to measure molecular transport through single nanopores. This experimental setup will allow for both biological and nonbiological membranes to be explored. We built the initial hardware and are developing the data-collection apparatus. We ...Sandia is conducting research in the general area of nanoscale structures, biomolecular materials, and their application in support of Sandia's microelectromechanical system (MEMS) technology....we designed an experimental test station that Sandia will use to measure molecular transport through single nanopores. This experimental setup will allow for both biological and nonbiological membranes to be explored. evaluated solid-state nanopore structures that were fabricated at Sandia using a unique edge-lithography approach. The solidstate nanopores are constructed from a silicon-based lithography process that results in orthogonal channels whose intersection creates a nanoscale pore feature. 
22132

\section{Magnetic Polysilicon MEMS Devices}

P. C. Galambos, M. Okandan

Microelectromechanical systems (MEMS) technology has been identified by federal government agencies as an area of national need and as a revolutionary emerging technology. Recently, MEMS showed great promise in the miniaturization of mechanical components in products from the most advanced weapons systems to automobiles. The goal of this project is to build a magnetically driven micropump in collaboration with Florida A\&M University-Florida State University. We will drive micromachines remotely utilizing magnetic field. In this technology, the MEMS devices are coated with magnetically responsive material and are driven using external, macroscopic magnets. The advantages of this approach are twofold: (1) there is no direct, visible, invasive connection between the macroscopic world and the microscopic world, and (2) the microscopic device becomes comparatively simpler to design due to the absence of energy-converting systems.

The main accomplishment of this project was the detailed design of several micropumps suitable for both magnetic and electrostatic actuation. We designed these micropumps for fabrication in polysilicon using the SUMMiT process.

The first pump concept is a type of gear pump that utilizes a planetary gear to generate areas of high and low pressure in a planar geometry. The external gear is driven either by an electrostatic torsional ratcheting actuator (TRA), developed by Sandia, an electrostatic comb drive, or an external magnet. The sun gear is offset from the center of the external (driven) gear. As the two planetary gears rotate around the sun gear, the area that they sweep out varies. Part of the swept area is reduced as the gears turn and therefore compresses trapped fluid and squeezes it out of the pump exit. Part of the swept area is increased as the gears turn and therefore pulls in fluid from the pump inlet. The pump inlet and exit are made through the rear of the silicon wafer using the Bosch etch process. We used a mechanistic model (Working Model 2D) to design this planetary gear system.

The second pump concept is a crescent gear pump concept. We used straight-toothed gears in this design for simplicity and because they have a better volumetric efficiency.
Microelectromechanical systems (MEMS) technology has been identified by federal government agencies as an area of national need and as a revolutionary emerging technology....

The main accomplishment of this project was the detailed design of several micropumps suitable for both magnetic and electrostatic actuation. We designed these micropumps for fabrication in polysilicon using the SUMMiT process. 
The final pump design concept is a type of viscous drag pump that is similar to a Von Karman viscous pump with a spiral sidewall attached. All three designs have several different parametric variations included in the modules to be fabricated. 
22136

\section{Autonomous Microexplosive Subsurface Tracing System}

M. C. Grubelich, S. H. Kravitz

The objective of the Autonomous Microexplosive Subsurface Tracing System is to image the location and geometry of hydraulically induced fractures in subsurface petroleum reservoirs. This system will be based on the insertion of a swarm of autonomous microexplosive packages during the fracturing process, with subsequent triggering of the energetic material (EM) to create an array of microseismic sources that can be detected and analyzed using existing seismic receiver arrays and analysis software. This technology has the potential for a major impact on the petroleum industry, which spends approximately \$1 billion per year on hydraulic fracturing operations in the U.S. alone. Improvements in hydraulic fracturing technology could result in 10\%-20\% cost savings in the fracturing operations and significantly increase U.S. producible reserves.

To realize the autonomous microexplosive technology, the following key research questions need to be addressed:

(1) How small a seismic signal can we detect and analyze?

(2) Can we trigger the explosive packages at appropriate times and under appropriate conditions and create an energy release that produces the right shape of seismic source within fracture/rock materials?

(3) Can we get sufficient volume of explosive and a reliable trigger mechanism into a package that is small enough to inject during the hydraulic fracturing process?

In Sandia's research into the second question, preliminary analysis identified a water-based initiation of an exothermic reaction as a reasonable solution to this question. Development of a specific trigger and EM combination depends on several factors, including physical and chemical conditions within hydraulic fracturing environments, rate and magnitude of energy release, and coupling of energy release with the surrounding fracture/rock environment. We are developing an apparatus to test the water-based initiation of an exothermic reaction and to determine if it will function correctly in the aqueous, high-pressure environment of a hydraulic fracture and have acceptable source characteristics.

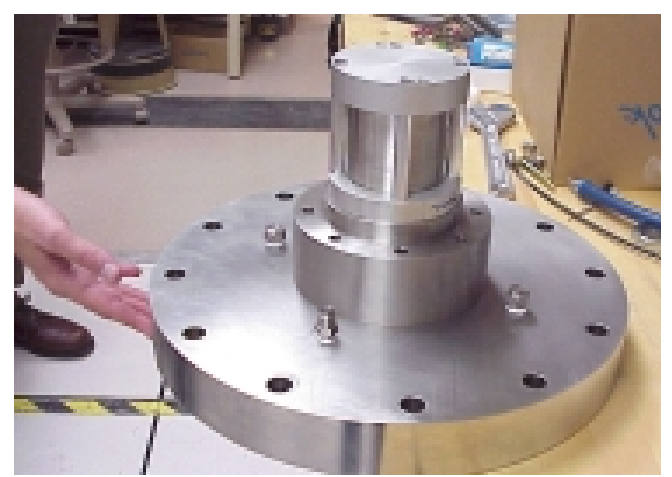

Top of the test section with the plunger injection apparatus attached to it. 
The focus of our work was the development of an apparatus to test the concept of microexplosive functioning. This apparatus is essentially a test chamber that is built to withstand the high pressure and dynamic loading of an explosive event, albeit a relatively small one. We built the test chamber to hold an autonomous microexplosive package, control the functioning of that package, and record the output of the subsequent event. The chamber is 10 inches inside diameter and 10 inches long, built of stainless steel with a design pressure rating of 5,000 psi. The end plates of the chamber are 2-inch-thick stainless steel attached with eight bolts.

The chamber can be used in many modes, but the first mode to be considered is that of reactive substances. The test chamber is built with an attached isolation disk that holds the reactive material between burst plugs. A compressed-airdriven ram pushes the material into the test chamber, allowing it to react with the resident fluid. To record the output of the package, we attached pressure sensors to the apparatus to record the subsequent waveform and rise time. We controlled the compressed-air ram remotely, as well as a drain valve to bleed pressure after the test.
The focus of our work was the development of an apparatus to test the concept of microexplosive functioning.

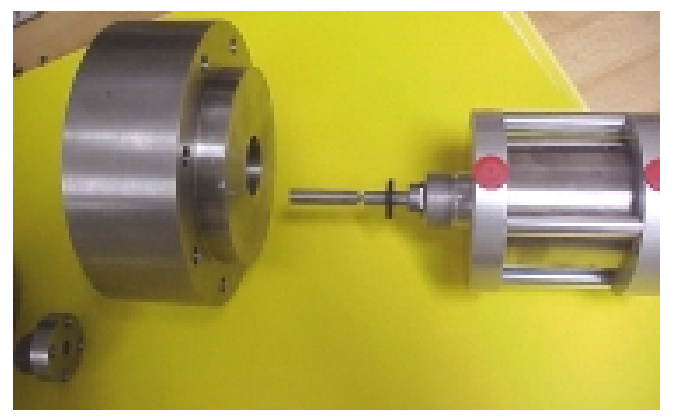

Plunger injection apparatus in more detail. 
22854

\section{New Metal-Organic Chemical Vapor Deposition Reactor Designs and Optimization}

N. A. Modine, R. R. Wixom

This project will advance the state of the art in new metal-organic chemical vapor deposition (MOCVD) reactor designs and development of next-generation diagnostic tools. A key strategic direction for Sandia and the DOE is development of integrated microsystems. This effort will stress the capabilities across the laboratory in microsystem component development and integration. A key element of this back-end integration at the silicon ( $\mathrm{Si}$ ) multichip module level will be an infrared (IR) vertical-cavity surface-emitting laser (VCSEL) to be produced via MOCVD. Unprecedented reproducibility and control of the MOCVD process will be required to certify the reliability and weapon-worthiness of the VCSEL devices. In collaboration with the University of Utah, we are designing and optimizing the next-generation MOCVD reactors and developing in situ diagnostic tools as process monitors.

We studied the (100) surface of gallium phosphorus (GaP) using the Vienna Ab Initio Simulation Package (VASP) first-principles electronic structure code. We trained in the use of planewave-based electronic structure methods, in general, and the VASP code, in particular. We used the local density approximation (LDA) to represent the exchange correlation potential, and we used Vanderbilt Ultrasoft Pseudopotentials to represent the atomic cores. We developed a procedure to accurately determine surface energies of reconstructed semiconductor surfaces. We based this scheme on the stable "extrapolate to zero thickness" approach developed by others. We studied the convergence of this procedure with respect to numerous technical parameters (e.g., planewave cutoff, k-point sampling, slab thickness, inter-slab vacuum thickness, dipole compensation scheme) and determined a set of parameters that would efficiently yield accurate results. We determined the surface energies of the B2 $(2 \times 4), c(4 \times 4)$, and $(4 \times 3)$ reconstructions of $\mathrm{GaP}$ as a function of the difference between the $\mathrm{Ga}$ and $\mathrm{P}$ chemical potentials. We determined the thermodynamically allowable range of chemical potential and constructed a phase diagram of the stable reconstructions. In agreement with experiment, we confirmed that the B2(2x4) reconstruction should be stable during organometallic vapor phase epitaxy (OMVPE), allowing for copper platinum (CuPt)
This project will advance the state of the art in new metalorganic chemical vapor deposition (MOCVD) reactor designs and development of next-generation diagnostic tools. A key strategic direction for Sandia and the DOE is development of integrated microsystems. This effort will stress the capabilities across the laboratory in microsystem component development and integration. 
ordering. We substituted varying amounts of antimony $(\mathrm{Sb})$ into the surface reconstructions and completed the initial steps toward the construction of a phase diagram as a function of $\mathrm{Sb}$ chemical potential. 
Composite-Wire Plasma Formation and Evolution

R. B. Spielman

Modeling of low-temperature (1-50 eV) and lowdensity $\left(<10^{17} \mathrm{~cm}^{-3}\right)$ current-carrying plasmas is difficult because of the interrelation of the plasma resistivity, joule heating, and plasma dynamics. Improvements in the known values of plasma resistivities in the parameter regimes of interest are helping this situation, but validations of the resistivity values when coupled with detailed code calculations are needed before real confidence in molecular hydrodynamics (MHD) codes can be achieved.

In collaboration with Cornell University, we are conducting a series of validation experiments in which we compare highly diagnosed wire explosions with predictions made by state-of-the-art MHD codes such as Sandia's ALEGRA code. These experiments will measure the detailed dynamics of wire explosions as well as the electrical heating caused by the applied current. We will compare these measured values against predictions made by the MHD codes.

To organize the work on comparing the experimental observations of single-wire explosions with the MHD codes, we collected time-resolved current and voltage traces during the heating and expansion phases of different wires as well as images framed during the expansion phase by $\mathrm{x}$-ray backlighting. We investigated several different materials: (a) insulated and bare 25-micron silver (Ag) wire, (b) bare 25micron gold $(\mathrm{Au})$ wire, $(\mathrm{c})$ bare 25 -micron copper $(\mathrm{Cu})$ wire, and (d) bare 25-micron zinc ( $\mathrm{Z}$ ) wire. These data are being used with the MHD codes to simulate the I-V characteristics and the attenuation and expansion rates indicated by the $\mathrm{x}$-ray images. In addition, we compared these measurements with MHD simulations.

We performed explosion studies on 17.8-micron alloy wire composed of $70 \% \mathrm{Ag}, 20 \% \mathrm{Cu}$, and $10 \% \mathrm{Au}$. During these experiments we made interesting observations using filtered x-ray diodes. We monitored diode signals during the liquification, vaporization, breakdown, and expansion phases of wire explosion. We used various filter densities to isolate the wavelength range of any observed radiation. While no significant $\mathrm{x}$-ray emission was recorded, a burst of light at the longest-wavelength sensitivity of the diode occurred at voltage ...we are conducting a series of validation experiments in which we compare highly diagnosed wire explosions with predictions made by state-of-the-art $M H D$ codes such as Sandia's ALEGRA code. These experiments will measure the detailed dynamics of wire explosions as well as the electrical heating caused by the applied current. 
collapse and then increased in intensity. This confirms the idea that essentially no plasma exists until voltage collapse and that after this time the current is shunted by this plasma, resulting in no further heating of the wire material.

A new low-inductance device for exploding single wires has become operational. The energy for driving the wires is provided by an 8-ohm, 30-foot transmission line charged to $15 \mathrm{kV}$. We took care to minimize the inductance of the wiremounting structure and the noise in the voltage and current monitors. Presently the voltage and current monitors are working well. Comparing the corrected voltage across and current through the wire, we can see four distinct phases of the wire heating and expansion. The wire used for the data in one phase had a 5-micron insulating coating; during the first phase, voltage built up across the insulation of the wire with no current flow. The insulation was finally punctured at about 4 $\mathrm{kV}$, and current began to flow in phase two. During this time we heated the wire, and its resistivity increased with time. The wire melted during this heating process. Phase three was characterized by a large voltage drop together with decreased load impedance. We attribute this voltage drop to plasma formation along the wire. During phase four, the resistance was below $1 \mathrm{ohm}$ and, although current on the order of a kiloamp was flowing, there was very little additional energy deposition. We are in the process of installing a laser interferometer channel for electron and neutral density measurements.

\section{Refereed}

Sinars, D. B., T. A. Shelkovenko, S. A. Pikuz, M. Hu, V. M. Romanova, K. M. Chandler, J. B. Greenly, D. A. Hammer, and B. R. Kusse. 2000. "The Effect of Insulating Coatings on Exploding Wire Plasma Formation." Phys. of Plasmas 7 (2) (February): 429_ 432.
This confirms the idea that essentially no plasma exists until voltage collapse and that after this time the current is shunted by this plasma, resulting in no further heating of the wire material. 
22996

\section{Scattering from Nanostructured Materials}

A. J. Hurd, K. Yu

Characterization strategies for some novel materials having new and sometimes surprising structure near the nanometer scale are in their infancy. Techniques will be developed specific to the determination of structure, including symmetry of nanostructured materials. These materials have a ceramic scaffold-sculpted with microscopic detail into complicated, ordered structures-surrounded by a polymer or vacuum phase. In this project, Sandia is performing smallangle $x$-ray scattering and reflectivity in collaboration with the University of New Mexico (UNM). The use of small-angle x-ray scattering is imperative, given the size $(10 \mathrm{~nm}-200 \mathrm{~nm})$ of the features. Fundamental questions exist about the form taken by the scaffold in evaporation-induced self-assembly (EISA), especially whether it is possible to make phases that are doubly connected so that a fluid can pass through the scaffold. Our goal is to develop crystallographic and powder protocols for films and particles of nanostructured silica.

Applications include sensors and separations in which a gas or liquid that invades the material interacts with it in some way, such as to separate into constituent molecules or to excite a sensor molecule attached to the scaffold. Such a sensor might be made sensitive to chem-bio agents, for example. A second application is for nanocomposite materials. Finely divided composites can have enhanced toughness, thermal insulation, and anisotropic electrical properties that can be exploited in microelectronics, optoelectronics, and protective coatings applications. Both energy and national security technologies require advanced materials that might be found by exploring nanostructured ceramics structures. Separation membranes for dividing mixed gases, such as methane and nitrogen $(N)$, have clear energy impact. Porous materials with high surface area and ordered pores have advantages for sensors of toxic and waste molecules. It is conceivable that thermal management materials, appropriate for thermal batteries or for radar systems, might apply to the nuclear stockpile.

EISA is a new technology pioneered by Sandia for the fabrication of films and materials with nanoscopic order (arrays of pores). These films have excellent prospects for use in sensors, membranes, pharmacy, and catalytic systems. We are
Sandia is performing smallangle $x$-ray scattering and reflectivity.... Our goal is to develop crystallographic and powder protocols for films and particles of nanostructured silica....EISA is a new technology pioneered by Sandia for the fabrication of films and materials with nanoscopic order (arrays of pores). 
studying an important potential application-ion exchange for environmental remediation.

Key aspects of the physics of pore formation are not well understood, and there exists a critical need for detailed structural information. Since the important structure is at a scale of $10 \mathrm{~nm}$ and larger, the technique of choice for sampling large numbers of pores is small-angle scattering, performed in collaboration with UNM.

We are addressing unresolved issues by design of critical experiments to measure mesophase order during processing. On a freestanding membrane from a sol-gel solution (peeled from a substrate or formed freestanding), we will study mesophase structure directly to determine the relationship between chemistry and order parameter.

Samples of block copolymer-templated materials show interesting structure at previously uncharted length scales. It appears that cubic arrangements of large pores, in the range of $100 \mathrm{~nm}$, are formed. In addition, we studied templated samples of layered double hydroxides by transmission electron microscopy (TEM) and some small-angle x-ray scattering (SAXS). We prepared a technical advance for these materials.
We are studying an important potential application-ion exchange for environmental remediation. 
22997

\section{The Liquid-Crystal Physics of Evaporation- Induced Self-Assembly}

C. J. Brinker, F. B. Van Swol

Evaporation-induced self-assembly (EISA) is a new technology, pioneered by Sandia for the fabrication of films with nanoscopic order (arrays of pores). These films have excellent prospects for use in sensors, membranes, pharmacy, and catalytic systems. However, key aspects of the physics of their formation are not well understood, and there exists a critical need for liquid crystal physics insights into the use of detergent solutions to template ceramics. EISA involves the evaporation of solutions containing dilute silica and surfactant. As the concentration climbs, the silica gels leave the silica to harden in the space surrounding the surfactant. Understanding these sequential transitions opens the possibility of controlling the structure of the resulting ceramic films via innovative tricks of the liquid crystal field, such as external fields and shear. This research will be completed in collaboration with the University of Colorado.

We developed plans for the optical system needed to study continuous self-assembly and implemented the design.

Among the accomplishments was the design and development of optical birefringence measurements for in situ determination of structure development during evaporationinduced self-assembly. The target design was determined to be an optical microscope with a horizontal optical path and a continuous dip-coating apparatus as a sample. Substrate birefringence will be an issue: flexible polymer tapes may not be adequate. If so, batch-type coating experiments on silica substrates will be the backup.

Theoretical work included discussions and preliminary calculation of the phases both seen and expected in scattering and imaging experiments. It may be necessary to seed the system with an additional molecule to boost birefringence.
Evaporation-induced selfassembly (EISA) is a new technology, pioneered by Sandia for the fabrication of films with nanoscopic order (arrays of pores)....Among the accomplishments was the design and development of optical birefringence measurements for in situ determination of structure development during evaporation-induced selfassembly. 
22998

\section{Electrochemical Interrogation of Cellular Membrane Transport Processes}

A. W. Flounders, R. V. Davalos

Electroporation is the controllable and reversible permeabilization of a cellular membrane by applying an electric field. Electroporation has been used as an electrically controlled technique for inserting materials into cells. For example, electroporation can be used to insert foreign deoxyribonucleic acid (DNA) into host cells to modify protein expression or to inject cytotoxic agents into cancerous cells to control tumor growth. To date, electroporation has been performed on large populations of cells and hence has been difficult to characterize. The ability to interrogate single cells enables significant advances in understanding electroporation by allowing controlled measurement of the electric field strength necessary for permeabilization and the flux of species through the membrane during electroporation.

In collaboration with researchers at the University of California-Berkley, Sandia is monitoring electroporation with picoamp sensitivity over a range of frequencies via impedance spectroscopy (IS). By analyzing the frequency response of a system, IS identifies a combination of ideal circuit elements that correspond to the pattern of interconnectivity observed during the experiment. These ideal circuit elements suggest physical mechanisms associated with the system. There is presently no accepted model of electroporation, and IS may provide significant insight into the physical mechanisms of the phenomenon. In addition, we will begin to correlate transport of cellular contents across an electropermeabilized membrane with applied voltage and pulse duration. Rather than trying to inject material into the cell, our efforts will focus on extracting material out of a cell-electroextraction. In its simplest form, this electroextraction process serves as a convenient method for removing the entire contents of a single cell.

Electroextraction may provide a technique for selective removal of specific cellular components or lead to a method for time-dependent analysis of cellular regulation and expression.

We trained Sandia personnel in all fabrication equipment and procedures and initiated fabrication of a separate SCED for use at Sandia. We set up all necessary impedance analysis equipment. The project will leverage a capital equipment purchase of a dielectric interface analysis
Rather than trying to inject material into the cell, our efforts will focus on extracting material out of a cell-electroextraction. In its simplest form, this electroextraction process serves as a convenient method for removing the entire contents of a single cell. Electroextraction may provide a technique for selective removal of specific cellular components or lead to a method for time-dependent analysis of cellular regulation and expression. 
system. This equipment is able to measure resistances in the teraohm range and should be ideal for membrane characterization prior to electroporation. In addition, we set up a custom LabView program and tested it for automated data collection.
This equipment is able to measure resistances in the teraohm range and should be ideal for membrane characterization prior to electroporation. 
24101

\section{A Micromethanol Steam Reformer for a Hydrogen-Based Microfuel Cell}

R. J. Shul

In collaboration with Lehigh University, Sandia sought to build a micromethanol steam reformer (MMSR) to convert methanol and water into hydrogen $(H)$, with carbon dioxide $\left(\mathrm{CO}_{2}\right)$ as the by-product. The $\mathrm{H}$ would be used as fuel. We developed a planar microfuel cell designed to use $H$ and air as its two fuels. Typically, $H$ is stored as a metal hydride, which contains less than $1.8 \% \mathrm{H}$ by weight. This low level of storage makes a microfuel cell appear volumetrically inefficient. An MMSR would have much less volume and would require replacement only of a small methanol fuel cartridge, rather than a bulky hydride container. An MMSR would be based on a well-known chemical reaction in which methanol would be heated to yield $\mathrm{H}$ and carbon monoxide $(\mathrm{CO})$. Since $\mathrm{CO}$ will poison the fuel-cell catalyst, it must be removed. A second reaction is to heat the gases with water (steam), which causes the $\mathrm{CO}$ to be converted to $\mathrm{CO}_{2}$ and more $\mathrm{H}$ to be produced. The gases would be filtered through porous palladium $(P d)$, which allows only $H$ to pass. The MMSR would utilize the technology developed in the ChemLab project, such as the micro-hotplate for creating steam and silicon ( $\mathrm{Si}$ ) microchambers for reactions and gas handling. The heat created by these reactions would be removed with our patented micro-heatpipe technology. This combination of unique Sandia capabilities makes a microsteam reformer a winning combination both technically and economically.

We initiated technical discussions for the design and fabrication of an MMSR to convert methanol and water into $\mathrm{H}$, with $\mathrm{CO}_{2}$ as the by-product. We used a schematic diagram of a methanol reformer developed by our collaborator. The design included an evaporator as well as a shift-reformer section for the on-chip system. The shift reformer incorporated a thin-film nitride membrane with lithographically created porosity and with a Pd thin film deposited on top of it. We would use the Pd thin film to prevent the reformate from containing $\mathrm{CO}$, which would damage the downstream fuel cell. Pressure required to maintain the mass flow through the system will be provided externally. Sensors and control systems will be required to maintain the necessary flow rates, temperatures, and pressures.
...Sandia sought to build a micromethanol steam reformer (MMSR) to convert methanol and water into hydrogen $(H)$, with carbon dioxide $\left(\mathrm{CO}_{2}\right)$ as the by-product. The $H$ would be used as fuel. Sandia developed a planar microfuel cell designed to use $H$ and air as its two fuels....This combination of unique Sandia capabilities makes a microsteam reformer a winning combination both technically and economically. 
We are ready to begin technical discussions for the design and fabrication of the heating units needed for both sections of the system. 
24102

Spatial Anomalies in Magnetically Insulated Vacuum Structures

J. P. Vandevender, L. Lorence, C. L. Ruiz

Sandia will investigate anomalies that might lead to a new energy source. Reviews by Sandia experts indicate that the anomalies are inconsistent with current knowledge and have no apparent explanation. Magnetically insulated transmission lines and ion diodes have sufficient magnetic flux between the cathode and anode to insulate electrons that originate on the cathode from reaching the anode. These devices have demonstrated excellent insulation efficiencies in operational facilities for over 20 years. However, magnetic insulation data from the Mite and HydraMite high-power magnetic-insulation experiments of the late 1970s show the following anomalies:

- Since electrons should originate at the cathode, they should reach the anode with approximately the full gap-energy of 2,000 KeV. However, there are seven times as many lowenergy (< $200 \mathrm{KeV})$ than high-energy $(\sim 2000 \mathrm{KeV})$ electrons collected at the anode.

- If the local measurements of loss-current density are representative of the entire line, the low-energy electron loss to the anode is an order-of-magnitude larger than the total charge lost between the input and the output.

- The time-resolved energy/nucleon of negative ions, as diagnosed with a time-of-flight (TOF) spectrometer through the anode, often disagrees with the full-gap voltage, e.g., proton energy of $500 \mathrm{KeV}$ with $2000 \mathrm{KV}$ across the vacuum gap.

- The time-integrated energy distribution of negative ions coming through the anode has several distinct peaks in energy, even though the potential across the gap is quite constant.

- The pattern of electron loss to the anode is approximately circular and without any indication of the expected magnetic deflection.

- The spatial distribution of the ion loss to the cathode is very circular and is sometimes hollow.

The anomalous data are most likely from a combination of diagnostic problems and yet-to-be-identified, but conventional, physical phenomena. Energy production is certainly one of the possible applications, but this could lead to a new fundamental understanding of physical phenomena that
Sandia will investigate anomalies that might lead to a new energy source....The anomalous data are most likely from a combination of diagnostic problems and yet-tobe-identified, but conventional, physical phenomena. Energy production is certainly one of the possible applications, but this could lead to a new fundamental understanding of physical phenomena that will impact the entire spectrum of Sandia's national security missions. 
will impact the entire spectrum of Sandia's national security missions.

The magnetically insulated transmission line on Hermes III accelerator operated at $0.7 \mathrm{MV} / \mathrm{cm}$, and the experiment showed the axially nonuniform pattern of electron return to the cathode that is consistent with the injection instability proposed by Bergeron and Poukey. The multilayer, spatially resolved diagnostic on the cathode may have shown one large ring. The previous assumption of axial homogeneity for the particle loss has been falsified.

We transferred the experiment to a specially modified magnetically insulated transmission line that was fabricated and installed for this experiment on Sphinx. The new structure provides the highest stressed vacuum line at Sandia and operates at the lowest cost. It could be useful for a variety of future power-flow experiments.

Sphinx experiment and supporting calculations (1) successfully reproduced the low-energy electron loss to the anode, reproduced the unexplained ring patterns on the cathode, and showed that they are not caused directly by ions; (2) demonstrated that the effects are not uniform along the transmission line; (3) plausibly resolved that the electrical diagnostics on the earlier experiments were consistent with the particle energy balance; and (4) showed that secondary electrons along the anode could not explain the low-energy electron loss.

The ring structures observed on the cathode would be consistent with massive supercharging of microparticles during transit across the gap, which could also explain the earlier results on MITE and HYDRAMITE, but such supercharging is many orders of magnitude larger than current computer codes predict.
We transferred the experiment to a specially modified magnetically insulated transmission line that was fabricated and installed for this experiment on Sphinx. The new structure provides the highest stressed vacuum line at Sandia and operates at the lowest cost. It could be useful for a variety of future power-flow experiments. 


\section{Physical Phenomena Underpinning Simulation}

\author{
The Physical Phenomena \\ Underpinning Simulation investment \\ area invests in experimental \\ research investigations of funda- \\ mental physical phenomena that are \\ complementary to sophisticated, \\ large-scale simulations. The \\ objectives of this investment area \\ are to improve the fidelity of large- \\ scale simulations, to strengthen the \\ experimental capabilities required \\ for confidence in the fidelity of \\ current and future models, and to \\ improve the integration of the \\ experimental and modeling efforts \\ at Sandia.
}

Research topics for this investment area include (1) the discovery, elucidation, or quantification of localized phenomena that must be incorporated into large-scale simulations, (2) the examination of physical phenomena under extreme conditions, (3) the study of interactions among complex or competing phenomena, and (4) the discovery of the underlying physical and/or chemical mechanisms governing materials performance as a function of time.

The "Energetic-Material Burn and Detonation at the Mesoscale" project is investigating material properties and reactions at the mesoscale to improve our understanding of the factors that govern the success or failure of an ignited energetic material to sustain and propagate a burn or detonation. The research efforts rely heavily on advances made in semiconductor manufacturing to create structures in which burning and detonations can occur at these very small scales. For example, burn channels of varying diameters have been created and loaded with energetic material in order to examine burning rates. In addition, the propagation of reactive waves in materials engineered with porosity but without traditional grain structure are being investigated to improve our understanding of detonations.

These experiments will provide insight into the controlling mechanisms associated with the reliability and safety of energetic materials that will be useful in the development of new classes of microcomponents. 
10719

\section{Radiation-Induced Prompt Photocurrents in Microelectronics: Physics}

P. E. Dodd, D. S. Walsh, W. Beezhold, R. S. Flores, B. L. Doyle, D.

L. Buller, J. R. Schwank, G. Vizkelethy, M. R. Shaneyfelt

The effects of photocurrents in nuclear weapons induced by proximal nuclear detonations are well known and remain a serious hostile environment threat for the U.S. stockpile. The focus of this project is to study the physical phenomena underlying prompt photocurrents in microelectronic devices and circuits. The goal of this project is to obtain an improved understanding of these phenomena and to incorporate improved models of photocurrent effects into simulation codes to assist designers in meeting hostile radiation requirements with minimum build and test cycles.

Sandia successfully applied analytical photocurrent models for low-level transients. At higher radiation dose rates, effects such as the variation of lifetime and carrier mobility with injection level, internal electric field distortions, and Auger recombination become important. These effects are typically ignored in analytical models. Numerical device simulators such as the Da Vinci three-dimensional (3-D) code work at a fundamental physical level and inherently handle phenomena such as electric field distortions and high injection conditions. However, Da Vinci, used at Sandia primarily for cosmic-ray upset analysis, has neither been rigorously applied nor validated for the study of x-ray and gamma-ray photocurrents.

A newly developed capability on our ion microbeam accelerator is the ability to supply ionizing radiation in selected microregions of a device. Achievable dose rates should approach those possible with conventional large-scale simulators such as Hermes III and Saturn. It is therefore possible to test the physics and models in Da Vinci at the device or subcircuit level in ways not previously possible. This project will benefit weapon-based integrated circuit (IC) development by ensuring that they are insensitive to hostile threats.

We successfully set up and demonstrated the first version of the photocurrent effects microscope (PEM). Ions used on the system so far include protons, helium (He), carbon (C), silicon $(\mathrm{Si})$, and chlorine $(\mathrm{Cl})$. We demonstrated dose rates on the order of one to a few hundred $\mathrm{Grad}(\mathrm{Si}) / \mathrm{s}$, with
The focus of this project is to study the physical phenomena underlying prompt photocurrents in microelectronic devices and circuits. The goal of this project is to obtain an improved understanding of these phenomena and to incorporate improved models of photocurrent effects into simulation codes to assist designers in meeting hostile radiation requirements with minimum build and test cycles....This project will benefit weapon-based integrated circuit (IC) development by ensuring that they are insensitive to hostile threats. 
pulsewidths of 200-250 ns.

We performed the first-ever PEM experiments using a commercially available diode. The measured response to a 0.1 $\operatorname{Trad}(\mathrm{Si}) / \mathrm{s}, 250 \mathrm{~ns}$ pulse persists beyond $1 \mu \mathrm{s}$. This extremely long storage time was an unexpected result and had not been seen in previous broad-beam testing. Da Vinci simulations correctly predict the enhanced storage time of the photocurrent pulse. The detailed simulation results showed that the carriers induced by the dose-rate pulse perturb the majority carrier concentration in the diode, and the potential contours are disturbed deep within the diode. This pushes the electric field deep into the device, and as it relaxes, charge is collected later.

We also performed PEM on n-channel silicon-oninsulator (SOI) metalorganic semiconductor field-effect transistors (MOSFETs) fabricated at Sandia and observed photocurrent responses that may be indicative of dose-rateinduced snapback. Initial sensitivity studies indicate that some physical parameters, such as minority carrier lifetime, have a large impact on the PEM signal, which is desirable from the standpoint of validation.

\section{Refereed}

Dodd, P., W. Beezhold, D. Walsh, G. Vizkelethy, J.

Schwank, M. Shaneyfelt, and B. Doyle. 2000.

"Device-Level Studies of Transient Radiation Response Using Numerical Simulations and Photocurrent Effects Microscopy." Paper presented to the 19th Hardened Electronics and Radiation Technology Conference, San Antonio, TX, March 2001.

Renfrow, S. N., W. Beezhold, P. E. Dodd, D. S. Walsh, and B. L. Doyle. 2000. "Device-Level Prompt Photocurrent Radiation Microscopy Using Sandia's Ion Microprobe Facility." J. Radiation Effects Res. and Eng.

Renfrow, S. N., W. Beezhold, P. E. Dodd, D. S. Walsh, and B. L. Doyle. 2000. "Device-Level Prompt Photocurrent Radiation Microscopy Using Sandia's Ion Microprobe Facility." Paper presented to the 18th Hardened Electronics and Radiation Technology Conference, Anaheim, CA, 21 March.
We performed the first-ever PEM experiments using a commercially available diode. 
10735

\section{Physical Basis for Interfacial Traction- Separation Models}

N. R. Moody, E. D. Reedy, Jr., M. S. Kent

Many weapon components contain interfaces between dissimilar materials where cracks can initiate and fail components. In recent years, many researchers in the fracture community have adopted a cohesive zone model for simulating crack propagation (based upon traction-separation [T-U] relations). Sandia is implementing this model. There is one important obstacle to using a cohesive zone modeling approach. Currently, $T$ - $U$ relations are chosen in an ad hoc manner. The goal of this project is to determine a physical basis for mesoscale-level T-U relations. We propose experiments that will elucidate the dependence of such relations on adhesive and bulk properties. Work will focus on epoxy/solid interfaces, although the approach is applicable to a broad range of materials. Sandwich-layered doublecantilevered beam (SDCB) and free-surface film nanoindentation fracture toughness tests will generate a unique set of data spanning length scales, applied mode mixities, and yield (plastic) zone constraint. The crucial roles of crack-tip plastic zone size and interfacial adhesion will be defined by varying epoxy-layer thickness and using coupling agents or special self-assembled monolayers (SAMs) in preparing the samples. The nature of the yield zone will be probed in collaborative experiments run at the Advanced Photon Source, and high-resolution optical methods will be used to measure crack-opening displacements of the SDCB specimens. This work will provide an understanding of the major mesoscale phenomena governing polymer/solid interfacial fracture and identify the essential features that must be incorporated into a $T$-U-based cohesive zone failure model. We believe that models using physically based $T$ - $U$ relations will provide a more accurate and widely applicable description of interface cracking than models using ad hoc relations.

Furthermore, these T-U relations will provide an essential tool for using models to tailor interface properties to meet design needs.

We developed procedures for making epoxy/aluminum (Al) interfaces with controlled bonding, began fracture toughness testing, demonstrated the feasibility of using a metal overlayer to induce the delamination of thin epoxy films, and
This work will provide an understanding of the major mesoscale phenomena governing polymer/solid interfacial fracture and identify the essential features that must be incorporated into a $T$-Ubased cohesive zone failure model. We believe that models using physically based T-U relations will provide a more accurate and widely applicable description of interface cracking than models using ad hoc relations. Furthermore, these $T$ $U$ relations will provide an essential tool for using models to tailor interface properties to meet design needs. 
began studies that lay the foundation for x-ray imaging of crack-tip deformation fields. One major accomplishment is the development of a method for preparing carefully controlled and reproducible epoxy/Al interfaces with SAM-mitigated surface interactions. This is a crucial step in our project since it provides a technique for varying interface adhesion levels (a primary variable affecting toughness) in a controlled manner.

Another accomplishment is the completion of an initial series of nanoindentation tests of spin-coated epoxy on aluminized glass. We identified failure modes and measured toughness values. We also determined epoxy film hardness and modulus as a function of film thickness. Furthermore, we developed a method to deposit a stressed-metal overlayer on a polymer film in collaboration with Washington State University. When a highly compressed overlayer is applied to a thin epoxy film, the nanoindentation method can be used to generate delamination. Without the overlayers, it is not possible to induce delamination in very thin films. Interfacial toughness is very sensitive to bulk deformation, and bulk deformation is related to film thickness. The use of specimens with metal overlayers allows us to study the effect of thickness variations that range from nanometers to millimeters.

We designed an improved asymmetric double cantilever beam (ADCB) fracture mechanics specimen to optimize toughness measurements over a large range of potential values. We also assessed the capability of Sandia's TAHOE and JAS3D codes to simulate subcritical interfacial crack growth using two common T-U relationships. This capability is needed to identify potentially viable T-U relationships that are consistent with experimental results.
One major accomplishment is the development of a method for preparing carefully controlled and reproducible epoxy/Al interfaces with SAM-mitigated surface interactions. This is a crucial step in our project since it provides a technique for varying interface adhesion levels (a primary variable affecting toughness) in a controlled manner.

\section{Other Communications}

Strojny, A., N. R. Moody, J. A. Emerson, and W. W. Gerberich. 2000. "Interfacial Fracture of Thin Polymer Films on Aluminum." Interfaces, Adhesion, and Processing in Polymer Sys., Proc. MRS Spring Mtg. 629 (San Francisco, CA, 24-28 April). 
10740

Diagnostics for Joining Solidification/ Microstructural Simulations

C. V. Robino, G. A. Knorovsky, J. A. Brooks, J. R. Michael, P. G.

Kotula, A. C. Hall

Solidification is an important aspect of welding, brazing, soldering, laser-engineered netshaping (LENSTM) fabrication, and casting. For these processes, solidification affects and, in many instances, controls the final microstructure and properties. The current trend toward utilizing large-scale process simulations and materials response models for model-based engineering is driving the development of new modeling techniques. However, the effective utilization of these models and simulations is currently limited by a lack of understanding of the subprocesses and interactions involved.

This project will identify key physical phenomena in, and expand and refine our mechanistic descriptions of, solidification in the Fe-Cr-Ni (iron chromium nickel) system. This system displays a wide range of behavior and is well known from a thermochemical perspective. We developed and will continue to refine new and expanded experimental techniques, particularly those needed for in situ measurement of the features of the process. We will further develop and/or redirect our experiments as suggested by model sensitivity studies and will further develop the data needed for continued development and comprehensive validation of the models.

We assembled a literature survey of solidification in the $\mathrm{Fe}-\mathrm{Ni}-\mathrm{Cr}$ system and systematically assessed it in terms of relevance to solidification mode transitions. This survey indicated that although phenomenological descriptions of the mode transfer have been proposed, these have not been verified experimentally, and model approaches have not been developed. We obtained and exercised a thermochemical computational database and compared it with observations from the literature and previous solidification data. We used these assessments to identify alloys that are likely to exhibit mode transitions at solidification velocities in an appropriate range for study. We conducted experimental trials, using spiral welds with both increasing velocity and decreasing velocity, to validate the model assessments and to determine if a hysteresis is associated with the transition velocity. We expect that the

This project will identify key physical phenomena in, and expand and refine our mechanistic descriptions of, solidification in the $\mathrm{Fe}-\mathrm{Cr}-\mathrm{Ni}$ (iron chromium nickel) system.... We developed and will continue to refine new and expanded experimental techniques, particularly those needed for in situ measurement of the features of the process. 
presence of a hysteresis will provide important information regarding the mechanism of mode changes.

We developed a plan for experimental techniques, including transition velocity experiments, microstructural analyses, orientation imaging, high-resolution solid/liquid interface imaging, rapid quenching experiments, controlled thermal gradient experiments, and high-resolution thermography. The goal of the plan was to coordinate the wide range of experimental techniques so that the experiments follow a logical and efficient progression.

The high-resolution light optical imaging of the solidification front in gas tungsten arc (GTA) welds that we conducted revealed very promising images of the solidification front at high travel speeds. The primary dendrite arms (20micron spacing) were clearly resolved by this technique. Secondary dendrite arms, growing perpendicular to the image plane, were also visible in the images. Dendrite arm spacing measurements taken from the same samples directly correspond to those in the video images, indicating that the video image features were not artifacts. The work indicates that direct imaging is very feasible and that quantitative information concerning the solidification front can be obtained. The images illustrate the complex nature of the solidification front and show that it is much more dynamic than is normally assumed in simulations, which typically assume a steady-state, invariant interface shape. For example, the dynamic nature of the interface indicates that the temporal resolution of the imaging system must be increased as much as possible, and work is currently under way to make these improvements. Minimal effort is needed to increase the temporal resolution to $1 \mathrm{msec}$ with available equipment. We encountered additional imaging difficulties because of the oxide that forms on the surface, and we are evaluating the feasibility of installing the system in an available glove box to alleviate this problem. These near-term enhancements should dramatically improve the system capability.

Orientation imaging microscopy (OIM) is a new analytical technique that allows for crystallographic mapping of large numbers of grains within a sample. We applied the technique to $\mathrm{Fe}-\mathrm{Ni}$-Cr laser welds to assess differences in the mechanism of austenitic versus ferritic solidification. For austenitic solidification from an austenitic base material, we found essentially all grains to be continuous across the fusion boundary, indicating epitaxial solidification. In contrast, for ferritic solidification from an austenitic base material, there are
We developed a plan for experimental techniques, including transition velocity experiments, microstructural analyses, orientation imaging, high-resolution solid/liquid interface imaging, rapid quenching experiments, controlled thermal gradient experiments, and highresolution thermography. The goal of the plan was to coordinate the wide range of experimental techniques so that the experiments follow a logical and efficient progression. 
many more grains (for essentially identical heat-flow conditions), and only a fraction of the orientations are continuous across the boundary. Analysis is continuing to determine if the misorientations across the boundary are systematically related to those of the underlying material (indicating pseudo-epitaxial solidification with an orientation relationship) or if the solidified grains are randomly oriented (indicating the nucleation of new ferrite grains) at the solidification front. In either case, OIM is providing important information for analysis of the mechanism of mode shift in these alloys and is essential information for accurate numerical descriptions.

OIM is providing important information for analysis of the mechanism of mode shift in these alloys and is essential information for accurate numerical descriptions. 
10741

\section{Effects of Microstructural Variables on the Shock-Wave Response of PZT 95/5}

R. E. Setchell, M. U. Anderson, E. L. Venturini, B. A. Tuttle, J. A. Voigt

Sandia has continuing responsibility for the design and production of neutron generators (NGs). Most generators use explosively driven power supplies in which shock-wave depoling of the ferroelectric ceramic PZT 95/5 provides the required high voltage for the neutron tube. Generator production will change soon to PZT 95/5 material prepared using a new "chem prep" process that is still under development. This process will introduce a porous microstructure that is significantly different from that found within most material presently in the stockpile. Past studies during component development showed that the porous microstructure can have a profound effect on performance. The rate for failures due to high-voltage breakdown at low temperatures is acceptably low only in materials made within a narrow density range through the addition of organic pore formers. More fundamentally, the dielectric and ferroelectric (FE) properties of the material and the physics of the FE-toantiferroelectric (AFE) phase transformation that governs the depoling process depend on microstructural properties in ways that are not well understood. Consequently, it is not yet apparent how to optimize microstructural features in "chem prep" material, and significant microstructural effects cannot be incorporated into the present efforts to numerically simulate generator operation. This project represents the first systematic study of the basic physics of microstructural effects on the electromechanical behavior of PZT 95/5. We are focusing on the effects of relatively homogeneous porosity by varying only the amount of a certain pore former added to powder from a particular "chem prep" production lot. Dielectric properties are being characterized for material samples at each of three densities, and the electromechanical responses of similar samples are being investigated under carefully controlled shock-loading conditions. Results to date demonstrate significant porosity effects and underscore the continuing need for systematically examining microstructural variables.
This project represents the first systematic study of the basic physics of microstructural effects on the electromechanical behavior of PZT 95/5. We are focusing on the effects of relatively homogeneous porosity by varying only the amount of a certain pore former added to powder from a particular "chem prep" production lot. 
Work focused on relatively homogeneous microstructures in which the bulk porosity resulting from the addition of Avicel pore formers was varied. We mixed powder from a particular production-scale lot of "chem prep" PZT 95/5 with $0.45,1.8$, and 4.0 weight percents of Avicel to yield three high-fire batches having densities of 7.61, 7.33, and $6.94 \mathrm{~g} / \mathrm{cc}$, respectively. We fabricated samples suitable for shock experiments and other characterizations from all of these batches, although the best yield of good samples was from the $7.33 \mathrm{~g} / \mathrm{cc}$ batch. This batch has a density that is very close to a particular mixed-oxide batch $(7.30 \mathrm{~g} / \mathrm{cc})$ that has been extensively characterized under the MAVEN program. We chose experimental conditions to allow results from the 7.33 $\mathrm{g} / \mathrm{cc}$ samples to be compared directly with the results from this mixed-oxide material that has a similar bulk porosity but a very different void morphology. In addition, we prepared a few samples with 0.90 weight percent of Avicel, resulting in samples having a density of $7.59 \mathrm{~g} / \mathrm{cc}$. Dielectric characterizations of "chem prep" materials have consisted of low-field measurements of the permittivity and loss as functions of hydrostatic pressure, temperature, and frequency. A significant change in permittivity as the material undergoes phase transitions allowed the phase diagrams to be mapped in temperature-pressure space. Shock experiments conducted on the $7.33 \mathrm{~g} / \mathrm{cc}$ material have determined a Hugoniot state, a release-wave velocity, and a transmitted wave structure in unpoled material at a shock pressure high enough (4.6 GPa) to show dynamic pore collapse effects. We also conducted experiments to examine the dynamic depoling of normally poled samples at two different shock pressures (0.9 and 2.4 GPa) under either short-circuit or high-field $(36 \mathrm{kV} / \mathrm{cm})$ conditions on this material. We conducted additional experiments to examine the dynamic depoling of samples having densities of 6.94 and $7.59 \mathrm{~g} / \mathrm{cc}$ under one of the conditions used in the experiments with $7.33 \mathrm{~g} / \mathrm{cc}$ material.

Comparisons between mixed-oxide and "chem prep" PZT 95/5 samples having similar bulk porosities but very different void morphologies show that there are relatively small but distinct differences in the depoling kinetics under fixed conditions for shock pressure and electric field. The "chem prep" material appears to have slower rates for depoling, which could be an issue in terms of meeting performance requirements. In addition, the "chem prep" material appears to have a lower value for yield strength, corresponding to earlier onset of pore collapse. Interpretation of these comparative
Comparisons between mixedoxide and "chem prep" PZT 95/ 5 samples having similar bulk porosities but very different void morphologies show that there are relatively small but distinct differences in the depoling kinetics under fixed conditions for shock pressure and electric field. 
results is challenged by the fact that there are other differences in these materials besides pore morphology. Comparisons between "chem prep" samples having different bulk porosities show strong effects for fixed impact conditions. Final shocked states (Hugoniot conditions) increase with decreasing porosity, as expected. Depoling currents appear to decrease more rapidly with increasing porosity than might be expected from corresponding decreases in shock speeds and remanent polarizations.

Results from this project indicate a continuing need to explore the effects of relatively homogeneous bulk porosity introduced by Avicel addition in "chem prep" PZT 95/5. In particular, the role of these voids in determining the rate at which a shock wave releases charge due to the FE-to-AFE phase transition needs to be understood more completely.
Results from this project

indicate a continuing need to explore the effects of relatively homogeneous bulk porosity introduced by Avicel addition in “chem prep" PZT 95/5. 


\section{2}

\section{Mechanisms of Dislocation-Grain-Boundary Interaction}

\section{L. Medlin, J. E. Houston, K. J. Gross}

Incorporating the localized atomistic and microscopic effects of internal interfaces on materials properties and longterm behavior is a significant challenge for large-scale materials simulations. Critical to improving such models is developing an improved understanding of the dislocationgrain-boundary interactions that ultimately control the interfacial response to strain. Such interactions impact many materials phenomena and properties, including slip transmission, boundary migration, recrystallization, and yield strength, and are a critical element in linking atomic structure to continuum behavior. In this project Sandia seeks to develop an experimentally based understanding of these dislocationgrain-boundary interactions.

We developed the experimental capability to produce $\mathrm{Au}\langle 110\rangle$ (gold) and $<111\rangle$ thin-film bicrystals and are now employing these specimens in our current experiments.

We are probing the mechanical response of grain boundaries using interfacial force microscopy (IFM) indentation measurements. Through these experiments, we directly observed grain-boundary sliding and measured the energy dissipation associated with this deformation process.

We observed the atomic structure of a high-angle grain boundary in a Au bicrystal using transmission electron microscopy (TEM). This work shows that an earlier dislocation-based model, developed originally for simpler boundaries, can be extended to boundaries of greater complexity.

We investigated dislocation-boundary interactions through in situ heating experiments in the TEM. These observations show how dislocations can cause a deviation from capillarity-driven grain-boundary motion.

\section{Sandia seeks to develop an experimentally based understanding of these dislocation-grain-boundary interactions.}
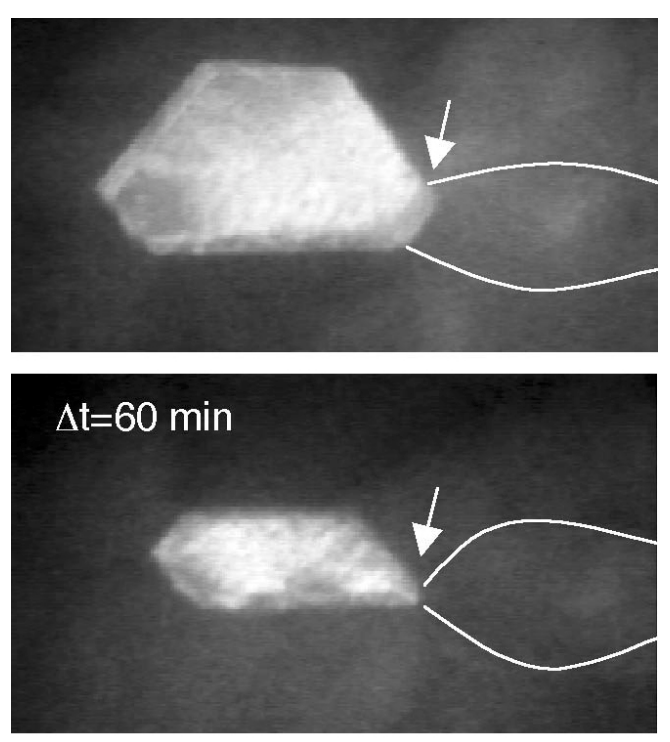

$\Delta \mathrm{t}=62 \mathrm{~min}$

$50 \mathrm{~nm}$

In situ TEM observations showing the time evolution of a faceted grain boundary in gold $\left(T=480^{\circ} \mathrm{C}\right)$. Depinning of the facet corner from a low-angle dislocation boundary (white line) results in a rapid reduction in island size. This observation provides a clear example of dislocation producing a deviation from capillarity-driven grain-boundary motion. 


\section{Refereed}

Jarausch, K. F., J. D. Kiely, J. E. Houston, and P. E. Russell. 2000. "Defect-Dependent Elasticity:

Nanoindentation as a Probe of Stress State." J. Mater.

Res. 15 (8) (August): 1693-1701.

\section{Other Communications}

Medlin, D. L. 2000. "Observation and Modeling of Grain-Boundary Dislocation Structure and Behavior." (Invited) Paper presented to the TMS Annual Meeting, Symposium on Dislocations and Microscale Plasticity Modeling, Nashville, TN, 12-16 March.

Medlin, D. L. 2000. "Structure and Dynamics of Grain-Boundary Defects." (Invited) Paper presented to the TMS Annual Meeting, Symposium on HighResolution Electron Microscopy in Materials Science, Nashville, TN, 12-16 March.

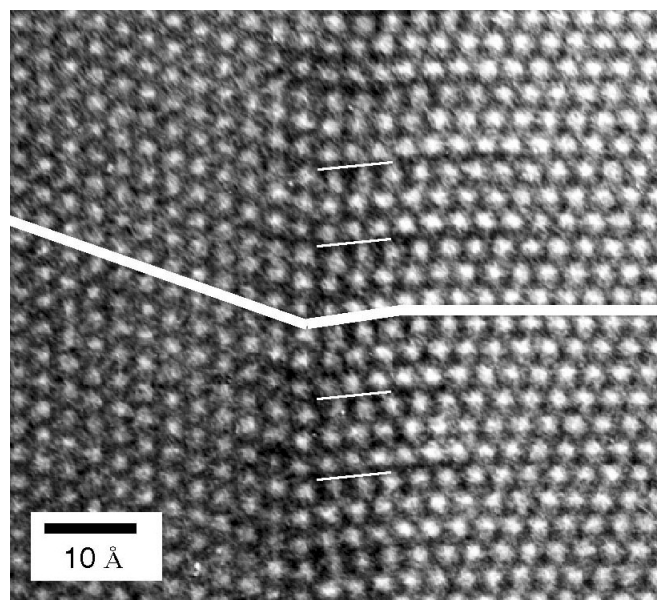

Atomic resolution image of $90^{\circ}(111) /(112)$ interface in a Au<110> film. Observations show that this boundary dissociates to form an intermediate layer of the $9 R$ phase. The interplanar bending and stacking fault distribution (white lines) is consistent with that predicted by a dislocation-based model that we developed for interface structure. 
10765

\section{Energetic-Material Burn and Detonation at the} Mesoscale

A. M. Renlund, S. H. Kravitz, M. R. Baer, K. L. Erickson, A. S. Tappan, P. K. Walker

The success or failure of an ignited energetic material (EM) to propagate a burn or detonation is determined by material properties and reactions at the mesoscale. Burn models cannot adequately account for surface-area burning effects without understanding burning at high pressure in small fissures. Similarly, rarefactions from sides and from grain boundaries are efficient at quenching shocks, leading to failure of a detonation wave at some critical diameter. Whether or not a detonation can be sustained at small diameters (submillimeter) may depend on engineering the EM without traditional grains while incorporating microporosity into the material. A novel and intriguing prospect is rapid energy release from binary EMs, traditional pyrotechnic and propellant materials that may have 3-5 times the energy density of conventional high explosives. If these materials can be engineered so that reactions can occur without strong influence from diffusion times to mix fuel and oxidizer, Sandia may develop new EMs capable of higher output.

This project applies innovative experiments to observe burning and detonation at the mesoscale. We rely heavily on advances made in semiconductor manufacturing to create structures in which burning and detonations can occur at these very small scales. For example, we will create burn channels of varying cross-sections, load the channels with EM, and monitor the burning rate using integrated gauges and highresolution optical diagnostics. For detonations, we will examine the propagation of reactive waves in materials engineered with porosity, but without the traditional grain structure used in macroscopic EM applications, and will study the novel nanoscale mixed materials. All material deposition and patterning techniques will be compatible with modern microelectronic fabrication technology.

State-of-the-art simulations of deflagration and detonation are now incorporating effects of microstructural elements. These experiments will provide insight into the controlling mechanisms associated with the reliability and safety of energetic components and address issues for using EMs to provide useful work in new classes of microcomponents.
If these materials can be engineered so that reactions can occur without strong influence from diffusion times to mix fuel and oxidizer, Sandia may develop new EMs capable of higher output....These experiments will provide insight into the controlling mechanisms associated with the reliability and safety of energetic components and address issues for using EMs to provide useful work in new classes of microcomponents. 
We selected nitrocellulose (NC) for our initial burn studies because of the data available for modeling and its wellbehaved burning characteristics. We successfully developed methods for spin-coating NC by varying nitration levels and solvent, with a major effort to develop "thick" films (> 30 microns) without an excessive number of coats. We patterned NC using plasma-enhanced chemical vapor deposition (PECVD). The process involved first coating the NC with silicon dioxide $\left(\mathrm{SiO}_{2}\right)$, applying a patterned photoresist, and performing plasma etching followed by an oxygen $(\mathrm{O})$ plasma to remove the photoresist. What remained was a well-defined patterned structure of $\mathrm{NC}$, topped by $\mathrm{SiO}_{2}$. The $\mathrm{NC}$ survived the processing without signs of chemical degradation. We first demonstrated PECVD on thin samples of NC while developing the techniques to fabricate thicker films. We are now extending this patterning technique to the explosive pentaerythritol tetranitrate (PETN) that is first deposited on a substrate via physical vapor deposition.

We fabricated test devices for initial burn experiments more simply than described above. Channels from 100 to 500 microns wide and 100 microns deep were cut into silica substrates. These were filled with $\mathrm{NC}$ using a syringe dispenser. In some cases a tungsten (W) film was placed in the channel prior to filling the NC to allow for a "hot spot" for laser ignition of the NC.

Ignition studies have begun on samples prepared by affixing a thin fused-silica slide over the substrate that confines the NC in channels. Pyrofuse was introduced at one end of the channel and ignited with electrical current. A second ignition scheme was to place a $\mathrm{W}$ filament at the bottom of the channel prior to $\mathrm{NC}$ deposition. We used laser heating of the $\mathrm{W}$ to ignite the NC. We are presently investigating various optical diagnostics that will be definitive in monitoring the propagation of burn at these scales.

Anticipating needs for better control of ignition leading to detonation, we fabricated complex semiconductor bridge (SCB) devices with active elements as small as 10 microns wide. These were tested and demonstrated to function at energies as low as 30 microJoules.
We successfully developed methods for spin-coating NC by varying nitration levels and solvent, with a major effort to develop "thick" films (> 30 microns) without an excessive number of coats. 
10788

Small-Scale Multiaxial Deformation Experiments on Solder for High-Fidelity Model Development

W. Y. Lu, D. A. La Van, T. E. Buchheit, H. E. Fang, T. J. Lim

Deformation of engineering materials is complex; the ambient temperature deformation of low-melting-point alloys is complicated by competing, thermally activated mechanisms. The interrelation between temperature, stress state, deformation, microstructural coarsening, and localization is not understood. This project will examine the following three areas, with a focus on understanding the underpinning physics.

(1) The failure of solder joints is often preceded by the formation of localized coarsened bands, but there is no fundamental understanding as to the energy criteria that govern coarsening or localization. Uniaxial tension and shear experiments have not revealed the underpinning mechanisms; it is not known whether resolved shear, hydrostatic, or deviatoric strains are the critical factors and how they interrelate with thermal activation.

(2) Deformation mechanism maps are widely applied in continuum-level deformation models. However, these maps do not differentiate between the effects of shear and tension. A thorough understanding of the role of multiaxial stress states on deformation mechanisms will lead to an understanding of critical deformation conditions.

(3) As joint size decreases, mechanical behavior changes from true polycrystalline behavior to a scale where bulk, size-independent constitutive relations no longer hold. Current models do not incorporate a length scale that would allow exploration of this effect. Experiments at a range of length scales, decreasing to the point where breakdown of continuum is found, will provide a foundation for the development of deformation models that account for size effects.

High precision and rigorous control of microstructures are necessary to succeed in these investigations. The resulting small-scale multiaxial experimental methodology will be an enabling technology for the microstructural evolution of multiphase alloys. We concentrated on developing small-scale and multiaxial experiments and starting size-effect research.
The resulting small-scale multiaxial experimental methodology will be an enabling technology for the microstructural evolution of multiphase alloys.

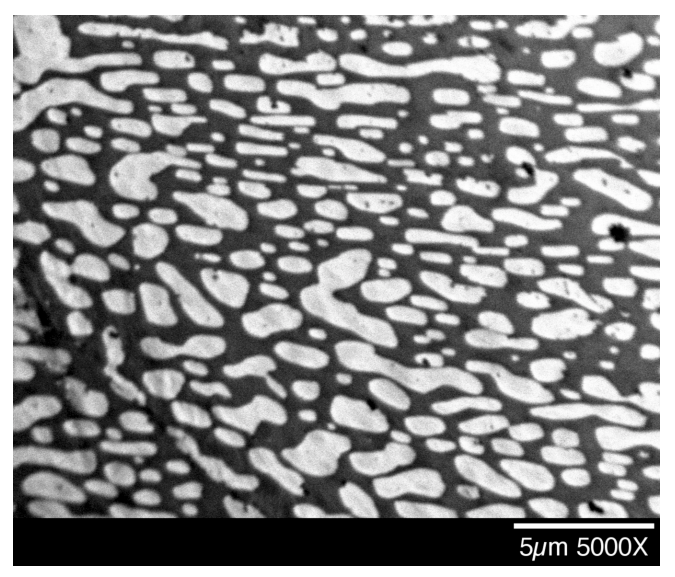

Solder microstructure results from 16 hours aging at $100^{\circ} \mathrm{C}$.

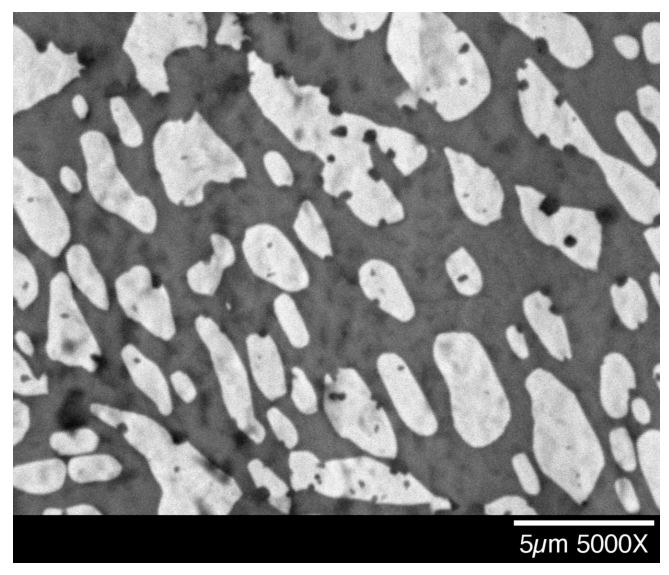

Solder microstructure results from 25 days aging at $100^{\circ} \mathrm{C}$. 
We developed techniques and fabricated ${ }^{63} \mathrm{Sn}-{ }^{37} \mathrm{~Pb}$ (tinlead) solder specimens that have multiple sizes and geometries. We cast the specimens between two brass rods and subjected them to different aging treatments to generate controlled microstructures. We used two $100^{\circ} \mathrm{C}$ aging treatments, 16 hours and 25 days, that generated microstructures with two distinctive $\mathrm{Pb}$-rich phase sizes. We set up tensile experiments on small specimens with various sizes and microstructures on the Tytron microforce testing system to address the effect of size or length scale in solder. The smallest specimen had a gage length of 350 microns. Preliminary results show that the effect of size or length scale on the ultimate strength is not obvious for solder size greater than 350 microns, though the strain to failure differs. In general, the specimens having coarser microstructures had lower ultimate strengths and higher failure strains. We are pursuing the investigation of a specimen size of 100 microns.

We designed and are fabricating a static-load tensiontorsion creep machine. We will use this unique multiaxial creep machine to develop an accurate deformation mechanism map of ${ }^{63} \mathrm{Sn}-{ }^{37} \mathrm{~Pb}$ solder. We designed the machine to accommodate a thin-wall tube-shaped specimen with a diameter of $6 \mathrm{~mm}$ and a wall thickness of $0.6 \mathrm{~mm}$. Static axial and shear loads can be applied independently, permitting creep test investigations among a variety of deformation paths. A general multiaxial deformation system that will test the same thin-wall tubular specimen is also in the design and development stages and will be ready for multiaxial experiments.

We have begun detailed microstructural analyses on the constituents of $\mathrm{Pb}-\mathrm{Sn}$ solder. Our focus has been on studying the role of the substructure evolution in Sn during deformation of $\mathrm{Pb}-\mathrm{Sn}$ solder.

We are working in collaboration with solder modeling researchers at Sandia and at the University of Michigan.

\section{Refereed}

Lim, T. J., and W. Y. Lu. 2000. "Length Scale Effect

on the Mechanical Properties of ${ }^{63} \mathrm{Sn}-{ }^{37} \mathrm{~Pb}$ Solder

Alloy." Symp. on Mechanics of SMT and Photonic

Structures, accepted
We designed and are fabricating a static-load tension-torsion creep machine. We will use this unique multiaxial creep machine to develop an accurate deformation mechanism map of ${ }^{63} \mathrm{Sn}-{ }^{37} \mathrm{~Pb}$ solder. 


\section{Appendix A: Project Number / Title Index}

Page Title

Number

32 Functional Materials for Microsystems: Smart Self-Assembled Photochromic Films

34 Innovative Experimental and Computational Diagnostics for Monitoring Corrosion in Weapons Environments

37 Self-Healing Molecular Assemblies for Control of Friction and Adhesion in MEMS

39 Linking Atomistic Computations with Phase-Field Modeling

10303

41 A Combinatorial Microlab Investigation of Critical Copper-Corrosion Mechanisms

10304

43 Self-Assembled Templates for Fabricating Novel Nanoarrays and Controlling Materials Growth

45 Wetting and Spreading Dynamics of Solder and Braze Alloys

10306

48 Improved Materials-Aging Diagnostics and Mechanisms Through 2-D Hyperspectral Imaging Methods and Algorithms

10307

52 Microscale Shock-Wave Physics Using Photonic Driver Techniques

54 Reactivity of Metal-Oxide Surfaces

57 Exploiting LENS ${ }^{\text {TM }}$ Technology

Through Novel Materials

60 Molecular Characterization of Energetic-Material Initiation

Project

Number

10300

10305

10308

10309
Page

Title

Project

Number

Number

63 Nonvolatile Protonic Memory

10312

79 Heterogeneous Simulation

10313

81 Volumetric Video Motion Sensing for Unobtrusive Human-Computer Interactions

10314

83 Hybrid Sparse-Dense Incomplete Factorization Preconditioners

10315

85 Advanced Large-Eddy Simulation Algorithms for Coupled-Flow Physics and Complex Geometry

10316

87 Molecular Simulation of Reacting Systems

10317

89 Massively Parallel Global Climate Model for Paleoclimate Applications

10318

91 From Atom-Picoseconds to CentimeterYears in Simulation and Experiment

10319

93 Parallel Combinatorial Optimization for Scheduling Problems

10320

96 Multilevel Techniques for Unstructured Grid Problems on Massively Parallel Computers

10321

99 Visual Explanation and Insight

10323

117 Monolithic Integration of VCSELs and Detectors for Microsystems

10325

119 Post-Processed Integrated Microsystems

10326

122 Development of Radiation-Hard Sensors for Space-Based Visible and Infrared Sensing Applications

10327 


\section{Appendix A: Project Number / Title Index}

Page Title

Number

124 Silicon Three-Dimensional Photonic Crystal and Its Applications

126 Monolithic Micromachined Variable Tuners for Rapid Prototyping and Optimization of Microwave Circuits

129 Quantum Tunneling Transistors for Practical Applications

10330

131 Development of Magnetically Excited Flexural Plate-Wave Devices for Implementation as Physical, Chemical, and Acoustic Sensors, and as Integrated Micropumps for Sensored Systems

10331

134 Novel Acoustically Driven Microoptoelectronic Devices

136 Photonics Integration Devices and Technologies

10333

139 Stress-Free Amorphous Diamond for High-Sensitivity Microsensors with Integrated Microstructures

185 Development of In Situ Diagnostics for Simultaneous Measurement of Transient Gas Species and Soot in Large Fires

188 Structural Simulations Using Multiresolution Material Models

190 Mechanisms of Adiabatic Shear Failure

10338

192 Evolvable Hardware 10339

194 Crack Nucleation and Growth: Combining Validated Atomistic and Continuum Modeling

Project

10328

10329

10335
201 High-Resolution Modeling of Multiscale Transient Phenomena in Turbulent Boundary Layers

10344

203 Dispersive Measurements of Velocity in Heterogeneous Materials

10345

206 A Physically Based Computational Method for Predicting Generalized Fracture

10346

212 Microdiagnostic MEMS Lab-on-aChip

10347

214 Real-Time Error Correction Using Electromagnetic-Bearing Spindles

10348

216 Thin-Film Deposition Processes Incorporating In Situ Monitoring Capabilities

10349

218 Solid-State Neutron Generator for Use in Nuclear Weapons

10351

220 Scripting for Video Inspection

10352

223 Advanced Machining Processes for Microfabrication

10353

225 Advanced Production Planning Models

10354

247 Weighted-Nearest-Neighbor (WNN) Decision Making for Data Mining

10355

249 Varying QoS for Fixed and Mobile Networks 


\section{Appendix A: Project Number / Title Index}

Page Title

Number

251

A Real-Time Decision-Support

Framework to Guide Facility

Response to Abnormal Events

253 Physical Model-Based Fusion of Sensor Array Data

10358

255 Large-Scale Distributed Information System Modeling and Simulation

258 High-Surety SCADA for the Critical

Energy Infrastructures

260 Agent-Based Mediation and Cooperative Information Systems

10361

262 Dynamically Self-Configurable Network Protocol

10362

264 Optical Backplane/Interconnect for Super-High-Speed Communication

266 PUSH Technology Demonstration

268 Controlling Information: Its Flow, Fusion, and Coordination

10365

271 Low-Power, Reduced-Computation, Public-Key Protocols

10366

273 Ten-to-One-Hundred-Gigabit/ Second Network-Enabling R\&D

283 Surface Decontamination of Bacterial Protein Toxins by RF Power

10368

284 Intense White-Light Pulse Propagation in Air Using Self-Guided Optical Filamentation: Applications to Remote Sensing and Countermeasures

10369

286 HPM Vulnerability Assessment and Tests

10370
Page Title

Number

293 Miniature Bioaerosol Concentrator

Project

Number

10371

295 Recognizing Partially Obscured Targets by Combining Multiple Data Sources Using Evidential Reasoning

10372

298 Computational Engineering of Sensor Materials and Integration with a Novel Biological Weapon Detection System

10373

300 Biological Weapon Detector Using Bioaffinity Array Impedance Analysis with Chemical Amplification Through Redox Recycling

10374

302 ATR / Exploitation Utilizing Ultrahigh-

Resolution, Complex SAR Imaging

10375

304 Thin-Skin Deployable Mirrors for Remote Sensing Systems

10376

307 Dispersible Granular Sensor (Smart Sand) for Landmine Detection Based on TNT Immunoassay

10377

309 Characterization of Underground Facilities in an Urban Environment

10378

311 Dexterous Robotic Manipulation of Hazardous Materials in Unstructured Environments

10379

313 Autonomous Dynamic Soaring Platform for Distributed Mobile Sensor Arrays

10380

315 Miniature UV Fluorescence-Based Biological Agent Sensor

10381

317 Enabling Human Skills with Cooperative Automation

10382 


\section{Appendix A: Project Number / Title Index}

Page Title
Number

319 Cloud to CAD

337 Adaptive 3-D Sensing

339 Designed Ionophores for Liquid-

Membrane Separation and

Extraction of Metal Ions

343 An Electromagnetic Imaging System for Environmental Site Reconnaissance

345 Advanced Geosphere Transport Simulation

362 Hybrid Processing of Measurable and Subjective Information in Surety Analysis

10388

364 Approximate Public-Key Authentication with Information Hiding

366 New Network Analysis Approaches to Evaluate Infrastructure Risk and Reliability

369 Improved Tools for Identifying and Quantifying Potentially Dangerous Human Actions

10391

371 Advanced Signal Processing for Thermal Flaw Detection

10392

374 Production Surety and Disruption Vulnerability Analysis

10393

376 An Optically Triggered Semiconductor Switch for Firing Systems

378 Source Code Assurance Tool

Project
Number
10383
10384

10385

10386

10387

10389

10390

10394

10395

\section{Broadening Mechanism in 2-D}

Excitonic and Electron Gases

425 Direct Fabrication of Multifunctional Nanocomposites Via Supramolecular Self-Assembly

10406

428 Information Extraction from Hyperspectral Images Obtained from Satellites

10407

430 Advanced Radiation Sources:

Rayleigh-Taylor Mitigation Via

Perturbation Reduction

10408

Project

Number

10396

10397

10398

10399

10401

10402

10403

10404

10405

433 Capillary Elastohydrodynamics in Manufacturing Processes 


\section{Appendix A: Project Number / Title Index}

\begin{tabular}{ll}
$\begin{array}{l}\text { Page } \\
\text { Number }\end{array}$ & \multicolumn{1}{c}{ Title } \\
$\mathbf{4 3 6}$ & $\begin{array}{l}\text { Real-Time Design of Improved } \\
\text { Powder-Pressing Dies Using Finite- } \\
\\
\end{array}$ \\
& Element Method Modeling
\end{tabular}

385 A Massively Parallel Microsimulation Model of Infrastructure Interdependency

142 AlGaN Materials Engineering for Integrated Multifunction Systems

439 Calculation and Interpretation of the Energies that Underlie TransitionMetal Surface Structure

102 Emergent Behavior of Large Swarms of Intelligent Agents

10690

441 Interfacial Reactions in Ceramic Systems

10692

404 Open Access (Nanosat)

209 Micromechanical Failure Analyses for Finite-Element Polymer Modeling

443 Poco Switch Tubes

10694

Project

Page

Title

Number

567 Radiation-Induced Prompt Photo-

10410

10687

10688

10689

232 Fabrication of Three-Dimensional Microstructures Using Soft

Lithography

10723

323 High-Speed 2-D Hadamard Transform Spectral Imager for IR Applications

10724

10693

350 A Microgas Chromatograph (mGC)Based Controller for EnergyIntensive Processes

10725

325 Micro-High-G Acceleration Recorder

10695

144 Semiconductor Current Filament Lasers

10696

321 Sparse Geophysical Networks for Monitoring Deep Targets

10697

409 Autonomous MicroChem Laboratory $\left(\mu \mathrm{ChemLab}{ }^{\mathrm{TM}}\right)$

10698

348 A Novel Microcombustor for Sensor and Thermal-Energy Management Applications in Microsystems
104 Algorithmic Advances in Computational Structural Biology

10728

444 Ultraminiaturization of RF Circuitry

10729

446 A Stronglink Prime Mover Based on an Electrokinetic Pump

10731

352 A MEMS Microelectric Generator

10732

147 SOI-Based High-Aspect-Ratio Si Bulk Micromachining for MEMS Applications 


\section{Appendix A: Project Number / Title Index}

Page Title

Number

66 Next-Generation Output-Based

Process Control: An Integration of

Modeling, Sensors, and Intelligent

Data Analysis

10734

569 Physical Basis for Interfacial

Traction-Separation Models

10735

327 Dynamic Range Imaging for Terrain

Mapping, Position Determination, and Obstacle Avoidance in Autono-

mous Navigation

10736

448 Laser-Assisted Microgas Metal Arc Welding

10739

571 Diagnostics for Joining Solidification/

Microstructural Simulations

10740

574 Effects of Microstructural Variables on the Shock-Wave Response of PZT $95 / 5$

10741

577 Mechanisms of Dislocation-GrainBoundary Interaction

10742

150 Defining the Frontiers of Vertical, External-Cavity, Surface-Emitting Lasers

10744

153 Photoresist Technology for $70 \mathrm{~nm}$ Lithography with $157 \mathrm{~nm}$ Radiation

10745

106 Predicting Function of Biological Macromolecules

10746

329 Induced Molecular Markers for Pathogen Detection: Microincubators for Rapid Toxin Expression

10747

354 Nanostructured Silicon Surfaces for Cost-Effective Photovoltaic Efficiency Improvements

10748
Page Title

Number

Project

155 Growth and Characterization of Quantum Dots and Quantum Dot Devices

10749

$158 \mathrm{THz}$ Technologies for Ultrahigh-

Data-Rate Communications

10750

234 Automatic Design of Practical Fixtures

10751

331 Distributed Reconfigurable Homogeneous Microrobotic Systems

10752

334 Robust Planning for Autonomous Navigation of Mobile Robots in Unstructured, Dynamic Environments

10753

68 Functional Materials for Electrochemomechanical Actuation of Microvalves and Micropumps

10754

238 Microreplication: Precision Metal Parts from Electroformed Master Molds

10755

160 New Architectures for Micro-TotalAnalytical Systems

10756

108 Large-Scale Nonlinear Optimization Arising from PDE Models

10757

388 Network Intrusion Detection Using Adaptive Critic Neural Networks

10758

390 Key Management Techniques for Authenticating Highly Secure SCADA Systems in the Electric Power Industry

10759

70 Making the Connection Between Microstructure and Mechanics 


\section{Appendix A: Project Number / Title Index}

Page Title

Number

392 Self-Assembled Tamper-Detection Seals

357 Control Strategies for Homogeneous Charge-Compression Ignition (HCCI) Engines

579 Energetic-Material Burn and Detonation at the Mesoscale

162 Heterogeneous Integration of Optoelectronic Arrays and Microelectronics

276 Intrusion Detection for Asynchronous Transfer Mode (ATM) Networks

10767

278 Conversational Policies for Multiagent Systems

10768

236 IMEMS Packaging and Interconnection Technology

10770

359 Silicon/Carbon Nanocomposites for Rechargeable Battery Applications

290 Intense Directed-Energy Theory Initiative

240 Assembly of LIGA Using Electric Fields

10773

164 Biosensors Based on the Electrical Impedance of Tethered Lipid Bilayers on Planar Electrodes

166 Miniature Sensors for BW Agents Using Fatty-Acid Profiles

10775

168 Enhanced-Sensitivity Acoustic-Wave Biosensor Arrays

Project
Number

10771

10774
Page

Title

Project

Number

Number

110 Parallel Methods for Coupling Circuitand Device-Scale Simulations

10777

112 A Java-Based Tool for Multifidelity

Modeling of Coupled Systems

10778

72 Switchable Hydrophobic-Hydrophilic Surfaces

10779

$74 \quad$ Nanostructured Materials Integrated in Microfabricated Optical Devices

10780

395 Virtual Reliability Realization

System

10781

171 High-Al-Content AlGaInN Devices for Next-Generation Electronic and Optoelectronic Applications

10782

173 Microfabrication of Electromagnetic Devices

10783

175 GaAs MOEMS Technology

10784

178 Integrated Microsensors for

Autonomous Microrobots

10785

180 Silicon-Integrated Planar

Microbatteries

10786

581 Small-Scale Multiaxial Deformation Experiments on Solder for High-

Fidelity Model Development

10788

76 All-Ceramic Thin-Film Battery

10789

$280 \quad$ Fuzzy Data Mining

10791

397 Ultrahigh-Resolution Radiography for Detailed Inspection of Weapon Components and Systems 


\section{Appendix A: Project Number / Title Index}

$\begin{aligned} & \text { Page } \\ & \text { Number }\end{aligned}$
$\mathbf{2 4 1} \quad \begin{aligned} & \text { Computer Numerically Controlled } \\ & \text { Micromachines }\end{aligned}$

244 Electromicrofluidic Packaging

450 Surface-Micromachined Mechanical Timer

182 High-Efficiency Optical MEMS by the Integration of Photonic Lattices with Surface MEMS

412 Molecular Integrated Microsystems (MIMS)

11130

114 Gamma-Ray Bursts and the Particle Mass Scale

11336

453 Pseudostationary Separation Materials for Highly Parallel Microseparations

455 Novel Coatings for Microelectromechanical (MEM) Devices

17230

458 Investigation of the Feasibility of RTL Concept for Fusion Energy

460 Rapid Discovery of Materials for Energy Systems

462 In Situ Formation of Reactive Barriers for Radionuclide, Heavy Metal, and Organic Stabilization

19991

464 MPP Direct Numerical Simulation of Diesel Autoignition

19993

466 A Functional Parallel Discrete-Event Simulation of a Snoopy Coprocessor Software Monitor

20407

468 New Complexing Agent for Co(II) Analysis
Page Tit

Number

Project

Number

470 Infrared Polarization for Target Identification

20410

472 Characterization of Polymer-Metal Interface Properties for LIGA-Based Microsystem Technologies

20411

474 Fault-Tolerant Computing Services for Heterogeneous Clusters

20412

476 DNA Microarray Technology

20413

479 Shock Response of Diamond Crystals

20414

480 The Use of Active Fiber Composites for the Health Monitoring of Wind Turbine Blades

20415

482 Information-Sharing Security

Module

20416

484 Adaptive Sensor Optimization and Cognitive Image Processing Using Autonomous Optical Neuroprocessors

21270

486 Integration of Sandia Microsensors to the Internet for Logistics Applications

21271

488 Automatic Target Recognition in Three Dimensions

21272

490 Modeling the Jini Infrastructure Protocol

21273

492 Intelligent Targeting for Remote Weapons

21275

494 Microfuel Reformer: Hydrogen for Microfuel Cells

21276 


\section{Appendix A: Project Number / Title Index}

\begin{tabular}{ll}
$\begin{array}{l}\text { Page } \\
\text { Number }\end{array}$ & \multicolumn{1}{c}{ Title } \\
$\mathbf{4 9 7}$ & $\begin{array}{l}\text { Advanced Techniques for Real- } \\
\text { Time Visualization of Data- } \\
\text { Intensive Missions }\end{array}$ \\
$\mathbf{4 9 9}$ & $\begin{array}{l}\text { Early Diagnosis of Human Exposure } \\
\text { to Biological Agents }\end{array}$ \\
$\mathbf{5 0 2}$ & $\begin{array}{l}\text { Multisensor Fusion for Characterizing } \\
\text { Underground Facilities (UGFs) }\end{array}$
\end{tabular}

504 Developing Land Surface-Temperature Maps from Multispectral Thermal Imager (MTI) Data

506 Enterprise Modeling of Medical Surveillance Network

21281

508 The Thermal History of Charred Materials by Raman Spectroscopy

21282

510 Optical Interconnections to Focal Plane Arrays

21283

512 Change Detection in Flash Memory

514 Study of Effectiveness of Spectro-

Polarization Parameters in Military

Target Detection

21287

516 Damaged Chip

21288

519 Engineered Window Glass for Architectural Surety Applications

521 Layered Pyrotechnic Smoke Materials for Barriers

523 Miniaturized Flying Penetrator 21311

525 Preconcentration of Volatile Chemical Agents and Explosives

Project
Number

21289

21312
Page

Number

Title

Project

527 Accelerated Beta Decay for the Reduction of Legacy Wastes

21545

529 Kinetic-Energy Lightning Generator

21548

531 Comprehensive Representation of Human Decision Process: Sensor Shooter Problem

21550

533 Spectroscopic Detection of Pathogens

21553

534 Technical Solutions to National Security Issues Based on Limited Natural Resources

21554

535 Sustainable Bioelectronic Microsystems: Life on a Chip

21557

537 Near-Real-Time Surveillance

Against Bioterror Attack

21558

539 Investigation of Modeling

Approaches for Nuclear Futures

21559

541 Understanding and Managing

Threats to the Social Fabric of the United States

22126

543 Physical and Functional Modeling of a Bioelectronic Microsystem

22127

544 Analytic Models for Cooperative Utilization of Critical Resources

22128

546 Cryopreservation of Biological

Tissue

22129

548 Investigation of Nanoscience

Technologies

22131
549 Magnetic Polysilicon MEMS

Devices 


\section{Appendix A: Project Number / Title Index}

$\begin{array}{lll}\text { Page } & \text { Title } & \text { Project } \\ \text { Number } & & \text { Number }\end{array}$

551 Autonomous Microexplosive

Subsurface Tracing System

553 New Metal-Organic Chemical Vapor

Deposition Reactor Designs and

Optimization

22854

555 Composite-Wire Plasma Formation and Evolution

557 Scattering from Nanostructured Materials

559 The Liquid-Crystal Physics of Evaporation-Induced Self-

Assembly

560 Electrochemical Interrogation of Cellular Membrane Transport

Processes

562 A Micromethanol Steam Reformer for a Hydrogen-Based Microfuel Cell

24101

564 Spatial Anomalies in Magnetically Insulated Vacuum Structures 
Materials Research Society: Graduate Student Award

American Chemical Society: Victor

K. Lamer for graduate research

1999 R\&D Award, R\&D Magazine

Finalist, 1999 Franz Edelman Award

for Management Science Achieve-

ment Institute for Operations Re-

search and the Management Sciences

(INFORMS)

DOE Basic Energy Science Award, 2000

Employee Recognition Team Award

Sandia Employee Recognition Team Award: $\mu$ ChemLab Grand Challenge Team

Recognition for Exceptional Service, U.S. Department of Energy, Office of Solar Energy Technologies, Energy Efficiency and Renewal Energy

ATM Forum Spotlight Award by ATM Forum for editing and technical work on ATM security specifications

IEEE Sidney Fernbach Award: Steve Attaway

Fellow of the American Society of Mechanical Engineers (ASME), 2000: Steve Attaway

First prize, SuParCup Competition, SuperComputing SC '99 Conference: Parallel Pronto3D Code

Eric Reissner Medal for excellence in computational mechanics research, 1998 International Conference on Computational Engineering Science: Steve Attaway
10328

10354

Self-Assembled Templates for Fabricating Novel Nanoarrays and Controlling Materials Growth

Silicon Three-Dimensional Photonic Crystal and Its Applications

Advanced Production Planning Models

Direct Fabrication of Multifunctional Nanocomposites Via Supramolecular Self-Assembly

Semiconductor Current Filament Lasers

10698 Autonomous MicroChem Laboratory ( $\mu$ ChemLab)

10748

10767

3532330

Computational Methods for Predicting the Response of Critical As-Built Infrastructure to Dynamic Loads (Architectural Surety®) (project ended in 1999) 


\section{Appendix B: Awards/Recognition List}

1999 R\&D 100 Award, R\&D Magazine

DOE Basic Energy Science Award, 2000
Low-Cost Cadmium Zinc Telluride Radiation Detectors Based on Electron-Transport-Only Designs

Tailorable, Visible, Room-Temperature Light Emission from $\mathrm{Si}, \mathrm{Ge}$, and $\mathrm{Si}-\mathrm{Ge}$ Nanoclusters 


\section{Appendix C: Project Performance Measures}

\begin{tabular}{|c|c|c|c|c|c|c|c|c|c|c|c|c|c|c|c|}
\hline & & \multicolumn{11}{|c|}{$\begin{array}{l}\text { Performance Statistics } \\
\text { (Number of) }\end{array}$} & \multicolumn{3}{|c|}{$\begin{array}{c}\text { Project } \\
\text { Qualitative } \\
\text { Assessment }\end{array}$} \\
\hline Project Title & Project \# & 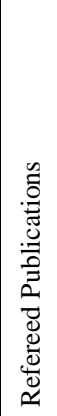 & 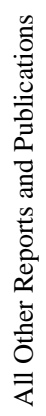 & 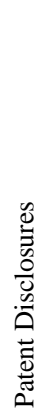 & 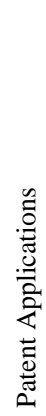 & $\frac{0}{0}$ & 0 & 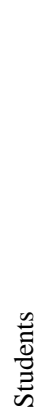 & 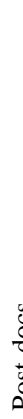 & & & & 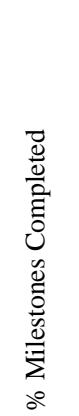 & 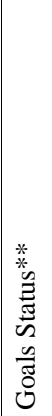 & 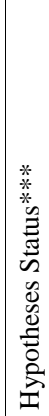 \\
\hline $\begin{array}{l}\text { Functional Materials for Microsystems: Smart Self-Assembled } \\
\text { Photochromic Films }\end{array}$ & 10300 & 5 & 3 & & & & & & & & & & $90 \%$ & 1 & 6 \\
\hline $\begin{array}{l}\text { Innovative Experimental and Computational Diagnostics for } \\
\text { Monitoring Corrosion in Weapons Environments }\end{array}$ & 10301 & & & & & & & & & & & & $95 \%$ & 2 & 7 \\
\hline $\begin{array}{l}\text { Self-Healing Molecular Assemblies for Control of Friction and } \\
\text { Adhesion in MEMS }\end{array}$ & 10302 & 5 & & & & & & 2 & 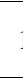 & & & & $90 \%$ & 1 & 7 \\
\hline Linking Atomistic Computations with Phase-Field Modeling & 10303 & & & & & & & & . & & & & $75 \%$ & 2 & 6 \\
\hline $\begin{array}{l}\text { A Combinatorial Microlab Investigation of Critical Copper- } \\
\text { Corrosion Mechanisms }\end{array}$ & 10304 & 3 & 2 & & & & & & & & & & $80 \%$ & 2 & 8 \\
\hline $\begin{array}{l}\text { Self-Assembled Templates for Fabricating Novel Nanoarrays and } \\
\text { Controlling Materials Growth }\end{array}$ & 10305 & 2 & & & & & & 2 & 2 & & & & $90 \%$ & 1 & 7 \\
\hline Wetting and Spreading Dynamics of Solder and Braze Alloys & 10306 & 17 & & & & & & & 2 & & & & $100 \%$ & 1 & 7 \\
\hline $\begin{array}{l}\text { Improved Materials-Aging Diagnostics and Mechanisms Through } \\
\text { 2-D Hyperspectral Imaging Methods and Algorithms }\end{array}$ & 10307 & 2 & 14 & 1 & 2 & & & & 2 & & & & $100 \%$ & 1 & 7 \\
\hline $\begin{array}{l}\text { Microscale Shock-Wave Physics Using Photonic Driver } \\
\text { Techniques }\end{array}$ & 10308 & & 1 & & & & & & & & & & $90 \%$ & 2 & 7 \\
\hline Reactivity of Metal-Oxide Surfaces & 10309 & 2 & & & & & & 1 & & & & & $100 \%$ & 1 & 6 \\
\hline Exploiting LENS Technology Through Novel Materials & 10310 & 6 & 2 & & & & & & & & & & $98 \%$ & 1 & 6 \\
\hline Molecular Characterization of Energetic-Material Initiation & 10311 & & 2 & & & & & & & & & & $85 \%$ & 2 & 6 \\
\hline Nonvolatile Protonic Memory & 10312 & 3 & & 2 & 1 & & & & 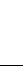 & & & & $75 \%$ & 11 & 7 \\
\hline Heterogeneous Simulation & 10313 & & & 1 & & & 1 & 1 & & & & & $100 \%$ & 1 & 6 \\
\hline $\begin{array}{l}\text { Volumetric Video Motion Sensing for Unobtrusive Human- } \\
\text { Computer Interactions }\end{array}$ & 10314 & 1 & & & 1 & & & 2 & & & & & $100 \%$ & 1 & 6 \\
\hline Hybrid Sparse-Dense Incomplete Factorization Preconditioners & 10315 & & 3 & & & & & & & & & & $100 \%$ & 1 & 7 \\
\hline $\begin{array}{l}\text { Advanced Large-Eddy Simulation Algorithms for Coupled-Flow } \\
\text { Physics and Complex Geometry }\end{array}$ & 10316 & & & & & & & & 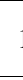 & & & & $95 \%$ & 1 & 7 \\
\hline Molecular Simulation of Reacting Systems & 10317 & 3 & & & & & & & & & & & $50 \%$ & 3 & 8 \\
\hline $\begin{array}{l}\text { Massively Parallel Global Climate Model for Paleoclimate } \\
\text { Applications }\end{array}$ & 10318 & & & & & & & & & & & & $100 \%$ & 1 & 7 \\
\hline
\end{tabular}




\section{Appendix C: Project Performance Measures}

\begin{tabular}{|c|c|c|c|c|c|c|c|c|c|c|c|c|c|c|c|}
\hline & & \multicolumn{11}{|c|}{$\begin{array}{l}\text { Performance Statistics } \\
\text { (Number of) }\end{array}$} & \multicolumn{3}{|c|}{$\begin{array}{l}\text { Project } \\
\text { Qualitative } \\
\text { Assessment }\end{array}$} \\
\hline Project Title & Project \# & 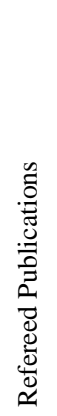 & 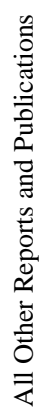 & 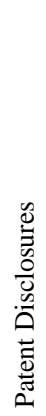 & 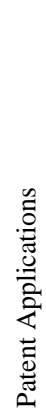 & 节 & ठิ & 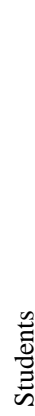 & 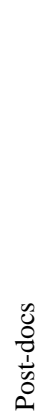 & 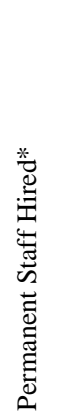 & $\begin{array}{l}n \\
z \\
0 \\
z \\
z \\
z\end{array}$ & 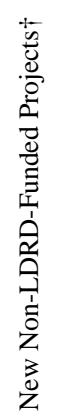 & 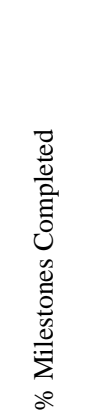 & 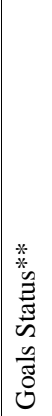 & 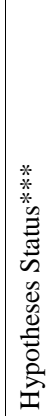 \\
\hline $\begin{array}{l}\text { From Atom-Picoseconds to Centimeter-Years in Simulation and } \\
\text { Experiment }\end{array}$ & 10319 & 4 & & & & & & 1 & 1 & & & & $100 \%$ & 1 & 6 \\
\hline Parallel Combinatorial Optimization for Scheduling Problems & 10320 & & 6 & & & & & & & & & & $70 \%$ & 3 & 7 \\
\hline $\begin{array}{l}\text { Multilevel Techniques for Unstructured Grid Problems on } \\
\text { Massively Parallel Computers }\end{array}$ & 10321 & 2 & & & & & & & & & & & $100 \%$ & 1 & 6 \\
\hline Visual Explanation and Insight & 10323 & 2 & 15 & & 2 & 1 & 1 & & & & & & $90 \%$ & 2 & 7 \\
\hline $\begin{array}{l}\text { Monolithic Integration of VCSELs and Detectors for } \\
\text { Microsystems }\end{array}$ & 10325 & & & & & & & & 1 & 1 & & & $75 \%$ & 3 & 7 \\
\hline Post-Processed Integrated Microsystems & 10326 & & & & & & & & & & & & $85 \%$ & 2 & 7 \\
\hline $\begin{array}{l}\text { Development of Radiation-Hard Sensors for Space-Based Visible } \\
\text { and Infrared Sensing Applications }\end{array}$ & 10327 & & & & & & & & & & & & $90 \%$ & 2 & 6 \\
\hline Silicon Three-Dimensional Photonic Crystal and Its Applications & 10328 & 2 & & & 1 & 1 & & & & & 1 & & $100 \%$ & 1 & 6 \\
\hline $\begin{array}{l}\text { Monolithic Micromachined Variable Tuners for Rapid } \\
\text { Prototyping and Optimization of Microwave Circuits }\end{array}$ & 10329 & & 2 & 1 & & & & 1 & & & & & $80 \%$ & 3 & 6 \\
\hline Quantum Tunneling Transistors for Practical Application & 10330 & 3 & & & & 1 & & & 1 & & & & $70 \%$ & 3 & 8 \\
\hline $\begin{array}{l}\text { Development of Magnetically Excited Flexural Plate-Wave } \\
\text { Devices for Implementation as Physical, Chemical, and Acoustic } \\
\text { Sensors, and as Integrated Micropumps for Sensored Systems }\end{array}$ & 10331 & & 2 & & & & & & & & & & $80 \%$ & 2 & 8 \\
\hline Novel Acoustically Driven Microoptoelectronic Devices & 10332 & & & & & & & & & & & & $90 \%$ & 1 & 6 \\
\hline Photonics Integration Devices and Technologies & 10333 & & & 1 & 1 & & & & 1 & & & & $90 \%$ & 11 & 7 \\
\hline $\begin{array}{l}\text { Stress-Free Amorphous Diamond for High-Sensitivity } \\
\text { Microsensors with Integrated Microstructures }\end{array}$ & 10334 & 4 & 1 & & & 1 & & & 1 & & & & $100 \%$ & 1 & 7 \\
\hline $\begin{array}{l}\text { Development of In Situ Diagnostics for Simultaneous } \\
\text { Measurement of Transient Gas Species and Soot in Large Fires }\end{array}$ & 10335 & & 3 & & & & & & 1 & & & & $80 \%$ & 2 & 7 \\
\hline Structural Simulations Using Multiresolution Material Models & 10336 & 2 & 1 & & & & & 5 & 0.5 & 0.5 & & & $90 \%$ & 2 & 6 \\
\hline Mechanisms of Adiabatic Shear Failure & 10338 & & & & & & & & 1 & & & & $80 \%$ & 2 & 7 \\
\hline Evolvable Hardware & 10339 & 1 & & & & & & & & & & & $10 \%$ & 11 & 7 \\
\hline $\begin{array}{l}\text { Crack Nucleation and Growth: Combining Validated Atomistic } \\
\text { and Continuum Modeling }\end{array}$ & 10340 & 3 & & & & & & & 2 & & & & $80 \%$ & 2 & 7 \\
\hline
\end{tabular}




\section{Appendix C: Project Performance Measures}

\begin{tabular}{|c|c|c|c|c|c|c|c|c|c|c|c|c|c|}
\hline & & \multicolumn{8}{|c|}{$\begin{array}{l}\text { Performance Statistics } \\
\text { (Number of) }\end{array}$} & \multicolumn{4}{|c|}{$\begin{array}{c}\text { Project } \\
\text { Qualitative } \\
\text { Assessment }\end{array}$} \\
\hline Project Title & Project \# & 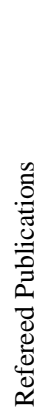 & 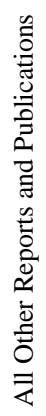 & 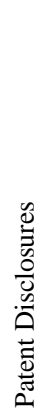 & 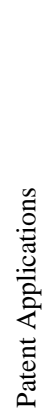 & $\frac{0}{0}$ & $\frac{0}{0}$ & 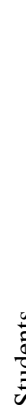 & & & 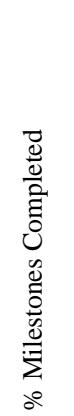 & 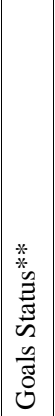 & 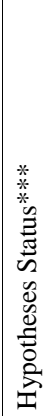 \\
\hline Applied Microfluidic Physics & 10341 & 4 & 4 & 1 & 1 & & & & & & $100 \%$ & 1 & 7 \\
\hline $\begin{array}{l}\text { Innovative Measurement Diagostics for Fluid/Solid and } \\
\text { Fluid/Fluid Interactions in Rotating Flowfields }\end{array}$ & 10343 & & 1 & & & & & & & & $85 \%$ & 2 & 7 \\
\hline $\begin{array}{l}\text { High-Resolution Modeling of Multiscale Transient Phenomena in } \\
\text { Turbulent Boundary Layers }\end{array}$ & 10344 & 1 & & & & & & & & & $90 \%$ & 2 & 6 \\
\hline Dispersive Measurements of Velocity in Heterogeneous Materials & 10345 & 2 & 1 & & & & & & & & $100 \%$ & 1 & 6 \\
\hline $\begin{array}{l}\text { A Physically Based Computational Method for Predicting } \\
\text { Generalized Fracture }\end{array}$ & 10346 & 4 & & & & & & 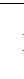 & & & $100 \%$ & 1 & 7 \\
\hline Microdiagnostic MEMS Lab-on-a-Chip & 10347 & 7 & & 3 & 2 & & & se & & & $90 \%$ & 2 & 7 \\
\hline $\begin{array}{l}\text { Real-Time Error Correction Using Electromagnetic-Bearing } \\
\text { Spindles }\end{array}$ & 10348 & 3 & & & & & & & & & $80 \%$ & 2 & 7 \\
\hline $\begin{array}{l}\text { Thin-Film Deposition Processes Incorporating In Situ Monitoring } \\
\text { Capabilities }\end{array}$ & 10349 & 1 & & & & & & . & & & $95 \%$ & 1 & 6 \\
\hline Solid-State Neutron Generator for Use in Nuclear Weapons & 10351 & & & & & & & 1 & & & $85 \%$ & 2 & 10 \\
\hline $\begin{array}{l}\text { Next-Generation Output-Based Process Control: An Integration } \\
\text { of Modeling, Sensors, and Intelligent Data Analysis }\end{array}$ & 10352 & & & & & & & & & & $85 \%$ & 2 & 8 \\
\hline Advanced Machining Processes for Microfabrication & 10353 & 2 & 3 & 1 & & & & 2 & & & $100 \%$ & 1 & 6 \\
\hline Advanced Production Planning Models & 10354 & 3 & 1 & 1 & & & & 1 & & & $100 \%$ & 1 & 7 \\
\hline $\begin{array}{l}\text { Weighted-Nearest-Neighbor (WNN) Decision Making for Data } \\
\text { Mining }\end{array}$ & 10355 & & & & & & & 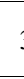 & & & $100 \%$ & 1 & 6 \\
\hline Varying QoS for Fixed and Mobile Networks & 10356 & & & & & & & & & & $100 \%$ & 1 & 7 \\
\hline $\begin{array}{l}\text { A Real-Time Decision-Support Framework to Guide Facility } \\
\text { Response to Abnormal Events }\end{array}$ & 10357 & & 2 & 1 & & & & & & & $80 \%$ & 2 & 7 \\
\hline Physical Model-Based Fusion of Sensor Array Data & 10358 & & & & & & & & & & $100 \%$ & 1 & 6 \\
\hline $\begin{array}{l}\text { Large-Scale Distributed Information System Modeling and } \\
\text { Simulation }\end{array}$ & 10359 & 2 & & & & & 1 & . & & & $100 \%$ & 1 & 8 \\
\hline High-Surety SCADA for the Critical Energy Infrastructures & 10360 & & 3 & & & & & & & & $100 \%$ & 1 & 7 \\
\hline Agent-Based Mediation and Cooperative Information Systems & 10361 & & & & & & & & & & $90 \%$ & 2 & 7 \\
\hline
\end{tabular}




\section{Appendix C: Project Performance Measures}

\begin{tabular}{|c|c|c|c|c|c|c|c|c|c|c|c|c|c|c|c|}
\hline & & \multicolumn{11}{|c|}{$\begin{array}{l}\text { Performance Statistics } \\
\text { (Number of) }\end{array}$} & \multicolumn{3}{|c|}{$\begin{array}{l}\text { Project } \\
\text { Qualitative } \\
\text { Assessment }\end{array}$} \\
\hline Project Title & Project \# & 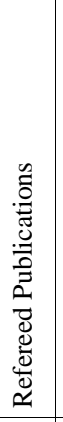 & 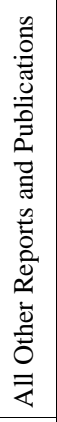 & 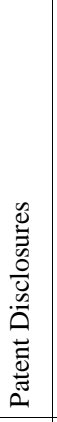 & 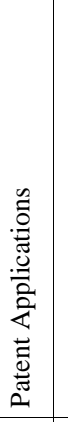 & 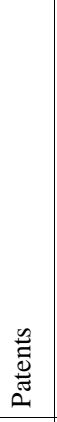 & 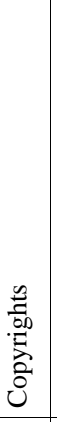 & $\begin{array}{l}\stackrel{0}{0} \\
\stackrel{0}{0} \\
\stackrel{\Xi}{0}\end{array}$ & 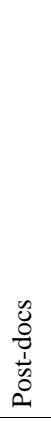 & 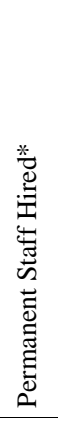 & 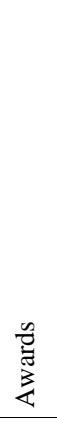 & 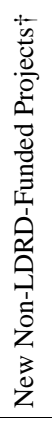 & 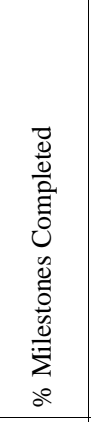 & 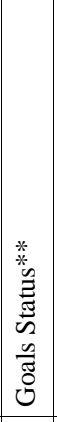 & 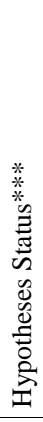 \\
\hline Dynamically Self-Configurable Network Protocol & 10362 & & & & & & & & & & & & $100 \%$ & 1 & 8 \\
\hline $\begin{array}{l}\text { Optical Backplane/Interconnect for Super-High-Speed } \\
\text { Communication }\end{array}$ & 10363 & & 1 & & & & & & & & & & $75 \%$ & 4 & 9 \\
\hline PUSH Technology Demonstration & 10364 & & & & & & & & & & & & $100 \%$ & 1 & 6 \\
\hline Controlling Information: Its Flow, Fusion, and Coordination & 10365 & & & & & & & & & & & & $100 \%$ & 1 & 7 \\
\hline Low-Power, Reduced-Computation, Public-Key Protocols & 10366 & & & & & & & & & & & & $100 \%$ & 1 & 6 \\
\hline Ten-to-One-Hundred-Gigabit/Second Network-Enabling R\&D & 10367 & & 6 & 3 & 1 & & & 1 & & & & & $95 \%$ & 1 & 8 \\
\hline $\begin{array}{l}\text { Surface Decontamination of Bacterial Protein Toxins by RF } \\
\text { Power }\end{array}$ & 10368 & & & 1 & & & & & & & & & $100 \%$ & 1 & 7 \\
\hline $\begin{array}{l}\text { Intense White-Light Pulse Propagation in Air Using Self-Guided } \\
\text { Optical Filamentation: Applications to Remote Sensing and } \\
\text { Countermeasures }\end{array}$ & 10369 & 5 & & & & & & 2 & & 1 & & & $100 \%$ & 2 & 7 \\
\hline HPM Vulnerability Assessment and Tests & 10370 & & & & & & & & & & & & $100 \%$ & 1 & 6 \\
\hline Miniature Bioaerosol Concentrator & 10371 & & & 2 & & & & & & 1 & & & $75 \%$ & 2 & 7 \\
\hline $\begin{array}{l}\text { Recognizing Partially Obscured Targets by Combining Multiple } \\
\text { Data Sources Using Evidential Reasoning }\end{array}$ & 10372 & & 4 & & & & & & & & & & $100 \%$ & 1 & 6 \\
\hline $\begin{array}{l}\text { Computational Engineering of Sensor Materials and Integration } \\
\text { with a Novel Biological Weapon Detection System }\end{array}$ & 10373 & & & & & & & & & & & & $90 \%$ & 2 & 6 \\
\hline $\begin{array}{l}\text { Biological Weapon Detector Using Bioaffinity Array Impedance } \\
\text { Analysis with Chemical Amplification Through Redox Recycling }\end{array}$ & 10374 & & & & & & & & & & & & $50 \%$ & 3 & 8 \\
\hline $\begin{array}{l}\text { ATR / Exploitation Utilizing Ultrahigh-Resolution, Complex } \\
\text { SAR Imaging }\end{array}$ & 10375 & & & & & & & & & & & & $95 \%$ & 2 & 7 \\
\hline Thin-Skin Deployable Mirrors for Remote Sensing Systems & 10376 & 2 & 3 & 2 & & & & 1 & & 3 & & & $100 \%$ & 1 & 7 \\
\hline $\begin{array}{l}\text { Dispersible Granular Sensor (Smart Sand) for Landmine } \\
\text { Detection Based on TNT Immunoassay }\end{array}$ & 10377 & & & & & & & & & & & & $85 \%$ & 4 & 7 \\
\hline $\begin{array}{l}\text { Characterization of Underground Facilities in an Urban } \\
\text { Environment }\end{array}$ & 10378 & 1 & & & & & & & & & & & $80 \%$ & 3 & 7 \\
\hline $\begin{array}{l}\text { Dexterous Robotic Manipulation of Hazardous Materials in } \\
\text { Unstructured Environments }\end{array}$ & 10379 & & & & & & & & & & & & $100 \%$ & 1 & 6 \\
\hline $\begin{array}{l}\text { Autonomous Dynamic Soaring Platform for Distributed Mobile } \\
\text { Sensor Arrays }\end{array}$ & 10380 & & & & & & & & & & & & $80 \%$ & 2 & 7 \\
\hline
\end{tabular}




\section{Appendix C: Project Performance Measures}

\begin{tabular}{|c|c|c|c|c|c|c|c|c|c|c|c|c|c|c|c|}
\hline & & \multicolumn{11}{|c|}{$\begin{array}{l}\text { Performance Statistics } \\
\text { (Number of) }\end{array}$} & \multicolumn{3}{|c|}{$\begin{array}{c}\text { Project } \\
\text { Qualitative } \\
\text { Assessment }\end{array}$} \\
\hline Project Title & Project \# & 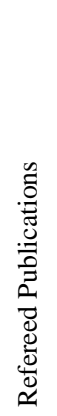 & 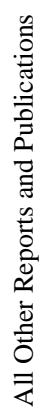 & 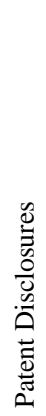 & 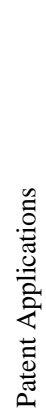 & $\frac{\tilde{\Xi}}{\tilde{\Xi}}$ & $\frac{0}{0}$ & 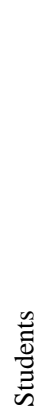 & $\begin{array}{l}y \\
y \\
y \\
\frac{1}{y} \\
0 \\
0\end{array}$ & 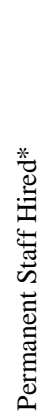 & 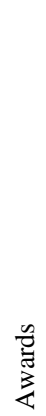 & 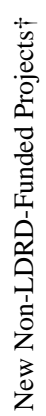 & 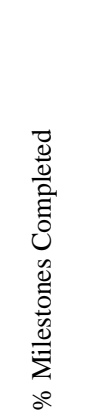 & 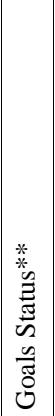 & 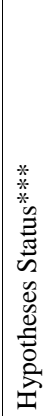 \\
\hline Miniature UV Fluorescence-Based Biological Agent Sensor & 10381 & & & & & & & & & & & & $50 \%$ & 3 & 8 \\
\hline Enabling Human Skills with Cooperative Automation & 10382 & & & & & & & & & & & & $100 \%$ & 1 & 6 \\
\hline Cloud to CAD & 10383 & & & & & & & & & & & & $100 \%$ & 1 & 6 \\
\hline Adaptive 3-D Sensing & 10384 & & & & & & & 1 & & & & & $100 \%$ & 1 & 7 \\
\hline $\begin{array}{l}\text { Designed Ionophores for Liquid-Membrane Separation and } \\
\text { Extraction of Metal Ions }\end{array}$ & 10385 & 13 & & 3 & & 1 & & 2 & 1 & & 1 & & $50 \%$ & 4 & 9 \\
\hline $\begin{array}{l}\text { An Electromagnetic Imaging System for Environmental Site } \\
\text { Reconnaissance }\end{array}$ & 10386 & & & & & & & & & & & & $50 \%$ & 3 & 9 \\
\hline Advanced Geosphere Transport Simulation & 10387 & 3 & 3 & & & & & & & & & & $80 \%$ & 2 & 7 \\
\hline $\begin{array}{l}\text { Hybrid Processing of Measurable and Subjective Information in } \\
\text { Surety Analysis }\end{array}$ & 10388 & 4 & & 1 & & & 1 & & & & & & $99 \%$ & 1 & 7 \\
\hline Approximate Public-Key Authentication with Information Hiding & 10389 & & & 1 & & & & 1 & & & & & $100 \%$ & 2 & 6 \\
\hline $\begin{array}{l}\text { New Network Analysis Approaches to Evaluate Infrastructure } \\
\text { Risk and Reliability }\end{array}$ & 10390 & & 3 & & & & & & & & & & $95 \%$ & 3 & 6 \\
\hline $\begin{array}{l}\text { Improved Tools for Identifying and Quantifying Potentially } \\
\text { Dangerous Human Actions }\end{array}$ & 10391 & & 1 & & & & & & & & & & $80 \%$ & 2 & 6 \\
\hline Advanced Signal Processing for Thermal Flaw Detection & 10392 & & & & & & & & & & & & $90 \%$ & 3 & 6 \\
\hline Production Surety and Disruption Vulnerability Analysis & 10393 & 1 & & & & & & & & & & & $100 \%$ & 1 & 7 \\
\hline An Optically Triggered Semiconductor Switch for Firing Systems & 10394 & 1 & & & & 1 & & & & & & & $100 \%$ & 1 & 6 \\
\hline Source Code Assurance Tool & 10395 & & 2 & 1 & & & & & & & & & $90 \%$ & 2 & 9 \\
\hline $\begin{array}{l}\text { Physical Models for Predicting the Effect of Atmospheric } \\
\text { Corrosion on Microelectronic Reliability }\end{array}$ & 10396 & 2 & 3 & & & & & 1 & & & & & $65 \%$ & 2 & 7 \\
\hline Security of Bulk Power Systems & 10397 & & & & & & & & & & & & $100 \%$ & 1 & 7 \\
\hline Engineering Complex Distributed Systems & 10398 & & & 4 & 1 & & & & & & & & $100 \%$ & 1 & 6 \\
\hline Information Collection & 10399 & & & & & & & & & & & & $97 \%$ & 1 & 7 \\
\hline
\end{tabular}




\section{Appendix C: Project Performance Measures}

\begin{tabular}{|c|c|c|c|c|c|c|c|c|c|c|c|c|c|c|c|}
\hline & & \multicolumn{11}{|c|}{$\begin{array}{l}\text { Performance Statistics } \\
\text { (Number of) }\end{array}$} & \multicolumn{3}{|c|}{$\begin{array}{c}\text { Project } \\
\text { Qualitative } \\
\text { Assessment }\end{array}$} \\
\hline Project Title & Project \# & 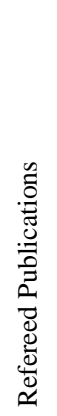 & 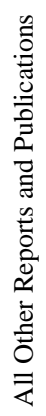 & 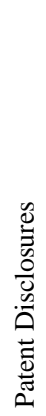 & 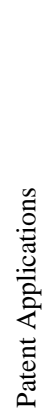 & 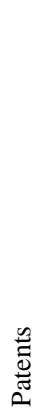 & $\frac{0}{0}$ & $\begin{array}{l}\text { : } \\
\text { 泀 } \\
\text { क }\end{array}$ & $\begin{array}{l}y \\
0 \\
0 \\
\frac{1}{d} \\
0 \\
0\end{array}$ & 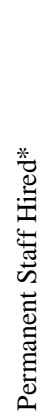 & 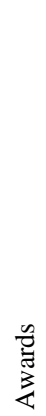 & 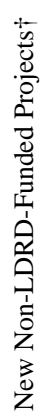 & 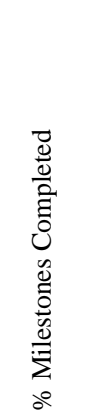 & 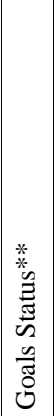 & 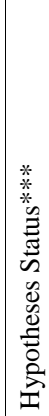 \\
\hline $\begin{array}{l}\text { Chemiresistors Based on Metal-Loaded Polymers for Solvent } \\
\text { Spill Detection }\end{array}$ & 10401 & & & & & & & & 1 & & & & $75 \%$ & 3 & 7 \\
\hline Advance Neutron-Tube Design and Producibility & 10402 & & & & & & & & & & & & $80 \%$ & 2 & 8 \\
\hline $\begin{array}{l}\text { Surface Hardening by Nanoparticle Precipitation and Atomic } \\
\text { Clustering in } \mathrm{Ni}(\mathrm{Al}, \mathrm{O})\end{array}$ & 10403 & & & & & & & & & & & & $100 \%$ & 1 & 6 \\
\hline Dynamical Properties of Polymers: Computational Modeling & 10404 & & & & & & & & & & & & $100 \%$ & 1 & 7 \\
\hline Broadening Mechanism in 2-D Excitonic and Electron Gases & 10405 & 1 & & & & & & & & & & & $100 \%$ & 1 & 9 \\
\hline $\begin{array}{l}\text { Direct Fabrication of Multifunctional Nanocomposites Via } \\
\text { Supramolecular Self-Assembly }\end{array}$ & 10406 & 1 & & 1 & 1 & & & 1 & 1 & & & & $90 \%$ & 1 & 7 \\
\hline $\begin{array}{l}\text { Information Extraction from Hyperspectral Images Obtained from } \\
\text { Satellites }\end{array}$ & 10407 & 1 & & & & & & & 1 & & & & $90 \%$ & 2 & 8 \\
\hline $\begin{array}{l}\text { Advanced Radiation Sources: Rayleigh-Taylor Mitigation Via } \\
\text { Perturbation Reduction }\end{array}$ & 10408 & 3 & & & & & & & & & & & $80 \%$ & 3 & 7 \\
\hline Capillary Elastohydrodynamics in Manufacturing Processes & 10409 & 3 & & & & & & & & & & & $100 \%$ & 1 & 7 \\
\hline $\begin{array}{l}\text { Real-Time Design of Improved Powder-Pressing Dies Using } \\
\text { Finite-Element Method Modeling }\end{array}$ & 10410 & 1 & 3 & 1 & & & 1 & & & & & & $100 \%$ & 1 & 6 \\
\hline $\begin{array}{l}\text { A Massively Parallel Microsimulation Model of Infrastructure } \\
\text { Interdependency }\end{array}$ & 10687 & & 3 & & & & & & & & & & $100 \%$ & 1 & 6 \\
\hline $\begin{array}{l}\text { AlGaN Materials Engineering for Integrated Multifunction } \\
\text { Systems }\end{array}$ & 10688 & 3 & & 3 & & & & 1 & & & & & $85 \%$ & 2 & 6 \\
\hline $\begin{array}{l}\text { Calculation and Interpretation of the Energies that Underlie } \\
\text { Transition-Metal Surface Structure }\end{array}$ & 10689 & 1 & & & & & & & & & & & $75 \%$ & 3 & 7 \\
\hline Emergent Behavior of Large Swarms of Intelligent Agents & 10690 & 1 & & & 1 & & & & & & & & $100 \%$ & 1 & 6 \\
\hline Interfacial Reactions in Ceramic Systems & 10692 & & & & & & & 2 & & & & & $85 \%$ & 2 & 7 \\
\hline Open Access (Nanosat) & 10693 & 24 & 6 & 1 & 2 & 1 & & & & & & & $75 \%$ & 2 & 8 \\
\hline $\begin{array}{l}\text { Micromechanical Failure Analyses for Finite-Element Polymer } \\
\text { Modeling }\end{array}$ & 10694 & 1 & & & & & & & & & & & $100 \%$ & 1 & 6 \\
\hline Poco Switch Tubes & 10695 & & & 1 & & & & & & & & & $100 \%$ & 1 & 6 \\
\hline Semiconductor Current Filament Lasers & 10696 & & 2 & 1 & 1 & & & & & & & & $95 \%$ & 1 & 6 \\
\hline
\end{tabular}




\section{Appendix C: Project Performance Measures}

\begin{tabular}{|c|c|c|c|c|c|c|c|c|c|c|c|c|c|c|c|}
\hline & & \multicolumn{11}{|c|}{$\begin{array}{l}\text { Performance Statistics } \\
\text { (Number of) }\end{array}$} & \multicolumn{3}{|c|}{$\begin{array}{l}\text { Project } \\
\text { Qualitative } \\
\text { Assessment }\end{array}$} \\
\hline Project Title & Project \# & 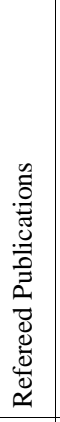 & 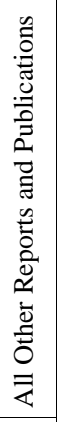 & 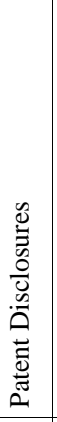 & 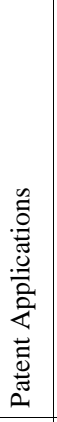 & 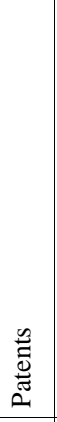 & 苛 & $\begin{array}{l}\stackrel{0}{0} \\
\stackrel{0}{0} \\
\stackrel{\Xi}{0}\end{array}$ & $\begin{array}{l}0 \\
0 \\
\frac{1}{1} \\
\frac{1}{0} \\
0\end{array}$ & 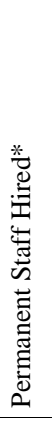 & 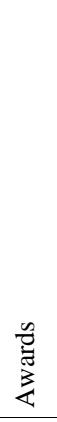 & 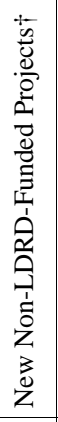 & 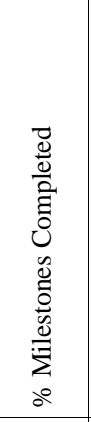 & 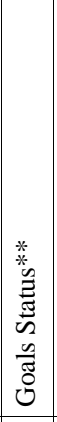 & 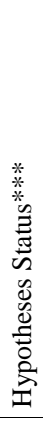 \\
\hline Sparse Geophysical Networks for Monitoring Deep Targets & 10697 & 2 & & & & & & & & & & & $100 \%$ & 1 & 6 \\
\hline Autonomous MicroChem Laboratory ( $\mu$ ChemLab) & 10698 & & 6 & 28 & 13 & 6 & & 9 & 8 & 3 & 1 & & $100 \%$ & 1 & 6 \\
\hline $\begin{array}{l}\text { A Novel Microcombustor for Sensor and Thermal-Energy } \\
\text { Management Applications in Microsystems }\end{array}$ & 10718 & & & & & & & 2 & 1 & 1 & & & $67 \%$ & 3 & 6 \\
\hline $\begin{array}{l}\text { Radiation-Induced Prompt Photocurrents in Microelectronics: } \\
\text { Physics }\end{array}$ & 10719 & 3 & & & & & & & & & & & $100 \%$ & 1 & 8 \\
\hline Proton Beam Directed-Energy Weapon & 10720 & & & & & & & & & & & & $100 \%$ & 1 & 7 \\
\hline Volumetric Displacement Control (VDC) of Manufacturing Tools & 10721 & 1 & & 2 & 3 & & & 2 & 1 & & & & $100 \%$ & 1 & 7 \\
\hline $\begin{array}{l}\text { Process-Based Quality Tools to Verify Cleaning and Surface } \\
\text { Preparation }\end{array}$ & 10722 & 2 & 1 & & & & & 3 & 1 & & & & $100 \%$ & 1 & 7 \\
\hline $\begin{array}{l}\text { Fabrication of Three-Dimensional Microstructures Using Soft } \\
\text { Lithography }\end{array}$ & 10723 & & & & & & & 1 & & & & & $100 \%$ & 1 & 7 \\
\hline $\begin{array}{l}\text { High-Speed 2-D Hadamard Transform Spectral Imager for IR } \\
\text { Applications }\end{array}$ & 10724 & 1 & & & & & & & & & & & $100 \%$ & 1 & 6 \\
\hline $\begin{array}{l}\text { A Microgas Chromatograph (GC)-Based Controller for Energy- } \\
\text { Intensive Processes }\end{array}$ & 10725 & & & & & & & 5 & 3 & & & & $95 \%$ & 2 & 7 \\
\hline Micro-High-G Acceleration Recorder & 10727 & & & & & & & & & & & & $90 \%$ & 1 & 7 \\
\hline Algorithmic Advances in Computational Structural Biology & 10728 & 2 & & & & & & 1 & & 4 & & & $100 \%$ & 1 & 7 \\
\hline Ultraminiaturization of RF Circuitry & 10729 & & & & & & & & & & & & $70 \%$ & 2 & 7 \\
\hline A Stronglink Prime Mover Based on an Electrokinetic Pump & 10731 & & & 1 & & & & & & 1 & & & $50 \%$ & 1 & 7 \\
\hline A MEMS Microelectric Generator & 10732 & & & & & & & & & & & & $75 \%$ & 2 & 7 \\
\hline $\begin{array}{l}\text { SOI-Based High-Aspect-Ratio Si Bulk Micromachining for } \\
\text { MEMS Applications }\end{array}$ & 10733 & & & & & & & & & & & & $90 \%$ & 1 & 7 \\
\hline $\begin{array}{l}\text { Next-Generation Output-Based Process Control: An Integration } \\
\text { of Modeling, Sensors, and Intelligent Data Analysis }\end{array}$ & 10734 & & & & & & & & 1 & 1 & & & $25 \%$ & 3 & 7 \\
\hline Physical Basis for Interfacial Traction-Separation Models & 10735 & & 1 & & & & & & 1 & & & & $90 \%$ & 2 & 7 \\
\hline $\begin{array}{l}\text { Dynamic Range Imaging for Terrain Mapping, Position } \\
\text { Determination, and Obstacle Avoidance in Autonomous } \\
\text { Navigation }\end{array}$ & 10736 & & & & & & & & & & & & $90 \%$ & 1 & 8 \\
\hline
\end{tabular}




\section{Appendix C: Project Performance Measures}

\begin{tabular}{|c|c|c|c|c|c|c|c|c|c|c|c|c|c|c|c|}
\hline & & \multicolumn{11}{|c|}{$\begin{array}{l}\text { Performance Statistics } \\
\text { (Number of) }\end{array}$} & \multicolumn{3}{|c|}{$\begin{array}{c}\text { Project } \\
\text { Qualitative } \\
\text { Assessment }\end{array}$} \\
\hline Project Title & Project \# & 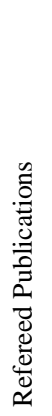 & 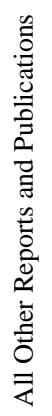 & 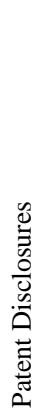 & 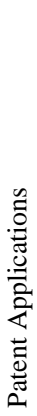 & 节 & ن & 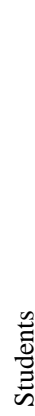 & $\begin{array}{l}\tilde{U} \\
0 \\
0 \\
\frac{1}{2} \\
0 \\
0\end{array}$ & 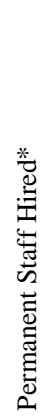 & $\begin{array}{l}n \\
z \\
0 \\
z \\
z \\
z\end{array}$ & 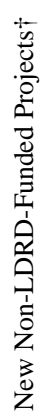 & 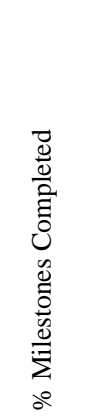 & 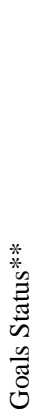 & 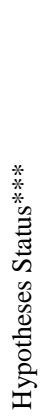 \\
\hline Laser-Assisted Microgas Metal Arc Welding & 10739 & & & & & & & 2 & & & & & $100 \%$ & 1 & 9 \\
\hline $\begin{array}{l}\text { Diagnostics for Joining Solidification/Microstructural } \\
\text { Simulations }\end{array}$ & 10740 & & & & & & & & 1 & & & & $95 \%$ & 1 & 7 \\
\hline $\begin{array}{l}\text { Effects of Microstructural Variables on the Shock-Wave } \\
\text { Response of PZT 95/5 }\end{array}$ & 10741 & & & & & & & & & & & & $80 \%$ & 2 & 7 \\
\hline Mechanisms of Dislocation-Grain-Boundary Interaction & 10742 & 1 & 2 & & & & & & 1 & & & & $100 \%$ & 2 & 7 \\
\hline $\begin{array}{l}\text { Defining the Frontiers of Vertical, External-Cavity, Surface- } \\
\text { Emitting Lasers }\end{array}$ & 10744 & & & & & & & 1 & & & & & $80 \%$ & 3 & 7 \\
\hline $\begin{array}{l}\text { Photoresist Technology for } 70 \mathrm{~nm} \text { Lithography with } 157 \mathrm{~nm} \\
\text { Radiation }\end{array}$ & 10745 & & & & & & & & & & & & $80 \%$ & 2 & 8 \\
\hline Predicting Function of Biological Macromolecules & 10746 & 1 & & & & & & & 1 & & & & $90 \%$ & 2 & 7 \\
\hline $\begin{array}{l}\text { Induced Molecular Markers for Pathogen Detection: } \\
\text { Microincubators for Rapid Toxin Expression }\end{array}$ & 10747 & & & & & & & 1 & 1 & & & & $80 \%$ & 2 & 8 \\
\hline $\begin{array}{l}\text { Nanostructured Silicon Surfaces for Cost-Effective Photovoltaic } \\
\text { Efficiency Improvements }\end{array}$ & 10748 & 1 & 1 & 1 & 1 & & & & & & 1 & & $75 \%$ & 1 & 7 \\
\hline $\begin{array}{l}\text { Growth and Characterization of Quantum Dots and Quantum Dot } \\
\text { Devices }\end{array}$ & 10749 & & & & & & & & 2 & & & & $90 \%$ & 2 & 7 \\
\hline THz Technologies for Ultrahigh-Data-Rate Communications & 10750 & 3 & 1 & 1 & 1 & & & & 1 & & & & $70 \%$ & 3 & 7 \\
\hline Automatic Design of Practical Fixtures & 10751 & 3 & 1 & & & & & 1 & & & 1 & & $90 \%$ & 2 & 7 \\
\hline Distributed Reconfigurable Homogeneous Microrobotic Systems & 10752 & & & & & & & & & & & & $100 \%$ & 2 & 7 \\
\hline $\begin{array}{l}\text { Robust Planning for Autonomous Navigation of Mobile Robots } \\
\text { in Unstructured, Dynamic Environments }\end{array}$ & 10753 & & & & & & & & & & & & $100 \%$ & 1 & 7 \\
\hline $\begin{array}{l}\text { Functional Materials for Electrochemomechanical Actuation of } \\
\text { Microvalves and Micropumps }\end{array}$ & 10754 & 1 & & 1 & & & & & 1 & & & & $80 \%$ & 3 & 7 \\
\hline $\begin{array}{l}\text { Microreplication: Precision Metal Parts from Electroformed } \\
\text { Master Molds }\end{array}$ & 10755 & & & & & & & & & 1 & & & $80 \%$ & 2 & 7 \\
\hline New Architectures for Micro-Total-Analytical Systems & 10756 & 1 & & 2 & & & & & & & & & $80 \%$ & 3 & 7 \\
\hline Large-Scale Nonlinear Optimization Arising from PDE Models & 10757 & 3 & 1 & & 2 & & & 3 & & & & & $75 \%$ & 3 & 7 \\
\hline $\begin{array}{l}\text { Network Intrusion Detection Using Adaptive Critic Neural } \\
\text { Networks }\end{array}$ & 10758 & & & & & & & 1 & & & & & $80 \%$ & 2 & 7 \\
\hline
\end{tabular}




\section{Appendix C: Project Performance Measures}

\begin{tabular}{|c|c|c|c|c|c|c|c|c|c|c|c|c|c|c|c|}
\hline & & \multicolumn{11}{|c|}{$\begin{array}{l}\text { Performance Statistics } \\
\text { (Number of) }\end{array}$} & \multicolumn{3}{|c|}{$\begin{array}{c}\text { Project } \\
\text { Qualitative } \\
\text { Assessment }\end{array}$} \\
\hline Project Title & Project \# & 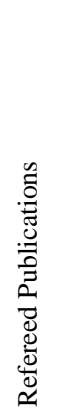 & 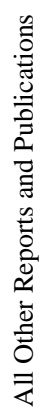 & 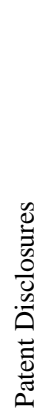 & 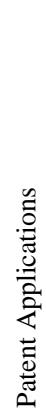 & $\stackrel{\mathscr{U}}{\tilde{D}}$ & 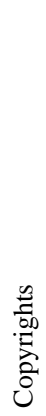 & 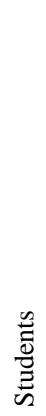 & $\begin{array}{l}\tilde{0} \\
0 \\
\frac{0}{1} \\
\frac{1}{0} \\
0\end{array}$ & 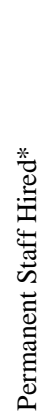 & $\begin{array}{l}n \\
z \\
0 \\
z \\
z \\
z\end{array}$ & 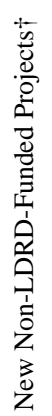 & 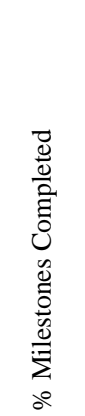 & 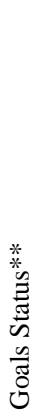 & 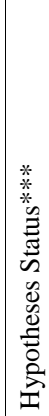 \\
\hline $\begin{array}{l}\text { Key Management Techniques for Authenticating Highly Secure } \\
\text { SCADA Systems in the Electric Power Industry }\end{array}$ & 10759 & & & & & & & 1 & & & & & $100 \%$ & 1 & 7 \\
\hline Making the Connection Between Microstructure and Mechanics & 10761 & 4 & & & & & & & & & & & $90 \%$ & 1 & 7 \\
\hline Self-Assembled, Tamper-Detection Seals & 10762 & & & & & & & & & & & & $95 \%$ & 1 & 8 \\
\hline $\begin{array}{l}\text { Control Strategies for Homogeneous Charge-Compression } \\
\text { Ignition (HCCI) Engines }\end{array}$ & 10763 & & & & 1 & & & & 2 & & & & $100 \%$ & 1 & 7 \\
\hline Energetic-Material Burn and Detonation at the Mesoscale & 10765 & & & & & & & & & 1 & & & $90 \%$ & 2 & 7 \\
\hline $\begin{array}{l}\text { Heterogeneous Integration of Optoelectronic Arrays and } \\
\text { Microelectronics }\end{array}$ & 10766 & & & & & & & 1 & & & & & $95 \%$ & 2 & 7 \\
\hline $\begin{array}{l}\text { Intrusion Detection for Asynchronous Transfer Mode (ATM) } \\
\text { Networks }\end{array}$ & 10767 & 1 & 5 & & & & & 1 & & & 1 & & $100 \%$ & 1 & 7 \\
\hline Conversational Policies for Multiagent Systems & 10768 & & & & & & & & & & & & $90 \%$ & 2 & 7 \\
\hline IMEMS Packaging and Interconnection Technology & 10770 & & & 1 & 4 & & & & & & & & $100 \%$ & 1 & 7 \\
\hline $\begin{array}{l}\text { Silicon/Carbon Nanocomposites for Rechargeable Battery } \\
\text { Applications }\end{array}$ & 10771 & & & 1 & & & & 1 & & & & & $90 \%$ & 2 & 7 \\
\hline Intense Directed-Energy Theory Initiative & 10772 & & 1 & & & & & 1 & 1 & & & & $100 \%$ & 1 & 7 \\
\hline Assembly of LIGA Using Electric Fields & 10773 & & & & & & & 1 & & & & & $75 \%$ & 2 & 7 \\
\hline $\begin{array}{l}\text { Biosensors Based on the Electrical Impedance of Tethered Lipid } \\
\text { Bilayers on Planar Electrodes }\end{array}$ & 10774 & & & & & & & & 1 & 1 & & & $100 \%$ & 1 & 7 \\
\hline Miniature Sensors for BW Agents Using Fatty-Acid Profiles & 10775 & & & & & & & & 1 & & & & $90 \%$ & 2 & 6 \\
\hline Enhanced-Sensitivity Acoustic-Wave Biosensor Arrays & 10776 & 5 & 2 & & & & & 2 & 1 & 2 & & & $100 \%$ & 1 & 7 \\
\hline $\begin{array}{l}\text { Parallel Methods for Coupling Circuit- and Device-Scale } \\
\text { Simulations }\end{array}$ & 10777 & & & & & & & & 1 & & & & $90 \%$ & 2 & 7 \\
\hline $\begin{array}{l}\text { A Java-Based Tool for Multifidelity Modeling of Coupled } \\
\text { Systems }\end{array}$ & 10778 & & 1 & & & & & & & & & & $90 \%$ & 2 & 7 \\
\hline Switchable Hydrophobic-Hydrophilic Surfaces & 10779 & & & & & & & 1 & 2 & & & & $80 \%$ & 1 & 8 \\
\hline $\begin{array}{l}\text { Nanostructured Materials Integrated in Microfabricated Optical } \\
\text { Devices }\end{array}$ & 10780 & 2 & & & & & & 1 & 1 & & & & $85 \%$ & 3 & 7 \\
\hline
\end{tabular}




\section{Appendix C: Project Performance Measures}

\begin{tabular}{|c|c|c|c|c|c|c|c|c|c|c|c|c|c|c|c|}
\hline & & \multicolumn{11}{|c|}{$\begin{array}{l}\text { Performance Statistics } \\
\text { (Number of) }\end{array}$} & \multicolumn{3}{|c|}{$\begin{array}{c}\text { Project } \\
\text { Qualitative } \\
\text { Assessment }\end{array}$} \\
\hline Project Title & Project \# & 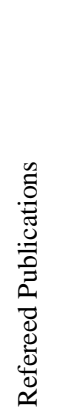 & 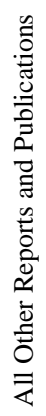 & 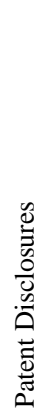 & 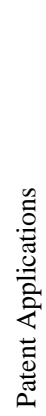 & 节 & ن & $\begin{array}{l}\text { : } \\
\text { 泀 } \\
\text { क }\end{array}$ & $\begin{array}{l}y \\
0 \\
0 \\
\frac{1}{d} \\
0 \\
0\end{array}$ & 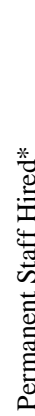 & 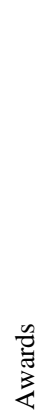 & 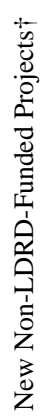 & 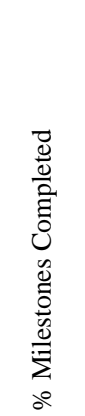 & 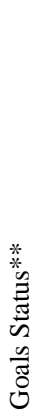 & 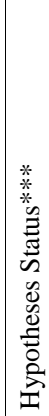 \\
\hline Virtual Reliability Realization System & 10781 & & & & & & & & & & & & $100 \%$ & 1 & 7 \\
\hline $\begin{array}{l}\text { High-Al-Content AlGaInN Devices for Next-Generation } \\
\text { Electronic and Optoelectronic Applications }\end{array}$ & 10782 & 5 & & 2 & & & & & & & & & $100 \%$ & 1 & 7 \\
\hline Microfabrication of Electromagnetic Devices & 10783 & & & 2 & 2 & & & 2 & & & & & $100 \%$ & 1 & 8 \\
\hline GaAs MOEMS Technology & 10784 & & 1 & 1 & & & & 1 & & & & & $80 \%$ & 2 & 7 \\
\hline Integrated Microsensors for Autonomous Microrobots & 10785 & & & & & & & & & & & & $100 \%$ & 1 & 7 \\
\hline Silicon-Integrated Planar Microbatteries & 10786 & & & & & & & & & & & & $90 \%$ & 1 & 7 \\
\hline $\begin{array}{l}\text { Small-Scale Multiaxial Deformation Experiments on Solder for } \\
\text { High-Fidelity Model Development }\end{array}$ & 10788 & 1 & & & & & & 1 & 2 & & & & $100 \%$ & 1 & 7 \\
\hline All-Ceramic Thin-Film Battery & 10789 & & & & & & & 2 & 1 & & & & $100 \%$ & 1 & 7 \\
\hline Fuzzy Data Mining & 10791 & & & & & & & & & 1 & & & $80 \%$ & 2 & 7 \\
\hline $\begin{array}{l}\text { Ultrahigh-Resolution Radiography for Detailed Inspection of } \\
\text { Weapon Components and Systems }\end{array}$ & 10792 & 4 & 4 & 2 & 1 & & & 1 & & & & & $100 \%$ & 1 & 7 \\
\hline Computer Numerically Controlled Micromachines & 10793 & & & & & & & 1 & & & & & $75 \%$ & 2 & 7 \\
\hline Electromicrofluidic Packaging & 10794 & & 1 & 1 & & & & 1 & & & & & $85 \%$ & 2 & 7 \\
\hline Surface-Micromachined Mechanical Timer & 10795 & & & & & & & & & & & & $90 \%$ & 2 & 7 \\
\hline $\begin{array}{l}\text { High-Efficiency Optical MEMS by the Integration of Photonic } \\
\text { Lattices with Surface MEMS }\end{array}$ & 10796 & & & 1 & & & & & & & & & $100 \%$ & 1 & 7 \\
\hline Molecular Integrated Microsystems (MIMS) & 11130 & 2 & 6 & 5 & 2 & 3 & & & 4 & 3 & & & $90 \%$ & 2 & 7 \\
\hline Gamma-Ray Bursts and the Particle Mass Scale & 11336 & 1 & & & & & & 1 & 1 & & & & $100 \%$ & 1 & 7 \\
\hline $\begin{array}{l}\text { Pseudostationary Separation Materials for Highly Parallel } \\
\text { Microseparations }\end{array}$ & 16167 & 9 & & & & & & 2 & & & & & $100 \%$ & 1 & 6 \\
\hline Novel Coatings for Microelectromechanical (MEM) Devices & 17230 & 5 & & & 2 & & & 2 & & & & & $80 \%$ & 2 & 8 \\
\hline Investigation of the Feasibility of RTL Concept for Fusion Energy & 19989 & 2 & 4 & & & & & & & & & & $100 \%$ & 1 & 6 \\
\hline
\end{tabular}




\section{Appendix C: Project Performance Measures}

\begin{tabular}{|c|c|c|c|c|c|c|c|c|c|c|c|c|c|c|c|}
\hline & & \multicolumn{11}{|c|}{$\begin{array}{l}\text { Performance Statistics } \\
\text { (Number of) }\end{array}$} & \multicolumn{3}{|c|}{$\begin{array}{c}\text { Project } \\
\text { Qualitative } \\
\text { Assessment }\end{array}$} \\
\hline Project Title & Project \# & 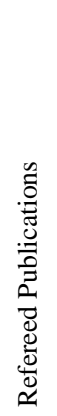 & 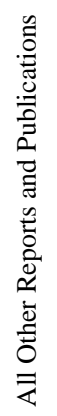 & 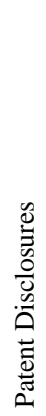 & 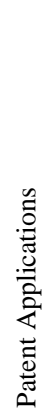 & $\stackrel{\mathscr{U}}{\tilde{D}}$ & $\begin{array}{l}\frac{0}{0} \\
\frac{0}{00} \\
\frac{2}{0} \\
0 \\
0\end{array}$ & $\begin{array}{l}\stackrel{0}{0} \\
\stackrel{0}{0} \\
\tilde{E}\end{array}$ & $\begin{array}{l}\tilde{0} \\
0 \\
\frac{0}{1} \\
\frac{1}{0} \\
0\end{array}$ & 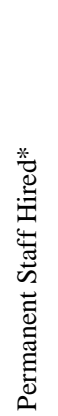 & $\begin{array}{l}n \\
z \\
0 \\
z \\
z \\
z\end{array}$ & 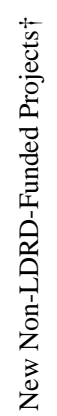 & 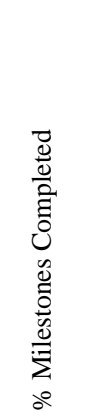 & 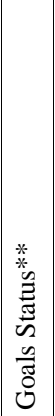 & 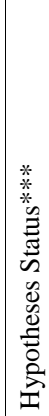 \\
\hline Rapid Discovery of Materials for Energy Systems & 19990 & & & & & & & 5 & 1 & & & & $95 \%$ & 2 & 6 \\
\hline $\begin{array}{l}\text { In Situ Formation of Reactive Barriers for Radionuclide, Heavy- } \\
\text { Metal, and Organic Stabilization }\end{array}$ & 19991 & 2 & & 3 & & & & 1 & & & & & $90 \%$ & 2 & 6 \\
\hline MPP Direct Numerical Simulation of Diesel Autoignition & 19993 & & & & & & & & 1 & 1 & & & $100 \%$ & 1 & 8 \\
\hline $\begin{array}{l}\text { A Functional Parallel Discrete-Event Simulation of a Snoopy } \\
\text { Coprocessor Software Monitor }\end{array}$ & 20407 & & & & & & & 1 & & & & & $100 \%$ & 1 & 6 \\
\hline New Complexing Agent for Co(II) Analysis & 20409 & & & & & & & 2 & & & & & $100 \%$ & 1 & 7 \\
\hline Infrared Polarization for Target Identification & 20410 & 1 & & & & & & 4 & & & & & $90 \%$ & 2 & 6 \\
\hline $\begin{array}{l}\text { Characterization of Polymer-Metal Interface Properties for } \\
\text { LIGA-Based Microsystem Technologies }\end{array}$ & 20411 & & & & & & & & & & & & $75 \%$ & 2 & 8 \\
\hline Fault-Tolerant Computing Services for Heterogeneous Clusters & 20412 & 1 & & & & & & 3 & & & & & $95 \%$ & 1 & 7 \\
\hline DNA Microarray Technology & 20413 & 3 & 2 & & & & & 10 & 1 & & & & $85 \%$ & 2 & 7 \\
\hline Shock Response of Diamond Crystals & 20414 & & & & & & & & & & & & $85 \%$ & 2 & 7 \\
\hline $\begin{array}{l}\text { The Use of Active Fiber Composites for the Health Monitoring of } \\
\text { Wind Turbine Blades }\end{array}$ & 20415 & & & & & & & 1 & & & & & $99 \%$ & 1 & 7 \\
\hline Information-Sharing Security Module & 20416 & & & & & & & 1 & & & & & $100 \%$ & 1 & 6 \\
\hline $\begin{array}{l}\text { Adaptive Sensor Optimization and Cognitive Image Processing } \\
\text { Using Autonomous Optical Neuroprocessors }\end{array}$ & 21270 & & & & & & & & & & & & $75 \%$ & 3 & 9 \\
\hline $\begin{array}{l}\text { Integration of Sandia Microsensors to the Internet for Logistics } \\
\text { Applications }\end{array}$ & 21271 & & 1 & 1 & & & & & & & & & $100 \%$ & 1 & 6 \\
\hline Automatic Target Recognition in Three Dimensions & 21272 & & & & & & & & & & & & $100 \%$ & 1 & 7 \\
\hline Modeling the Jini Infrastructure Protocol & 21273 & & & & & & & & & & & & $100 \%$ & 1 & 6 \\
\hline Intelligent Targeting for Remote Weapons & 21275 & & & & & & & & & & & & $100 \%$ & 1 & 6 \\
\hline Microfuel Reformer: Hydrogen for Microfuel Cells & 21276 & & & & & & & 1 & & & & & $100 \%$ & 1 & 7 \\
\hline $\begin{array}{l}\text { Advanced Techniques for Real-Time Visualization of Data- } \\
\text { Intensive Missions }\end{array}$ & 21277 & & & & & & & & & & & & $90 \%$ & 1 & 7 \\
\hline
\end{tabular}




\section{Appendix C: Project Performance Measures}

\begin{tabular}{|c|c|c|c|c|c|c|c|c|c|c|c|c|c|c|c|}
\hline & & \multicolumn{11}{|c|}{$\begin{array}{l}\text { Performance Statistics } \\
\text { (Number of) }\end{array}$} & \multicolumn{3}{|c|}{$\begin{array}{c}\text { Project } \\
\text { Qualitative } \\
\text { Assessment }\end{array}$} \\
\hline Project Title & Project \# & 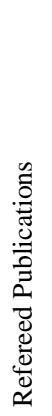 & 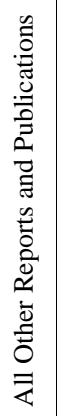 & 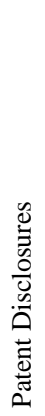 & 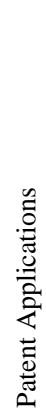 & 节 & 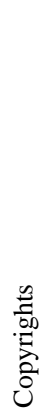 & 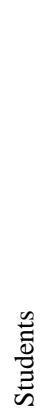 & $\begin{array}{l}\tilde{0} \\
0 \\
\frac{1}{0} \\
0 \\
0\end{array}$ & 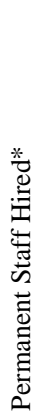 & $\begin{array}{l}n \\
z \\
0 \\
z \\
z \\
z\end{array}$ & 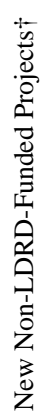 & 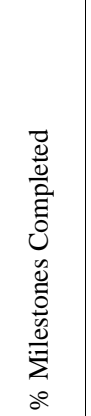 & 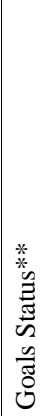 & 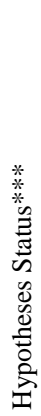 \\
\hline Early Diagnosis of Human Exposure to Biological Agents & 21278 & & & & & & & & & & & & $80 \%$ & 2 & 7 \\
\hline $\begin{array}{l}\text { Multisensor Fusion for Characterizing Underground Facilities } \\
\text { (UGFs) }\end{array}$ & 21279 & & & & & & & & & & & & $100 \%$ & 2 & 6 \\
\hline $\begin{array}{l}\text { Developing Land Surface-Temperature Maps form Multispectral } \\
\text { Thermal Imager (MTI) Data }\end{array}$ & 21280 & & & & & & & & & & & & $50 \%$ & 4 & 9 \\
\hline Enterprise Modeling of Medical Surveillance Network & 21281 & & & & & & & & & & & & $100 \%$ & 1 & 7 \\
\hline $\begin{array}{l}\text { The Thermal History of Charred Materials by Raman } \\
\text { Spectroscopy }\end{array}$ & 21282 & & & & & & & & & & & & $100 \%$ & 1 & 6 \\
\hline Optical Interconnections to Focal Plane Arrays & 21283 & & & & & & & & & & & & $100 \%$ & 1 & 8 \\
\hline Change Detection in Flash Memory & 21285 & & & & & & & & & & & & $100 \%$ & 1 & 9 \\
\hline $\begin{array}{l}\text { Study of Effectiveness of Spectro-Polarization Parameters in } \\
\text { Military Target Detection }\end{array}$ & 21287 & & & & & & & & & & & & $75 \%$ & 4 & 10 \\
\hline Damaged Chip & 21288 & & & & & & & & & & & & $100 \%$ & 1 & 6 \\
\hline Engineered Window Glass for Architectural Surety Applications & 21289 & 2 & 2 & 1 & & & & 1 & & & & & $75 \%$ & 2 & 7 \\
\hline Layered Pyrotechnic Smoke Materials for Barriers & 21290 & & & & & & & & 1 & & & & $100 \%$ & 2 & 6 \\
\hline Miniaturized Flying Penetrator & 21311 & & 1 & & & & & & & & & & $100 \%$ & 1 & 6 \\
\hline Preconcentration of Volatile Chemical Agents and Explosives & 21312 & & & & & & & & & & & & $100 \%$ & 1 & 8 \\
\hline Accelerated Beta Decay for the Reduction of Legacy Wastes & 21545 & & & & & & & & & & & & $100 \%$ & 1 & 10 \\
\hline Kinetic-Energy Lightning Generator & 21548 & & & & & & & & & & & & $70 \%$ & 2 & 8 \\
\hline $\begin{array}{l}\text { Comprehensive Representation of Human Decision Process: } \\
\text { Sensor Shooter Problem }\end{array}$ & 21550 & & & & 1 & & & & & & & & $90 \%$ & 1 & 8 \\
\hline Spectroscopic Detection of Pathogens & 21553 & & & & & & & & 1 & & & & $100 \%$ & 1 & 8 \\
\hline $\begin{array}{l}\text { Technical Solutions to National Security Issues Based on Limited } \\
\text { Natural Resources }\end{array}$ & 21554 & & & 2 & 2 & & & 1 & & & & & $100 \%$ & 1 & 8 \\
\hline Sustainable Bioelectronic Microsystems: Life on a Chip & 21557 & & & & & & & 2 & 2 & & & & $80 \%$ & 4 & 8 \\
\hline
\end{tabular}




\section{Appendix C: Project Performance Measures}

\begin{tabular}{|c|c|c|c|c|c|c|c|c|c|c|c|c|c|c|c|}
\hline & & \multicolumn{11}{|c|}{$\begin{array}{l}\text { Performance Statistics } \\
\text { (Number of) }\end{array}$} & \multicolumn{3}{|c|}{$\begin{array}{c}\text { Project } \\
\text { Qualitative } \\
\text { Assessment }\end{array}$} \\
\hline Project Title & Project \# & 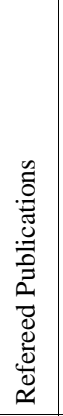 & 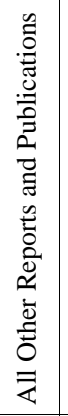 & 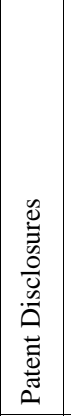 & 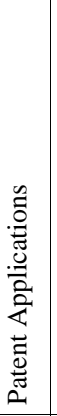 & 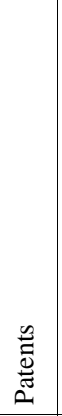 & 莣 & $\frac{0}{0}$ & $\begin{array}{l}\tilde{u} \\
0 \\
0 \\
i \\
0 \\
0 \\
0\end{array}$ & 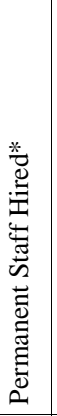 & 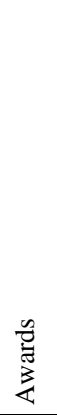 & 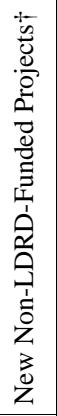 & 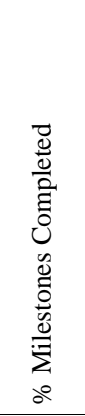 & 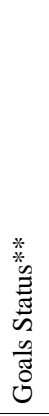 & 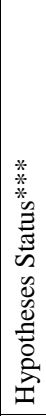 \\
\hline Near-Real-Time Surveillance Against Bioterror Attack & 21558 & & 2 & & & & & & & & & & $75 \%$ & 3 & 7 \\
\hline Investigation of Modeling Approaches for Nuclear Futures & 21559 & & & & & & & & & & 1 & & $100 \%$ & 2 & 6 \\
\hline $\begin{array}{l}\text { Understanding and Managing Threats to the Social Fabric of the } \\
\text { United States }\end{array}$ & 22126 & & 1 & & & & & & & & & & $90 \%$ & 2 & 7 \\
\hline $\begin{array}{l}\text { Physical and Functional Modeling of a Bioelectronic } \\
\text { Microsystem }\end{array}$ & 22127 & & & & & & & & & & & & $100 \%$ & 1 & 7 \\
\hline Analytic Models for Cooperative Utilization of Critical Resources & 22128 & & 1 & & & & & & & & & & $100 \%$ & 1 & 7 \\
\hline Cryopreservation of Biological Tissue & 22129 & 1 & & & & & & 1 & & & & & $80 \%$ & 2 & 7 \\
\hline Investigation of Nanoscience Technologies & 22131 & & & 1 & & & & & & & & & $75 \%$ & 2 & 7 \\
\hline Magnetic Polysilicon MEMS Devices & 22132 & & & & 5 & & & 1 & & & & & $100 \%$ & 1 & 7 \\
\hline Autonomous Microexplosive Subsurface Tracing System & 22136 & & & & & & & & & & & & $100 \%$ & 1 & 7 \\
\hline $\begin{array}{l}\text { New Metal-Organic Chemical Vapor Deposition Reactor Designs } \\
\text { and Optimization }\end{array}$ & 22854 & & & & & & & 1 & & & & & $80 \%$ & 3 & 7 \\
\hline Composite-Wire Plasma Formation and Evolution & 22995 & 1 & & & & & & 2 & & & & & $100 \%$ & 1 & 9 \\
\hline Scattering from Nanostructured Materials & 22996 & & & 1 & & & & & 1 & & & & $50 \%$ & 2 & 9 \\
\hline $\begin{array}{l}\text { The Liquid-Crystal Physics of Evaporation-Induced Self- } \\
\text { Assembly }\end{array}$ & 22997 & & & & & & & 1 & & & & & $50 \%$ & 3 & 7 \\
\hline $\begin{array}{l}\text { Electrochemical Interrogation of Cellular Membrane Transport } \\
\text { Processes }\end{array}$ & 22998 & & & & & & & & & & & & $25 \%$ & 3 & 7 \\
\hline $\begin{array}{l}\text { A Micromethanol Steam Reformer for a Hydrogen-Based } \\
\text { Microfuel Cell }\end{array}$ & 24101 & & & & & & & & & & & & $80 \%$ & 3 & 7 \\
\hline Spatial Anomalies in Magnetically Insulated Vacuum Structures & 24102 & & & & & & & 2 & & & & & $100 \%$ & 1 & 8 \\
\hline & & 250 & 161 & 100 & 58 & 16 & 5 & 139 & 79 & 32 & 10 & 0 & & & \\
\hline
\end{tabular}

$\dagger$ Identification of new non-LDRD-funded projects is now completed in the fiscal year following the annual report.

* Permanent Staff Hired. Laboratories operate under manpower cap. There are no increases in permanent staff. 


\section{Appendix C: Project Performance Measures}

\begin{tabular}{|c|c|c|c|c|c|c|c|c|c|c|c|c|c|c|c|}
\hline & & \multicolumn{11}{|c|}{$\begin{array}{l}\text { Performance Statistics } \\
\text { (Number of) }\end{array}$} & \multicolumn{3}{|c|}{$\begin{array}{l}\text { Project } \\
\text { Qualitative } \\
\text { Assessment }\end{array}$} \\
\hline Project Title & Project \# & 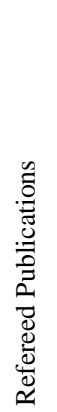 & 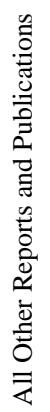 & 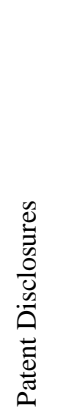 & 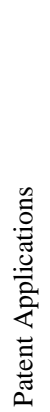 & $\frac{n}{\tilde{\Xi}}$ & 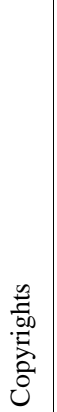 & 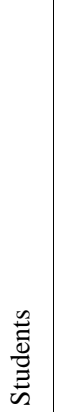 & $\begin{array}{l}0 \\
0 \\
0 \\
0 \\
1 \\
0 \\
0 \\
0\end{array}$ & 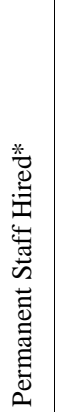 & 量 & 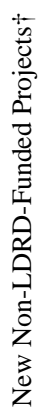 & 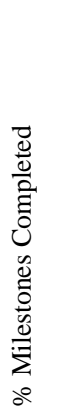 & 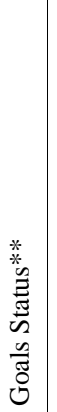 & 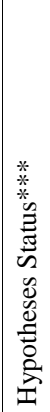 \\
\hline
\end{tabular}

** Goals Status

1 - Goals Met

2 - Goals Substantially Met

3 - Goals Partially Met

4 - Goals Substantially Modified

5 - Goals Not Met

11 - Project Terminated

***Hypothesis Status

6 - Hypotheses Proved

7 - Hypotheses Remains Unchanged

8 - Hypotheses Modified

9 - Hypotheses Redefined

10 - Hypotheses Disproved

11 - Project Terminated 


\section{Appendix D: DOE Critical Technologies}

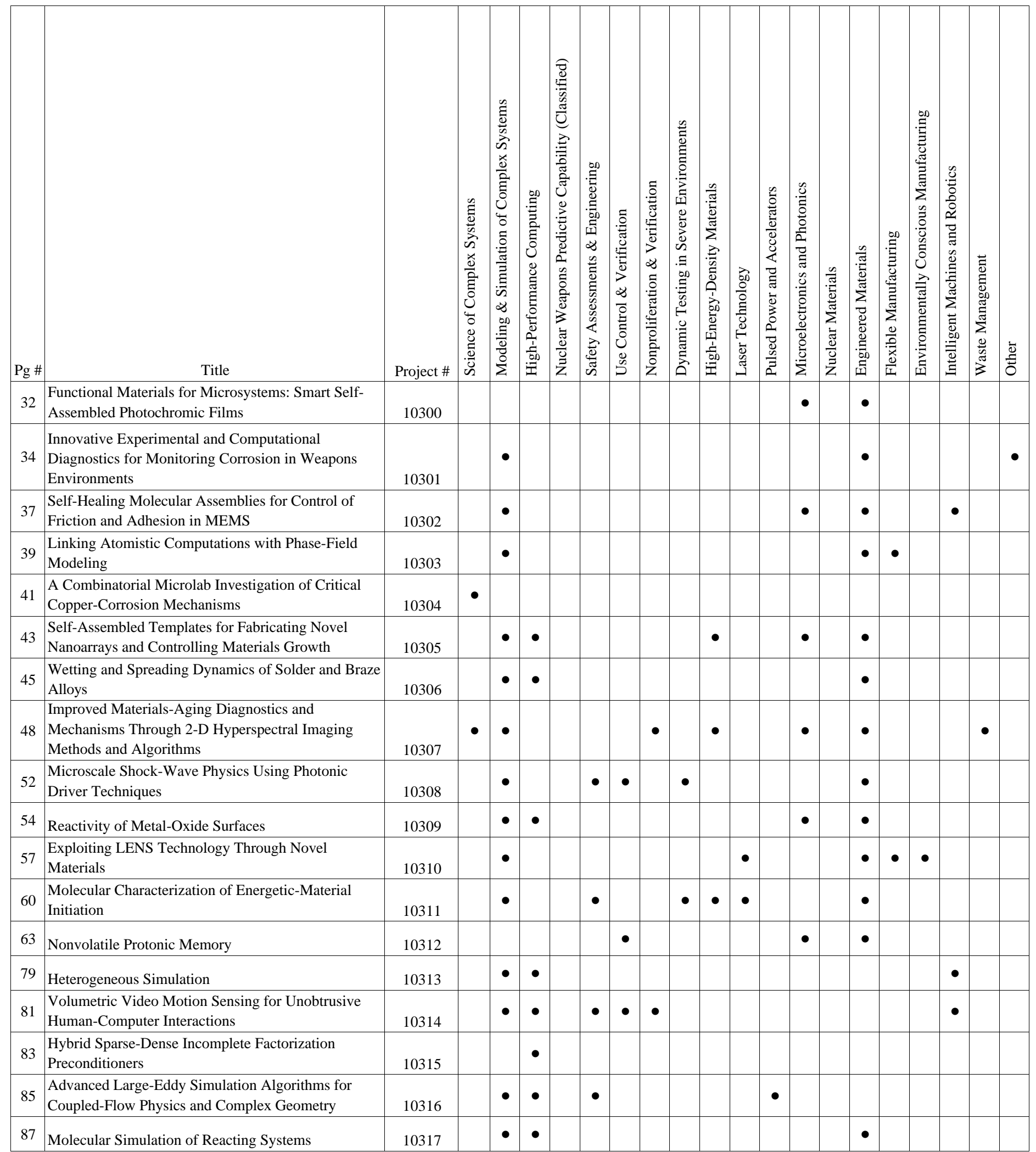




\section{Appendix D: DOE Critical Technologies}

\begin{tabular}{|c|c|c|c|c|c|c|c|c|c|c|c|c|c|c|c|c|c|c|c|c|c|}
\hline Pg \# & Title & Project \# & 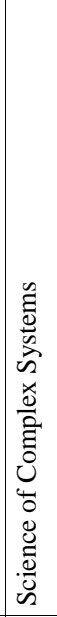 & 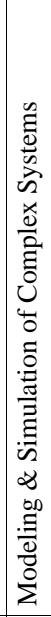 & 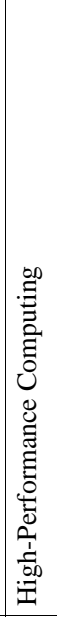 & 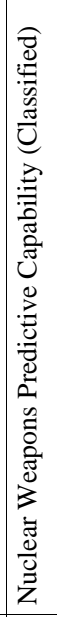 & 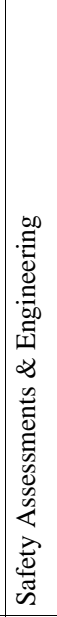 & 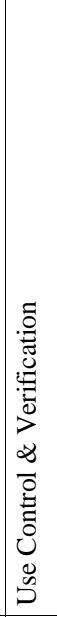 & 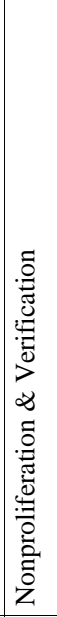 & 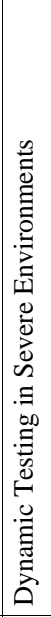 & 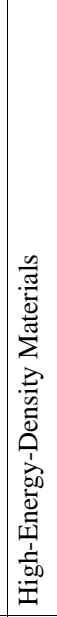 & 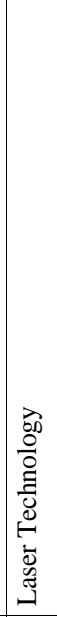 & 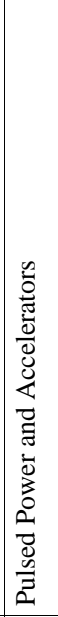 & 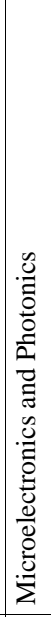 & 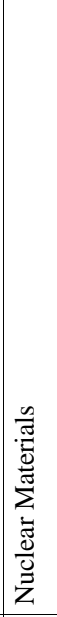 & 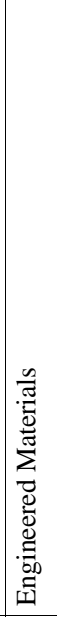 & 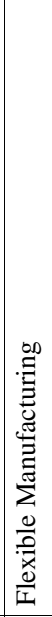 & 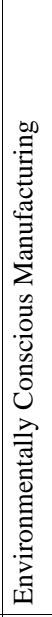 & 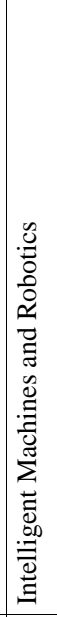 & 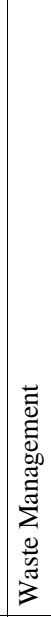 & 离 \\
\hline 89 & $\begin{array}{l}\text { Massively Parallel Global Climate Model for } \\
\text { Paleoclimate Applications }\end{array}$ & 10318 & & $\bullet$ & $\bullet$ & & & & & & & & & & & & & & & & \\
\hline 91 & $\begin{array}{l}\text { From Atom-Picoseconds to Centimeter-Years in } \\
\text { Simulation and Experiment }\end{array}$ & 10319 & & $\bullet$ & $\bullet$ & & & & & & & & & & & & & & & & \\
\hline 93 & $\begin{array}{l}\text { Parallel Combinatorial Optimization for Scheduling } \\
\text { Problems }\end{array}$ & 10320 & & $\bullet$ & • & & & & & & & & & & & & & & & & \\
\hline 96 & $\begin{array}{l}\text { Multilevel Techniques for Unstructured Grid } \\
\text { Problems on Massively Parallel Computers }\end{array}$ & 10321 & & & $\bullet$ & & & & & & & & & & & & & & & & \\
\hline 99 & Visual Explanation and Insight & 10323 & & & & & & & & & & & & & & & & & & & \\
\hline 117 & $\begin{array}{l}\text { Monolithic Integration of VCSELs and Detectors for } \\
\text { Microsystems }\end{array}$ & 10325 & & & & & & & & & & $\bullet$ & & • & & & & & & & \\
\hline 119 & Post-Processed Integrated Microsystems & 10326 & & & & & & & & & & & & $\bullet$ & & & & & $\bullet$ & & \\
\hline 122 & $\begin{array}{l}\text { Development of Radiation-Hard Sensors for Space- } \\
\text { Based Visible and Infrared Sensing Applications }\end{array}$ & 10327 & & & & & & & $\bullet$ & & & & & $\bullet$ & & & & & & & \\
\hline 124 & $\begin{array}{l}\text { Silicon Three-Dimensional Photonic Crystal and Its } \\
\text { Applications }\end{array}$ & 10328 & & & & & & & & & & & & $\bullet$ & & & & & & & \\
\hline 126 & $\begin{array}{l}\text { Monolithic Micromachined Variable Tuners for Rapid } \\
\text { Prototyping and Optimization of Microwave Circuits }\end{array}$ & 10329 & & & & & & & $\bullet$ & & & & & - & & & & & - & & \\
\hline 129 & $\begin{array}{l}\text { Quantum Tunneling Transistors for Practical } \\
\text { Application }\end{array}$ & 10330 & & & & & & & & & & & & $\bullet$ & & & & & & & \\
\hline 131 & $\begin{array}{l}\text { Development of Magnetically Excited Flexural Plate- } \\
\text { Wave Devices for Implementation as Physical, } \\
\text { Chemical, and Acoustic Sensors, and as Integrated } \\
\text { Micropumps for Sensored Systems }\end{array}$ & 10331 & & & & & & & • & & & & & $\bullet$ & & & & & $\bullet$ & & \\
\hline 134 & $\begin{array}{l}\text { Novel Acoustically Driven Microoptoelectronic } \\
\text { Devices }\end{array}$ & 10332 & & $\bullet$ & & & & & & & & & & $\bullet$ & & $\bullet$ & & & & & \\
\hline 136 & Photonics Integration Devices and Technologies & 10333 & & & $\bullet$ & & & & & & & $\bullet$ & & - & & $\bullet$ & & & & & \\
\hline 139 & $\begin{array}{l}\text { Stress-Free Amorphous Diamond for High-Sensitivity } \\
\text { Microsensors with Integrated Microstructures }\end{array}$ & 10334 & & & & & & & & & & & & $\bullet$ & & $\bullet$ & & & $\bullet$ & & \\
\hline 185 & $\begin{array}{l}\text { Development of In Situ Diagnostics for Simultaneous } \\
\text { Measurement of Transient Gas Species and Soot in } \\
\text { Large Fires }\end{array}$ & 10335 & & - & & & $\bullet$ & & & $\bullet$ & & $\bullet$ & & & & $\bullet$ & & & & & \\
\hline 188 & $\begin{array}{l}\text { Structural Simulations Using Multiresolution Material } \\
\text { Models }\end{array}$ & 10336 & & $\bullet$ & 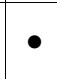 & & & & & & & & & & & $\bullet$ & & & & & \\
\hline
\end{tabular}




\section{Appendix D: DOE Critical Technologies}

\begin{tabular}{|c|c|c|c|c|c|c|c|c|c|c|c|c|c|c|c|c|c|c|c|c|c|}
\hline $\mathrm{Pg} \#$ & Title & Project \# & 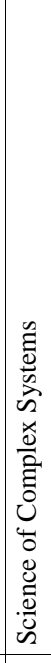 & 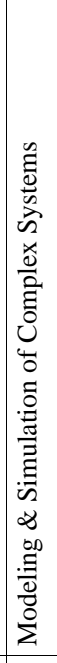 & 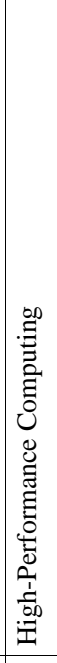 & 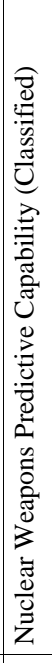 & 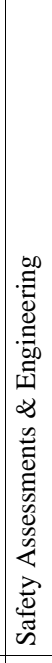 & 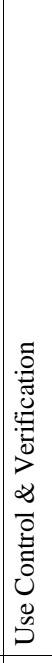 & 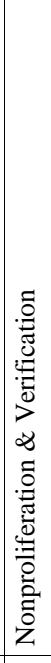 & 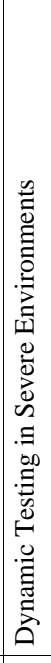 & 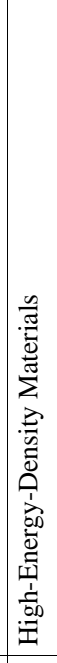 & 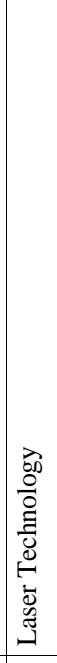 & 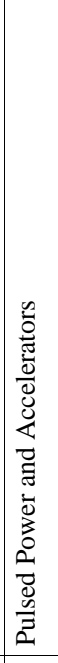 & 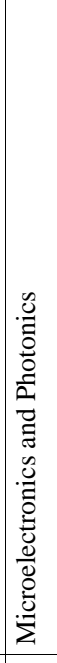 & 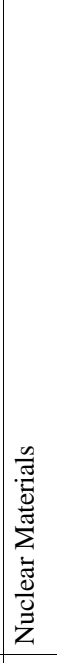 & 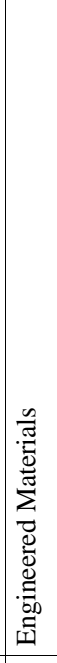 & 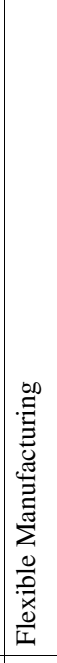 & 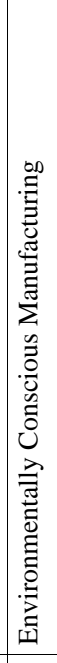 & 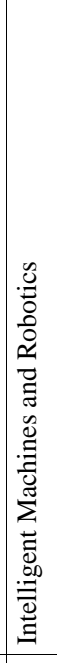 & 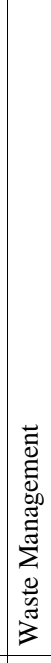 & 离 \\
\hline 190 & Mechanisms of Adiabatic Shear Failure & 10338 & & $\bullet$ & & & & & & $\bullet$ & & & & & & $\bullet$ & & $\bullet$ & & & \\
\hline 192 & Evolvable Hardware & 10339 & & $\bullet$ & $\bullet$ & & & & & & & & & $\bullet$ & & & & & $\bullet$ & & \\
\hline 194 & $\begin{array}{l}\text { Crack Nucleation and Growth: Combining Validated } \\
\text { Atomistic and Continuum Modeling }\end{array}$ & 10340 & $\bullet$ & $\bullet$ & $\bullet$ & & & & & & & & & & & $\bullet$ & & & & & \\
\hline 196 & Applied Microfluidic Physics & 10341 & $\bullet$ & $\bullet$ & & & & & & & & & & $\bullet$ & & & & & & & \\
\hline 198 & $\begin{array}{l}\text { Innovative Measurement Diagostics for Fluid/Solid } \\
\text { and Fluid/Fluid Interactions in Rotating Flowfields }\end{array}$ & 10343 & $\bullet$ & - & & $\bullet$ & & & & & & & & • & & & & & & & \\
\hline 201 & $\begin{array}{l}\text { High-Resolution Modeling of Multiscale Transient } \\
\text { Phenomena in Turbulent Boundary Layers }\end{array}$ & 10344 & & - & & & & & & & & & & & & & & & & & \\
\hline 203 & $\begin{array}{l}\text { Dispersive Measurements of Velocity in } \\
\text { Heterogeneous Materials }\end{array}$ & 10345 & $\bullet$ & $\bullet$ & • & & & & & - & $\bullet$ & $\bullet$ & - & & & • & & & & & \\
\hline 206 & $\begin{array}{l}\text { A Physically Based Computational Method for } \\
\text { Predicting Generalized Fracture }\end{array}$ & 10346 & - & - & $\bullet$ & & - & & & & & & & & & $\bullet$ & & & & & \\
\hline 212 & Microdiagnostic MEMS Lab-on-a-Chip & 10347 & • & • & & & • & • & • & • & & • & & $\bullet$ & & & $\bullet$ & $\bullet$ & • & & \\
\hline 214 & $\begin{array}{l}\text { Real-Time Error Correction Using Electromagnetic- } \\
\text { Bearing Spindles }\end{array}$ & 10348 & $\bullet$ & $\bullet$ & & & & & & & & & & & & & - & & & & \\
\hline 216 & $\begin{array}{l}\text { Thin-Film Deposition Processes Incorporating In Situ } \\
\text { Monitoring Capabilities }\end{array}$ & 10349 & & & & & & & & & & • & & & & $\bullet$ & & & & & \\
\hline 218 & $\begin{array}{l}\text { Solid-State Neutron Generator for Use in Nuclear } \\
\text { Weapons }\end{array}$ & 10351 & $\bullet$ & & & & & & & & & & & & & & & & & & • \\
\hline 220 & Scripting for Video Inspection & 10352 & & & & & & & & & & & & & & $\bullet$ & $\bullet$ & $\bullet$ & $\bullet$ & & \\
\hline 223 & Advanced Machining Processes for Microfabrication & 10353 & & & & & & & & & & $\bullet$ & & & & & & & & & \\
\hline 225 & Advanced Production Planning Models & 10354 & & $\bullet$ & $\bullet$ & & & & & & & & & & & & $\bullet$ & $\bullet$ & & & \\
\hline 247 & $\begin{array}{l}\text { Weighted-Nearest-Neighbor (WNN) Decision Making } \\
\text { for Data Mining }\end{array}$ & 10355 & & $\bullet$ & $\bullet$ & & & • & - & & & & & & $\bullet$ & & & & & & \\
\hline 249 & Varying QoS for Fixed and Mobile Networks & 10356 & & $\bullet$ & $\bullet$ & & & & & & & & & & & & & & & & \\
\hline 251 & $\begin{array}{l}\text { A Real-Time Decision-Support Framework to Guide } \\
\text { Facility Response to Abnormal Events }\end{array}$ & 10357 & & $\bullet$ & $\bullet$ & & $\bullet$ & & $\bullet$ & & & & & & $\bullet$ & & & - & • & & • \\
\hline 253 & Physical Model-Based Fusion of Sensor Array Data & 10358 & & & & & & & & & & & & & & & & & & & \\
\hline 255 & $\begin{array}{l}\text { Large-Scale Distributed Information System Modeling } \\
\text { and Simulation }\end{array}$ & 10359 & & $\bullet$ & 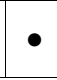 & & & & & & & & & & & & & & & & \\
\hline
\end{tabular}




\section{Appendix D: DOE Critical Technologies}

\begin{tabular}{|c|c|c|c|c|c|c|c|c|c|c|c|c|c|c|c|c|c|c|c|c|c|}
\hline $\operatorname{Pg} \#$ & Title & Project \# & 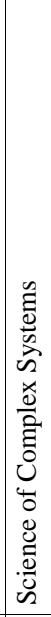 & 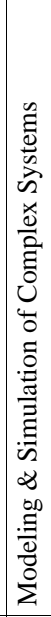 & 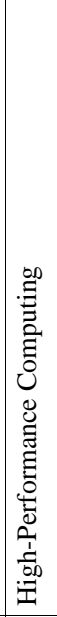 & 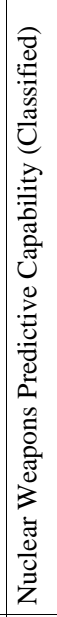 & 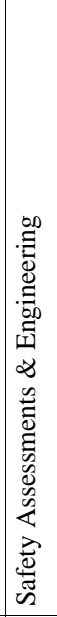 & 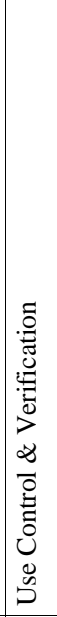 & 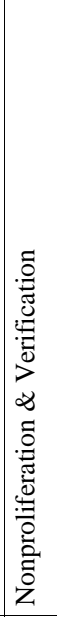 & 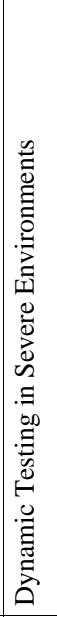 & 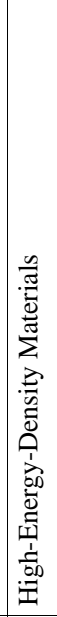 & 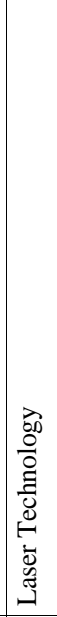 & 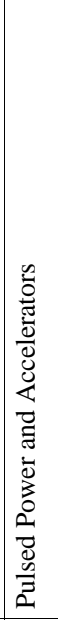 & 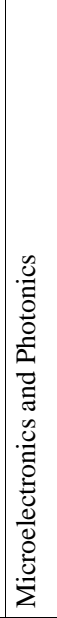 & 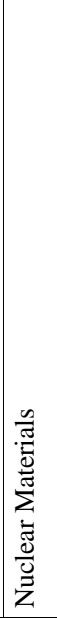 & 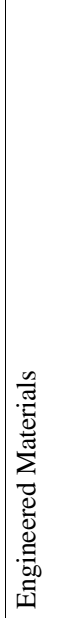 & 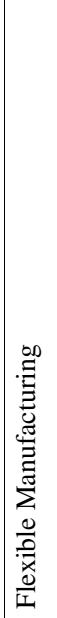 & 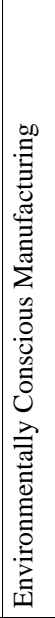 & 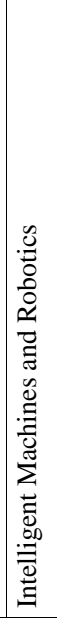 & 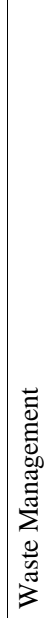 & $\bar{\Phi}$ \\
\hline 258 & $\begin{array}{l}\text { High-Surety SCADA for the Critical Energy } \\
\text { Infrastructures }\end{array}$ & 10360 & $\bullet$ & $\bullet$ & & & & & & $\bullet$ & & & & & & & & & $\bullet$ & & \\
\hline 260 & $\begin{array}{l}\text { Agent-Based Mediation and Cooperative Information } \\
\text { Systems }\end{array}$ & 10361 & $\bullet$ & - & & & & & & & & & & & & & & & $\bullet$ & & \\
\hline 262 & Dynamically Self-Configurable Network Protocol & 10362 & & $\bullet$ & $\bullet$ & & & & $\bullet$ & & & & & & & & & & $\bullet$ & & \\
\hline 264 & $\begin{array}{l}\text { Optical Backplane/Interconnect for Super-High-Speed } \\
\text { Communication }\end{array}$ & 10363 & & & $\bullet$ & & & & & & & & & - & & & & & & & \\
\hline 266 & PUSH Technology Demonstration & 10364 & & & & & & $\bullet$ & & & & & & & & & & & & & \\
\hline 268 & $\begin{array}{l}\text { Controlling Information: Its Flow, Fusion, and } \\
\text { Coordination }\end{array}$ & 10365 & $\bullet$ & • & & & & & & & & & & & & & & & • & & \\
\hline 271 & $\begin{array}{l}\text { Low-Power, Reduced-Computation, Public-Key } \\
\text { Protocols }\end{array}$ & 10366 & & & & & & • & $\bullet$ & & & & & & & & & & & & \\
\hline 273 & $\begin{array}{l}\text { Ten-to-One-Hundred-Gigabit/Second Network- } \\
\text { Enabling R\&D }\end{array}$ & 10367 & & & • & & & & & & & & & • & & & & & & & \\
\hline 283 & $\begin{array}{l}\text { Surface Decontamination of Bacterial Protein Toxins } \\
\text { by RF Power }\end{array}$ & 10368 & & & & & & & & & & & & & & & & & & & - \\
\hline 284 & $\begin{array}{l}\text { Intense White-Light Pulse Propagation in Air Using } \\
\text { Self-Guided Optical Filamentation: Applications to } \\
\text { Remote Sensing and Countermeasures }\end{array}$ & 10369 & & & & & & & & & & $\bullet$ & & $\bullet$ & & & & & & & \\
\hline 286 & HPM Vulnerability Assessment and Tests & 10370 & & & & & & & $\bullet$ & & & & $\bullet$ & $\bullet$ & & & & & & & \\
\hline 293 & Miniature Bioaerosol Concentrator & 10371 & & & & & & & $\bullet$ & & & & & & & & & & & & $\bullet$ \\
\hline 295 & $\begin{array}{l}\text { Recognizing Partially Obscured Targets by } \\
\text { Combining Multiple Data Sources Using Evidential } \\
\text { Reasoning }\end{array}$ & 10372 & & & & & & & $\bullet$ & & & & & & & & & & $\bullet$ & & \\
\hline 298 & $\begin{array}{l}\text { Computational Engineering of Sensor Materials and } \\
\text { Integration with a Novel Biological Weapon Detection } \\
\text { System }\end{array}$ & 10373 & & $\bullet$ & & & & & & & & & & & & $\bullet$ & & & & & \\
\hline 300 & $\begin{array}{l}\text { Biological Weapon Detector Using Bioaffinity Array } \\
\text { Impedance Analysis with Chemical Amplification } \\
\text { Through Redox Recycling }\end{array}$ & 10374 & & & & & & & $\bullet$ & & & & & $\bullet$ & & & & & & & \\
\hline 302 & $\begin{array}{l}\text { ATR / Exploitation Utilizing Ultrahigh-Resolution, } \\
\text { Complex SAR Imaging }\end{array}$ & 10375 & & 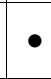 & & & & & $\bullet$ & & & & & & & & & & & & \\
\hline 304 & $\begin{array}{l}\text { Thin-Skin Deployable Mirrors for Remote Sensing } \\
\text { Systems }\end{array}$ & 10376 & & $\bullet$ & & & & & $\bullet$ & & & & & 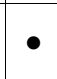 & & • & & & & & \\
\hline
\end{tabular}




\section{Appendix D: DOE Critical Technologies}

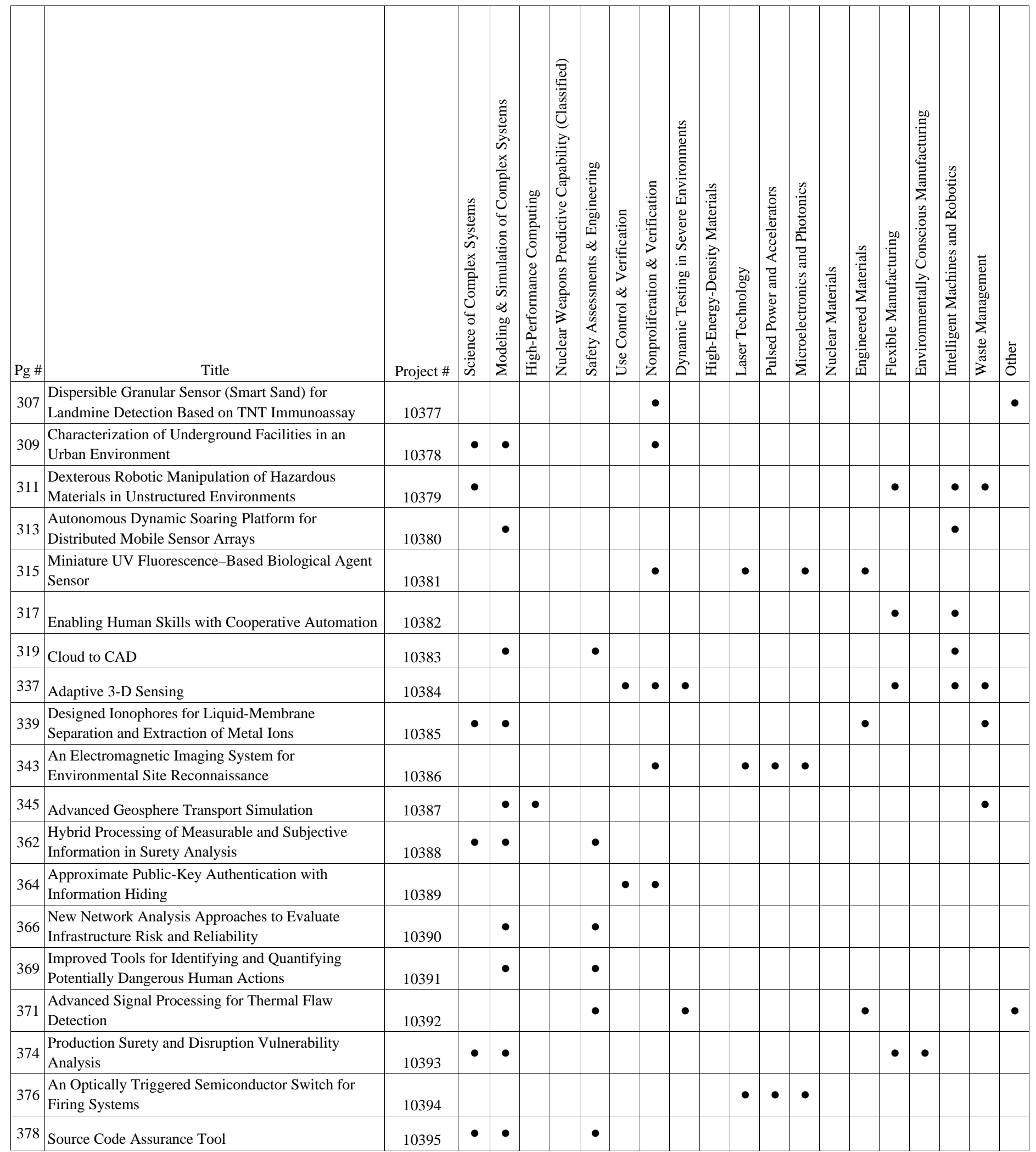




\section{Appendix D: DOE Critical Technologies}

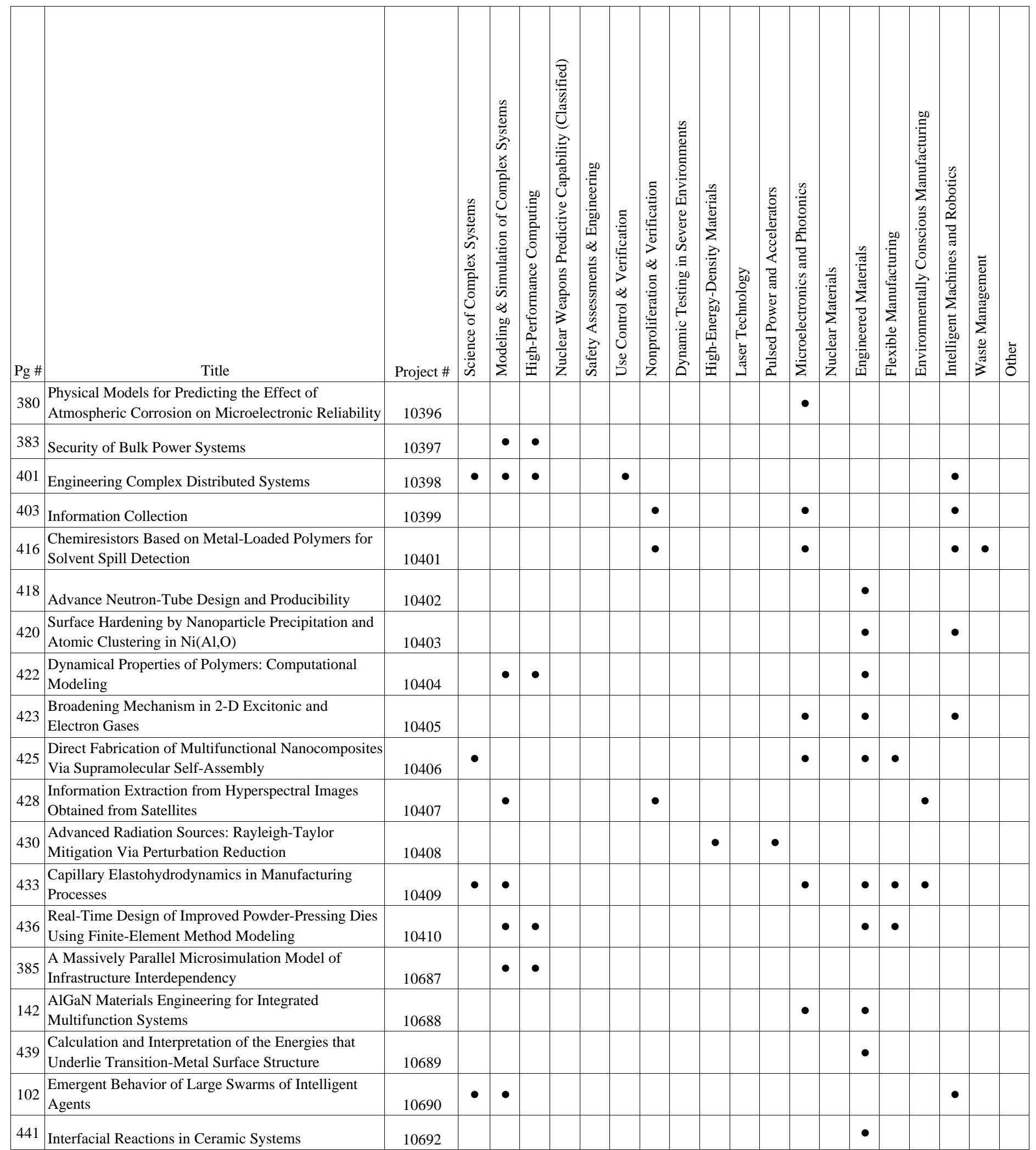




\section{Appendix D: DOE Critical Technologies}

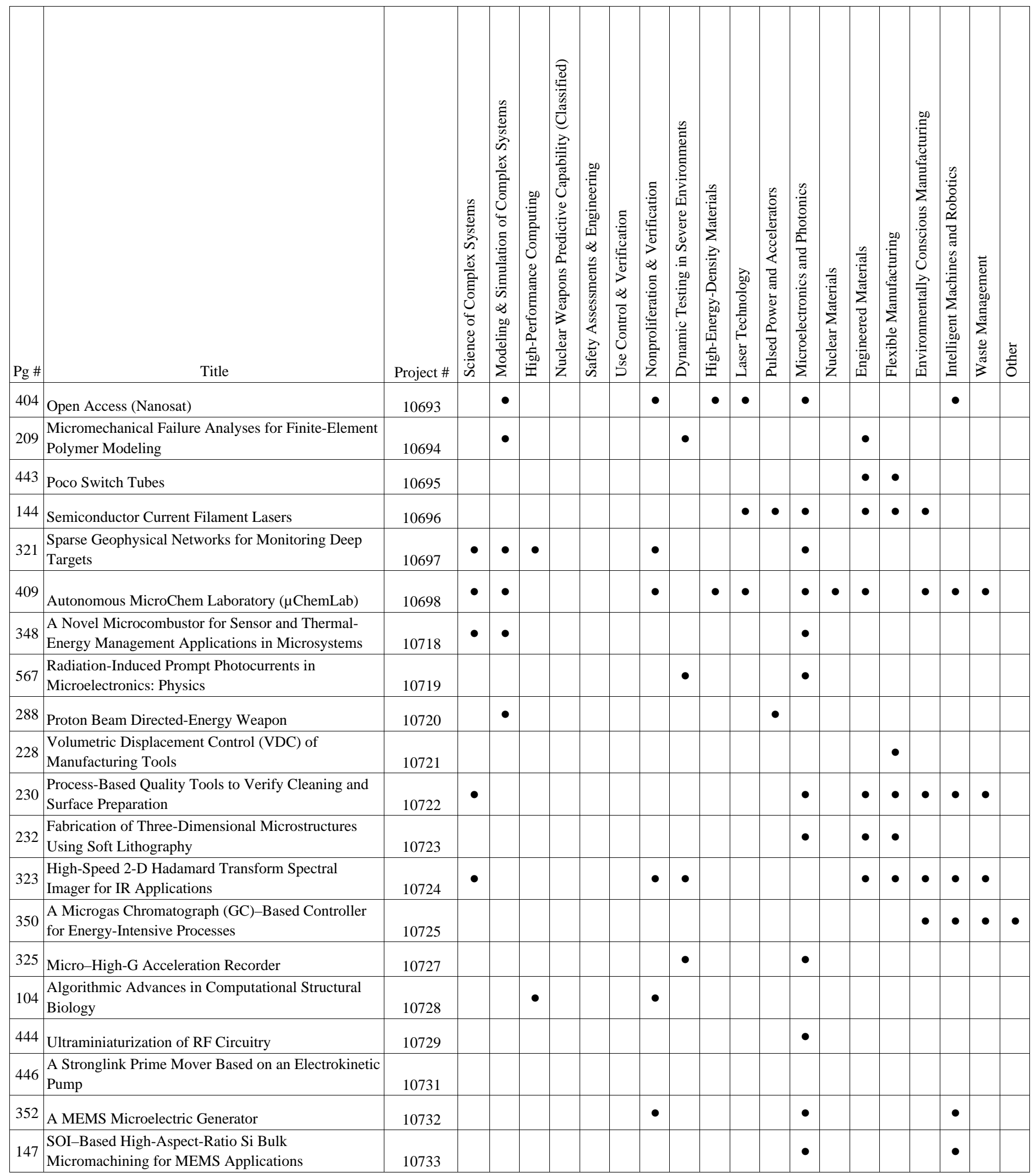




\section{Appendix D: DOE Critical Technologies}

\begin{tabular}{|c|c|c|c|c|c|c|c|c|c|c|c|c|c|c|c|c|c|c|c|c|c|}
\hline $\operatorname{Pg} \#$ & Title & Project \# & 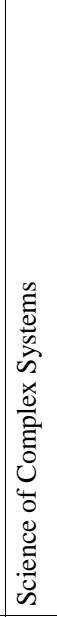 & 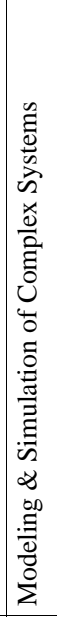 & 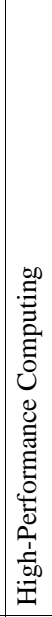 & 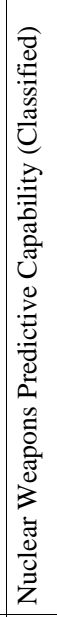 & 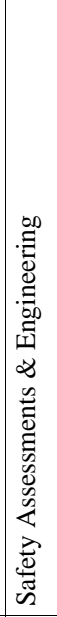 & 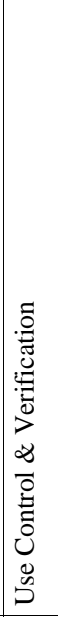 & 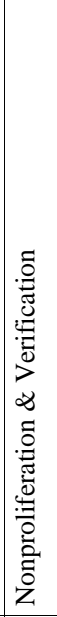 & 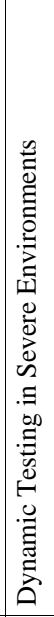 & 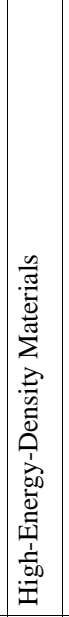 & 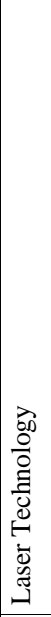 & 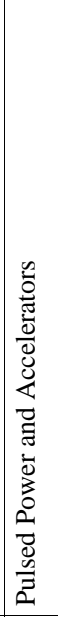 & 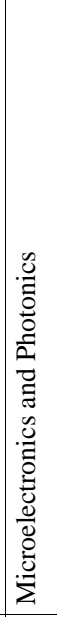 & 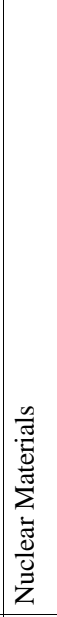 & 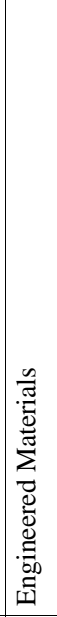 & 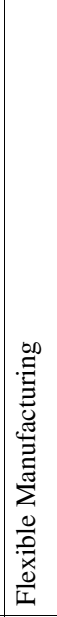 & 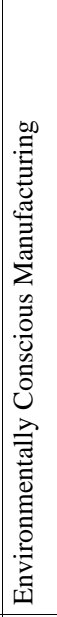 & 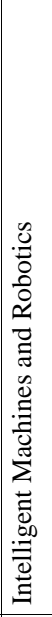 & 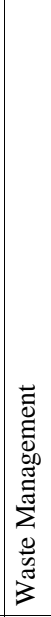 & $\bar{\Xi}$ \\
\hline 66 & $\begin{array}{l}\text { Next-Generation Output-Based Process Control: An } \\
\text { Integration of Modeling, Sensors, and Intelligent Data } \\
\text { Analysis }\end{array}$ & 10734 & & $\bullet$ & & & & & & & & & & & & $\bullet$ & $\bullet$ & & & & \\
\hline 569 & $\begin{array}{l}\text { Physical Basis for Interfacial Traction-Separation } \\
\text { Models }\end{array}$ & 10735 & & $\bullet$ & & & & & & & & & & & & $\bullet$ & & & & & \\
\hline 327 & $\begin{array}{l}\text { Dynamic Range Imaging for Terrain Mapping, } \\
\text { Position Determination, and Obstacle Avoidance in } \\
\text { Autonomous Navigation }\end{array}$ & 10736 & & & & & & $\bullet$ & $\bullet$ & $\bullet$ & & & & $\bullet$ & & & & & $\bullet$ & & \\
\hline 448 & Laser-Assisted Microgas Metal Arc Welding & 10739 & $\bullet$ & & & & & & & & & $\bullet$ & & & & & $\bullet$ & & & & \\
\hline 571 & $\begin{array}{l}\text { Diagnostics for Joining Solidification/Microstructural } \\
\text { Simulations }\end{array}$ & 10740 & & $\bullet$ & - & & & & & & & & & & & $\bullet$ & $\bullet$ & & & & \\
\hline 574 & $\begin{array}{l}\text { Effects of Microstructural Variables on the Shock- } \\
\text { Wave Response of PZT 95/5 }\end{array}$ & 10741 & & $\bullet$ & & & & & & & • & & & & & $\bullet$ & & & & & \\
\hline 577 & $\begin{array}{l}\text { Mechanisms of Dislocation-Grain-Boundary } \\
\text { Interaction }\end{array}$ & 10742 & & $\bullet$ & & & & & & & & & & & & $\bullet$ & $\bullet$ & & & & \\
\hline 150 & $\begin{array}{l}\text { Defining the Frontiers of Vertical, External-Cavity, } \\
\text { Surface-Emitting Lasers }\end{array}$ & 10744 & & & & & & & & & & $\bullet$ & & - & & - & & & & & \\
\hline 153 & $\begin{array}{l}\text { Photoresist Technology for } 70 \mathrm{~nm} \text { Lithography with } \\
157 \mathrm{~nm} \text { Radiation }\end{array}$ & 10745 & & & $\bullet$ & & & $\bullet$ & & & & & & $\bullet$ & & $\bullet$ & & & & & - \\
\hline 106 & Predicting Function of Biological Macromolecules & 10746 & $\bullet$ & $\bullet$ & $\bullet$ & & & & & & & & & & & & & & & & \\
\hline 329 & $\begin{array}{l}\text { Induced Molecular Markers for Pathogen Detection: } \\
\text { Microincubators for Rapid Toxin Expression }\end{array}$ & 10747 & & & & & & & - & & & • & & & & $\bullet$ & & & & & \\
\hline 354 & $\begin{array}{l}\text { Nanostructured Silicon Surfaces for Cost-Effective } \\
\text { Photovoltaic Efficiency Improvements }\end{array}$ & 10748 & & & & & & & & & & & & $\bullet$ & & $\bullet$ & & • & & & \\
\hline 155 & $\begin{array}{l}\text { Growth and Characterization of Quantum Dots and } \\
\text { Quantum Dot Devices }\end{array}$ & 10749 & $\bullet$ & & & & & & & & & $\bullet$ & & $\bullet$ & & $\bullet$ & & & & & \\
\hline 158 & $\begin{array}{l}\text { THz Technologies for Ultrahigh-Data-Rate } \\
\text { Communications }\end{array}$ & 10750 & & & & & & & & & & & & $\bullet$ & & & & & & & \\
\hline 234 & Automatic Design of Practical Fixtures & 10751 & $\bullet$ & $\bullet$ & • & & & & & & & & & & & & $\bullet$ & & $\bullet$ & & \\
\hline 331 & $\begin{array}{l}\text { Distributed Reconfigurable Homogeneous } \\
\text { Microrobotic Systems }\end{array}$ & 10752 & & 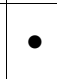 & & & & & - & & & & & 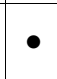 & & & $\bullet$ & & $\bullet$ & & \\
\hline 334 & $\begin{array}{l}\text { Robust Planning for Autonomous Navigation of } \\
\text { Mobile Robots in Unstructured, Dynamic } \\
\text { Environments }\end{array}$ & 10753 & & & & & & & & & & - & & & & & & & $\bullet$ & & \\
\hline
\end{tabular}




\section{Appendix D: DOE Critical Technologies}

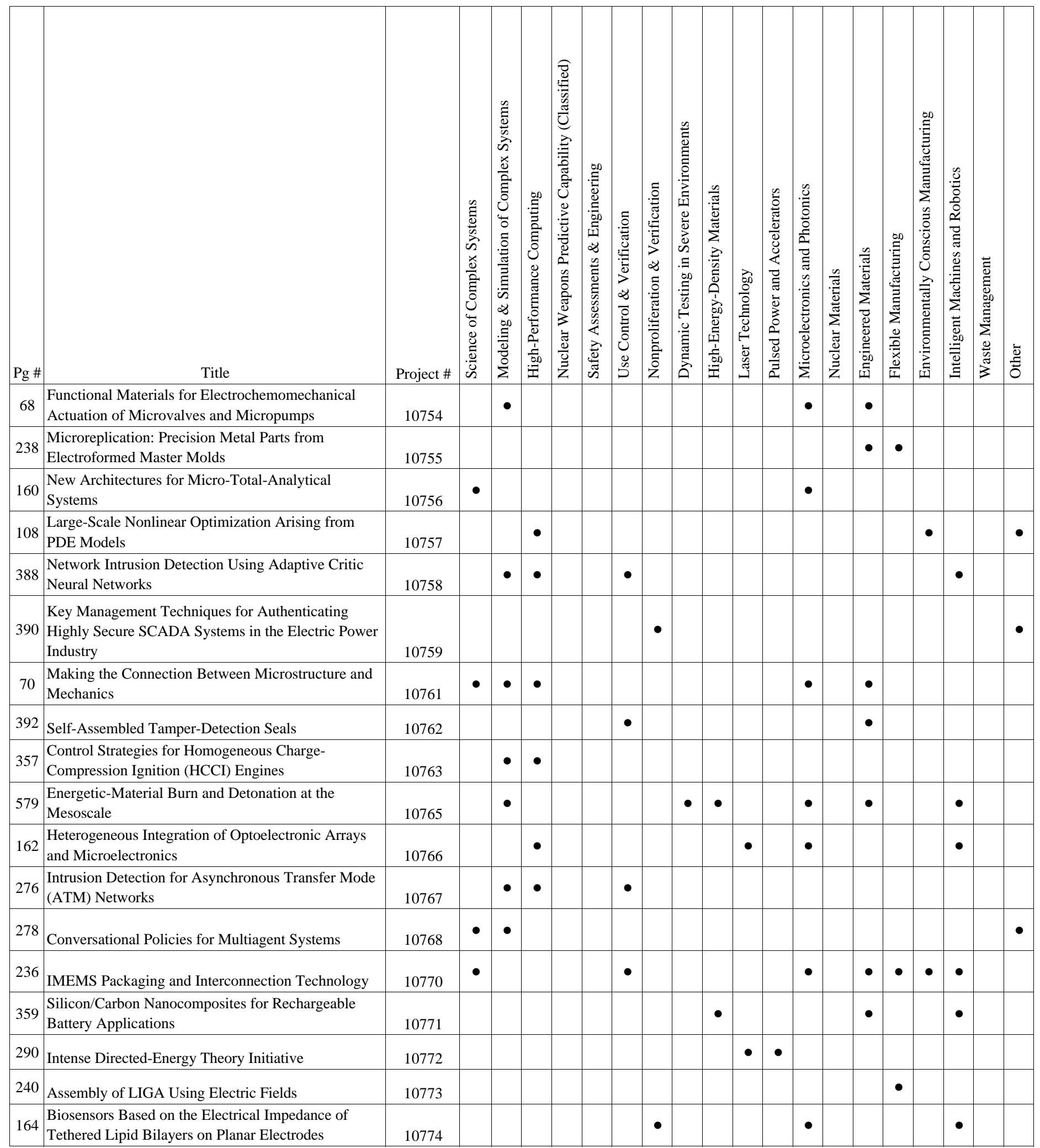




\section{Appendix D: DOE Critical Technologies}

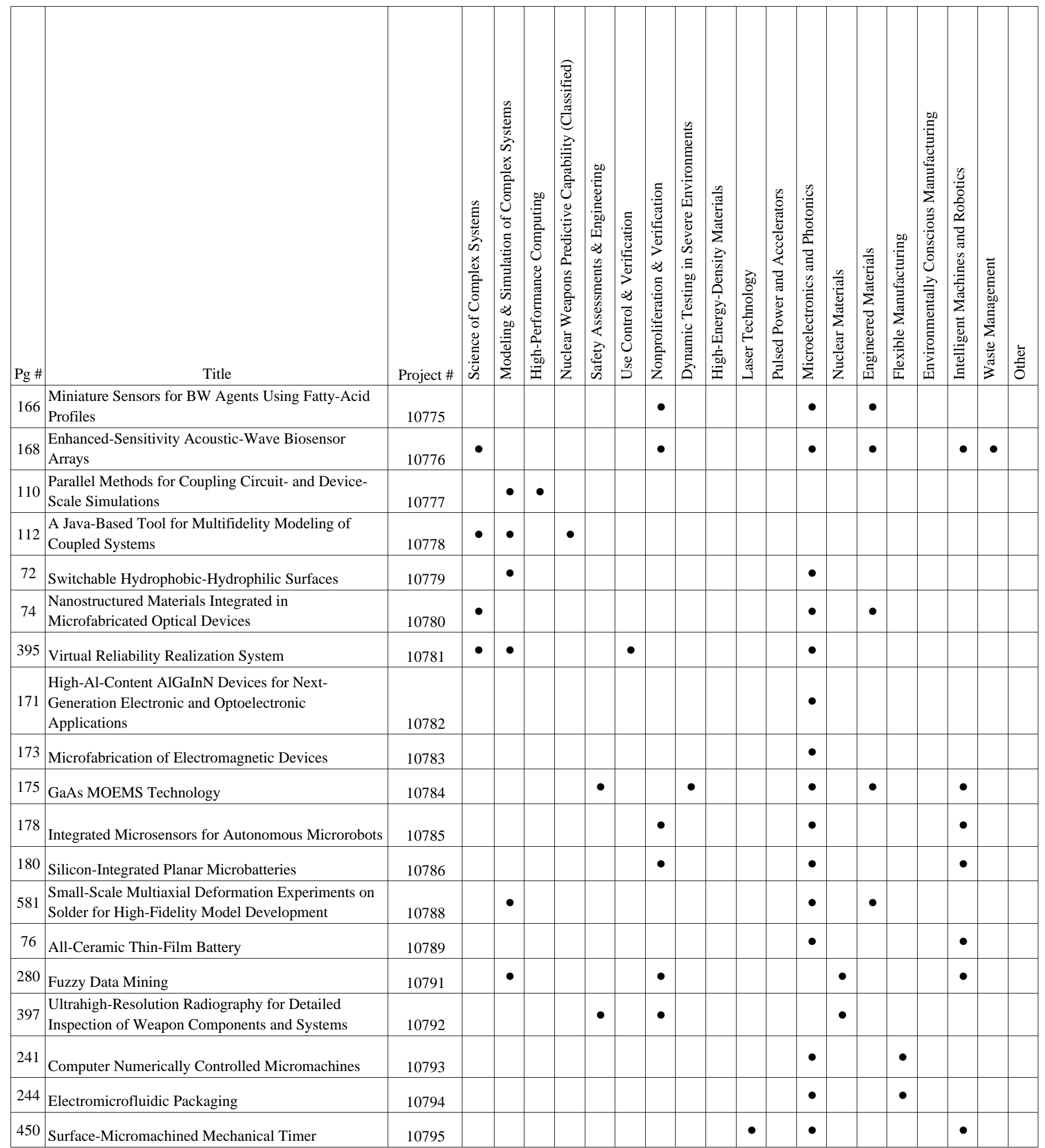




\section{Appendix D: DOE Critical Technologies}

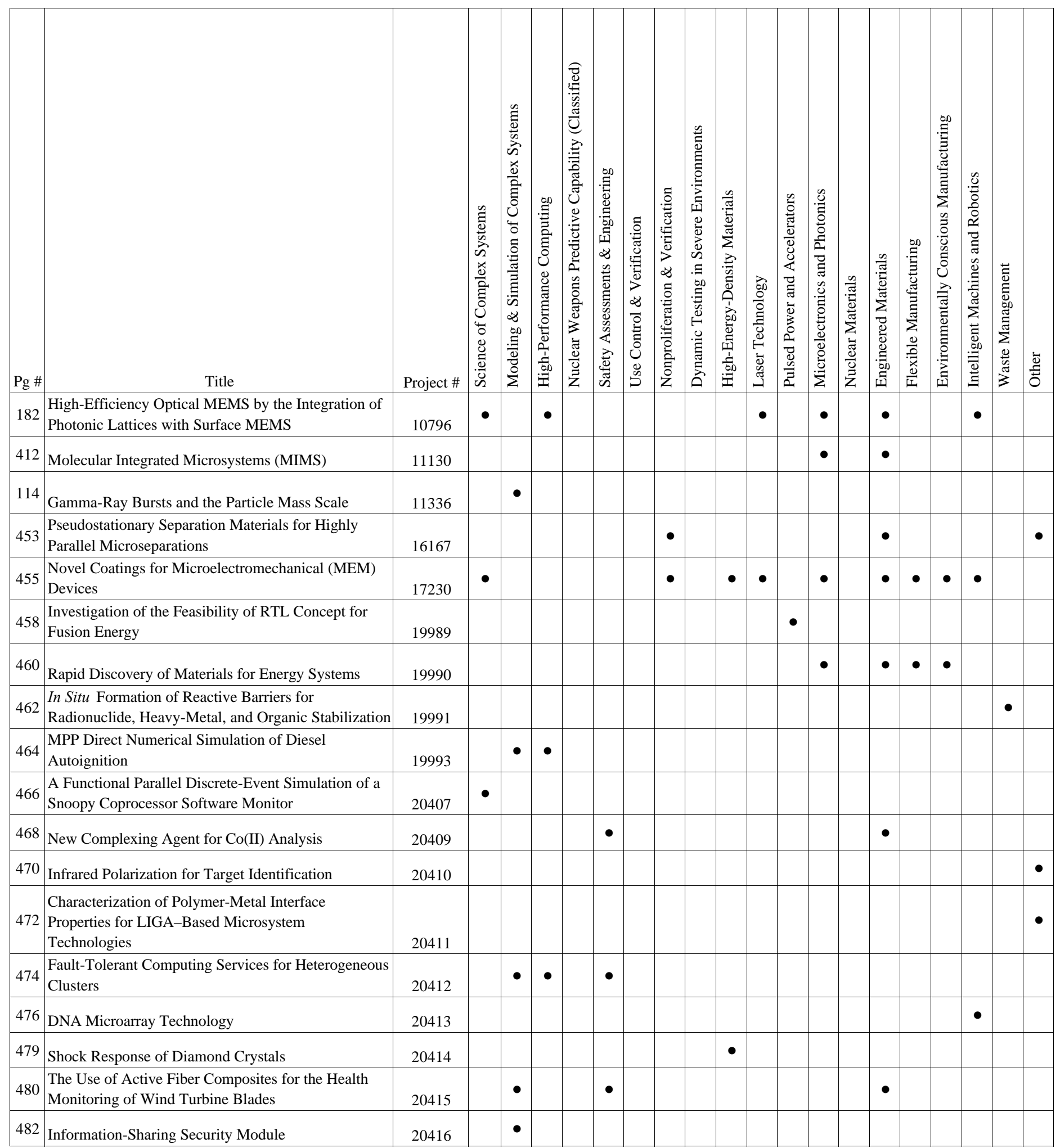




\section{Appendix D: DOE Critical Technologies}

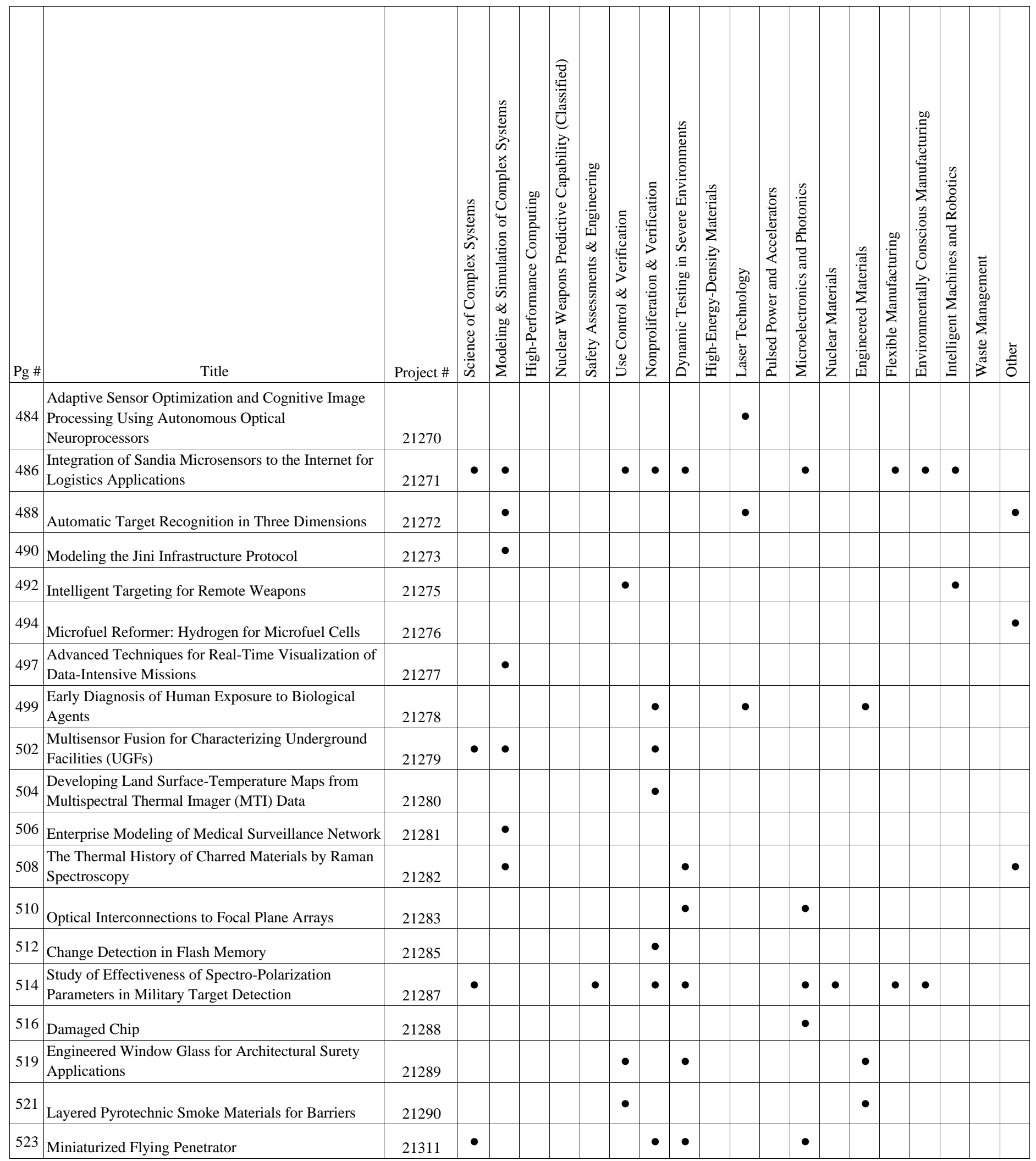




\section{Appendix D: DOE Critical Technologies}

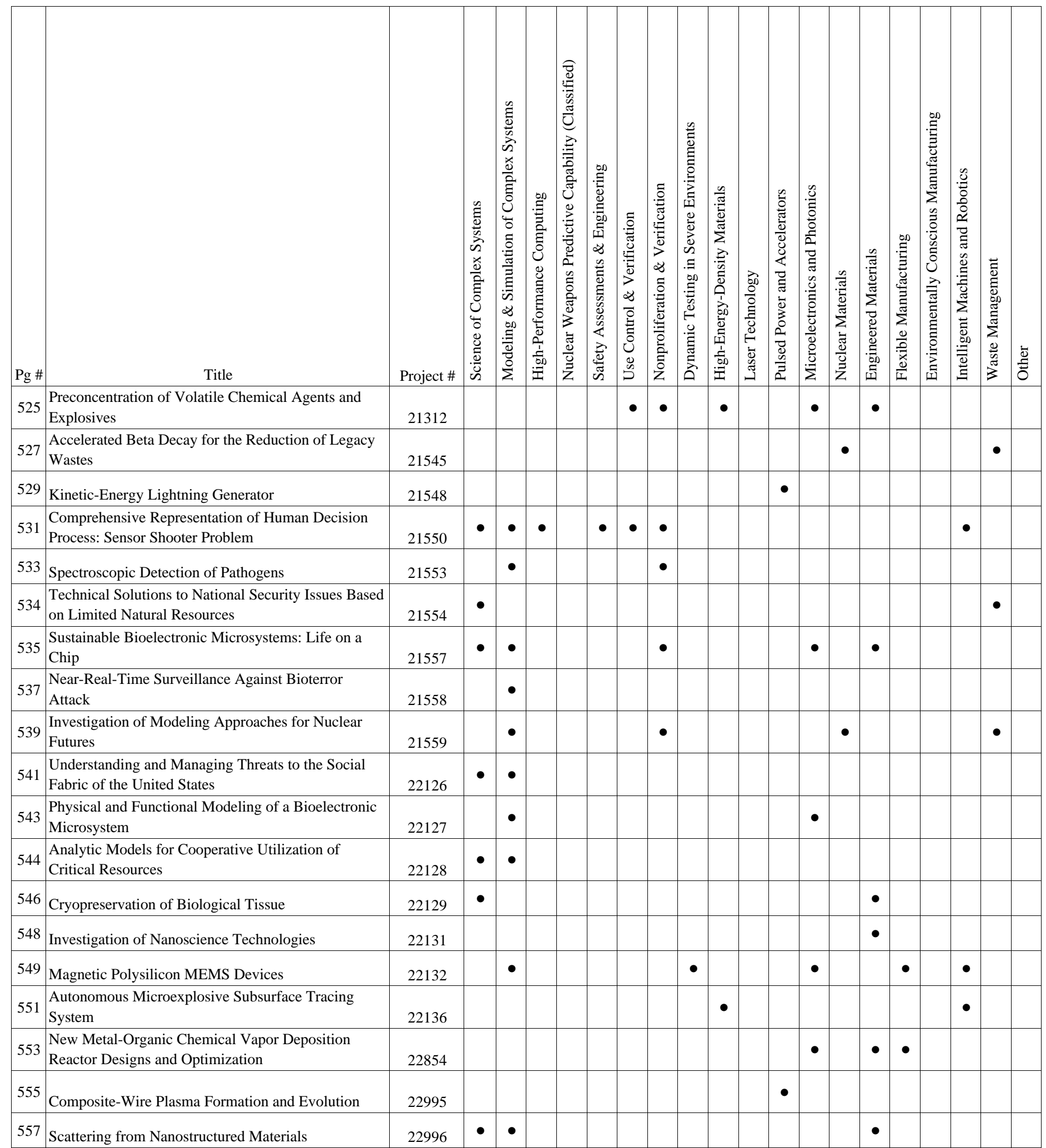




\section{Appendix D: DOE Critical Technologies}

\begin{tabular}{|c|c|c|c|c|c|c|c|c|c|c|c|c|c|c|c|c|c|c|c|c|c|}
\hline $\operatorname{Pg} \#$ & Title & Project \# & 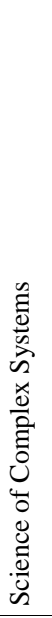 & 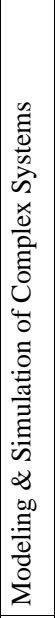 & 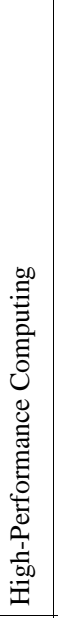 & 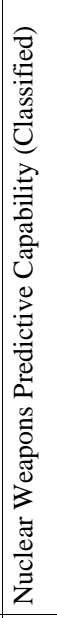 & 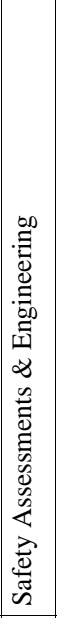 & 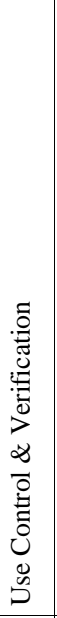 & 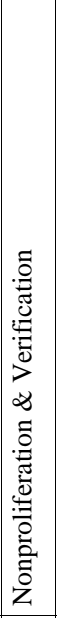 & 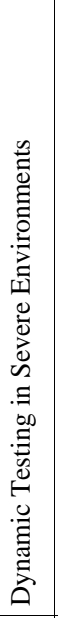 & 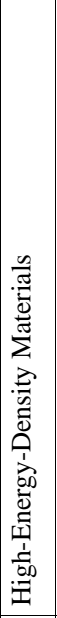 & 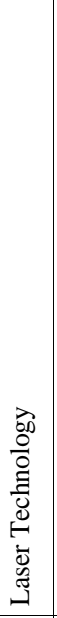 & 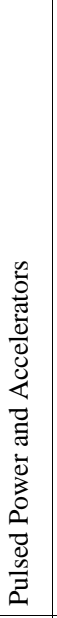 & 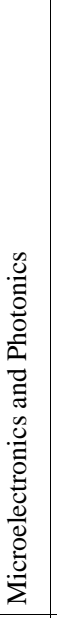 & 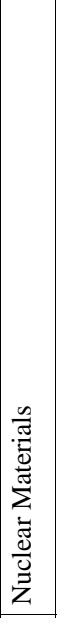 & 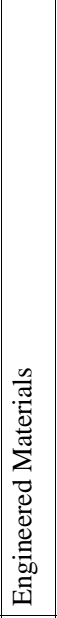 & 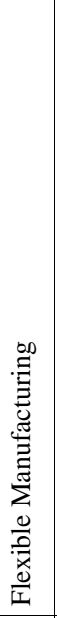 & 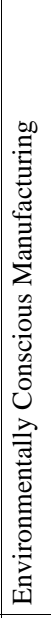 & 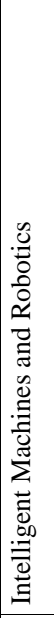 & 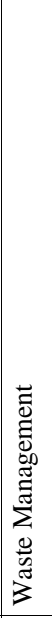 & 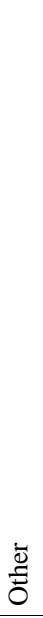 \\
\hline 559 & $\begin{array}{l}\text { The Liquid-Crystal Physics of Evaporation-Induced } \\
\text { Self-Assembly }\end{array}$ & 22997 & $\bullet$ & • & & & & & & & & & & & & $\bullet$ & & & & & \\
\hline 560 & $\begin{array}{l}\text { Electrochemical Interrogation of Cellular Membrane } \\
\text { Transport Processes }\end{array}$ & 22998 & & & & & & & & & & & & $\bullet$ & & $\bullet$ & & & & & \\
\hline 562 & $\begin{array}{l}\text { A Micromethanol Steam Reformer for a Hydrogen- } \\
\text { Based Microfuel Cell }\end{array}$ & 24101 & & & & & & & & & & & & $\bullet$ & & & & & & & \\
\hline 564 & $\begin{array}{l}\text { Spatial Anomalies in Magnetically Insulated Vacuum } \\
\text { Structures }\end{array}$ & 24102 & & & & & & & & & & & & & & & & & & & \\
\hline
\end{tabular}




\section{Appendix E: Major National Programs}

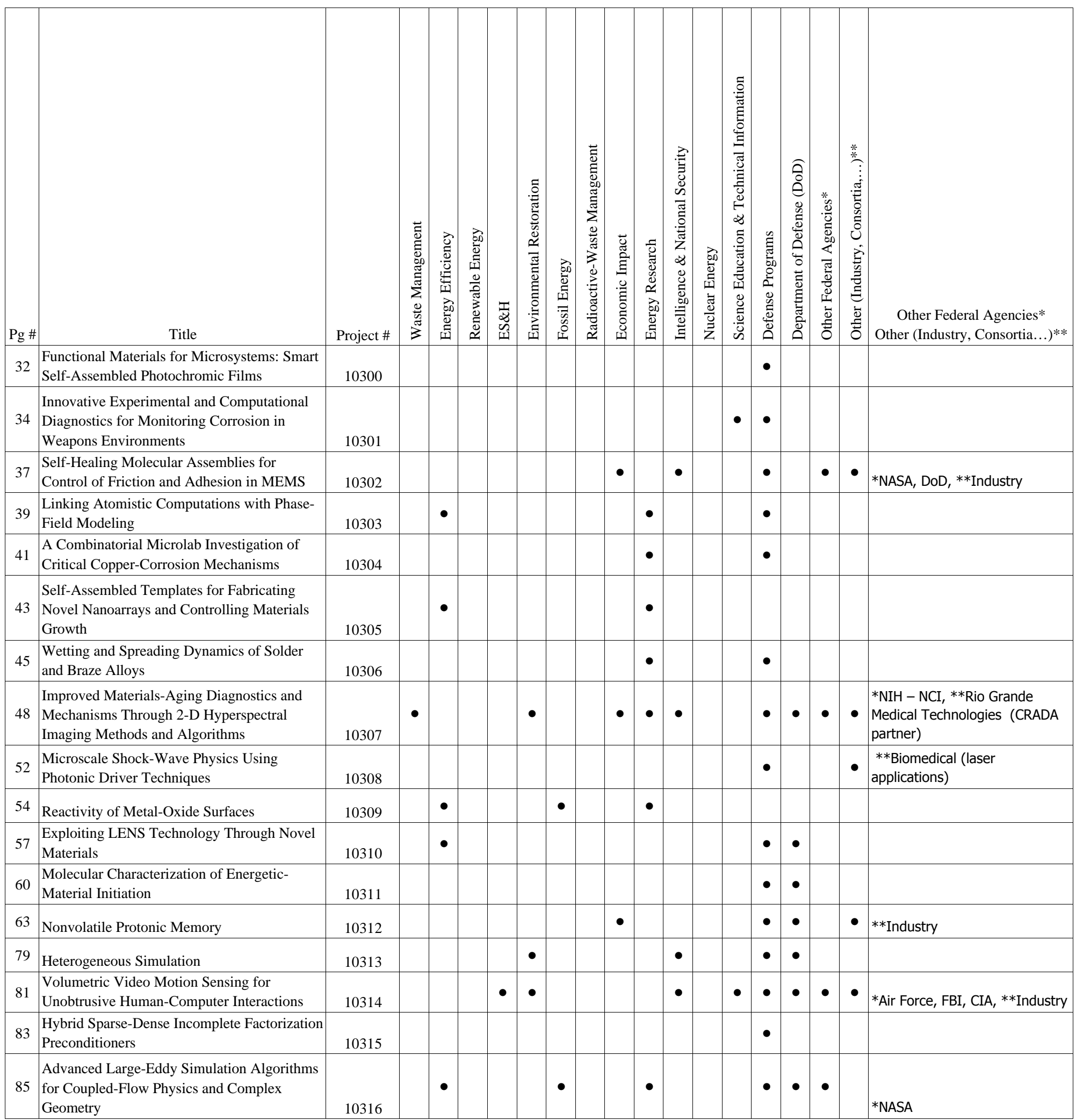




\section{Appendix E: Major National Programs}

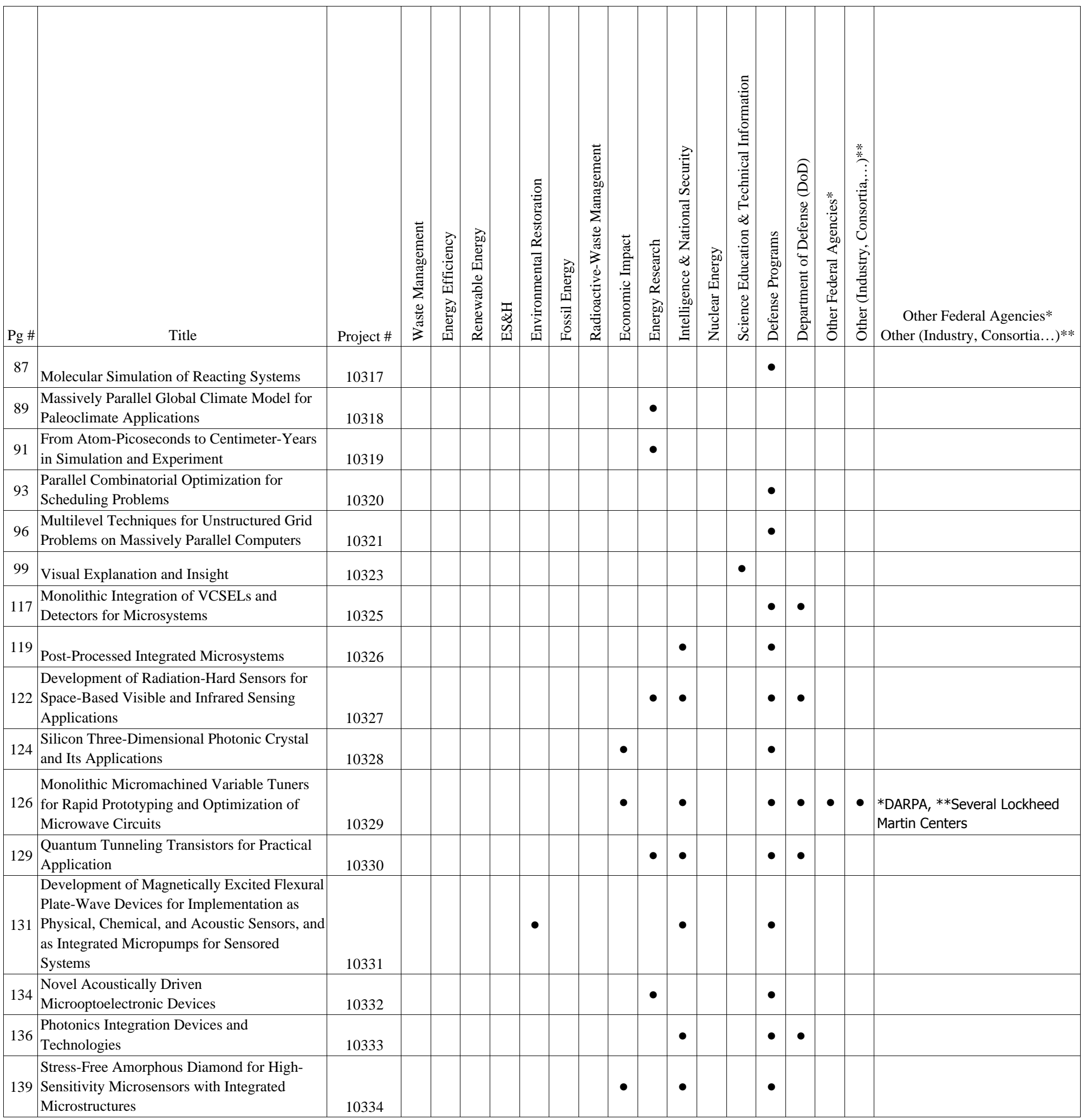




\section{Appendix E: Major National Programs}

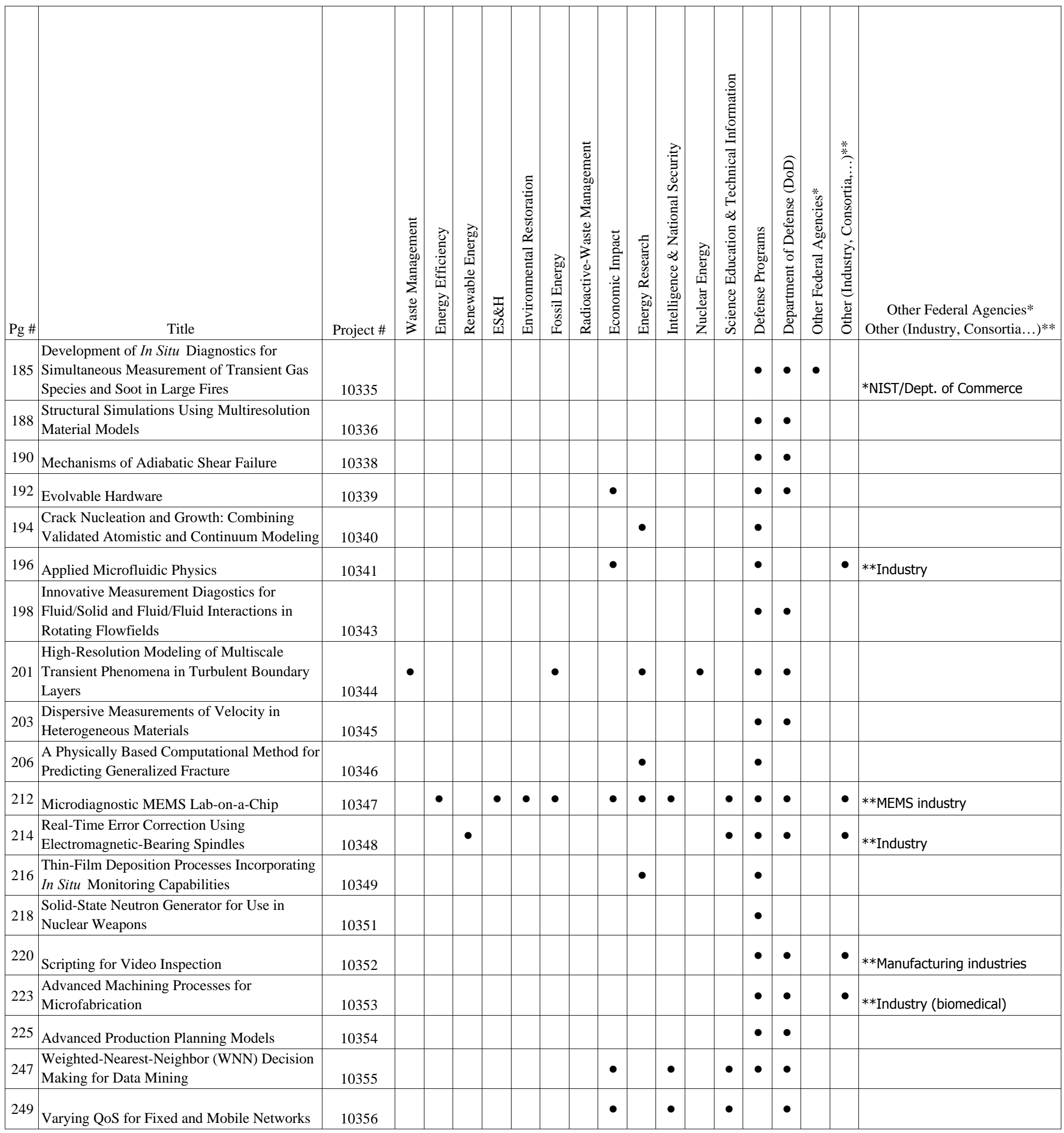




\section{Appendix E: Major National Programs}

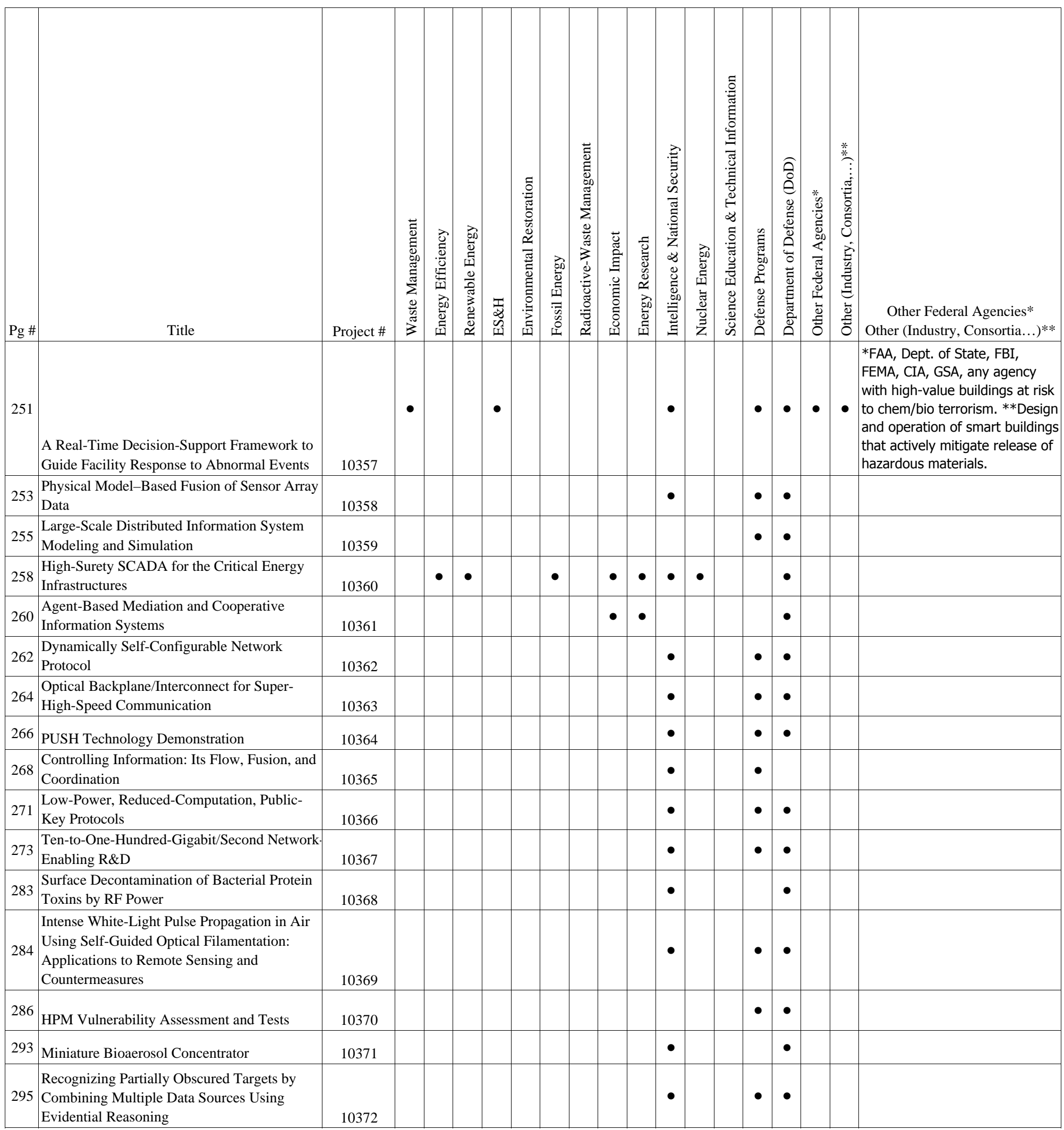




\section{Appendix E: Major National Programs}

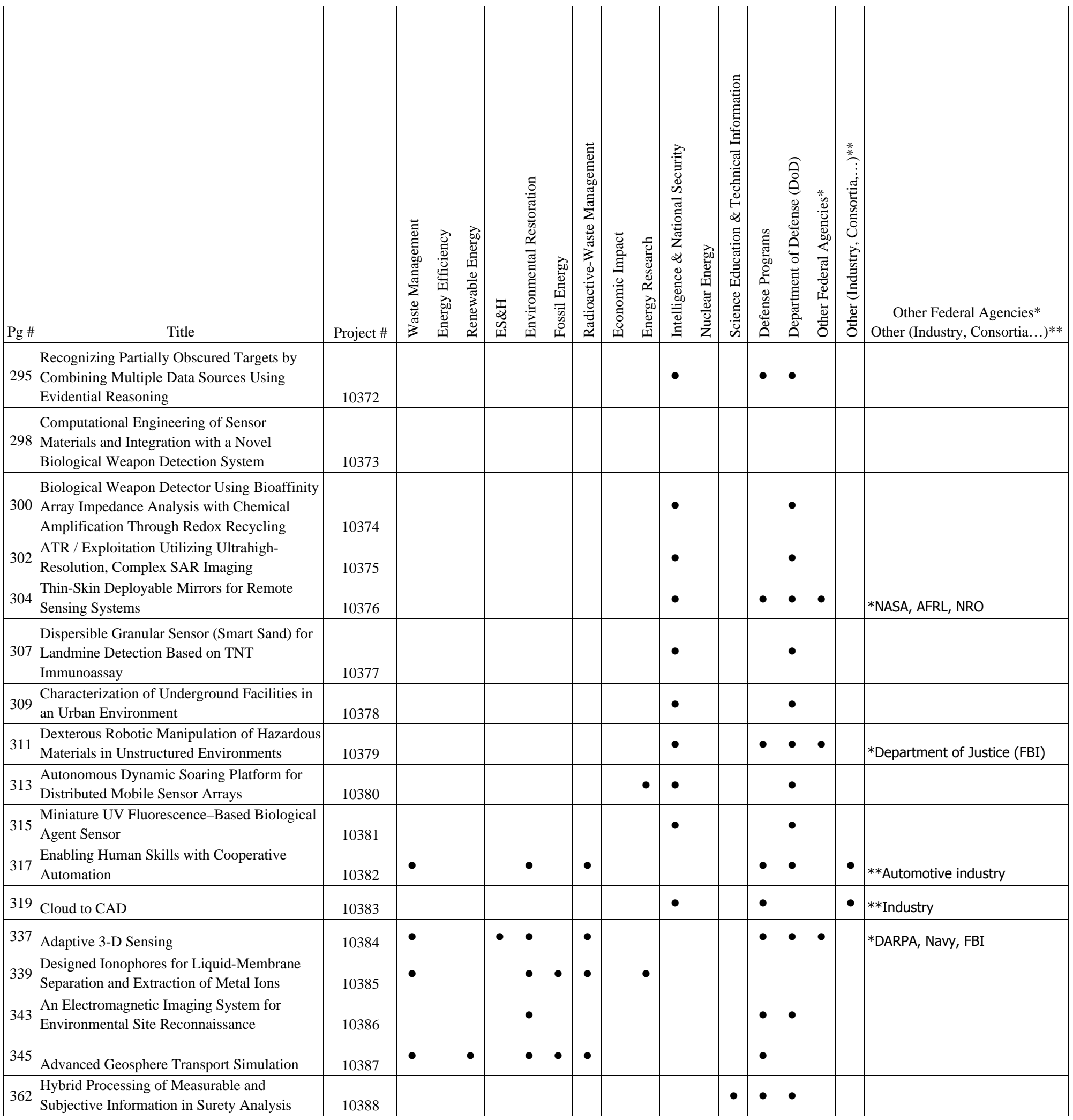




\section{Appendix E: Major National Programs}

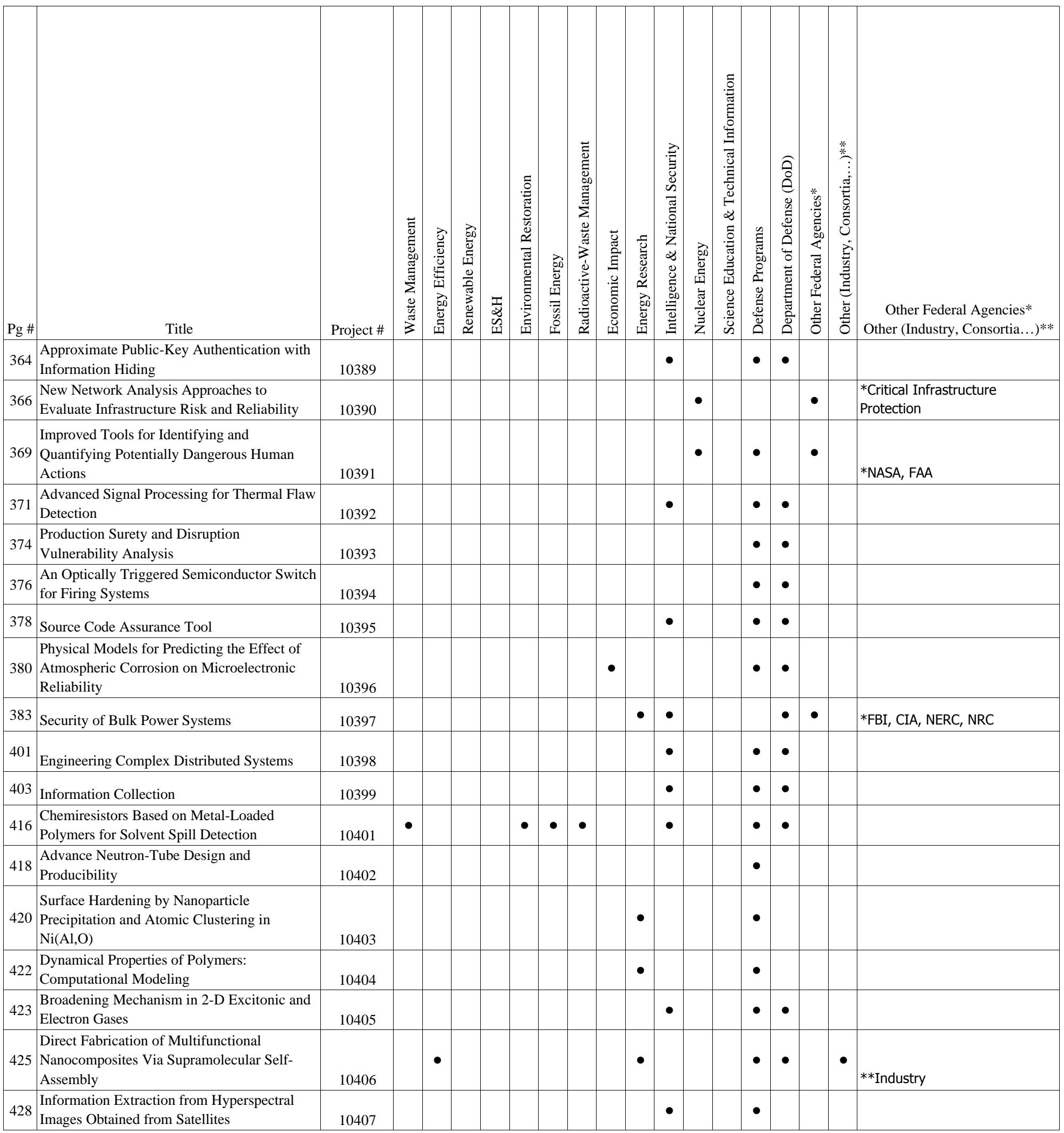




\section{Appendix E: Major National Programs}

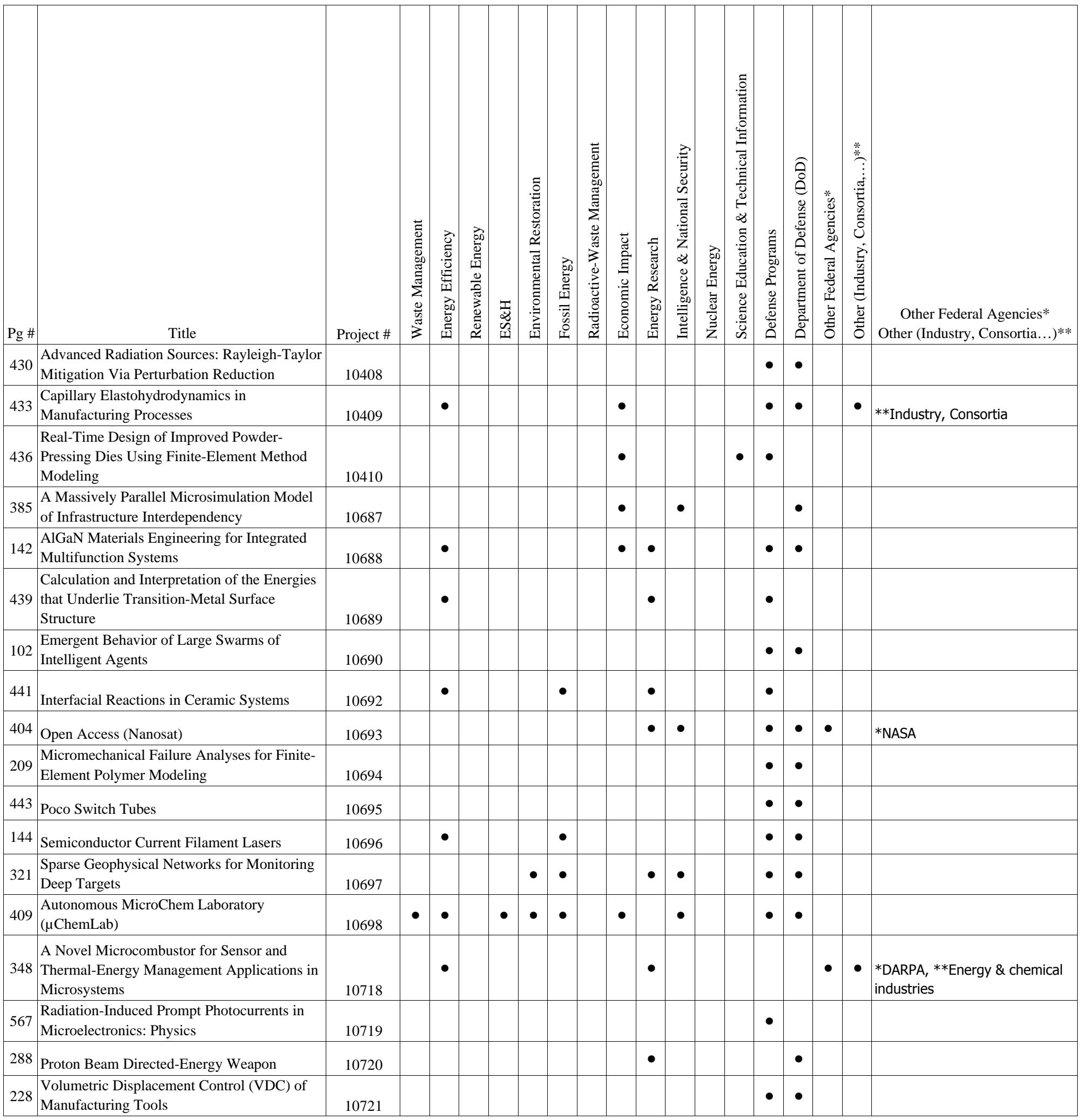




\section{Appendix E: Major National Programs}

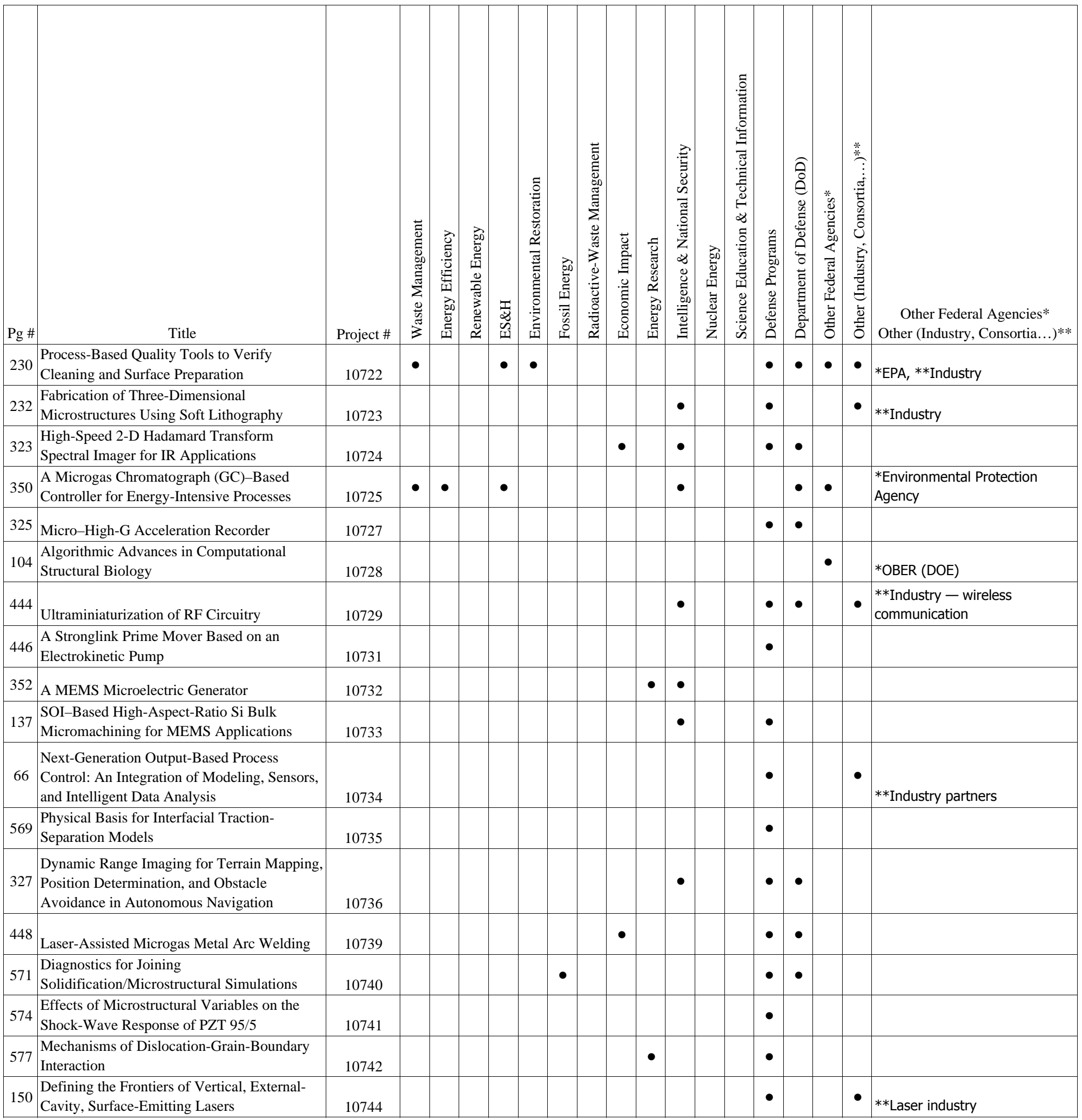




\section{Appendix E: Major National Programs}

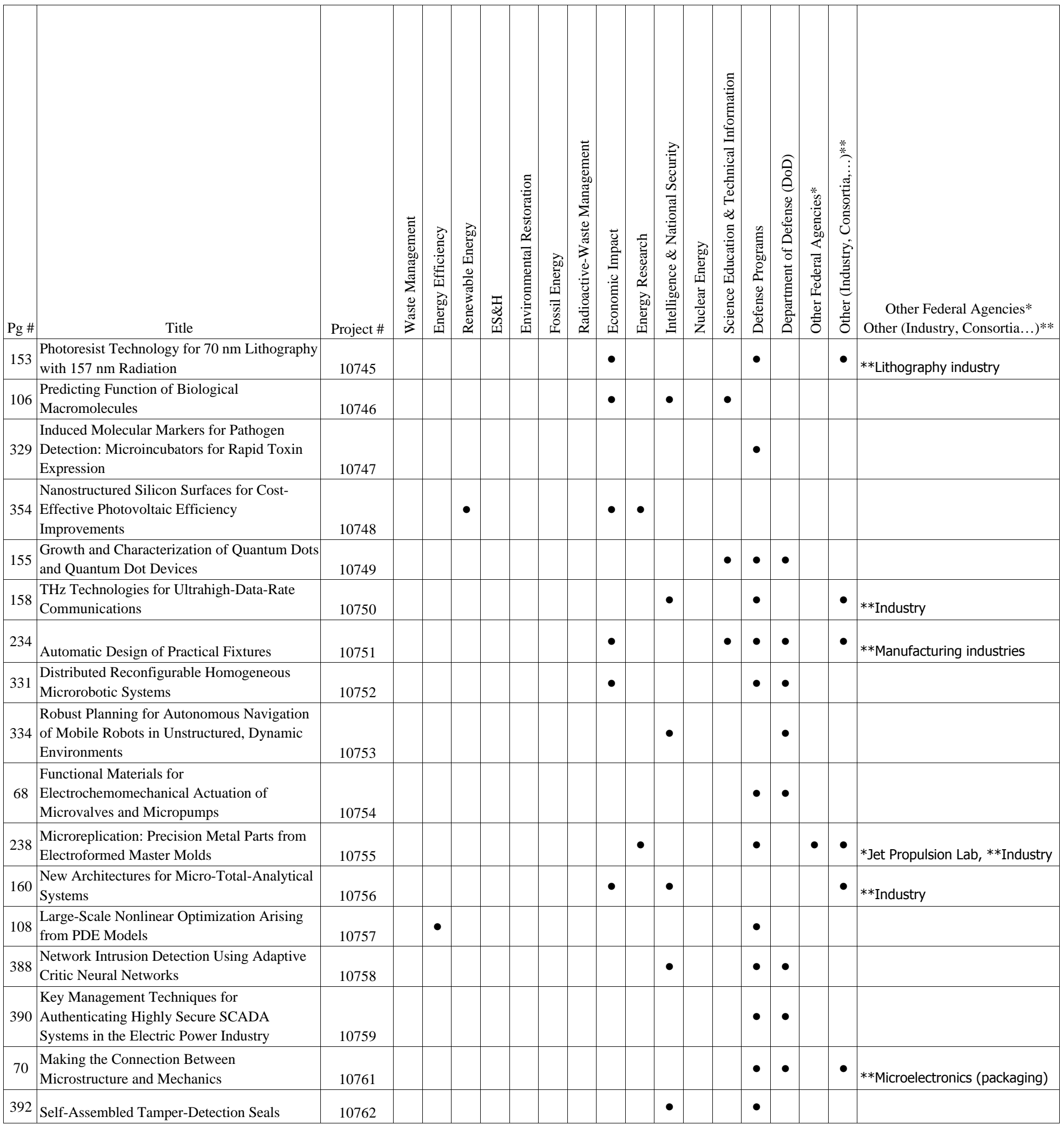




\section{Appendix E: Major National Programs}

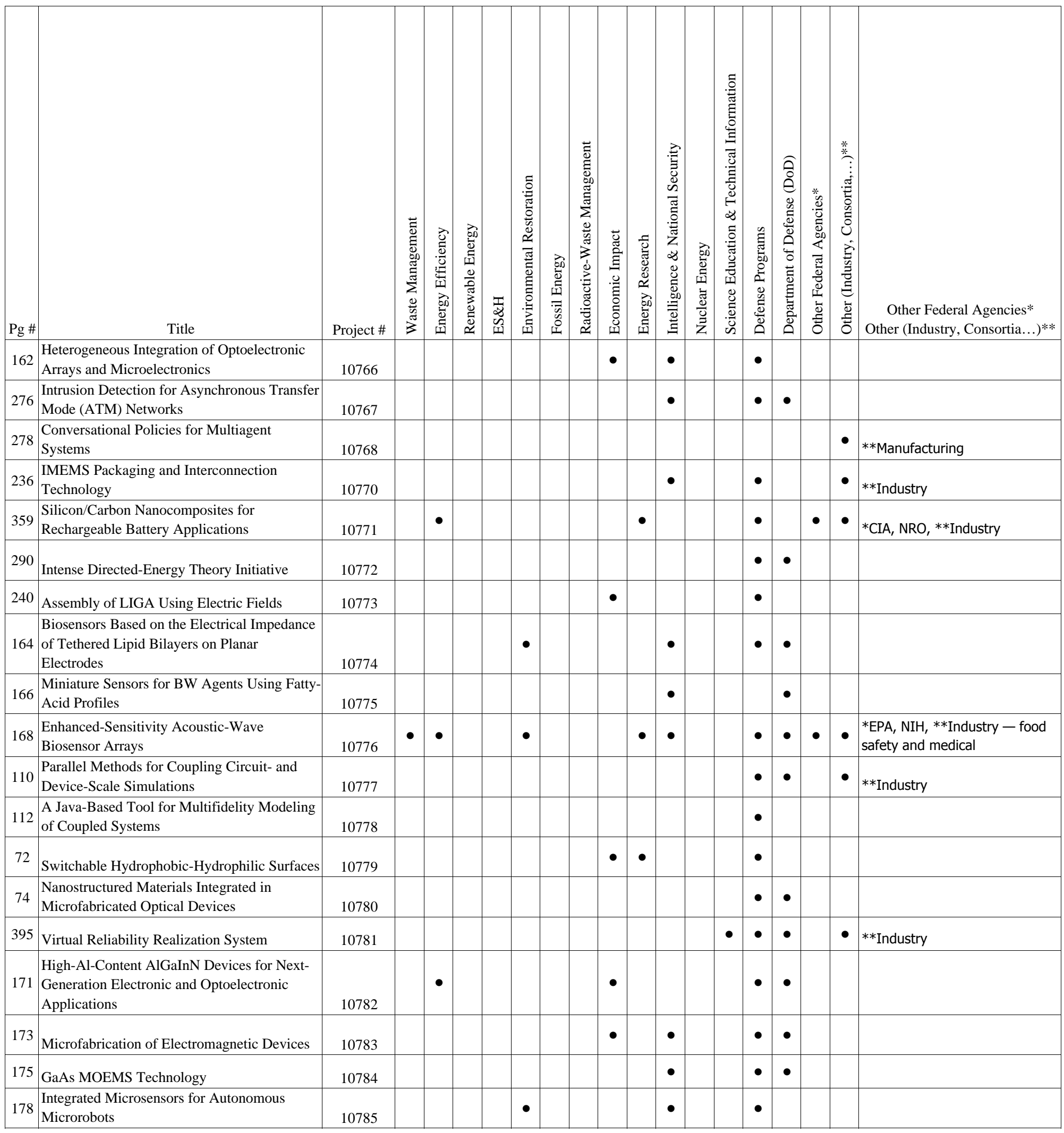




\section{Appendix E: Major National Programs}

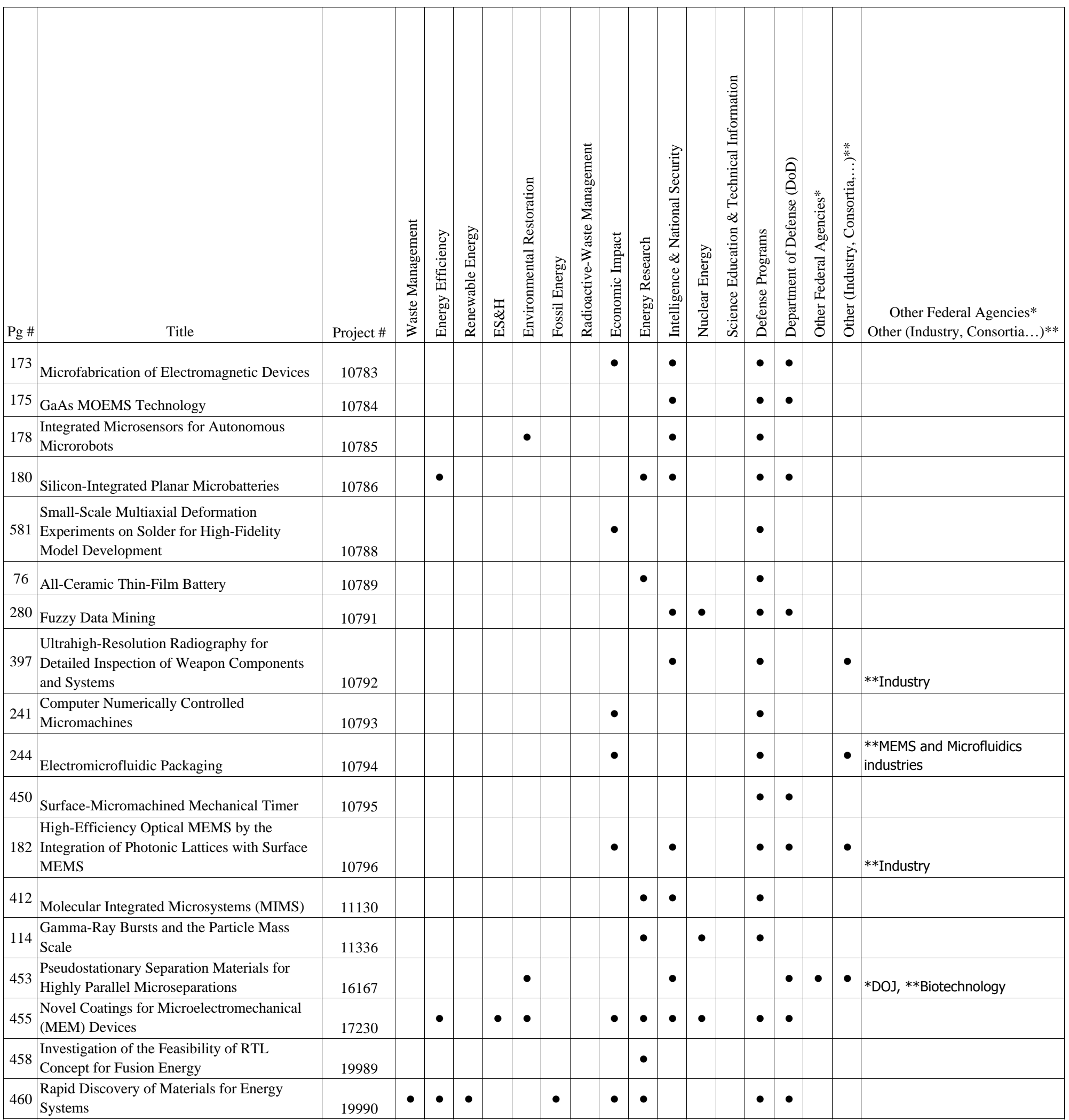




\section{Appendix E: Major National Programs}

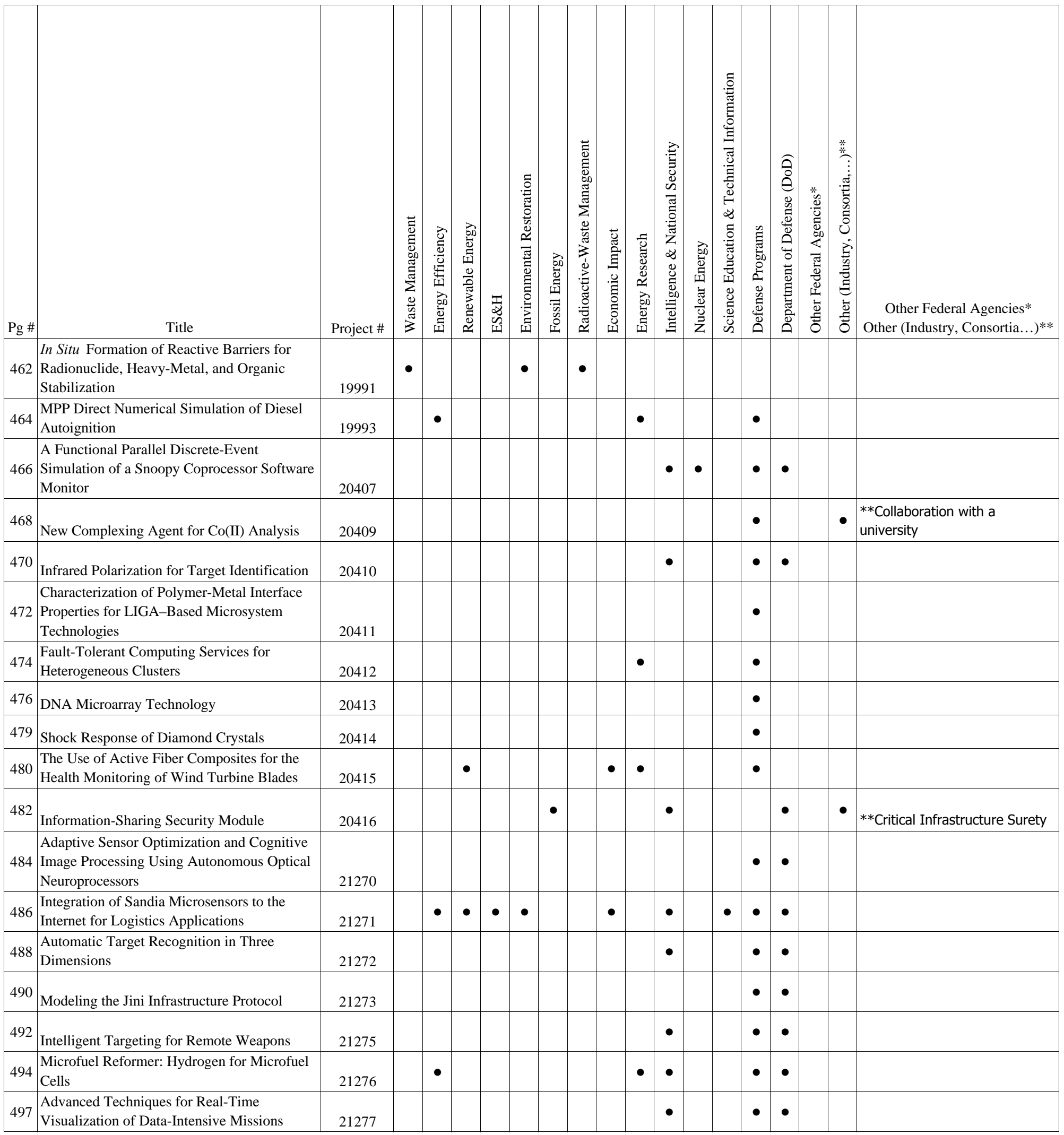




\section{Appendix E: Major National Programs}

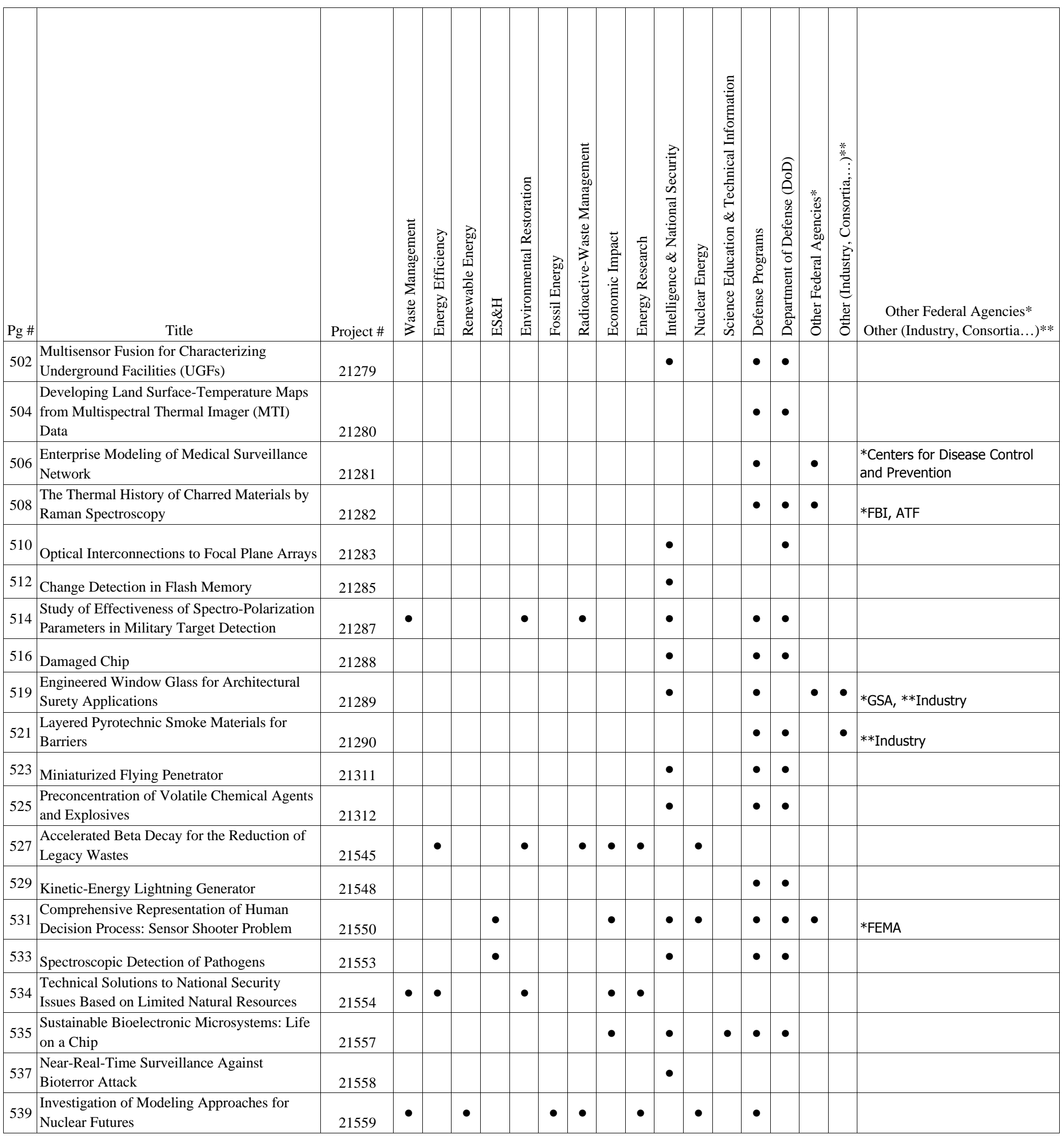




\section{Appendix E: Major National Programs}

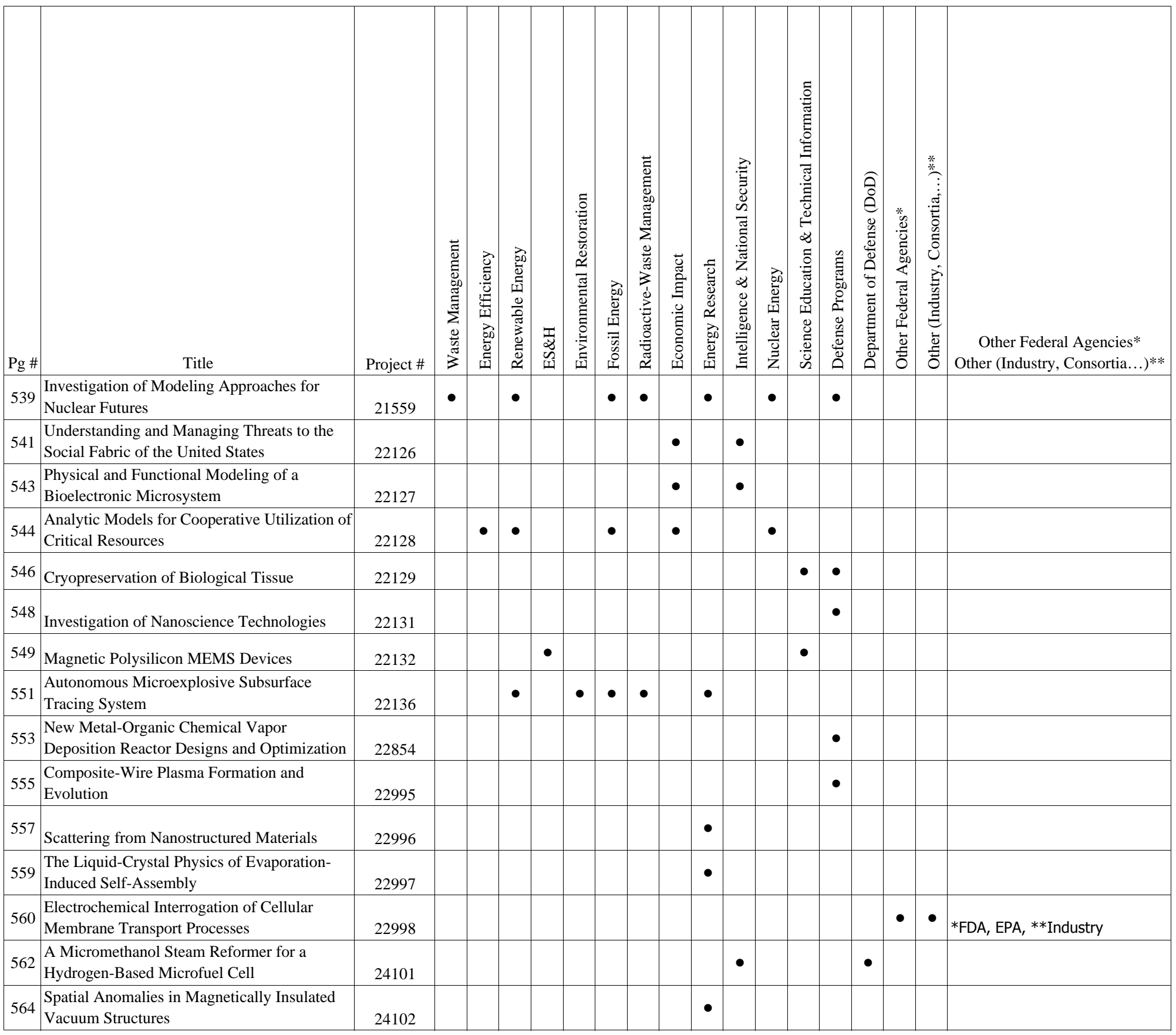




\section{Appendix F: Dual-Benefit Areas and Single-Use Categories}

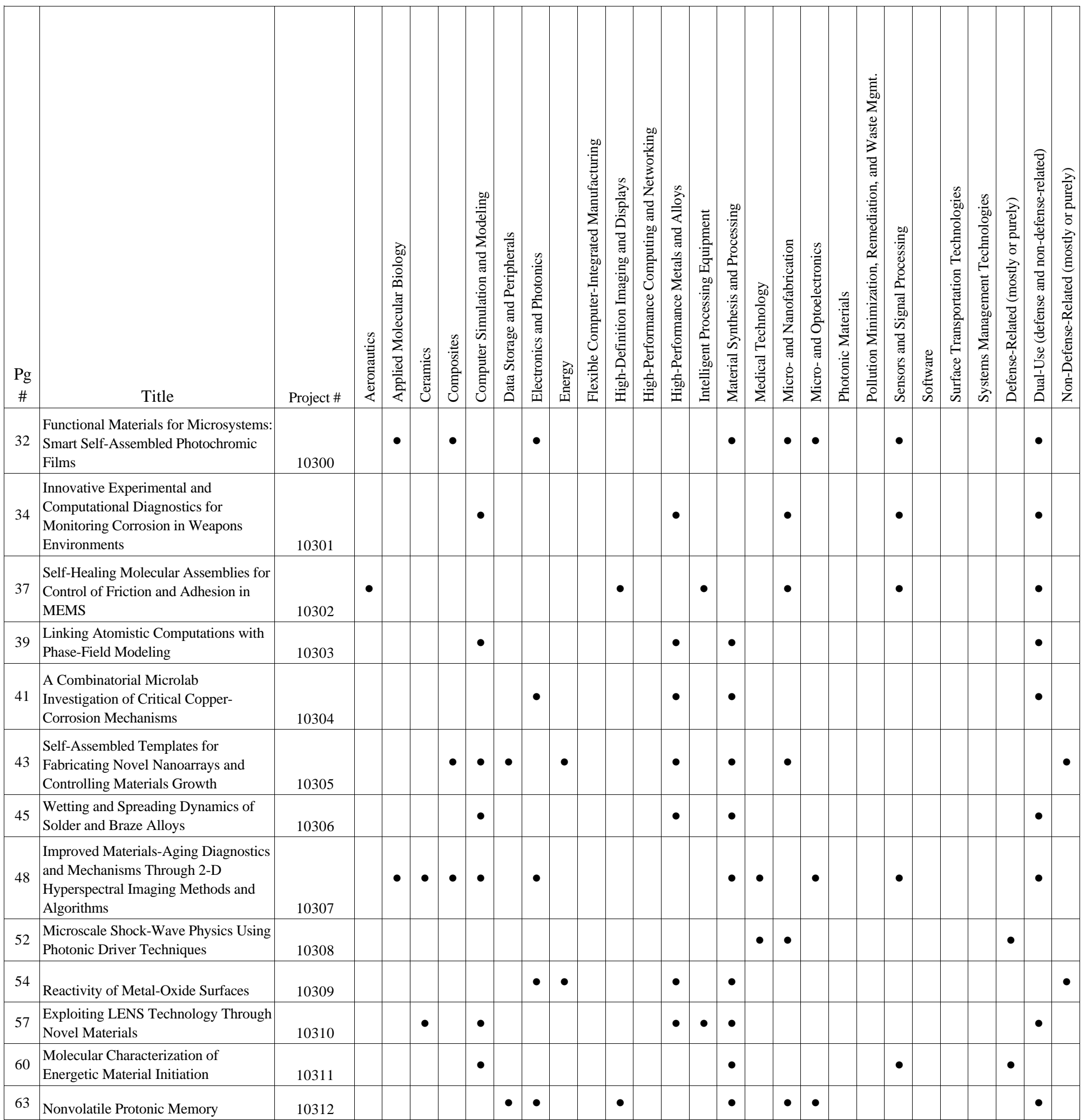




\section{Appendix F: Dual-Benefit Areas and Single-Use Categories}

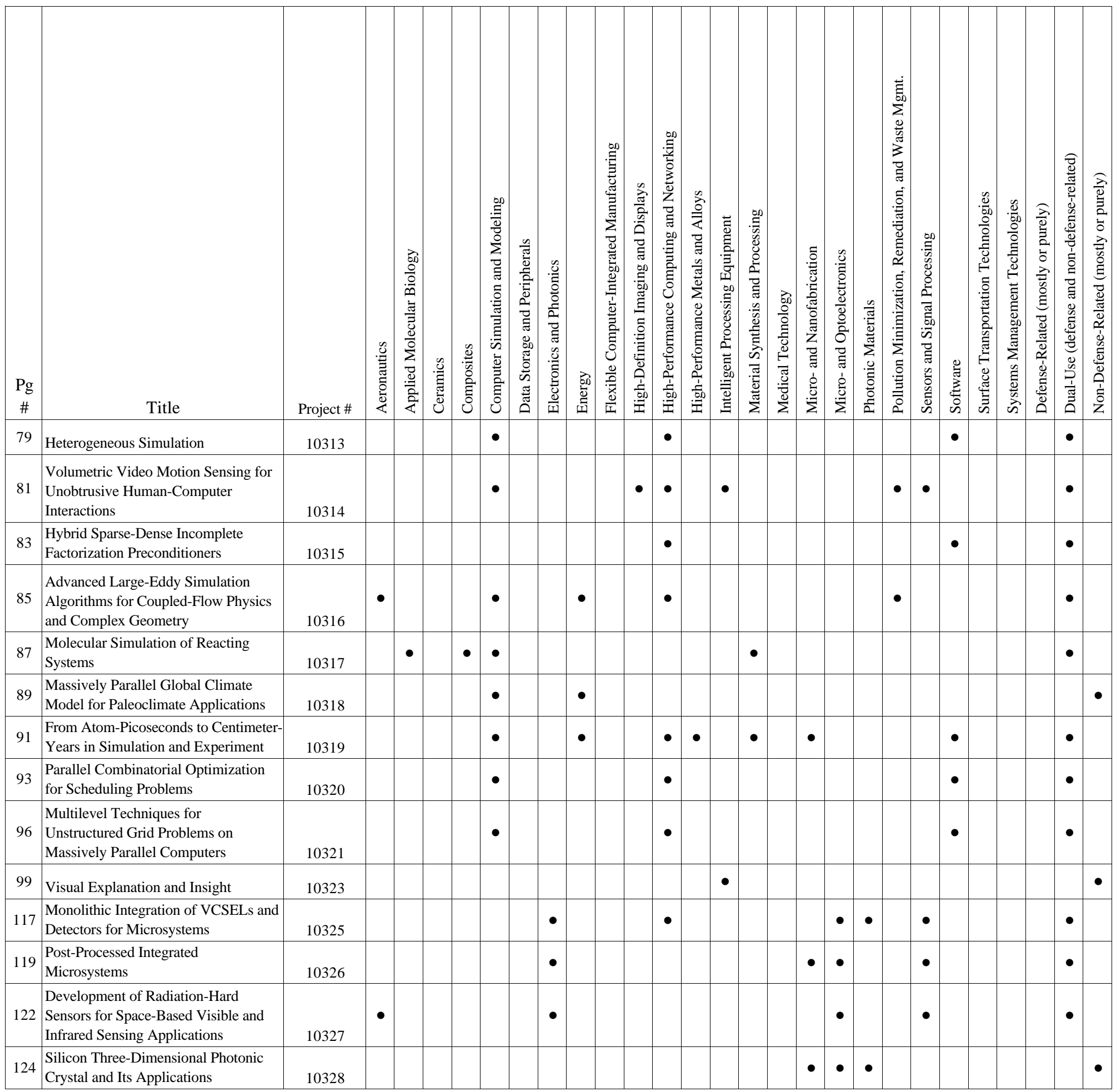




\section{Appendix F: Dual-Benefit Areas and Single-Use Categories}

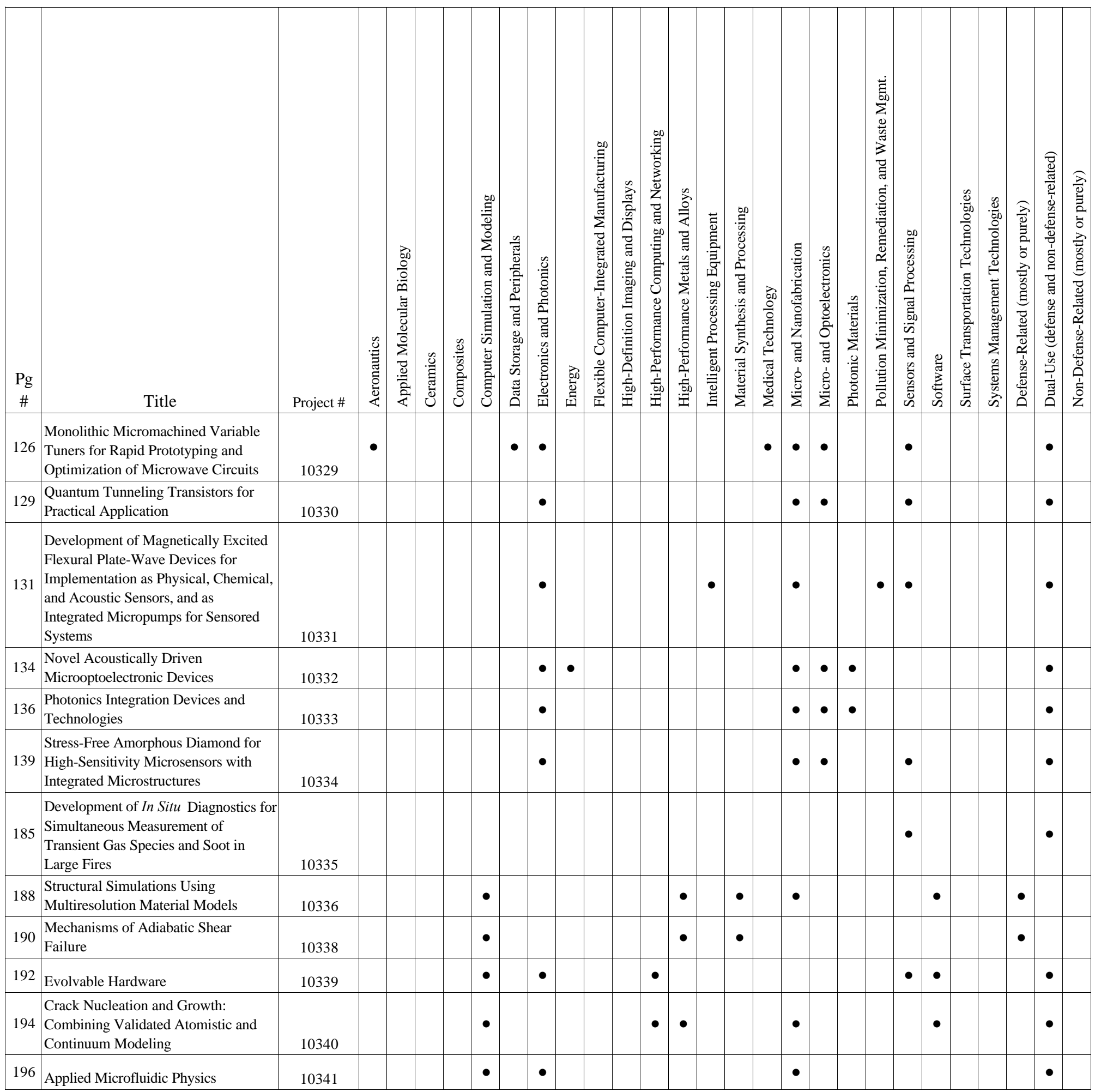




\section{Appendix F: Dual-Benefit Areas and Single-Use Categories}

\begin{tabular}{|c|c|c|c|c|c|c|c|c|c|c|c|c|c|c|c|c|c|c|c|c|c|c|c|c|c|c|c|c|}
\hline $\begin{array}{c}\mathrm{Pg} \\
\#\end{array}$ & Title & Project \# & 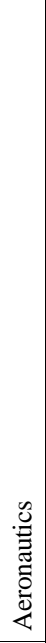 & 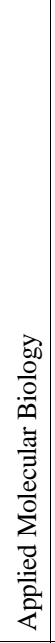 & 䓠 & 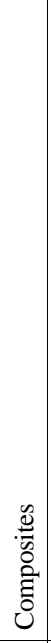 & 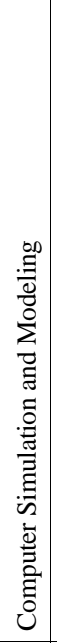 & 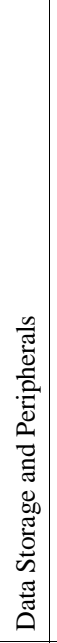 & 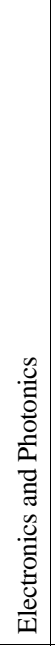 & $\begin{array}{c}\overrightarrow{0} 0 \\
\text { 島 }\end{array}$ & 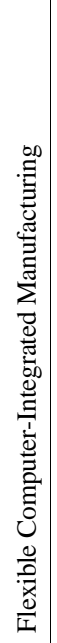 & 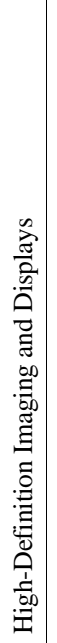 & 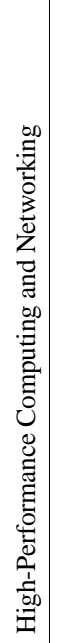 & 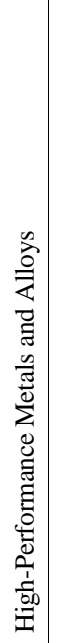 & 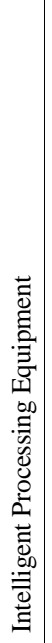 & 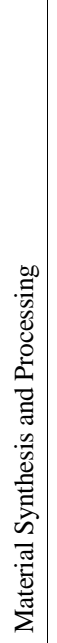 & 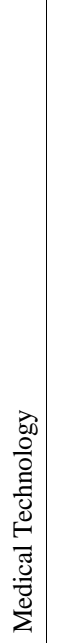 & 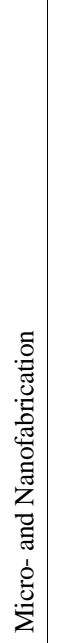 & 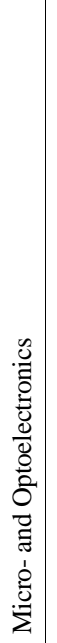 & 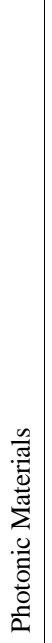 & 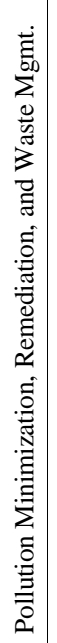 & 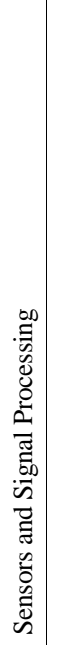 & 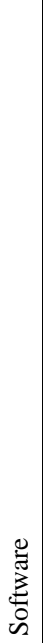 & 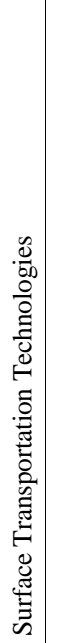 & 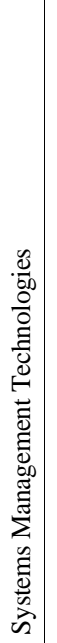 & 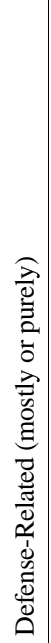 & 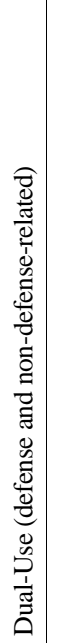 & 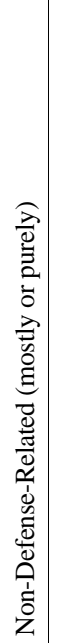 \\
\hline 198 & $\begin{array}{l}\text { Innovative Measurement Diagostics for } \\
\text { Fluid/Solid and Fluid/Fluid } \\
\text { Interactions in Rotating Flowfields }\end{array}$ & 10343 & $\bullet$ & & & & $\bullet$ & & $\bullet$ & & & & & & & & & $\bullet$ & $\bullet$ & & & $\bullet$ & & & & & $\bullet$ & \\
\hline 201 & $\begin{array}{l}\text { High-Resolution Modeling of } \\
\text { Multiscale Transient Phenomena in } \\
\text { Turbulent Boundary Layers }\end{array}$ & 10344 & $\bullet$ & & & & $\bullet$ & & & $\bullet$ & & & & & & $\bullet$ & & & & & $\bullet$ & & & & & & $\bullet$ & \\
\hline 203 & $\begin{array}{l}\text { Dispersive Measurements of Velocity } \\
\text { in Heterogeneous Materials }\end{array}$ & 10345 & & & - & - & $\bullet$ & & & & & & - & & & & & & $\bullet$ & & & & & & & & $\bullet$ & \\
\hline 206 & $\begin{array}{l}\text { A Physically Based Computational } \\
\text { Method for Predicting Generalized } \\
\text { Fracture }\end{array}$ & 10346 & & & $\bullet$ & $\bullet$ & $\bullet$ & & & & & & & $\bullet$ & & & & & & & & & $\bullet$ & & & & $\bullet$ & \\
\hline 212 & $\begin{array}{l}\text { Microdiagnostic MEMS Lab-on-a- } \\
\text { Chip }\end{array}$ & 10347 & - & - & $\bullet$ & & - & & - & & $\bullet$ & $\bullet$ & $\bullet$ & & $\bullet$ & $\bullet$ & & $\bullet$ & $\bullet$ & $\bullet$ & & $\bullet$ & $\bullet$ & $\bullet$ & $\bullet$ & & - & \\
\hline 214 & $\begin{array}{l}\text { Real-Time Error Correction Using } \\
\text { Electromagnetic-Bearing Spindles }\end{array}$ & 10348 & & & & & - & & & & & & & & $\bullet$ & & $\bullet$ & $\bullet$ & & & & & & $\bullet$ & & & $\bullet$ & \\
\hline 216 & $\begin{array}{l}\text { Thin-Film Deposition Processes } \\
\text { Incorporating In Situ Monitoring } \\
\text { Capabilities }\end{array}$ & 10349 & & & & & & & & & & & & & & - & & - & & & & & & & & & - & \\
\hline 218 & $\begin{array}{l}\text { Solid-State Neutron Generator for Use } \\
\text { in Nuclear Weapons }\end{array}$ & 10351 & & & & & - & & & & & & & & & & & $\bullet$ & & & & & & & & - & & \\
\hline 220 & Scripting for Video Inspection & 10352 & $\bullet$ & & & & & & & & • & & & & • & & & $\bullet$ & & & & & & & & & $\bullet$ & \\
\hline 223 & $\begin{array}{l}\text { Advanced Machining Processes for } \\
\text { Microfabrication }\end{array}$ & 10353 & - & $\bullet$ & - & & & & & & & & & & & & - & $\bullet$ & & & & & & & & & $\bullet$ & \\
\hline 225 & Advanced Production Planning Models & 10354 & & & & & - & & & & - & & & & & & & & & & & & $\bullet$ & & & & $\bullet$ & \\
\hline 247 & $\begin{array}{l}\text { Weighted-Nearest-Neighbor (WNN) } \\
\text { Decision Making for Data Mining }\end{array}$ & 10355 & & & & & • & & & & & & & & $\bullet$ & & & & & & & • & $\bullet$ & & & & - & \\
\hline 249 & $\begin{array}{l}\text { Varying QoS for Fixed and Mobile } \\
\text { Networks }\end{array}$ & 10356 & & & & & - & & & & & & - & & & & & & & & & & & & $\bullet$ & & $\bullet$ & \\
\hline 251 & $\begin{array}{l}\text { A Real-Time Decision-Support } \\
\text { Framework to Guide Facility Response } \\
\text { to Abnormal Events }\end{array}$ & 10357 & & & & & - & & & & & & - & & • & & & & & & - & - & $\bullet$ & & - & & $\bullet$ & \\
\hline 253 & $\begin{array}{l}\text { Physical Model-Based Fusion of } \\
\text { Sensor Array Data }\end{array}$ & 10358 & & & & & & & & & & & & & & & & & & & & & & & & & 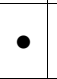 & \\
\hline
\end{tabular}




\section{Appendix F: Dual-Benefit Areas and Single-Use Categories}

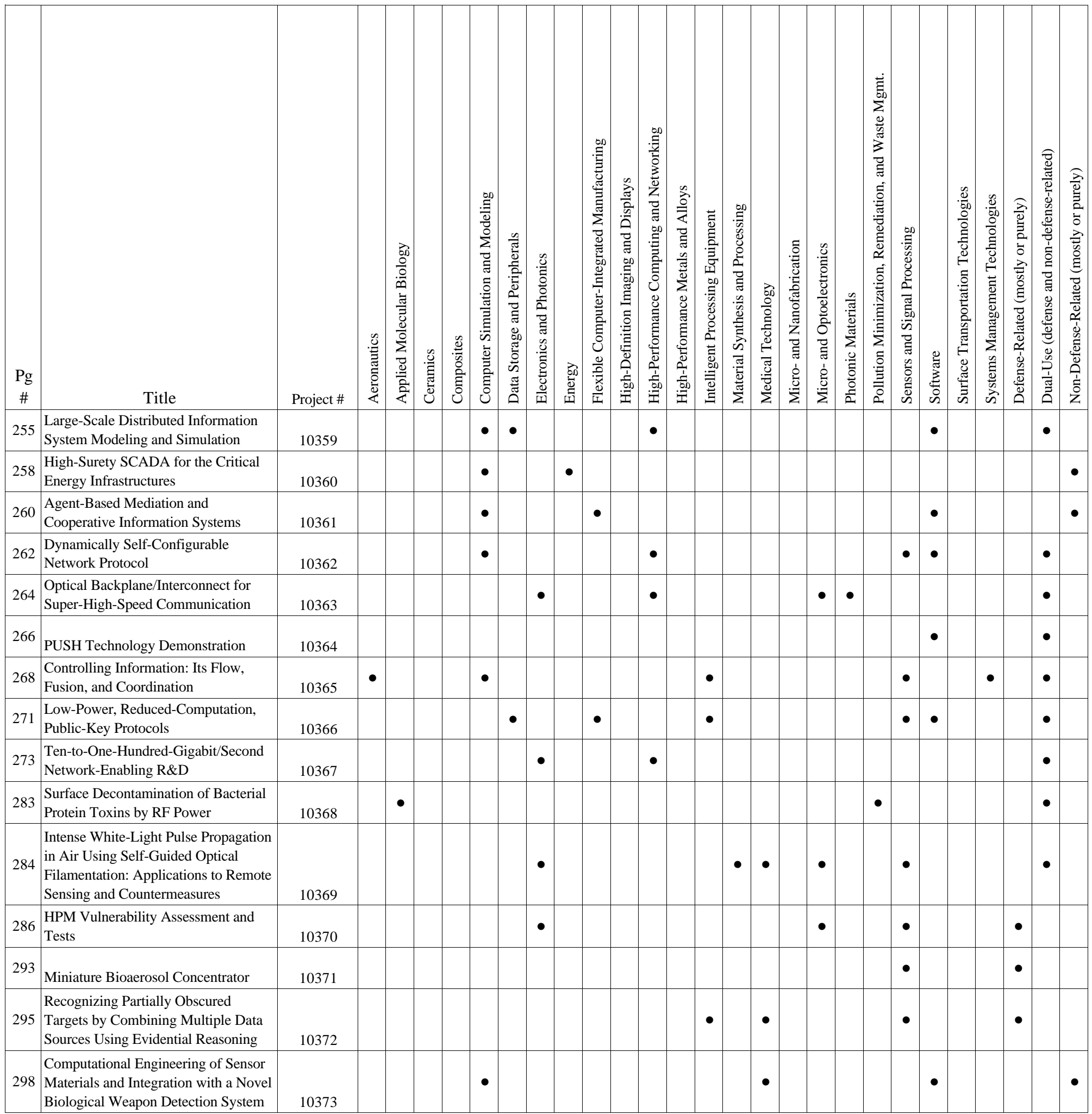




\section{Appendix F: Dual-Benefit Areas and Single-Use Categories}

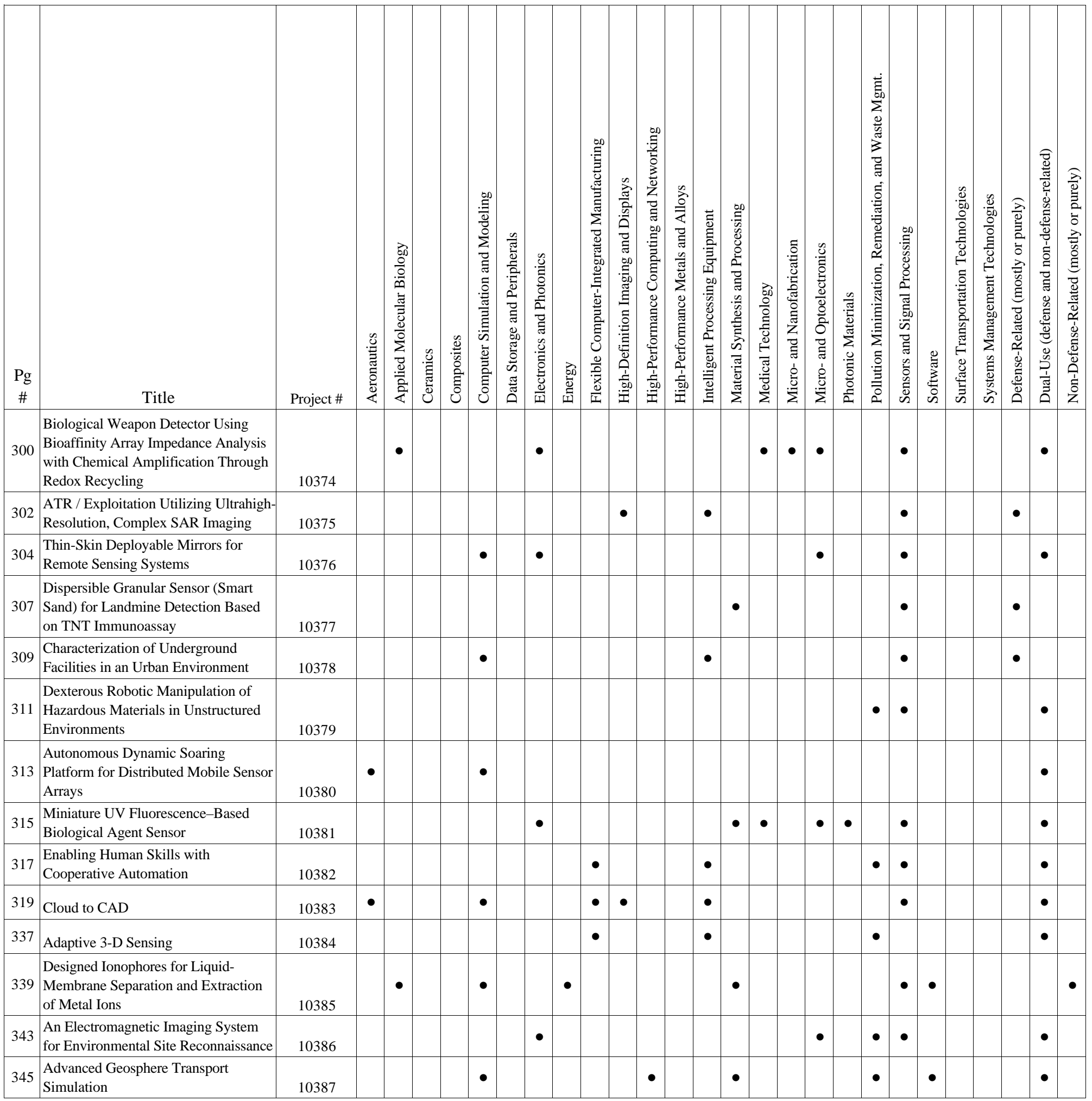




\section{Appendix F: Dual-Benefit Areas and Single-Use Categories}

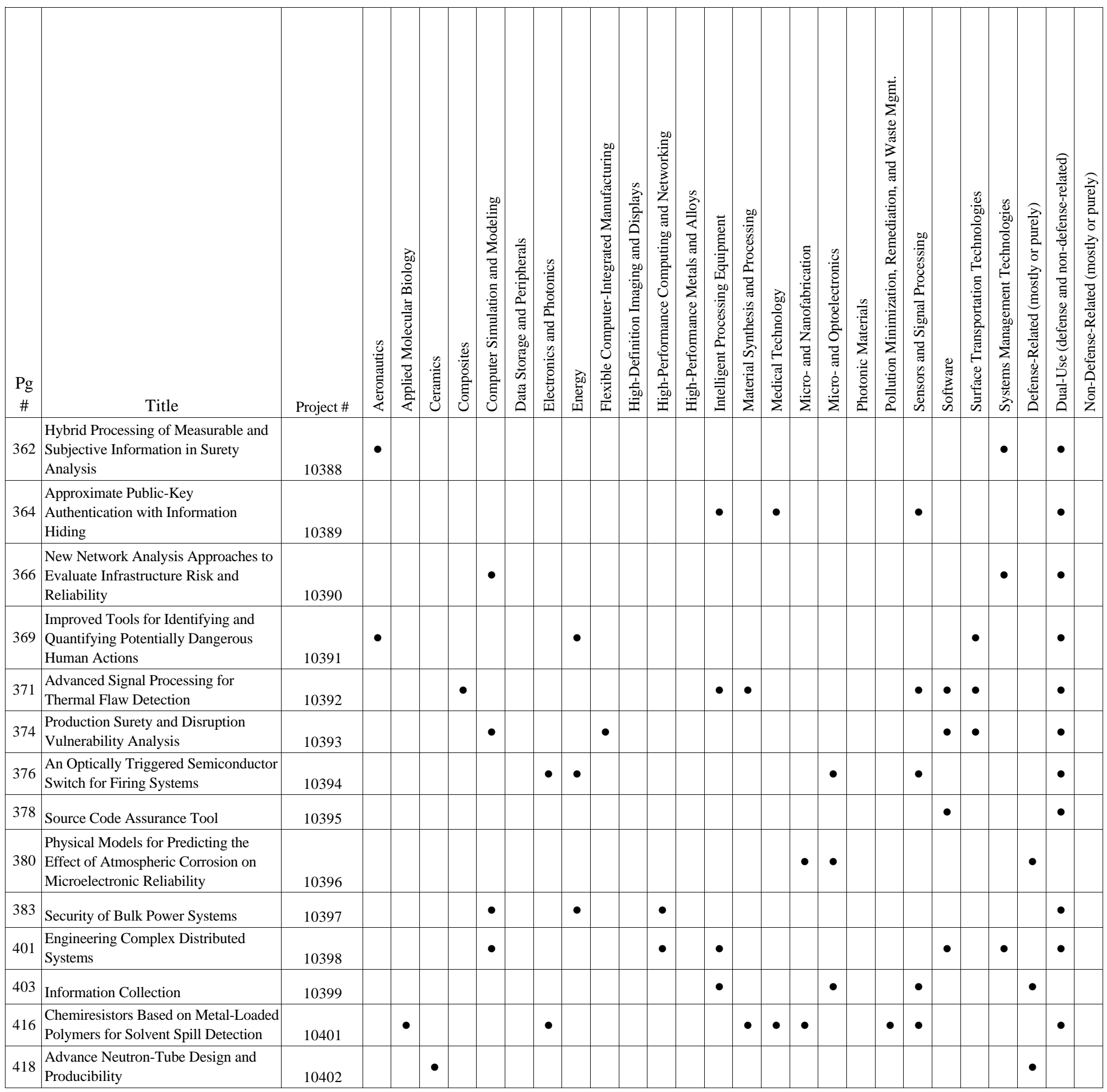




\section{Appendix F: Dual-Benefit Areas and Single-Use Categories}

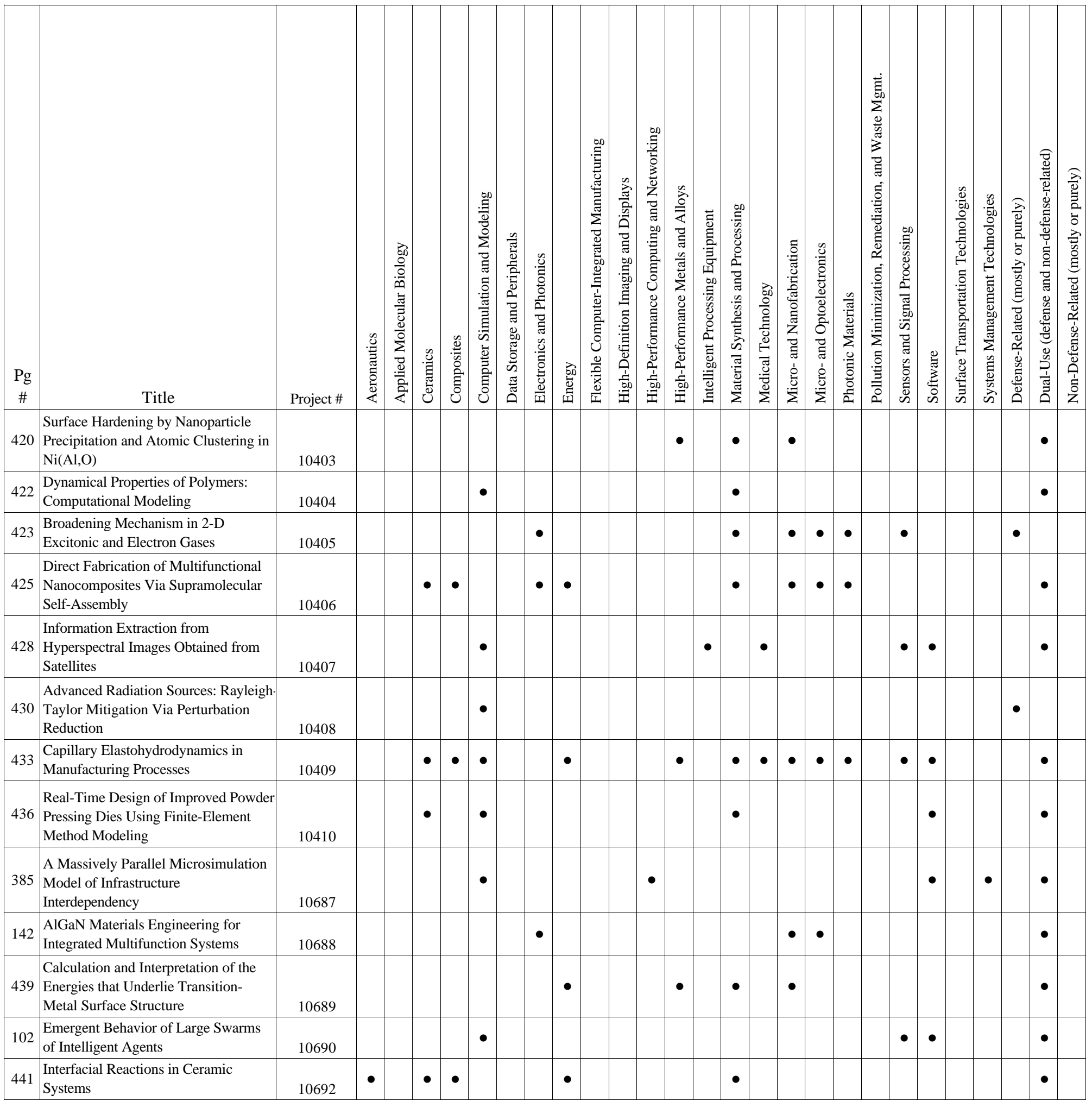




\section{Appendix F: Dual-Benefit Areas and Single-Use Categories}

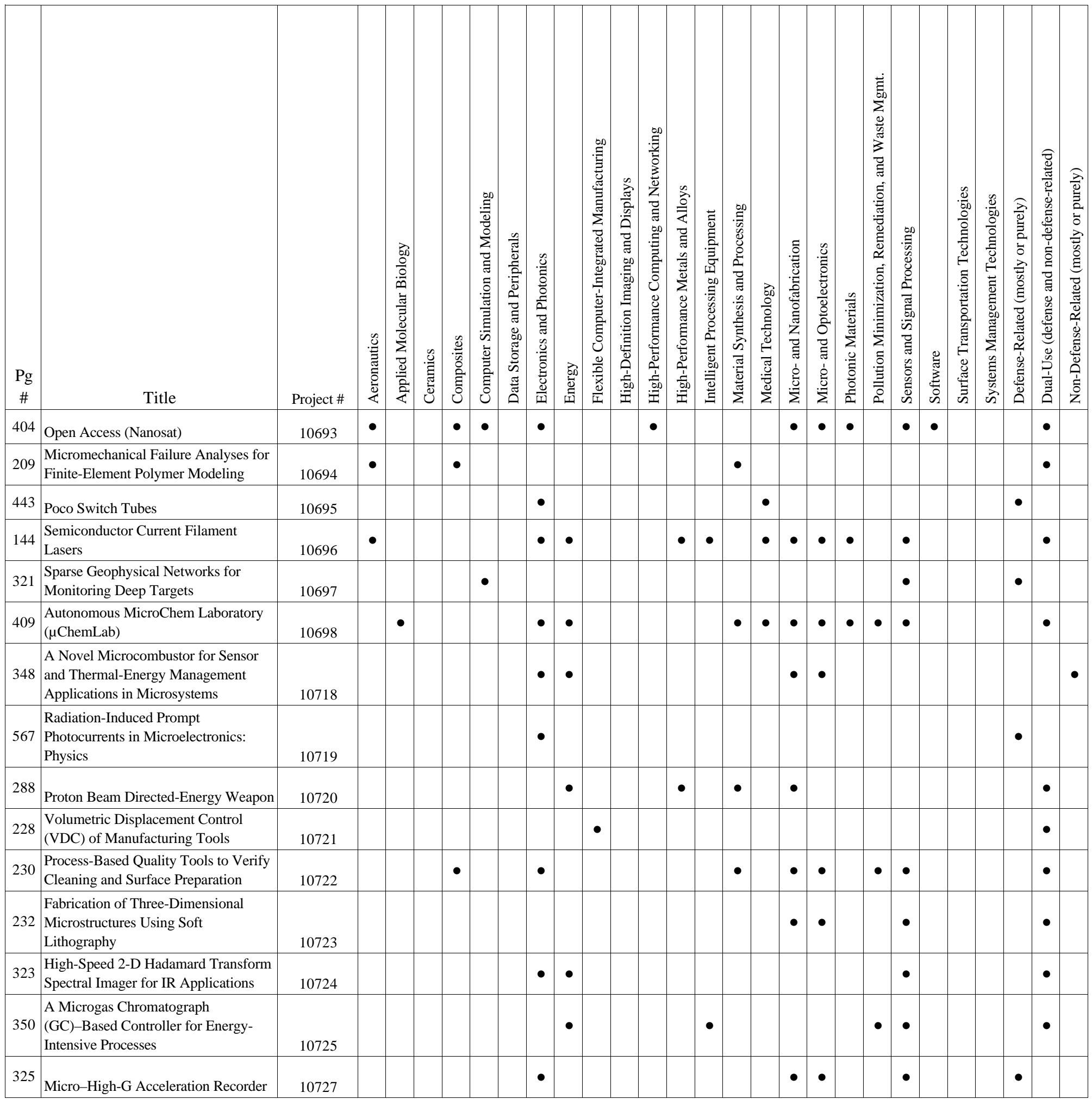




\section{Appendix F: Dual-Benefit Areas and Single-Use Categories}

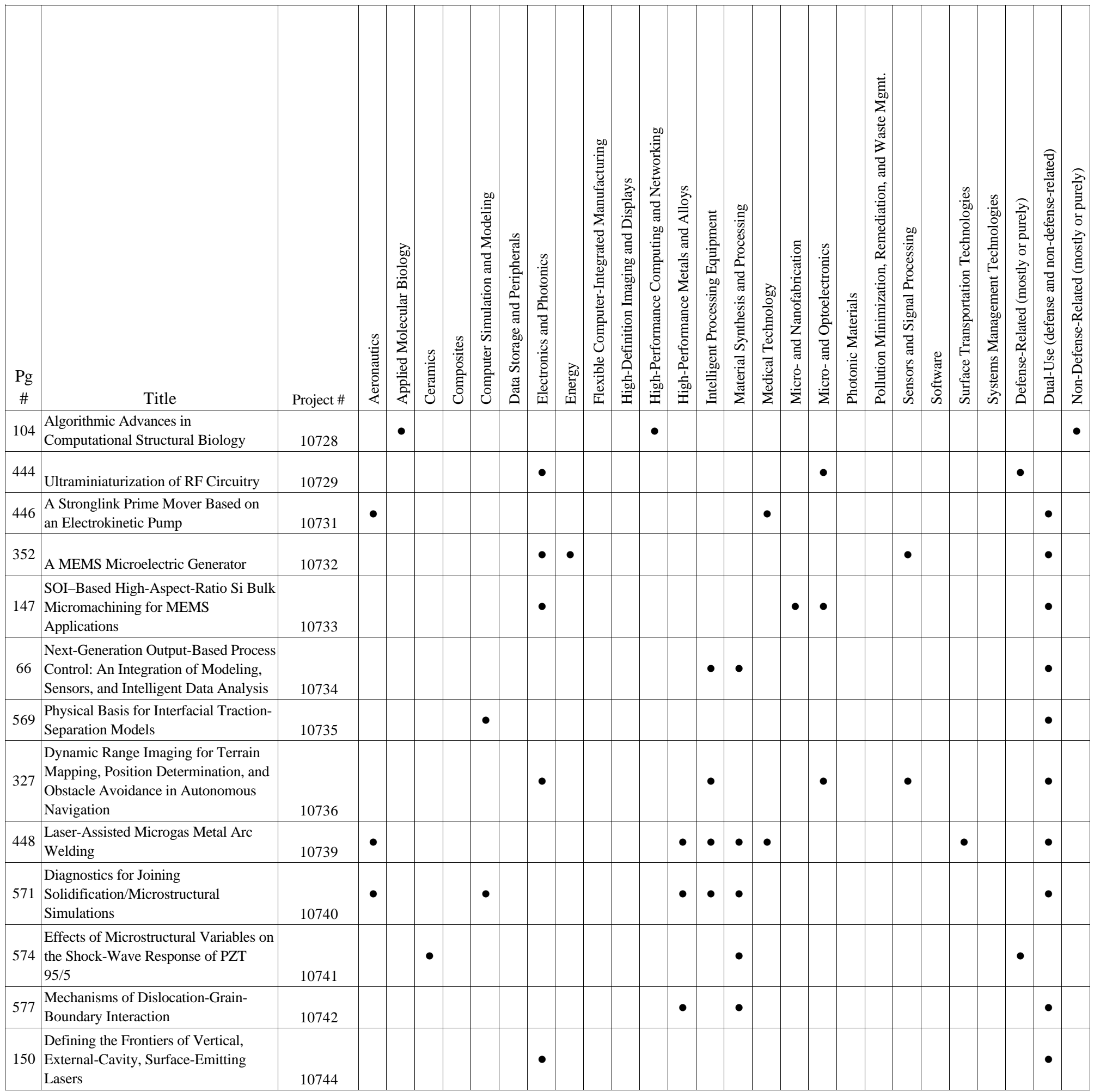




\section{Appendix F: Dual-Benefit Areas and Single-Use Categories}

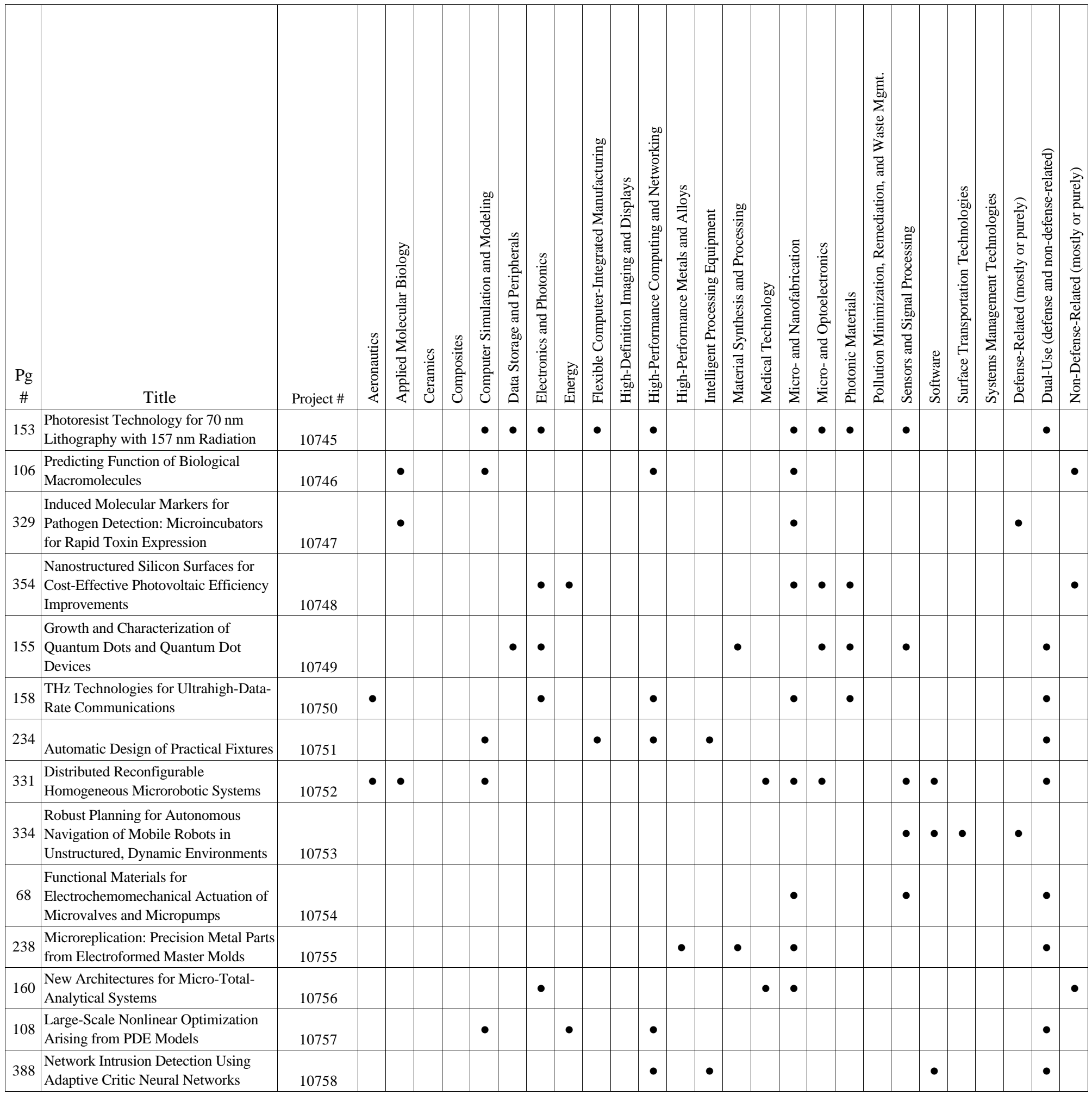




\section{Appendix F: Dual-Benefit Areas and Single-Use Categories}

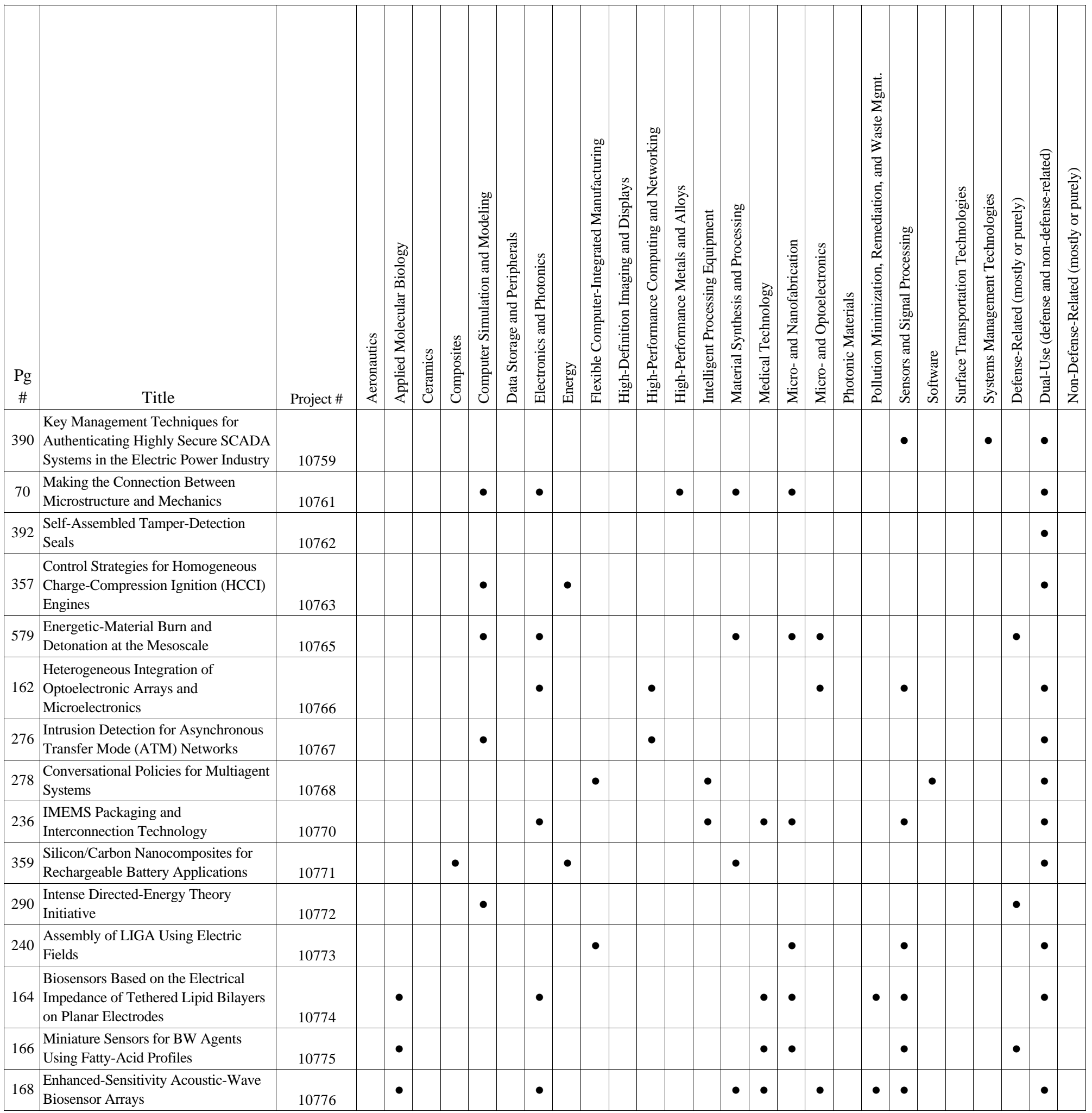




\section{Appendix F: Dual-Benefit Areas and Single-Use Categories}

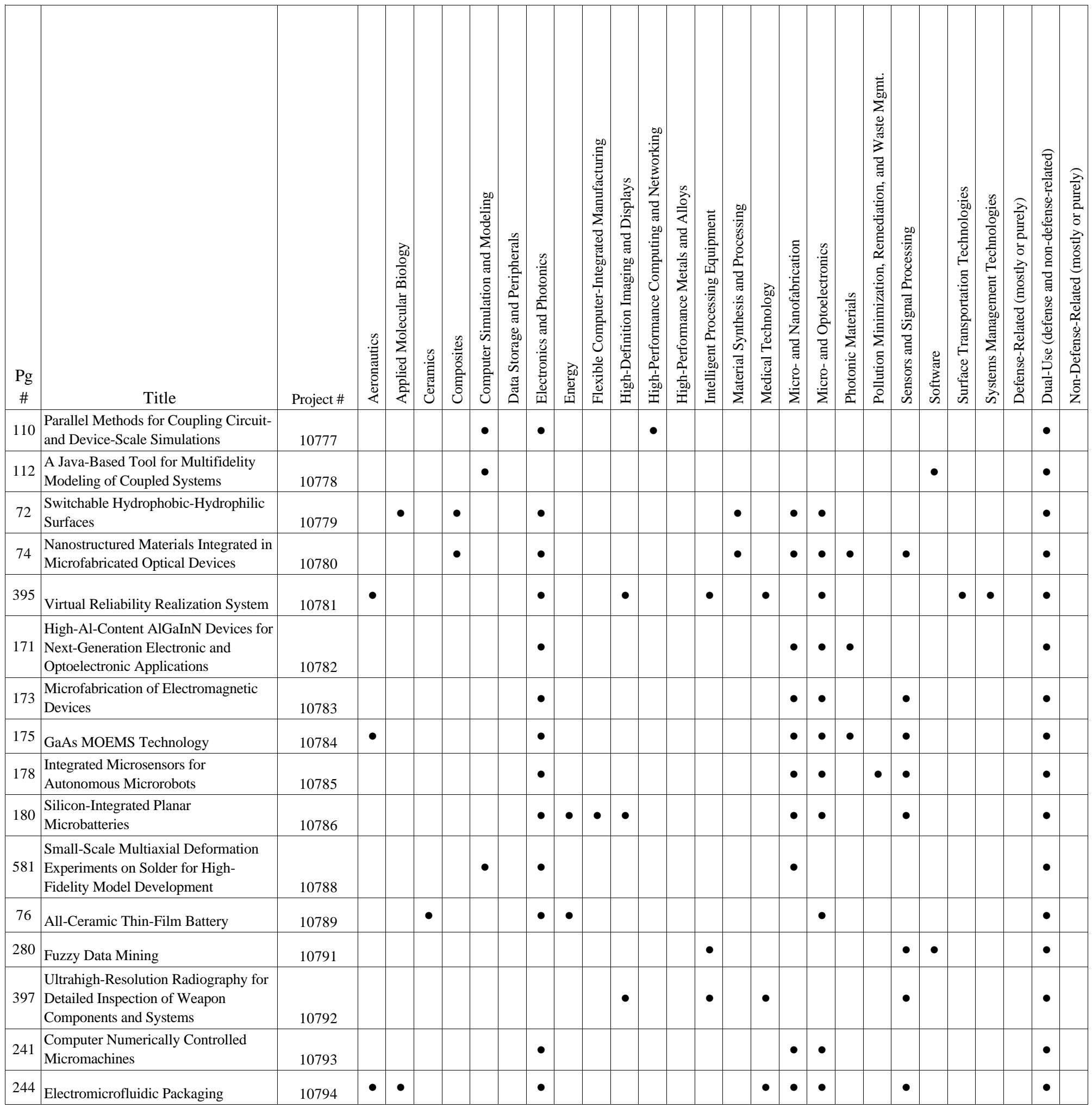




\section{Appendix F: Dual-Benefit Areas and Single-Use Categories}

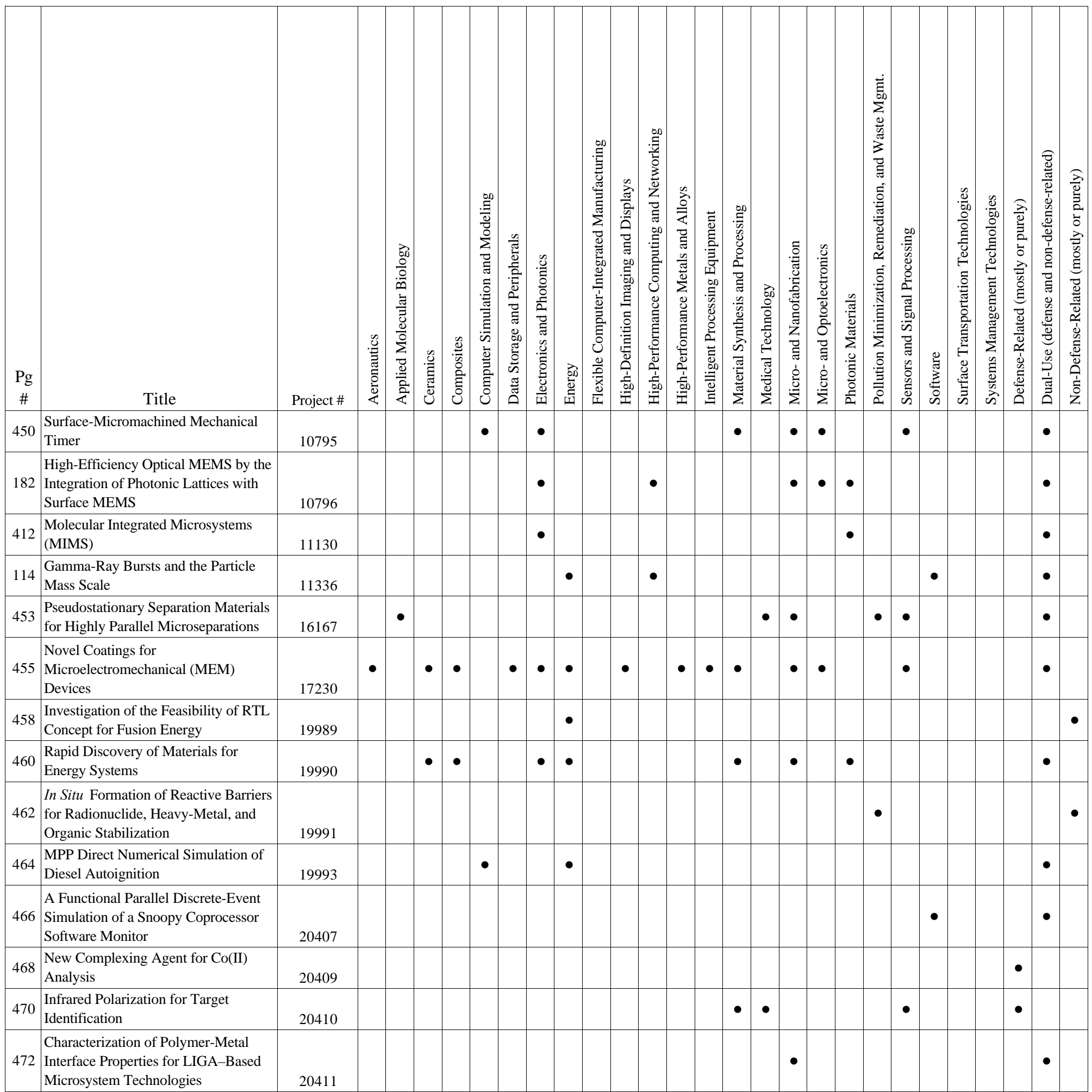




\section{Appendix F: Dual-Benefit Areas and Single-Use Categories}

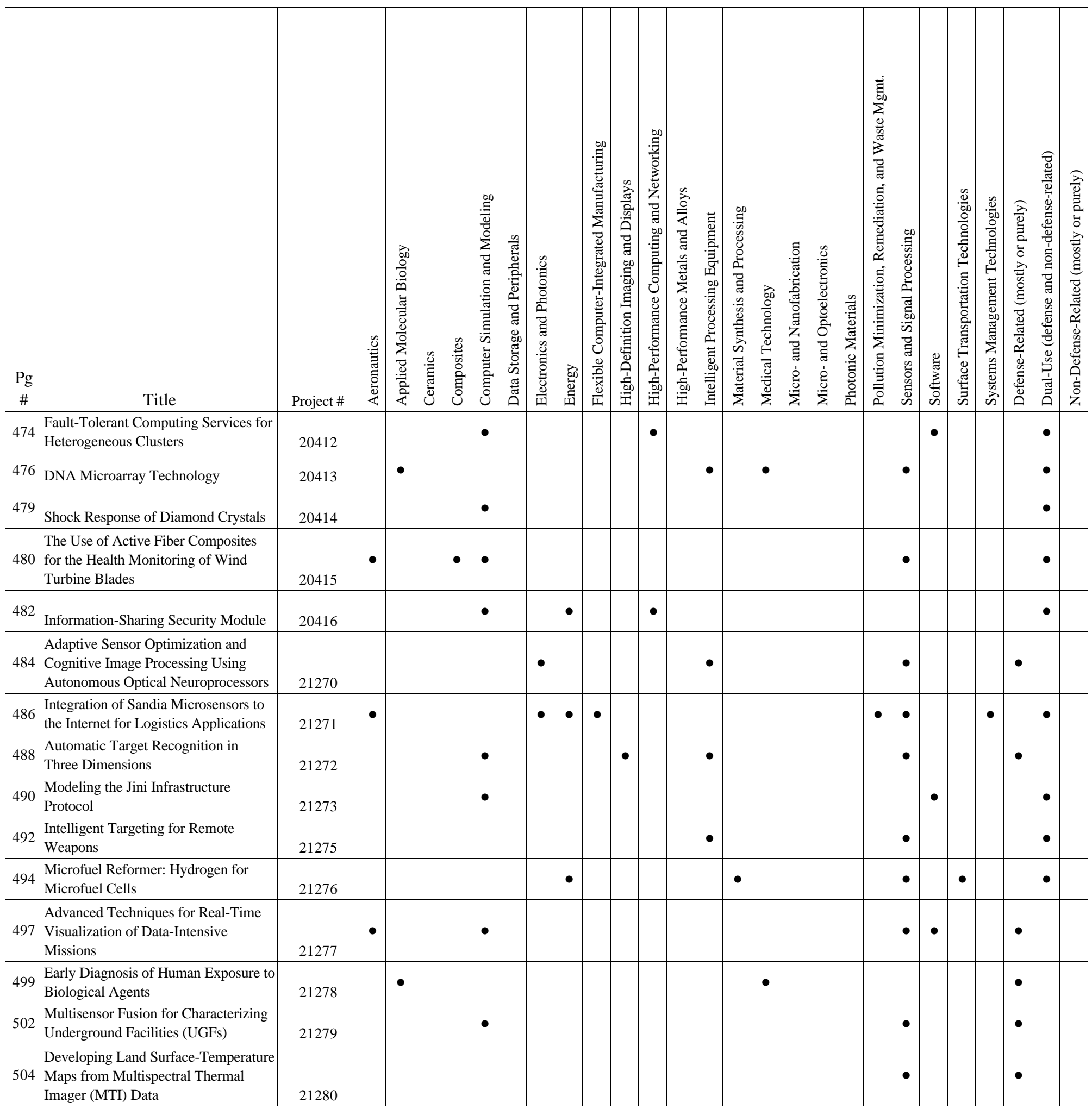




\section{Appendix F: Dual-Benefit Areas and Single-Use Categories}

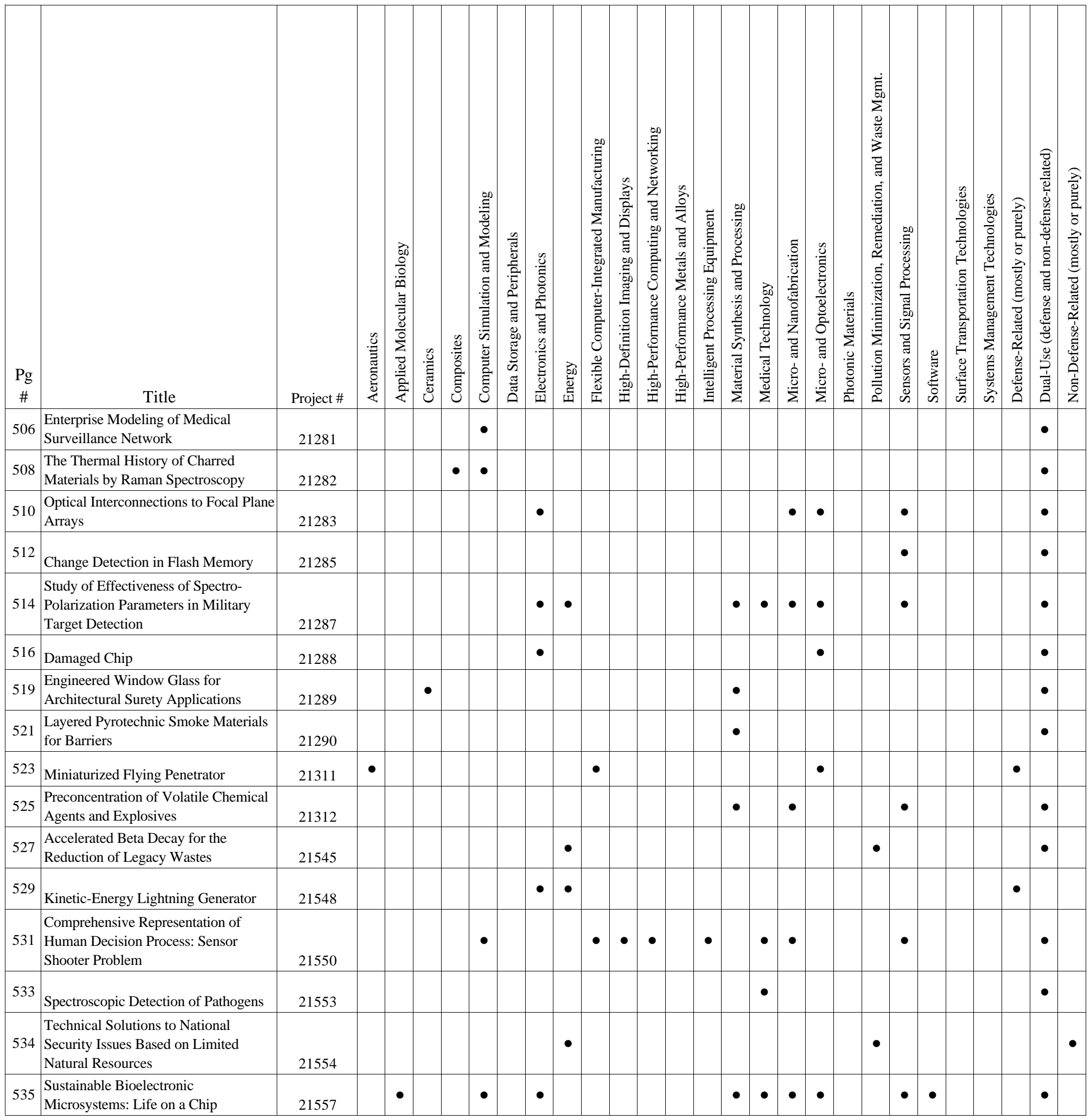




\section{Appendix F: Dual-Benefit Areas and Single-Use Categories}

\begin{tabular}{|c|c|c|c|c|c|c|c|c|c|c|c|c|c|c|c|c|c|c|c|c|c|c|c|c|c|c|c|c|}
\hline $\begin{array}{c}\mathrm{Pg} \\
\#\end{array}$ & Title & Project \# & 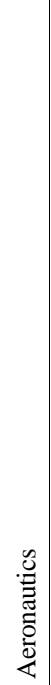 & 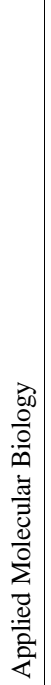 & 苍 & 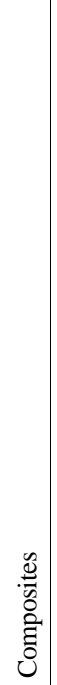 & 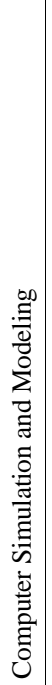 & 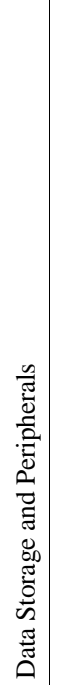 & 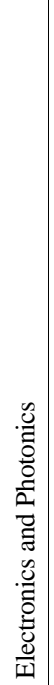 & $\begin{array}{c}\overrightarrow{00} \\
\vec{\Phi} \\
\overrightarrow{|c|}\end{array}$ & 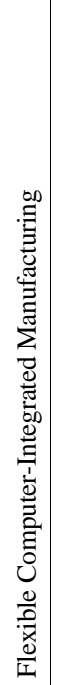 & 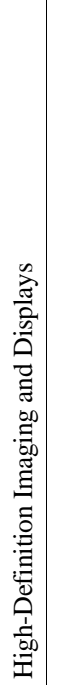 & 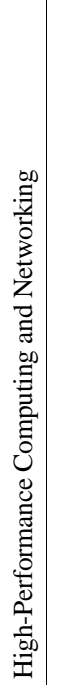 & 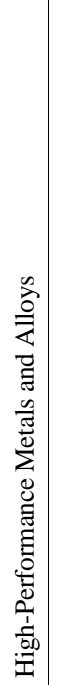 & 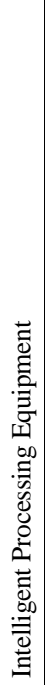 & 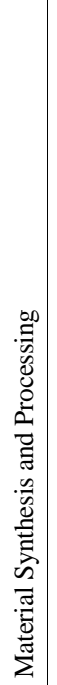 & 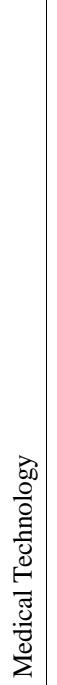 & 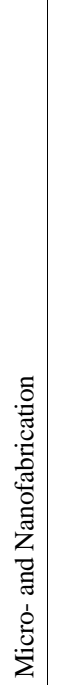 & 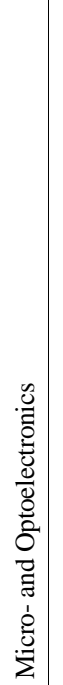 & 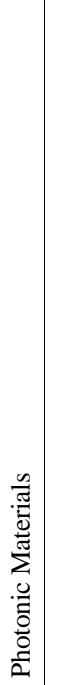 & 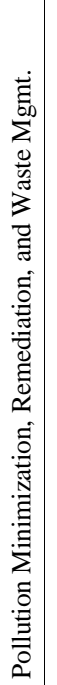 & 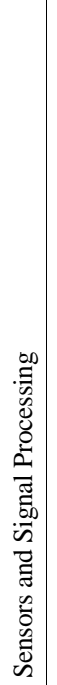 & 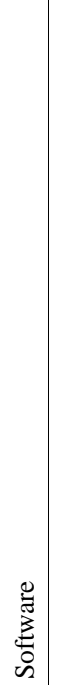 & 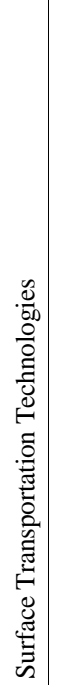 & 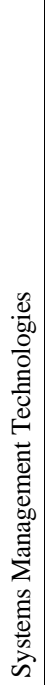 & 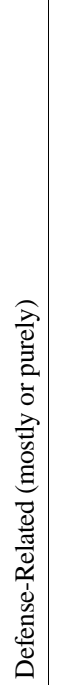 & 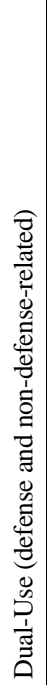 & 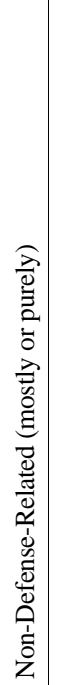 \\
\hline 537 & $\begin{array}{l}\text { Near-Real-Time Surveillance Against } \\
\text { Bioterror Attack }\end{array}$ & 21558 & & & & & $\bullet$ & & & & & & & & & & $\bullet$ & & & & & $\bullet$ & • & & & $\bullet$ & & \\
\hline 539 & $\begin{array}{l}\text { Investigation of Modeling Approaches } \\
\text { for Nuclear Futures }\end{array}$ & 21559 & & & & & $\bullet$ & & & • & & & & & & & & & & & & & & & & & $\bullet$ & \\
\hline 541 & $\begin{array}{l}\text { Understanding and Managing Threats } \\
\text { to the Social Fabric of the United } \\
\text { States }\end{array}$ & 22126 & & & & & - & & & & & & & & & & & & & & & & - & & - & $\bullet$ & & \\
\hline 543 & $\begin{array}{l}\text { Physical and Functional Modeling of a } \\
\text { Bioelectronic Microsystem }\end{array}$ & 22127 & & & & & • & & & & & & $\bullet$ & & & & - & & & & & & $\bullet$ & & & & - & \\
\hline 544 & $\begin{array}{l}\text { Analytic Models for Cooperative } \\
\text { Utilization of Critical Resources }\end{array}$ & 22128 & & & & & - & & & - & & & & & & & & & & & & & $\bullet$ & & - & & & $\bullet$ \\
\hline 546 & Cryopreservation of Biological Tissue & 22129 & & & & & - & & & & & & & & & & & & & & & & & & & & $\bullet$ & \\
\hline 548 & $\begin{array}{l}\text { Investigation of Nanoscience } \\
\text { Technologies }\end{array}$ & 22131 & & & & & & & & & & & & & & & & & & & & - & & & & & - & \\
\hline 549 & Magnetic Polysilicon MEMS Devices & 22132 & & • & & & & & $\bullet$ & & & & & & $\bullet$ & & & $\bullet$ & & & & $\bullet$ & & & & & $\bullet$ & \\
\hline 551 & $\begin{array}{l}\text { Autonomous Microexplosive } \\
\text { Subsurface Tracing System }\end{array}$ & 22136 & & & & & & & & - & & & & & & & & & & & - & - & & & & & & - \\
\hline 553 & $\begin{array}{l}\text { New Metal-Organic Chemical Vapor } \\
\text { Deposition Reactor Designs and } \\
\text { Optimization }\end{array}$ & 22854 & & & & & & & - & & & & & & & $\bullet$ & & & - & & & & & & & & - & \\
\hline 555 & $\begin{array}{l}\text { Composite-Wire Plasma Formation } \\
\text { and Evolution }\end{array}$ & 22995 & & & & & & & & & & & & & & & & & & & & & & & & - & & \\
\hline 557 & $\begin{array}{l}\text { Scattering from Nanostructured } \\
\text { Materials }\end{array}$ & 22996 & & & - & $\bullet$ & - & & & & & & & & & & & & & & & & & & & & - & \\
\hline 559 & $\begin{array}{l}\text { The Liquid-Crystal Physics of } \\
\text { Evaporation-Induced Self-Assembly }\end{array}$ & 22997 & & - & - & - & & & & & & & & & & $\bullet$ & & & & $\bullet$ & & & & & & & - & \\
\hline 560 & $\begin{array}{l}\text { Electrochemical Interrogation of } \\
\text { Cellular Membrane Transport } \\
\text { Processes }\end{array}$ & 22998 & & $\bullet$ & & & & & & & & & & & & & & $\bullet$ & & & & & & & & & & $\bullet$ \\
\hline 562 & $\begin{array}{l}\text { A Micromethanol Steam Reformer for } \\
\text { a Hydrogen-Based Microfuel Cell }\end{array}$ & 24101 & & & & & & & & & & & & & & & & 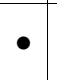 & & & & & & & & & $\bullet$ & \\
\hline
\end{tabular}




\section{Appendix F: Dual-Benefit Areas and Single-Use Categories}

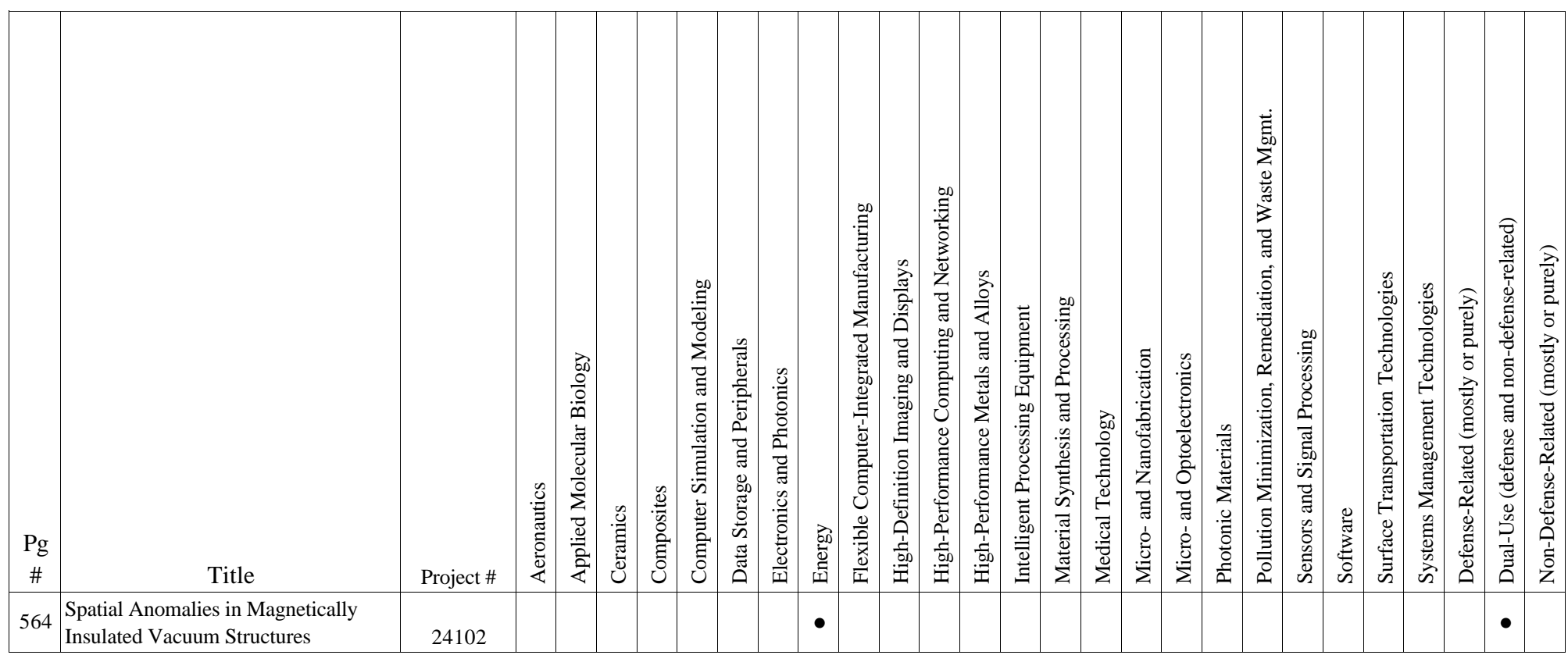




\section{Author and Title Index}

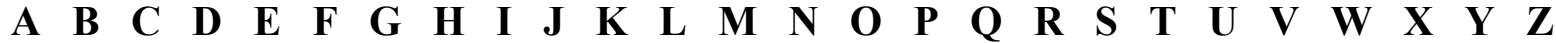

A

Abate, M. L.

Cooperative Utilization of Critical Resources, 544

Abnormal, 251

Accelerate

Decay, 527

Recorder, 325

Access (nanosat), 404

Acid, 166

Ackermann, M. R.

Autonomous Optical Neuroprocessors, 484

Acoustic

Biosensor array, 168

Microoptoelectronic, 134

Plate-wave device, 131

Actuation, 68

Adams, D. P.

Advance Neutron-Tube Design, 418

Advanced Machining for Microfabrication, 223

Fabrication of 3-D Microstructures, 232

Numerically Controlled Micromachines, 241

Thin-Film Deposition Processes, 216

Verify Cleaning and Surface Preparation, 230

Adaptive

3-D sensing, 337

Critic neural networks, 388

Sensor optimization, 484

Adhesion, 37

Adiabatic, 190

Adkins, D. R.

Autonomous MicroChem Laboratory, 409

Flexural Plate-Wave Devices, 131

Microsensors for Autonomous Microrobots, 178

Adolf, D. B.

Finite-Element Polymer Modeling, 209

Structural Simulations Using Models, 188 Agent

Biological warfare, 166

Complexing, 468

Conversational policy, 278

Cooperative information system, 260

Human exposure, 499

Large swarm, 102

Preconcentration, 525

Sensor, 315

Air (propagation), 284

Alam, M. K.

Spectroscopic Detection of Pathogens, 533

Alford, W. J.

External-Cavity Surface-Emitting Lasers, 150

AlGaInN, 171
AlGaN, 142

Algorithm,

Large-eddy simulation, 85

Materials-aging, 48

Structural Biology, 104

Allan, B. A.

Numerical Simulation of Diesel Autoignition, 464

Allen, J. J.

Numerically Controlled Micromachines, 241

Allendorf, S. W.

Transient Gas and Soot in Fires, 185

Allerman, A. A.

External-Cavity Surface-Emitting Lasers, 150

GaAs MOEMS Technology, 175

Integration of VCSELs and Detectors, 117

Open Access (Nanosat), 404

Optoelectronic Arrays and Microelectronics, 162

Photonics Integration Devices, 136

Allman, R. E.

External-Cavity Surface-Emitting Lasers, 150

Alloys, 45

Amatucci, V. A.

Diagnostics for Interactions in Rotating Flowfields, 198

Ames, A. L.

Cloud to CAD, 319

Amorphous, 139

Amplification, 300

Anderson, M. U.

Shock-Wave Response of PZT 95/5, 574

Anderson, R. A.

Laser-Assisted Microgas Metal Arc Welding, 448

Nonvolatile Protonic Memory, 63

Anderson, R. J.

Robotic Manipulation of Hazardous Materials, 311

Anomalies, 564

Anthes, J. P.

Information Collection, 403

$\mathrm{THz}$ for High-Data-Rate Communications, 158

Antolak, A. J.

Radiography for Inspection of Weapons, 397

Arc (welding), 448

Architecture

Micro-total-analytical systems, 160

Surety, 519

Arguello, J. G., Jr.

Design of Improved Powder-Pressing Dies, 436

Armour, D. L.

Assembly of LIGA Using Electric Fields, 240

Open Access (Nanosat), 404

Arnold, D. W.

Autonomous MicroChem Laboratory, 409 


\section{Author and Title Index}

Array

Bioaffinity, 300

Biosensor, 168

DNA, 476

Mobile sensor, 313

Nano, 43

Optical interconnects, 510

Optoelectronic, 162

Sensor data fusion, 253

Ashbaugh, D. M.

Advanced Signal Processing, 371

Ashby, C. I. H.

Stress-Free Amorphous Diamond, 139

Ashcraft, G. W.

Techniques for Real-Time Visualization, 497

Ashley, C. S.

Smart Sand for Landmine Detection, 307

Ashurst, W. T.

Modeling of Multiscale Transient Phenomena, 201

Assemble

LIGA, 240

See also: Self-assemble

Assessment (vulnerability), 286

Assurance (source code), 378

Asynchronous Transfer Mode, 276

ATM

See Asynchronous Transfer Mode

Atmospheric, 380

Atomic, 420

Atomistic

Crack nucleation, 194

Phase-field modeling, 39

Atom-Picoseconds, 91

ATR

See Automatic (Target recognition)

Attack (bioterror), 537

Authenticate

Public-key, 364

SCADA, 390

Autoignition, 464

Automatic

Design, 234

Target recognition (3-D), 488

Target recognition (SAR), 302

Automation, 317

Autonomous

Microrobot, 178

Navigation, 327

Avoidance, 327

Axline, R. M.

Information Collection, 403

Open Access (Nanosat), 404
B

Baca, A. G.

High-Al-Content AlGaInN Devices, 171

Semiconductor Current Filament Lasers, 144

Semiconductor Switch for Firing Systems, 376

Backplane, 264

Bacon, L. D.

HPM Vulnerability Assessment and Tests, 286

Bacterial, 283

Baer, M. R.

Dispersive Measurements of Velocity, 203

Energetic Material Burn at the Mesoscale, 579

Bailey, C. G.

Autonomous MicroChem Laboratory, 409

Baker, A. B.

Modeling Approaches for Nuclear Futures, 539

Ballard, W. P.

Radiography for Inspection of Weapons, 397

Barbour, J. C.

Critical Copper-Corrosion Mechanisms, 41

Monitoring Corrosion in Weapons Environments, 34

Barney, P. S.

Electromagnetic-Bearing Spindles, 214

Barriers

Reactive, 462

Smoke, 521

Bartel, L. C.

Information Collection, 403

Monitoring Deep Targets, 321

Bartelt, N. C.

Self-Assembled Templates, 43

Barton, D. C. Model of Infrastructure Interdependency, 385

Bartram, M. E.

Self-Assembled Templates, 43

Bateman, V. I.

Micro-High-G Acceleration Recorder, 325

Battaile, C. C.

Atmospheric Corrosion of Microelectronics, 380

Microstructure and Mechanics, 70

Monitoring Corrosion in Weapons Environments, 34

Battery

Ceramic, 76

Micro, 180

Rechargeable, 359

Bauer, K. C.

Intrusion Detection for ATM Networks, 276

Beam (proton), 288

Beauchamp, E. K.

Engineered Window Glass, 519

Beaver, C. L.

Information-Sharing Security Module, 482

Low-Power Public-Key Protocols, 271

SCADA for Critical Energy Infrastructures, 258 


\section{Author and Title Index}

Beezhold, W.

Radiation-Induced Prompt Photocurrents, 567

Behavior (emergent), 102

Behrens, R., Jr.

Molecular Markers for Pathogen Detection, 329

Benavides, G. L.

Advanced Machining for Microfabrication, 223

Electromicrofluidic Packaging, 244

Numerically Controlled Micromachines, 241

Bennett, P. C.

Robotic Manipulation of Hazardous Materials, 311

Bentley, A. E.

SCADA for Critical Energy Infrastructures, 258

Beresh, S. J.

Diagnostics for Interactions in Rotating Flowfields, 198

Berg, T. M.

Controlling Information, 268

Bernstein, A. C.

Intense White-Light Pulse Propagation in Air, 284

Berry, N. M.

Controlling Information, 268

Model-Based Fusion of Sensor Array Data, 253

Policies for Multiagent Systems, 278

Bertram, L. A.

Laser-Assisted Microgas Metal Arc Welding, 448

Beta (decay), 527

Beyeler, W. E.

Modeling Approaches for Nuclear Futures, 539

Biefeld, R. M.

Characterization of Quantum Dots, 155

Bieg, L. F.

Numerically Controlled Micromachines, 241

VDC of Manufacturing Tools, 228

Bilayer (lipid), 164

Bioaerosol, 293

Bioaffinity, 300

Bioelectronic

Modeling, 543

Sustainable, 535

Biological

Agents, 499

Cryopreservation, 546

Fluorescence, 315

Macromolecules, 106

Structural, 104

Warfare, 166

Weapon detection, 298

See also: Bioaerosol

See also: Bioaffinity

See also: Bioelectronic

See also: Biosensor

See also: Bioterror

Biosensor

Lipid bilayers, 164

Acoustic-wave, 168

Bioterror, 537

Bitsie, F.

Microdiagnostic MEMS Lab-on-a-Chip, 212
Open Access (Nanosat), 404

Black, K. L.

Information Collection, 403

Blades (turbine), 480

Bliss, D. E.

Autonomous Optical Neuroprocessors, 484

Intense White-Light Pulse Propagation in Air, 284

Boebert, W. E.

PUSH Technology Demonstration, 266

Boettcher, G. E.

Poco Switch Tubes, 443

Boggs, P. T.

Nonlinear Optimization from PDE Models, 108

Bolton, W. R., Jr. Information Collection, 403

Boney, C. M. Improved Materials-Aging Diagnostics, 48

Boslough, M. B. E.

Distributed Mobile Sensor Arrays, 313

Global Climate Model for Paleoclimates, 89

Boundary

Interaction, 577

Turbulent, 201

Bow, W. J.

ATR/Exploitation SAR Imaging, 302

Automatic Target Recognition in 3-D, 488

Boyack, K. W.

Distributed Mobile Sensor Arrays, 313

Visual Explanation and Insight, 99

Boye, L. A.

Information Collection, 403

Land Surface-Temperature Maps, 504

Boyle, T. J.

All-Ceramic Thin-Film Battery, 76

Brainard, J. P.

Advance Neutron-Tube Design, 418

Braithwaite, J. W.

Atmospheric Corrosion of Microelectronics, 380

Critical Copper-Corrosion Mechanisms, 41

Branch, D. W.

Acoustic-Wave Biosensor Arrays, 168

Brandt, L. D.

Facility Response to Abnormal Events, 251

Bray, B. K.

ATR/Exploitation SAR Imaging, 302

Braze (alloys), 45

Breiland, W. G.

Critical Copper-Corrosion Mechanisms, 41

Brenkosh, J. P.

Varying QoS for Fixed and Mobile Networks, 249

Briggs, R. D. Integration of VCSELs and Detectors, 117

Brinker, C. J.

Evaporation-Induced Self-Assembly, 559

Information Collection, 403

Microfabricated Optical Devices, 74

Molecular Integrated Microsystems, 412

Self-Assembled Templates, 43 


\section{Author and Title Index}

Smart Sand for Landmine Detection, 307

Smart Self-Assembled Photochromic Films, 32

Supramolecular Self-Assembly, 425

Sustainable Bioelectronic Microsystems, 535

Brockmann, J. E.

Facility Response to Abnormal Events, 251

Brooks, J. A.

Exploiting LENS Technology, 57

Solidification/Microstructural Simulations, 571

Brozik, S. M.

Acoustic-Wave Biosensor Arrays, 168

Biological Weapon Detector, 300

Biosensors Based on Electrical Impedance, 164

Microfabricated Optical Devices, 74

Molecular Integrated Microsystems, 412

Sustainable Bioelectronic Microsystems, 535

Brunett, B. A.

Radiography for Inspection of Weapons, 397

Buchheit, T. E.

Microstructure and Mechanics, 70

Solder for High-Fidelity Model Development, 581

Bulk

Micromachining, 147

Power systems, 383

Buller, D. L.

Radiation-Induced Prompt Photocurrents, 567

Bunker, B. C.

Molecular Integrated Microsystems, 412

Bunker, B. C.

Hydrophobic-Hydrophilic Surfaces, 72

Burn (energetic-materials), 579

Burns, A. R.

Investigation of Nanoscience Technologies, 548

Microfabricated Optical Devices, 74

Molecular Integrated Microsystems, 412

Smart Self-Assembled Photochromic Films, 32

Bursts, 114

Buss, R. J.

Nanostructured Silicon Surfaces, 354

BW

see Biological Warfare

Byrne, R. H.

Microsensors for Autonomous Microrobots, 178

\section{C}

$\mathrm{CAD}$

See Computer aided design

Calton, T. L.

Distributed Reconfigurable Microrobotics, 331

Cameron, S. M.

Intense White-Light Pulse Propagation in Air, 284

Representation of Human Decision Process, 531

Campbell, D. V.

Radiation-Hard Sensors, 122

Campbell, P. L.

Source Code Assurance Tool, 378

Capillary, 433
Carlson, D. C.

ATR/Exploitation SAR Imaging, 302

Carlson, J. J.

Adaptive 3-D Sensing, 337

Intelligent Targeting for Remote Weapons, 492

Unobtrusive Human-Computer Interactions, 81

WNN Decision Making for Data Mining, 247

Carlson, R. E.

SCADA for Critical Energy Infrastructures, 258

SCADA Systems in the Electric Industry, 390

Carpick, R. W.

Smart Self-Assembled Photochromic Films, 32

Carr, R. D.

Combinatorial Optimization for Scheduling, 93 Computational Structural Biology, 104

Casalnuovo, S. A

AlGaN Materials Engineering, 142

Microsensors for Autonomous Microrobots, 178

Molecular Integrated Microsystems, 412

Post-Processed Integrated Microsystems, 119

Cashen, J. J.

Radiography for Inspection of Weapons, 397

Castro, J. P.

Tool for Modeling of Coupled Systems, 112

Cavity (external), 150

Cederberg, J. G.

Characterization of Quantum Dots, 155

Celina, M. C.

Improved Materials-Aging Diagnostics, 48

Cell

Membrane transport, 560

Microfuel reformer, 494

Steam reformer, 562

Centimeter-Years, 91

Ceramic

Battery, 76

Interfacial, 441

Cernosek, R. W.

Acoustic-Wave Biosensor Arrays, 168

Sandia Microsensors to the Internet, 486

Chambers, R. S.

Finite-Element Polymer Modeling, 209

Structural Simulations Using Models, 188

Charge

Compression, 357

See also: Rechargeable

Charred, 508

Chavez, J. C.

Open Access (Nanosat), 404

Chavez, L. M

Information-Sharing Security Module, 482

Chemical

Amplification, 300

Preconcentration, 525

Sensors, 131

Vapor, 553

See also: Electrochemical 


\section{Author and Title Index}

Chemiresistors, 416

Chen, H. Y.

Optical Backplane/Interconnect, 264

Chen, J. H.

Control Strategies for HCCI Engines, 357

Numerical Simulation of Diesel Autoignition, 464

Chhabildas, L. C.

Dispersive Measurements of Velocity, 203

Chinn, D. A.

Chip

Actuation of Microvalves and Micropumps, 68

Damaged, 516

Lab-on-a-chip, 212

Life on a chip, 535

Choquette, K. D.

Characterization of Quantum Dots, 155

Integration of VCSELs and Detectors, 117

Chow, W. W.

Characterization of Quantum Dots, 155

External-Cavity Surface-Emitting Lasers, 150

Photonics Integration Devices, 136

Semiconductor Current Filament Lasers, 144

Christenson, T. R.

Bulk Micromachining for MEMS, 147

MEMS Microelectric Generator, 352

Microfabrication of Electromagnetic Devices, 173

Post-Processed Integrated Microsystems, 119

Circuit

Microwave, 126

RF, 444

Simulation, 110

Claassen, J. P.

Monitoring Deep Targets, 321

Clark, D. F.

Neutron Generators for Nuclear Weapons, 218

Clay, R. L.

Numerical Simulation of Diesel Autoignition, 464

Cleaning (surface), 230

Clem, P. G.

Microfabrication of Electromagnetic Devices, 173

Climate, 89

Cloud, 319

Cluster

Atomic, 420

Computer, 474

Coatings, 455

Cochran, J. M.

Approximate Public-Key Authentication, 364

Network Intrusion Detection, 388

Code, 378

Cognitive (image processing), 484

Cole, E. I., Jr.

Damaged Chip, 516

Collins, M. J.

Network Intrusion Detection, 388
Combinatorial

Corrosion, 41

Scheduling, 93

Communication

THz, 158

Optical, 264

Complex

Agent, 468

Distributed systems, 401

Geometry, 85

Imaging, 302

Composite

Fiber, 480

Plasma, 555

See also: Nanocomposite

Compression (charge), 357

Computation

Atomistic, 39

Diagnostic, 34

Fracture, 206

Polymer, 422

Public-key, 271

Strucutural biology, 104

Weapon detection, 298

Computer

Aided design, 319

Clusters, 474

Micromachines, 241

Unstructured grid, 96

VVMD, 81

Concentrator

Bioaerosol, 293

See also: Preconcentration

Conrad, S. H.

Modeling Approaches for Nuclear Futures, 539

Continuum (modeling), 194

Control

HCCI, 357

Information, 268

Materials growth, 43

MEMS, 37

Microgas, 350

Micromachines, 241

Process, 66

VDC, 228

Copper, J. A.

Hybrid Processing in Surety Analysis, 362

Cooperative

Automation, 317

Information systems, 260

Resources, 544

Copper, 41

Coprocessor, 466 


\section{Author and Title Index}

Corrosion

Microelectronic, 380

Microlab, 41

Monitoring, 34

Countermeasure, 284

Couple

Circuit, 110

Flow, 85

Modeling, 112

Crack, 194

Crawford, M. H.

External-Cavity Surface-Emitting Lasers, 150

High-Al-Content AlGaInN Devices, 171

Miniature UV Biological Agent Sensor, 315

Smart Self-Assembled Photochromic Films, 32

Critic (neural network), 388

Critical

Corrosion, 41

Infrastructure, 258

Resources, 544

Crocker, R. W

Information Collection, 403

Microfuel Reformer, 494

Miniature Bioaerosol Concentrator, 293

Cryopreservation, 546

Crystal

Diamond, 479

Liquid, 559

Silicon, 124

Cummings, E. B.

Applied Microfluidic Physics, 196

Self-Assembled Tamper-Detection Seals, 392

Current

Laser, 144

See also: Photocurrent

Curro, J. G.

Dynamical Properties of Polymers, 422

Hydrophobic-Hydrophilic Surfaces, 72

Curry, R. E.

PUSH Technology Demonstration, 266

Cygan, R. T.

All-Ceramic Thin-Film Battery, 76

\section{D}

Daily, M. R.

Autonomous MicroChem Laboratory, 409

Information Collection, 403

Damaged (chip), 516

Dandini, V. J.

Identifying Dangerous Human Actions, 369

Daniels, J. W.

Hadamard Transform Spectral Imager, 323

Data

Analysis, 66

Array, 253

Communication, 158

Map, 504
Mining, 280

Target, 295

Visualization, 497

WNN, 247

Davalos, R. V.

Cellular Membrane Transport Processes, 560

Davidson, G. S.

DNA Microarray, 476

Evolvable Hardware, 192

Davis, J. P.

Dispersive Measurements of Velocity, 203

Dawson, D. B.

Mechanisms of Adiabatic Shear Failure, 190

De Boer, M. P.

Microdiagnostic MEMS Lab-on-a-Chip, 212

Novel Coatings for MEM Devices, 455

Stress-Free Amorphous Diamond, 139

Dean, L. B.

One-Hundred-Gigabit/Second Networks, 273

Optical Backplane/Interconnect, 264

Self-Configurable Network Protocol, 262

Dec, J. E.

Control Strategies HCCI Engines, 357

Decay, 527

Decision

Human, 531

Response, 251

WNN, 247

Decontamination, 283

Deformation, 581

Deland, S. M.

Fuzzy Data Mining, 280

Denison, G. J.

Electromagnetic Imaging System, 343

HPM Vulnerability Assessment and Tests, 286

Dentinger, P. M.

Self-Assembled Tamper-Detection Seals, 392

Deoxyribonucleic acid, 476

Deployable (sensor), 304

Deposition

Monitor, 216

Vapor, 553

Derzon, M. S.

Kinetic-Energy Lightning Generator, 529

National Security Issues/Limited Resources, 534

Reduction of Legacy Wastes, 527

RTL Concept for Fusion Energy, 458

Design

Automatic, 234

Deposition reactor, 553

Dies, 436

Ionophores, 339

Neutron-tube, 418

Desjardin, P. E.

Advanced Large-Eddy Simulation Algorithms, 85

Detect

Bioaffinity, 300

Biological weapon, 298 


\section{Author and Title Index}

Change, 512

Intrusion, 276

Landmine, 307

Microsystem, 117

Network intrusion, 388

Pathogens (spectroscopic), 533

Pathogen (markers), 329

Solvent, 416

Tamper, 392

Target, 514

Thermal flaw, 371

Detonation, 579

Device

AlGaInN, 171

Electromagnetic, 173

MEM (novel coating), 455

MEM (polysilicon), 549

Microoptoelectronic, 134

Optical, 74

Photonic, 136

Plate-wave, 131

Quantum dot, 155

Simulation, 110

Dexterous, 311

Diagnosis

Computational, 34

Fire, 185

Flowfield, 198

Human, 499

Materials-aging, 48

Micro, 212

Simulation, 571

Diamond

Microsensor, 139

Shock response, 479

Diegert, C. F.

Unobtrusive Human-Computer Interactions, 81

Dies (powder-pressing), 436

Diesel, 464

Dike, J. J.

Mechanisms of Adiabatic Shear Failure, 190

Dillinger, J. D.

Varying QoS for Fixed and Mobile Networks, 249

Directed-Energy

Intense, 290

Proton, 288

Discrete (event), 466

Dislocation (grain-boundary), 577

Dispersive

Smart sand, 307

Velocity, 203

Displacement, 228

Distributed

Array, 313

Information, 255

Microrobotic, 331

System, 401
DNA

See Deoxyribonucleic acid

Dodd, P. E.

Radiation-Induced Prompt Photocurrents, 567

Dohner, J. L.

Flexural Plate-Wave Devices, 131

Douglas, M. R. Advanced Radiation Sources, 430

Doyle, B. L.

Radiation-Induced Prompt Photocurrents, 567

Draelos, T. J.

Approximate Public-Key Authentication, 364

Low-Power Public-Key Protocols, 271

Network Intrusion Detection, 388

Draper, B. L.

Nonvolatile Protonic Memory, 63

Drewien, C. A. Improved Materials-Aging Diagnostics, 48

Driver (photonic), 52

Drummond, T. J. Damaged Chip, 516

Duckett, E. B. Microsensors for Autonomous Microrobots, 178

Dugger, $\mathrm{M}$. T.

Self-Healing Molecular Assemblies, 37

Dulleck, G. R., Jr. Autonomous MicroChem Laboratory, 409

Dunbar, T. D.

Nonvolatile Protonic Memory, 63

Dunlap, S. K.

Dynamic Range Imaging for Terrain Mapping, 327

Radiation-Hard Sensors, 122

Dupree, S. A. Information Collection, 403

Duran, F. A. Infrastructure Risk and Reliability, 366

Dyck, C. W.

Micro-High-G Acceleration Recorder, 325

Monolithic Micromachined Variable Tuners, 126

Dykhuizen, R. C.

Cryopreservation of Biological Tissue, 546

Output-Based Process Control, 66

Dynamic

Evironment, 334

Imaging, 327

Network protocol, 262

Platform, 313

Polymers, 422

Solder, 45

$\mathbf{E}$

Edwards, D. M.

Facility Response to Abnormal Events, 251

Efficiency

MEMS, 182

Photovoltaic, 354 


\section{Author and Title Index}

Eidson, E. D.

Model of Infrastructure Interdependency, 385

Eisler, G. R.

Autonomous Navigation of Mobile Robots, 334

Elastohydrodynamics, 433

Elbring, G. J.

Information Collection, 403

Monitoring Deep Targets, 321

Underground Facilities in an Urban Area, 309

Eldred, M. S.

Nonlinear Optimization from PDE Models, 108

Electric

Field, 240

Impedance, 164

Power, 390

Electrochemical, 560

Electrochemomechanical, 68

Electrode (planar), 164

Electroformed, 238

Electrokinetic, 446

Electromagnetic

Imaging, 343

Microfabrication, 173

Spindles, 214

Electromicrofluidic, 244

Electron (gases), 423

Electronic

AlGaInN, 171

See also: Bioelectronic

See also: Optoelectronic

See also: Microelectromechanical

See also: Microelectronic

Emerson, J. A.

Fabrication of 3-D Microstructures, 232

Verify Cleaning and Surface Preparation, 230

Energetic

Initation, 60

Mesoscale, 579

Energy

Fusion, 458

Infrastructure, 258

Lightning, 529

Management, 348

Process, 350

Surface, 439

System, 460

Theory, 290

Weapon, 288

Engi, D.

Threats to the Social Fabric of the US, 541

Engine, 357

Ensz, M. T.

VDC of Manufacturing Tools, 228

Environment

Reconnaissance, 343

Robot, 334
Unstructured, 311

Urban, 309

Weapon, 34

Eras, K.

Microfabrication of Electromagnetic Devices, 173

Erickson, J. C.

Radiography for Inspection of Weapons, 397

Erickson, K. L.

Atmospheric Corrosion of Microelectronics, 380

Energetic Material Burn at the Mesoscale, 579

Error (correction), 214

Erven, R. J.

Diagnostics for Interactions in Rotating Flowfields, 198

Eskridge, S. E.

Distributed Reconfigurable Microrobotics, 331

Espinoza, J.

Self-Configurable Network Protocol, 262

Source Code Assurance Tool, 378

Threats to the Social Fabric of the US, 541

Evaporation, 559

Even, W. R., Jr.

Self-Assembled Tamper-Detection Seals, 392

Event

Abnormal, 251

Simulation, 466

Evolution (plasma), 555

Evolvable (hardware), 192

Ewsuk, K. G.

Design of Improved Powder-Pressing Dies, 436

Excitonic (gases), 423

Exploit

ATR, 302

LENS, 57

Explosive

Micro, 551

Preconcentration, 525

Exposure, 499

Extraction

Information, 428

Metal ions, 339

$\mathbf{F}$

Fabrication

Microstructures, 323

Nanocomposite, 425

Template, 43

See also: Microfabricate

Factorization, 83

Failure

Analysis, 209

Shear, 190

Fang, H. E.

Solder for High-Fidelity Model Development, 581

Farnsworth, A. V., Jr.

Microscale Shock-Wave Physics, 52 


\section{Author and Title Index}

Farrell, D. E.

PUSH Technology Demonstration, 266

Fatty acid, 166

Faucett, D. L.

Underground Facilities in an Urban Area, 309

Fault (tolerant), 474

Feddema, J. T.

Assembly of LIGA Using Electric Fields, 240

Distributed Reconfigurable Microrobotics, 331

Dynamic Range Imaging for Terrain Mapping, 327

Scripting for Video Inspection, 220

Feibelman, P. J.

Self-Assembled Templates, 43

Transition-Metal Surface Structure, 439

Ferko, S. M.

Miniature Bioaerosol Concentrator, 293

Fiber (monitoring), 480

Fidelity

Solder, 581

See also: Multifidelity

Filament, 144

Filamentation, 284

Film

Ceramic, 76

Deposition, 216

Photochromic, 32

Finite-Element

Dies, 436

Polymer, 209

Fintschenko, Y.

Micro-Total-Analytical Systems, 160

Fire, 185

Firing (system), 376

Fischer, A. J.

Characterization of Quantum Dots, 155

Fischer, G. J.

Information Collection, 403

Fixed (network), 249

Fixture (design), 234

Flash (memory), 512

Flaw (detection), 371

Fleming, J. G.

AlGaN Materials Engineering, 142

High-Efficiency Optical MEMS, 182

Silicon 3-D Photonic Crystal, 124

$\mathrm{THz}$ for High-Data-Rate Communications, 158

Flexural plate-wave device, 131

Flores, R. S.

Radiation-Induced Prompt Photocurrents, 567

Floro, J. A.

Thin-Film Deposition Processes, 216

Flounders, A. W.

Biological Weapon Detector, 300

Cellular Membrane Transport Processes, 560

Flow

Coupled, 85

Information, 268
Flowfield, 198

Fluid

flowfield, 198

See also: Electromicrofluidic

See also: Microfluidic

Fluorescence, 315

Flying (penetrator), 523

Focal (plane), 510

Foiles, S. M.

Crack Nucleation and Growth, 194

Follstaedt, D. M.

AlGaN Materials Engineering, 142

Forester, J. A. Identifying Dangerous Human Actions, 369

Formation

Plasma, 555

Reactive barriers, 462

See also: Deformation

Forsythe, J. C.

Representation of Human Decision Process, 531

Fossum, A. F.

Design of Improved Powder-Pressing Dies, 436

Foster, A. D. Miniaturized Flying Penetrator, 523

Foulk, J. W., III Method for Predicting Generalized Fracture, 206

Fracture, 206

Framework (decision), 251

Friction (MEMS), 37

Friedmann, T. A.

Stress-Free Amorphous Diamond, 139

Frink, L. J. D. Function of Biological Macromolecules, 106

Frye-Mason, G. C.

Autonomous MicroChem Laboratory, 409

Controller for Energy-Intensive Processes, 350

Microcombustor for Sensor Management, 348

Miniature Sensors for BW Agents, 166

Fuerschbach, P. W.

Laser-Assisted Microgas Metal Arc Welding, 448

Fusion

Data, 253

Energy, 458

Information, 268

Sensor, 502

Fuzzy (data mining), 280

G

GaAs, 175

Galambos, P. C.

Electromicrofluidic Packaging, 244

Magnetic Polysilicon MEMS Devices, 549

Micro-Total-Analytical Systems, 160

Gallup, D. R.

SCADA Systems in the Electric Industry, 390

Gamma-Ray, 114 


\section{Author and Title Index}

Garbin, H. D

Monitoring Deep Targets, 321

Underground Facilities in an Urban Area, 309

Garcia, E. J.

GaAs MOEMS Technology, 175

Gardner, D. R.

Tool for Modeling of Coupled Systems, 112

Gardner, J. T.

Information Collection, 403

PUSH Technology Demonstration, 266

Gardner, T. J.

Microcombustor for Sensor Management, 348

Garrett, S. E.

IMEMS Packaging and Interconnection, 236 Gas

Electron, 423

Fire, 185

See also: Microgas

Geib, K. M.

Characterization of Quantum Dots, 155

Integration of VCSELs and Detectors, 117

Optoelectronic Arrays and Microelectronics, 162

Gelbard, F.

Facility Response to Abnormal Events, 251

Generator

Lightning, 529

Microelectric, 352

Neutron, 218

Gentry, S. M.

Military Target Detection, 514

Geometry (complex), 85

Geophysical (network), 321

Geosphere, 345

Giron, J. W.

Techniques for Real-Time Visualization, 497

Glass, 519

Glass, S. J.

Engineered Window Glass, 519

Global (climate), 89

Goeke, R. S.

Advance Neutron-Tube Design, 418

Thin-Film Deposition Processes, 216

Goldsby, M. E.

Distributed Information Systems, 255

Fault-Tolerant Computing Services, 474

Goldsmith, S. Y.

Agent-Based Mediation, 260

Engineering Complex Distributed Systems, 401

Gonzales, R. A.

Low-Power Public-Key Protocols, 271

Goods, S. H.

Actuation of Microvalves and Micropumps, 68

Mechanisms of Adiabatic Shear Failure, 190

Polymer-Metal Interface Properties, 472
Gordon, S. P.

Facility Response to Abnormal Events, 251

Gottlieb, E.

Automatic Design of Practical Fixtures, 234

Heterogeneous Simulation, 79

Granfield, R. J.

SCADA for Critical Energy Infrastructures, 258

Granular, 307

Gray, P. C.

Gamma-Ray Bursts, 114

Intense Directed-Energy Theory Initiative, 290

Green, K. K.

Automatic Target Recognition in 3-D, 488

Greenholt, C. J. Pyrotechnic Smoke Materials for Barriers, 521

Grest, G. S.

Wetting and Spreading Dynamics of Alloys, 45

Grid (unstructured), 96

Griffiths, S. K. Applied Microfluidic Physics, 196

Gritzo, L. A

Transient Gas and Soot in Fires, 185

Groom, K. N.

Controller for Energy-Intensive Processes, 350

Gross, K. J.

Dislocation-Grain-Boundary Interaction, 577

Nanocomposites for Rechargeable Batteries, 359

Gross, R. J.

Automatic Design of Practical Fixtures, 234

Grotbeck, C. L.

Hadamard Transform Spectral Imager, 323

Growth

Crack, 194

Materials, 43

Quantum Dots, 155

Grubelich, M. C.

Microexplosive Subsurface Tracing System, 551

Pyrotechnic Smoke Materials for Barriers, 521

Guess, T. R.

Finite-Element Polymer Modeling, 209

Guide

Optical filamentation, 284

Response, 251

Guilinger, T. R.

AlGaN Materials Engineering, 142

Global Climate Model for Paleoclimates, 89

Gupta, V. P.

National Security Issues/Limited Resources, 534

Gutierrez, D. Electromicrofluidic Packaging, 244

Gutierrez, W. T. Miniaturized Flying Penetrator, 523 


\section{Author and Title Index}

H

Haaland, D. M.

Hadamard Transform Spectral Imager, 323

Improved Materials-Aging Diagnostics, 48

Information Extraction from Satellites, 428

Output-Based Process Control, 66

Spectroscopic Detection of Pathogens, 533

Hadamard transform spectral imager, 323

Hafich, M. J.

Characterization of Quantum Dots, 155

Hall, A. C.

Solidification/Microstructural Simulations, 571

Hamilton, J. C.

Atom-Picoseconds to Centimeter-Years, 91

Crack Nucleation and Growth, 194

Hammerand, D. C.

Structural Simulations Using Models, 188

Han, J.

AlGaN Materials Engineering, 142

High-Al-Content AlGaInN Devices, 171

Hance, B. G.

Smart Sand for Landmine Detection, 307

Hansche, B. D.

Advanced Signal Processing, 371

Hardening, 420

Hardware, 192

Harff, N. E

Quantum Tunneling Transistors, 129

Hargis, P. J., Jr.

Miniature UV Biological Agent Sensor, 315

Harmony, D. W.

ATR/Exploitation SAR Imaging, 302

Harris, D. L.

Modeling Approaches for Nuclear Futures, 539

Modeling the Jini Infrastructure Protocol, 490

Self-Configurable Network Protocol, 262

Harrison, K. L.

Change Detection in Flash Memory, 512

Hart, W. E.

Advanced Production Planning Models, 225

Combinatorial Optimization for Scheduling, 93

Nonlinear Optimization from PDE Models, 108

Production Surety Analysis, 374

Hasselbrink, E. F.

Micro-Total-Analytical Systems, 160

Molecular Integrated Microsystems, 412

Stronglink Prime Mover, 446

Hazardous materials, 311

HCCI

see Homogeneous charge-compression ignition

Heath, C. E.

Laser-Assisted Microgas Metal Arc Welding, 448

Heller, E. J.

Autonomous MicroChem Laboratory, 409

Microsensors for Autonomous Microrobots, 178

Henderson, C. C.

Photoresist Technology, 153
Henderson, C. L.

Virtual Reliability Realization System, 395

Hendrickson, B. A.

Visual Explanation and Insight, 99

Henfling, J. F.

Diagnostics for Interactions in Rotating Flowfields, 198

Hennigan, G. L.

Tool for Modeling of Coupled Systems, 112

Hensinger, D. M.

Distributed Reconfigurable Microrobotics, 331

Henson, T. D.

Thin-Skin Deployable Mirrors for Sensing, 304

Herdman, C. M.

Microfuel Reformer, 494

Heroux, M. A.

Incomplete Factorization Preconditioners, 83

Herrera, G. V.

Nonvolatile Protonic Memory, 63

Heterogeneous

Clusters, 474

Integration, 162

Materials, 203

Simulation, 79

Hewson, J. C.

Control Strategies HCCI Engines, 357

High-power microwave, 286

Hillaire, R. G.

Controlling Information, 268

Model-Based Fusion of Sensor Array Data, 253

Hilton, N. R.

Neutron Generators for Nuclear Weapons, 218

Hinckley, M. K.

Optical Interconnections to Focal Plane Arrays, 510

Radiation-Hard Sensors, 122

Hjalmarson, H. P.

Function of Biological Macromolecules, 106

Semiconductor Current Filament Lasers, 144

Semiconductor Switch for Firing Systems, 376

Hoekstra, R. J.

Parallel Methods for Simulations, 110

Hoffman, E. L.

Modeling of Medical Surveillance Network, 506

Hoffman, M.

Land Surface-Temperature Maps, 504

Hohlfelder, R. J.

Stress-Free Amorphous Diamond, 139

Holm, E. A.

Microstructure and Mechanics, 70

Holswade, S. C.

Semiconductor Switch for Firing Systems, 376

Holzrichter, M. W.

Underground Facilities in an Urban Area, 309

Homogeneous

Charge-compression ignition, 357

Microrobot, 331 


\section{Author and Title Index}

Hopkins, P. L.

Advanced Geosphere Transport Simulation, 345

Horstemeyer, M. F.

Atom-Picoseconds to Centimeter-Years, 91

Hosking, F. M.

Phase-Field Modeling, 39

Wetting and Spreading Dynamics of Alloys, 45

Hough, P. D.

Fault-Tolerant Computing Services, 474

Houston, J. E.

Crack Nucleation and Growth, 194

Dislocation-Grain-Boundary Interaction, 577

Howard, M. S.

Miniaturized Flying Penetrator, 523

Hoyt, J. J.

Phase-Field Modeling, 39

HPM

See High-power microwave

Hubbard, G. L.

Transient Gas and Soot in Fires, 185

Huber, D. L.

Hydrophobic-Hydrophilic Surfaces, 72

Hughes, R. C.

Biological Weapon Detector, 300

Biosensors Based on Electrical Impedance, 164

Chemiresistors for Solvent Spill Detection, 416

Microsensors for Autonomous Microrobots, 178

Sustainable Bioelectronic Microsystems, 535

Human

Biological agents, 499

Cooperative automation, 317

Dangerous actions, 369

Decision, 531

VVMD sensing, 81

Hurd, A. J.

Scattering from Nanostructured Materials, 557

Hutchinson, R. L. Intrusion Detection for ATM Networks, 276

Hutchinson, S. A.

Parallel Methods for Simulations, 110

Hwang, R. Q.

Reactivity of Metal-Oxide Surfaces, 54

Wetting and Spreading Dynamics of Alloys, 45

Hybrid

Preconditioners, 83

Surety, 362

Hydrogen

Microfuel cell, 494

Steam reformer, 562

Hydrophilic, 72

Hydrophobic, 72

Hyperspectral

Imaging, 48

Information, 428
I

Identify

Action, 369

Target, 470

Ignition

Engine, 357

See also: Autoignition

Image

Electromagnetic, 343

Hyperspectral (2-D), 48

IR, 323

Map, 504

Navigation, 327

Process, 484

SAR, 302

Satellite, 428

IMEMS

See Integrated microelectromechanical systems

Immunoassay, 307

Impedance, 164

Induce

Marker, 329

Photocurrent, 567

Self-assembly, 559

Information

Collection, 403

Control, 268

Cooperative, 260

Distributed, 255

Extraction, 428

Hiding, 364

Sharing, 482

Surety, 362

Infrared

Hadamard, 323

Polarization, 470

Sensor, 122

Infrastructure

Energy, 258

Evaluate, 366

Interdependency, 385

Protocol, 490

Ingersoll, D.

All-Ceramic Thin-Film Battery, 76

Nanocomposites for Rechargeable Batteries, 359

Open Access (Nanosat), 404

Initiation

Material, 60

Theory, 290

Insight (visual), 99

Inspection

Video, 220

Weapon, 397 


\section{Author and Title Index}

Insulate, 564

Integrate

Internet, 486

MEMS, 182

Microbattery, 180

Microrobot, 178

Microsystem (post-processed), 119

Microsystem (VCSEL), 117

Microstructure, 139

MIMS, 412

Multifunction system, 142

Optical device, 74

Optoelectronics, 162

Photonics, 136

Process control, 66

Sensor, 131

Weapon detection, 298

Integrated microelectromechanical systems, 236

Intelligent

Agent, 102

Data analysis, 66

Targeting, 492

Interface

Ceramic, 441

Polymer-metal, 472

Traction-separation, 569

Internet (microsensor), 486

Interrogation, 560

Intrusion

Detection (ATM), 276

Detection (neural), 388

Ionophores, 339

Ions, 339

IR

See Infrared

Istrail, $\mathrm{S}$.

Novel Biological Weapon Detection System, 298 Ivey, M. D.

Controller for Energy-Intensive Processes, 350

\section{$\mathbf{J}$}

Jacobus, M. J.

Change Detection in Flash Memory, 512

James, R. B.

Radiography for Inspection of Weapons, 397

Janek, R. P.

Biological Weapon Detector, 300

Microreplication from Electroformed Molds, 238

Miniature Bioaerosol Concentrator, 293

Janssen, C. L.

Photoresist Technology, 153

JAVA, 112

Jenkin, C. L.

Fuzzy Data Mining, 280

Jennison, D. R.

Reactivity of Metal-Oxide Surfaces, 54

Wetting and Spreading Dynamics of Alloys, 45
Jensen, K. M.

Distributed Mobile Sensor Arrays, 313

Intelligent Targeting for Remote Weapons, 492

WNN Decision Making for Data Mining, 247

Jini, 490

Johnsen, H. A

Transient Gas and Soot in Fires, 185

Johnson, D. J.

Open Access (Nanosat), 404

Johnson, D. K.

Visual Explanation and Insight, 99

Johnson, M. M.

Modeling of Medical Surveillance Network, 506

Johnson, W. A.

Assembly of LIGA Using Electric Fields, 240

Johnston, A. M.

Approximate Public-Key Authentication, 364

Computational Structural Biology, 104

SCADA Systems in the Electric Industry, 390

Joining (simulation), 571

Jojola, A. A.

MEMS Microelectric Generator, 352

Jokiel, B., Jr.

Numerically Controlled Micromachines, 241

VDC of Manufacturing Tools, 228

Jones, D. A

Advanced Production Planning Models, 225

Combinatorial Optimization for Scheduling, 93

Production Surety Analysis, 374

Jones, E. D.

Acoustically Driven Microoptoelectronics, 134

Broadening Mechanism in Gases, 423

Characterization of Quantum Dots, 155

Jones, J. S.

Evolvable Hardware, 192

Jungst, R. G.

MEMS Microelectric Generator, 352

K

Karas, T. H.

Surveillance Against Bioterror Attack, 537

Kataoka, D. E. Modeling of Medical Surveillance Network, 506

Kay, R. R. Radiation-Hard Sensors, 122

Kaye, R. J. Kinetic-Energy Lightning Generator, 529

Keenan, M. R. Military Target Detection, 514

Keese, D. L. Information Collection, 403

Keiter, E. R.

Parallel Methods for Simulations, 110

Kellogg, G. L. Wetting and Spreading Dynamics of Alloys, 45

Kelly, M. J. AlGaN Materials Engineering, 142 


\section{Author and Title Index}

Kemme, S. A.

Microfabricated Optical Devices, 74

Molecular Integrated Microsystems, 412

Nanostructured Silicon Surfaces, 354

Optoelectronic Arrays and Microelectronics, 162

Kennedy, C. A.

Control Strategies for HCCI Engines, 357

Kennicott, L. A.

Fuzzy Data Mining, 280

Kent, M. S.

Hydrophobic-Hydrophilic Surfaces, 72

Interfacial Traction-Separation Models, 569

Kerstein, A. R.

Advanced Large-Eddy Simulation Algorithms, 85

Control Strategies for HCCI Engines, 357

Modeling of Multiscale Transient Phenomena, 201

Key

Authentication, 364

Protocol, 271

Key, S. W.

Structural Simulations Using Models, 188

Kim, T. J.

Open Access (Nanosat), 404

Kinetic

Lightning, 529

See also: Electrokinetic

Kjeldgaard, E. A.

Combinatorial Optimization for Scheduling, 93

Klarkowski, J. R. Open Access (Nanosat), 404

Klassen, S. E.

New Complexing Agent for Co(II) Analysis, 468

Klein, P. A.

Crack Nucleation and Growth, 194

Method for Predicting Generalized Fracture, 206

Klem, J. F.

Characterization of Quantum Dots, 155

Knorovsky, G. A.

Solidification/Microstructural Simulations, 571

Knudson, M. D.

Dispersive Measurements of Velocity, 203

Shock Response of Diamond Crystals, 479

Knudson, R. T.

Ultraminiaturization of RF Circuitry, 444

Koch, M. W.

Automatic Target Recognition in 3-D, 488

Multisensor Fusion for Characterizing UGFs, 502

Recognizing Partially Obscured Targets, 295

Surveillance Against Bioterror Attack, 537

Koegler, W. S.

Numerical Simulation of Diesel Autoignition, 464

Koehler, F. W. Improved Materials-Aging Diagnostics, 48

Kohler, S. M.

Miniaturized Flying Penetrator, 523

Koller, M. H.

Visual Explanation and Insight, 99
Korellis, J. S.

Actuation of Microvalves and Micropumps, 68

Kottenstette, R. J.

Autonomous MicroChem Laboratory, 409

Microcombustor for Sensor Management, 348

Preconcentration of Volatile Chemical, 525

Kotula, P. G.

Solidification/Microstructural Simulations, 571

Kozlowski, D. M.

Robotic Manipulation of Hazardous Materials, 311

Kramer, D. K.

Damaged Chip, 516

Kravitz, S. H.

Autonomous MicroChem Laboratory, 409

Biological Weapon Detector, 300

Energetic Material Burn at the Mesoscale, 579

Hydrophobic-Hydrophilic Surfaces, 72

Microexplosive Subsurface Tracing System, 551

Open Access (Nanosat), 404

Post-Processed Integrated Microsystems, 119

Silicon-Integrated Planar Microbatteries, 180

Krumel, L. J.

Hadamard Transform Spectral Imager, 323

Krygowski, T. W.

Micro-Total-Analytical Systems, 160

Kurtz, S. R.

Characterization of Quantum Dots, 155

Kushmerick, J. G.

Hydrophobic-Hydrophilic Surfaces, 72

$\mathbf{L}$

La Van, D. A.

Solder for High-Fidelity Model Development, 581

Stress-Free Amorphous Diamond, 139

Ladd, M. D.

Monitoring Deep Targets, 321

Underground Facilities in an Urban Area, 309

Landmine, 307

Lanes, C. E.

Open Access (Nanosat), 404

Lanes, K. R.

Open Access (Nanosat), 404

Laser

External-cavity, 150

Filament, 144

Welding, 448

Laser-engineered net shaping, 57

Lattice (photonic), 182

Lau, H. S. J.

Miniature Bioaerosol Concentrator, 293

Lauffer, J. P.

Electromagnetic-Bearing Spindles, 214

Lawton, C. R.

Advanced Production Planning Models, 225

Production Surety Analysis, 374 


\section{Author and Title Index}

Layer

Boundary, 201

Smoke, 521

See also: Bilayer

Lebien, S. M.

Dynamic Range Imaging for Terrain Mapping, 327

Lee, S. R.

AlGaN Materials Engineering, 142

High-Al-Content AlGaInN Devices, 171

Legacy (waste), 527

Lemke, P. A.

Verify Cleaning and Surface Preparation, 230 LENS $^{\mathrm{TM}}$

See Laser-engineered net shaping

Lewis, P. R.

Autonomous MicroChem Laboratory, 409

Miniature Sensors for BW Agents, 166

LIGA

See Lithography

Lightning, 529

Lim, T. J.

Solder for High-Fidelity Model Development, 581

Lin, S. Y.

High-Efficiency Optical MEMS, 182

Photonics Integration Devices, 136

Silicon 3-D Photonic Crystal, 124

THz for High-Data-Rate Communications, 158

Lindner, D. L.

Autonomous MicroChem Laboratory, 409

Link, H. E.

Agent-Based Mediation, 260

Engineering Complex Distributed Systems, 401

Lipid, 164

List, G. F.

Advanced Production Planning Models, 225

Production Surety Analysis, 374

Lithography

LIGA (Electric field), 240

LIGA (polymer-metal), 472

Photoresist, 153

Soft, 232

Little, C. Q.

Adaptive 3-D Sensing, 337

Cloud to CAD, 319

Lo, C. S.

Finite-Element Polymer Modeling, 209

Lockhart, R. R.

Micro-High-G Acceleration Recorder, 325

Loehman, R. E.

Interfacial Reactions in Ceramic Systems, 441

Logistics, 486

Long, K. R.

Nonlinear Optimization from PDE Models, 108

Lorence, L.

Magnetically Insulated Vacuum Structures, 564
Loubriel, G. M.

Electromagnetic Imaging System, 343

HPM Vulnerability Assessment and Tests, 286

Semiconductor Switch for Firing Systems, 376

Loy, D. A.

Controller for Energy-Intensive Processes, 350

Materials for Energy Systems, 460

Molecular Integrated Microsystems, 412

Lu, W. Y.

Solder for High-Fidelity Model Development, 581

Luck, J. P.

Unobtrusive Human-Computer Interactions, 81

Luk, T. S.

Intense White-Light Pulse Propagation in Air, 284

Lukens, L. L.

Surface-Micromachined Mechanical Timer, 450

Lund, J. C.

Neutron Generators for Nuclear Weapons, 218

Lundgren, R. G. Micro-High-G Acceleration Recorder, 325

Lyo, S. K.

Quantum Tunneling Transistors, 129

Silicon 3-D Photonic Crystal, 124

M

Machining

Microfabrication, 223

See also: Micromachine

Macromolecules, 106

Magnetic

Excited, 131

Insulated, 564

Polysilicon, 549

See also: Electromagnetic

Malczynski, L. A.

Modeling Approaches for Nuclear Futures, 539

Management

Microcombustor, 348

SCADA, 390

Threat, 541

Manginell, R. P

Autonomous MicroChem Laboratory, 409

Hydrophobic-Hydrophilic Surfaces, 72

Microcombustor for Sensor Management, 348

Miniature Sensors for BW Agents, 166

Mani, S. S.

AlGaN Materials Engineering, 142

Mann, G. A.

Modeling Approaches for Nuclear Futures, 539

Manufacture

Elastohydrodynamics, 433

Tools, 228

Map

Navigation, 327

Temperature, 504 


\section{Author and Title Index}

Mar, A.

Semiconductor Current Filament Lasers, 144

Semiconductor Switch for Firing Systems, 376

Marder, B. M.

Kinetic-Energy Lightning Generator, 529

Margolis, S. B.

Microcombustor for Sensor Management, 348

Marker (molecular), 329

Marron, L. C.

Distributed Mobile Sensor Arrays, 313

Martin, J. W.

Thin-Skin Deployable Mirrors for Sensing, 304

Martin, L. E.

Spectroscopic Detection of Pathogens, 533

Martinez, M. J.

Advanced Geosphere Transport Simulation, 345

Mass (particle), 114

Massively parallel processor

Autoignition, 464

Grid, 96

Infrastructure, 385

Paleoclimate, 89

Master (mold), 238

Matzke, C. M.

Autonomous MicroChem Laboratory, 409

Mazarakis, M. G.

Intense Directed-Energy Theory Initiative, 290

Proton Beam Directed-Energy Weapon, 288

McConnell, T. D.

Change Detection in Flash Memory, 512

McDonald, T. S.

Underground Facilities in an Urban Area, 309

McKenna, S. A.

Surveillance Against Bioterror Attack, 537

Mechanical

Microstructure, 70

Timer, 450

See also: Electrochemomechanical

See also: Microelectromechanical

See also: Micromechanical

Mechanism

Boundary, 577

Broadening, 423

Corrosion, 41

Diagnostic, 48

Shear, 190

Mediation, 260

Medical (surveillance), 506

Medlin, D. L.

Dislocation-Grain-Boundary Interaction, 577

Melgaard, D. K. Improved Materials-Aging Diagnostics, 48

Melius, C. F.

Model-Based Fusion of Sensor Array Data, 253

Melof, B. M.

Pyrotechnic Smoke Materials for Barriers, 521
Membrane

Cellular, 560

Separation, 339

Memory

Flash, 512

Protonic, 63

MEMS

See Microelectromechanical systems

Mendenhall, F. T.

Information Collection, 403

Mesoscale, 579

Metal

Ion, 339

LIGA, 472

Part, 238

Polymer, 416

Reactivity, 54

Stabilization, 462

Transition, 439

Vapor, 553

Welding, 448

Michael, J. R.

Atmospheric Corrosion of Microelectronics, 380

Solidification/Microstructural Simulations, 571

Michalski, J. T.

Network Intrusion Detection, 388

Varying QoS for Fixed and Mobile Networks, 249

Microarray, 476

Microbattery, 180

MicroChem, 409

Microcombustor, 348

Microdiagnostic, 212

Microelectromechanical systems

Coating, 455

Generator, 352

Microdiagnostic, 212

Micromachining, 147

Optical, 182

Polysilicon, 549

Self-healing, 37

See also: Integrated microelectromechanical systems

Microelectronic

Generator, 352

Integration, 162

Photocurrent, 567

Reliability, 380

Microexplosive, 551

Microfabricate

Electromagnetic, 173

Machining, 223

Optical, 74

Microfluidic

Physics, 196

See also: Electromicrofluidic

Microfuel

Reformer, 494

Steam, 562 


\section{Author and Title Index}

Microgas

Chromatograph, 350

Welding, 448

Microincubators, 329

Microlab, 41

Micromachine

Bulk, 147

Computer-controlled, 241

Timer, 450

Tuner, 126

Micromechanical, 209

Micromethanol, 562

Microoptoelectronic, 134

Microoptoelectromechanical systems, 175

Micropump

Electrochemomechanical, 68

Sensor, 131

Microreplication, 238

Microrobot

Autonomous, 178

Distributed, 331

Microscale, 52

Microsensor

Diamond, 139

Internet, 486

Microrobot, 178

Microseparation, 453

Microsimulation, 385

Microstructure

Diamond, 139

Lithography, 232

Mechanics, 70

PZT 95/5, 574

Simulation, 571

Microsystem

Bioelectronic (model), 543

Bioelectronic (sustainable), 535

Detector, 117

Film, 32

LIGA, 472

Microcombustor, 348

MIMS, 412

Post-processes, 119

Micro-Total-Analytical, 160

Microvalves, 68

Microwave, 126

Military (target), 514

Miller, A. K.

Information Collection, 403

Miller, D. P.

Identifying Dangerous Human Actions, 369

Miller, G. J., Jr.

Neutron Generators for Nuclear Weapons, 218

Miller, J. C.

Energetic-Material Initiation, 60

Miller, J. E.

Designed Ionophores Separation, 339

National Security Issues/Limited Resources, 534
Miller, R. D.

Low-Power Public-Key Protocols, 271

MIMS

See Molecular Integrated Microsystems

Miniature

Concentrator, 293

Penetrator, 523

Sensor (Fatty-acid), 166

Sensor (UV), 315

See also: Ultraminiaturization

Mining (Data)

Fuzzy, 280

WNN, 247

Mirror, 304

Missert, N. A.

Critical Copper-Corrosion Mechanisms, 41

Monitoring Corrosion in Weapons Environments, 34

Self-Assembled Templates, 43

Mitchell, M. A.

Flexural Plate-Wave Devices, 131

Mitigation (Rayleigh-Taylor), 430

Mobile

Network, 249

Robot, 334

Sensor, 313

Mode (ATM), 276

Modine, N. A.

Chemical Vapor Deposition Reactor Designs, 553

Module (security), 482

MOEMS

See Microoptoelectromechanical systems

Moen, C. D.

Unstructured Grid Problems, 96

Mold (parts), 238

Molecular

Characterization, 60

Integrated microsystems, 412

Markers, 329

Self-healing, 37

Simulation, 87

See also: Supramolecular

Monitor

Blade, 480

Corrosion, 34

Software, 466

Target, 321

Thin-film, 216

Monolithic

Integration, 117

Tuner, 126

Montague, S.

Micro-Total-Analytical Systems, 160

Moody, N. R.

Interfacial traction-separation, 569

Thin-Film Deposition Processes, 216

Verify Cleaning and Surface Preparation, 230

Moon, J. S.

Quantum Tunneling Transistors, 129 


\section{Author and Title Index}

Moore, R. C.

In Situ Formation of Reactive Barriers, 462

Morales, A. M.

Miniature UV Biological Agent Sensor, 315

Neutron Generators for Nuclear Weapons, 218

Stronglink Prime Mover, 446

Morgan, C. H.

Miniature Sensors for BW Agents, 166

Morse, W. D.

Intelligent Targeting for Remote Weapons, 492

Motion (sensing), 81

Mover (stronglink), 446

Mowery, D. L.

Microcombustor for Sensor Management, 348

Mowry, C. D.

Miniature Sensors for BW Agents, 166

Moya, M. M.

MPP

Recognizing Partially Obscured Targets, 295

See Massively parallel processor

MTI

See Multispectral thermal imager

Muguira, M. R.

WNN Decision Making for Data Mining, 247

Multiagent, 278

Multiaxial, 581

Multifidelity, 112

Multifunction

$\mathrm{AlGaN}, 142$

Nanocomposite, 425

Multiresolution, 188

Multiscale, 201

Multisensor, 502

Multispectral thermal imager, 504

Murata, K. K.

Facility Response to Abnormal Events, 251

Murphy, M. B.

Information Collection, 403

Murphy-Dye, B. D.

Engineering Complex Distributed Systems, 401

Myers, R. L.

Fabrication of 3-D Microstructures, 232

Myers, S. M., Jr.

Surface Hardening, 420

\section{$\mathbf{N}$}

Najm, H. N.

Numerical Simulation of Diesel Autoignition, 464

Nanoarray, 43

Nanocomposite

Battery, 359

Self-assembly, 425

Nanoparticle, 420

Nanosat (nanosatellite), 404

Nanoscience, 548
Nanostructure

Optical, 74

Photovoltaic, 354

Scattering, 557

Napolitano, L. M.

Molecular Integrated Microsystems, 412

Navigation

Autonomous (mapping), 327

Autonomous (Robot), 334

Neiser, R. A., Jr.

Output-Based Process Control, 66

Nellums, R. O.

Automatic Target Recognition in 3-D, 488

Dynamic Range Imaging for Terrain Mapping, 327

Network

Analysis, 366

ATM, 276

Geophysical, 321

Gigabit, 273

Medical, 506

Mobile, 249

Neural, 388

Self-configurable, 262

Neumann, W. D.

Information-Sharing Security Module, 482

SCADA Systems in the Electric Industry, 390

Neural (network), 388

Neuroprocessors, 484

Neutron

Generator, 218

Tube, 418

Nevers, J. A.

Integration of VCSELs and Detectors, 117

Open Access (Nanosat), 404

Newton, C. S.

Engineered Window Glass, 519

Ngola, S. M.

Molecular Markers for Pathogen Detection, 329

$\mathrm{Ni}(\mathrm{Al}, \mathrm{O}), 420$

Nilson, R. H. Applied Microfluidic Physics, 196

Nonlinear, 108

Nonvolatile, 63

Nuclear

Future, 539

Weapon, 218

Nucleation, 194

Numerical

Micromachine, 241

Simulation, 464

$\mathbf{O}$

O'Bannon, D. S.

PUSH Technology Demonstration, 266

Obscured (target), 295 


\section{Author and Title Index}

Obstacle (navigation), 327

Okandan, M.

Magnetic Polysilicon MEMS Devices, 549

Micro-Total-Analytical Systems, 160

Oliver, A. D.

Fabrication of 3-D Microstructures, 232

MEMS Microelectric Generator, 352

Stronglink Prime Mover, 446

Olson, C. L.

Intense Directed-Energy Theory Initiative, 290

O'Malley, M. W.

Semiconductor Current Filament Lasers, 144

Oppel, F. J., III

Heterogeneous Simulation, 79

Optical

Communication, 264

Filamentation, 284

Firing, 376

Interconnection, 510

MEMS, 182

Microfabricate, 74

Neuroprocessor, 484

Optimization

Circuit, 126

Nonlinear, 108

Reactor, 553

Schedule, 93

Sensor, 484

Optoelectronic

AlGaInN, 171

Microelectronic, 162

See also: Microoptoelectronic

Organic

Barrier, 462

Reactor, 553

O'Toole, E. J.

Virtual Reliability Realization System, 395

Ottesen, C. W.

Ultraminiaturization of RF Circuitry, 444

Ottesen, D. K.

Transient Gas and Soot in Fires, 185

Owen, T. E.

Techniques for Real-Time Visualization, 497

\section{$\mathbf{P}$}

Packaging

Electromicrofluidic, 244

IMEMS, 236

Paez, T. L.

Advanced Signal Processing, 371

Paleoclimate, 89

Pancerella, C. M.

Policies for Multiagent Systems, 278

Parallel

Grid, 96

Infrastructure, 385

Method, 110
Microseparation, 453

Paleoclimate, 89

Scheduling, 93

Software monitor, 466

Partial differential equation, 108

Particle

Mass, 114

See also: Nanoparticle

Pathogen

Microincubator, 329

Spectroscopic, 533

Paul, J.

National Security Issues/Limited Resources, 534

Paul, P. H.

Stronglink Prime Mover, 446

PDE

See Partial differential equation

Penetrator (flying), 523

Perturbation, 430

Peterson, K. A.

Electromicrofluidic Packaging, 244

IMEMS Packaging and Interconnection, 236

Pfeifer, K. B.

Sandia Microsensors to the Internet, 486

Pfohl, J. S.

Information Collection, 403

Phase-Field, 39

Phelan, J. R.

Miniaturized Flying Penetrator, 523

Phenomena (transient), 201

Phillips, C. A.

Advanced Production Planning Models, 225

Combinatorial Optimization for Scheduling, 93

Production Surety Analysis, 374

Phillips, L. R.

Agent-Based Mediation, 260

Engineering Complex Distributed Systems, 401

Photochromic, 32

Photocurrent, 567

Photonic

Crystal, 124

Driver, 52

Integration, 136

Lattice, 182

Photoresist, 153

Photovoltaic, 354

Physics

Coupled-flow, 85

Liquid-crystal, 559

Microfluidic, 196

Shock-wave, 52

Pierson, L. G.

One-Hundred-Gigabit/Second Networks, 273

Optical Backplane/Interconnect, 264

Planar

Electrode, 164

Microbattery, 180

Plane (arrays), 510 


\section{Author and Title Index}

Planning

Model, 225

Navigation, 334

Plantenga, T. D.

Distributed Information Systems, 255

Modeling of Medical Surveillance Network, 506

Plasma (formation), 555

Plate-Wave, 131

Platform (autonomous), 313

Platzberger, M. R.

Techniques for Real-Time Visualization, 497

Plimpton, S. J.

Atom-Picoseconds to Centimeter-Years, 91

Computational Structural Biology, 104

Heterogeneous Simulation, 79

Plummer, K. W.

Ultra-Miniaturization of RF Circuitry, 444

Plut, T. A.

Monolithic Micromachined Variable Tuners, 126

Poco (sprytrons), 443

Polarization

Infrared, 470

Spectro, 514

Polosky, M. A.

Bulk Micromachining for MEMS, 147

GaAs MOEMS Technology, 175

High-Efficiency Optical MEMS, 182

Polymer

Chemiresistor, 416

LIGA, 472

Modeling (computational), 422

Modeling (failure), 209

Polysilicon, 549

Post-Processed (integrated), 119

Poteet, H. M.

Information Collection, 403

Potter, B. G., Jr.

Molecular Integrated Microsystems, 412

Potter, K. S.

Miniature UV Biological Agent Sensor, 315

Molecular Integrated Microsystems, 412

Powder (pressing), 436

Power

Bulk, 383

Electric, 390

Protocol, 271

RF, 283

Prasad, M. R.

Information Collection, 403

Precipitation (nanoparticle), 420

Precision (parts), 238

Preconcentration, 525

Preconditioner, 83

Predict

Corrosion, 380

Fracture, 206

Macromolecule, 106

Pressing (dies), 436
Prevender, T. S.

Information Collection, 403

Production

Planning, 225

Surety, 374

Propagation (light), 284

Protein, 283

Proton

Beam, 288

Memory, 83

Prototypingm, 126

Pryor, R. J.

Emergent Behavior of Intelligent Agents, 102

Model of Infrastructure Interdependency, 385

Representation of Human Decision Process, 531

Pseudostationary, 453

Public-Key

Authentication, 364

Protocol, 271

Pulse, 284

Pump

Electrokinetic, 446

See also: Micropump

PUSH (web casting), 266

Pyrotechnic, 521

PZT 95/5, 574

Q

QoS

See Quality (of service)

Quality

Of service, 249

Tools, 230

Quantum

Dot, 155

Tunneling, 129

$\mathbf{R}$

Rader, D. J.

Miniature Bioaerosol Concentrator, 293

Radiation

Hard, 122

Photocurrent, 567

Photoresist, 153

Source, 430

Radio-frequency

Circuitry, 444

Decontamination, 283

Radiography, 397

Radionuclide, 462

Rahimian, K.

All-Ceramic Thin-Film Battery, 76

Rakestraw, D. J.

Autonomous MicroChem Laboratory, 409

Raman (spectroscopy), 508

Ramsey, J. L., Jr.

Advanced Geosphere Transport Simulation, 345 


\section{Author and Title Index}

Rao, R. R. Incomplete Factorization Preconditioners, 83

Ray, J.

Numerical Simulation of Diesel Autoignition, 464

Raybourn, E. M.

Representation of Human Decision Process, 531

Rayleigh-Taylor, 430

Raymond, T. D.

External-Cavity Surface-Emitting Lasers, 150

React

Barrier, 462

Ceramic, 441

Surface, 54

System, 87

Reactor (deposition), 553

Real-Time

Design, 436

Response, 251

Spindle, 214

Surveillance, 537

Visualization, 497

Reasoning, 295

Reber, C. A.

Electromicrofluidic Packaging, 244

IMEMS Packaging and Interconnection, 236

Rechargeable (battery), 359

Recognition

Target (3-D), 488

Target (obscured), 295

Reconfigurable, 331

Reconnaissance, 343

Recorder, 325

Recyclable transmission line, 458

Recycling, 300

Redmond, J. M.

Electromagnetic-Bearing Spindles, 214

Thin-Skin Deployable Mirrors for Sensing, 304

Redox, 300

Reduce

Computation, 271

Perturbation, 430

Waste, 527

Reedy, E. D., Jr.

Finite-Element Polymer Modeling, 209

Interfacial traction-separation, 569

Verify Cleaning and Surface Preparation, 230

Reeves, P. C.

Advanced Geosphere Transport Simulation, 345

Reformer (microfuel)

Hydrogen, 494

Steam, 562

Regueiro, R. A.

Mechanisms of Adiabatic Shear Failure, 190

Reliability

Infrastructure, 366

Microelectronic, 380
Remote

Sensing (filamentation), 284

Sensing (mirror), 304

Weapon, 492

Renk, T. J.

Proton Beam Directed-Energy Weapon, 288

Renlund, A. M.

Energetic-Material Burn at the Mesoscale, 579

Energetic-Material Initiation, 60

Reno, J. L.

Acoustically Driven Microoptoelectronics, 134

GaAs MOEMS Technology, 175

Quantum Tunneling Transistors, 129

Renzi, R. F.

Autonomous MicroChem Laboratory, 409

Miniature Bioaerosol Concentrator, 293

Representation (decision process), 531

Resolution

Modeling, 201

See also: Multiresolution

See also: Ultrahigh (resolution)

Resource, 544

Response

Facility, 251

Shock, 479

Shock-wave, 574

Rexroth, P. E

Modeling Approaches for Nuclear Futures, 539

$\mathrm{RF}$

See Radio-frequency

Rhykerd, C. L., Jr.

Preconcentration of Volatile Chemical, 525

Rice, S. F. Microfuel Reformer, 494

Rieb, D. A.

PUSH Technology Demonstration, 266

Rieger, D. J. Optoelectronic Arrays and Microelectronics, 162

Rienstra, J. L.

Optical Interconnections to Focal Plane Arrays, 510

Radiation-Hard Sensors, 122

Rinne, R. L.

Representation of Human Decision Process, 531

Risk (infrastructure), 366

Robertson, P. J.

One-Hundred-Gigabit/Second Networks, 273

Open Access (Nanosat), 404

Optical Backplane/Interconnect, 264

Robinett, R. D., III

Information Collection, 403

Robino, C. V.

Solidification/Microstructural Simulations, 571

Robinson, A. C.

Unstructured Grid Problems, 96

Robinson, D. G.

Atmospheric Corrosion of Microelectronics, 380 Security of Bulk Power Systems, 383 


\section{Author and Title Index}

Robot

Dexterous, 311

Mobile, 334

See also: Microrobot

Robust (planning), 334

Rochau, G. E.

RTL Concept for Fusion Energy, 458

Rodgers, M. S. High-Efficiency Optical MEMS, 182

Rodriguez, M. A. All-Ceramic Thin-Film Battery, 76

Nanocomposites for Rechargeable Batteries, 359

Roe, D. C.

Computational Structural Biology, 104

Function of Biological Macromolecules, 106

Model-Based Fusion of Sensor Array Data, 253

Novel Biological Weapon Detection System, 298

Rohwer, T. A.

Micro-High-G Acceleration Recorder, 325

Rollstin, L. R.

Miniaturized Flying Penetrator, 523

Romero, A. M.

Neutron Generators for Nuclear Weapons, 218

Romero, J. A.

Thin-Film Deposition Processes, 216

Ross, J. R.

Transient Gas and Soot in Fires, 185

Rotating (flowfields), 198

RTL

See Recyclable transmission line

Ruby, D. S.

Nanostructured Silicon Surfaces, 354

Ruffner, J. A.

All-Ceramic Thin-Film Battery, 76

Reactivity of Metal-Oxide Surfaces, 54

Ruiz, C. L.

Magnetically Insulated Vacuum Structures, 564

\section{$\mathbf{S}$}

Sa, T. J.

Modeling of Medical Surveillance Network, 506

Salazar, J. S.

Recognizing Partially Obscured Targets, 295

Salinger, A. G.

Function of Biological Macromolecules, 106

Sand (smart), 307

Sandoval, C. E.

Ultraminiaturization of RF Circuitry, 444

Santangelo, P. J.

Transient Gas and Soot in Fires, 185

SAR

See Synthetic aperture radar

Sasaki, D. Y.

Acoustic-Wave Biosensor Arrays, 168

Biosensors Based on Electrical Impedance, 164

Microfabricated Optical Devices, 74

Molecular Integrated Microsystems, 412
Self-Healing Molecular Assemblies, 37

Smart Self-Assembled Photochromic Films, 32

Satellites, 428

Sault, A. G.

Nonvolatile Protonic Memory, 63

Reactivity of Metal-Oxide Surfaces, 54

Savignon, D. J.

Micro-High-G Acceleration Recorder, 325

SCADA

Scale

See Supervisory control and data aquisition

Device, 110

Information, 255

Mass, 114

Optimization, 108

See also: Mesoscale

See also: Microscale

See also: Multiscale

Scattering, 557

Scheduling, 93

Schmid, A. K.

Crack Nucleation and Growth, 194

Self-Assembled Templates, 43

Schmidt, R. C.

Advanced Large-Eddy Simulation Algorithms, 85

Modeling of Multiscale Transient Phenomena, 201

Schmitt, D. J.

Adaptive 3-D Sensing, 337

Enabling Human Skills, 317

Schoeneman, J. L.

Open Access (Nanosat), 404

Schoeniger, J. S.

Biological Weapon Detector, 300

Human Exposure to Biological Agents, 499

Molecular Integrated Microsystems, 412

Molecular Markers for Pathogen Detection, 329

Novel Biological Weapon Detection System, 298

Smart Sand for Landmine Detection, 307

Schoenwald, D. A.

Model of Infrastructure Interdependency, 385

Schroder, K. L.

Miniature UV Biological Agent Sensor, 315

Schroeppel, R. C.

Information-Sharing Security Module, 482

Low-Power Public-Key Protocols, 271

Schubert, W. K.

Flexural Plate-Wave Devices, 131

Post-Processed Integrated Microsystems, 119

Stress-Free Amorphous Diamond, 139

Schunk, P. R.

Elastohydrodynamics in Manufacturing, 433

Incomplete Factorization Preconditioners, 83

Unstructured Grid Problems, 96

Schwank, J. R.

Radiation-Induced Prompt Photocurrents, 567

Scott, M. W.

Information Collection, 403

Scripting, 220 


\section{Author and Title Index}

Seager, C. H.

Nonvolatile Protonic Memory, 63

Seal (tamper-detection), 392

Sears, M. P.

Function of Biological Macromolecules, 106

Security

Information-sharing, 482

National, 534

Power, 383

SCADA, 390

Self-Assemble

Physics, 559

Nanoarray, 43

Nanocomposite, 425

Film, 32

Seal, 392

See also: Self-Healing

Self-Configurable, 262

Self-Guided, 284

Self-Healing, 37

Semiconductor

Laser, 144

Switch, 376

Senglaub, M. E.

Modeling the Jini Infrastructure Protocol, 490

Sensitivity

Biosensor, 168

Microsensor, 139

Sensor

Adaptive, 337

Array, 253

Fatty-acid, 166

Materials, 298

Microcombustor, 348

Mirror, 304

Mobile, 313

Optical, 484

Plate-wave, 131

Process control, 66

Radiation-hard, 122

Remote, 284

Shooter, 531

Smart sand, 307

UV, 315

VVMD, 81

See also: Biosensor

See also: Microsensor

See also: Multisensor

Separation

Interfacial, 569

Liquid-membrane, 339

See also: Microseparation

Setchell, R. E.

Microscale Shock-Wave Physics, 52

Shock-Wave Response of PZT 95/5, 574

Shaddix, C. R.

Transient Gas and Soot in Fires, 185
Shadid, J. N.

Incomplete Factorization Preconditioners, 83

Unstructured Grid Problems, 96

Shaneyfelt, M. R.

Radiation-Induced Prompt Photocurrents, 567

Shawver, D. M.

Information Collection, 403

Shear (failure), 190

Shelnutt, J. A.

Designed Ionophores Separation, 339

Shen, H. C.

Open Access (Nanosat), 404

Shepodd, T. J.

Molecular Integrated Microsystems, 412

Shinn, N. D.

Wetting and Spreading Dynamics of Alloys, 45

Shock (crystals), 479

Shock-Wave

Photonic, 52

PZT 95/5, 574

Shokair, I. R.

Model-Based Fusion of Sensor Array Data, 253

Shooter, 531

Shul, R. J.

Bulk Micromachining for MEMS, 147

Flexural Plate-Wave Devices, 131

High-Al-Content AlGaInN Devices, 171

Micromethanol Steam Reformer, 562

Open Access (Nanosat), 404

Post-Processed Integrated Microsystems, 119

Stress-Free Amorphous Diamond, 139

$\mathrm{Si}$

See Silicon

Sicking, C. W.

Monitoring Deep Targets, 321

Underground Facilities in an Urban Area, 309

Signal (processing), 371

Silicon

Crystal, 124

On-insulator, 147

Microbattery, 180

Photovoltaic, 354

Rechargeable battery, 359

See also: Polysilicon

Simmermacher, T. W.

Health Monitoring of Wind Turbine Blades, 480

Simmons, J. A.

Quantum Tunneling Transistors, 129

$\mathrm{THz}$ for High-Data-Rate Communications, 158

Simonson, K. M.

ATR/Exploitation SAR Imaging, 302

Multisensor Fusion for Characterizing UGFs, 502

Simonson, R. J.

Information Collection, 403

Simpson, R. L.

Thermal History of Charred Materials, 508 


\section{Author and Title Index}

Simulation

Atom-picosecond, 91

Autoignition, 464

Coprocessor, 466

Geosphere, 345

Hetrogeneous, 79

Information, 255

Large-eddy, 85

Microstructural, 571

Molecular, 87

Scale, 110

Structural, 188

See also: Microsimulation

Simultaneous (measurement), 185

Sinclair, M. B.

Microdiagnostic MEMS Lab-on-a-Chip, 212

Microfabricated Optical Devices, 74

Singh, A. K.

Highly Parallel Microseparations, 453

Molecular Integrated Microsystems, 412

Smart Sand for Landmine Detection, 307

Sleefe, G. E.

Monitoring Deep Targets, 321

Sloan, G. R.

Ultraminiaturization of RF Circuitry, 444

Sloan, L. R.

Monolithic Micromachined Variable Tuners, 126

$\mathrm{THz}$ for High-Data-Rate Communications, 158

Ultraminiaturization of RF Circuitry, 444

Sloane, M. C.

Automatic Design of Practical Fixtures, 234

Slutz, S. A.

RTL Concept for Fusion Energy, 458

Small, D. E.

Adaptive 3-D Sensing, 337

Enabling Human Skills, 317

Intelligent Targeting for Remote Weapons, 492

Unobtrusive Human-Computer Interactions, 81

Smart

Film, 32

Sand, 307

Smith, D. A

Hadamard Transform Spectral Imager, 323

Smith, J. L.

Land Surface-Temperature Maps, 504

Smith, M. F.

Output-Based Process Control, 66

Smith, N. F.

Microdiagnostic MEMS Lab-on-a-Chip, 212

Smith, T. M.

Advanced Large-Eddy Simulation Algorithms, 85

Smithpeter, C. L.

Automatic Target Recognition in 3-D, 488

Dynamic Range Imaging for Terrain Mapping, 327

Smoke (barrier), 521

Snavely, J. A.

Modeling of Medical Surveillance Network, 506
Sniegowski, J. J.

Bulk Micromachining for MEMS, 147

Snoopy (coprocessor), 466

Snyder, L. A.

Information-Sharing Security Module, 482

Soaring, 313

Sobel, A. L.

Intense White-Light Pulse Propagation in Air, 284

Social (fabric), 541

Software, 466

SOI

See Silicon-on-insulator

Solder

Deformation, 581

Dynamics, 45

Solidification, 571

Solid-State, 218

Solution (security), 534

Solvent, 416

Somerday, B. P.

Polymer-Metal Interface Properties, 472

Soot, 185

Sorensen, N. R.

Atmospheric Corrosion of Microelectronics, 380

Source

Code, 378

Data, 295

Radiation, 430

Space-Based, 122

Spahn, O. B.

GaAs MOEMS Technology, 175

Photonics Integration Devices, 136

Sparse

Network, 321

Preconditioner, 83

Spatial (anomalies), 564

Species (gas), 185

Spectral

Imager, 323

See also: Hyperspectral

See also: Multispectral thermal imager

Spectro-Polarization, 514

Spectroscopy

Pathogen, 533

Raman, 508

Speed

Communication, 264

Imager, 323

Spencer, D. D.

Information Collection, 403

Land Surface-Temperature Maps, 504

Spencer, F. W.

Output-Based Process Control, 66

Spielman, R. B.

Composite-Wire Plasma Formation, 555

RTL Concept for Fusion Energy, 458

Spill (detection), 416 


\section{Author and Title Index}

Spindles, 214

Spires, S. V.

Agent-Based Mediation, 260

Engineering Complex Distributed Systems, 401

Spletzer, B. L.

Distributed Mobile Sensor Arrays, 313

Emergent Behavior of Intelligent Agents, 102

Information Collection, 403

Springer, C.

Function of Biological Macromolecules, 106

Model-Based Fusion of Sensor Array Data, 253

Stabilization, 462

Stalker, K. T.

Information Collection, 403

Miniaturized Flying Penetrator, 523

Stallard, B. R.

Land Surface-Temperature Maps, 504

Stamber, K. L.

Model of Infrastructure Interdependency, 385

Stamp, J. E.

SCADA for Critical Energy Infrastructures, 258

Varying QoS for Fixed and Mobile Networks, 249

Stamps, J. F.

Autonomous MicroChem Laboratory, 409

Stansfield, S. A.

Information Collection, 403

Steam, 562

Stephens, J. J., Jr.

Wetting and Spreading Dynamics of Alloys, 45

Stevens, M. J.

Actuation of Microvalves and Micropumps, 68

Function of Biological Macromolecules, 106

Self-Healing Molecular Assemblies, 37

Stewart, H. D.

Nonvolatile Protonic Memory, 63

Stone, R. G.

Engineered Window Glass, 519

Stress-Free, 139

Stronglink, 446

Structural

Biology, 104

Simulation, 188

Surface, 439

Vacuum, 564

See also: Microstructure

See also: Nanostructure

See also: Unstructured

Sturgis, B. R.

Techniques for Real-Time Visualization, 497

Stygar, W. A.

RTL Concept for Fusion Energy, 458

Subsurface (tracing), 551
Sullivan, C. T.

Bulk Micromachining for MEMS, 147

GaAs MOEMS Technology, 175

Integration of VCSELs and Detectors, 117

Monolithic Micromachined Variable Tuners, 126

Open Access (Nanosat), 404

Optical Backplane/Interconnect, 264

Photonics Integration Devices, 136

Sullivan, J. P.

Critical Copper-Corrosion Mechanisms, 41

Stress-Free Amorphous Diamond, 139

Supervisory control and data acquisition

Authenticate, 390

Surety, 258

Supramolecular, 425

Surety

Analysis, 362

Glass, 519

Production, 374

SCADA, 258

Surface

Decontamination, 283

Hardening, 420

Laser, 150

Map, 504

MEMS, 182

Metal-oxide, 54

Preparation, 230

Silicon, 354

Structure, 439

Switchable, 72

Timer, 450

See also: Subsurface

Surveillance

Bioterror, 537

Medical, 506

Sustainable, 535

Swarms, 102

Swartzentruber, B. S.

Reactivity of Metal-Oxide Surfaces, 54

Sweatt, W. C.

Information Collection, 403

Infrared Polarization for Target Identification, 470

Open Access (Nanosat), 404

Sweet, J. N.

Atmospheric Corrosion of Microelectronics, 380

Switch

Firing, 376

Poco, 443

Surface, 72

Synthetic aperture radar, 302 


\section{Author and Title Index}

$\mathbf{T}$

Tadros, M. E.

National Security Issues/Limited Resources, 534

Tallant, D. R.

Thermal History of Charred Materials, 508

Tamper (detection), 392

Tappan, A. S.

Energetic-Material Burn at the Mesoscale, 579

Energetic-Material Initiation, 60

Target

Deep, 321

Detection, 514

Identification, 470

Obscured, 295

Recognition, 488

Weapon, 492

Tarman, T. D.

Intrusion Detection for ATM Networks, 276

Optical Backplane/Interconnect, 264

Varying QoS for Fixed and Mobile Networks, 249

Tarver, E. E., III

Radiography for Inspection of Weapons, 397

Taylor, J. G.

Land Surface-Temperature Maps, 504

Temperature (maps), 504

Template, 43

Terrain, 327

Tethered (lipid bilayers), 164

Thai, T. Q.

Change Detection in Flash Memory, 512

PUSH Technology Demonstration, 266

Thermal

Flaw, 371

Imager, 504

Microsystem, 348

Thin-Film

Battery, 76

Deposition, 216

Thin-Skin, 304

Thomas, E. V.

Approximate Public-Key Authentication, 364

Improved Materials-Aging Diagnostics, 48

Low-Power Public-Key Protocols, 271

Output-Based Process Control, 66

Thomas, G. A.

Information Collection, 403

Thompson, A. P.

Atom-Picoseconds to Centimeter-Years, 91

Molecular Integrated Microsystems, 412

Molecular Simulation of Reacting Systems, 87

Thorne, L. R.

Information Collection, 403

Threat, 541
Three-Dimensional

Lithography, 232

Sensing, 337

Silicon, 124

Target, 488

THz (10 ${ }^{12}$ Hertz), 158

Tigges, C. P.

Bulk Micromachining for MEMS, 147

Microfabrication of Electromagnetic Devices, 173

Flexural Plate-Wave Devices, 131

Monolithic Micromachined Variable Tuners, 126

Tikare, V.

Phase-Field Modeling, 39

Timer, 450

Timlin, J. A.

Spectroscopic Detection of Pathogens, 533

Tissue, 546

TNT

See Trinitrotoluene

Torgerson, M. D.

SCADA Systems in the Electric Industry, 390

Toth, R. P.

Surface Decontamination of Protein Toxins, 283

Toxin, 283

Tracing, 551

Traction, 569

Trahan, M. W.

Intense Directed-Energy Theory Initiative, 290

Transfer (ATM), 276

Transform, 323

Transient

Gas, 185

Phenomena, 201

Transistors, 129

Transition-Metal, 439

Transport

Cellular, 560

Geosphere, 345

Triggered, 376

Trinitrotoluene, 307

Trinkle, J. C.

Automatic Design of Practical Fixtures, 234

Trott, W. M.

Dispersive Measurements of Velocity, 203

Microscale Shock-Wave Physics, 52

Tsao, S. S. I. AlGaN Materials Engineering, 142

Tube

Neutron, 418

Poco, 443

Tucker, M. D.

National Security Issues/Limited Resources, 534

Tuminaro, R. S.

Unstructured Grid Problems, 96 


\section{Author and Title Index}

Tuner, 126

Tunneling (transistors), 129

Turbine, 480

Turbulent, 201

Turnquist, M. A.

Advanced Production Planning Models, 225

Production Surety Analysis, 374

Tuttle, B. A.

Shock-Wave Response of PZT 95/5, 574

U

UGFs

See Underground facilites

Ultrahigh

Data-rate, 158

Resolution (Radiography), 397

Resolution (SAR), 302

Ultraminiaturization, 444

Ultraviolet, 315

Underground facilites

Multisensor, 502

Urban, 309

Unstructured

Environment (dynamic), 334

Environment (hazardous), 311

Grid, 96

Urban (UGF), 309

Urbina, A.

Advanced Signal Processing, 371

UV

See Ultraviolet

V

Vacuum, 564

Validated, 194

Valley, M. T.

Advanced Signal Processing, 371

Van Bloemen Waanders, B. G.

Nonlinear Optimization from PDE Models, 108

Van Leeuwen, B. P.

Self-Configurable Network Protocol, 262

Van Swol, F. B.

Evaporation-Induced Self-Assembly, 559

Molecular Simulation of Reacting Systems, 87

Smart Self-Assembled Photochromic Films, 32

Vandernoot, V. A.

Human Exposure to Biological Agents, 499

Molecular Markers for Pathogen Detection, 329

Vandevender, J. P.

Magnetically Insulated Vacuum Structures, 564

Vanecek, C. W.

Stronglink Prime Mover, 446

Vapor (chemical), 553

Vawter, G. A.

Photonics Integration Devices, 136

Semiconductor Current Filament Lasers, 144
VCSELS

See Vertical-cavity surface-emitting laser VDC

See Volumetric displacement control

Velocity, 203

Venturini, E. L.

Shock-Wave Response of PZT 95/5, 574

Verify, 230

Vernon, G. E.

Surface-Micromachined Mechanical Timer, 450

Vertical-cavity surface-emitting laser

External, 150

Microsystem, 117

Video

Inspection, 220

Sensing, 81

Virtual, 395

Visible, 122

Visual, 99

Visualization, 497

Vizkelethy, G.

Radiation-Induced Prompt Photocurrents, 567

Voigt, J. A.

All-Ceramic Thin-Film Battery, 76

Shock-Wave Response of PZT 95/5, 574

Volatile

Memory, 63

Preconcentration, 525

Volponi, J. V.

Molecular Markers for Pathogen Detection, 329

Volumetric

Displacement control, 228

Video, 81

Voth, T. E. Advanced Large-Eddy Simulation Algorithms, 85

Vulnerability

HPM, 286

Production, 374

W

Wagner, J. S.

Intense Directed-Energy Theory Initiative, 290

Modeling of a Bioelectronic Microsystem, 543

Walck, M. C.

Information Collection, 403

Walker, P. K.

Energetic-Material Burn at the Mesoscale, 579

Wall, F. D.

Monitoring Corrosion in Weapons Environments, 34

Wally, K.

Biological Weapon Detector, 300

Microfuel Reformer, 494

Miniature Bioaerosol Concentrator, 293

Miniature Sensors for BW Agents, 166

Walsh, D. S

Radiation-Induced Prompt Photocurrents, 567 


\section{Author and Title Index}

Walsh, E. J.

Fault-Tolerant Computing Services, 474

Walther, E. A.

WNN Decision Making for Data Mining, 247

Wampler, B. L. Acoustically Driven Microoptoelectronics, 134

Wampler, W. R. AlGaN Materials Engineering, 142

Warne, L. K. Assembly of LIGA Using Electric Fields, 240

Warren, M. E.

Autonomous MicroChem Laboratory, 409

Microfabricated Optical Devices, 74

Waste, 527

Waymire, D. R. Approximate Public-Key Authentication, 364

Weapon

Bioaffinity, 300

Corrosion, 34

Detection, 298

Energy, 288

Inspection, 397

Nuclear, 218

Remote, 492

Webb, E. B., III

Wetting and Spreading Dynamics of Alloys, 45

Weber, T. M.

Assembly of LIGA Using Electric Fields, 240

Wehlburg, C. M.

Hadamard Transform Spectral Imager, 323

Improved Materials-Aging Diagnostics, 48

Information Extraction from Satellites, 428

Wehlburg, J. C.

Hadamard Transform Spectral Imager, 323

Improved Materials-Aging Diagnostics, 48

Thin-Skin Deployable Mirrors for Sensing, 304

Weighted-Nearest-Neighbor, 247

Welding, 448

Wellman, G. W.

Microstructure and Mechanics, 70

Wendt, J. R.

Characterization of Quantum Dots, 155

Photonics Integration Devices, 136

Quantum Tunneling Transistors, 129

Wessendorf, K. O.

Information Collection, 403

Post-Processed Integrated Microsystems, 119

Wetting, 45

Wheeler, D. R.

Molecular Integrated Microsystems, 412

Photoresist Technology, 153

Wheelis, W. T.

Open Access (Nanosat), 404

Whinnery, L. L., Jr. Actuation of Microvalves and Micropumps, 68

White, R. L.

Acoustically Driven Microoptoelectronics, 134
Whitehurst, H. O.

Identifying Dangerous Human Actions, 369

White-Light, 284

Wick, D. V. Autonomous Optical Neuroprocessors, 484

Wilde, M. G.

PUSH Technology Demonstration, 266

Willan, C. C.

AlGaN Materials Engineering, 142

Williams, A. B.

Unstructured Grid Problems, 96

Wilson, C. W.

Scripting for Video Inspection, 220

Wimmer, S. A. Method for Predicting Generalized Fracture, 206

Wind, 480

Window, 519

Winter, V. L.

Simulation of Coprocessor Software Monitor, 466

Wire, 555

Witzke, E. L.

Intrusion Detection for ATM Networks, 276

One-Hundred-Gigabit/Second Networks, 273

Optical Backplane/Interconnect, 264

Wixom, R. R.

Chemical Vapor Deposition Reactor Designs, 553

WNN

See Weighted-nearest-neighbor

Wong, C. C.

External-Cavity Surface-Emitting Lasers, 150

Wright, A. F.

Critical Copper-Corrosion Mechanisms, 41

High-Al-Content AlGaInN Devices, 171

$\mathrm{Wu}, \mathrm{B} . \mathrm{C}$.

Microfuel Reformer, 494

$\mathrm{Wu}, \mathrm{J}$.

Autonomous MicroChem Laboratory, 409

Wunsch, S. E.

Modeling of Multiscale Transient Phenomena, 201

Wylie, B. N.

Visual Explanation and Insight, 99

Wyss, G. D.

Infrastructure Risk and Reliability, 366

$\mathbf{X}$

Xavier, P. G.

Automatic Design of Practical Fixtures, 234

Cloud to CAD, 319

Heterogeneous Simulation, 79

Y

Yang, $\mathrm{P}$.

Advanced Machining for Microfabrication, 223

Yee, A. A.

Monitoring Deep Targets, 321

Yee, M. L.

Multisensor Fusion for Characterizing UGFs, 502 
Yelton, W. G.

Microfabrication of Electromagnetic Devices, 173

Yocky, D. A.

Recognizing Partially Obscured Targets, 295

Yoder, J. R.

Land Surface-Temperature Maps, 504

Yoshimura, A. S.

Distributed Information Systems, 255

Modeling of Medical Surveillance Network, 506

Young, M. F.

Tool for Modeling of Coupled Systems, 112

Young, W. F.

Intrusion Detection for ATM Networks, 276

Self-Configurable Network Protocol, 262

$\mathrm{Yu}, \mathrm{K}$.

Scattering from Nanostructured Materials, 557

\section{$\mathbf{Z}$}

Zaidi, S. H.

Nanostructured Silicon Surfaces, 354

Zavadil, K. R.

Critical Copper-Corrosion Mechanisms, 41

Self-Assembled Templates, 43

Zeuch, D. H.

Design of Improved Powder-Pressing Dies, 436

Zimmerer, D. J.

Evolvable Hardware, 192

Zimmerman, J. A.

Crack Nucleation and Growth, 194

Zutavern, F. J.

Reduction of Legacy Wastes, 527

Semiconductor Current Filament Lasers, 144 